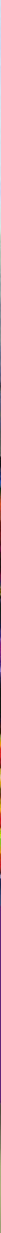


Vice-Presidente da República no Exercício do Cargo de Presidente da República Michel Miguel Elias Temer Lulia

Ministro do Planejamento, Desenvolvimento e Gestão

Dyogo Henrique de Oliveira (interino)

INSTITUTO BRASILEIRO

DE GEOGRAFIA E

ESTATÍSTICA - IBGE

Presidente

Paulo Rabello de Castro

Diretor-Executivo

Fernando J. Abrantes

ÓRGÃOS ESPECÍFICOS SINGULARES

Diretoria de Pesquisas

Roberto Luís Olinto Ramos

Diretoria de Geociências

Wadih João Scandar Neto

Diretoria de Informática

José Sant Anna Bevilaqua (em exercício)

Centro de Documentação e Disseminação de Informações

David Wu Tai

Escola Nacional de Ciências Estatísticas

Maysa Sacramento de Magalhães

UNIDADE RESPONSÁVEL

Centro de Documentação e Disseminação de Informações

Gerência de Relações Institucionais

Isabela Mateus de Araujo Torres 
Ministério do Planejamento, Desenvolvimento e Gestão Instituto Brasileiro de Geografia e Estatística - IBGE

\section{Brasil em números}

Brazil in figures

ISSN 1808-1983

Brasil núm., Rio de Janeiro, v. 24, p. 1-464, 2016 
ISSN 1808-1983

(C) IBGE. 2016

As opiniões emitidas nesta publicação são de exclusiva e inteira responsabilidade do(s) autor(es), não exprimindo, necessariamente, o ponto de vista do IBGE.

\author{
Capa/Cover - Marcelo Thadeu Rodrigues, Gerência de Editoração - CDDI. \\ Museu Pinacoteca do Estado do Rio Grande do Norte/Pinacotheca of the State of \\ Rio Grande do Norte \\ Na Bodega de Zé de Deus/At Zé de Deus'tavern, 2014 \\ Assis Costa, Currais Novos-RN
}

Projeto gráfico editorial / Printing Project - Luiz Carlos Chagas Teixeira, Gerência de Editoração - CDDI.

Impressão / Printing - Centro de Documentação e Disseminação de Informações - CDDI.

Brasil em números = Brazil in figures / IBGE. Centro de Documentação

e Disseminação de Informações. - Vol. 1 (1992- ). - Rio de Janeiro :

IBGE, 1992-

Anual.

Publicações anteriores: "O Brasil em números" = ISSN 0524-2010, v.1 e v.2 $(1960,1966)$ e "Brasil: séries estatísticas retrospectivas" = ISSN 00680842, v.1 e v.2 (1970, 1977).

Título e texto também em inglês: Brazil in figures = ISSN 0103-9970.

ISSN 1808-1983

1. Brasil - Estatística. I. IBGE. Centro de Documentação e Disseminação de Informações.

Gerência de Biblioteca e Acervos Especiais CDU 31(81)(05)

RJ-IBGE/92-15(rev. 2011)

PERIÓDICO

Impresso no Brasil / Printed in Brazil 


\section{Agradecimentos}

\section{Acknowledgments}

O IBGE agradece aos colaboradores abaixo relacionados que, com seus textos analíticos e comentários, enriqueceram o conteúdo desta obra.

Alexandre Marinho

Alexsandro Ferreira Cardoso da Silva

Alvino Moser

Ana Paula Camilo Pereira

Ângela Lúcia Ferreira

Antonio Rioyei Higa

Carlos Henrique Vasconcellos Horn

Clésio Lourenço Xavier

Cleverson Vitório Andreoli

Danielle Carusi Machado

Fabiana De Nadai Andreoli

Fagner Alexandre Nunes de França

Fernanda Paixão

Fernando Álvares Salis

Fernando Sarti

Fernando Tadeu de Miranda Borges

Gabriela Soares

Gerson Rosenberg

laperi Araujo

Igor Stemler

Jean-Paul Prates
IBGE would like to thank the following collaborators for their analyses and comments that enriched this publication.

Joacir Rufino de Aquino

João Agra Neto

Luana Junqueira Dias Myrrha

Luís Alessandro Câmara

Marcia Ribeiro Dias

Marco Antônio Freitas de Hollanda

Cavalcanti

Maria de Fátima Alves de Matos

Maria Inês Cunha Miranda

Milko Matijascic

Milton Pinto

Moacir Guilhermino da Silva

Nelson Chalfun Homsy

Neri dos Santos

Pierre Ohayon

Rafael Kuster Oliveira

Rafael Oliveira Fonseca

Santiago Falluh Varella

Sergio Schneider

Suzy dos Santos

Wilker Ricardo de Mendonça Nóbrega 


\section{Museu Pinacoteca do Estado do Rio Grande do Norte}

por laperi Araujo - Presidente do

Conselho Estadual de Cultura

\section{Um panorama da arte potiguar}

A Pinacoteca do Estado do Rio Grande do Norte está instalada no Palácio Potengi desde 1996 quando o poder executivo do Estado mudou sua sede para o Centro Administrativo em Lagoa Nova, Natal.

O Palácio Potengi foi construído entre 1865 e 1873 para sediar o Tribunal do Júri o Tesouro estadual e a Assembleia provincial, com base em estudo arquitetônico do engenheiro Ernesto Amorim, sendo Governador do Estado Olinto José Meira. O prédio tem linhas neoclássicas e situa-se no centro histórico da capital Natal. Foi sede do Governo estadual somente a partir de 1902. Foi tombado como Patrimônio Histórico Nacional em 1965.

Por mais de noventa anos o prédio serviu a esta função. Desativado de suas funções executivas foi transformado em Palácio da Cultura, abrigando a Pinacoteca, constituída por obras de artistas do Estado que formavam o acervo do patrimônio estadual, notadamente da Fundação José Augusto, responsável pela política cultura do Rio Grande do Norte.

\section{A Pinacoteca foi criada com o objetivo} de recolher, conservar, expor, divulgar e promover a produção das artes visuais mediante exposições, realização de cursos e palestras. Dispõe de um rico
Pinacotheca of the State of

Rio Grande do Norte

\author{
by laperi Araujo - President of the State \\ Council of Culture
}

\section{A panoramic view of the potiguar ${ }^{1}$ art}

The Pinacotheca of the State of Rio Grande do Norte was set in the Palácio Potengi in 1996 when the executive power was transferred to the Administrative Center in Lagoa Nova, Natal.

Palácio Potengi was built between 1865 and 1873 during Olinto José Meira's term as governor, to be the headquarters of the Jury's Court, of the State Treasury and of the Provincial Assembly, following the architectonic study of engineer Ernesto Amorim. The building has neoclassic trends and is located in the historic center of the capital Natal. It was not before 1902 that it became the headquarters of the State Government. It was declared a National Cultural Heritage in 1965.

For more than ninety years the building was used for this executive function. Then it was transformed into a Palace of Culture, hosting the Pinacotheca, with the works of the local artists which formed the state heritage asset, mainly that of the Fundação José Augusto, responsible for the cultural policy of Rio Grande do Norte.

The Pinacotheca was created with the purpose of receiving, conserving, showing, disseminating and promoting the production of visual arts through exhibitions, courses and lectures. It

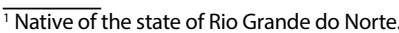


acervo em seus seis acervos expositivos, além do mobiliário do antigo Palácio do Governo, cuja ala governamental foi preservada. Possui várias salas de exposições, ocupadas periodicamente mediante edital público. A configuração arquitetônica do edifício torna tecnicamente possível receber exposições de grande porte e permitir a realização de exposições simultâneas, além da exibição permanente do seu acervo.

\section{A Pinacoteca do Estado também é um} equipamento de Educação recebendo cerca de 1.000 visitantes/mês, a maioria grupos de estudantes da rede pública e privada da Capital e do interior, acompanhados por professores que discutem as obras do acervo e sua inserção na cultura do estado.

No seu acervo pode-se contemplar obras dos principais artistas do Estado, inclusive dos considerados precursores das artes no Rio Grande do Norte como Moura Rabelo, Hostílio Dantas, Newton Navarro e Dorian Gray, esses dois últimos considerados os introdutores da arte moderna no Estado em 1949.

A diversidade criativa é uma das marcas do acervo da Pinacoteca, onde artistas como Abraham Palatinik, Antonio Parreiras, Fayga Ostrower, Cícero Dias, Rubens Gershman, Tarsila do Amaral, Raul Córdula, Maria do Santíssimo, laponi, Thomé Filgueira, Aécio Emerenciano, Leopoldo Nelson, Tulio Fernandes, Manxa, Jordão, Ana Antunes, laperi, Vicente Vitoriano, dentre outros, demonstram a pluralidade estética da criação artística no estado convergindo para diferentes expressões dos vários estilos de criação das artes. houses a rich collection divided into six exhibitions, as well as the ancient furniture of the Palace of Government - whose governmental hall has been preserved. The several exhibition rooms are occupied by means of periodical public bidding. The architectonic configuration of the building makes it technically able to receive large and simultaneous exhibitions, besides the permanent exposure of its collection.

The State Pinacotheca is also an education tool as it receives around 1,000 visitors per month. Most of them, students from public and private schools from the capital or rural areas, monitored by teachers that discuss the artworks and their relevance to the culture of the state.

The collection has the works of the main artists of the State, including those considered the art precursors in Rio Grande do Norte, as Moura Rabelo, Hostílio Dantas, Newton Navarro and Dorian Gray. The last two ones are taken as the introducers of modern art in the State in 1949.

Creative diversity is one of the marks of the Pinacotheca's collection, in which artists such as Abraham Palatinik, Antonio Parreiras, Fayga Ostrower, Cícero Dias, Rubens Gershman, Tarsila do Amaral, Raul Córdula, Maria do Santíssimo, laponi, Thomé Filgueira, Aécio Emerenciano, Leopoldo Nelson, Tulio Fernandes, Manxa, Jordão, Ana Antunes, laperi, Vicente Vitoriano, among others, demonstrate the aesthetic plurality of the artistic creation in the State converging towards different expressions of the several styles of art creation. 
Algumas características do acervo diferenciam a Pinacoteca do Estado. Um grande número de pintores naifs com sua arte embasada na cultura popular, até por ser uma expressão in sita, natural e espontânea sem necessidade de uma formação acadêmica. Nesse particular laponi (1942-1984) e Maria do Santíssimo (1890-1974) pelo renome nacional que conseguiram com exposições no Brasil e no exterior se constituem nos dois dos maiores nomes da arte primitiva do acervo.

Da mesma forma, o entalhe em madeira em grandes painéis vazados é peculiar à arte do Rio Grande do Norte pela expressão dos artistas Manxa (1947- 2014) e Jordão que integrados na expressão naif recortaram em madeira, alguns com espaços em laminas de cobre marchetadas, personagens, ofícios e toda flora tropical do Rio Grande do Norte.

Constam ainda do acervo coleções de xilogravuras, litogravuras esculturas e instalações e objetos de artistas populares como Luzia Dantas e Zé da China.

O acervo das artes visuais da Pinacoteca é mantido pela Fundação José Augusto foi formado gradativamente, ao longo dos anos na sua grande maioria por doação dos artistas. Este acervo circula pelas mais de 40 Casas de Cultura no interior do estado, recebendo um grande público de alunos das escolas públicas em visita comentada por técnicos e acompanhada por professores de educação artística.

As manifestações artísticas do Rio Grande do Norte demonstram a intensa criatividade do seu povo. Artistas que
Some characteristics make the collection of the Pinacotheca rather distinctive. A great number of naif painters with their art based on the popular culture, which is in itself a natural, in situ and spontaneous expression, with no need for an academic degree. In this respect, laponi (1942-1984) and Maria do Santíssimo (1890-1974), with the national recognition achieved with their exhibitions in Brazil and abroad, are the two greatest exponents of the primitive art of the collection.

Similarly, the wood carving in large pierced panels is peculiar to the art of Rio Grande do Norte thanks to artists such as Manxa (1947-2014) and Jordão that, as practitioners the naif expression, carved in wood, sometimes with spaces in copper inlays, characters, crafts and all tropical flora of Rio Grande do Norte.

The collection also includes wood engravings, lithographs, sculptures, as well as the facilities and objects of popular artists like Luzia Dantas and Zé da China.

The visual arts collection of the Pinacotheca is supported by the Fundação José Augusto and was gradually brought together over the years, mostly as donations from the artists. This collection also goes around the 40 Cultural Houses in the countryside of the state, being seen by many public schools' students, with the guidance of technicians and art education teachers.

The artistic expressions of Rio Grande do Norte show the great creativity of its people. Most of the artists came out 
saíram em sua maioria do anonimato pela intensa atividade que exerciam cumprindo a destinação de sua própria criatividade. Muitos produzem com materiais rústicos como a primitiva Maria do Santíssimo que usava anilina aplicada com palitos de coqueiro em folhas de papel pautado e cartolina. Outros usam esmalte sintético que sobram das pinturas de portas, mas em todos, a cor tropical e intensa, firmada na luminosidade nordestina, explode nas expressões dos sentimentos do povo. Religiosidade, festas e labores como se fizesse, uma crônica permanente da vida do povo potiguar. Uma arte comprometida com os sentimentos e sofrimentos do povo, mas radiosamente bela e vitoriosa. of anonymity due to the intense activity exerted as the fulfillment of own their creativity's fate. Many of them produce their works with rustic material as the primitive Maria do Santíssimo, who used aniline applied with coconut tree sticks on sheets of lined paper and cardboard. Some others use synthetic enamel leftovers from door paintings, but, in all of them, the tropical intense colors, fixed in the northeastern luminosity, blow out in the expressions of people's feelings. Religiosity, parties and labors are presented as a permanent chronicle of the local people's lives. An art committed to the feelings and sufferings of the people, but brightly beautiful and victorious.

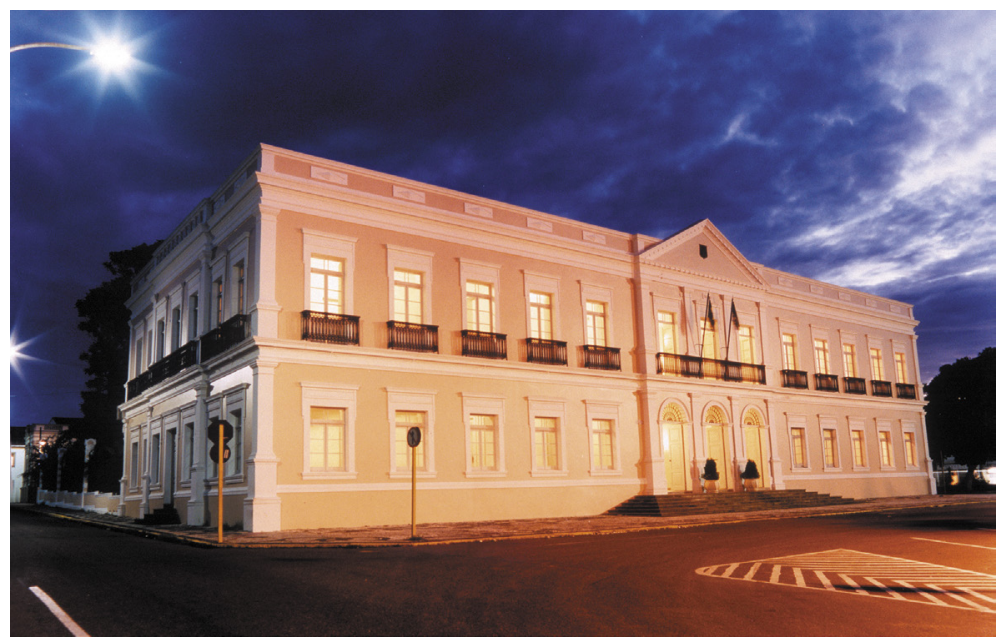

Palácio Potengi - Pinacoteca do Estado do Rio Grande do Norte Palácio Potengi - Pinacotheca of the State of Rio Grande do Norte Fotografia / Photograph: Humberto Lopes 
Obras e imagens cedidas pela Pinacoteca do Estado do Rio Grande do Norte reproduzidas neste volume Works and images reproduced in this publication under permission of the Pinacoteca do Estado do Rio Grande do Norte

Capa / Cover

Na Bodega de Zé de Deus/At Zé de Deus'

tavern, 2014

Assis Costa, Currais Novos-RN

Acrílica sobre tela/ Acrylic on canvas

$80 \times 100 \mathrm{~cm}$

Museu Pinacoteca do Estado do Rio Grande

do Norte/ Pinacotheca of the State of Rio

Grande do Norte

Palácio Potengi / Palácio Potengi

Fotografia / Photograph: Humberto Lopes

\section{Uma Breve História do Brasil / A Brief}

History of Brazil

Degradê III/Color Gradient III, 1989

Deusdeth Avelino de Araújo, Patu-RN, 1953

Desenho em cor/ Drawing in color

$39 \times 43 \mathrm{~cm}$

\section{Território / Territory}

Painel com motivo regional/Panel with regional motifs, 1979

Ziltamir Sebastião Soares de Maria 'Manxa', São

Vicente-RN, 1946

Madeira entalhada/Carved wood

250x350m

População / Population

Farra da Bola/Ball-playing revelry, 2014

Wagner Di Oliveira, Assu-RN, 1976

Acrílica sobre tela/Acrylic on canvas

$100 \times 100 \mathrm{~cm}$

Habitação/ Housing

Casario/Row houses, 1972

Dorian Gray Caldas, Natal-RN, 1930

Acrílica sobre tecido/ Acrylic on fabric

$150 \times 160 \mathrm{~cm}$
Saúde / Health

Ceia Larga/Broad supper, 1995

Assis Marinho, Canoas de Cubati-PB, 1960

Crayon/Crayon

$70 \times 128 \mathrm{~cm}$

\section{Previdência Social / Social Security}

Motor I/Engine I, 1988

Francisco Iran Dantas, Currais Novos-RN, 1960

Tinta cerâmica sobre fórmica/Ceramic paint

on formica

$27 \times 21 \mathrm{~cm}$

\section{Educação / Education}

Brincadeira de roda/Ring a ring o' roses, 1981

Júlio César Revoredo Serafim, Natal-RN,1959

Óleo sobre Eucatex/Oil on Eucatex

$39 \times 55 \mathrm{~cm}$

Trabalho / Labor

Salina/Saltern, 1985

Newton Navarro Bilro, Natal -RN, 1925-1992

Guache sobre tela/Gouache on canvas

$96 \times 66 \mathrm{~cm}$

Participação Política / Political Participation Natureza I/Nature I, 1978

Fernando Henrique de Oliveira Gurgel, NatalRN, 1958

Óleo sobre tela/Oil on canvas

$37 \times 28 \mathrm{~cm}$

\section{Preços / Prices}

Progressão de ripas de madeira, sem título/

Wooden slat, untitled, 2004

Abraham Palatnik, Natal-RN, 1928

Ripas pintadas com tinta acrílica/Slats painted

in acrylic

$82,5 \times 98 \mathrm{~cm}$ 
Contas Nacionais / National Accounts

Banda/Marching band, 2014

Geicifran Francisco de Assis Azevedo, Jardim

do Seridó-RN

Madeira/Wood

\section{Agropecuária / Agriculture}

Sem título, s.d./Untitled, n.d.

Iaponi Araújo, São Vicente-RN, 1942 - Rio de

Janeiro, 1996

Óleo sobre tela/Oil on canvas

$115 \times 147 \mathrm{~cm}$

Indústria / Industry

Casario rio/Row houses river flow, 1982

Dorian Gray Caldas, Natal-RN, 1930

Acrílica sobre tecido colado em Eucatex/Acrylic on fabric glued with Eucatex

$79 \times 100 \mathrm{~cm}$

\section{Energia / Energy}

Salinas/Cajus, s.d./Salterns/cashews, n.d.

Iraken Marques de Lima, Natal-RN, 1937

Lã tapeçaria/Wool tapestry

$80 \times 143 \mathrm{~cm}$

\section{Comércio / Trade}

Lavrador, s.d./Farmer, n.d.

Ziltamir Sebastião Soares de Maria 'Manxa', São

Vicente-RN, 1946

Madeira entalhada/Carved wood

$105 \times 40 \mathrm{~cm}$

\section{Transportes / Transportation}

A Ponte de Igapó/The Igapó's bridge, 1980

Thomé Soares Filgueira, Natal-RN,1938

Óleo sobre tela/Oil on canvas

$75 \times 130 \mathrm{~cm}$

\section{Turismo / Tourism}

Igreja Casario, s.d./Church row houses, n.d.

Tânia Maria de Oliveira Monte Melo, Natal-RN,1950

Prata boliviana em relevo/Bolivian embossed silver $59 \times 80 \mathrm{~cm}$

\section{Comunicações / Communication}

Sefus Coisa/Sefus Gõis, 1974

Leopoldo Nelson de Sousa Leite, Natal-RN, 1940-1994

Óleo sobre tela/Oil on canvas

$81 \times 65 \mathrm{~cm}$

\section{Finanças / Finances}

O Forte/The Fort, 1979

Newton Navarro Bilro, Natal-RN, 1025-1992

Nanquim/Indian ink

$22 \times 32 \mathrm{~cm}$

\section{Comércio Exterior / Foreign Trade \\ Sem Título/Untitled, 1994 \\ J. Medeiros, João Pessoa-PB, 1958 \\ Óleo sobre tela/Oil on canvas \\ $50 \times 80 \mathrm{~cm}$}

Ciência e Tecnologia / Science and Technology

Câmara Cascudo/Câmara Cascudo, 1971

Newton Navarro Bilro, Natal-RN, 1925-1992

Nanquim sobre papel/Indian ink on paper

\section{Poder Judiciário / Judicial Power}

Julgamento de Padre Miguelinho/Father

Miguelinho's trial, 1918

Antônio Diogo da Silva Parreiras, Niterói-RJ,

1860-1937

Óleo sobre tela/Oil on canvas

$159 \times 250 \mathrm{~cm}$

\section{Meio Ambiente / Environment}

Cajueiro nas Dunas/Cashew tree on the dunes, 1987

Vatenor de Oliveira Silva, Natal-RN, 1953

Óleo sobre tela/Oil on canvas

$93 \times 139 \mathrm{~cm}$ 


\section{Mini Bios dos artistas/ Mini Bios of artists}

\section{Abraham Palatnik}

Abraham Palatnik, (Natal/RN, 1928). Artista cinético. Pintor. Desenhista. Pioneiro em arte cinética no Brasil, suas obras contêm instalações elétricas que criam movimentos e jogos de luzes. Integrou o primeiro núcleo de artistas abstratos do Rio de Janeiro. No ano seguinte, iniciou suas pesquisas no campo da luz e do movimento, responsáveis por seu reconhecimento como um dos pioneiros da Arte cinética, após a menção especial do júri internacional, na I Bienal Internacional de São Paulo, em 1951. Suas obras integram coleções particulares e importantes museus europeus e norte americanos. Integrou o Grupo Frente, aproximando-se da poética visual dos concretos e neoconcretos. Desde então, vem desenvolvendo um trabalho que une pesquisa visual e rigor matemático.

\section{Antônio Diogo da Silva Parreiras}

Antônio Diogo da Silva Parreiras, (Niterói/RJ - 18601937). Pintor. Desenhista. Ilustrador. A partir de 1899 parreiras deixa de dedicar-se às paisagens e dedica-se a pinturas de cenas históricas para o poder público. Entre elas se destacam Proclamação da República, Morte de Estácio de Sá e Prisão de Tiradentes, trabalhos que aumentam sua notoriedade no Brasil. A partir de 1906, Parreiras vive entre Paris e Niterói, mantem seu ateliê na França onde trabalha e expõe com regularidade. O artista falece em 1937, em Niterói.

\section{Assis Costa}

Assis Costa, (Currais Novos/RN). Pintor. Escultor. O artista plástico Assis Costa nasceu em Currais Novos / RN, onde possui o seu atelier. Inicia o seu desenvolvimento artístico na década de 1990, com o curso de desenho e pintura ministrado pelo artista José Antônio. Contudo, desde 2002, passa temporadas no Rio Grande do Sul (Gramado, Nova Petrópolis e Pelotas) no cargo de diretor artístico de eventos culturais. A sua obra como artista plástico é multifacetada: o mesmo desenvolve além da pintura em tela (técnicas óleo e/ou acrílica), também a escultura, orbitando no universo das artes pelas suas diversas expressões como a poesia, a música e os quadrinhos. Assis Costa, em sua estética moderna,
Abraham Palatnik, (Natal/RN, 1928). Kinetic artist. Painter. Draftsman. A pioneer kinetic artist in Brazil, whose works contain electric circuits that create movement and light shows. He was part of the first group of abstract artists of Rio de Janeiro. He later took up research in light and movement, which gained him recognition as a kinetic artist pioneer, after a special mention by the international jury in the 1st International Biennial of São Paulo, in 1951. His works feature in private collections and in important European and North American museums. He was a member of Grupo Frente, coming closer to the visual poetics of concrete and neoconcrete artists. Since then his works have combined visual research and mathematical rigor.

Antônio Diogo da Silva Parreiras, (Niterói/RJ - 1860 1937). Painter. Draftsman. Illustrator. In 1899 Parreiras leaves landscapes behind and starts painting historical scenes for the public power. Worth of special mention are Proclamation of the Republic, Death of Estácio de Sá and The Enprisonment of Tiradentes, works that increased his acknowledgment in Brazil. From 1906 on he would live between Paris and Niterói, keeping his atelier in France, $a$ country where he used to work and have exhibitions on a regular basis. The artist died in 1937, in Niterói.

Assis Costa, (Currais Novos/RN). Painter. Sculptor. Plastic artist Assis Costa was born in Currais Novos / RN, where his atelier is located. He starts his artistic development in the 1990s, with a painting and drawing course tutored by artist José Antônio. Nevertheless, since 2002, he goes to Rio Grande do Sul (Gramado, Nova Petrópolis and Pelotas) from time to time as an artistic director for cultural events. His work as a plastic artist is multifaceted: besides painting on canvas (with either oil and/or acrylic techniques) he is also a sculptor and a man surrounded by art expressions such as poetry, music and comics. Assis Costa, in his modern aesthetics, deals with themes of everyday life, popular culture, iconic figures of Saint 
versa sobre temáticas que aludem ao cotidiano, a cultura popular, as figuras emblemáticas de São Francisco de Assis, Dom Quixote e Sancho, buscando sempre um traço próprio que diretamente o liga à paisagem do Seridó, região onde vive. (https://www. blogger.com/profile/10442326624674329477 Texto retirado do blog do autor em 19/07/2016).

\section{Assis Marinho}

Assis Marinho, (Canoas de Cubati/PB,1960). Pintor. Usa o lápis cera ou aquarela, para dramatizar e enaltecer as suas figuras, com a mesma determinação de recriar as origens vividas entre o rio e o mar, nesta faixa de mangue das margens da cidade ou da praia da Redinha. Sua pintura fixa de preferência os pescadores, feirantes, catraieiros, mangalheiros, barqueiros, numa biotipologia fantástica e de surpreendentes efeitos realistas. Olhos da miséria que nos olham de frente, mãos e pés deformados pelo trabalho árduo é o que mais surpreende.

\section{Deusdeth Avelino de Araújo}

Deusdeth Avelino de Araújo, (Patu/RN,1953).

Médico. Pintor. Sobressai-se no artista Avelino sua consciência participativa na construção dos valores da Arte Processo, da poesia visual, da comunicação eletiva e quase sempre objetiva dos artistas do grupo. Utilizando ideogramas, colagens, apelos, recados, poemas, o artista Avelino recria os valores morais de uma sociedade em crise. Na sua pintura de cavalete, Avelino se permite à divagação onírica e ao niilismo habilidoso, acentuado pelas formas puras. Nessa fase, sua arte desata suas fantasias e o coloca mais próximo dos expressionistas abstratos.

\section{Dorian Gray Caldas}

Dorian Gray Caldas, (Natal/RN,1930). Pintor. Tapeceiro. Desenhista. Escultor. Poeta. Crítico de arte. Um misto de talento e disciplina, cultura e técnica, inteligência e sensibilidade. Em 1950, fez juntamente com Newton Navarro e Ivon Rodrigues o "Salão de Arte Moderna" o evento ficou como o marco do movimento modernista no campo das Artes Plásticas do Estado. A partir daí expôs em todo o Brasil e em diversos países do mundo, o que lhe rendeu importantes prêmios como o Grand
Francis of Assisi, Don Quixote and Sancho, forever in search of a particular trace that can connect him to the landscapes of Seridó, his home. (https://www.blogger. com/profile/10442326624674329477 - Text taken from the author's blog on 19/07/2016).

Assis Marinho, (Canoas de Cubati/PB,1960). Painter. He uses crayons or watercolor to exalt his figures and make them more dramatic, with the same determination he has for recreating his origins, between the sea and the river, in a mangrove city outskirt or on Redinha beach. His painting is more often focused on fishermen, fair vendors, skiff rowers, mangalheiros (sellers of craftsmanship and family farm goods), boatmen, in a fantastic biotypology of surprising realistic effects. Misery eyes staring at us, hands and feet deformed from hard work are very striking in his production.

Deusdeth Avelino de Araújo, (Patu/RN,1953). Physician. Painter. What stands out in Avelino as an artist is his participative conscience in the construction of the values of Process Art, of visual poetry, of elective and almost always objective communication of the artists of the group. By using ideograms, collages, appeals, messages, poems, artistic Avelino recreates moral values of a society in crisis. In his easel painting, Avelino allows himself an oneiric

Dorian Gray Caldas, (Natal/RN,1930). Painter. Rug weaver. Draftsman. Sculptor. Poet. Art critic. A mix of talent and discipline, intelligence and sensitivity. In 1950, together with Newton Navarro and Ivon Rodrigues, he created the "Modern Art Salon", the event later regarded as the landmark of modernist plastic arts movement in the state. From then on he presented exhibitions all over Brazil and in several countries around the world, and won important prizes such as the Belgium Grand Prix, Portinari 
Prix da Bélgica, Prêmio Portinari, SESC Centenário de Pintura, Prêmio de Aquisição no BIRD e na Alemanha, dentre outros. Sua obra artística encontra-se em acervos e instituições culturais do país e do exterior.

\section{Fernando Henrique de Oliveira Gurgel}

Fernando Henrique de Oliveira Gurgel, (Natal/RN, 1958). Pintor. Iniciou seus estudos no Parque Lage, no Rio de Janeiro, em 1981. Participou de mostras em diversas cidades brasileiras e na Alemanha, Espanha, EUA e Portugal. Coleciona prêmios e participações em salões de arte na sua cidade e em outras capitais do Brasil.

\section{Francisco Iran Dantas}

Francisco Iran Dantas, (Currais Novos/RN, 1960). Pintor. Ligado a propostas expressionistas, vivíssimas da arte contemporânea. "Sua arte é fruto de sua personalidade forte e vocação nata" diz Dorian Gray. Artista seridoense. Iran Dantas canta e conta a alma do seu povo, transbordando de ingenuidade, quermesses, feiras, procissões, retalhos do cotidiano que ele retrata em seus trabalhos.

\section{Geicifran Francisco de Assis Azevedo}

Geicifran Francisco de Assis Azevedo, (Jardim do Seridó/RN, 1988). Despertou para arte ainda criança, quando observava o trabalho do seu pai, também artesão, esculpindo em madeira umburana. $\mathrm{Na}$ adolescência tornou-se um grande admirador da arte de dois outros artesãos jardinenses, Júlio Cassiano e Neném de Chicó, e desse último recebeu orientações técnicas. A partir de 2006 passou a produzir esculturas encomendadas, especialmente peças sacras populares e ex-votos. Paralelamente também produzia peças sobre costumes folclóricos regionais e do cotidiano seridoense, todas tendo como matéria-prima a madeira umburana.

\section{Iaponi Araújo}

laponi Araújo, (São Vicente-RN, 1942-1996). Pintor. Radicado no Rio de Janeiro desde 1967 não esqueceu as vertentes populares de sua terra natal, recriando os temas do imaginário popular, as estórias do povo. Sua obra, de grande aceitação pela crítica e pelo público, é
Prize, SESC Painting Centennial, Acquisition Prize at IBRD and in Germany, and many more. His artistic production can be found in collections and cultural institutions here and abroad.

Fernando Henrique de Oliveira Gurgel, (Natal/RN, 1958). Painter. He started his studies at Parque Lage, in Rio de Janeiro, in 1981 and participated in exhibitions in Brazilian cities and in Germany, Spain, the United States and Portugal. He collects prizes and participations in art salons in his hometown and in other capitals of Brazil.

Francisco Iran Dantas, (Currais Novos/RN, 1960).

Painter. Connected to expressionist, vivid initiatives of contemporary art. "His art comes from his strong personality and an innate call" says Dorian Gray. An artist from the Seridó area, Iran Dantas reveals and exalts the soul of his people, flooding with naiveté, popular festivities, fairs, religious processions, pieces of everyday life that he portrays in his works.

Geicifran Francisco de Assis Azevedo, (Jardim do Seridó) $R N$, 1988). Awakened for the arts while still a child, the artist used to watch his father, who was also an artisan, making sculptures out of umburana wood. In his teenage years he fell in love with the art of other two artisans from his city, Júlio Cassiano and Neném de Chicó, having even received some technical guidance from the latter. In 2006 he started to make sculptures on demand, mainly to produce sacred pieces and ex-votos. At the same time he would produce works on regional folklore and customs and on life at Jardim do Seridó. Umburana wood was always used as the raw material for these works.

laponi Araújo (São Vicente-RN, 1942-1996). Painter. Based in Rio de Janeiro since 1967, he did not leave behind the popular features of his hometown, recreating themes of popular imagination, stories of the people. His works, widely welcomed by critics and the audience, 
testemunho da sua inventiva e da qualidade artística e documental da qual é portadora. Participou de várias coletivas e individuais. Residiu em Londres, Inglaterra, entre 1970 e 1972.

\section{Iraken Marques de Lima}

Iraken Marques de Lima, (Natal/RN, 1937). Pintor. Tapeceiro. Desenhista. Iraken pertence à geração dos anos 60. Participou do grupo do L'atelier, primeira galeria de arte particular de Natal, em 1971 começou a dedicar-se à tapeçaria, expondo suas belas folhagens tropicais. Ressalte-se a preferência pela flora e a fauna, o tropicalismo exuberante de suas composições aliadas ao seu ritmo de composição e equilíbrio.

\section{J. Medeiros}

J. Medeiros, (João Pessoa/PB, 1958). Pintor. Poeta. Formado em Educação Artística pela Universidade Federal do Rio Grande do Norte (UFRN), é um dos mais importantes poetas visuais brasileiros. É autor do livro/ poema "Progressão", editado pela Stempelplates, de Amsterdã, na Holanda, em 1978, e "Geração Alternativa", pela editora Amarela/PROFINC, Natal-RN, 1997. Organizou várias exposições internacionais de poesia visual na cidade de Natal, e participou como artista convidado do MAM da Bahia e do Brazilian Northeast Festival of Contemporary Art, realizado em Nova lorque em 1996.

\section{Júlio César Revoredo Serafim}

Júlio César Revoredo Serafim, (Natal/RN,1959).

Pintor. Escultor. Cenógrafo. Desenhista. Sobre César Revorêdo Luís da Câmara Cascudo diz: "Uma pintura movimentada, de cores vivas, sobre a natureza humana e social. Uma fixação legítima de sensibilidade e pessoal. Documentário mais de sua mentalidade do que de sua visão. Vê-lo é viajar por uma inteligência espontânea e nobre que nos deu a alegria de ser nossa".

\section{Leopoldo Nelson de Souza Leite}

Leopoldo Nelson de Souza Leite, (Natal/RN,19401994). Desenhista. Pintor. Médico. Dedica-se à pintura a partir de 1963. Em 1967, voltou a trabalhar com nanquim, realizou exposições individuais na Galeria "Xaria" (1965) e no Museu de História (1967), Natal. represents his creativity and artistic and documental quality. He took part in many collective and individual exhibitions and lived in London, England, between 1970 and 1972.

Iraken Marques de Lima, (Natal/RN, 1937). Painter. Rug weaver. Draftsman. Iraken is part of the 1960s' generation. He participated in the L'atelier group, the first private art gallery in Natal, and started in tapestry in 1971 by displaying his beautiful tropical foliage. A highlight in Lima's work is his preference for flora and fauna, the magnificent tropicalism of his creations combined with a unique balance in his composition.

J. Medeiros, (João Pessoa/PB, 1958). Painter. Poet. With a degree in Art Education from the Federal University of Rio Grande do Norte (UFRN), he is one of the most important visual poets in Brazil. He is author of the book/poem "Progressão" (Progression), edited by Stempelplates, from Amsterdan, in the Netherlands, in 1978, and "Geração Alternativa" (Alternative Generation), by the Amarelal PROFINC publishing house, Natal-RN, 1997. He organized many international exhibitions of visual poetry in Natal, and was an invited artist at MAM in Bahia and at the Brazilian Northeast Festival of Contemporary Art, held in New York in 1996.

Júlio César Revoredo Serafim, (Natal/RN, 1959). Painter. Sculptor. Cenographer. Draftsman. About César Revorêdo, Luís da Câmara Cascudo says: "Painting in movement, of bright colors, about human and social nature. A legitimate personal fixation of sensibility. A documentary based on his mentality rather than on his vision. Watching it is traveling through a spontaneous and noble intelligence that we are fortunate to call ours."

Leopoldo Nelson de Souza Leite, (Natal/RN,1940-1994). Draftsman. Painter. Physician. He dedicated his life to art since 1963. In 1967 he went back to the Indian ink tecnique and performed individual exhibitions in the "Xaria" Gallery (1965) and at the History Museum (1967), 
Participou, entre outras mostras coletivas, da $1^{\text {a }}$ Feira de Artes Plásticas do Rio Grande do Norte (Natal, 1966) e do Arte Jovem Norte-rio-grandense (Recife, 1968). A seu respeito disse laperí em 1968: “Apaixonado pelas coisas do povo, seu principal tema, sem focalizar o subdesenvolvimento, utiliza-o num protesto sob forma de esquálidas figuras do submundo do lixo e das favelas".

\section{Newton Navarro Bilro}

Newton Navarro Bilro, (Natal/RN, 1928-1992). Pintor. Poeta. Jornalista. Desenhista. Contista. Escritor.

Frequentou o curso livre da Escola de Belas-Artes do Recife e o "atelier" de Hélio Feijó (1948). Também foi discípulo de Aldemir Tavares e, estudou gravura com Oswaldo Goeldi. Em Natal, juntamente com Dorian Gray Caldas, liderou, na década de 1950, movimento em prol da atualização do Rio Grande do Norte no setor das Artes Plásticas. Participou de coletivas e individuais no país e exterior. Seu traço ágil, inventivo, e de criação fulgurante, com um estilo preciso, $\mathrm{e}$ domínio técnico, sua figura se estiliza. O vaqueiro, o marujo, a rendeira, o cangaceiro se transformam em figuras newtonianas com personalidade indiscutível.

\section{Tânia Maria de Oliveira Monte Melo}

Tânia Maria de Oliveira Monte Melo, (Natal/RN,1950). Pintora. Ceramista. Tânia definiu-se pela porcelana. Usando o cima-vidrado para os seus conjuntos, vasos, placas, pratos. E o faz com perfeição técnica. Disciplina sua vida em função da Arte milenar da porcelana. $\mathrm{O}$ resultado só pode ser esse que hoje se vê. Um trabalho cuja qualidade é indiscutível.

\section{Thomé Soares Filgueira}

Thomé Soares Filgueira, (Natal/RN,1938-2008). Pintor. Professor. Em 1957, ganha seu primeiro prêmio de pintura em Natal. Thomé tem permanecido fiel às suas origens: o campo e o rio. Expôs na Califórnia em 1964. Com a experiência vivida nos EUA, guardou a influência dos artistas americanos do começo do século, sem apegar-se a nenhum vestígio do modernismo da megalópole. Continuou a fazer sua pintura com o tácito compromisso com a verdade
Natal. Leopoldo Leite took part, among other collective exhibitions, in the 1st plastic Art Fair of Rio Grande do Norte (Natal, 1966) and of the Young People's Art of Rio Grande do Norte (Recife, 1968). About him, said laperi, in 1968: "In love with the people's issues, his main theme, without focusing on underdevelopment, uses it in a protest shaped as squalid figures from the underworld of trash and slums".

Newton Navarro Bilro, (Natal/RN, 1928-1992). Painter. Poet. Journalist. Draftsman. Writer. He attended the course of the School of Fine Arts of Recife and the atelier of Hélio Feijó (1948). A disciple of Aldemir Tavares, Newton Navarro studied engraving with Oswaldo Goeldi. In Natal, together with Dorian Gray Caldas, in the 1960 's, he led a movement for the update of Rio Grande do Norte's Plastic Arts. He participated in collective and individual exhibitions in the country and abroad. His agile, inventive trace, of bright creation, with precise style and technical domain, his stylish figures. The cowboy, the sailor, the lacemaker, the cangaceiros (nomadic bandits in the 1920s and 1930s' northeastern Brazil) are turned into Newtonian figures of undebatable personality.

Tânia Maria de Oliveira Monte Melo, (Natal/RN, 1950). Painter. Ceramist. Tânia has chosen porcelain. She uses glaze on her kits, vases, plates and dishes, and does that with technical perfection. Her life goes round the ancient porcelain art and we see the inevitably good results of it. A work of undeniable excellence.

Thomé Soares Filgueira, (Natal/RN,1938-2008). Painter. Professor. In 1957, he won his first prize in painting in Natal. Faithful to his origins: the field and the river. He made an exhibition in California in 1964. With the experience acquired in the USA, he was able to benefit from the influence of American artists of the beginning of the century, while setting apart from any trace of the modernism of the megalopolis. His paintings kept a tacit commitment to the truth and origins without being 
e origens sem ser cópia servil ou representação

superficial, pintura densa da terra e dos homens.

\section{Vatenor de Oliveira Silva}

Vatenor de Oliveira Silva, (Natal/RN, 1953). Pintor. Paisagista, sobretudo, Vatenor usa a cor pura nos espaços da tela, sugerindo mais do que mostrando. Interpreta sensivelmente o caju da sua terra e de suas raízes.

\section{Wagner Di Oliveira}

Wagner Di Oliveira, (Assu/RN, 1976). Pintor. Desenhista. Escultor. Autodidata. Prefere utilizar pincéis chatos e médios e tinta pura, sem adição de tocantes ou óleo de linhaça como diluentes. Para o desenho, utiliza a caneta esferográfica preta e costuma trabalhar com o pastel oleoso e o lápis grafite. Sua obra é diversificada, pois já se dedicou à escultura criando figuras em papel machê. (Texto adaptado de http://tudodeassu. blogspot.com.br/2014/11/wagner-di-oliveira-pintor. html, acessado em 27 jul. 2016).

\section{Ziltamir Sebastião Soares de Maria (Manxa)}

Ziltamir Sebastião Soares de Maria (Manxa), (São Vicente/RN,1946-2012). Gravador. Entalhador. Escultor. Teve por muitos anos uma atuação marcante na vida artística do Estado, numa profícua atividade artísticoartesanal. Tendo editado álbuns de seu assunto predileto, a literatura de cordel, incursionando pelo trabalho mural e obras de encomenda, Manxa evoluiu sua Arte de talhar, destacando-se logo pela força das suas composições, seus mitos, suas lendas populares, seus faunos, esculpidos, esculturais, numa afirmação inequívoca do seu talento nato. Sua maior obra no Estado pode ser vista e admirada no Banco do Brasil S/A, Natal-RN. a mere copy or a superficial representation; a dense painting of men and land.

Vatenor de Oliveira Silva, (Natal/RN, 1953). Painter. A landscaper, mainly, Vatenor uses pure color on the screen, suggesting more than actually revealing. With great sensitivity, he interprets the cashew of his land and his roots.

Wagner Di Oliveira, (Assu/RN, 1976). Painter. Draftsman. Sculptor. Self-taught artist. He prefers to use flat and medium brushes and plain unmixed paint, without linseed oil to dilute it. For the drawing, he uses his black pen and often works with oil pastel and graphite pencil. His work is very diverse for he has already dedicated to sculpture creating figures in paper mache. (Adapted from http://tudodeassu.blogspot.com.br/2014/11/wagner-dioliveira-pintor.html, cited July 27, 2016).

Ziltamir Sebastião Soares de Maria (Manxa), (São Vicente/RN,1946-2012). Engraver. Woodcarver. Sculptor. For many years he had important participation in the artistic life of the State, with a productive artisanal/ artisitc activity. Having edited albums of his favorite topic, cordel literature, and experiencing mural art and works on demand, Manxa saw the evolution of his woodcarving art, with highlight to the strength of his compositions, his myths, popular legends, his fauns, either carved or sculptured, in a confirmed reinforcement of his innate talent. His biggest work in the state can be seen at Banco do Brasil S/A, Natal-RN. 



\section{Sumário Contents}

Apresentação/Presentation .......................................................................................................

Uma Breve História do Brasil/A Brief History of Brazil .............................................................35

Território/Territory ...................................................................................................................4

População/Population ............................................................................................................61

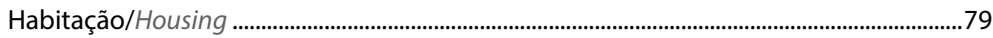

Saúde/Health ............................................................................................................................91

Previdência Social/Social Security …………………………………………………………... 105

Educação/Education ……………………………………………………………………….... 123

Trabalho/Labor ........................................................................................................... 149

Participação Política/Political Participation ......................................................................... 171

Preços/Prices........................................................................................................................... 185

Contas Nacionais/National Accounts .................................................................................... 201

Agropecuária/Agriculture .................................................................................................... 213

Indústria/Industry .............................................................................................................. 233

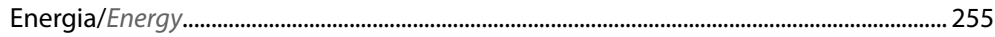

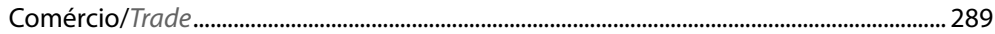

Transportes/Transportation ………….............................................................................. 305

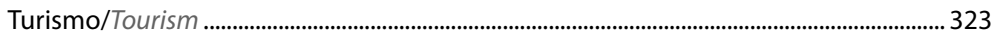

Comunicações/Communications ............................................................................................ 339

Finanças/Finances................................................................................................................. 361

Comércio Exterior/Foreign Trade ............................................................................................. 387

Ciência e Tecnologia/Science and Technology ..................................................................... 399

Meio Ambiente/Environment .................................................................................................. 433 


\section{Tabelas}

\section{Território}

1.1 - Área total do País - 2015 .54

1.2 - Evolução político-administrativa do País - 1940/2014 56

1.3 - Pontos mais altos do País - 2016 ..58

1.4 - Localização geográfica dos Municípios das Capitais e distância a Brasília - 2015 .59

1.5 - Pontos extremos do País e suas distâncias - 2015 .60

População

2.1 - População residente, por situação do domicílio e sexo - 2010 . .72

2.2 - Indicadores demográficos - 2010.........74

2.3 - Projeções de população e taxas - 2000-2017. .76

\section{Habitação}

3.1 - Domicílios particulares permanentes, pessoas residentes em domicílios particulares permanentes e média de pessoas, por domicílio particular permanente e dormitório em domicílio particular permanente 2013 .90

\section{Saúde}

4.1 - Óbitos de residentes, por sexo, segundo as 10 principais causas - 2014 100

4.2 - Cobertura vacinal, por Unidades da Federação - 2015 101

4.3 - Internações, mortalidade hospitalar e média de permanência no Sistema Único de Saúde - SUS - 2015. .102

\section{Previdência Social}

\section{1 - Recebimentos da Previdência} Social - 2003-2014

\section{Tables}

\section{Territory}

1.1 - Total area of Brazil - 2015 54

1.2 - Administrative evolution of Brazil 1940/2014. 56

1.3 - Highest points in Brazil - 2016.........58

1.4 - Geographic location of the Municipalities of the Capital and distance to Brasília - 2015 59

1.5 - Extreme points of Brazil and their distances - 2015 .60

\section{Population}

2.1 - Resident population, by urban/rural housing unit and sex - 2010. .72

2.2 - Demographic indicators - 2010 .....74

2.3 - Population projections and rates - 2000-2017 .76

\section{Housing}

3.1 - Permanent private housing units, persons living in permanent private housing units, and average number of persons, per permanent private housing unit and per bedroom in permanent private housing unit 2013

\section{Health}

4.1 - Deaths of residents, by sex and 10 leading causes of death - 2014 100

4.2 - Immunization coverage by Federation Unit - 2015 101

4.3 - Hospitalization, deaths in hospitals and average length of stay in the National Health System - SUS - 2015.

\section{Social Security}

\section{1 - Brazilian social security} revenues - 2003-2014 
5.2 - Pagamentos da Previdência Social - 2003-2014

5.3 - Distribuição dos benefícios ativos, urbano e rural - 2011-2014

5.4 - Benefícios concedidos pela previdência social - 2011-2014 ... 119

5.5 - Quantidade de pessoas físicas contribuintes do Regime Geral de Previdência Social - RGPS, por categoria - 2004-2014 120

5.6 - Percentual da população, segundo a cobertura de previdência pública e BPC por grupos de idade - Brasil 2004/2014.

5.7 - Percentual da renda domiciliar mensal paga pela previdência pública e BPC sobre a renda domiciliar total segundo decis de renda Brasil - 2004/2014

\section{Educação}

6.1 - Taxa de analfabetismo das pessoas de 10 anos ou mais de idade, por sexo, segundo os grupos de idade 2009/2014

6.2 - Média de anos de estudo das pessoas de 10 anos ou mais de idade, por sexo, segundo os grupos de idade 2009/2014

6.3 - Distribuição das pessoas de 25 anos ou mais de idade, por Grandes Regiões, por Grandes Regiões, segundo o sexo e o nível de instrução - 2014 143

6.4 - Distribuição das pessoas que frequentavam escola ou creche, por Grandes Regiões, segundo o nível e a rede de ensino que frequentavam 2014. 145

6.5 - Taxa de frequência a creche das crianças de 0 a 3 anos de idade, por sexo, segundo as Grandes Regiões 2009/2014
5.2 - Brazilian social security payments - 2003-2014

5.3 - Distribution of urban and rural benefits - 2011-2014

5.4 - Benefits granted by social security - 2011-2014

5.5 - Number of people contributing to the General Regime of Social Security - RGPS, by category - 20042014 120

5.6 - Percentual share of the population according to public pension and BPC coverage and age group - Brazil - 2004/2014 121

5.7 - Percentual from household income share paid by public pensions or BPC according to deciles - Brazil - 2004/2014

\section{Education}

6.1 - Iliteracy rate of persons 10 years old and over, by sex and age groups - 2009/2014

6.2 - Average of years of schooling of persons 10 years old and over, by sex and age groups - 2009/2014

6.3 - Distribution of persons 25 years old and over, by Major Regions, sex and level of schooling 2014

6.4 - Distribution of persons attending school or nursery school, by Major Regions, level of education and network attended 2014

6.5 - Attendance rate to nursery school of children 0 to 3 years old, by sex and Major Regions 2009/2014 146 
6.6 - Taxa de escolarização das pessoas de 4 anos ou mais de idade, por Grandes Regiões, segundo os grupos de idade e sexo - 2014 .... 147

Trabalho

7.1 - Distribuição das pessoas de 15 anos ou mais de idade, ocupadas, por Grandes Regiões, segundo algumas características - 2014 162

7.2 - Distribuição das pessoas de 15 anos ou mais de idade, ocupadas, por Grandes Regiões, segundo os grupamentos de atividade do trabalho principal - 2014

7.3 - Média anual da taxa de desocupação das pessoas de 10 anos ou mais de idade, por principais regiões metropolitanas 2006-2015. 165

7.4 - Variação anual do rendimento médio mensal real habitual de categorias selecionadas das pessoas ocupadas, por principais regiões metropolitanas - período 2014-2015

\section{Participação Política}

8.1 - Média de eleitores por seção, seções e eleitores existentes - 2014

8.2 - Distribuição percentual dos resultados da apuração para presidente - $2^{\circ}$ turno - 2014

8.3 - Candidatos eleitos, por partido político - 2014

\section{Preços}

9.1 - Índice Nacional de Preços ao Consumidor Amplo - IPCA - 2015
6.6 - Schooling rate of persons 4 years old and over, by Major Regions, age groups and sex - 2014

\section{Labor}

7.1 - Distribution of employed persons 15 years old and over, by Major Regions and some characteristics 2014

7.2-Distribution of employed persons 15 years old and over, by Major Regions and groups of activity in the main work - 2014

7.3 - Annual average unemployment rate of persons 10 years and over, by Metropolitan Areas - 2006-2015

7.4 - Annual variation of average real usual monthly income of selected categories of employed persons, by main metropolitan areas - 2014-2015

\section{Political Participation}

8.1 - Average voters by polling sections, zones and voters - 2014

8.2 - Percentage distribution of vote cast for President 2nd round - 2014

8.3 - Candidates elected by political parties - 2014

\section{Prices}

9.1 - Extended National Consumer Price Index - IPCA - 2015 
9.2 - Variação acumulada no ano do Índice Nacional de Preços ao Consumidor - INPC e do Índice Nacional de Preços ao Consumidor Amplo - IPCA - 2002-2015 196

9.3 - Custo médio, número-índice e variação acumulada no ano, na construção civil, segundo as Grandes Regiões e Unidades da Federação - 2015

9.4 - Variação acumulada no ano do Índice Nacional da Construção Civil - 2006-2015 198

\section{Contas Nacionais}

10.1 - Principais agregados macroeconômicos - 2013-2015......208

10.2 - Participação percentual dos impostos e do valor adicionado, a preços básicos no Produto Interno Bruto - PIB, e dos setores de atividade, no valor adicionado a preços básicos - 2013-2015 ....... 209

10.3 - Composição do Produto Interno Bruto - PIB, sob a ótica da despesa - 2013-2015. 210

10.4 -Variação da taxa trimestral do Produto Interno Bruto - PIB, por setor de atividade - 2014-2015 211

10.5 - Principais relações macroeconômicas - 2013-2015

\section{Agropecuária}

11.1 - Principais produtos agrícolas, segundo valor da produção e principal Unidade da Federação produtora - 2014 226

11.2 - Efetivo dos rebanhos e das aves 2013-2014 227

11.3 - Quantidade e valor dos produtos de origem animal e variação anual - 2013-2014. 228
9.2 - Cumulative change in the year of the Extended National Consumer Price Index - IPCA and of the National Consumer Price Index INPC - 2002-2015 196

9.3 - Average cost, index-number and cumulative change in the year in civil construction, by Major Regions and Federation Units - 2015 197

9.4 - Cumulative change in the year of the National Index of Civil Construction - 2006-2015 198

\section{National Accounts}

10.1 - Main macroeconomic aggregates 2013-2015 208

10.2 - Percentage participation of taxes and of value added at basic prices in the Gross Domestic Product - GDP, and of the sectors of activity, in value added at basic prices - 2013-2015 209

10.3 - Composition of Gross Domestic Product - GDP, considering expenditures - 2013-2015 ........... 210

10.4 - Quarterly rate change of the Gross Domestic Product - GDP, by sector of activity - 2014-2015 211

10.5 - Main macroeconomic relationships - 2013-2015 212

\section{Agriculture}

11.1 - Major agricultural crops, according to the value of production and main producer Federation Unit - 2014 226

11.2 - Number of livestock and poultry on farms - 2013-2014 227

11.3 - Amount and value of products of animal origin and annual variation - 2013-2014. 228 
11.4 - Produção madeireira da extração vegetal e da silvicultura - 20132014

Indústria

12.1 - Produção industrial, segundo as seções e atividades de indústria 2013-2015

12.2 - Produção industrial e grau de intensidade de energia elétrica 2013-2015

12.3 - Produção industrial - 2012-2015 248

12.4 - Variáveis selecionadas das unidades locais industriais de empresas industriais com 5 ou mais pessoas ocupadas, segundo as Grandes Regiões e as Unidades da Federação - 2013 249

\section{Energia}

13.1 - Dados gerais de energia - 20122014.

13.2 - Geração de energia elétrica - 20132014

13.3 - Produção de petróleo e oferta interna de energia, por países selecionados - 2014 283

13.4 - Potencial hidrelétrico, segundo as bacias hidrográficas - 2015 283

\section{Comércio}

14.1 - Dados gerais do comércio - 2013 300

14.2 - Número de empresas, pessoal ocupado, salários e receita total, segundo as divisões do comércio 2013 301

14.3 - Participação dos segmentos do comércio - 2013 301

Transportes

15.1 - Extensão das malhas viárias do País - 2015
11.4 - Production from wood wild crop harvesting and silviculture - 20132014 229

Industry

12.1 - Industrial output, according to industry sectors and activities 2013-2015 245

12.2 - Industrial output and degree of intensity of electricity consumption - 2013-2015 247

12.3 - Industrial output - 2012-2015 248

12.4 - Selected variables from industrial local units with 5 or more employed persons, by Major Regions and Federtion Units - 2013 249

\section{Energy}

13.1 - General data on energy - 20122014

13.2 - Generation of electricity - 20132014

13.3 - Petroleum production and total primary energy supply, by selected countries - 2014 283

13.4 - Hydroelectric potential, according to river basins - 2015 283

\section{Trade}

14.1 - General data of trade - 2013

14.2 - Number of companies, employed persons, salaries and total revenue, according to trade divisions - 2013 301

14.3 - Participation of trade segments 2013 301

\section{Transportation}

15.1 - Road network extention of Brazil 2015 
15.2 - Dados gerais do transporte ferroviário - 2015

Turismo

16.1 - Chegadas de turistas no Brasil 2012-2014

16.2 - Chegadas de turistas no Brasil, por Unidades da Federação de acesso 2012-2014 334

16.3 - Agências de turismo cadastradas no CADASTUR - 2014 335

\section{Comunicações}

17.1 - Organização dos Correios e Telégrafos - 2011-2015 355

17.2 - Tráfego postal - 2011-2015 ......... 356

17.3 - Telefones em serviço - 2015 ....... 357

17.4 - Televisão e radiodifusão - 20112015 358

17.5 - Banda larga fixa, por Grandes Regiões e Unidades da Federação 2012-2015 359

17.6 - Banda larga móvel, por Grandes Regiões e Unidades da Federação 2012-2015 360

\section{Finanças}

18.1 - Evolução da dívida líquida do setor público - 2004-2015 375

18.2 - Evolução da dívida bruta do Governo Geral - 2009-2015. 376

18.3 - Necessidades de financiamento do setor público - 2012-2015 377

18.4 - Dívida líquida do setor público 2012-2015 378

18.5 - Dívida líquida e superávit primário, por região - 2012-2015 379

18.6 - Despesa liquidada da União 2010-2015 380
15.2 - General data of railway transportation - 2015.

\section{Tourism}

16.1 - Arrivals of tourists to Brazil - 20122014 332

16.2 - Tourist arrivals to Brazil, by Federation Unit of access - 20122014

16.3 - Travel and tourism agencies registered in CADASTUR - 2014335

\section{Communications}

17.1 - Organization of the Postal and Telegraph Services - 2011-2015355

17.2 - Postal traffic - 2011-2015 ............. 356

17.3 - Telephones in service - 2015 ..... 357

17.4 - Television and radio broadcasting - 2011-2015. 358

17.5 - Fixed broadband acess, by Major Rergions and Federative Units 2012-2015 359

17.6 - Mobile broadband access, by Major Regions and Federative Units 2012-2015 360

\section{Finances}

18.1 - Public sector net debt evolution 2004-2015 375

18.2 - General government gross deb evolution - 2009-2015 376

18.3 - Public sector net borrowing 2012-2015

18.4 - Public sector net debt - 2012-2015 378

18.5 - Net debt and primary surplus, according to Region - 2012-2015 379

18.6 - Government settled expenditure 2010-2015 380 
18.7 - Despesa liquidada da União, por áreas de atuação/funções - 20142015 381

18.8 - Despesas com o pessoal da União 2001-2015

18.9 - Número de servidores públicos federais - 2001-2015 383

\section{Comércio Exterior}

19.1 - Balanço de pagamentos - 20132015. 396

19.2 - Exportação - 2013-2015 397

19.3 - Importação - 2013-2015. 397

Ciência e Tecnologia

20.1 - Investimentos nacionais em pesquisa e desenvolvimento, por setores, em relação ao Produto Interno Bruto PIB - 2012-2013. 409

20.2 - Recursos dos governos estaduais aplicados em ciência e tecnologia 2008-2013 410

20.3 - Indicadores selecionados dos cursos de pós-graduação - 20012014 411

20.4 - Instituições, grupos de pesquisa, pesquisadores e doutores em ciência e tecnologia - 2004/2014

20.5 - Artigos brasileiros e do mundo publicados em periódicos científicos internacionais indexados pela Scopus e percentual do Brasil em relação ao mundo em número de artigos e de citações recebidas 2000-2013

20.6 - Pedidos depositados e decisões dos processos sobre patentes 2010-2015

Poder Judiciário/

21.1 - Informações de estrutura, recursos humanos e litigiosidade - 20102014 430
18.7 - Government settled expenditure, according to practice areas - 20142015 381

18.8 - Federal personnel expendtitures 2001-2015 382

18.9 - Number of federal public employees - 2001-2015 383

Foreign Trade

19.1 - Balance of payments - 2013-2015 396

19.2 - Exports - 2013-2015 397

19.3 - Imports - 2013-2015 397

Science and Technology

20.1 - National investments in research and development, by sectors, vis-à-vis Gross Domestic Product GDP - 2012-2013 409

20.2 - State government resources invested in science and technology - 2008-2013. 410

20.3 - Selected indicators in master's and doctoral programs - 20012014

20.4 - Institutions, research groups, researchers and doctors in science and technology - 2004/2014

20.5 - Brazilian and World papers published in international scientific journals indexed by Scopus and Brazilian relative contribution to World publication and citation 2000-2013

20.6 - Patent applications and decisions of patent trials - 20102015

Judicial Power

21.1 - Information onstructure, human resources and litigiousness - 20102014 430 
21.2 - Informações de estrutura, recursos humanos e litigiosidade, por ramos de Justiça - 2014

Meio Ambiente

22.1 - Número de violações do padrão primário nacional de qualidade do ar de PM10, nas Regiões Metropolitanas de Salvador (Camaçari), Belo Horizonte, Vitória, Rio de Janeiro, São Paulo, Curitiba e Porto Alegre - 1995-2013 450

22.2 - Média anual da Demanda Bioquímica de Oxigênio - DBO, em corpos de água selecionados, nas Unidades da Federação de Pernambuco, Bahia, Espírito Santo, Minas Gerais, Rio de Janeiro, São Paulo, Paraná e Rio Grande do Sul 2003-2013 451

22.3 - Número e área das Unidades de Conservação Federais - 2014 .... 453

\section{Gráficos}

\section{População}

2.1 - Composição relativa da população residente, por sexo e grupos de idade - 1991/2010. . .70

2.2 - Projeção da população - 2000/2020..70

2.3 - Esperança de vida ao nascer 1930/2016

2.4 - Taxas brutas de natalidade e mortalidade - 2000-2016 . .71

2.5 - Taxa média geométrica de crescimento anual - 1940/2010 .....77

\section{Habitação}

3.1 - Distribuição dos domicílios particulares permanentes, por condição de ocupação - 2014 . .88
21.2 - Information on structure, human resources and litigiousness, by courts of justice - 2014 431

\section{Environment}

22.1 - Number of violations of the national primary standard of air quality of PM10 in the metropolitan areas of Salvador (Camaçari), Belo Vitoria, Rio de Janeiro, São Paulo, Curitiba and Porto Alegre -1995-2013 450

22.2 - Annual Average of Biochemical Oxygen Demand - BOD, in selected water bodies, in the F.U. of Pernambuco, Bahia, Espírito Santo, Minas Gerais, Rio de Janeiro, São Paulo, Paraná and Rio Grande do Sul - 2003-2013 451

22.3 - Number and areas of Federal Conservation Units - 2014 453

\section{Graphs}

\section{Population}

2.1 - Relative composition of the resident population, by sex and age groups 1991/2010

2.2 - Population projections - 2000/2020 ..70

2.3 - Life expectancy at birth - 1930/2016

2.4 - Crude birth and death rates - 20002016

2.5 - Average geometric rate of annual increase - 1940/2010

\section{Housing}

3.1 - Distribution of permanent private housing units, by tenure - 2014 
3.2 - Distribuição dos domicílios particulares permanentes, por existência de rede geral de abastecimento de água, segundo as Grandes Regiões - 2014 . .88

3.4 - Distribuição dos domicílios particulares permanentes, por existência de serviço de coleta de lixo, segundo as Grandes Regiões - 2014

3.3 - Distribuição dos domicílios particulares permanentes, por tipo de esgotamento sanitário, segundo as Grandes Regiões - 2014. 89

\section{Saúde}

4.1 - Casos notificados de Aids 2002-2014

\section{Educação}

6.1 - Taxa de analfabetismo da população de 15 anos ou mais de idade, por situação do domicílio - 2005/2014 146

6.2 - Taxa de analfabetismo das pessoas de 15 anos ou mais de idade - 2009/2014 148

6.3 - Média de anos de estudo da população de 10 anos ou mais de idade, por grupos de idade - 2014... 148

Trabalho

7.1 - Distribuição percentual das pessoas de 15 anos ou mais de idade, ocupadas, por classes de rendimento mensal de trabalho - 2014 165

7.2 - Taxa de atividade das pessoas de 15 anos ou mais de idade, por grupo de idade, segundo o sexo-2014 167

\section{3 - Taxa de desocupação das} pessoas de 10 anos ou mais de idade - 2007-2015
3.2 - Distribution of permanent private housing units, by presence of water supply system - 2014

3.4 - Distribution of urban permanent private housing units, by presence of refuse disposal service - 2014

3.3 - Distribution of permanent private housing units, by type sewage disposal and Major Regions - 2014

\section{Health}

4.1 - Aids cases reported 2002-2014. 103

\section{Education}

6.1 - Illiteracy rate of the population 15 years old and over by urban/rural housing unit - 2005/2014. 146

6.2 - Illiteracy rate of the population 15 years old and over - 2009/2014 148

6.3 - Average of years of schooling of the population 10 years old and over, by age groups - 2014 148

\section{Labor}

7.1 - Percentage distribution of employed persons 15 years old and over, by ranges of monthly earnings $-2014$ 165

7.2 - Labor force participation rate of persons 15 years old and over, by sex -2014

7.3 - Unemployment rate of persons 10 years old and over - 2007-2015 167 
7.4 - Distribuição das pessoas de 15 anos ou mais de idade, ocupadas, por Grandes Regiões, segundo a contribuição para instituto de previdêndia - 2014 168

7.5 - Percentual das pessoas ocupadas na indústria no total da população de 15 anos ou mais de idade, ocupada, por Grandes Regiões - 2014 168

7.6 - Variação anual do rendimento médio mensal real habitual de categorias selecionadas de pessoas ocupadas - período 2014-2015... 169

\section{Participação Política}

8.1 - Deputados Federais eleitos, por partido político - 2014 183

8.2 - Governadores eleitos, por partido político - 2014 183

\section{Preços}

9.1 - Variação mensal do Índice Nacional de Preços ao Consumidor Amplo IPCA - 2015 196

9.2 - Variação mensal do Índice Nacional da Construção Civil - 2014-2015... 198

9.3 - Custo total por metro quadrado, parcela de materiais e mão-de-obra - dez. 2015 199

9.4 - Variação mensal das parcelas de materiais e de mão-de-obra na composição do custo Nacional da Construção Civil - 2015 199

9.5 - Variação acumulada do Custo Nacional e Custos Regionais da Construção Civil - 2015 200

\section{Agropecuária}

11.1 - Área plantada, quantidade produzida e valor da produção de cereais, leguminosas e oleaginosas - 2004-2014.
7.4 - Percentage distribution of employed persons 15 years old and over, by Major Regions, according to the participation in social security - 2014 168

7.5 - Percentage of employed persons in industry in the total employed population 15 years old and over, by Major Regions - 2014 168

7.6 - Annual percentage variation of average real usual monthly income by selected categories of employed persons - period 2014-2015 169

\section{Political Participation}

8.1 - Congressmen elected, by political parties - 2014 183

8.2 - Governors elected, by political parties - 2014

\section{Prices}

9.1 - Monthly change of the Extended National Consumer Price Index IPCA - 2015 196

9.2 - Monthly change of the National Index of Civil Construction - 2014-2015 .... 198

9.3 - Total cost per square meter, portion of materials and labor force Dec. 2015 199

9.4 - Monthly change of the Portion of materials and labor force in the composition of the National Cost of Civil Construction - 2015 199

9.5 - Cumlative change of the National and Regional Costs of Civil Construction - 2015. 200

\section{Agriculture}

11.1 - Planted area, amount produced and value of production of cereals, legumes and oilseeds - 2004-2014 
11.2 - Participação das Unidades da Federação no valor da produção agrícola - 2014 230

11.3 - Evolução da capacidade útil de armazenagem - 2005-2015 231

11.4 - Peso das carcaças - 2009-2014 231

11.5 - Participação das principais espécies na produção da piscicultura - 2014 .232

11.6 - Área total existente em 31.12 dos efetivos da silvicultura, por Grandes Regiões - 2014 232

Indústria

12.1 - Taxas anuais de crescimento da produção industrial, por grandes categorias econômicas - 20122015

12.2 - Produção de aço, por países selecionados - 2014 253

12.3 - Produção de aço da América Latina - 2014.

\section{Energia}

13.1 - Produção de energia primária 2002-2014

13.2 - Evolução da oferta interna de energia - 2002-2014

13.3 - Evolução do consumo final de energia, por fonte - 2002-2014

13.4 - Evolução do consumo final de energia, por setor - 2002-2014

13.5 - Dependência externa de energia 2002-2014 286

13.6 - Evolução do consumo final de energia em relação ao valor agregado, por setor - 2003-2014
11.2 - Brazilian states participation in the value of agricultural production 2014 230

11.3 - Evolution of storage capacity 2005-2015

11.4 - Weight of carcasses - 2009-2014

11.5 - Participation of the main species in the production of fisch farming 2014 232

11.6 - Total area existing in 31.12 of silviculture effective, by Major Regions - 2014 232

\section{Industry}

12.1 - Annual growth rates of industrial output, by major economic categories - 2012-2015

12.2 - Production of steel, by selected countries - 2014

12.3 - Production of steel, in Latin America - 2014 253

\section{Energy}

13.1 - Primary energy production - 20022014 284

13.2 - Evolution of primary energy supply - 2002-2014 284

13.3 - Evolution of final energy consumption, by source - 20022014 285

13.4 - Evolution of final energy consumption, by sector - 20022014 285

13.5 - Dependence on foreign energy 2002-2014 286

13.6 - Evolution of final energy consumption in relation to the value added, by sector - 2003-2014 286 
13.7 - Participação de fontes renováveis na matriz energética - 2013-2014 287

\section{Comércio}

14.1 - Participação dos segmentos na receita total do comércio varejista e de veículos - 2013 302

14.2 - Participação dos segmentos na receita total do comércio atacadista - 2013 302

14.3 - Participação das empresas, por faixas de pessoal ocupado, na receita operacional líquida do comércio 2013 303

14.4 - Evolução da receita operacional líquida, por faixas de pessoal ocupado - 2012-2013 303

14.5 - Taxa acumulada de desempenho no comércio varejista - 2007-2014 .....304

14.6 - Participação das empresas, por faixas de pessoal ocupado, no total de pessoal ocupado do comércio - 2013 304

\section{Transportes}

15.1 - Matriz de transporte de cargas, por modalidade Brasil - 2015 318

15.2 - Habitantes por autoveículo em alguns países - 2001/2013 319

15.3 - Movimento de carga, por tipo de navegação - 2005-2013 320

15.4 - Evolução da quantidade de voos 2005-2014

15.4 - Evolução da quantidade de passageiros transportada - 20052014.

15.6 - Carga aérea transportada - 20052014

\section{Turismo}

16.1 - Dez cidades brasileiras mais visitadas, pelos turistas estrangeiros para lazer - 2013-2014. 335
13.7 - Share of renewables sources in the energy matrix - 2013-2014. 287

\section{Trade}

14.1 - Participation of segments in total revenue of retail and vehicle trade $-2013$ 302

14.2 - Participation of segments in wholesale trade receipts - 2013 302

14.3 - Participation of companies, by ranges of employed persons, in net operating revenue of trade 2013 303

14.4 - Evolution of net operating revenue, by ranges of employed persons - 20122013

14.5 - Cumulative performance rate of retail trade - 2007-2014 304

14.6 - Participation of companies in total employed persons in trade, by ranges of employed persons 2013 304

\section{Transportation}

15.1 - Cargo transportation matrix Brazil - 2015

15.2 - Inhabitants per vehicle in selected countries - 2001/2013

15.3 - Cargo movement, by type of navigation - 2005-2013 320

15.4 - Evolution of the number of flights - 2005-2014. 320

15.4 - Evolution of the number of passangers transported - 20052014

15.6 - Air cargo transported - 2005-2014

\section{Tourism}

16.1 - Top ten Brazilian cities visited by foreign tourists - 2013-2014 
16.2 - Despesa da balança de pagamentos da conta-turismo 2007-2014 336

16.3 - Receita da balança de pagamentos da conta-turismo - 2007-2014 .. 336

16.4 - Taxa de câmbio e saldo da balança de pagamentos da Conta-Turismo - 2005-2014

\section{Comunicações}

17.1 - Evolução dos terminais telefônicos - 2007-2015 356

Finanças

18.1 - Dívida do setor público - 20052015

18.2 - Dívida líquida do setor público 2005-2015 384

18.3 - Prazo médio dos títulos federais 2005-2015 385

18.4 - Dívida líquida dos governos regionais - 2005-2015 385

\section{Comércio Exterior}

19.1 - Comércio exterior - 2008-2015 398

19.2 - Reservas internacionais - 20012015 398

\section{Ciência e Tecnologia}

20.1 - Dispêndios do governo federal em pesquisa e desenvolvimento, por instituições - 2013

20.2 - Artigos brasileiros publicados em periódicos científicos internacionais indexados pela Scopus e respectivo percentual em relação ao mundo 2000-2013

Poder Judiciário/

21.1 - Percentual de processos, tramitados por ramos de Justiça - 2014 433

21.2 - Percentual da despesa, por ramos de Justiça - 2014. 433
16.2 - Expenditure in balance of payments of tourism account 2007-2014 336

16.3 - Receipt in balance of payments of tourism account - 2007-2014 .... 336

16.4 - Exchangerate and balance of the Account of Turism - 2005-2014 337

\section{Communications}

17.1 - Telephone lines in service - 2007-2015 356

\section{Finances}

18.1 - Public debt - 2005-2015 384

18.2 - Public Sector Net Debt - 2005-2015 384

18.3 - Average term of federal securities 2005-2015 385

18.4 - Net debt of regional governments - 2005-2015. 385

\section{Foreign Trade}

19.1 - Foreign trade - 2008-2015 398

19.2 - International reserves - 2001-2015

Science and Technology

20.1 - Federal government expenditures on research and development, by institution - 2013 413

20.2 - Brazilian papers published in international scientific journals indexed by Scopus and respective percent contribution in relation to the World - 2000-2013 414

Judicial Power

21.1 - Percentage of cases proceeded by courts of justice - 2014 433

21.2 - Percentage of expenditures by courts of justice - 2014 .433 
Meio Ambiente

22.1 - Desflorestamento bruto anual na Amazônia Legal - 1992/2015..... 454

22.2 - Focos de calor no Brasil, na Amazônia Legal e em Unidades de Conservação, Parques e Terras Indígenas - 2008/2015 454

22.3 - Comercialização de agrotóxicos e afins, por área plantada - Brasil 2001/2013 .455

22.4 - Proporção de material reciclado em atividades industriais selecionadas - 1994/2014 456

\section{Quadro}

Participação Política

8.1 - Partidos políticos com votação - 2014 182

\section{Mapas}

\section{Território}

1.1 - Mapa político do Brasil .55

1.2 - Pontos extremos e pontos mais altos do Brasil 60

\section{Environment}

22.1 - Annual Gross Deflorestation in Legal Amazon - 1992/2015 ........ 454

22.2 - Fire outbreaks in Brazil, Legal Amazon and in Conservation Units, Indigenous Lands 2008/2015 454

22.3 - Commercialization of pesticides and the like, by planted area -

Brazil - 2001/2013. 455

22.4 - Proportion of recycled material in selected industrial activities 1994/2014 456

\section{Figure}

\section{Political Participation}

8.1 - Political parties with votes - 2014 182

\section{Maps}

\section{Territory}

1.1 - Political map of Brazil .55

1.2 - Extreme points and highest points in Brazil

\section{Convenções / Symbols used}

... Dado numérico não disponível; Figure not available;

.. Não se aplica dado numérico; Not applicable;

- Dado numérico igual a zero não resultante de arredondamento; Zero not resulting from rounding;

0; 0, 0 Dado numérico igual a zero resultante de arredondamento de um dado numérico originalmente positivo. Originally positive numerical data rounded to zero. 



\section{Apresentação}

\section{Foreword}

Prezado leitor

Você tem em mãos o Brasil em Números Edição 2016 - publicação editada anualmente pelo IBGE contendo informações que aprofundam nosso conhecimento sobre importantes aspectos da realidade brasileira.

É um valioso instrumento de consulta e de base para análises e planejamentos em diversas esferas.

Cada tema abordado recebeu a contribuição de destacados especialistas na área, que enriquecem e ressaltam com seus comentários os dados, tabelas e gráficos apresentados.

Desta vez, a publicação é ilustrada com obras de arte do Museu Pinacoteca do Estado do Rio Grande do Norte, localizado no município de Natal. Cada capítulo se inicia com imagens das obras de arte presentes no Museu, nos fazendo viajar por dentro dele e pela rica e diversificada cultura de seus artistas regionais!

E não para por aí: o Brasil em Números é compacto em seu formato, leve e fácil de levar! Por ser uma publicação bilíngue, português e inglês, isso amplia ainda mais o impacto e a abrangência das informações.
Dear reader

In your hands is the 2016 edition of Brazil in Figures. This annual publication issued by the IBGE brings information that can help us improve knowledge about relevant aspects of the Brazilian reality.

It is a valuable reference material that can support analyses and planning initiatives at several spheres.

Each of the topics presented herein has had the contribution of renowned specialists whose commentaries have enriched and reinforced the data from tables and graphs.

The current edition is illustrated with art works from the Museum Pinacotheca of the State of Rio Grande do Norte, located in the municipality of Natal. An image showing one of the works displayed in the Museum opens each chapter and guides us inside the rich and diverse culture expressed by the artists from that region.

And that is not all: Brazil in Figures is a compact publication, light and easy to carry! And being bilingual, in Portuguese and English, the book has increased reach and coverage. 
Para maiores detalhes, você pode visitar a página do IBGE na Internet: www.ibge. gov.br

Carregue consigo um Brasil de informações e leve-o para onde quiser!
Further information on Brazil in Figures is available on the IBGE website: www. ibge.gov.br

Take Brazil with you and a world of information about it wherever you go!

Paulo Rabello de Castro

Presidente do IBGE/President of IBGE 


\section{Uma Breve História do Brasil A Brief History of Brazil}

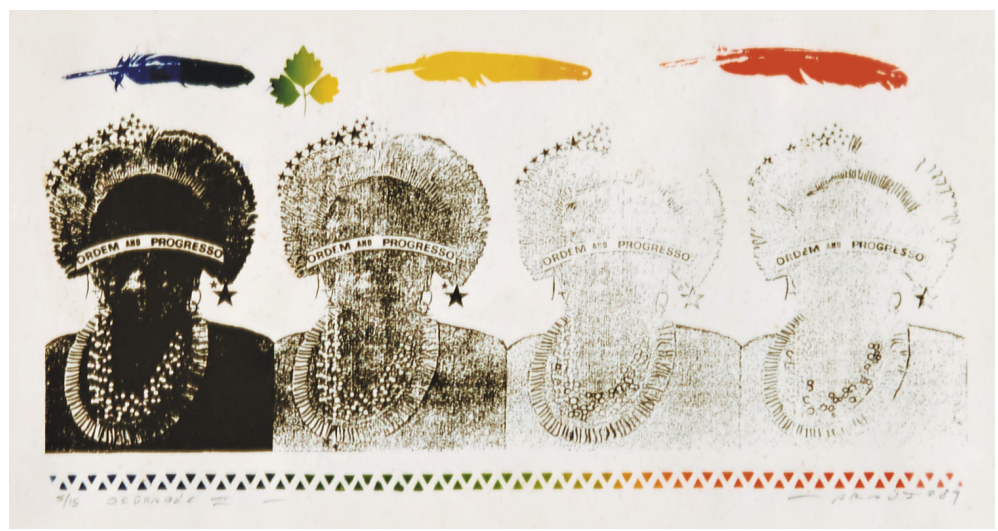

Degradê III/ Color Gradient III, 1989 Deusdeth Avelino de Araújo 



\section{Uma Breve História do Brasil}

\section{A Brief History of Brazil}

\section{Os saberes indígenas nas cartografias culturais brasileiras}

No início, diversas etnias indígenas, com uma infinidade de línguas, formas especiais de subsistência, criação de significados, inventando o mundo. As etnias indígenas dão cores ao Brasil. Tudo isso muito fascinante e pouco divulgado. O Brasil parece não conhecer o Brasil. O coração brasileiro tem no seu pulsar a presença das etnias indígenas. Essas etnias clamam pela regularização de seus direitos territoriais. As culturas indígenas estão presentes nas cartografias identitárias brasileiras. Suas manifestações revelam-se na identificação das plantas, nas vozes dos animais, das aves, e nos conhecimentos da natureza.

O guaraná, típica fruta brasileira tem sua história focada numa lenda indígena. Conta a lenda que um pequeno índio, líder de uma tribo amazônica, ao adentrar sozinho pela mata, fora picado por uma cobra, tendo sobrado dos seus órgãos apenas os olhos, que ao serem descobertos pelos entes queridos, foram os mesmos recolhidos com tristeza e dor, trazidos para a aldeia, e enterrados.

\section{Indigenous knowledge in Brazilian cultural cartography}

At first, many indigenous ethnic groups, with a multitude of languages, special forms of livelihood, and creation of meaning, were inventing the world. The indigenous ethnic groups give color to Brazil. All this is very fascinating and little known. Brazil seems not to know Brazil. The Brazilian heart has in its pulsing the presence of indigenous groups. These ethnic groups call for the regularization of their territorial rights. Indigenous cultures are present in Brazil's cartography of identity. Their manifestations are revealed in the identification of plants, in animal and bird sounds, and in the knowledge of nature.

Guaraná, a typically Brazilian fruit, has its story centered on an indigenous legend. According to this legend, a small Indian leader of an Amazon tribe, when entering the woods alone, was bitten by a snake, and from his body only the eyes were left. These, when found by his loved ones, were collected in sadness and pain, and brought to the village to be buried. The tears of the 
As lágrimas dos índios, que choraram a morte do menino, regaram o lugar onde os olhos foram sepultados, brotando dali o guaraná, que tem sua fruta muito parecida com os olhos do guerreiro, e que por isso alimenta a crença de que quando alguém bebe o guaraná com água, adquire a força do índio, garante longevidade à vida, mais memória e vitalidade para participar do aumento da população, conforme contavam com mais detalhes as vovós. Trata-se de uma história que mantém viva a presença dos índios dentro de cada brasileiro.

As culturas indígenas manifestamse na cultura brasileira na forma espontânea de expressão, no hábito de dar e receber presentes, na relação saudável e pura entre corpo e alma, nas danças, expressões lingüísticas e comidas. Foram as etnias indígenas, com suas curiosidades, as fornecedoras das chaves para a interiorização do território brasileiro. As suas redes embalam sonhos, e dão forças para que os sofrimentos possam ser suportados. Suas redes guardam tramas, que por sua vez levam à inspiração das invenções tecnológicas. A invenção do computador em rede aproximou as populações mundiais, forneceu sentido à vida, $\mathrm{e}$ a solidão parece ter sido salva pela presença, mesmo que virtual das tribos de internautas.

Sem os saberes indígenas dificilmente as culturas dos chegantes teriam penetrado e ampliado o território brasileiro, vencido os acidentes geográficos, mapeado os lugares, e conseguido instrumentos para adentrar nas matas numa fase em que a medicina era precária, diante das desconhecidas doenças tropicais. Uma terra marcada por muitos caminhos
Indians, who wept the boy's death, watered the place where the eyes were buried, making the guaraná, whose fruit is very similar to the warrior eyes, sprout. Therefore, the legend fuels the belief that drinking guaraná with water gives Indians strength, ensures longevity, and enhances memory and vitality to contribute to population growth, as told in greater detail by the grannies. It is a story that keeps alive the presence of Indians in every Brazilian.

Indigenous cultures manifest themselves in Brazilian culture in the spontaneous form of expression, in the habit of giving and receiving gifts, in the healthy and pure relationship between body and soul, in dances, linguistic expressions, and food. It was the indigenous ethnic groups, with their curiosity, that unlocked the keys to the movement towards the interior of Brazil. Their hammocks swing dreams and - provide the necessary support to endure suffering. Their hammocks are woven with threads in networks, which on their turn lead to the inspiration of technological inventions. The invention of the computer network brought the world closer together, gave meaning to life, and loneliness seems to have been replaced by the presence, even when virtual, of tribes of Internet users.

If not for the indigenous knowledge, hardly would the cultures of newcomers have penetrated and expanded over Brazil, overcome the land challenges, mapped places and come up with instruments to enter the woods at a stage where medicine was precarious in the face of unknown tropical diseases. A land marked by 
e descaminhos, mas embalada pela coragem das culturas indígenas. $\mathrm{O}$ alento e a alegria da cultura brasileira têm o perfume das flores e frutas apresentadas pelos saberes indígenas. Uma população dizimada que foi, mas que ainda assim conseguiu resistir às doenças e violências sofridas.

Ter as culturas indígenas nas veias da cultura brasileira significa gozar da plenitude da vida e gostar das águas doces e salgadas para aliviar o calor dos trópicos. $O$ encontro das etnias indígenas com os chegantes deve ter sido um momento mágico e de difícil descrição, diversas civilizações juntas olhando umas para as outras com os seus diferentes saberes e modos de enxergar o tempo. Todas devem ter ficado surpreendidas pelas diferenças, vindo desde então, a presença das diferenças na cultura brasileira, diferenças essas "superadas" pela resistência, mesmo quando proibidas. A superação definitiva das diferenças talvez ainda seja o maior dos desafios da cultura brasileira. Toda barreira cultural acaba por criar diversas outras barreiras. $O$ desenvolvimento pode tornar-se refém das barreiras culturais. As barreiras culturais afastam populações, produzem guerras. As barreiras culturais existentes na América do Sul clamam por uma maior integração.

Os chegantes, depois do encontro com os índios, passado os primeiros encantamentos, os grandes choques na luta pelo poder do espaço, a formação das primeiras povoações, 0 estabelecimento da vida com "sentido", propuseram, com o apoio dos índios a base para a formação da sociedade brasileira. Não foi fácil, nem continua many paths and detours, but powered by the courage of indigenous cultures. The impetus and the joy of Brazilian culture have the scent of flowers and fruits brought by indigenous knowledge. Although the Indian population was decimated, it still managed to resist disease and violence.

Having the indigenous cultures in the veins of Brazilian culture means enjoying the fullness of life and appreciating the fresh and salt water to relieve the heat of the tropics. The meeting of indigenous ethnic groups with new-comers must have been a magical moment, several civilizations together looking at each other, carrying different knowledge and different ways of interpreting their time. All of these civilizations must have been amazed at the differences, which, since then, have placed diversity in the Brazilian culture - differences which were "overcome" by resistance, even when they were prohibited. The definitive overcoming of differences may still be the greatest challenge of the Brazilian culture. Every cultural barrier ends up creating a number of other barriers. Development may become a hostage of cultural barriers. Cultural barriers separate populations, produce wars. The existing cultural barriers in South America call for greater integration.

The new-comers, after the encounter with the Indians, past the initial thrill, the major fight for space, the establishment of the first settlements, the establishment of life with a "meaning", proposed, with the support of the Indians, the basis for the formation of Brazilian society. It was not easy then, nor is it now, but 
sendo, mas a luta e a construção são permanentes.

Embora a língua oficial do Brasil seja o português cabe destacar que no território brasileiro existem mais de duzentos e setenta e quatro línguas faladas pelos índios. São muitos os desafios ainda por serem aliviados. As diferenças desde o primeiro encontro dos índios com os chegantes foram reduzidas, mas ainda não superadas. Todo trauma leva tempo para se recompor na cartografia das relações humanas. Trata-se de um processo longo que o Brasil ainda terá pela frente, mas que deve ser buscado.

Os chegantes, tendo os índios como "anfitriões", conseguiram, conforme enfatizado, interiorizar o Brasil. Cuiabá, capital do Estado de Mato Grosso, uma cidade do século XVIII, com traçados típicos do período colonial, resulta dessa conjugação cultural entre índios e chegantes. Uma população no coração bororo do território brasileiro, que conseguiu reinventar-se no tempo, e que apresenta no bordado do seu espaço regional diversas outras etnias indígenas. São esses, portanto, os saberes acumulados indígenas nas cartografias culturais brasileiras. the struggle and construction are permanent.

Although the official language of Brazil is Portuguese, it is worth noting that in our territory there are more than two hundred and seventy four languages spoken by Indians. There are still many challenges to face. The differences since the first encounter, of Indians with new-comers, have been reduced but not yet overcome. On the map of human relations, recovery from trauma takes time. It is a long process that Brazil still has to go through, but that needs to be pursued.

The new-comers, having with the Indians as "hosts", succeeded, as emphasized, in the movement towards the interior of Brazil. Cuiabá, capital of Mato Grosso, an 18th century city with typical traces of the colonial period, follows that cultural combination between Indians and new-comers. A population in the bororo heart of Brazil, which managed to reinvent itself in time, and has, in the embroidery of their regional space, several other indigenous groups. This is, therefore, the indigenous knowledge collected in Brazilian cultural cartography.

Fernando Tadeu de Miranda Borges

Professor Titular da Faculdade de Economia da Universidade Federal de Mato Grosso Bacharel em Ciências Econômicas pela FE-UFMT - Faculdade de Economia da Universidade Federal de Mato Grosso Mestre em Economia pela FEA-USP - Faculdade de Economia, Administração e Contabilidade da Universidade de São Paulo

Doutor em História Social pela FFLCH-USP

- Faculdade de Filosofia, Letras e Ciências
Full Professor in the School of Economics of the Federal University of Mato Grosso Bachelor's degree in Economic Sciences from the FE-UFMT Master's degree in Economics from FEA-USP Ph.D. in Social History from FFLCH-USP Professor of the Postgraduate Program in History and Postgraduate Program in 
Humanas da Universidade de São Paulo Docente do Programa de Pós-Graduação em História e do Programa de Pós-Graduação em Agronegócios e Desenvolvimento Regional da Universidade Federal de Mato Grosso Membro da Academia Mato-Grossense de Letras, do Instituto Histórico e Geográfico de Mato Grosso e da Sociedade de Amigos de Rondon
Agribusiness and Regional Development of the Federal University of Mato Grosso

Member of the Mato Grosso Academy of Letters, of the Historical and Geographical Institute of Mato Grosso and Rondon's Friends Society 



\section{Território}

\section{Territory}

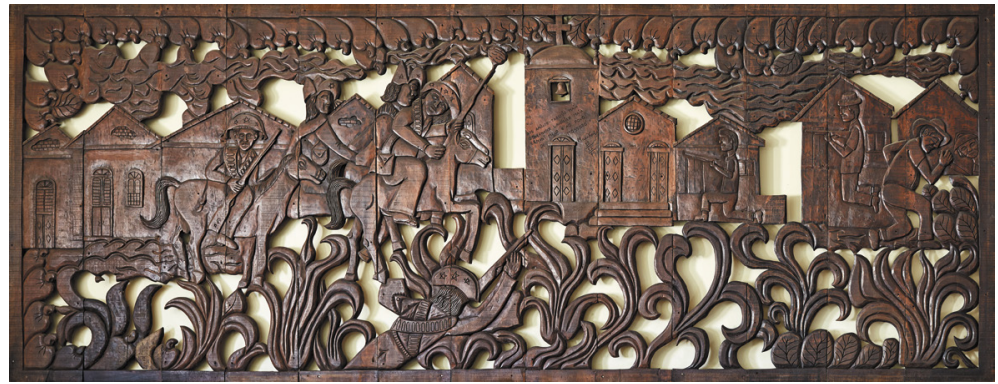

Painel com motivo regional/ Panel with regional motifs, 1979 Ziltamir Sebastião Soares de Maria 'Manxa' 



\section{Território}

Território é o produto histórico do trabalho humano, que resulta na construção de um domínio ou de uma delimitação do vivido territorial, assumindo múltiplas formas e determinações: econômicas, administrativas, culturais e jurídicas. Desta forma, o território ganha uma identidade, não em si mesma, mas na coletividade que nele vive e o produz. Ele é todo, um concreto, mas ao mesmo tempo flexível dinâmico e contraditório por isso dialético, rico de possibilidades que só realizam quando impressas e espacializadas no próprio território (Mitidiero, 1999).

Conceito-chave da geografia, a subjetividade da definição de Território pode levar a diversas acepções políticas, econômicas e culturais que, de forma entrelaçada, tentam explicar a dinâmica de um espaço em construção. Ao observar os números apresentados nesta publicação ficam mais evidentes os aspectos político-administrativos e físicos do território brasileiro, base fundamental para que a sociedade crie seus lugares. Esta, portanto, será a abordagem deste preâmbulo que pretende comentar os dados apresentados, situando assim o

\section{Territory}

A territory is the historical product of human labor, resulting in the construction of a domain or of a delimitation of the lived-in territory, with multiple forms and determinations: economic, administrative, cultural and legal. So, the territory wins an identity that is not enclosed in itself, but defined in the collectivity that produces it and lives in it. It is a unit, concrete, but at the same time flexible, dynamic and contradictory, and, thus, dialectic, full of possibilities which only become real when printed and spatialized on the territory itself (Mitidiero, 1999).

A key concept in geography, the subjectivity of the Territory definition can lead to several political, economic and cultural meanings which, being entwined, try to explain the dynamics of a space under construction. The figures presented in this publication evidence the political-administrative and physical aspects of the Brazilian territory, the fundamental basis for the creation of places in society. This is the approach of the introduction that follows, in which I intend to comment on the data presented, showing 
território brasileiro diante de grandes possibilidades marcadas por diversos contrastes, das quais, uma das mais significativas está em sua exuberante riqueza natural e biodiversidade.

\section{Brasil - território político- administrativo}

O Estado Brasileiro adota a federação como sistema de governo, congregando 26 estados autônomos reunidos para constituir o Estado Federal. Apesar das raízes dessa configuração remontarem o sistema de divisão em Capitanias Hereditárias, implantadas desde o Brasil Colônia, o Federalismo só foi implantado efetivamente no Brasil, com a instalação da República e, mais especificamente, com a promulgação da Constituição Federal de 1891. É importante destacar que essa transição se deu de forma lenta e nem sempre pacífica - vários foram os movimentos nativistas, revoltas e guerras que, na busca por emancipação, moldaram a atual configuração da República Federativa do Brasil. O território, com dimensões continentais, mantido íntegro desde sua formação, impunha uma organização descentralizada em contraposição ao unitarismo característico na Monarquia Parlamentar, implantado após a independência brasileira.

Ao longo de sua história o território brasileiro passou, gradativamente, por grandes transformações até chegar à atual organização espacial interna (Mapa 1.1). A subdivisão em unidades autônomas, que possibilitassem a administração efetiva das políticas públicas, resultou na divisão atual. that the Brazilian territory has ahead of it many possibilities marked by several contrasts, among which we can mention its magnificent natural richness and biodiversity.

\section{Brazil - political-administrative territory}

Brazil has adopted the federation as its system of government. Ours encompasses a group of 26 autonomous states which form the Federal State. Although this configuration resembles the Hereditary Captaincy system, adopted for the division of the Brazilian territory in colonial times, Federalism was only definitively implemented in Brazil after the proclamation of the Republic, most specifically with the promulgation of the Federal Constitution of 1891. It is worth mentioning the transition was gradual and not always pacific - there were several nativist movements, riots and wars which, searching for emancipation, shaped the current configuration of Brazil's Federal Republic. Having been kept intact since its creation, our territory, with its continental dimensions, required a more decentered type of organization, opposite to the unitarian character of the Parliamentary Monarch that followed the Brazilian independence.

Throughout history, the Brazilian territory has gone through gradual changes before reaching its current spatial organization (Map 1.1). A subdivision into autonomous units, enabling the effective administration of public policies has led to the present ordering. 
Ainda no período imperial foram feitos rearranjos como punição a participação de províncias em revoltas anti-imperiais. Assim foi desmembrada a Comarca do Rio São Francisco, originalmente pertencente à Pernambuco e anexada a Minas Gerais e, posteriormente, de forma definitiva à Bahia em 1827. A criação da província do Paraná (1853), desmembrada de São Paulo, teve como motivação a participação desta última, na revolta de 1842.

A partir da década de 1940 a divisão territorial do Brasil passa a levar em consideração além dos aspectos físicos, os socioeconômicos, permanecendo assim nas cinco regiões atuais, contudo, entre a década de 1940 até 2014, a organização interna entre os estados e municípios assumiu várias transformações durante esses 74 anos (Tabela 1.2).

Durante a Segunda Guerra Mundial (1943), foram criados seis territórios estratégicos, dos quais três foram alçados a condição de estados posteriormente sendo Rondônia em 1982, Roraima e Amapá em 1988. O território de Fernando de Noronha foi extinto e seu território incorporado ao estado de Pernambuco em 1988. Ponta Porã e lguaçú retornaram em 1946 a seus estados de origem.

Em 1960 foi criado o Distrito Federal, a partir de um desmembramento do estado Goiás e, simultaneamente, o antigo Distrito Federal foi transformado em Guanabara, incorporado em 1975 como capital do estado do Rio de Janeiro. Em 1962, o território do Acre, incorporado como território pelo Tratado de Petrópolis (1903) foi elevado a condição de estado.
Early in imperial times some rearrangements took place as punishment for provinces taking part in riots against the empire. This way, the District of São Francisco River, which originally belonged to Pernambuco, was dismembered and annexed into Minas Gerais, and later on, in 1827, into Bahia. The creation of the Paraná Province (1853), dismembered from São Paulo, was fostered by the latter's participation in the 1842 riot.

From the 1940s on, the territory division of Brazil would start to consider not only physical, but also socioeconomic aspects. That is still seen in the five current Major Regions. Nevertheless, many changes were observed in the internal organization of states and municipalities in the 74 years between 1940 and 2014 (Table 1.2).

During the Second World War (1943), six strategic territories were created, being three of them later turned into states: Rondônia, in 1982, Roraima and Amapá, in 1988. The territory of Fernando de Noronha was extinguished and its land was annexed into the state of Pernambuco in 1988. Ponta Porã and Iguaçu returned to their states of origin in 1946.

The Federal District was created in 1960 , as a result of a separation of part of Goiás. Simultaneously, the former Federal District became the state of Guanabara, which, in 1975, was made capital of the state of Rio de Janeiro. In 1962, the territory of Acre, annexed by means of the Treaty of Petrópolis (1903) was officially turned into a state. 
Mais recentemente o estado do Mato Grosso foi desmembrado dando origem a uma nova unidade denominada Mato Grosso do Sul (1977) e Goiás foi subdividido criando o estado do Tocantins (1988). Com isso chega-se a configuração atual, com 26 estados e um 01 distrito federal, somando assim 27 unidades da federação.

Durante muitos anos o processo de ocupação se deu de forma mais intensa nas áreas costeiras, aonde foram se instalando as principais cidades e muitas das capitais dos estados. A partir da construção de Brasília, aprofunda-se o processo de interiorização iniciado no século XVI, com o movimento de entradas e bandeiras. Juntamente com a industrialização do país, incrementase o processo de urbanização e a população, essencialmente rural, passa a residir nas áreas urbanas. Esse fenômeno continua nas décadas de 1970, 1980 e 1990, dando origem a várias regiões metropolitanas e cidades superpopulosas como São Paulo.

É importante destacar o processo de descentralização iniciado com a república perde força no período da ditadura militar (1964-1986) e volta a ter ênfase a partir da promulgação da Constituição de 1988. Esse fenômeno pode ser percebido com muita clareza na Tabela 1.2, onde se destacam dois momentos de inflexão na quantidade de municípios brasileiros, quais sejam: i. entre as década de 1950 e 1960 e ii. entre as décadas de 1980 e 1990 . É perceptível o incremento no número de municípios, o que demonstra uma tendência à emancipação políticoadministrativa.
The state of Mato Grosso has been dismembered quite recently, resulting in a new unit called Mato Grosso do Sul (1977); Goiás was subdivided to create the state of Tocantins (1988). These two states complete the current configuration of the Brazilian territory, with 26 states and one Federal District, amounting to 27 Federation Units.

For years settlement was more concentrated in coastal areas, where the main cities and many state capitals were being founded. After the construction of Brasília, the inland movement which had begun in the XVI century, with the movement called "Entradas e Bandeiras" (expeditions aimed at territory exploration), gains force. Together with the industrialization of Brazil, urbanization rises as the once primarily rural population starts living in urban areas. This phenomenon goes on in the following decades, 1970s, 1980s, 1990s and accounts for the birth of many metropolitan areas and overpopulated cities such as São Paulo.

It is important to mention that the process of decentralization which started with the republic would weaken during the military dictatorship (1964-1986) and only recover after the promulgation of the 1988 Constitution. This phenomenon can be seen clearly in Table 1.2, which highlights two inflection points in the number of Brazilian municipalities: i. between the 1950s and the 1960s and ii. between the 1980s and the 1990s. The noticeable increase in the number of municipalities reflects a trend to political and administrative emancipation. 
O número de municípios, que em 1940 abrangia 1.574, triplicou em quantidade até 2014 , com 5.570. Um aumento de 3.996 municípios distribuídos nas cinco regiões. Sendo a maior parte dos municípios concentrados nas regiões Nordeste (33.3\%), Sudeste (26.7\%), Sul (20.0\%) e Centro-Oeste e Norte com os menores números de municípios, $13.3 \%$ e $6.7 \%$, respectivamente. Ressaltando assim, que a região Norte, que detêm a maior parte do território brasileiro, com 3.853.669.768 km2 de área, representando $45 \%$ é a menos povoada.

Além da divisão político-administrativa o Brasil apresenta um agrupamento de estados em regiões, estas determinadas muito mais em função das características naturais do que em aspectos políticos. Nesse caso o território brasileiro, vasto e variado, está agrupado em cinco grandes regiões como pode ser visto nas Tabelas 1.1 e 1.2 .

\section{Brasil - território físico}

Observando os dados disponibilizados pelas tabelas e mapas do IBGE confirma-se o conceito de que o Brasil é um país com dimensões continentais. Ocupa uma área de $8.515767,049 \mathrm{~km}^{2}$ (Tabela 1.1), configurando-se como o quinto maior país do mundo em área territorial. Com forma triangular sua maior dimensão norte-sul é de 4.378,41 $\mathrm{Km}$, indo da nascente do Rio Ailã em Uiramutã (Roraima) ao Arroio Chuí em Santa Vitória do Palmar (Rio Grande do Sul). No sentido latitudinal (lesteoeste), a maior dimensão é de $4.326,63$ $\mathrm{Km}$, tendo como pontos extremos a Ponta do Seixas em João Pessoa (Paraíba) e a nascente do Rio Moa no município de Mâncio Lima (Acre).
The number of municipalities, 1,574 in 1940 , had tripled by 2014 , reaching 5,570, an increase of 3,996 municipalities distributed among five Major Regions. Most of the municipalities were located in the Northeast (33.3\%), Southeast (26.7\%), South $(20.0 \%)$ and Central West and North, these with the smallest number of municipalities, $13.3 \%$ and $6.7 \%$, respectively. Figures show the North Region, whose 3,853,669,768 km2 of area make up $45 \%$ of the Brazilian territory, is the least populated one.

Besides the political-administrative division, Brazil has states grouped into regions that are much closer in terms of natural features than of political aspects. The vast and diverse Brazilian territory is subdivided into five Major Regions, as shown in Tables 1.1 and 1.2.

\section{Brazil - physical territory}

By observing the data made available in the IBGE tables and maps, we reaffirm that Brazil is a country of continental dimensions. It has an area of 8,515 $767.049 \mathrm{~km}^{2}$ (Table 1.1), being the fifth biggest country in the world in territory size. With a triangular shape, its longest distance between the North and the South is $4,378,41 \mathrm{Km}$ and separates the source of the Ailã River in Uiramutã (Roraima) from the Chuí Creek in Santa Vitória do Palmar (Rio Grande do Sul). In latitudinal direction (east-west), the longest distance in Brazil is 4,326.63 $\mathrm{Km}$, having as extreme points Ponta do Seixas in João Pessoa (Paraíba) and the source of the Moa river in the municipality of Mâncio Lima (Acre). 
Sua localização geográfica, o coloca na posição de único país do mundo atravessado pela Linha do Equador e pelo Trópico de Capricórnio, possuindo terras tanto no hemisfério sul (93\%) quanto em terras no hemisfério norte (7\%) caracterizando-se assim como um país tropical (92\%). Situado $100 \%$ a oeste do Meridiano de Greenwich, ocupa a porção centro-oriental e $47 \%$ de território da América do Sul.

Devido sua grande dimensão territorial, o Brasil apresenta uma variação de fusos horários. O primeiro fuso está duas horas atrasado em relação ao Meridiano de Greenwich, -2GMT, abrange particularmente as ilhas do Oceano Atlântico; o segundo fuso está atrasado em relação a Greenwich três horas, -3GMT, é considerado o mais importante fuso por abranger grande parte do território brasileiro, bem como as grandes regiões de desenvolvimento do país, incluindo o Distrito Federal e a capital Brasília, sendo assim, o horário oficial do país; o terceiro fuso, -4GMT, abrange alguns estados a oeste: Mato Grosso, Mato Grosso do Sul, Rondônia, Roraima e a maior parte dos estados do Pará e Amazonas; o quarto fuso, -5GMT, abrange uma pequena parte oeste do Amazonas e o estado do Acre.

O Brasil não é um país de grandes altitudes. Isso pode ser explicado pela antiguidade do seu território, que sofreu o ataque dos agentes da erosão.

Segundo o IBGE, quase $99 \%$ do nosso país é formado por terrenos de menos de $1.200 \mathrm{~m}$ de altitude, sendo que $41 \%$ têm de 0 a $200 \mathrm{~m}$ e $58,5 \%$ chegam até 1.200 $\mathrm{m}$. Os pontos mais altos do país são o pico da Neblina (2.995,3 m) e o pico 31
The country's geographical location places it as the only one in the world crossed by the Equator and by the Tropic of Capricorn, with land both in the southern (93\%) and the northern (7\%) hemispheres, being, thus, characterized as a tropical country (92\%). Situated $100 \%$ to the west of the Greenwich Meridian, it takes up the central eastern area and $47 \%$ of the South American territory.

Due to its large territory, Brazil uses some different time zones. The first time zone is two hours behind Greenwich Mean Time, -2GMT: that includes mainly the islands of the Atlantic Ocean. The second time zone is three hours behind Greenwich Mean Time, -3GMT; it is considered the most important one, since it covers most of the Brazilian territory, including the Federal District and Brasilia, the capital, and represents the official time in the country. The third time zone, - 4GMT, covers some western states: Mato Grosso, Mato Grosso do Sul, Rondônia, Roraima and a big amount of Pará and Amazonas. The fourth time zone, -5GMT, encompasses a small portion of the Amazon and the whole state of Acre.

Brazil is not famous for its high altitudes. A possible explanation is the old age of its territory, and the effect of erosion on it.

According to the IBGE, almost 99\% of our country is formed by land below $1,200 \mathrm{~m}$ altitude, being $41 \%$ from 0 to $200 \mathrm{~m}$ and $58.5 \%$ up to $1,200 \mathrm{~m}$. The highest peaks in the country are Pico da Neblina $(2,995,3$ 
de Março (2.974,2 m) (Figura 1.3) ambos localizados no extremo norte do estado do Amazonas e fazendo fronteira com a Venezuela. Com exceção do Monte Roraima $(2.734,1 \mathrm{~m})$, situado no estado de Roraima, e faz fronteira com a Guiana e Venezuela, os demais pontos mais altos do Brasil encontram-se localizados nos estado de Minas Gerais, Espírito Santos, Rio de Janeiro e São Paulo.

No Brasil, ao longo do tempo geológico, sob os efeitos climáticos variáveis ocorreram amplos conjuntos de relevos com características particulares, cujas as feições embora diversas, guardam entre si, as relações comuns com a estrutura geológica a partir do qual se formaram. Estão representadas por diversas unidades geomorfológicas, como as planícies, depressões, tabuleiros, chapadas patamares, planaltos e serras. As depressões e planaltos são as formas de relevo que representam a maior parte do território brasileiro.

Além destas características o Brasil é formado por seis grandes biomas: Amazônico, Caatinga, Cerrado, Mata Atlântica, Pampa e Pantanal. Cada bioma possui diferentes tipos de vegetação e de fauna, bem como diversos tipos de uso e desenvolvimento socioeconômico. O bioma Amazônico, possui grande destaque por abranger mais de $40 \%$ de área do território brasileiro, com cerca de 4.196.943 km2 (IBGE,2004), possui a maior bacia hidrográfica do mundo: cobre cerca de 6 milhões de $k m 2$ e tem 1.100 afluentes. Seu principal rio, o Amazonas, que nasce nos Andes, corta a região para desaguar no Oceano Atlântico, lançando ao mar cerca de $200.000 \mathrm{m3} / \mathrm{s}$, além dos demais rios que compõe a bacia, são m) and 31 de Março (2,974,2 m)

(Figure 1.3), both located in the north of Amazonas and bordering Venezuela. Except for Mount Roraima $(2,734,1 \mathrm{~m})$, situated in the state of Roraima, and bordering the French Guyana, the other high points are found in Minas Gerais, Espírito Santo, Rio de Janeiro and São Paulo.

In Brazil, throughout geologic time and under variable climate effects, there have been diverse relief groups with particular characteristics. In spite of their diverse features, they share their geological structure of origin. These are represented by several geomorphologic units, such as plains, depressions, tablelands, plateaus and mountains.

Depressions and plateaus are the most common forms of relief all over the Brazilian territory.

Besides these characteristics, Brazil is formed by six major biomes: Amazon, Caatinga, Cerrado, Atlantic Forest, Pampa and Pantanal. Each biome has a different type of vegetation and fauna, as well as different types of use and socioeconomic development. The Amazon biome is a highlight since it encompasses more than $40 \%$ of the Brazilian territory, with about 4,196,943 km2 (IBGE,2004); it has the largest water basin in the world, covering about 6 million $\mathrm{km} 2$, with more than 1,100 tributary rivers.

Amazonas, the main one, starts in the Andes and crosses the area to flow out in the Atlantic Ocean, reaching the sea at $200,000 \mathrm{~m} 3 / \mathrm{s}$. Together with other rivers that form the basin, 
responsáveis por $72 \%$ dos recursos hídricos do território brasileiro. Apesar de toda essa riqueza a Amazônia, há uma contradição, pois possui baixo índices de desenvolvimento socioeconômico, baixa densidade demográfica e aumento na urbanização em relação as demais regiões do país. Colocando-o assim, numa posição estratégica em termos de desenvolvimento econômico.

Nas demais regiões do país as características físicas, como os ventos fortes constantes, a baixa precipitação e sol o ano todo, proporcionam a região do nordeste, sobretudo o estado do Rio Grande do Norte, a produção do sal marinho, representando $95 \%$ do total de produção do país.

Nestes aspectos, devido sua posição geográfica, próximo a Linha do Equador ao norte, o Brasil recebe a influência dos ventos alísios de nordeste e sudeste, proporcionando a constância dos ventos durante todo o ano. Tal condição tem favorecido o crescente aumento de Parques Eólicos, a exemplo, a costa nordestina para a geração de energia do país.

É sobre essa base física e essa organização política-administrativa que se distribui a diversidade de uma população rica por diferenças sociais e culturais que reflete a essência da nação brasileira. A redemocratização política e os avanços na distribuição de renda, ocorridos nas últimas décadas, certamente contribuem para a democratização do território - fenômeno fundamental para a articulação do povo com o seu território. Certamente será um longo percurso, com muitas idas e vindas, mas com resultados inequivocamente positivos para o País. it is responsible for $72 \%$ of the water resources of the Brazilian territory. In spite of all its richness, the Amazon is a contradictory region: it has low levels of socioeconomic development, low demographic density and increased urbanization in comparison with other parts of the country, and may have, thus, a strategic role in terms of economic development.

Physical features, such as nonconstant strong wind, low precipitation and sunshine throughout the year make the Northeast Region, for example, especially Rio Grande do Norte, a big producer of salt, with $95 \%$ of the overall output in the country.

In this respect, due to its geographic location, close to the Equator, Brazil is subject to trade winds from the Northeast and from the Southeast, leading to constant wind blow throughout the year. This condition has favored the growing increase of Wind Farms for electricity generation, as seen, for example, on the Northeast coastline.

This physical base and politicaladministrative organization support the diversity of a population rich in social and cultural differences which reflect the essence of the Brazilian nation. The political redemocratization and the advances in income distribution, seen in the last few decades, certainly add to the democratization of the territory - a fundamental phenomenon for the articulation between people and their land. There is certainly a long way to go, a way full of ups and downs, but which will bring about undoubtedly positive results for the country. 


\section{Luís Alessandro Câmara}

Graduado em Arquitetura e Urbanismo

(UFRN/1992), Especialista em Transporte e

Tráfego Urbano (UFRN/1994), Mestre em

Desenvolvimento Urbano (UFPE/1998)

e Doutor em Desenvolvimento Urbano

(UFPE/2012). Atualmente é Professor Associado

I, lotado no Departamento de Engenharia Civil da UFRN. Entre 1999 e 2011 colaborou com o setor de Engenharia de Tráfego do DETRAN/ RN. Enquanto pesquisador atua principalmente com os temas: Mercado Imobiliário, Ciclos Imobiliários, Incorporações Imobiliárias, Transportes e Tráfego Urbano.
Bachelor in Architecture and Urban Planning, currently Associate Professor I at the Civil Engineering Department of UFRN. Between 1999 and 2011 he was a collaborator at the Traffic Engineering Sector of DETRAN/RN. As a researcher, he deals mainly with the following topics: Real Estate Market, Real Estate Cycles and Real Estate Incorporations, Transportation and Urban Traffic.

\section{Maria de Fátima Alves de Matos}

Atualmente está no Pós-Doutorado no Programa de Pós-Graduação de Engenharia Civil pela Universidade Federal do Rio Grande do Norte. Realizou o Doutorado pelo Programa de Pós-Graduação em Geodinâmica e Geofísica - PPGG, da Universidade Federal do Rio Grande do Norte - UFRN, com doutorado-sandwich no Laboratório Nacional de Engenharia Civil de Portugal - LNEC em 2010-2011. Mestre em Geodinâmica pelo Programa de Pós-Graduação em Geodinâmica e Geofísica - PPGG, pela Universidade Federal do Rio Grande do Norte - UFRN. Graduada em Licenciatura Plena e Bacharelado em Geografia pela Universidade Federal do Amapá. Atua na área de Geociências, com ênfase em Modelagem de Ondas Oceânicas, Hidrodinâmica Costeira, Sensoriamento Remoto e Geotecnologias Aplicadas.
Currently a postdoctoral student at the Graduate Program in Civil Engineering of the Federal University of Rio Grande do Norte. She got her $P h D$ degree from the Graduate Program in Geodynamics and Geophysics - PPGG - of the Federal University of Rio Grande do Norte - UFRN, having gotten a scholarship from the National Laboratory of Civil Engineering of Portugal LNEC in 2010-2011. Master in Geodynamics from the Graduate Program in Geodynamics and Geophysics - PPGG - of the Federal University of Rio Grande do Norte - UFRN. Bachelor in Teaching and in Geography from the Federal University of Amapá. Working in the field of Geosciences, with emphasis on the Modeling of Ocean Waves, Coastal Hidrodynamics, Remote Sensing and Applied Geotechnologies.

Translated by: Aline Milani Romeiro Pereira 
Tabela 1.1 - Área total do País - 2015

Table 1.1 - Total area of Brazil - 2015

(continua/to be continued)

\begin{tabular}{|c|c|c|c|}
\hline \multirow{3}{*}{$\begin{array}{c}\text { Grandes Regiões } \\
\text { e } \\
\text { Unidades da Federação/ } \\
\text { Major Regions and Federation Units }\end{array}$} & \multicolumn{3}{|c|}{$\begin{array}{l}\text { Área total/ } \\
\text { Total area }\end{array}$} \\
\hline & \multirow{2}{*}{$\begin{array}{l}\text { Absoluta } \\
\left(\mathrm{km}^{2}\right) / \\
\text { Absolute } \\
\left(\mathrm{km}^{2}\right)\end{array}$} & \multicolumn{2}{|c|}{$\begin{array}{l}\text { Relativa (\%)/ } \\
\text { Relative (\%) }\end{array}$} \\
\hline & & $\begin{array}{l}\text { Brasil/ } \\
\text { Brazil }\end{array}$ & $\begin{array}{l}\text { Regiões/ } \\
\text { Regions }\end{array}$ \\
\hline Brasil/ Brazil & 8515767,049 & 100,00 & \\
\hline Norte/North & 3853669,768 & 45,25 & 100,00 \\
\hline Rondônia & 237590,543 & 2,79 & 6,17 \\
\hline Acre & 164123,739 & 1,93 & 4,26 \\
\hline Amazonas & 1559148,890 & 18,31 & 40,46 \\
\hline Roraima & 224303,187 & 2,63 & 5,82 \\
\hline Pará & 1247954,320 & 14,65 & 32,38 \\
\hline Amapá & 142828,520 & 1,68 & 3,71 \\
\hline Tocantins & 277720,569 & 3,26 & 7,21 \\
\hline Nordeste/Northeast & 1554291,744 & 18,25 & 100,00 \\
\hline Maranhão & 331936,948 & 3,90 & 21,36 \\
\hline Piauí & 251611,932 & 2,95 & 16,19 \\
\hline Ceará & 148886,308 & 1,75 & 9,58 \\
\hline Rio Grande do Norte & 52811,126 & 0,62 & 3,40 \\
\hline Paraíba & 56469,744 & 0,66 & 3,63 \\
\hline Pernambuco & 98076,109 & 1,15 & 6,31 \\
\hline Alagoas & 27848,003 & 0,33 & 1,79 \\
\hline Sergipe & 21918,493 & 0,26 & 1,41 \\
\hline Bahia & 564733,081 & 6,63 & 36,33 \\
\hline Sudeste/Southeast & 924616,968 & 10,86 & 100,00 \\
\hline Minas Gerais & 586519,727 & 6,89 & 63,43 \\
\hline Espírito Santo & 46096,925 & 0,54 & 4,99 \\
\hline Rio de Janeiro & 43777,954 & 0,51 & 4,73 \\
\hline São Paulo & 248222,362 & 2,91 & 26,85 \\
\hline Sul/South & 576773,368 & 6,77 & 100,00 \\
\hline Paraná & 199307,945 & 2,34 & 34,56 \\
\hline Santa Catarina & 95733,978 & 1,12 & 16,60 \\
\hline Rio Grande do Sul & 281731,445 & 3,31 & 48,85 \\
\hline
\end{tabular}


Tabela 1.1 - Área total do País - 2015

Table 1.1 - Total area of Brazil - 2015

(conclusão/concluded)

\begin{tabular}{|c|c|c|c|}
\hline \multirow{3}{*}{$\begin{array}{c}\text { Grandes Regiões } \\
\text { e } \\
\text { Unidades da Federação/ } \\
\text { Major Regions and Federation Units }\end{array}$} & \multicolumn{3}{|c|}{$\begin{array}{l}\text { Área total/ } \\
\text { Total area }\end{array}$} \\
\hline & \multirow{2}{*}{$\begin{array}{l}\text { Absoluta } \\
\left(\mathrm{km}^{2}\right) / \\
\text { Absolute } \\
\left(\mathrm{km}^{2}\right)\end{array}$} & \multicolumn{2}{|c|}{$\begin{array}{c}\text { Relativa (\%)/ } \\
\text { Relative (\%) }\end{array}$} \\
\hline & & $\begin{array}{l}\text { Brasil/ } \\
\text { Brazil }\end{array}$ & $\begin{array}{l}\text { Regiões/ } \\
\text { Regions }\end{array}$ \\
\hline Centro-Oeste/Central West & 1606415,201 & 18,86 & 100,00 \\
\hline Mato Grosso do Sul & 357145,534 & 4,19 & 22,23 \\
\hline Mato Grosso & 903378,292 & 10,61 & 56,24 \\
\hline Goiás & 340111,376 & 3,99 & 21,17 \\
\hline Distrito Federal/Federal District & 5779,999 & 0,07 & 0,36 \\
\hline
\end{tabular}

Fonte/Source: IBGE, Diretoria de Geociências, Coordenação de Estruturas Territoriais, Malha Municipal 2014.

\section{Mapa 1.1 - Mapa político do Brasil Map 1.1 - Political map of Brazil}

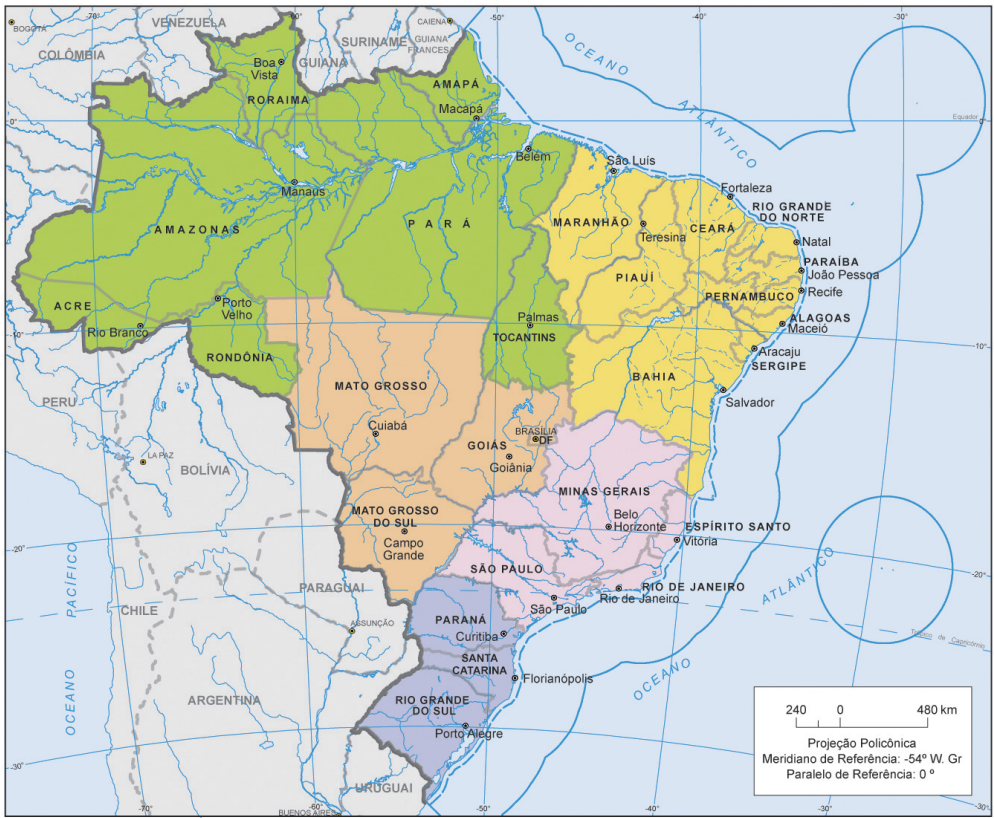

Fonte/Source: IBGE, Diretoria de Geociências. 
Tabela 1.2 - Evolução político-administrativa do País - 1940/2014

Table 1.2 - Administrative evolution of Brazil - 1940/2014

\begin{tabular}{|c|c|c|c|c|c|c|}
\hline \multirow{2}{*}{$\begin{array}{l}\text { Grandes Regiões e } \\
\text { Unidades da Federação/ } \\
\text { Major Regions and } \\
\text { Federation Units }\end{array}$} & \multicolumn{6}{|c|}{$\begin{array}{l}\text { Municípios criados e instalados (Até 01.09)/ } \\
\text { Municipalities created and installed (Until Sept. 1st) }\end{array}$} \\
\hline & $\begin{array}{c}1940 \\
(1)\end{array}$ & 1950 & 1960 & 1970 & 1980 & 1990 \\
\hline Brasil/Brazil & 1574 & 1889 & 2766 & 3952 & 3974 & 4491 \\
\hline Norte/North & 88 & 99 & 120 & 143 & 153 & 298 \\
\hline Rondônia & - & 2 & 2 & 2 & 7 & 23 \\
\hline Acre & 7 & 7 & 7 & 7 & 12 & 12 \\
\hline Amazonas & 28 & 25 & 44 & 44 & 44 & 62 \\
\hline Roraima & - & 2 & 2 & 2 & 2 & 8 \\
\hline Pará & 53 & 59 & 60 & 83 & 83 & 105 \\
\hline Amapá & - & 4 & 5 & 5 & 5 & 9 \\
\hline Tocantins & - & - & - & - & - & 79 \\
\hline Nordeste/Northeast & 584 & 609 & 903 & 1376 & 1375 & 1509 \\
\hline Maranhão & 65 & 72 & 91 & 130 & 130 & 136 \\
\hline Piauí & 47 & 49 & 71 & 114 & 114 & 118 \\
\hline Ceará & 79 & 79 & 142 & 142 & 141 & 178 \\
\hline Rio Grande do Norte & 42 & 48 & 83 & 150 & 150 & 152 \\
\hline Paraíba & 41 & 41 & 88 & 171 & 171 & 171 \\
\hline Pernambuco & 85 & 91 & 103 & 165 & 165 & (2) 168 \\
\hline Alagoas & 33 & 37 & 69 & 94 & 94 & 97 \\
\hline Sergipe & 42 & 42 & 62 & 74 & 74 & 74 \\
\hline Bahia & 150 & 150 & 194 & 336 & 336 & 415 \\
\hline Sudeste/Southeast & 641 & 845 & 1085 & 1410 & 1410 & 1432 \\
\hline Minas Gerais & 288 & 386 & 483 & 722 & 722 & 723 \\
\hline Espírito Santo & 32 & 33 & 37 & 53 & 53 & 67 \\
\hline Rio de Janeiro & 51 & 57 & 62 & 64 & 64 & 70 \\
\hline São Paulo & 270 & 369 & 503 & 571 & 571 & 572 \\
\hline Sul/South & 181 & 224 & 414 & 717 & 719 & 873 \\
\hline Paraná & 49 & 80 & 162 & 288 & 290 & 323 \\
\hline Santa Catarina & 44 & 52 & 102 & 197 & 197 & 217 \\
\hline Rio Grande do Sul & 88 & 92 & 150 & 232 & 232 & 333 \\
\hline Centro-Oeste/Central West & 80 & 112 & 244 & 306 & 317 & 379 \\
\hline Mato Grosso do Sul & - & - & - & - & 55 & 72 \\
\hline Mato Grosso & 28 & 35 & 64 & 84 & 38 & 95 \\
\hline Goiás & 52 & 77 & 179 & 221 & 223 & 211 \\
\hline Distrito Federal/Federal District & - & - & 1 & 1 & 1 & 1 \\
\hline
\end{tabular}


Tabela 1.2 - Evolução político-administrativa do País - 1940/2014

Table 1.2 - Administrative evolution of Brazil - 1940/2014

(conclusão/concluded)

\begin{tabular}{|c|c|c|c|c|c|c|}
\hline \multirow{2}{*}{$\begin{array}{l}\text { Grandes Regiões e } \\
\text { Unidades da Federação/ } \\
\text { Major Regions and } \\
\text { Federation Units }\end{array}$} & \multicolumn{6}{|c|}{$\begin{array}{c}\text { Municípios criados e instalados (Até 01.09)/ } \\
\text { Municipalities created and installed (Until Sept. 1st) }\end{array}$} \\
\hline & 2000 & 2008 & 2009 & 2010 & 2013 & 2014 \\
\hline Brasil/Brazil & 5507 & 5565 & 5565 & 5565 & 5570 & 5570 \\
\hline Norte/North & 449 & 449 & 449 & 449 & 450 & 450 \\
\hline Rondônia & 52 & 52 & 52 & 52 & 52 & 52 \\
\hline Acre & 22 & 22 & 22 & 22 & 22 & 22 \\
\hline Amazonas & 62 & 62 & 62 & 62 & 62 & 62 \\
\hline Roraima & 15 & 15 & 15 & 15 & 15 & 15 \\
\hline Pará & 143 & 143 & 143 & 143 & 144 & 144 \\
\hline Amapá & 16 & 16 & 16 & 16 & 16 & 16 \\
\hline Tocantins & 139 & 139 & 139 & 139 & 139 & 139 \\
\hline Nordeste/Northeast & 1787 & 1794 & 1794 & 1794 & 1794 & 1794 \\
\hline Maranhão & 217 & 217 & 217 & 217 & 217 & 217 \\
\hline Piauí & 221 & 224 & 224 & 224 & 224 & 224 \\
\hline Ceará & 184 & 184 & 184 & 184 & 184 & 184 \\
\hline Rio Grande do Norte & 166 & 167 & 167 & 167 & 167 & 167 \\
\hline Paraíba & 223 & 223 & 223 & 223 & 223 & 223 \\
\hline Pernambuco & (2) 185 & (2) 185 & (2) 185 & (2) 185 & (2) 185 & (2) 185 \\
\hline Alagoas & 101 & 102 & 102 & 102 & 102 & 102 \\
\hline Sergipe & 75 & 75 & 75 & 75 & 75 & 75 \\
\hline Bahia & 415 & 417 & 417 & 417 & 417 & 417 \\
\hline Sudeste/Southeast & 1666 & 1668 & 1668 & 1668 & 1668 & 1668 \\
\hline Minas Gerais & 853 & 853 & 853 & 853 & 853 & 853 \\
\hline Espírito Santo & 77 & 78 & 78 & 78 & 78 & 78 \\
\hline Rio de Janeiro & 91 & 92 & 92 & 92 & 92 & 92 \\
\hline São Paulo & 645 & 645 & 645 & 645 & 645 & 645 \\
\hline Sul/South & 1159 & 1188 & 1188 & 1188 & 1191 & 1191 \\
\hline Paraná & 399 & 399 & 399 & 399 & 399 & 399 \\
\hline Santa Catarina & 293 & 293 & 293 & 293 & 295 & 295 \\
\hline Rio Grande do Sul & 467 & 496 & 496 & 496 & 497 & 497 \\
\hline Centro-Oeste/Central West & 446 & 466 & 466 & 466 & 467 & 467 \\
\hline Mato Grosso do Sul & 77 & 78 & 78 & 78 & 79 & 79 \\
\hline Mato Grosso & 126 & 141 & 141 & 141 & 141 & 141 \\
\hline Goiás & 242 & 246 & 246 & 246 & 246 & 246 \\
\hline Distrito Federal/Federal District & 1 & 1 & 1 & 1 & 1 & 1 \\
\hline
\end{tabular}

Fonte/Source: IBGE, Diretoria de Geociências, Coordenação de Estruturas Territoriais, Banco de Estruturas Territoriais.

(1) Unidades administrativas em 01.07. (2) Inclui o Distrito Estadual de Fernando de Noronha./

(1) Administrative units on July 1st. (2) Includes the State District of Fernando de Noronha. 
Tabela 1.3 - Pontos mais altos do País - 2016

Table 1.3 - Highest points in Brazil - 2016

\begin{tabular}{|c|c|c|c|}
\hline $\begin{array}{l}\text { Topônimos/ } \\
\text { Toponyms }\end{array}$ & $\begin{array}{c}\text { Unidades da Federação/ } \\
\text { Federative Units }\end{array}$ & $\begin{array}{l}\text { Localização/ } \\
\text { Location }\end{array}$ & $\begin{array}{l}\text { Altitude }(\mathrm{m}) / \\
\text { Altitude }(\mathrm{m})\end{array}$ \\
\hline $\begin{array}{l}\text { Pico da Neblina (1) / } \\
\text { Neblina Peak (1) }\end{array}$ & Amazonas & $\begin{array}{l}\text { Serra Imeri/ } \\
\text { Imeri Range }\end{array}$ & 2995,3 \\
\hline $\begin{array}{l}\text { Pico } 31 \text { de Março (1) / } \\
31 \text { de Março Peak (1) }\end{array}$ & Amazonas (2) & $\begin{array}{l}\text { Serra Imeri/ } \\
\text { Imeri Range }\end{array}$ & 2974,2 \\
\hline $\begin{array}{l}\text { Pico da Bandeira (1) / } \\
\text { Bandeira Peak (1) }\end{array}$ & Minas Gerais/Espírito Santo & $\begin{array}{l}\text { Serra do Caparaó/ } \\
\text { Caparaó Range }\end{array}$ & 2891,3 \\
\hline $\begin{array}{l}\text { Pedra da Mina (1) / } \\
\text { Mina Rock (1) }\end{array}$ & Minas Gerais/São Paulo & $\begin{array}{l}\text { Serra da Mantiqueira/ } \\
\text { Mantiqueira Range }\end{array}$ & 2798,1 \\
\hline $\begin{array}{l}\text { Pico das Agulhas Negras (1) / } \\
\text { Agulhas Negras Peak (1) }\end{array}$ & Minas Gerais/Rio de Janeiro & $\begin{array}{l}\text { Serra do Itatiaia/ } \\
\text { Itatiaia Range }\end{array}$ & 2790,9 \\
\hline $\begin{array}{l}\text { Pico do Cristal (1) / } \\
\text { Cristal Peak (1) }\end{array}$ & Minas Gerais & $\begin{array}{l}\text { Serra do Caparaó/ } \\
\text { Caparaó Range }\end{array}$ & 2769,1 \\
\hline $\begin{array}{l}\text { Monte Roraima/ } \\
\text { Roraima Mount }\end{array}$ & Roraima (2) (3) & $\begin{array}{l}\text { Serra do Pacaraima/ } \\
\text { Pacaraima Range }\end{array}$ & 2734,1 \\
\hline $\begin{array}{l}\text { Morro do Couto/ } \\
\text { Couto Mount }\end{array}$ & Rio de Janeiro & $\begin{array}{l}\text { Serra das Prateleiras/ } \\
\text { Prateleiras Range }\end{array}$ & 2680,0 \\
\hline $\begin{array}{l}\text { Pedra do Sino de Itatiaia/ } \\
\text { Sino de Itatiaia Rock }\end{array}$ & Minas Gerais & $\begin{array}{l}\text { Serra da Mantiqueira/ } \\
\text { Mantiqueira Range }\end{array}$ & 2670,0 \\
\hline $\begin{array}{l}\text { Pico dos Três Estados/ } \\
\text { Três Estados Peak }\end{array}$ & $\begin{array}{l}\text { São Paulo/Minas Gerais/ } \\
\text { Rio de Janeiro }\end{array}$ & $\begin{array}{l}\text { Serra da Mantiqueira/ } \\
\text { Mantiqueira Range }\end{array}$ & 2665,0 \\
\hline $\begin{array}{l}\text { Pedra do Altar/ } \\
\text { Altar Rock }\end{array}$ & Rio de Janeiro & $\begin{array}{l}\text { Serra da Mantiqueira/ } \\
\text { Mantiqueira Range }\end{array}$ & 2665,0 \\
\hline
\end{tabular}

Fonte/Source: IBGE, Diretoria de Geociências, Coordenação de Cartografia, Cadastro de Pontos mais Altos do Brasil e Coordenação de Geodésia, Projeto Pontos Culminantes.

Nota: Foram considerados os pontos com altitude superior a 2500 metros./Note: Only the points over 2,500 meters were included.

(1) Projeto Pontos Culminantes, 2004. (2) Fronteira com a Venezuela. (3) Fronteira com a Guiana./

(1) Highest Points Project. (2) Venezuela border. (3) Guyana border. 


\section{Tabela 1.4 - Localização geográfica dos Municípios das Capitais e distância a Brasília - 2015}

Table 1.4 - Geographic location of the Municipalities of the Capital and distance to Brasília - 2015

\begin{tabular}{|c|c|c|c|c|}
\hline \multirow{2}{*}{$\begin{array}{c}\text { Municípios das Capitais/ } \\
\text { Municipalities of } \\
\text { the capital }\end{array}$} & \multicolumn{2}{|c|}{$\begin{array}{l}\text { Localização geográfica/ } \\
\text { Geographic location }\end{array}$} & \multicolumn{2}{|c|}{$\begin{array}{l}\text { Distância a Brasilia }(\mathrm{km}) / \\
\text { Distance to Brasília }(\mathrm{Km})\end{array}$} \\
\hline & $\begin{array}{l}\text { Latitude/ } \\
\text { Latitude }\end{array}$ & $\begin{array}{l}\text { Longitude/ } \\
\text { Longitude }\end{array}$ & $\begin{array}{l}\text { Em reta (1)/ } \\
\text { In straight (1) }\end{array}$ & $\begin{array}{l}\text { Rodoviária (2)/ } \\
\text { Road (2) }\end{array}$ \\
\hline Porto Velho (RO) & $-08^{\circ} 46^{\prime} 08^{\prime \prime}$ & $-63^{\circ} 49^{\prime} 53^{\prime \prime}$ & 1899,274 & 2589 \\
\hline Rio Branco (AC) & $-09^{\circ} 58^{\prime} 42^{\prime \prime}$ & $-67^{\circ} 48^{\prime} 38^{\prime \prime}$ & 2254,440 & 3123 \\
\hline Manaus (AM) & $-03^{\circ} 08^{\prime} 05^{\prime \prime}$ & $-60^{\circ} 01^{\prime} 24^{\prime \prime}$ & 1930,977 & 3490 \\
\hline Boa Vista (RR) & $+02^{\circ} 49^{\prime} 00^{\prime \prime}$ & $-60^{\circ} 40^{\prime} 14^{\prime \prime}$ & 2493,498 & 4275 \\
\hline Belém (PA) & $-01^{\circ} 27^{\prime} 35^{\prime \prime}$ & $-48^{\circ} 29^{\prime} 16^{\prime \prime}$ & 1586,825 & 2120 \\
\hline Macapá (AP) & $+00^{\circ} 02^{\prime} 20^{\prime \prime}$ & $-51^{\circ} 03^{\prime} 27^{\prime \prime}$ & 1785,482 & ... \\
\hline Palmas (TO) & $-10^{\circ} 09^{\prime} 48^{\prime \prime}$ & $-48^{\circ} 21^{\prime} 04^{\prime \prime}$ & 624,968 & 973 \\
\hline São Luís (MA) & $-02^{\circ} 31^{\prime} 55^{\prime \prime}$ & $-44^{\circ} 17^{\prime} 53^{\prime \prime}$ & 1518,782 & 2157 \\
\hline Teresina (PI) & $-05^{\circ} 05^{\prime} 11^{\prime \prime}$ & $-42^{\circ} 48^{\prime} 19^{\prime \prime}$ & 1308,258 & 1789 \\
\hline Fortaleza (CE) & $-03^{\circ} 43^{\prime} 26^{\prime \prime}$ & $-38^{\circ} 35^{\prime} 24^{\prime \prime}$ & 1678,967 & 2378 \\
\hline Natal (RN) & $-05^{\circ} 45^{\prime} 03^{\prime \prime}$ & $-35^{\circ} 15^{\prime} 08^{\prime \prime}$ & 1771,537 & 2422 \\
\hline João Pessoa (PB) & $-07^{\circ} 08^{\prime} 58^{\prime \prime}$ & $-34^{\circ} 52^{\prime} 24^{\prime \prime}$ & 1710,633 & 2245 \\
\hline Recife (PE) & $-08^{\circ} 03^{\prime} 46^{\prime \prime}$ & $-34^{\circ} 53^{\prime} 20^{\prime \prime}$ & 1653,087 & 2220 \\
\hline Maceió (AL) & $-09 \circ 39^{\prime} 39^{\prime \prime}$ & $-35^{\circ} 42^{\prime} 06^{\prime \prime}$ & 1486,483 & 1928 \\
\hline Aracaju (SE) & $-10^{\circ} 54^{\prime} 26^{\prime \prime}$ & $-37^{\circ} 02^{\prime} 54^{\prime \prime}$ & 1292,271 & 1652 \\
\hline Salvador (BA) & $-13^{\circ} 00^{\prime} 53^{\prime \prime}$ & $-38^{\circ} 29^{\prime} 17^{\prime \prime}$ & 1059,129 & 1446 \\
\hline Belo Horizonte (MG) & $-19^{\circ} 56^{\prime} 15^{\prime \prime}$ & $-43^{\circ} 55^{\prime} 35^{\prime \prime}$ & 621,660 & 716 \\
\hline Vitória (ES) & $-20^{\circ} 19^{\prime} 13^{\prime \prime}$ & $-40^{\circ} 19^{\prime} 20^{\prime \prime}$ & 944,449 & 1238 \\
\hline Rio de Janeiro (RJ) & $-22^{\circ} 52^{\prime} 36^{\prime \prime}$ & $-43^{\circ} 13^{\prime} 40^{\prime \prime}$ & 924,132 & 1148 \\
\hline São Paulo (SP) & $-23^{\circ} 34^{\prime} 03^{\prime \prime}$ & $-46^{\circ} 34^{\prime} 13^{\prime \prime}$ & 871,516 & 1015 \\
\hline Curitiba (PR) & $-25^{\circ} 25^{\prime} 59^{\prime \prime}$ & $-49^{\circ} 16^{\prime} 19^{\prime \prime}$ & 1076,837 & 1366 \\
\hline Florianópolis (SC) & $-27^{\circ} 35^{\prime} 16^{\prime \prime}$ & $-48^{\circ} 32^{\prime} 51^{\prime \prime}$ & 1307,669 & 1673 \\
\hline Porto Alegre (RS) & $-30^{\circ} 01^{\prime} 48^{\prime \prime}$ & $-51^{\circ} 13^{\prime} 43^{\prime \prime}$ & 1613,107 & 2027 \\
\hline Campo Grande (MS) & $-20^{\circ} 27^{\prime} 29^{\prime \prime}$ & $-54^{\circ} 36^{\prime} 57^{\prime \prime}$ & 879,221 & 1134 \\
\hline Cuiabá (MT) & $-15^{\circ} 34^{\prime} 12^{\prime \prime}$ & $-56^{\circ} 04^{\prime} 24^{\prime \prime}$ & 877,803 & 1133 \\
\hline Goiânia (GO) & $-16^{\circ} 40^{\prime} 24^{\prime \prime}$ & $-49^{\circ} 15^{\prime} 21^{\prime \prime}$ & 175,659 & 209 \\
\hline Brasília (DF) & $-15^{\circ} 47^{\prime} 39^{\prime \prime}$ & $-47^{\circ} 53^{\prime} 16^{\prime \prime}$ & - & - \\
\hline
\end{tabular}

Fonte/Source: IBGE, Diretoria de Geociências, Coordenação de Estruturas Territoriais.

(1) Coordenadas Geográficas no datum SIRGAS2000 - (Sedes Municipais) do Cadastro de Localidades Selecionadas 2010 - e a Distância a Brasília em linha reta foram obtidas através modelo elipsoidal.

(2) Dados do Departamento Nacional de Infraestrutura de Transportes - DNIT.

(1) Geographic Coordinates in datum SIRGAS 2000 - (Municipal Headquarters) of Cadastro de Localidades Selecionadas 2010 - and distance to Brasilia straight were obtained ellipsoidal model. (2) Data from the National Department of Transport Infrastructure - DNIT. 
Tabela 1.5 - Pontos extremos do País e suas distâncias - 2015

Table 1.5 - Extreme points of Brazil and their distances - 2015

\begin{tabular}{|c|c|c|c|c|}
\hline \multirow{2}{*}{$\begin{array}{c}\text { Extre-mo/ } \\
\text { Extreme } \\
\text { points }\end{array}$} & \multicolumn{2}{|c|}{$\begin{array}{l}\text { Coordenadas geográficas/ } \\
\text { Geographical coordinates }\end{array}$} & \multirow{2}{*}{$\begin{array}{l}\text { Localização/ } \\
\text { Location }\end{array}$} & \multirow{2}{*}{$\begin{array}{c}\text { Distância } \\
(\mathrm{km}) / \\
\text { Distance } \\
(\mathrm{km})\end{array}$} \\
\hline & $\begin{array}{l}\text { Latitude/ } \\
\text { Latitude }\end{array}$ & $\begin{array}{l}\text { Longitude/ } \\
\text { Longitude }\end{array}$ & & \\
\hline Norte/ & $+05^{\circ} 16^{\prime} 19^{\prime \prime}$ & $-60^{\circ} 12^{\prime} 45^{\prime \prime}$ & Nascente do rio Ailã (Uiramutã - RR)/ & \\
\hline $\begin{array}{l}\text { North } \\
\text { Sul/ }\end{array}$ & $-33^{\circ} 45^{\prime} 07^{\prime \prime}$ & $-53^{\circ} 23^{\prime} 50^{\prime \prime}$ & $\begin{array}{l}\text { Source of Ailã river (Uiramutã - RR) } \\
\text { Arroio Chuí (Santa Vitória do Palmar - RS)/ } \\
\text { Chuí Brook (Santa Vitória do Palmar - RS) }\end{array}$ & 4378,41 \\
\hline Leste/ & $-07^{\circ} 09^{\prime} 18^{\prime \prime}$ & $-34^{\circ} 47^{\prime} 34^{\prime \prime}$ & Ponta do Seixas (Cabo Branco/João Pessoa - PB)/ & \\
\hline $\begin{array}{l}\text { East } \\
\text { Oeste/ } \\
\text { West }\end{array}$ & $-07^{\circ} 32^{\prime} 09^{\prime \prime}$ & $-73^{\circ} 59^{\prime} 26^{\prime \prime}$ & $\begin{array}{l}\text { Point of Seixas (Cape Branco/João Pessoa - PB) } \\
\text { Nascente do rio Moa (Mâncio Lima - AC)/ } \\
\text { Source of Moa river (Mâncio Lima - AC) }\end{array}$ & 4326,63 \\
\hline
\end{tabular}

Fonte/Source: IBGE, Diretoria de Geociências, Coordenação de Estruturas Territoriais, Malha Municipal 2014. Nota: Coordenadas Geográficas no datum SIRGAS2000, com distâncias em linha reta obtidas através do modelo elipsoidal./Note: Geographic Coordinates in datum SIRGAS 2000 with distances in a straight line obtained from theellipsoidal model.

Mapa 1.2 - Pontos extremos e pontos mais altos do Brasil Map 1.2 - Extreme points and highest points in Brazil

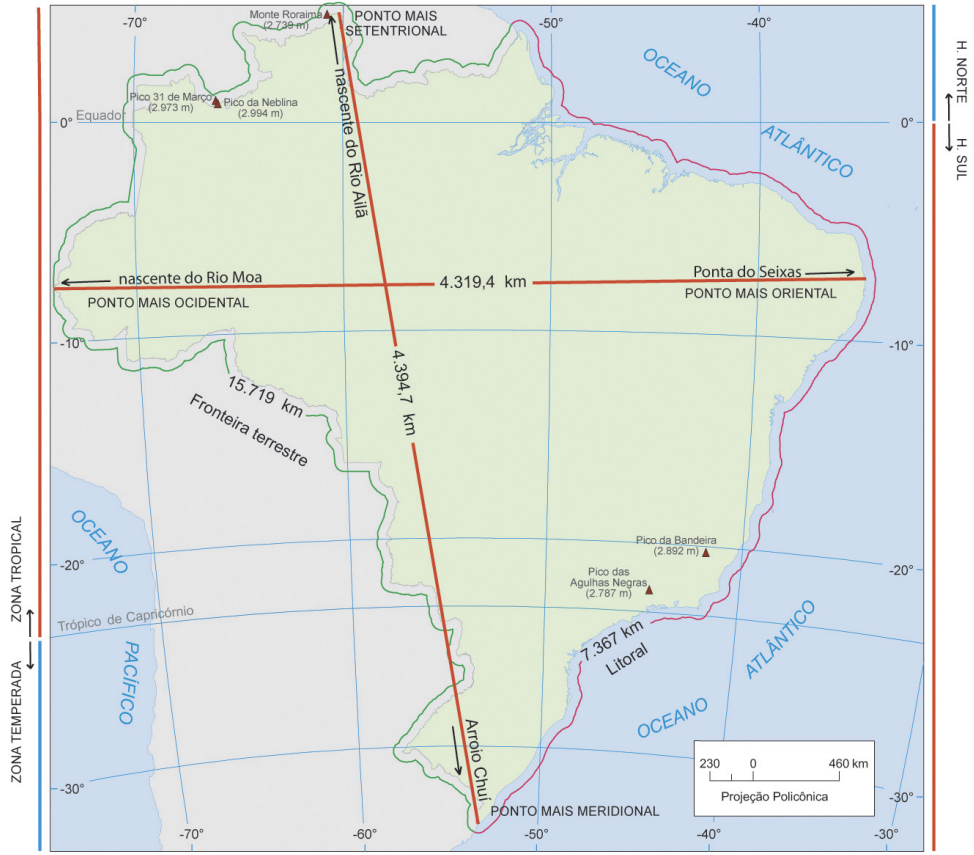

Fonte/Source: IBGE, Diretoria de Geociências. 


\section{População Population}

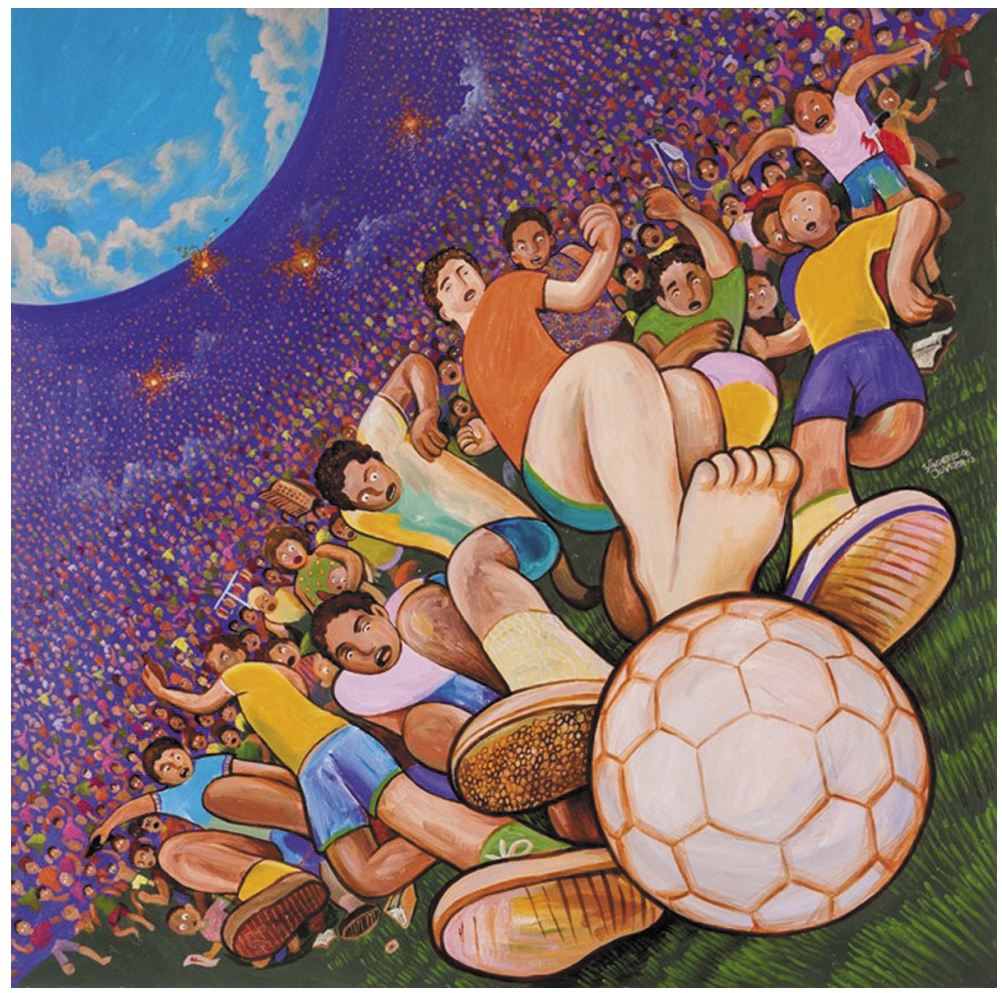

Farra da Bola/Ball-playing revelry, 2014 Wagner Di Oliveira 



\section{População}

\section{Population}

Até meados do século $X X$, grande parte dos líderes mundiais acreditava que a população dos países em desenvolvimento, incluindo o Brasil, vivenciaria a famosa explosão demográfica. Esperavam que os níveis de fecundidade fossem permanecer elevados nesses países, concomitante à redução da mortalidade, o que acarretaria em taxas de crescimento populacional muito expressivas. A maior preocupação da época era o crescimento acelerado da população mundial mais carente. Contudo, para a surpresa de muitos, esse fenômeno não se concretizou, pois, assim como a redução da mortalidade se espalhou para o resto do mundo, o declínio da fecundidade também tomou o mesmo caminho.

A redução dos níveis da fecundidade e da mortalidade vem acontecendo em ritmo diferenciado entre as nações e esse processo é conhecido como a transição demográfica, definida como a passagem de uma população jovem e quase-estável, com taxas de fecundidade e mortalidade em níveis elevados e praticamente constantes, para uma população quase-estável e mais envelhecida, com baixos
Before the first half of the 20th century, most world leaders used to believe that the population in developing countries, including Brazil, would undergo the muchdiscussed population explosion. They expected the fertility rates to remain high in those countries. Such factor, coupled with decreasing mortality, would result in very significant population growth rates. The biggest concern at that time was the accelerated growth of the world's poorest population. However, surprisingly enough, this phenomenon did not take place, since, just as decreased mortality, the decline of fertility rates spread all over the rest of the world.

The reduction in fertility and mortality rates has been progressing at different speeds among the nations. This process is known as demographic transition and consists of the change of a young, almost stable population, with high and constant fertility and mortality rates, into an older, almost stable population, with low fertility and mortality rates. Regardless the 
níveis de fecundidade e mortalidade. Independentemente do ritmo e do momento dessas mudanças, as consequências serão as mesmas: 0 aumento no tamanho da população, em ritmos decrescentes, e o envelhecimento populacional.

Esse fenômeno ocorreu primeiro em alguns países da Europa, a partir da segunda metade do século XVIII, e nas demais regiões do mundo, a partir do século XX. O Brasil vem vivenciando esse processo desde 1940, quando a mortalidade começou a declinar, como consequência da melhoria nas condições de saúde, proporcionada pelos avanços tecnológicos, melhoria do saneamento básico e a difusão da informação.

Inicialmente, as crianças foram as que mais se beneficiaram dessas melhorias, o que acarretou uma significativa redução na mortalidade infantil. Em 1940, 160 óbitos infantis aconteciam a cada 1000 nascidos vivos, em 2000, esse indicador assume um valor consideravelmente menor, 29 óbitos infantis por 1000 nascidos vivos. Nos anos 2000, a redução da Pobreza e da Extrema Pobreza proporcionada por um conjunto de políticas (política de Salário Mínimo, crescimento do emprego formal, concessão de Aposentadorias e Benefícios de Prestação Continuada e expansão do Programa Bolsa Família), contribuiu significativamente para o declínio da mortalidade a infantil. Em 2016, estima-se que apenas 13,3 óbitos infantis ocorrerão a cada 1000 nascidos vivos.

Com o passar dos anos, as melhorias da saúde vem impactando positivamente todos os grupos etários, ou seja, todas as idades se beneficiaram do declínio da mortalidade. $E$ a evolução da expectativa de vida ao nascer no Brasil evidencia pace and time of these changes, the consequences will always be the same: population growth at a decreasing pace and a population aging process.

This phenomenon firstly occurred in some European countries, starting in the second half of the 18 th century, and, in the other regions of the world, from the 20 th century on. Brazil has been undergoing this process since 1940, when mortality rates started to decline as a consequence of the improvement in health conditions due to technological advances, better sanitation and information dissemination. At first, such improvements were very beneficial to children, causing a significant reduction in infant mortality. In 1940,160 children died in each 1,000 live births; in 2000, this indicator fell considerably: 29 infant deaths in each 1,000 live births. In the 2000s, the reduction of Poverty and Extreme Poverty, made possible by a number of public policies (Minimum Wages policy, more formal jobs, granting of Retirement Pensions as well as Continuous Cash Benefits and the expansion of the "Bolsa Família" Program), has significantly contributed to the reduction of the infant mortality rate. It is estimated that just 13.3 infant deaths will occur in each 1,000 live births in 2016.

As the years go by, improvements in the health system have been causing a positive impact on all the age groups, that is to say that people of all ages took advantage of the mortality rate decline. The evolution 
tais ganhos, pois o tempo médio de vida de um brasileiro nascido vivo passou 42,7 anos, em 1940, para 75,7 anos em 2016, considerando que o nascido vivo experimentasse ao longo de toda a sua vida as taxas específicas de mortalidade do Brasil vigentes em cada um dos respectivos anos. A taxa bruta de mortalidade é um indicador que não reflete claramente o efeito do declínio da mortalidade, porque sofre efeito da distribuição etária da população. Nos anos 2000, por exemplo, os níveis de mortalidade no Brasil reduziram ao longo do tempo, mas a população foi se concentrando nas idades onde a intensidade da mortalidade era maior, consequentemente, a taxa bruta de mortalidade quase não se alterou, passou de 6,7 óbitos por mil habitantes em 2000 para 6,1 óbitos por 1000 habitantes em 2016.

A taxa de fecundidade total, que corresponde ao número médio de filhos por mulher, começou a declinar no Brasil entre 1960 e 1970 . A entrada da mulher no mercado de trabalho e a disseminação dos métodos contraceptivos entre outros motivos culminaram na redução do número de filhos por mulher. Em 1960 a taxa de fecundidade total brasileira era de 6,28 filhos por mulher e em 2006 já havia alcançado a taxa de 2,04 filhos por mulher, nível abaixo da reposição que no Brasil é de 2,1 filhos por mulher. Estima-se que em 2016, a média de filhos por mulher seja de 1,69 . No ano de 2015 , surgiu a hipótese de que o zika vírus tenha alguma associação com a microcefalia. Esse fato fez com que muitos países latino-americanos, incluindo o Brasil, os governos recomendassem o adiamento da gravidez. Potencialmente, essa of life expectancy at birth in Brazil demonstrates this advance, since the average lifespan of a Brazilian born alive infant went from 42.7, in 1940 , to 75.7 , in 2016 , considering that the born alive infant would undergo the specific mortality rates in force in Brazil in each of those years, respectively. As it suffers the impact of the age distribution of the population, the gross mortality rate does not clearly reflect the results generated by decreased mortality. In the 2000 s, for instance, the mortality trends of Brazil decreased along time, but the population was concentrated in the age group where the mortality levels were higher. As a consequence, the gross mortality rate almost did not change: it went from 6.7 deaths per thousand inhabitants in 2000 to 6.1 deaths per 1,000 inhabitants in 2016 .

The total fertility rate, which corresponds to the average number of children per women, started to decline in Brazil between 1960 and 1970. The entry of woman in the labor market and the dissemination of contraceptive methods, among other factors, culminated in the reduction of the number of children per woman. In 1960, the total fertility rate in Brazil was 6.28 children per woman, whereas, in 2006, it reached 2.04 children per woman - below the replacement rate, which, in Brazil, is 2.1 children per woman. In 2016 , the estimated average number of children per woman is 1.69. In 2015, the hypothesis that the Zika virus would be linked to microcephaly was brought up. Such association has led authorities in many Latin-American countries, including Brazil, to recommend that women postpone pregnancy. This 
recomendação aliada ao medo da microcefalia pode ter afetado ainda mais os níveis da fecundidade brasileira.

O processo de urbanização no Brasil ocorreu concomitante às mudanças na mortalidade e na fecundidade, contribuindo para o declínio de ambas. A concentração das pessoas nos centros urbanos permitiu que a informação fosse difundida de forma mais rápida, tanto no que diz respeito aos cuidados com a saúde, como em relação aos métodos contraceptivos. Além disso, os centros urbanos concentraram e ainda concentram os serviços de saúde e incorporam a mulher no mercado de trabalho. De acordo com o Censo Demográfico de 2010 , mais de $84 \%$ da população brasileira já residem em áreas urbanas, porém essa proporção não é homogênea entre as grandes regiões brasileiras. Nas regiões Norte e Nordeste, cerca de $73 \%$ da população residem em centros urbanos, ao passo que no Sudeste essa percentagem é de $92,95 \%$. Apesar das disparidades, pode ser afirmar que em todas as regiões brasileiras a proporção de pessoas residindo nas cidades é mais elevada do que no campo.

Em decorrência das mudanças nas trajetórias da mortalidade e da fecundidade, a população brasileira experimentou oscilações significativas na taxa de crescimento populacional. Entre 1940 e 1960, a redução da taxa bruta mortalidade concomitante a manutenção dos níveis da natalidade fizeram a população brasileira alcançar uma taxa de crescimento de $3 \%$ ao ano. A partir da década de 1970, a população brasileira continuou a crescer, embora em ritmo declinante, devido à redução rápida e generalizada do nível da fecundidade. recommendation, coupled with the fear of microcephaly, has probably affected even more the fertility levels in Brazil.

The urbanization process in Brazil occurred together with the trend changes in mortality and fertility rates, contributing, thus, to the reduction of both. The population concentration in urban centers made way for faster information dissemination, either concerning health care or contraceptive methods. Besides, urban centers used to concentrate and still do concentrate health services and have a lot of women in the workforce. According to the 2010 Population Census, more than $84 \%$ of the Brazilian population lives in urban areas; however, this proportion is not the same among the Major Regions in Brazil. In the North and Northeast Regions, nearly $73 \%$ of the population lives in urban centers, whereas, in the Southeast, this percentage is $92.95 \%$. Despite this gap, it could be said that the proportion of people living in the cities is higher than in the countryside in all Major Regions.

Due to the changes in the mortality and fertility trends, the Brazilian population has undergone significant oscillations in the growth rate. Between 1940 and 1960, the reduction of the gross mortality rate together with the stable levels of fertility made the Brazilian population reach a $3 \%$ growth rate per year. From the 1970 s on, the Brazilian population kept on growing, though at a declining pace because of the fast and generalized reduction of the fertility rate. However, the gross 
Entretanto, a taxa bruta de natalidade não reduziu em proporção semelhante ao que ocorreu com a fecundidade, uma vez que um grande número de mulheres de coortes anteriores à queda da fecundidade, ainda se encontravam em idade reprodutiva. Consequentemente, as taxas de crescimento correntes não sofreram por completo o impacto do declínio da fecundidade. Na década de 1970, a taxa de fecundidade total (TFT) caiu 25,9\%, ao passo que a taxa de crescimento teve a redução de apenas 14\%. Um declínio mais significativo de $22 \%$, na taxa de crescimento foi observado somente no período entre 1980 e 1991. A população brasileira continuou vivenciando um crescimento positivo, porém desacelerado. Entre os censos de 2000 e 2010, a taxa de crescimento anual foi de $1,17 \%$.

Como a redução da mortalidade e da fecundidade não ocorreu no mesmo ritmo e momento em todas as regiões brasileiras, as taxas de crescimento dos estados e regiões são variadas. De acordo com o Censo Demográfico de 2010, as taxas de crescimento das regiões Norte e Centro-Oeste foram as maiores, 2,09\% e $1,91 \%$ ao ano, respectivamente. A Região Sul foi a que apresentou a menor taxa de crescimento, 0,87\% ao ano, seguida pela região Sudeste, 1,05\% ao ano, e pela Região Nordeste, $1,07 \%$ ao ano. È importante ressaltar que, como no Brasil a migração interna ainda é significativa, as diferenças regionais das taxas de crescimento também são influenciadas pelos fluxos migratórios.

A redução dos níveis de mortalidade e fecundidade também afetaram diretamente a estrutura etária da população brasileira, tendo como principal consequência o envelhecimento birth rate did not decline at the same proportion as fertility, since a great number of women of cohorts prior to the fertility drop were still at a reproductive age. As a consequence, the current growth rates were not completely affected by the impact of decreasing fertility. In the 1970s, the total fertility rate (TFR) fell $25.9 \%$, while the population growth rate had just a $14 \%$ reduction. A more significant decline of $22 \%$ in the growth rate was only to be seen in the period between 1980 and 1991. Then, the Brazilian population still underwent a positive growth, but decelerated. Between the 2000 and the 2010 Census, the annual growth rate was $1.17 \%$.

As the reduction of the mortality and fertility rates did not occur at the same pace and time in all the Brazilian Regions, the growth rates vary among the states and Regions. According to the 2010 Population Census, the growth rates of the North and Central-West Regions were the highest ones, $2.09 \%$ and $1.91 \%$ per year, respectively. The South Region had the lowest growth rate, $0.87 \%$ per year, followed by the Southeast Region, $1.05 \%$ per year, and by the Northeast Region, $1.07 \%$ per year. It is important to highlight that in Brazil the regional differences in the growth rates are also influenced by the migration flows, as the internal migration is still significant here.

The reduction of the mortality and fertility rates has also directly affected the age structure of the Brazilian population, having as its main consequence the aging of the 
populacional. Nesse processo, o declínio da fecundidade teve um impacto maior sobre a estrutura etária se comparado à queda da mortalidade, pois afetou continuamente a base da pirâmide, reduzindo a participação relativa das crianças e aumentando a participação das demais idades. Portanto, ao contrário de que muitos imaginam, os efeitos da mortalidade foram secundários para o processo de envelhecimento populacional, sendo o declínio da fecundidade o grande protagonista. Mas no futuro próximo, se a fecundidade se mantiver constante e a redução da mortalidade se concentrar nas idades avançadas, as quais ainda tem potencial para cair, a mortalidade poderá assumir o papel de principal agente envelhecedor para a estrutura etária brasileira.

Grande parte das demandas de uma população é determinada por sua composição etária e por sexo. No contexto de 1940-1991, quando a população brasileira se concentrava nas idades infantis e jovens, as maiores demandas eram na educação e saúde, uma vez que essa população necessitava de um número maior de vagas nas escolas e creches, bem como necessitava de maiores cuidados com a saúde para sobreviver aos elevados riscos de morte nas idades infantis. Atualmente, a população se concentra nas idades jovens e adultas, apresentando maiores demandas relacionadas à habitação, à capacitação dos jovens para o mercado de trabalho e à empregabilidade dessa mão de obra em abundância.

$\mathrm{Na}$ verdade, estamos vivenciando um momento bastante oportuno para um maior desenvolvimento econômico do país, conhecido como a janela de population. In this process, the fertility drop had a greater impact on the age structure than the mortality drop, for the former continuously affects the basis of the pyramid, reducing the relative participation of children and increasing the participation of the other age groups. Therefore, contrary to what many believe, the effects of mortality were secondary in the population aging process, since the fertility decline has played the main part. Nevertheless, in the near future, if fertility keeps stable and the reduction of mortality concentrates at the older ages, where it still holds the potential to decrease, mortality might take over the role of main aging agent in the Brazilian age structure.

Most of the population demands are set by its age and sex composition. In the context of 1940-1991, when the Brazilian population used to concentrate at age ranges of children and youngsters, the greatest demands were education and health, since that population needed a greater number of schools and daycare centers, as well as effective health care systems to survive the high death risks at infant age. Nowadays, the population concentrates at the age brackets of youngsters and adults, with more demands related to housing, capacity building programs for youngsters entering the labor market and the employability of the abundant workforce.

As a matter of fact, this time we are living gives us the chance to enhance the economic development of the country - the so-called window of 
oportunidades. A maior proporção de pessoas em idade economicamente ativa em relação às crianças e idosos se apresenta como um bônus demográfico capaz de elevar a produtividade e a riqueza da nação, se as políticas públicas foram adequadamente direcionadas. Investir na qualidade da educação das gerações futuras, as quais têm sido cada vez menores, também é uma política essencial para o aproveitamento desse bônus, pois vai garantir um capital humano qualificado capaz de contribuir para o aumento da produtividade do país. Infelizmente, até o momento aproveitamos muito pouco dessa janela de oportunidades, que se fechará quando a população entre 15 e 64 anos de idade começar a reduzir, fenômeno previsto para o ano de 2020.A previsão é de que em 2050 quase $30 \%$ da população brasileira esteja com mais de 60 anos de idade. Portanto, tendemos para uma população envelhecida, que deverá vivenciar taxas de crescimento negativas, cujas demandas se concentrarão na saúde e na previdência. Nesse sentido, é de extrema importância o planejamento de políticas públicas desde o atual momento, para que possamos enfrentar os desafios futuros da melhor forma possível. opportunity. The high proportion of economically active population in relation to children and the elderly represents a demographic dividend capable of increasing the nation's productivity and wealth, if the public policies are adequately managed. Investing in quality education for the future generations, which tend to gradually decrease, is also an essential way of profiting from this dividend, since it will guarantee a skilled workforce, able to contribute to the productivity increase in the country. Unfortunately, up to the moment, we have not taken much advantage of this window of opportunity, which will close when the population aged between 15 and 65 starts to decrease - a phenomenon expected for the year 2020 . The forecast is that, by the year 2050 , almost $30 \%$ of the Brazilian population will be older than 60 years of age. Therefore, we tend to become an aged population, undergoing negative growth rates and with demands concentrated on health and social security. In this context, planning public policies today is extremely relevant in order to enable us to face tomorrow's challenges in the best way we can.

Luana Junqueira Dias Myrrha

Atuária pela Universidade Federal de Minas Gerais (UFMG)

Mestre e Doutora em Demografia pela UFMG Coordenadora da graduação em Ciências Atuariais e Professora do Programa de PósGraduação em Demografia da Universidade Federal do Rio Grande do Norte (UFRN).
Actuary from the Federal University of Minas Gerais (UFMG)

Master and PhD in Demography from UFMG Coordinator of the Actuarial Sciences School and Professor in the Postgraduate Program in Demography of the Federal University of Rio Grande do Norte (UFRN). 


\section{Gráfico 2.1 - Composição relativa da população residente, por sexo e grupos de idade - 1991/2010}

Graph 2.1 - Relative composition of the resident population, by sex and age groups - 1991/2010

80 anos e mais/ 80 years and over 75 a 79 anos $/ 75$ to 79 years old 70 a 74 anos/70 to 74 years old 65 a 69 anos $/ 65$ to 69 years old 60 a 64 anos/ 60 to 64 years old 55 a 59 anos $/ 55$ to 59 years old 50 a 54 anos $/ 50$ to 54 years old 45 a 49 anos $/ 45$ to 49 years old 40 a 44 anos $/ 40$ to 44 years old 35 a 39 anos/ 35 to 39 years old 30 a 34 anos/ 30 to 34 years old 25 a 29 anos/ 25 to 29 years old 20 a 24 anos/20 to 24 years old 15 a 19 anos/15 to 19 years old 10 a 14 anos $/ 10$ to 14 years old 5 a 9 anos $/ 5$ to 9 years old 0 a 4 anos $/ 0$ to 4 years old

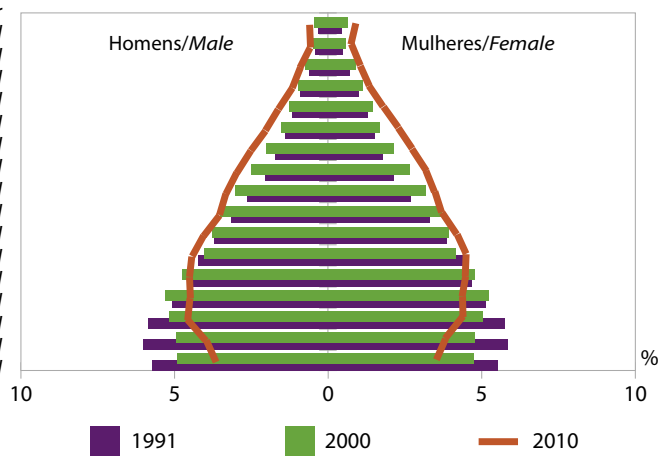

Fonte/Source: IBGE, Censo Demográfico 1991/2010.

\section{Gráfico 2.2 - Projeção da população - 2000/2020 Graph 2.2 - Population projections - 2000/2020}

80 anos e mais/ 80 years and over 75 a 79 anos $/ 75$ to 79 years old 70 a 74 anos $/ 70$ to 74 years old 65 a 69 anos/65 to 69 years old 60 a 64 anos/ 60 to 64 years old 55 a 59 anos $/ 55$ to 59 years old 50 a 54 anos $/ 50$ to 54 years old 45 a 49 anos $/ 45$ to 49 years old 40 a 44 anos $/ 40$ to 44 years old 35 a 39 anos $/ 35$ to 39 years old 30 a 34 anos $/ 30$ to 34 years old 25 a 29 anos $/ 25$ to 29 years old 20 a 24 anos $/ 20$ to 24 years old 15 a 19 anos $/ 15$ to 19 years old 10 a 14 anos/ 10 to 14 years old 5 a 9 anos $/ 5$ to 9 years old 0 a 4 anos $/ 0$ to 4 years old

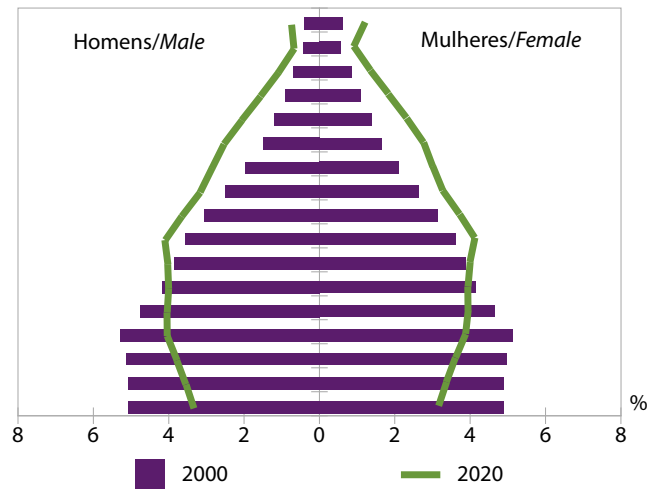

Fonte/Source: IBGE, Diretoria de Pesquisas, Coordenação de População e Indicadores Sociais, Projeção da População do Brasil por Sexo e Idade para o Período 2000-2060 - Revisão 2013. 


\section{Gráfico 2.3 - Esperança de vida ao nascer - 1930/2016}

Graph 2.3 - Life expectancy at birth - 1930/2016

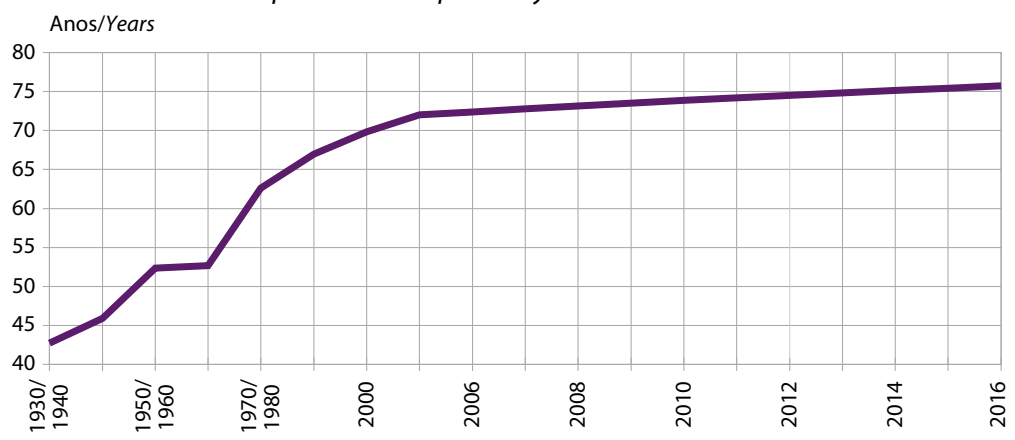

Fonte/Source: IBGE, Diretoria de Pesquisas, Coordenação de População e Indicadores Sociais, Tábuas Construídas e Projeção da População do Brasil por Sexo e Idade para o Período 2000-2060 - Revisão 2013.

\section{Gráfico 2.4 - Taxas brutas de natalidade e mortalidade - 2000-2016 Graph 2.4 - Crude birth and death rates - 2000-2016}

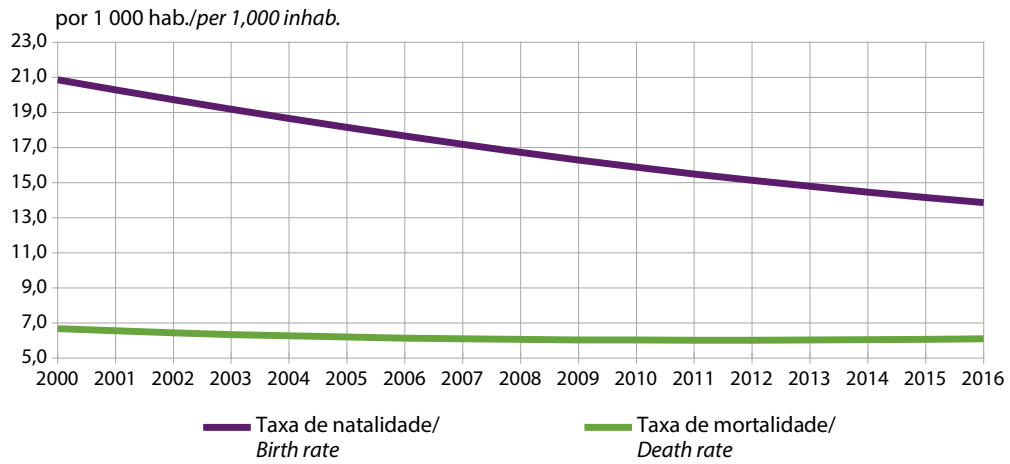

Fonte/Source: IBGE, Diretoria de Pesquisas, Coordenação de População e Indicadores Sociais, Projeção da População do Brasil por Sexo e Idade para o Período 2000-2060 - Revisão 2013. 
Tabela 2.1 - População residente, por situação do domicílio e sexo - 2010

Table 2.1 - Resident population, by urban/rural housing unit and sex - 2010

(continua/to be continued)

\begin{tabular}{|c|c|c|c|c|c|}
\hline \multirow{3}{*}{$\begin{array}{l}\text { Grandes Regiões } \\
\text { e } \\
\text { Unidades da Federação/ } \\
\text { Major Regions and } \\
\text { Federation Units }\end{array}$} & \multicolumn{5}{|c|}{ População residente/ Resident population } \\
\hline & \multirow{2}{*}{$\begin{array}{l}\text { Total/ } \\
\text { Total }\end{array}$} & \multicolumn{2}{|c|}{$\begin{array}{c}\text { Situação do domicílio/ } \\
\text { Housing unit }\end{array}$} & \multicolumn{2}{|c|}{$\begin{array}{c}\text { Sexo/ } \\
\text { Sex }\end{array}$} \\
\hline & & $\begin{array}{l}\text { Urbana/ } \\
\text { Urban }\end{array}$ & $\begin{array}{l}\text { Rural/ } \\
\text { Rural }\end{array}$ & $\begin{array}{c}\text { Homens/ } \\
\text { Male }\end{array}$ & $\begin{array}{l}\text { Mulheres/ } \\
\text { Female }\end{array}$ \\
\hline $\begin{array}{l}\text { Brasil/ } \\
\text { Brazil }\end{array}$ & 190755799 & 160925804 & 29829995 & 93406990 & 97348809 \\
\hline $\begin{array}{l}\text { Norte/ } \\
\text { North }\end{array}$ & 15864454 & 11664509 & 4199945 & 8004915 & 7859539 \\
\hline Rondônia & 1562409 & 1149180 & 413229 & 795157 & 767252 \\
\hline Acre & 733559 & 532279 & 201280 & 368324 & 365235 \\
\hline Amazonas & 3483985 & 2755490 & 728495 & 1753179 & 1730806 \\
\hline Roraima & 450479 & 344859 & 105620 & 228859 & 221620 \\
\hline Pará & 7581051 & 5191559 & 2389492 & 3821837 & 3759214 \\
\hline Amapá & 669526 & 601036 & 68490 & 335135 & 334391 \\
\hline Tocantins & 1383445 & 1090106 & 293339 & 702424 & 681021 \\
\hline $\begin{array}{l}\text { Nordeste/ } \\
\text { Northeast }\end{array}$ & 53081950 & 38821258 & 14260692 & 25909046 & 27172904 \\
\hline Maranhão & 6574789 & 4147149 & 2427640 & 3261515 & 3313274 \\
\hline Piauí & 3118360 & 2050959 & 1067401 & 1528422 & 1589938 \\
\hline Ceará & 8452381 & 6346569 & 2105812 & 4120088 & 4332293 \\
\hline Rio Grande do Norte & 3168027 & 2464991 & 703036 & 1548887 & 1619140 \\
\hline Paraíba & 3766528 & 2838678 & 927850 & 1824379 & 1942149 \\
\hline Pernambuco & 8796448 & 7052210 & 1744238 & 4230681 & 4565767 \\
\hline Alagoas & 3120494 & 2297860 & 822634 & 1511767 & 1608727 \\
\hline Sergipe & 2068017 & 1520366 & 547651 & 1005041 & 1062976 \\
\hline Bahia & 14016906 & 10102476 & 3914430 & 6878266 & 7138640 \\
\hline
\end{tabular}


Tabela 2.1 - População residente, por situação do domicílio e sexo - 2010

Table 2.1 - Resident population, by urban/rural housing unit and sex - 2010

(conclusão/concluded)

\begin{tabular}{|c|c|c|c|c|c|}
\hline \multirow{3}{*}{$\begin{array}{l}\text { Grandes Regiões } \\
\text { e } \\
\text { Unidades da Federação/ } \\
\text { Major Regions and } \\
\text { Federation Units }\end{array}$} & \multicolumn{5}{|c|}{ População residente/ Resident population } \\
\hline & \multirow{2}{*}{$\begin{array}{l}\text { Total/ } \\
\text { Total }\end{array}$} & \multicolumn{2}{|c|}{$\begin{array}{c}\text { Situação do domicílio/ } \\
\text { Housing unit }\end{array}$} & \multicolumn{2}{|c|}{$\begin{array}{l}\text { Sexo/ } \\
\text { Sex }\end{array}$} \\
\hline & & $\begin{array}{l}\text { Urbana/ } \\
\text { Urban }\end{array}$ & $\begin{array}{l}\text { Rural/ } \\
\text { Rural }\end{array}$ & $\begin{array}{c}\text { Homens/ } \\
\text { Male }\end{array}$ & $\begin{array}{l}\text { Mulheres/ } \\
\text { Female }\end{array}$ \\
\hline $\begin{array}{l}\text { Sudeste/ } \\
\text { Southeast }\end{array}$ & 80364410 & 74696178 & 5668232 & 39076647 & 41287763 \\
\hline Minas Gerais & 19597330 & 16715216 & 2882114 & 9641877 & 9955453 \\
\hline Espírito Santo & 3514952 & 2931472 & 583480 & 1731218 & 1783734 \\
\hline Rio de Janeiro & 15989929 & 15464239 & 525690 & 7625679 & 8364250 \\
\hline São Paulo & 41262199 & 39585251 & 1676948 & 20077873 & 21184326 \\
\hline $\begin{array}{l}\text { Sul/ } \\
\text { South }\end{array}$ & 27386891 & 23260896 & 4125995 & 13436411 & 13950480 \\
\hline Paraná & 10444526 & 8912692 & 1531834 & 5130994 & 5313532 \\
\hline Santa Catarina & 6248436 & 5247913 & 1000523 & 3100360 & 3148076 \\
\hline Rio Grande do Sul & 10693929 & 9100291 & 1593638 & 5205057 & 5488872 \\
\hline $\begin{array}{l}\text { Centro-Oeste/ } \\
\text { Central-West }\end{array}$ & 14058094 & 12482963 & 1575131 & 6979971 & 7078123 \\
\hline Mato Grosso do Sul & 2449024 & 2097238 & 351786 & 1219928 & 1229096 \\
\hline Mato Grosso & 3035122 & 2482801 & 552321 & 1549536 & 1485586 \\
\hline Goiás & 6003788 & 5420714 & 583074 & 2981627 & 3022161 \\
\hline $\begin{array}{l}\text { Distrito Federal } \\
\text { Federal District }\end{array}$ & 2570160 & 2482210 & 87950 & 1228880 & 1341280 \\
\hline
\end{tabular}

Fonte/Source: IBGE, Censo Demográfico 2010. 
Tabela 2.2 - Indicadores demográficos - 2010

Table 2.2 - Demographic indicators - 2010

(continua/to be continued)

\begin{tabular}{|c|c|c|c|c|}
\hline $\begin{array}{l}\text { Grandes Regiões } \\
\text { e } \\
\text { Unidades da } \\
\text { Federação/ } \\
\text { Major Regions and } \\
\text { Federation Units }\end{array}$ & $\begin{array}{l}\text { Taxa de } \\
\text { urbani- } \\
\text { zação } \\
(\%) / \\
\text { Urbanization } \\
\text { rate (\%) }\end{array}$ & $\begin{array}{c}\text { Taxa média } \\
\text { geométrica de } \\
\text { crescimento } \\
\text { anual } \\
(2000 / 2010) / \\
\text { Average geometric } \\
\text { rate of annual } \\
\text { Increase } \\
(2000 / 2010)\end{array}$ & $\begin{array}{c}\text { Densidade } \\
\text { demográfica } \\
\left(\text { hab./ } \mathrm{km}^{2}\right) / \\
\text { Demographic } \\
\text { density } \\
\text { (inhab. } / \mathrm{km}^{2} \text { ) }\end{array}$ & $\begin{array}{l}\text { Coeficiente } \\
\text { de } \\
\text { mascu- } \\
\text { linidade/ } \\
\text { Ratio of } \\
\text { males to } \\
\text { females }\end{array}$ \\
\hline $\begin{array}{l}\text { Brasil/ } \\
\text { Brazil }\end{array}$ & 84,36 & 1,17 & 22,43 & 95,95 \\
\hline $\begin{array}{l}\text { Norte/ } \\
\text { North }\end{array}$ & 73,53 & 2,09 & 4,12 & 101,85 \\
\hline Rondônia & 73,55 & 1,25 & 6,58 & 103,64 \\
\hline Acre & 72,56 & 2,78 & 4,47 & 100,85 \\
\hline Amazonas & 79,09 & 2,16 & 2,23 & 101,29 \\
\hline Roraima & 76,55 & 3,34 & 2,01 & 103,27 \\
\hline Pará & 68,48 & 2,04 & 6,07 & 101,67 \\
\hline Amapá & 89,77 & 3,45 & 4,69 & 100,22 \\
\hline Tocantins & 78,80 & 1,80 & 4,98 & 103,14 \\
\hline $\begin{array}{l}\text { Nordeste/ } \\
\text { Northeast }\end{array}$ & 73,13 & 1,07 & 34,15 & 95,35 \\
\hline Maranhão & 63,08 & 1,52 & 19,81 & 98,44 \\
\hline Piauí & 65,77 & 0,93 & 12,40 & 96,13 \\
\hline Ceará & 75,09 & 1,30 & 56,76 & 95,10 \\
\hline Rio Grande do Norte & 77,81 & 1,33 & 59,99 & 95,66 \\
\hline Paraíba & 75,37 & 0,90 & 66,70 & 93,94 \\
\hline Pernambuco & 80,17 & 1,06 & 89,63 & 92,66 \\
\hline Alagoas & 73,64 & 1,01 & 112,33 & 93,97 \\
\hline Sergipe & 73,52 & 1,49 & 94,35 & 94,55 \\
\hline Bahia & 72,07 & 0,70 & 24,82 & 96,35 \\
\hline
\end{tabular}


Tabela 2.2 - Indicadores demográficos - 2010

Table 2.2 - Demographic indicators - 2010

(conclusão/concluded)

\begin{tabular}{|c|c|c|c|c|}
\hline $\begin{array}{l}\text { Grandes Regiões } \\
\text { e } \\
\text { Unidades da } \\
\text { Federação/ } \\
\text { Major Regions and } \\
\text { Federation Units }\end{array}$ & $\begin{array}{l}\text { Taxa de } \\
\text { urbani- } \\
\text { zação } \\
\text { (\%)/ } \\
\text { Urbanization } \\
\text { rate (\%) }\end{array}$ & $\begin{array}{c}\text { Taxa média } \\
\text { geométrica de } \\
\text { crescimento } \\
\text { anual } \\
(2000 / 2010) / \\
\text { Average geometric } \\
\text { rate of annual } \\
\text { Increase } \\
\text { (2000/2010) }\end{array}$ & $\begin{array}{c}\text { Densidade } \\
\text { demográfica } \\
\left(\text { hab. } / \mathrm{km}^{2}\right) / \\
\text { Demographic } \\
\text { density } \\
\text { (inhab. } / \mathrm{km}^{2} \text { ) }\end{array}$ & $\begin{array}{c}\text { Coeficiente } \\
\text { de } \\
\text { mascu- } \\
\text { linidade/ } \\
\text { Ratio of } \\
\text { males to } \\
\text { females }\end{array}$ \\
\hline $\begin{array}{l}\text { Sudeste/ } \\
\text { Southeast }\end{array}$ & 92,95 & 1,05 & 86,92 & 94,64 \\
\hline Minas Gerais & 85,29 & 0,91 & 33,41 & 96,85 \\
\hline Espírito Santo & 83,40 & 1,27 & 76,25 & 97,06 \\
\hline Rio de Janeiro & 96,71 & 1,06 & 365,23 & 91,17 \\
\hline São Paulo & 95,94 & 1,09 & 166,25 & 94,78 \\
\hline $\begin{array}{l}\text { Sul/ } \\
\text { South }\end{array}$ & 84,93 & 0,87 & 48,58 & 96,32 \\
\hline Paraná & 85,33 & 0,89 & 52,40 & 96,56 \\
\hline Santa Catarina & 83,99 & 1,55 & 65,29 & 98,48 \\
\hline Rio Grande do Sul & 85,10 & 0,49 & 39,79 & 94,83 \\
\hline $\begin{array}{l}\text { Centro-Oeste/ } \\
\text { Central-West }\end{array}$ & 88,80 & 1,91 & 8,75 & 98,61 \\
\hline Mato Grosso do Sul & 85,64 & 1,66 & 6,86 & 99,25 \\
\hline Mato Grosso & 81,80 & 1,94 & 3,36 & 104,30 \\
\hline Goiás & 90,29 & 1,84 & 17,65 & 98,66 \\
\hline Distrito Federal/Federal District & 96,58 & 2,28 & 444,07 & 91,62 \\
\hline
\end{tabular}

Fonte/Source: IBGE, Censo Demográfico 2010. 
Tabela 2.3 - Projeções de população e taxas - 2000-2017

Table 2.3 - Population projections and rates - 2000-2017

\begin{tabular}{|c|c|c|c|c|c|c|}
\hline $\begin{array}{l}\text { Ano/ } \\
\text { Year }\end{array}$ & $\begin{array}{l}\text { População/ } \\
\text { Population }\end{array}$ & $\begin{array}{c}\text { Taxa } \\
\text { bruta } \\
\text { de natalidade } \\
\text { (por } 1000 \text { hab.)/ } \\
\text { Crude live } \\
\text { birth rate } \\
\text { (per 1,000inhab.) }\end{array}$ & $\begin{array}{c}\text { Taxa } \\
\text { bruta } \\
\text { de mortalidade } \\
\text { (por } 1000 \text { hab.)/ } \\
\text { Crude } \\
\text { death rate } \\
\text { (per 1,000inhab.) }\end{array}$ & $\begin{array}{l}\text { Esperança } \\
\text { de vida } \\
\text { ao nascer/ } \\
\text { Expectation } \\
\text { of life } \\
\text { at birth }\end{array}$ & $\begin{array}{c}\text { Taxa de } \\
\text { mortalidade } \\
\text { infantil } \\
\text { (por } 1000 \\
\text { nascidos } \\
\text { vivos)/ Infant } \\
\text { mortality rate } \\
\text { (per 1,000 } \\
\text { live births) }\end{array}$ & $\begin{array}{l}\text { Taxa } \\
\text { de } \\
\text { fecundi- } \\
\text { dade } \\
\text { total/ } \\
\text { Total } \\
\text { fertility } \\
\text { rate }\end{array}$ \\
\hline 2000 & 173448346 & 20,86 & 6,67 & 69,83 & 29,02 & 2,39 \\
\hline 2001 & 175885229 & 20,28 & 6,56 & 70,28 & 27,48 & 2,32 \\
\hline 2002 & 178276128 & 19,73 & 6,45 & 70,73 & 26,04 & 2,26 \\
\hline 2003 & 180619108 & 19,19 & 6,35 & 71,16 & 24,68 & 2,20 \\
\hline 2004 & 182911487 & 18,66 & 6,27 & 71,58 & 23,39 & 2,14 \\
\hline 2005 & 185150806 & 18,15 & 6,20 & 71,99 & 22,18 & 2,09 \\
\hline 2006 & 187335137 & 17,65 & 6,14 & 72,39 & 21,04 & 2,04 \\
\hline 2007 & 189462755 & 17,18 & 6,10 & 72,77 & 19,98 & 1,99 \\
\hline 2008 & 191532439 & 16,72 & 6,07 & 73,15 & 18,99 & 1,95 \\
\hline 2009 & 193543969 & 16,29 & 6,05 & 73,51 & 18,07 & 1,91 \\
\hline 2010 & 195497797 & 15,88 & 6,03 & 73,86 & 17,22 & 1,87 \\
\hline 2011 & 197397018 & 15,50 & 6,02 & 74,20 & 16,43 & 1,83 \\
\hline 2012 & 199242462 & 15,13 & 6,03 & 74,52 & 15,69 & 1,80 \\
\hline 2013 & 201032714 & 14,79 & 6,04 & 74,84 & 15,02 & 1,77 \\
\hline 2014 & 202768562 & 14,47 & 6,06 & 75,14 & 14,40 & 1,74 \\
\hline 2015 & 204450649 & 14,16 & 6,08 & 75,44 & 13,82 & 1,72 \\
\hline 2016 & 206081432 & 13,87 & 6,11 & 75,72 & 13,29 & 1,69 \\
\hline 2017 & 207660929 & 13,59 & 6,15 & 75,99 & 12,81 & 1,67 \\
\hline
\end{tabular}

Fonte/Source: IBGE, Diretoria de Pesquisas, Coordenação de População e Indicadores Sociais, Projeção da População do Brasil por Sexo e Idade para o Período 2000-2060 - Revisão 2013. 
Gráfico 2.5 - Taxa média geométrica de crescimento anual - 1940/2010 Graph 2.5 - Average geometric rate of annual increase - 1940/2010

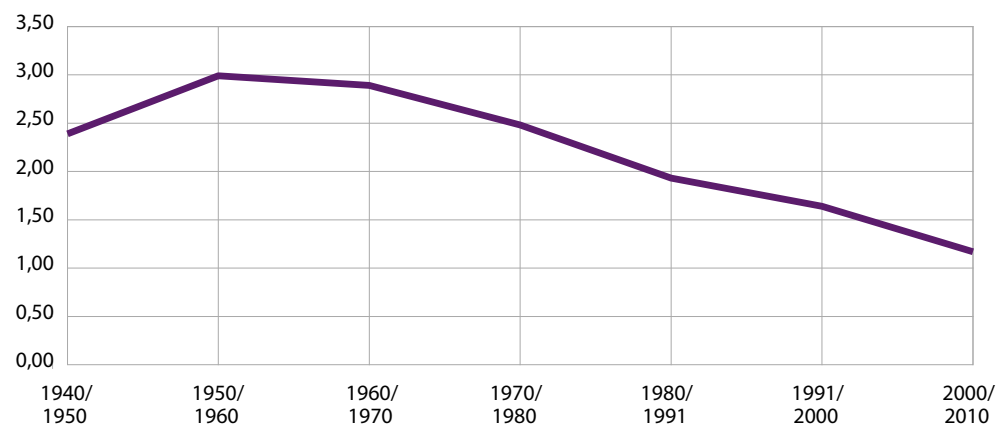

Fonte/Source: IBGE, Censo Demográfico 1940/2010. 



\section{Habitação}

\section{Housing}

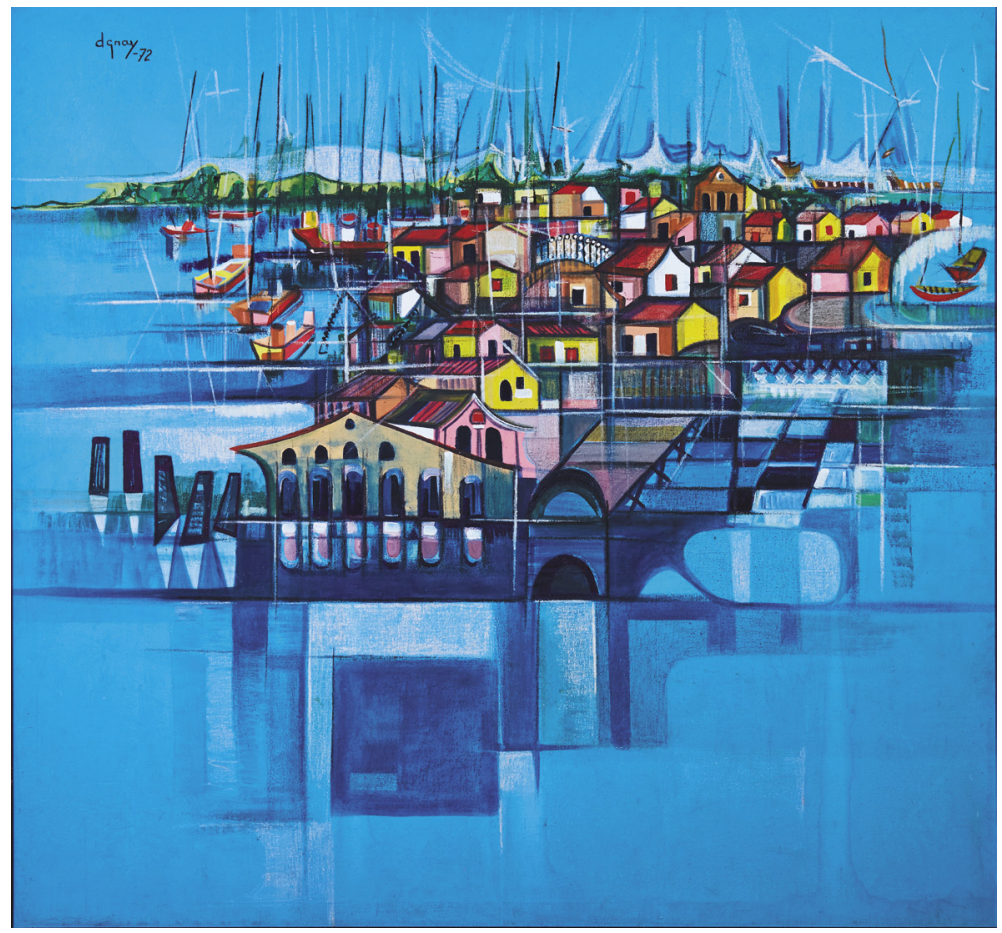

Casario/ Row houses, 1972

Dorian Gray Caldas 



\section{Habitação}

\section{Housing}

A habitação é um dos principais setores que mais contribui à melhoria dos padrões de qualidade de vida, saneamento ambiental e segurança das famílias, não apenas pela moradia em si, mas pelos diversos serviços a ela associados. Na história habitacional brasileira, há momentos de forte crescimento da produção formal e informal como nos anos de 1970 e 1980 - e outros de retração dos investimentos públicos e privados, resultando em descompassos entre o ritmo da urbanização e a oferta de novas moradias.

Um dos resultados desse descompasso foi o aumento do Déficit Habitacional que, por sua vez, contribuiu para a persistência do quadro geral de desigualdade social no Brasil em décadas recentes. A retomada, em meados dos anos 2000 , dos investimentos urbanos em habitação, representa um novo momento dessa trajetória, associada ao aumento de renda familiar dos setores menos favorecidos. As famílias tendem a se beneficiar com uma produção habitacional que privilegie e amplie o acesso à segurança, à saúde, entre outros.
Housing is one of the main sectors considered for measuring the improvement of life quality, environmental sanitation and family safety standards, not only because of the dwelling unit itself, but of the many services associated with it. The history of housing in Brazil has been marked by periods of significant increase of formal and informal production - in the 1970s and 1980s, for example - and periods of reduction of public and private investments, which have led to an unbalance between the pace of urbanization and the offer of new housing opportunities.

One of the observed outcomes is the increase of the Housing Shortage, which has helped maintain the scenario of widespread social inequality in Brazil in recent decades. In the mid-2000s, the restart of investments in urban housing, together with the increase of household income among the less privileged, represented a new moment in history. Families tend to benefit from a housing production plan focused on promoting and expanding the access to security and health, among other necessities. 
Desde a emergência, no início do século XXI, de marcos legais e políticas direcionadas à Política Urbana (por exemplo, a Lei Federal 10.257/2001), o tema da habitação ganhou mais espaço no debate social brasileiro, bem como as políticas setoriais - Saneamento Ambiental, principalmente. $O$ esforço em associar uma retomada da produção de moradia no Brasil esteve relacionado, por um lado, com a redefinição de uma Política Habitacional abrangente e, por outro, com o incremento do sistema de crédito às empresas e às famílias no acesso ao financiamento do imóvel. Tais faces da produção habitacional ganharam destaque ainda maior com o Programa Minha Casa Minha Vida, em 2009, e com o Programa de Aceleração do Crescimento (PAC), como parte do esforço em ampliar os investimentos em obras e serviços de infraestrutura urbana. Assim, os dados da Pesquisa Nacional por Amostra de Domicílios (PNAD) de 2014, aqui analisados, permitem avaliar, de modo mais aproximado, "como anda" a habitação brasileira e quão distantes estamos de alcançar os objetivos expressos nos marcos normativos nacionais.

Em 2014, a PNAD estimou um aumento de $2,9 \%$ no número de domicílios particulares permanentes no Brasil, alcançando 67 milhões de unidades - um incremento de 1,9 milhão de domicílios em relação ao ano anterior de 2013. A distribuição relativa dessa variação não alterou, de modo significativo, a distribuição por regiões, sendo que o Sudeste ainda lidera o estoque de domicílios com $43,45 \%$ do total, em 2014 , com aumento de 2,8\% em relação à 2013; logo depois, a Região Nordeste aparece com $26,20 \%$ dos domicílios no
Since the emergence of legal marks and measures directed to Urban Policy (for example, Federal Law no. 10,257/2001) in the beginning of the 21 st century, the issue of housing has gained relevance in the Brazilian social debate. The same is true for sector policies - especially Environmental Sanitation. The efforts made towards an associated recovery of housing production in Brazil were related, on the one hand, to the redefinition of a more comprehensive Housing Policy and, on the other, to the increased granting of credit for the financing of real estate by companies and families. These facets of housing production have become even more visible with the advance of Minha Casa Minha Vida (My House, My Life, a national social housing program created in 2009), and of PAC (Growth Acceleration Program), both resulting from efforts directed to the expansion of investments in works of urban infrastructure and services. As a result, data from the 2014 National Household Sample Survey (PNAD), analyzed herein, allow a closer evaluation of the current status of Brazilian housing and indicate how far we still are from the objectives established in the national normative frameworks.

In 2014, PNAD estimated an increase of $2.9 \%$ in the number of permanent private housing units in Brazil, which reached 67 million - a total 1.9 million housing units more than in 2013. The relative distribution of this change did not exert a significant effect on the distribution by Region: the Southeast keeps the leading position in terms of stock of housing units, with $43.45 \%$ of the total and an increase of $2.8 \%$ from 2013 to 2014. It is followed by the Northeast Region, with $26.20 \%$ of the housing units in Brazil, and then by the 
Brasil, seguido pela Região Sul (15,33\%), Centro-Oeste $(7,66 \%)$ e Norte $(7,37 \%)$.

Embora todas as regiões tenham ampliado o número de domicílios, percebe-se uma moderada alteração na posição relativa das Regiões Norte e Sul, quando comparados os anos 2013 e 2014, com pequeno aumento relativo dessa participação. Isso se explica pelo crescimento, em 2014, do número de domicílios particulares permanentes na Região Norte $(4,0 \%)$ e Região Sul $(3,1 \%)$, acima das demais regiões que cresceram 2,8\% em relação à 2013. A média de pessoas por domicílio no Brasil foi de 3,9, sendo que a Região Norte apresentou a maior média com 4,6 e a Região Sul a menor média, com 3,5 pessoas por domicílio.

Ao considerar a propriedade da habitação (Gráfico 3.1), o Brasil alcançou 73,7\% de domicílios próprios contra $18,5 \%$ de alugados e $7,4 \%$ cedidos. Tais números revelam variação negativa de $0,6 \%$ dos domicílios próprios e o consequente aumento de $0,6 \%$ de domicílios alugados, quando comparamos os números apresentados em 2013 e 2014. Referentes aos declarados como próprios, $5,3 \%$ estavam em aquisição, o que significa elevação de 0,2\% em relação à 2013.0 aumento do percentual de domicílios alugados indica um reforço ao setor rentista na moradia brasileira. Estes dados acompanham o padrão recente, registrado pelas PNAD, de crescimento dos domicílios alugados que, em 2004, respondia por $15,4 \%$ (PNAD, 2014). ${ }^{1}$

\footnotetext{
${ }^{1}$ Ao analisar historicamente, vemos que - segundo o Censo Demográfico do IBGE - há uma reversão no ritmo de queda observado desde os anos de 1960 - quando os domicílios alugados alcançaram $27,61 \%$ no Brasil.
}

South (15.33\%), Central-West (7.66\%) and North Region (7.37\%).

Although all the Regions have expanded the number of housing units, it is possible to observe some changes in the relative positions of the North and South when comparing figures of 2013 and 2014, with a slight relative increase in their participation. That can be explained by the rising number of permanent private housing units in the North Region (4.0\%) and in the South Region (3.1\%) in 2014, above the level of the other ones, representing an increment of $2.8 \%$ over 2013 . The average number of persons per housing unit in Brazil was 3.9, and the biggest and the smallest of these figures were recorded by the North and by the South Region: 4.6 and 3.5 persons per housing unit, respectively.

With regard to tenure (Graph 3.1), the results show Brazil had $73.7 \%$ of private housing units versus $18.5 \%$ of rented ones and $7.4 \%$ of lent ones. These figures evidence the negative change of private housing units by $0.6 \%$ and the subsequent increase of rented housing units, by the same rate, in comparison with figures of 2013 and 2014. As to those declared as private housing units, $5.3 \%$ were mortgaged, meaning a rise of $0.2 \%$ in relation to 2013 . The increased percentage of rented housing units points to a reinforcement of rent in the Brazilian housing segment. These data are in accordance with the latest pattern presented by PNAD of growth in rented homes, which accounted for only $15.4 \%$ in 2004 (PNAD, 2014)'.

\footnotetext{
'In a diachronic analysis, we can see that - according to the IBGE Population Census - a reversal in the pace of decrease has been observed since the 1960s when rented housing units totaled $27.61 \%$ in Brazil.
} 
Ainda referente aos domicílios alugados, observa-se alteração no padrão de oferta da moradia - considerando os anos de 2013 e 2014 - e na produção pública, associada a uma melhoria de renda da população, com possíveis impactos no mercado secundário residencial; outra hipótese pode ser a diversificação das opções no mercado para as famílias mais jovens, que retardam a entrada na transação de lançamentos imobiliários.

Ao abordar a infraestrutura disponível a esses domicílios, a PNAD 2014 revela um quadro diferenciado às regiões brasileiras. Percebe-se, para todo o Brasil, pequenas alterações indicando alguma melhora nos indicadores de abastecimento de água $(85,4 \%)$ e esgotamento sanitário considerado adequado (76,8\%). Por um lado, tal situação é positiva se compararmos com a PNAD de 2004 , quando $47,9 \%$ dos domicílios eram atendidos pela rede geral de esgotamento sanitário, contra $57,6 \%$ em 2014 - dez pontos percentuais em uma década.

Entretanto, ao observar a distribuição regional vemos que há ainda muito a investir, pois na Região Norte apenas 12,9\% dos domicílios têm acesso à rede geral de coleta (Gráfico 3.3) enquanto, $41,1 \%$ à fossa séptica e $35 \%$ dispõem apenas de fossa rudimentar. A Região Sudeste, apresenta números mais relevantes uma vez que $85,4 \%$ dos domicílios possuem acesso à rede geral, $6,0 \%$ apresentam fossa séptica e $5,1 \%$ fossa rudimentar. Na sequência, temos as regiões Sul, com $42,1 \%$ ligados à rede coletora, $39,4 \%$ com fossa séptica e $16,6 \%$ com fossa rudimentar; a região Centro-Oeste apresenta $42,5 \%$ de rede geral, $17,5 \%$ dos domicílios com fossa
Considering the years of 2013 and 2014 , changes in the pattern of offers and in the public production have been noticed in relation to rented housing units, associated with an improvement in the earnings of the population and its possible impacts on the secondary housing market. Another possibility is the diversification of offers directed to young families, who take longer before engaging in real estate negotiations.

As it presents the infrastructure available in these housing units, PNAD 2014 unveils existing differences among the Major Regions of Brazil. All over the country, improvements come from slight changes in indicators of water supply (85.4\%) and of proper sewerage (76.8\%). At first, a comparison between results of PNAD 2004 , when $47.9 \%$ of the housing units had access to a public sewer system, and of 2014, when this percentage increased to $57.6 \%$, indicates a positive scenario - ten percentage points in ten years.

Nevertheless, a more careful look into the regional distribution shows there is still a lot to invest in, because in the North Region only $12.9 \%$ of the municipalities have access to the public sewer system (Graph 3.3), whereas $41.1 \%$ make use of sceptic tanks and another $35 \%$ have only rudimentary cesspits. Figures for the Southeast Region are more relevant, since $85.4 \%$ of the housing units have access to the sewage system, $6.0 \%$ have sceptic tanks, and $5.1 \%$, rudimentary cesspits. Other results were: South Region, with $42.1 \%$ of the housing units accessing the public system, $39.4 \%$ with sceptic tanks and $16.6 \%$ with rudimentary 
séptica e 39,3\% fossa rudimentar; e, de modo intermediário, a Região Nordeste, com $37,6 \%$ de domicílios com rede coletora de esgoto, $23,4 \%$ com fossa séptica e $29,9 \%$ contando apenas com a fossa rudimentar ${ }^{2}$.

Em relação ao abastecimento de água, $84,5 \%$ possuem acesso à rede geral contra $14,6 \%$ que não dispõe desse serviço. Ao comparar com a PNAD 2013, observamos que houve o incremento de quase dois milhões de domicílios com rede geral de abastecimento de água. A Região Nordeste $(79,9 \%)$ e a Região Norte $(60,3 \%)$ são as regiões com menores percentuais de atendimento, se comparadas com os indicadores das regiões Sul $-87,9 \%$, Centro-Oeste $(85,2 \%)$ e Sudeste $(92,1 \%)$. Considerando que as políticas de Saneamento Básico avançaram, ao examinar a última década, há ainda um grande desafio no atendimento às regiões onde o nível de precariedade em saneamento é maior; tais diferenças reforçam o grau de desigualdade regional, impactando de forma direta os estados e os municípios - especialmente aqueles com baixa capacidade de investimento próprio.

Outro componente do saneamento básico que influencia a qualidade de vida da população é a coleta de lixo (Gráfico 3.4), aspecto que, quando associado a um abastecimento adequado de água e uma coleta do esgoto, contribui para uma melhor qualidade de vida da população. A PNAD 2014 revela que, para todo o Brasil, $89,9 \%$ do lixo era coletado - sendo que desse total, $6,7 \%$ de modo indireto. Por regiões, volta a desigualdade dos indicadores com a

\footnotetext{
${ }^{2}$ No gráfico 3.3, temos que os dados "Outros" também incluem a Fossa Rudimentar.
}

cesspits; Central-West, with $42.5 \%$ using the general system, $17.5 \%$, sceptic tanks, and $39.3 \%$, rudimentary cesspits, and, with intermediate results, the Northeast Region, with $37.6 \%$ of the housing units accessing the public sewer system, $23.4 \%$, using sceptic tanks and $29.9 \%$, rudimentary cesspits ${ }^{2}$.

Regarding water supply, $84.5 \%$ have access to the general system versus $14.6 \%$ that do not have this type of service. A comparison with PNAD 2013 shows an increment of almost two million housing units with a general water supply system. The Northeast Region (79.9\%) and the North Region (60.3\%) are the ones with the smallest percentages in comparison with the indicators of the South $-87.9 \%$, Central-West (82.5\%) and Southeast Regions (92.1\%). Considering the advance of Basic Sanitation policies, an analysis of the last decade shows the supply of these services is still a challenge in areas with precarious sanitation conditions. The existing differences highlight inequalities between the Major Regions and have a direct impact on states and municipalities - mainly on those with low investment capacity.

Waste collection (Graph 3.4) is another component of basic sanitation that affects quality of life. When combined with adequate water supply and proper sewerage, it can contribute to the improvement of a population's living conditions. According to PNAD 2014, $89.9 \%$ of the waste was collected all over the country $-6.7 \%$ in an indirect way. An analysis by Region evidences inequalities once more: in the Northeast Region, $21 \%$ of the housing units had

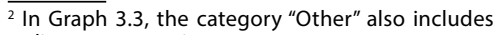
rudimentary cesspits. 
Região Nordeste com $21 \%$ de domicílios sem esse serviço, seguido pela Região Norte com 20,9\%; no caminho inverso, na Região Sudeste apenas 3,8\% dos domicílios não possuem coleta, assim como são menores os números das regiões Sul $(6,1 \%)$ e Centro-Oeste $(8,1 \%)$.

Deste modo, a PNAD 2014 nos mostra que os avanços nas condições de Habitação no Brasil apresentam avanços, em alguns indicadores, mas continua a persistente desigualdade regional, fruto de um padrão histórico de concentração de investimentos e maior renda familiar das regiões Sul e Sudeste, contra os menores percentuais nas regiões Norte e Nordeste. Tal padrão, entretanto, sofre modificações positivas com ganhos relativos nessas duas regiões, às vezes superiores à média nacional. Mas há ainda um caminho longo a percorrer para a definitiva superação do atraso estrutural na área de saneamento ambiental e combate ao Déficit Habitacional. Políticas que reforcem programas e projetos de acesso à terra urbanizada, regularização fundiária, universalização do acesso ao esgotamento sanitário - com tratamento adequado - podem indicar uma maior qualidade de vida, na próxima década, para o conjunto da população brasileira. to do without this service; in the North Region, $20.9 \%$. On the other extreme, in the Southeast Region, only $3.8 \%$ of the housing units did not have waste collection; also $6.1 \%$ in the South and $8.1 \%$ in the Central-West Region.

PNAD 2014 shows that housing conditions in Brazil have advanced in terms of some indicators, but regional inequalities still remain. They result from a historical trend of concentration of investments and from the occurrence of higher household income in the South and in the Southeast Regions, versus smaller figures in the North and Northeast. This pattern has undergone positive changes with relative gains in these two, sometimes even above the national average. Nevertheless, overcoming the structural delay in environmental sanitation and combating the housing shortage will still take us long. Policies that support programs and projects for access to urbanized land, land regularization, universal access to sewer systems including proper treatment - can pave the way for better living conditions for the Brazilian population as a whole in the next decade.

\section{Alexsandro Ferreira Cardoso da Silva}

Arquiteto e Urbanista, Doutor em Arquitetura e Urbanismo, Professor do Departamento de Políticas Públicas e do Programa de PósGraduação em Estudos Urbanos e Regionais da Universidade Federal do Rio Grande do Norte.
Architect and Urban Planner, PhD in Architecture and Urban Planning, Professor of the Department of Public Policies and of the Postgraduate Program in Urban and Regional Studies at the Federal University of Rio Grande do Norte. 


\section{Angela Lúcia Ferreira}

Arquiteta e Urbanista, Doutora em Geografia, Professora do Departamento de Arquitetura e do Programa de Pós-Graduação em Arquitetura e Urbanismo da Universidade Federal do Rio Grande do Norte. Bolsista de Produtividade em Pesquisa do CNPq.
Architect and Urban Planner, PhD in Geography, Professor of the Architecture Department and of the Postgraduate Program in Architecture and Urban Planning of the Federal University of Rio Grande do Norte. Fellow Researcher in Research Productivity at the National Counsel of Technological and Scientific Development - CNPq.

Translated by: Aline Milani Romeiro Pereira 


\section{Gráfico 3.1 - Distribuição dos domicílios particulares permanentes, por condição de ocupação - 2014}

Graph 3.1 - Distribution of permanent private housing units, by tenure - 2014

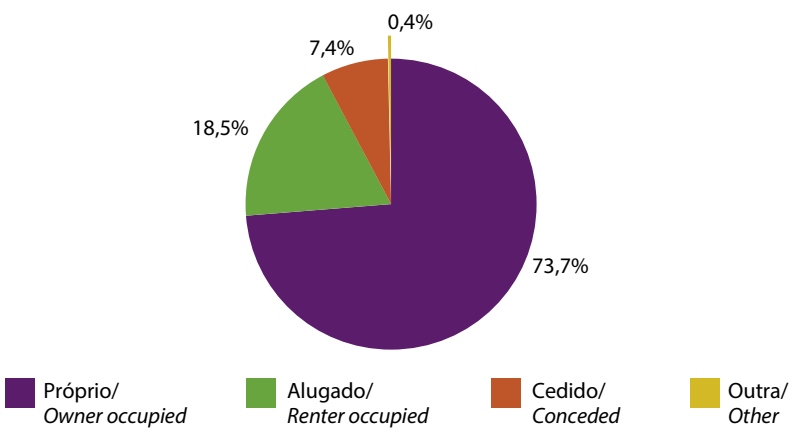

Fonte/Source: IBGE, Diretoria de Pesquisas, Coordenação de Trabalho e Rendimento, Pesquisa Nacional por Amostra de Domicílios 2014.

\section{Gráfico 3.2 - Distribuição dos domicílios particulares permanentes, por existência de rede geral de abastecimento de água, segundo as Grandes Regiões - 2014}

Graph 3.2 - Distribution of permanent private housing units, by presence of water supply system and Major Regions - 2014

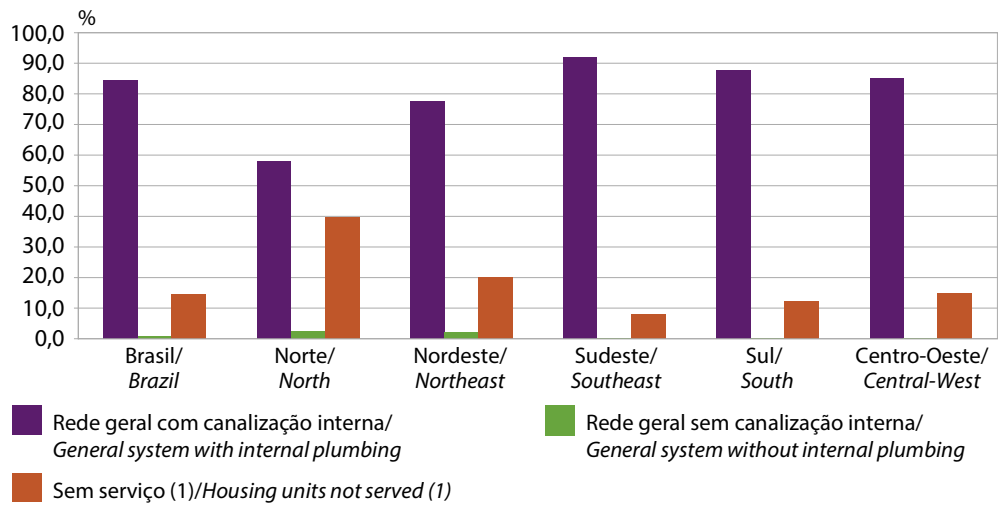

Fonte/Source: IBGE, Diretoria de Pesquisas, Coordenação de Trabalho e Rendimento, Pesquisa Nacional por Amostra de Domicílios 2014.

(1) Domicílios com abastecimento de água através de poço ou nascente ou outras formas./ (1) Housing units with water supply through wells or wellspring or other types. 


\section{Gráfico 3.3 - Distribuição dos domicílios particulares permanentes, por tipo de esgotamento sanitário, segundo as Grandes Regiões - 2014}

Graph 3.3 - Distribution of permanent private housing units, by type sewage disposal and Major Regions - 2014

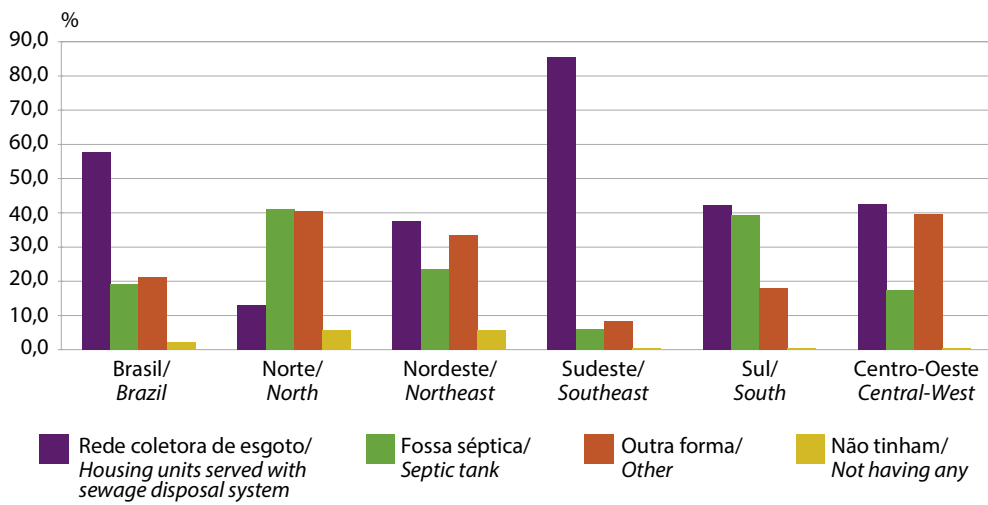

Fonte/Source: IBGE, Diretoria de Pesquisas, Coordenação de Trabalho e Rendimento, Pesquisa Nacional por Amostra de Domicílios 2014.

\section{Gráfico 3.4 - Distribuição dos domicílios particulares permanentes, por existência de serviço de coleta de lixo, segundo as Grandes Regiões - 2014}

Graph 3.4 - Distribution of permanent private housing units, by existence of garbage collection service and Major Regions - 2014

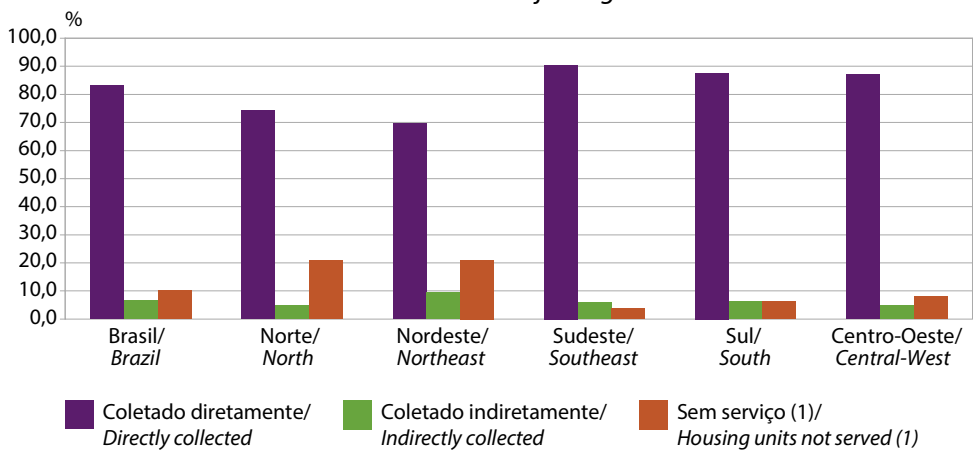

Fonte/Source: IBGE, Diretoria de Pesquisas, Coordenação de Trabalho e Rendimento, Pesquisa Nacional por Amostra de Domicílios 2014.

(1) Domicílio com lixo queimado ou enterrado e outros./(1) Housing unit with garbage burnt, buried and others. 


\section{Tabela 3.1 - Domicílios particulares permanentes, pessoas}

residentes em domicílios particulares permanentes e média

de pessoas, por domicílio particular permanente e

dormitório em domicílio particular permanente - 2013

Table 3.1 - Permanent private housing units, persons residents

in permanent private housing units, and average number of persons, per

permanent private housing unit and per bedroom in permanent private housing unit - 2013

\begin{tabular}{|c|c|c|c|c|}
\hline \multirow[b]{2}{*}{$\begin{array}{l}\text { Grandes Regiões/ } \\
\text { Major Regions }\end{array}$} & \multirow[b]{2}{*}{$\begin{array}{l}\text { Domicílios } \\
\text { particulares } \\
\text { permanentes/ } \\
\text { Permanent } \\
\text { private } \\
\text { housing units } \\
\text { (1 } 000 \\
\text { domicílios) }\end{array}$} & \multirow{2}{*}{$\begin{array}{l}\text { Pessoas } \\
\text { residentes } \\
\text { em domicílios } \\
\text { particulares } \\
\text { permanentes/ } \\
\text { Persons } \\
\text { residents in } \\
\text { permanent } \\
\text { private } \\
\text { housing units } \\
\text { (1 } 000 \text { pessoas) }\end{array}$} & \multicolumn{2}{|c|}{$\begin{array}{c}\text { Média de pessoas/ } \\
\text { Average number of persons }\end{array}$} \\
\hline & & & $\begin{array}{l}\text { Por domicílio } \\
\text { particular } \\
\text { permanente/ } \\
\text { Per permanent } \\
\text { private } \\
\text { housing unit }\end{array}$ & $\begin{array}{l}\text { Por dormitório } \\
\text { em domicílio } \\
\text { particular } \\
\text { permanente/ } \\
\text { Per bedroom } \\
\text { in permanent } \\
\text { private } \\
\text { housing unit }\end{array}$ \\
\hline Brasil/Brazil & 65130 & 201020 & 3,9 & 1,7 \\
\hline Norte/North & 4748 & 16952 & 4,6 & 2,0 \\
\hline Nordeste/Northeast & 17078 & 55817 & 4,1 & 1,8 \\
\hline Sudeste/Southeast & 28347 & 84474 & 3,7 & 1,7 \\
\hline Sul/South & 9966 & 28762 & 3,5 & 1,6 \\
\hline Centro-Oeste/Central-West & 4991 & 15015 & 3,7 & 1,7 \\
\hline
\end{tabular}

Fonte/Source: IBGE, Diretoria de Pesquisas, Coordenação de Trabalho e Rendimento, Pesquisa Nacional por Amostra de Domicílios 2013. 


\section{Saúde}

\section{Health}

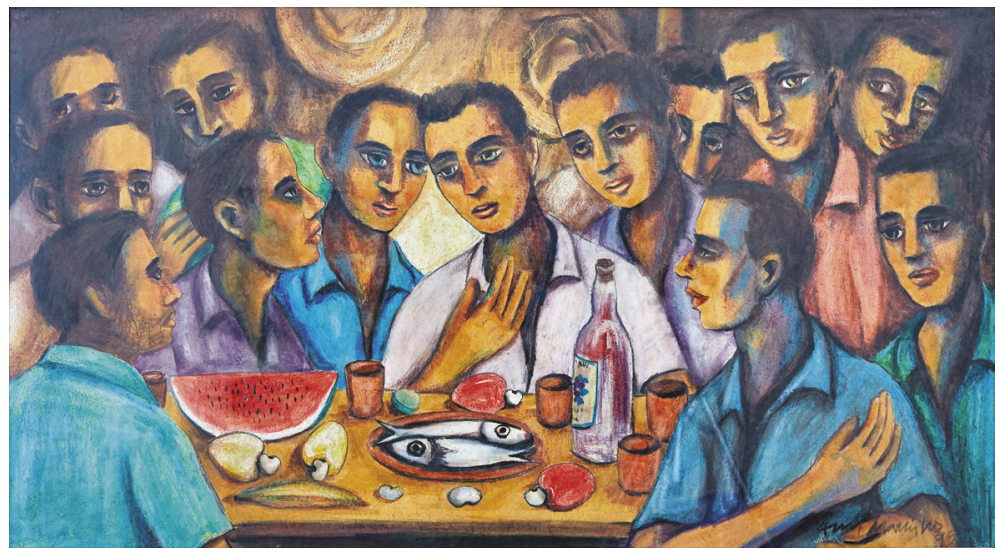

Ceia Larga/ Broad supper, 1995

Assis Marinho 



\section{Saúde}

\section{O quadro geral}

O Brasil administra um dos maiores e mais abrangentes sistemas de saúde pública do mundo, o Sistema Único de Saúde - SUS. O SUS presta assistência à saúde para aproximadamente $95 \%$ da população brasileira, incluindo algo em torno de $70 \%$ dos cuidados secundários e $90 \%$ dos cuidados mais complexos. O gasto total em saúde atinge aproximadamente $9,0 \%$ do PIB. Ao contrário dos países desenvolvidos, o gasto público brasileiro em saúde representa apenas algo em torno de $45 \%$ do gasto total. Como o gasto público ainda é baixo, aproximadamente 48,6 milhões de beneficiários de planos privados de assistência médica (os chamados planos de saúde) foram contabilizados pela Agência Nacional de Saúde Suplementar - ANS em maio 2016. Esse número revela uma queda em relação aos 50,0 milhões de beneficiários de planos de saúde registrados em maio do ano de 2015. Os planos de saúde são bens normais, cuja posse (ou consumo) aumentam quando a renda aumenta. Como a queda da posse de planos de saúde se repete mensalmente nos últimos doze meses, poderíamos atribuir

\section{Health}

\section{Overview}

Brazil manages one of the biggest and most comprehensive public health systems around the world, the Brazilian Unified Health System (Sistema Único de Saúde - SUS). SUS provides health care services to an estimated 95 per cent of the Brazilian population, including roughly 70 percent of secondary care and 90 percent of complex care. Total health spending amounts to about $9.0 \%$ of the GDP. However, unlike most developed countries, the Brazilian public health spending reaches only nearly $45 \%$ of the total expenditure. As public spending is still low, about 48.6 million beneficiaries of private health care plans were recorded in May 2016. Although the number of health insurance beneficiaries is still high for a country that has a public health system, a steady decline has been observed since 2015. This figure represents a decrease in relation to the 50.0 million health insurance beneficiaries registered in May 2015. Health insurance plans are quite normal goods for which demand increases when income increases. The recurrent decrease in the last 12 months could be attributable, at least in part, 
o fenômeno, ao menos em parte, às quedas do produto interno bruto e da renda disponível da população, causadas pela recessão econômica que o país atravessa.

\section{A Assistência Básica}

No Brasil, a Atenção Básica (AB) é norteada pela Política Nacional de Atenção Básica (PNAB) e almeja descentralização e proximidade do cidadão. Deveria ser a principal porta de entrada e o centro de comunicação com toda a Rede de Atenção à Saúde. Iniciada no ano de 1995, com o nome de Programa Saúde da Família - PSF, a atual Estratégia de Saúde da Família (ESF) é a principal estratégia de orientação e de execução da $A B$ no Brasil. A ESF pretende concentrar a atenção das unidades básicas de saúde nas famílias, para integrar as ações preventivas com a assistência médica e hospitalar. A ESF funciona por meio de equipes compostas por um médico, um enfermeiro, um auxiliar de enfermagem e quatro a seis agentes comunitários de saúde e por equipes de saúde bucal. As equipes trabalham em unidades de Saúde da Família que atuam em áreas geográficas definidas, e com responsabilidade por, no máximo, 4.000 pessoas para cada equipe. Em dezembro de 2015, a ESF estava implantada em 5.463 municípios com 40.162 equipes atingindo $63,72 \%$ de cobertura da população brasileira. Contava também com 266.217 Agentes Comunitários de Saúde atendendo a $66,65 \%$ da população brasileira. O Brasil alcançou a marca de 24.467 equipes de saúde bucal implantadas em 5.014 municípios brasileiros o que representa uma cobertura de cerca de $40 \%$ da população nacional. to declines in the gross domestic product and in the available income of the population, caused by the economic recession the country is facing.

\section{Primary Health Care}

Aiming at improving the accessibility to the system as well as to increase SUS effectiveness, the Brazilian health authorities have made efforts to change the health assistance model, which was eminently curative, to a more preventive approach. Born in 1994, the Family Health Strategy - (Estratégia de Saúde da Familia-ESF) is the main strategy for Primary Health Care activities in Brazil. The ESF focuses on the Basic Health Units (Unidades Básicas de Saúde) and on families, to integrate preventive medical care and hospital care. The ESF works through teams consisting of a doctor, a nurse, a nursing assistant and four to six community health agents and oral health teams. Each team working in Family Health Units operates in defined geographical areas and is assigned up to 4,000 people. The approximately 40,162 family health teams, in December 2015 , were present in 5,463 of the 5,565 municipalities in the country, reaching $63.72 \%$ of the population. Also, 266,217 Community Agents were taking care of $66.65 \%$ of the population. Brazil had a total 24,467 oral health teams settled in 5,014 municipalities, which means a $40 \%$ coverage of the national territory. 
O Programa Nacional de Imunização, que tem quase 40 anos, é um programa de saúde pública bem sucedido, e que distribui anualmente mais de 300 milhões de vacinas, soros e imunoglobulinas, fatos que contribuíram, por exemplo, com a erradicação da varíola, da febre amarela urbana, e da poliomielite, além da redução dos casos e mortes derivadas do sarampo, da rubéola, do tétano, da difteria e da coqueluche. Sua cobertura está acima de $95 \%$ da população, e as principais vacinas são fornecidas gratuitamente pelo SUS. O Brasil é autossuficiente na produção de várias vacinas.

Por outro lado, o saneamento básico ainda não foi universalizado, e as condições de moradia em muitas localidades não são satisfatórias. O IBGE em sua Síntese de Indicadores Sociais: uma análise das condições de vida da população brasileira (2014) informa que $18,3 \%$ das crianças de 0 a 14 anos de idade residentes em domicílios particulares permanentes não possuem acesso à rede geral de abastecimento de água e apenas 55,5\% têm esgotamento sanitário de rede geral ou fossa séptica. No Sistema Nacional de Informações sobre Saneamento (SNIS, 2014) ainda observamos que somente $39 \%$ dos esgotos do país são tratados. Algumas doenças infecciosas e parasitárias ainda preocupam, como é o caso das doenças transmitidas pelo mosquito Aedes Aegypti (dengue, chikungunya, e Zika); da doença de Chagas; da malária; e da tuberculose.

\section{A mortalidade geral}

A principal causa de mortalidade, no Brasil, são as doenças do aparelho circulatório que responderam, no ano de 2014 , por $25,6 \%$ das causa mortis em homens e 30,3\% das
The National Immunization Program, in effect for almost 40 years, is a very successful public health program. The immunization coverage by the Unified Health System - SUS reaches more than $95 \%$ of the population, and the main vaccines are provided freely by the government. The Program distributes over 300 million vaccines, serums and immunoglobulins. Brazil is selfsufficient in the production of several vaccines and has achieved the eradication of smallpox, the urban yellow fever and polio, as well as reduction in cases and deaths from measles, rubella, tetanus, diphtheria and whooping cough. In its Summary of Social Indicators: an analysis of the Brazilian population's life conditions (2014), the IBGE reports that $18.3 \%$ of the children aged 0 to 14 , living in permanent private housing units, do not have access to the general water supply system and that only $55.5 \%$ have access to sewer collection by means of the general system or of a sceptic tank. However, because sanitation has not been universalized and housing conditions in some areas are not satisfactory, some infectious and parasitic diseases remain a concern, such as dengue fever; Zika fever; Chagas disease; malaria; and tuberculosis.

\section{Overall mortality}

The main cause of death in Brazil for men and women is cardiovascular diseases, which account for $25.6 \%$ of male deaths 
causa mortis em mulheres. Para os homens, a segunda maior causa são as causas externas, principalmente as violências e os acidentes de trânsito, que respondem por $18,5 \%$ dos óbitos e, para as mulheres, as neoplasias são a segunda maior causa, que respondem por $17,8 \%$ do total de óbitos. A taxas de mortalidade infantil vem decrescendo consistentemente no Brasil, passando de 29,02 por 1.000 nascidos vivos no ano de 2000 para 13,82 por 1.000 nascidos vivos no ano de 2015 . A mortalidade materna ainda é elevada, com coeficiente de 54 por 100 mil nascidos vivos no ano de 2014 enquanto tal coeficiente, em países desenvolvidos, está em torno de 4 a 15 mortes maternas por 100 mil crianças nascidas vivas.

\section{Quadro geral das internações}

As principais causas de internação no Brasil são aquelas relacionadas às doenças do aparelho respiratório, seguida das do aparelho circulatório. $O$ número total de internações no Brasil é elevado, totalizando 11.258 .221 no ano de 2015. Deve-se descontar, desse total, uma parcela substancial da grande quantidade das internações relacionadas com a gravidez, o parto e o puerpério (Capítulo XV da CID-10), que impactam os dados da Clínica Obstétrica e a Clínica Pediátrica. Além da Obstetrícia e da Pediatria, duas grandes clínicas são, recorrentemente, responsáveis pela maioria das internações: a Clínica Médica, que respondeu por 4.010 .099 internações e a Clínica Cirúrgica, que apresentou 3.553.522 internações no ano de 2015.

A média de permanência, ou prazo médio de internação, no ano de 2015, registrado no SUS, foi de 5,6 dias. Esse não é um número elevado, quando comparado and for $30.3 \%$ of female deaths. Among men, the second major cause ( $18.5 \%$ of the total deaths) is external, mostly homicides and traffic accidents. Among women, cancer is the second leading cause, accounting for $17,8 \%$ of all deaths. Brazil has a high infant mortality rate, but it has consistently diminished from 29.2 per 1,000 live births in 2000 to 13.82 per 1,000 live births in 2008. Unfortunately, maternal mortality is still high, with a coefficient of 54 per 100 thousand live births in 2014, whereas the ratio in developed countries is around 4 to 15 maternal deaths per 100,000 live births.

\section{Overview of hospital stays}

The main causes of hospitalization in Brazil are those related to respiratory diseases, followed by diseases of the circulatory system. The total number of hospital admissions in Brazil is very big, amounting to $11,258,221$ in 2015. It includes the large number of hospitalizations related to pregnancy, childbirth and the puerperium (Chapter $X V$ of the ICD-10), which greatly impact Obstetrics and Pediatrics data and that must be discounted from the total figures. In addition to Obstetrics and Pediatrics, two large specialties are recurrently responsible for the majority of hospital admissions: General practice, which accounted for 4,010,099 hospitalizations and Surgery, which had 3,553,522 admissions in 2015.

In 2015, the average length of stay registered in the SUS was 5.6 days. This figure is quite similar to the gross average of OECD countries. 
aos países da OCDE. O prazo médio de internação é um indicador normalmente utilizado como indicador de eficiência, e que vem declinando ao longo dos anos em todo o mundo, em função da adoção procedimentos cirúrgicos cada vez menos invasivos, e da tendência a evitar a manutenção de pessoas em hospitais caso não haja estrita necessidade. $O$ prazo de internação varia muito, não somente com a gravidade e o tipo de internação, mas também de acordo com diversas características dos sistemas de saúde, como a remuneração dos prestadores de serviços.

A taxa de mortalidade hospitalar é um indicador muito complexo, que também varia bastante de acordo com as especialidades, as gravidades dos casos, e a qualidade dos hospitais. Existe uma probabilidade não desprezível de os casos mais graves serem direcionados aos melhores hospitais (selection), o que pode levar a interpretações errôneas das taxas de mortalidade hospitalar em análises onde haja ausência de controles ex-ante dos riscos envolvidos.

\section{A AIDS no Brasil}

De acordo com o Boletim

Epidemiológico HIV- AIDS do Ministério da Saúde do ano de 2015, desde o início da epidemia de AIDS no Brasil, no início da década de 1980 , até junho de 2015 , foram registrados no país 798.366 casos da doença. No ano de 2015 foram notificados 15.181 novos casos de HIV/AIDS no país. Pela primeira vez em sete anos, a taxa de detecção por 100.000 habitantes caiu para menos de 20 casos, passando a ser a menor taxa de detecção dos últimos 12 anos (19,7 casos por 100.000 habitantes). $O$
The average length of stay (ALOS) is an indicator frequently used as a proxy for efficiency. It has been declining over the years all over the world, due to the adoption of less invasive surgical procedures. The general trend to avoid the unnecessary maintenance of people in hospitals for long periods of time also has brought the ALOS down. The average length of time varies a lot, not only to the severity and type of hospitalization, but also according to different characteristics of health systems, including the payment system.

Hospital mortality rate is a very complex indicator. It varies according to the medical specialty, the severity of cases and the quality of hospitals. There is a rather significant probability of more serious cases being sent to higher-qiuality hospitals, which can lead to misinterpretations of hospital mortality rates in analyses which do not include ex-ante risk assessment.

\section{AIDS in Brazil}

According to the 2015 issue of the HIVAIDS Epidemiological Bulletin of the Brazilian Ministry of Health, from the virus inception in the early eighties, up to June $2015,798,366$ cases of the disease were registered in the country. During the year 2015, there were 15,181 new cases of AIDS reported in Brazil. For the first time within seven years the ratio per 100,000 inhabitants dropped to less than 20 cases (19.7 cases per 100,000 inhabitants), becoming the lowest detection rate in the last 12 years. Brazil has been successful in 
Boletim afirma que a epidemia brasileira está concentrada em populações mais vulneráveis que respondem pela maioria de casos novos do HIV em todo país, como gays e homens que fazem sexo com homens, travestis e transexuais, pessoas que usam drogas ilícitas, e profissionais do sexo.

A taxa de detecção dos últimos dez anos tem apresentado diferenças entre os sexos e entre as faixas etárias. Revertendo uma tendência observada no período entre os anos de 1980 e 2005, observa-se, a partir do ano de 2009, uma redução da participação relativa dos casos de aids em mulheres no total de casos e aumento da participação relativa nos casos em homens. O fenômeno está refletido na razão de sexos, que passou a ser de 19 casos de AIDS em homens para cada 10 casos em mulheres no ano de 2014. Comparando-se a distribuição proporcional dos casos de aids segundo raça/cor por sexo desde 2005 até 2014, observa-se que não existe diferença nas proporções de brancos, amarelos, pardos e indígenas segundo sexo. A exceção ocorre entre os pretos, nos quais a proporção de homens é inferior a proporção observada nas mulheres. A via sexual continua sendo a principal forma de transmissão da doença, a despeito das campanhas para uso de preservativos. Uma informação importante é que, no Brasil, no ano de $2013,33,5 \%$ da população brasileira de 15 a 64 anos haviam realizado o teste de HIV pelo menos uma vez na vida, e aproximadamente $13 \%$ haviam realizado o teste nos 12 meses anteriores à pesquisa. achieving declining prevalence of Aids after the implementation of a national program for AIDS and HIV control, which includes free treatment and free distribution of medicines. Even though we can observe a significant prevalence in all social groups, the AIDS epidemic seems to be concentrated in certain sub-populations living in vulnerable situations or in those exposed to risk behaviors: homosexuals; transvestites; users of illicit drugs; and sex workers.

The detection rate of the last ten years has been presenting a gap between the sexes and among the age groups. Offsetting a trend seen from 1980 to 2005 , a reduction in the relative contribution of the AIDS cases in women to the total cases and also an increase in the relative contribution of men could be noticed from 2009 on. The phenomenon can be seen in the sex ratio, which became 19 cases of AIDS in men for each 10 female cases in 2014. By comparing the proportional distribution of AIDS cases by race and gender from 2005 to 2014, it is observed that there is no difference in the share of white, yellow, multiracial and Indian by sex. The exception occurs between the blacks, with a bigger proportion of women in relation to men. Despite campaigns for the use of condoms, sexual intercourse remains as the main form of spread of the disease. A valuable piece of information is that, in Brazil, in 2013, 33.5\% of the population 15-64 years of age had taken an HIV test at least once in life, and about $13 \%$ had undergone the test in the 12 months prior to the survey. 
Alexandre Marinho

Possui graduação em Bacharel em Ciências

Bachelor in Economic Sciences from the State

Econômicas pela Universidade do Estado do

Rio de Janeiro (1985), Mestrado em Economia

pela Fundação Getúlio Vargas - RJ (1993)

e Doutorado em Economia pela Fundação

Getúlio Vargas - RJ (1996). Atualmente é

Técnico de Planejamento e Pesquisa do

Instituto de Pesquisa Econômica Aplicada -

IPEA e Professor Associado da Faculdade de

Ciências Econômicas da UERJ

University of Rio de Janeiro - UERJ (1985),

Master in Economics from the Getúlio Vargas

Foundation - RJ (1993) and PhD in Economics from the Getúlio Vargas Foundation - RJ (1996).

He is currently a Technician in Planning and Research at the Institute for Applied Economic Research - IPEA and Associate Professor of the Department of Economic Sciences at UERJ. 
Tabela 4.1 - Óbitos de residentes, por sexo, segundo as

10 principais causas - 2014

Table 4.1 - Deaths of residents, by sex and 10 leading causes of death - 2014

\begin{tabular}{|c|c|c|c|}
\hline $\begin{array}{l}\text { Causas de óbitos/ } \\
\text { Causes of death }\end{array}$ & $\begin{array}{l}\text { Total (1)/ } \\
\text { Total (1) }\end{array}$ & $\begin{array}{l}\text { Homens/ } \\
\text { Male }\end{array}$ & $\begin{array}{l}\text { Mulheres/ } \\
\text { Female }\end{array}$ \\
\hline $\begin{array}{l}\text { Total/ } \\
\text { Total }\end{array}$ & 1222381 & 691157 & 530447 \\
\hline $\begin{array}{l}\text { Doenças do aparelho circulatório/ } \\
\text { Circulatory System Diseases }\end{array}$ & 337611 & 176871 & 160656 \\
\hline $\begin{array}{l}\text { Neoplasias (tumores)/ } \\
\text { Neoplasm (tumors) }\end{array}$ & 200979 & 106699 & 94240 \\
\hline $\begin{array}{l}\text { Causas externas/ } \\
\text { External Causes }\end{array}$ & 155610 & 128138 & 27288 \\
\hline $\begin{array}{l}\text { Doenças do aparelho respiratório/ } \\
\text { Respiratory system diseases }\end{array}$ & 138487 & 71315 & 67114 \\
\hline $\begin{array}{l}\text { Sintomas, Sinais e Achados Anormais de Exames Clínicos e } \\
\text { de Laboratório não Classificados em Outra Parte/ } \\
\text { Syntoms, signs and ill-defined conditions not elsewhere } \\
\text { classified }\end{array}$ & 73682 & 41971 & 31604 \\
\hline $\begin{array}{l}\text { Doenças endócrinas nutricionais e metabólicas/ } \\
\text { Endocrine, nutritional and metabolic diseases }\end{array}$ & 73229 & 33278 & 39934 \\
\hline $\begin{array}{l}\text { Doenças do aparelho digestivo/ } \\
\text { Digestive System Diseases }\end{array}$ & 62453 & 38817 & 23615 \\
\hline $\begin{array}{l}\text { Algumas doenças infecciosas e parasitárias/ } \\
\text { Certain infectious and parasitic diseases }\end{array}$ & 51971 & 29663 & 22283 \\
\hline $\begin{array}{l}\text { Doenças do aparelho geniturinário/ } \\
\text { Genitourinary Diseases }\end{array}$ & 32406 & 15631 & 16757 \\
\hline $\begin{array}{l}\text { Doenças do sistema nervoso/ } \\
\text { Nervous System Diseases }\end{array}$ & 32101 & 14640 & 17450 \\
\hline $\begin{array}{l}\text { Outros/ } \\
\text { Others }\end{array}$ & 63852 & 34134 & 29506 \\
\hline
\end{tabular}

Fonte/Source: Informações de saúde. Mortalidade: dados preliminares. In: Brasil. Ministério da Saúde. Datasus. Brasília, DF, [2015]. Disponível em/Avaible from: <http://www2.datasus.gov.br/DATASUS/ index.php>. Acesso em: fev. 2016/Cited: Feb. 2016.

Nota: As causas de óbitos descritas correspondem ao Capítulo CID-10./ Note: Causes of death presented according to Chapter ICD-10.

(1) Inclusive óbitos de sexo não informado./ (1) Includes deaths of sex not reported. 
Tabela 4.2 - Cobertura vacinal, por Unidades da Federação - 2015

Table 4.2 - Immunization coverage by Federative Unit - 2015

\begin{tabular}{|c|c|c|c|c|c|}
\hline $\begin{array}{c}\text { Unidades da Federação/ } \\
\text { Federative Units }\end{array}$ & $\begin{array}{c}\text { BCG / } \\
\text { BCG vaccine } \\
\text { (against } \\
\text { tuberculosis) }\end{array}$ & $\begin{array}{l}\text { Contra } \\
\text { hepatite B/ } \\
\text { Against } \\
\text { hepatite B }\end{array}$ & $\begin{array}{c}\text { Poliomielite/ } \\
\text { Against } \\
\text { poliomyelitis }\end{array}$ & $\begin{array}{c}\text { Tríplice } \\
\text { viral D1/ } \\
\text { Triple viral } \\
\text { vaccine D1 }\end{array}$ & $\begin{array}{c}\text { Tetra/ } \\
\text { Tetra } \\
\text { vaccine }\end{array}$ \\
\hline Brasi/Brazil & 101,64 & 94,84 & 95,59 & 86,24 & 66,67 \\
\hline Norte/North & 98,32 & 83,72 & 85,89 & 75,7 & 57,89 \\
\hline Rondônia & 110,73 & 104,56 & 104,04 & 99,49 & 92,57 \\
\hline Acre & 100,48 & 79,84 & 80,51 & 69,46 & 45,95 \\
\hline Amazonas & 108,74 & 93,48 & 103,03 & 84,7 & 75,51 \\
\hline Roraima & 110,55 & 96,83 & 111,83 & 102,05 & 86,9 \\
\hline Pará & 88,25 & 71,99 & 70,6 & 64,48 & 40,17 \\
\hline Amapá & 109,67 & 77,61 & 79,16 & 69,11 & 65,35 \\
\hline Tocantins & 93,99 & 96,92 & 94,23 & 81,16 & 54,15 \\
\hline Nordeste/Northeast & 101,15 & 92,65 & 96,78 & 83,85 & 69,88 \\
\hline Maranhão & 98,6 & 88,65 & 96,99 & 77,65 & 67,77 \\
\hline Piauí & 83,45 & 80,11 & 78,74 & 73,89 & 58,75 \\
\hline Ceará & 106,54 & 100,09 & 106,33 & 99,62 & 72,61 \\
\hline Rio Grande do Norte & 104,15 & 89,08 & 95,79 & 80,52 & 72,9 \\
\hline Paraíba & 98,45 & 84,75 & 85,63 & 75,62 & 51,05 \\
\hline Pernambuco & 108,08 & 102,77 & 107,61 & 85,56 & 72,99 \\
\hline Alagoas & 97,12 & 89,54 & 91,48 & 88,12 & 72,44 \\
\hline Sergipe & 104,48 & 92,76 & 92,33 & 83,85 & 73,04 \\
\hline Bahia & 99,04 & 89,97 & 92,82 & 80,9 & 73,17 \\
\hline Sudeste/Southeast & 102,66 & 99,79 & 98,88 & 91,73 & 69,51 \\
\hline Minas Gerais & 98,38 & 97,81 & 94,23 & 89,59 & 53,16 \\
\hline Espírito Santo & 96,93 & 99,4 & 97,65 & 88,89 & 73,76 \\
\hline Rio de Janeiro & 113,5 & 103,01 & 105,39 & 97,42 & 74,8 \\
\hline São Paulo & 101,02 & 99,48 & 98,56 & 90,81 & 74,11 \\
\hline Sul/South & 103,42 & 96,78 & 93,28 & 86,78 & 63,29 \\
\hline Paraná & 104,25 & 99,47 & 95,04 & 91,13 & 58,41 \\
\hline Santa Catarina & 107,75 & 103,63 & 100,09 & 93,85 & 73,42 \\
\hline Rio Grande do Sul & 99,75 & 89,45 & 87,02 & 77,48 & 62,24 \\
\hline Centro-Oeste/Central-We: & 99,87 & 89,88 & 92,08 & 80,88 & 58,87 \\
\hline Mato Grosso do Sul & 124,51 & 114,95 & 116,45 & 101,69 & 70,66 \\
\hline Mato Grosso & 106,63 & 100,22 & 100,96 & 87,04 & 76,89 \\
\hline Goiás & 95,29 & 83,63 & 84,53 & 77,45 & 42,84 \\
\hline Distrito Federal & 78,16 & 67,06 & 74,45 & 61,09 & 60,35 \\
\hline
\end{tabular}

Fonte/Source: Informações de saúde. Assistência à saúde. Imunizações. In: Brasil. Ministério da Saúde. Datasus. Brasília, DF, [2015]. Disponível em/ Available from: <http://www2.datasus.gov.br/DATASUS/ index.php>. Acesso em: fev. 2016/ Cited:Feb. 2016. 
Tabela 4.3 - Internações, mortalidade hospitalar e média de permanência no Sistema Único de Saúde - SUS - 2015

Table 4.3 - Hospitalization, deaths in hospitals and average length of stay in the National Health System - SUS - 2015

\begin{tabular}{|c|c|c|c|}
\hline $\begin{array}{l}\text { Especialidades/ } \\
\text { Specialty }\end{array}$ & $\begin{array}{l}\text { Internações/ } \\
\text { Hospitalization }\end{array}$ & $\begin{array}{c}\text { Taxa de } \\
\text { mortalidade } \\
\text { hospitalar/ } \\
\text { hospital mortality } \\
\text { rates(\%) }\end{array}$ & $\begin{array}{l}\text { Média de } \\
\text { permanência/ } \\
\text { Average } \\
\text { length of stay }\end{array}$ \\
\hline Total/Total & 11258221 & 4,20 & 5,6 \\
\hline Clínica cirúrgica/Surgery & 3553522 & 2,15 & 4,1 \\
\hline Obstetrícia/Obstetrics & 2168355 & 0,03 & 2,5 \\
\hline Clínica médica/Internal medicine & 4010099 & 9,19 & 6,2 \\
\hline $\begin{array}{l}\text { Cuidados prolongados (crônicos)/Long-term care } \\
\text { (chronic) }\end{array}$ & 21294 & 29,04 & 110,1 \\
\hline Psiquiatria/Psychiatry & 166311 & 0,31 & 45,8 \\
\hline Tisiologia/Phthisiology & 6403 & 7,7 & 34,0 \\
\hline Pediatria/Pediatrics & 1185092 & 1,7 & 6,0 \\
\hline Reabilitação/Rehabilitation & 20032 & 0,04 & 8,7 \\
\hline Clínica cirúrgica - hospital dia/Surgery - day hospital & 89286 & 0,01 & 0,2 \\
\hline Aids - hospital dia/ Aids - day hospital & 7163 & 0,04 & 19,6 \\
\hline $\begin{array}{l}\text { Fibrose cística - hospital-dia/Cystic Fibrosis - day } \\
\text { hospital }\end{array}$ & 6 & - & 14,2 \\
\hline $\begin{array}{l}\text { Pós-transplante - hospital dia/ After transplant - day } \\
\text { hospital }\end{array}$ & 5648 & 0,69 & 9,2 \\
\hline Geriatria - hospital-dia/Geriatrics - day hospital & 345 & - & 23,7 \\
\hline $\begin{array}{l}\text { Saúde mental - hospital-dia/ Mental heatlh - day } \\
\text { hospital }\end{array}$ & 14292 & 0,03 & 27,7 \\
\hline Saúde mental - clínico/ Mental heatlh - clinical & 10373 & 0,34 & 11,2 \\
\hline
\end{tabular}

Fonte/Source: Informações de saúde. Internações hospitalares do [Sistema Único de Saúde] - SUS. In: Brasil. Ministério da Saúde. Ministério da Saúde. Datasus. Brasília, DF, [2015]. Disponível em/Available from: <http://www2.datasus.gov.br/DATASUS/index.php>. Acesso em: fev. 2016/Cited: Feb. 2016. 
Gráfico 4.1 - Casos notificados de AIDS - 2004-2014

Graph 4.1 - AIDS cases reported - 2004-2014

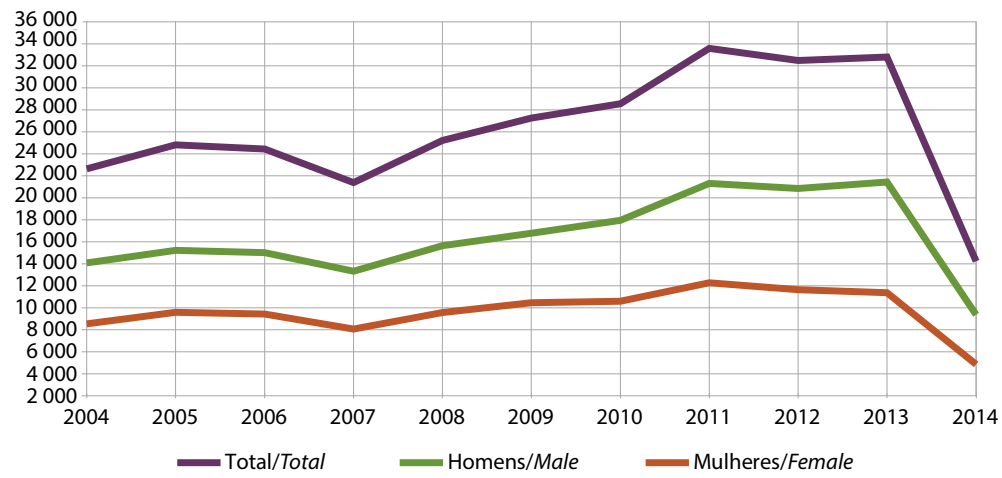

Fonte/Source: Informações de saúde. Epidemiológicas e morbidade. In: Brasil. Ministério da Saúde. Datasus. Brasília, DF, [2015]. Disponível em/Available from: <http://www2.datasus.gov.br/DATASUS/ index.php>. Acesso em: fev. 2016/Cited: Feb. 2016. 



\section{Previdência Social Social Security}

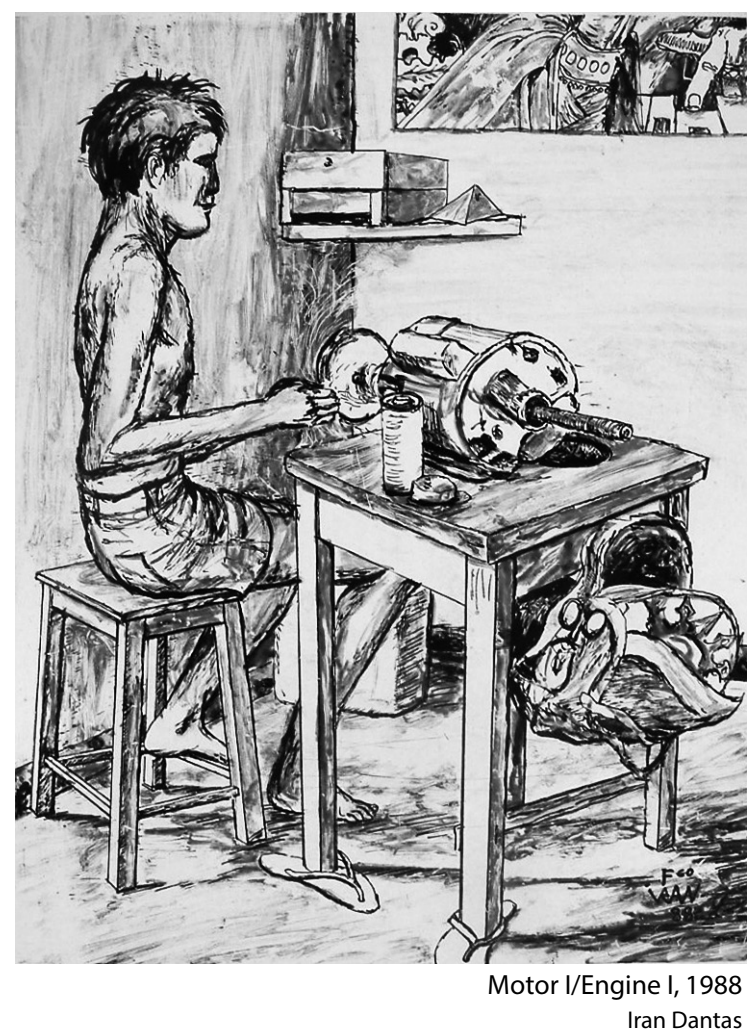





\section{Previdência Social}

\section{Social Security}

Não é difícil perceber a raiz dos desafios ao analisar a cobertura da previdência no Brasil, pois nos benefícios para idosos ela supera a marca de $95 \%$ da população com 65 anos ou mais de idade desde meados anos 1990 e; nesse mesmo período, o contingente de contribuintes para a previdência em relação à população em idade ativa oscilou em torno de 35\%, em função do nível de atividade da econômica.

A Constituição Federal promulgada em 1988 - CF/88 - teve como singularidade a introdução do Capítulo da Ordem Social. Os legisladores da CF/88 criaram a Seguridade Social, ao reconhecer o número restrito de contribuintes e a dificuldades para financiar o pagamento de benefícios. Separar o direito à atenção do Estado para políticas de saúde e assistência da condição de contribuinte da previdência foi a marca da CF/88. A Seguridade Social abrange as políticas de saúde, assistência e previdência e dispõe de um orçamento próprio e independente do fiscal, composto por múltiplas fontes de incidência como: folha salarial, lucro líquido, faturamento, loterias e, entre 1993 e 2007, movimentação financeira. Os
A brief analysis of the social security coverage in Brazil makes clear the origin of our challenges in this field. Pensions for the elderly have benefited more than $95 \%$ of the population aged 65 and over since the mid 1990s. In this same period, the percentage of contributors to social security in relation to the working age population has been around $35 \%$, considering our level of economic activity.

The Federal Constitution promulgated in $1988-\mathrm{CF} / 88$ - had as a distinguishing feature the inclusion of a chapter on Social Order. CF/88 legislators created Social Security in recognition of the limited number of contributors and of the difficulties faced to pay security benefits. Distinguishing between one's right to receive care from the State in terms of health policies and their status as a social security contributor was one of the landmarks of $C F / 88$. Social security encompasses health, welfare and security policies and has its own budget, not associated with the fiscal budget, but with inputs from multiple sources, such as: wage bill, net profits, revenues, lotteries and, between 1993 and 2007, financial transactions. The 
recursos do Orçamento da Seguridade Social - OSS, por deliberação da CF/88 devem custear as despesas de saúde, assistência e previdência, e, em caso de insuficiência podem ter suas alíquotas majoradas ou criar novas fontes para custear as despesas. Ao longo dos anos, as mutações da legislação e as reformas constitucionais, alteraram as formas de funcionamento, mas, a regra do OSS não foi revertida desde 1988.

No âmbito da previdência a $\mathrm{CF} / 88$ estabeleceu regras mais generosas para a concessão de benefícios para trabalhadores rurais, com aposentadorias por idade concedidas aos 60 anos e às mulheres, que têm direito a contribuir cinco anos a menos e se aposentar cinco anos antes. $O$ piso de benefícios passou a ser o equivalente a um salário mínimo. As pessoas idosas e as com incapacidade para o trabalho em famílias com renda per capita inferior a um quarto do salário mínimo passaram a fazer jus ao piso de um salário mínimo na modalidade de Benefícios de Prestação Continuada - BPC. O BPC é da órbita da assistência social, mas $O$ agente pagador é o INSS - Instituto Nacional do Seguro Social. O INSS foi um órgão vinculado à previdência desde os seus primórdios, mas, em maio de 2016, passou a se referir ao Ministério do Desenvolvimento Social e Agrário, o responsável, até então, pelas políticas de assistência.

Um aspecto a destacar é o fato da análise a seguir se concentrar no Regime Geral de Previdência Social - RGPS. O RGPS envolve os trabalhadores da iniciativa privada, de empresas estatais ou de economia mista e os servidores públicos da maioria dos municípios. Trata-se do maior contingente de segurados
$\mathrm{CF} / 88$ states that the resources from the Social Security Budget - OSS must be used to pay for health, welfare and social security expenses, and in case these resources are not enough their shares can be incremented or new sources can be created to pay for these expenses. Throughout the years, changes in the legislation and constitutional reforms have altered the operation of the OSS, but not its rules.

In the realm of social security, $\mathrm{CF} / 88$ established more generous rules towards the granting of benefits for rural workers, with old-age retirement at 60 , and for women, who retire five years before men. The minimum benefit provision is now equivalent to one minimum wage. The elderly and persons unable to work in families with per capita household earnings below one fourth of a minimum wage were entitled to receive benefit provisions of one minimum wage, as part of the Continuous Cash Benefit Program - BPC. BPC is a measure related to social protection, but it is paid by the INSS - National Institute for Social Security. The INSS had been connected with social security since its start, but in May 2016 it was transferred to the Ministry of Social and Agrarian Development, which had been in charge of assistance policies until then.

It is important to highlight that the analysis is focused on the General Social Security System - RGPS. RGPS encompasses workers from the private initiative, from state or mixed economy companies and also civil servants from most municipalities. It covers the majority of social security 
da previdência, mas não o único, pois existem, também, os Regimes Próprios de Previdência - RPP - e os Regimes de Previdência Complementar - RPC. Os RPP envolvem os regimes de previdência da União, Estados, das capitais e, em geral, dos maiores municípios. Os RPP possuem planos de benefícios e custeio diferentes, podendo pagar prestações de maior valor. Os RPC se baseiam na capitalização das contribuições, sendo diferentes do regime de repartição simples do RGPS, onde as contribuições correntes financiam o pagamento de benefícios. Os RPC são complementares, pois seus rendimentos representam aportes suplmentares àqueles pagos pela previdência. As informações referentes aos RPP e, sobretudo, RPC pouco influem na análise que se segue.

Diante do exposto até aqui, fica mais simples analisar as tabelas em anexo cuja fonte são os dados do Anuário Estatístico da Previdência Social - AEPS. A tabela 5.1 apresenta a estrutura de custeio do RGPS e a informação mais importante diz respeito à participação dos recursos coletados por contribuições de empregados e empregadores sobre a folha salarial e aquela referente à transferência dos demais recursos. Entre 2003 e 2014, a proporção da participação incidente sobre a folha variou entre $66 \%$ e $79 \%$ do total do custeio, ao passo que nas outras fontes oscilou inversamente entre $33 \%$ e $21 \%$. Rendimentos financeiros e outras fontes têm pouca relevância atualmente.

Essa trajetória se explica pelo desempenho econômico que, nesse período, apresentou bons níveis de atividade, quando comparado aos anos 1990. Isso resultou em aumento da pensioners, but it is not the only system available: there are also Private Social Security Systems - RPP and Complementary Social Security Systems - RPC. The RPP include the security systems of the Federation, of States, of capitals and of most municipalities, and have different benefit and security plans, ranked by price. The RPCs are based on the capitalization of contributions, and do not not follow the revenue sharing system of the RGPS, in which current contributions fund the pay of benefits. The RPCs are complementary because their revenues constitute additional contributions to benefits from social security. Information on the RPPs and, mainly, on the RPCs adds very little to the following analysis.

Considering what has been said so far, it is easier to analyze the attached tables with data from the Social Security Yearbook - AEPS. Table 5.1 presents the cost structure of the RGPS. The most relevant piece of information refers to the participation of resources collected as contributions from workers and employers' payroll or relative to the transfer of other resources. Between 2003 and 2004, the proportion of participation on the payroll ranged between $66 \%$ and $79 \%$ of the total pay, whereas in terms of other sources it ranged between $33 \%$ and $21 \%$. Financial income and other sources have little relevance nowadays.

That results from the economic performance which recorded good levels of activity in the period, when compared to activity in the $1990 \mathrm{~s}$. There was subsequent increase 
arrecadação sobre a folha salarial, que se elevou de $5 \%$ para $6,8 \%$ sobre o PIB, ao passo que as transferências via Tesouro Nacional representaram $2,8 \%$ do PIB em 2003 e 1,8\%, em 2014. A reversão, marcada pela forte retração do PIB após 2014 devem inverter essa trajetória, requerendo maiores aportes de outras fontes da seguridade a serem repassadas via Tesouro Nacional.

A tabela 5.2 apresenta a distribuição das despesas, seguindo a sistemática do fluxo de caixa da tabela 5.1. A tabela 5.2 revela que ocorreu uma importante evolução nas despesas com benefícios, sobretudo do BPC (assistenciais, como vimos) e pelos Encargos Previdenciários da União EPU (de incidência marginal). $O$ total de despesas do BPC e EPU representou 4,1\% em 2003 e atingiu 8,1\% em 2014. Assim, o maior aumento nas despesas não se deveu a benefícios previdenciários. Os benefícios da alçada do RGPS se reduziram de $87 \%$ para $82 \%$ do total de pagamentos. Os gastos com pessoal e custeio também se reduziram. $O$ que cresceu bastante foi o repasse a terceiros que representa os recursos arrecadados sobre folha salarial e transferidos ao chamado "sistema S" (como SENAC ou SENAI) que são instituições de cunho social e formação profissional das confederações de empregadores do Brasil.

Ao comparar as tabelas 5.1 e 5.2, existem elementos dignos de atenção. A arrecadação de contribuições sobre a folha salarial cresceu $34,6 \%$ em termos de sua participação sobre o PIB, enquanto os pagamentos de benefícios of tax collection based on payroll from $5 \%$ to $6.8 \%$ over GDP, whereas transfers from the National Treasury represented $2.8 \%$ of the GDP in 2003 and $1.8 \%$ in 2014. The reversal, characterized by strong retraction of GDP after 2014 is expected to invert this process, and requires bigger contributions from other sources of social security to be transferred from the National Treasury.

Table 5.2 shows the distribution of expenditure, folowing the cash flow system of Table 5.1. Table 5.2 shows there has been significant advance in the expenditure on benefits, mainly from the BPC - Continuous Cash Benefit Program (for welfare, as seen before) and from the Social Security Charges of the Federal Government (marginal). Total BPC and EPU expenditure represented $4.1 \%$ in 2003 and reached $8.1 \%$ in 2014 . So, the biggest increase in expenditure did not come from social security benefits. The participation of benefits encompassed by the General Social Security System fell from $87 \%$ to $82 \%$ of the total payments. Expenditure on personnel and costs also decreased. However, significant gain was observed in transfers to third parties, which represent the resources collected as part of payroll and transferred to the so-called "S-system" (like SENAC OR SENAI): institutions of a social nature and directed to professional qualification of employer's associations in Brazil.

A comparison between Tables 5.1 and 5.2 highlights some important elements. Payroll contribution increased $34.6 \%$ in terms of participation in GDP, whereas benefits from the RGPS increased by $14.6 \%$. 
do RGPS aumentaram em $14,6 \%$. Ao comparar as necessidades de financiamento via confrontação desses dois quesitos, os valores se reduziram de 1,2\% em 2003 para $0,4 \%$ sobre o PIB em 2014. A crise econômica dos anos seguintes deve reverter muito rapidamente esse cenário.

A tabela 5.3 distingue o pagamento de benefícios das esferas urbana e rural. Existem 29\% de beneficiários rurais contra $71 \%$ de urbanos. Entre os rurais ocorreu uma concentração de benefícios na esfera previdenciária. Esse universo é marcado por forte inserção do regime de economia familiar, que possui regras de custeio singulares, devendo pagar tributos que incidem sobre o resultado da comercialização da safra e, para fins de elegibilidade, comprovar o exercício regular de atividades rurais.

Segundo a tabela 5.3, o contingente populacional que recebe benefícios previdenciários se concentra em aposentadorias e pensões. Isso ocorre porque são esses os benefícios vitalícios, da mesma forma que os do BPC, ao contrário dos auxílios, que são concedidos por tempo determinado. Existe uma forte presença de pensões por morte, pois, no Brasil, o viúvo tem direito de acesso à mesma, ainda que receba aposentadoria ou rendimentos decorrentes do trabalho, ao contrário do que ocorre em muitos outros países. Por fim, é preciso destacar que existe, no universo urbano, uma importante presença de benefícios assistenciais, revelando que muitos não conseguem comprovar contribuições por, pelo menos, quinze anos. Isso indica ser elevado o contingente de trabalhadores afetados pela precarização das relações de trabalho. No universo rural isso não ocorre porque as regras de elegibilidade não estão centradas no esforço
When these two are compared in terms of net borrowing, we see figures fell from $1.2 \%$, in 2003 , to $0.4 \%$ above GDP in 2014 . The economic crisis of the following years is expected to reverse this scenario very fast.

Table 5.3 separates urban and rural benefits, with $71 \%$ of beneficiaries for the former and $29 \%$ for the latter. There was concentration of social security benefits in the rural sphere, which is mainly characterized by family economy and its specific cost regulation. They pay taxes according to crop sales and, prove, for eligibility purposes, the regular exercise of rural activities.

According to table 5.3, the greatest share of the benefits paid to the population is concentrated on retirement and pensions. That happens because these are lifetime benefits, the same as those of BPC, opposite to aids, which are granted on a pre-determined time basis.

There is a high occurrence of death benefits, which is a right of widowed persons in Brazil, differently from what happens in many other countries. Finally, it is worth highlighting that the urban scenario has a significant participation in welfare benefits, so many people do not succeed in proving they have contributed to social security for at least 15 years. That indicates that a big number of workers have been affected by the low quality of job relations. The situation is different in the rural area, for eligibility rules are 
contributivo e se baseiam no exercício regular dessa atividade.

Em relação ao perfil de benefícios, a tabela 5.4 tornou mais precisas algumas das informações precedentes ao tratar da concessão de benefícios, ou seja, aqueles que passaram a ser pagos num determinado ano, ao passo que, na tabela 5.3, o universo refletia todos os benefícios pagos (ativos). Entre os benefícios concedidos são os auxíliosdoença que aparecem com destaque, 0 que é compreensível, pois eles refletem o movimento ocorrido ao longo de um ano.

A tabela 5.4 revela que os benefícios ordinários, ou seja, as aposentadorias por tempo de contribuição representam apenas $16 \%$ do total de benefícios vitalícios ou $6 \%$ do total geral. Isso demonstra que a precarização do mercado de trabalho, marcado pela informalidade e por elevada rotatividade impede a maioria dos segurados de contribuir por 30 ou 35 anos. Prova disso é que as aposentadorias por idade, que, no caso urbano, exigem 15 ou mais anos de contribuição representam $33 \%$ dos benefícios de prestação vitalícia e os assistenciais, $18 \%$. As pensões por morte se apropriam de $21 \%$ e as aposentadorias por invalidez de quase $10 \%$ do total de benefícios de prestação vitalícia. A escassa participação da aposentadoria ordinária e a elevada participação daqueles que contribuíram parcialmente ou bem pouco revela ser muito difícil estabelecer um equilíbrio contábil baseado em contribuições que incidam apenas sobre a folha salarial. $O$ quadro se agrava em períodos de baixo crescimento do PIB.

A tabela 5.5 destaca a evolução do número de contribuintes e seu not based on contributions, but on the regular exercise of rural activity.

In relation to the profile of benefits, table 5.4 makes more precise some of the preceding information on the granting of benefits, that is, those that began to be paid in a given year, whereas, in table 5.3 , the universe reflected all benefits paid (active ones). Among the benefits granted, sickness benefits are highlighted, which makes sense, since they reflect the movement occurred throughout a year.

Table 5.4 shows that ordinary benefits, that is, retirement pensions for length of service, represent only $16 \%$ of lifetime benefits or $6 \%$ of total benefits. That reflects the precariousness of the job market, characterized by informality and a high level of turnover which prevents most associates from paying contributions for 30 or 35 years. Proof of that is that age retirement, which, in urban areas, requires 15 or more years of contribution, represents $33 \%$ of lifetime benefits, and $18 \%$ of welfare benefits. Death pensions make up $21 \%$ and retirement ones almost $10 \%$ of the total benefits of lifetime pensions. The scarce participation of ordinary retirement and the high level of participation of persons who have contributed partially or very little shows how difficult it is have an accounting balance based on payroll contributions. This scenario becomes worser in periods of low GDP growth.

Table 5.5 highlights the evolution of the number of contributors and 
perfil entre 2004 e 2014, reiterando a importância do desempenho do PIB como elemento essencial para melhorar o desempenho financeiro da previdência. $O$ número de contribuintes se elevou em quase $70 \%$ no período, um valor muito superior, segundo dados do IBGE, ao da População em Idade Ativa - PIA - (17\%) ou da população ocupada (19\%). A proporção de contribuintes sobre a população economicamente ativa - PEA -aumentou de $46 \%$ para $62 \%$, um patamar excepcional na série histórica. No Brasil, segundo a tabela 5.5, e emprego representa $76 \%$ das ocupações, ao passo que em países de maior desenvolvimento humano esse total supera a barreira dos $90 \%$. Isso se explica pelo fato de o Brasil possuir muitos profissionais autônomos, representando $21 \%$ do total dos ocupados em 2014. Os trabalhadores autônomos ou domésticos, nos países com maior desenvolvimento humano, representam uma fração mínima, ao contrário do Brasil, o que ajuda a explicar o reduzido contingente de contribuintes por aqui.

A tabela 5.6 revela, com base em dados do IBGE, como a previdência no Brasil possui uma cobertura quase universal para idosos com 65 anos de idade ou mais, ou seja, mais de $96 \%$ da população total, uma situação privilegiada. Entre idosos, o nível de cobertura pouco evoluiu entre 2004 e 2014 porque os efeitos positivos da $\mathrm{CF} / 88$ se consolidaram nos anos 1990. No entanto, na população com idades entre 21 e 64 anos e, mais ainda, nas faixas etárias inferiores, o problema da não cobertura por parte da previdência exige atenção. Para a primeira faixa, o patamar supera $29 \%$ e entre os com idade inferiores a 21 anos, representa their profile between 2004 and 2014, reinforcing the role of GDP as an essential element to improve the financial performance of social security. The number of contributors rose by almost $70 \%$ in the period, a much higher figure, according to the IBGE, than that of the Working Age Population - WAP - (17\%) or the employed population (19\%). The proportion of contributors in relation to the economically-active population - EAP - increased from $46 \%$ to $62 \%$, an incredible level in the time series. In Brazil, according to table 5.5, employees represent $76 \%$ of the occupations, whereas in countries of higher human development index this figure surpasses $90 \%$. That can be explained by the fact that Brazil has many selfemployed workers, accounting for $21 \%$ of the total employed population in 2014. Self-employed or domestic workers, in countries of high human development, represent a minimum fraction, opposite to Brazil, and that accounts for the reduced number of contributors here.

Based on the IBGE data, table 5.6 shows how social security in Brazil has an almost universal coverage for the elderly aged 65 and over, more than $96 \%$ of the total population, undoubtedly a very privileged situation. Among the elderly, the level of coverage grew little between 2004 and 2014 because the positive effects of $\mathrm{CF} / 88$ were consolidated in the 1990 s. However, in the population aged 21 to 64 , mainly among younger age groups, the problem of noncoverage demands attention. In the first range, the level surpasses $29 \%$ and among persons under 21 years of 
mais de $42 \%$. Vale destacar que os dados apresentam também a cobertura por dependência, ou seja, dos cônjuges e dos filhos menores de 21 anos idade, que por lei são elegíveis a pensões por morte e se mantém protegidos em caso de morte do segurado titular, contribuinte ou aposentado.

Entre as faixas de idade até 64 anos houve progresso entre 2004 e 2014. Entre aqueles com até 20 anos de idade, os não cobertos se reduziram de $50 \%$ para $42 \%$ e naqueles com idades entre 21 e 64 anos de $39 \%$ para $29 \%$. Esse efeito se deve ao maior crescimento da economia que teve efeitos amplamente positivos sobre o mercado de trabalho. Isso elevou o número de segurados contribuintes e de seus dependentes legais.

Na tabela 5.7 é possível medir os impactos dos rendimentos pagos pela previdência e pelo BPC sobre a renda domiciliar divididos em decis, onde o primeiro é composto por domicílios com rendimentos menores, enquanto no décimo se dá o oposto. Segundo dados do IBGE, os decis 1, 3 e 4 foram os que apresentaram a maior participação de rendimentos da previdência comparada à média brasileira de $16 \%$. Isso se deve aos impactos do BPC para os domicílios no decil 1 , enquanto nos decis 3 e 4 se inserem, sobretudo, os indivíduos que recebem em torno de um salário mínimo. Os decis de menor renda revelam que a previdência possui um maior impacto sobre os rendimentos médios e isso valeu até o decil 7, sendo que essa proporção aumentou entre 2004 e 2014. Isso se deveu à recomposição do valor do piso previdenciário de um salário mínimo e, em menor medida, dos benefícios de age, it represents more than $42 \%$. It is worth highlighting that these data present coverage by dependence, that is, of spouses and of children under 21 years of age, who are eligible to death pensions and remain protected in case of death of the second head contributor, either retired or not.

Considering age groups up to 64 , there were advances between 2004 and 2014. Among persons aged up to 20 , non-assisted persons fell from $50 \%$ to $42 \%$ and among those aged between 21 and 64 , from $39 \%$ to $29 \%$. This effect comes from the bigger increase of economy, which had widely positive effects on the job market. That raised the number of contributors and of their legal dependents.

In table 5.7 , it is possible to measure the impacts of incomes paid by social security and by the BPC over household income divided into deciles, where the first one is formed by housing units with smaller income, whereas the tenth presents the opposite. According to data from the IBGE, deciles 1, 3 and 4 were the ones presenting the biggest participation of income from social security compared with the Brazilian average of $16 \%$. That occurred due to impacts of BPC on housing units in decile 3 , whereas deciles 3 and 4 include mostly persons who earn approximately one minimum wage. Deciles of smaller income show that social security exerts a bigger impact on average yields and that was true up to decile 7 , considering that this proportion increased between 2004 and 2014. That happened due to the recomposition of the social security factor for one minimum wage, and, to 
valor maior. Para os três decis de maior renda, a evolução positiva da economia deve ter elevado as demais parcelas da renda e isso explica a participação menor da previdência em 2014 comparada a de 2004.

\section{A conjugação do cenário caracterizado} por uma virtual universalização da cobertura na esfera dos beneficiários e o restrita na de contribuintes sobre a PIA, PEA ou população ocupada gera desafios para fins de custeio. $O$ senso comum deduz que o pagamento de benefícios deve ser bancado em sua íntegra pelas contribuições dos trabalhadores e empregadores sobre a folha salarial para a previdência. Mas, diante do cenário brasileiro, essa condição parece ser impossível ao confrontar a profunda dicotomia de cobertura entre contribuintes, muito restrita e beneficiários, virtualmente universal para os idosos. A situação se tornou mais grave após 2014, devido ao envelhecimento da população e, principalmente, à rápida deterioração da economia brasileira, que precisa ser revertida para poder reequilibrar as finanças da previdência.

Com a dura crise econômica que se instalou após 2014, um novo ciclo de reformas retornou à agenda pública e a referente à previdência tem merecido destaque no Brasil. Ao cotejar os gastos do INSS com a arrecadação sobre a folha salarial é apresentado um argumento revelando que essa situação é insustentável sob o prisma atuarial. Existem dificuldades de fato e os problemas da previdência não são poucos. No entanto, deixar de considerar as diferentes situações de contribuição dos segurados num mercado de trabalho marcado pela precarização há décadas a lower extent, to higher value benefits. For the three deciles of highest incomes, the positive evolution of economy must have increased the income parcels and that explains the reduced participation of social security in 2014 versus 2004 levels.

The conjugation of a scenario characterized by a virtual universalization of coverage for beneficiaries and restricted in terms of contributors from WAP, EAP, or the employed population causes costing difficulties. Common sense suggests that social security benefits must be thoroughly paid by means of payroll contributions from workers and employers. Given the Brazilian scenario, this condition seems to be impossible: there is a dichotomy of coverage between contributors, which is very restricted, and beneficiaries, with virtually universal access for the elderly. The situation has become more serious after 2014, due to population ageing and mainly to the fast decay of the Brazilian economy, which needs to be recovered before social security balance is reached again.

With the serious economic crisis which has taken the segment after 2014, a new cycle of reforms has come to public light and social security has gained more attention in Brazil. The argument adopted when contrasting the expenditure of the INSS with the payroll benefit is that the current actuarial status of things is not affordable. Certainly there are differences and social security has been facing many problems. However, not considering the different scenarios of associate participation in a job market marked for decades by precarization can generate 
pode gerar propostas que desprezem a peculiaridade da situação social brasileira e, para resolver um problema, gera novas dificuldades, talvez piores. Além disso, existem regras de elegibilidade que beneficiam os mais abastados e que merecem atenção por não seguir os preceitos clássicos das políticas sociais. Promover reformas da previdência para estimular o desenvolvimento exige lidar com cenários complexos e multifacetados e que não se resumem apenas ao mero equilíbrio do fluxo de caixa. proposals that disregard the peculiar situation of Brazil and might create new difficulties, instead of helping solve the existing ones. In addition to that, there are eligibility rules which benefit wealthier classes and which must be looked at since they do not follow classic premises of social policies. Promoting social security reforms to stimulate development requires dealing with complex and multifaceted settings which are merely limited to balancing our cash flow.

\section{Milko Matijascic}

Técnico de Planejamento e Pesquisa pelo IPEA. Doutor em Economia pela UNICAMP Universidade Estadual de Campinas desde 2002 - e Bachelier en Sciences Économiques et Sociales pela Academia de Poitiers (França, 1983). Integra, desde 2008, o comitê Científico da AISS - Associação Internacional de Seguridade Social - e da Revista Internacional de Seguridade Social. Foi Assessor Especial do Ministro da Previdência Social em 2004/05.
Technician in Planning and Research at IPEA. $\mathrm{PhD}$ in Economics from the State University of Campinas - UNICAMP since 2002 and Bachelor in Social and Economic Sciences from the Poitiers Academy (France, 1983). He has been a member of the Scientific Committee of the International

Social Security Association - ISSA and of the International Social Security Review since 2008. He was a Special Adviser of the Minister of Social Security in 2004-2005. 
Tabela 5.1 - Recebimentos da Previdência Social - 2003-2014

Table 5.1 - Brazilian social security revenues - 2003-2014

\begin{tabular}{|c|c|c|c|c|c|c|}
\hline & \multirow[b]{2}{*}{$\begin{array}{l}\text { Ano/ } \\
\text { Year }\end{array}$} & \multicolumn{5}{|c|}{ Recebimentos (1 $000000 \mathrm{R} \$) /$ Revenues $(1,000,000 \mathrm{R} \$)$} \\
\hline & & $\begin{array}{l}\text { Total/ } \\
\text { Total }\end{array}$ & $\begin{array}{c}\text { Próprios/ } \\
\text { Contributions }\end{array}$ & $\begin{array}{c}\text { Transferências } \\
\text { da União/ } \\
\text { Federal transfers }\end{array}$ & $\begin{array}{l}\text { Rendimentos } \\
\text { financeiros/ } \\
\text { Financial } \\
\text { revenues }\end{array}$ & $\begin{array}{l}\text { Outros/ } \\
\text { Others }\end{array}$ \\
\hline 2003 & & 122229 & 86587 & 35038 & 385 & 219 \\
\hline 2004 & & 152684 & 101126 & 48947 & 932 & 1679 \\
\hline 2005 & & 172720 & 115956 & 55879 & 187 & 698 \\
\hline 2006 & & 201757 & 133015 & 67373 & $(-) 3$ & 1371 \\
\hline 2007 & & 216489 & 153788 & 63074 & 404 & (-) 777 \\
\hline 2008 & & 243831 & 180346 & 59512 & 150 & 3822 \\
\hline 2009 & & 273524 & 200618 & 72940 & (-) 383 & 348 \\
\hline 2010 & & 312641 & 233513 & 79029 & (-) 192 & 292 \\
\hline 2011 & & 351545 & 271406 & 79580 & 145 & 414 \\
\hline 2012 & & 396684 & 304881 & 92712 & (-) 1196 & 287 \\
\hline 2013 & & 431684 & 340004 & 92647 & (-) 1285 & 318 \\
\hline 2014 & & 471807 & 374017 & 99701 & (-) 2127 & 215 \\
\hline
\end{tabular}

Fonte/Source: Ministério da Previdência Social, Coordenação Geral de Estatística.

Tabela 5.2 - Pagamentos da Previdência Social - 2003-2014

Table 5.2 - Brazilian social security payments - 2003-2014

\begin{tabular}{|c|c|c|c|c|c|c|}
\hline \multirow[b]{2}{*}{$\begin{array}{l}\text { Ano/ } \\
\text { Year }\end{array}$} & \multicolumn{6}{|c|}{ Pagamentos (1 $000000 \mathrm{R} \$$ ) / Payments $(1,000,000 \mathrm{R} \$)$} \\
\hline & $\begin{array}{l}\text { Total/ } \\
\text { Total }\end{array}$ & $\begin{array}{c}\text { Benefícios } \\
\text { do RGPS/ } \\
\text { Social security } \\
\text { benefits }\end{array}$ & $\begin{array}{l}\text { LOAS + EPU/ } \\
\angle O A S+E P U\end{array}$ & $\begin{array}{l}\text { Pessoal/ } \\
\text { Personnel }\end{array}$ & $\begin{array}{l}\text { Custeio/ } \\
\text { Costing }\end{array}$ & $\begin{array}{c}\text { Transferências } \\
\text { a terceiros/ } \\
\text { Transfers to } \\
\text { third parties }\end{array}$ \\
\hline 2003 & 123361 & 107135 & 5063 & 3774 & 1533 & 5857 \\
\hline 2004 & 150654 & 125751 & 8168 & 6948 & 2427 & 7360 \\
\hline 2005 & 171798 & 146010 & 9999 & 4541 & 3727 & 7521 \\
\hline 2006 & 200511 & 165585 & 12333 & 5873 & 7225 & 9495 \\
\hline 2007 & 221942 & 185293 & 15015 & 6196 & 2061 & 13377 \\
\hline 2008 & 242592 & 199562 & 17054 & 6929 & 2398 & 16649 \\
\hline 2009 & 272656 & 224876 & 19987 & 7366 & 2191 & 18236 \\
\hline 2010 & 312124 & 254859 & 23294 & 8982 & 3877 & 21112 \\
\hline 2011 & 343922 & 281438 & 26168 & 8921 & 2397 & 24999 \\
\hline 2012 & 386692 & 316590 & 30575 & 8790 & 2210 & 28528 \\
\hline 2013 & 435649 & 357003 & 34896 & 8967 & 2614 & 32169 \\
\hline 2014 & 481797 & 394201 & 39142 & 9575 & 3420 & 35459 \\
\hline
\end{tabular}

Fonte/Source: Ministério da Previdência Social, Coordenação Geral de Estatística. 
Tabela 5.3 - Distribuição dos benefícios ativos,

urbano e rural - 2011-2014

Table 5.3 - Distribution of urban and rural benefits - 2011-2014

\begin{tabular}{|c|c|c|c|c|}
\hline Benefícios/ Benefits & 2011 & 2012 & 2013 & 2014 \\
\hline Total/Total & 28909419 & 29883423 & 31028250 & 32028710 \\
\hline Urbano/Urban & 20333324 & 21082324 & 21980637 & 22774297 \\
\hline Previdenciários/ Social security & 15786414 & 16370813 & 17069525 & 17706670 \\
\hline Aposentadorias/ Retirement pensions & 9923854 & 10338862 & 10788462 & 11231112 \\
\hline Pensões por morte/ Survivor pensions & 4648802 & 4773408 & 4904697 & 5018901 \\
\hline Auxílios/Cash aid & 1156549 & 1197125 & 1309928 & 1390098 \\
\hline Outros/Others & 57209 & 61418 & 66438 & 66559 \\
\hline Assistenciais/Social assistance & 3750033 & 3912069 & 4095090 & 4244544 \\
\hline $\begin{array}{l}\text { Amparos assistenciais/Income } \\
\text { assistance }\end{array}$ & 3600473 & 3777778 & 3975413 & 4137995 \\
\hline $\begin{array}{l}\text { Rendas mensais vitalícias/Lifelong } \\
\text { monthly income }\end{array}$ & 136072 & 121383 & 107351 & 94845 \\
\hline Outros/Others & 13488 & 12908 & 12326 & 11704 \\
\hline Acidentários/ Work-related injuries & 785210 & 787604 & 804389 & 811849 \\
\hline Aposentadorias/ Retirement pensions & 156859 & 164087 & 171186 & 177644 \\
\hline Pensões/Survivor pensions & 119386 & 117861 & 116216 & 114187 \\
\hline Auxílios/Cash aid & 508965 & 505656 & 516987 & 520018 \\
\hline $\begin{array}{l}\text { Encargos Previdenciários da União - EPU/ } \\
\text { Treasury Owed Pensions - EPU }\end{array}$ & 11667 & 11838 & 11633 & 11234 \\
\hline Rural/Rural & 8576095 & 8801099 & 9047613 & 9254413 \\
\hline Previdenciários/ Social security & 8427887 & 8664518 & 8920967 & 9137399 \\
\hline Aposentadorias/ Retirement pensions & 6105570 & 6279789 & 6460330 & 6614693 \\
\hline Pensões por morte/ Survivor pensions & 2155077 & 2206732 & 2261015 & 2305020 \\
\hline Auxílios/Cash aid & 154758 & 167024 & 187319 & 206480 \\
\hline Outros/Others & 12482 & 10973 & 12303 & 11206 \\
\hline Assistenciais/Social assistance & 115916 & 104178 & 93228 & 83550 \\
\hline $\begin{array}{l}\text { Amparos assistenciais/Income } \\
\text { assistance }\end{array}$ & - & - & - & - \\
\hline $\begin{array}{l}\text { Rendas mensais vitalícias/Lifelong } \\
\text { monthly income }\end{array}$ & 115916 & 104178 & 93228 & 83550 \\
\hline Outros/Others & - & - & - & \\
\hline Acidentários/ Work-related injuries & 32292 & 32403 & 33418 & 33464 \\
\hline Aposentadorias/ Retirement pensions & 11021 & 11497 & 11936 & 12400 \\
\hline Pensões/Survivor pensions & 4279 & 4215 & 4139 & 4058 \\
\hline Auxílios/Cash aid & 16992 & 16691 & 17343 & 17006 \\
\hline
\end{tabular}

Fonte/Source: Ministério da Previdência Social, Coordenação Geral de Estatística. 
Tabela 5.4 - Benefícios concedidos pela previdência social - 2011-2014

Table 5.4 - Benefits granted by social security - 2011-2014

\begin{tabular}{|c|c|c|c|c|}
\hline Benefícios/ Benefits & 2011 & 2012 & 2013 & 2014 \\
\hline Total/Total & 4767039 & 4957681 & 5207629 & 5211030 \\
\hline Previdenciários/Social security & 4077115 & 4295870 & 4513432 & 4554542 \\
\hline Aposentadorias/ Retirement pensions & 1061724 & 1102424 & 1162345 & 1150880 \\
\hline Idade/Old age & 580716 & 621515 & 654523 & 645687 \\
\hline Invalidez/ Disability & 183301 & 182818 & 193562 & 189651 \\
\hline Tempo de contribuição/ Lenght of contribution & 297707 & 298091 & 314260 & 315542 \\
\hline Pensões por morte/ Survivor pensions & 396278 & 399295 & 414675 & 409245 \\
\hline Auxílio-doença/ Sickness benefit & 2022613 & 2158346 & 2273074 & 2328151 \\
\hline Salário-maternidade/ Maternity leave & 568438 & 604815 & 628409 & 631687 \\
\hline Outros/ Others & 28062 & 30990 & 34929 & 34579 \\
\hline Assistenciais/Social assistance & 341643 & 327414 & 355509 & 343990 \\
\hline $\begin{array}{l}\text { Amparos assistenciais - LOAS/Income } \\
\text { assistance }\end{array}$ & 341288 & 327037 & 355165 & 343669 \\
\hline Idoso/Old age & 155353 & 153024 & 169138 & 160204 \\
\hline Portador de deficiência/ Impaired people & 185935 & 174013 & 186027 & 183465 \\
\hline $\begin{array}{l}\text { Pensões mensais vitalícias/ Lifelong } \\
\text { monthly pensions }\end{array}$ & 352 & 376 & 344 & 321 \\
\hline Rendas mensais vitalícias/ Lifelong & & & & \\
\hline monthly income & 3 & 1 & 0 & 0 \\
\hline Idoso/ Old age & 0 & 1 & 0 & 0 \\
\hline Invalidez/Impaired people & 3 & 0 & 0 & 0 \\
\hline Acidentários/ Work-related injuries & 346501 & 333398 & 338122 & 312192 \\
\hline Aposentadorias/ Retirement pensions & 11108 & 11433 & 11655 & 10877 \\
\hline Pensão por morte/ Survivor pensions & 751 & 614 & 497 & 412 \\
\hline Auxílio-doença/ Sickness benefit & 319445 & 305208 & 304217 & 279868 \\
\hline Auxílio-acidente/ Injury aid & 15068 & 16012 & 21563 & 20883 \\
\hline $\begin{array}{l}\text { Auxílio-suplementar/ Supplemental } \\
\text { income }\end{array}$ & 129 & 131 & 190 & 152 \\
\hline Encargos Previdenciários da União - EPU/ & 1780 & 999 & 566 & 306 \\
\hline Treasury Owed Benefits - EPU & & & & \\
\hline
\end{tabular}

Fonte/Source: Ministério da Previdência Social, Coordenação Geral de Estatística. 
Tabela 5.5 - Quantidade de pessoas físicas contribuintes do Regime Geral de Previdência Social - RGPS, por categoria - 2004-2014

Table 5.5 - Number of people contributing to the General Regime of Social Security - RGPS, by category - 2004-2014

\begin{tabular}{llrrr}
\hline $\begin{array}{c}\text { Ano/ } \\
\text { Year }\end{array}$ & \multicolumn{1}{l}{$\begin{array}{l}\text { Total/ } \\
\text { Total }\end{array}$} & $\begin{array}{c}\text { Empregado/ } \\
\text { Employee }\end{array}$ & $\begin{array}{c}\text { Outro Contribuinte/ } \\
\text { Other contributor }\end{array}$ & $\begin{array}{c}\text { Empregado e Outro } \\
\text { Contribuinte/ } \\
\text { Employee and Other } \\
\text { contributor }\end{array}$ \\
\hline 2004 & 42084323 & 32200411 & 8766902 & 1117010 \\
2005 & 45035035 & 34687001 & 9099704 & 1248330 \\
2006 & 46676737 & 36158570 & 9262079 & 1256088 \\
2007 & 49936338 & 38788600 & 9710280 & 1437458 \\
2008 & 53964928 & 42076251 & 10235457 & 1653220 \\
2009 & 55877835 & 43439321 & 10684737 & 1753777 \\
2010 & 60197924 & 46683012 & 11548708 & 1966204 \\
2011 & 64109870 & 49508175 & 12428273 & 2173422 \\
2012 & 67246063 & 51609519 & 13333407 & 2303137 \\
2013 & 69669481 & 53381148 & 13981592 & 2306741 \\
2014 & 71493806 & 54152097 & 14917515 & 2424194 \\
\hline
\end{tabular}

Fonte/Source : Anuário estatístico da previdência social - AEPS 2013. Brasília, DF: Ministério da Previdência Social, v. 22, [2014]. Disponível em: <http://www.mtps.gov.br/dados-abertos/dados-da-previdencia/ previdencia-social-e-inss>. Acesso em: abr. 2016. 
Tabela 5.6 - Percentual da população, segundo a cobertura de previdência pública e BPC por grupos de idade - Brasil - 2004/2014

Table 5.6 - Percentual share of the population according to public pension and BPC coverage and age group - Brazil - 2004/2014

\begin{tabular}{|c|c|c|c|c|c|}
\hline \multirow{3}{*}{$\begin{array}{l}\text { Cobertura/ } \\
\text { Coverage }\end{array}$} & \multicolumn{5}{|c|}{ Grupos de idade/age groups } \\
\hline & \multirow{2}{*}{ Total } & Até 20/ & 21 a 64/ & 65 ou mais/ & 70 ou mais/ \\
\hline & & Until 20 & 21 to 64 & 65 or more & 70 or more \\
\hline \multicolumn{6}{|c|}{2004} \\
\hline Total/Total & 100,0 & 100,0 & 100,0 & 100,0 & 100,0 \\
\hline Contribuintes (1)/Contributors (1) & 20,7 & 4,6 & 35,6 & 1,3 & 0,4 \\
\hline Beneficiários (2)/Beneficiaries (2) & 10,8 & 0,4 & 9,2 & 86,4 & 90,5 \\
\hline \multicolumn{6}{|l|}{ Contribuintes e beneficiários (3)/ } \\
\hline Contributors and beneficiaries (3) & 0,8 & 0,0 & 1,2 & 1,8 & 1,2 \\
\hline \multicolumn{6}{|l|}{ Dependentes legais (4)/ } \\
\hline Legal dependents (4) & 26,7 & 45,2 & 14,9 & 6,5 & 4,9 \\
\hline Não cobertos (5)/Non covered (5) & 41,1 & 49,8 & 39,1 & 4,0 & 3,0 \\
\hline \multicolumn{6}{|c|}{2014} \\
\hline Total/Total & 100,0 & 100,0 & 100,0 & 100,0 & 100,0 \\
\hline Contribuintes (1)/Contributors (1) & 28,8 & 7,0 & 45,9 & 1,9 & 0,5 \\
\hline Beneficiários (2)/Beneficiaries (2) & 13,7 & 0,6 & 9,6 & 86,6 & 91,4 \\
\hline \multicolumn{6}{|l|}{ Contribuintes e beneficiários/ } \\
\hline Contributors and beneficiaries & 1,1 & 0,0 & 1,5 & 2,6 & 1,5 \\
\hline \multicolumn{6}{|l|}{ Dependentes legais (3)/ } \\
\hline Legal dependents (3) & 25,2 & 50,2 & 13,9 & 5,2 & 3,7 \\
\hline Não cobertos (4)/Non covered (4) & 31,2 & 42,3 & 29,1 & 3,8 & 2,9 \\
\hline
\end{tabular}

Fonte/Source: IBGE, Diretoria de Pesquisas, Coordenação de Trabalho e Rendimento, Pesquisa Nacional por Amostra de Domicílios 2004/2014.

(1) Contribuintes individuais para instituto de previdência em pelo menos um dos trabalhos. (2) Beneficiários: aposentados, pensionistas ou beneficiários do BPC. (3) Dependentes legais: dependentes de contribuintes ou beneficiários. (4) Não cobertos./(1) Contributora to the public pension system in one or more occupations. (2) Beneficiaries: pensioners, survivor pensioners and BPC pensions.(3) Dependents: legaç dependents from contributors and beneficiaries. (4) Non covered. 
Tabela 5.7 - Percentual da renda domiciliar mensal paga pela previdência pública e BPC sobre a renda domiciliar total segundo decis de renda

Brasil - 2004/2014

Table 5.7- Percentual from household income share paid by public pensions or BPC according to deciles - Brazil - 2004/2014

\begin{tabular}{|c|c|c|c|}
\hline $\begin{array}{l}\text { Decil/ } \\
\text { Deciles }\end{array}$ & $\begin{array}{l}\text { Percentual da renda } \\
\text { da previdência e BPC/ } \\
\text { Percentual share from } \\
\text { pensions and BPC }\end{array}$ & $\begin{array}{c}\text { Renda domiciliar mensal } \\
\text { média/ } \\
\text { Monthly average } \\
\text { household income - } R \$\end{array}$ & $\begin{array}{c}\text { Renda domiciliar } \\
\text { mensal média paga } \\
\text { pela previdência e BPC/ } \\
\text { Monthly average } \\
\text { household income paid } \\
\text { by social security and } \\
B P C-R \$\end{array}$ \\
\hline \multicolumn{4}{|c|}{2004} \\
\hline Total/Total & 16,0 & 2381 & 380 \\
\hline 1 & 18,8 & 319 & 60 \\
\hline 2 & 13,1 & 556 & 73 \\
\hline 3 & 18,5 & 787 & 146 \\
\hline 4 & 20,6 & 991 & 204 \\
\hline 5 & 18,3 & 1258 & 231 \\
\hline 6 & 16,2 & 1568 & 254 \\
\hline 7 & 15,8 & 1988 & 314 \\
\hline 8 & 16,1 & 2645 & 426 \\
\hline 9 & 16,1 & 3893 & 626 \\
\hline 10 & 15,0 & 9802 & 1467 \\
\hline \multicolumn{4}{|c|}{2014} \\
\hline Total/Total & 16,3 & 3307 & 540 \\
\hline 1 & 28,4 & 519 & 147 \\
\hline 2 & 14,4 & 902 & 130 \\
\hline 3 & 24,2 & 1281 & 310 \\
\hline 4 & 28,3 & 1579 & 447 \\
\hline 5 & 19,1 & 1972 & 377 \\
\hline 6 & 18,8 & 2402 & 453 \\
\hline 7 & 16,9 & 2972 & 501 \\
\hline 8 & 16,2 & 3786 & 615 \\
\hline 9 & 14,6 & 5256 & 767 \\
\hline 10 & 13,4 & 12396 & 1657 \\
\hline
\end{tabular}

Fonte/Source: IBGE, Diretoria de Pesquisas, Coordenação de Trabalho e Rendimento, Pesquisa Nacional por Amostra de Domicílios 2004/2014. 


\section{Educação \\ Education}

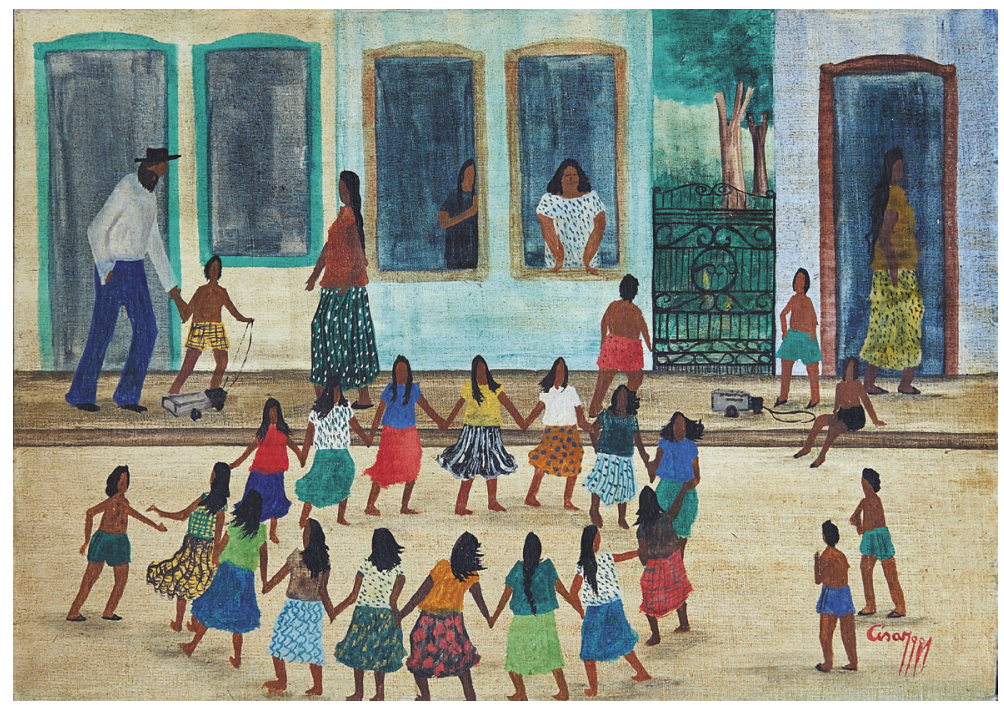

Brincadeira de roda/Ring a ring o' roses, 1981 Júlio César Revoredo Serafim 



\section{Educação}

\section{Education}

\section{Considerações iniciais}

\section{O que há de novo em termos teóricos para melhorar a Educação?}

Para responder esta questão, em recente publicação ${ }^{\prime}$, a UNESCO salienta a necessidade de repensar a educação e a aprendizagem, frente às mudanças que vem ocorrendo no mundo atual, caracterizadas por novos níveis de complexidade e de contradição. Segundo essa publicação, a educação deve preparar indivíduos e comunidades para as tensões geradas por tais mudanças, tornando-os capazes de se adaptar e de responder a elas. Na realidade, a educação ainda está ancorada na era industrial e não conseguiu integrar as mudanças da era digital para preparar o cidadão para enfrentar os novos desafios do século $X X I$. Somente para se ter uma ideia, os estudantes que nasceram em 2001 e que estão hoje no ensino básico (antigo $2^{\circ}$ Grau), já nasceram convivendo com o Google $^{\oplus}$, Facebook ${ }^{\circledR}$, Twitter ${ }^{\oplus}$, Smartfones e Tablets, mas essas tecnologias não estão incorporadas como meios para apoiar a

${ }^{1}$ Rethinking education: towards a global common good? Brasília: UNESCO, 2016.

\section{Initial considerations}

\section{What is new, in theoretical terms, to improve Education?}

To answer this question, a recent ${ }^{1}$ UNESCO publication points out the need to rethink education and learning before the changes that are happening in the world today, characterized by new levels of complexity and contradiction. According to that publication, education should prepare individuals and communities for the tensions generated by such changes, enabling them to adapt and respond to them. In reality, education is still anchored in the industrial era and has not incorporated the changes of the digital era so as to prepare citizens to face the new challenges of the 21 st century. Just for us to have an idea, students who were born in 2001 and are in high school (former 2nd Degree) today have been getting along with Google ${ }^{\oplus}$, Facebook ${ }^{\circledast}$, Twitter ${ }^{\oplus}$, Smartphones and Tablets, yet these technologies are not incorporated as a means to support education.

1 Rethinking education: towards a global common good? Brasília: UNESCO, 2016. 
educação. Como salienta Michel Serres², as novas tecnologias nos condenam a ser mais inteligentes e a mudar nossa relação com o saber.

De fato, as novas tecnologias mudam a nossa relação com o saber, atingindo uma imensa quantidade de estudantes, de pais e de professores, exigindo uma evolução nos métodos e técnicas de ensino e de aprendizagem. Assim aconteceu com a descoberta da escrita e também com a descoberta da impressão. Agora, o mesmo deve acontecer com a descoberta do digital. O que era texto está se tornando, cada vez mais, hipertexto e essa evolução deve ser incorporada na educação. Todavia, é preciso salientar, aprendizagem é uma atividade que se processa na primeira pessoa, ou como observa Eduardo Chaves (1999)3), "para dentro do indivíduo". Ou, em outros termos, não são as tecnologias ou a Internet que ensinam, é o indivíduo que aprende. Em contrapartida, com o avanço das neurociências foi possível lançar uma nova perspectiva no processo de aquisição do conhecimento, isto é, no processo de aprendizagem. Hoje, com a ajuda de equipamentos de última geração tecnológica, como o PET/Scan (Tomografia por Emissão de Pósitrons), é possível avaliar, de forma detalhada, a perfusão sanguínea e a atividade (consumo de oxigênio) de diferentes regiões do cérebro humano.

Assim, as neurociências têm se desenvolvido de forma significativa,

\footnotetext{
${ }^{2}$ SERRES, Michel. Petite Poucette. Paris: Le Pommier, 2012.

${ }^{3}$ CHAVES, Eduardo. Tecnologia na Educação, Ensino a Distância e Aprendizagem Mediada pela Tecnologia: Conceituação Básica. Revista de Educação. PUCCampinas, Vol. 3, n 7, pp. 29-43, novembro de 1999.
}

As stressed by Michel Serres ${ }^{2}$, new technologies condemn us to be more intelligent and to change our relation with knowledge.

Indeed, new technologies change our relation with knowledge, hitting a huge number of students, parents and teachers and requiring an evolution in the methods and techniques of teaching and learning. This has happened with the discovery of writing and with the discovery of press printing. Now, the same should happen with the discovery of digital information. What was once a text has increasingly become a hypertext, and this evolution should be incorporated in education. Nevertheless, it should be pointed out that learning is an activity processed in the first person or, as noticed by Eduardo Chaves (1999)3, "inside the individual". Or, in other words, it is not technology or Internet that teaches, but it is the individual who learns. In contrast, the advance of neurosciences has made it possible to cast new light on the process of knowledge acquisition, that is to say, in the process of learning. Today, the use of stateof-the-art equipment like PET/Scan (Positron Emission Tomography), allows the detailed measurement of the blood perfusion and of the activity (oxygen consumption) in different areas of the human brain.

Thereby, neurosciences have been improving significantly, providing a 2 SERRES, Michel. Petite Poucette. Paris: Le Pommier,
2012.
${ }^{3}$ CHAVES, Eduardo. Tecnologia na Educação, Ensino a
Distância e Aprendizagem Mediada pela Tecnologia:
Conceituação Básica. Revista de Educação. PUC-
Campinas, Vol. 3, n 7, pp. 29-43, novembro de 1999. 
permitindo uma melhor compreensão do funcionamento do cérebro humano. De fato, o século XX permitiu à humanidade desvendar 0 corpo humano. Da mesma forma, o século XXI permitirá à humanidade desvendar o cérebro humano. Assim, ressonâncias magnéticas funcionais e tomografias computadorizadas ajudam os pesquisadores das neurociências a desvendar o funcionamento cerebral e a aquisição do conhecimento. A partir dessas pesquisas é possível simular como o cérebro humano aprende, analisa, armazena, recorda e perde a linguagem. Surge, então, um novo paradigma para a explicação da aquisição do conhecimento: o conexionismo.

Segundo Siemens $(2004)^{4}$, o conexionismo apresenta-se, então, como um novo paradigma da cognição humana que se coloca entre o objetivismo do behaviorismo e o pragmatismo do cognitivismo ortodoxo. De um lado, o paradigma behaviorista concebe que o comportamento humano é determinado pelo ambiente no qual o sujeito cognoscente está inserido, pelos estímulos que ele recebe do meio ambiente. De outro lado, 0 paradigma cognitivista concebe que $o$ comportamento humano é definido pelo modelo mental que o sujeito cognoscente possui, construído ao longo de sua existência. O conexionismo fundamentase nos estudos do cérebro humano, desenvolvidos pelas neurociências, procurando explicações para a aquisição do conhecimento, a partir das atividades das células nervosas, os neurônios. $\mathrm{Na}$

\footnotetext{
${ }^{4}$ Siemens, G. (2004, December). Connectivism: A theory for the digital age. Disponível em http://www. elearnspace.org/Articles/connectivism.htm . Acesso em 04 de junho de 2015.
}

better understanding of the human brain. Actually, the 20th century allowed humanity to unravel the human body. Likewise, the 21st century will allow humanity to unravel the human brain. Thus, functional magnetic resonances and computerized tomographies help neuroscience researchers to understand the cerebral function and the acquisition of knowledge. From these researches, it is possible to simulate how the human brain learns, analyzes, stores, recalls and loses language. A new paradigm now emerges to explain the acquisition of knowledge: connectivism.

According to Siemens (2004) connectivism appears as a new paradigm of the human cognition that stands between behaviorism's objectivism and orthodox cognitivism's pragmatism. On the one hand, the behaviorist paradigm conceives that human behavior is determined by the environment in which the cognizant individual is inserted, by the stimuli he/she receives from the environment. On the other hand, the cognitivist paradigm conceives that human behavior is determined by the mental model the cognizant individual has built along his/ her existence. Connectivism is based on studies of the human brain developed by neurosciences, when searching for an explanation for the acquisition of knowledge

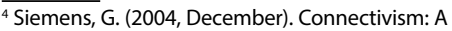
theory for the digital age. Available from http:// www.elearnspace.org/Articles/connectivism.htm. Cited on June 4, 2015.
} 
realidade, o objetivo do conexionismo é entender a cognição humana por meio de simulações das atividades neuronais, baseada nos seguintes princípios: i) Processamento em paralelo; ii) Formação de conceitos ad hoc; iii) Peso sináptico; iv) Plasticidade cerebral. Assim, o paradigma conexionista explica o armazenamento, o processamento e a recuperação da informação. Segundo este paradigma, a linguagem é aprendida por intermédio da experiência (POERSCH, 2003) ${ }^{5}$, ou seja, por intermédio da interação do indivíduo com o ambiente, com artefatos e, também, com outros indivíduos. Para entender a aquisição e/ou aprendizagem da linguagem é necessário compreender o funcionamento e a ligação dessas células nervosas. A aprendizagem, segundo a visão conexionista, se dá justamente por meio de mudanças físico-químicas entre essas células nervosas, designadas sinapses. $O$ cérebro humano se compõe de uma estrutura - os neurônios - e de um processo - as sinapses. Os sistemas conexionistas são formados por nódulos que imitam os neurônios humanos e são interligados em redes neurais que armazenam e processam dados. Rede neural é um conceito que simula o processamento de dados de maneira semelhante ao cérebro humano. $\mathrm{O}$ cérebro é tido como um processador altamente complexo e que realiza processamentos de maneira paralela. Para isso, ele organiza sua estrutura, ou seja, os neurônios, de forma que eles realizem o processamento necessário. Isso é feito numa velocidade extremamente alta e não existe qualquer computador no mundo capaz de realizar o que o cérebro humano faz.

\footnotetext{
${ }^{5}$ POERSCH , José Marcelino. Simulações conexionistas: a inteligência artificial moderna. Linguagem em (Dis) curso, Tubarão, v. 4, n. 2, 2003.
}

from the activities of the nerve cells, the neurons. In reality, the objective of connectivism is to understand human cognition by means of simulations of neuronal activities, based on the following principles: i) Parallel processing; ii) Formation of ad hoc concepts; iii) Synaptic weight; iv) Brain plasticity. Thereby, the connectivist paradigm explains the storage, processing and recovery of information. According to this paradigm, language is learned through the experience (POERSCH, 2003) $)^{5}$, that is to say, through the interaction of the individual with the environment, artifacts and other individuals. To understand the acquisition and/ or learning of the language, it is necessary to understand how these nerve cells work and link with one other. According to the connectivist vision, learning is made right through physical-chemical changes among the nerve cells, called synapses. The human brain comprises a structure - the neurons - and a process - the synapses. Connectivist systems are made by nodes that simulate human neurons and are interlinked in neural networks that store and process data. Neural network is a concept that simulates data processing like the human brain. The brain is considered a highly complex processor that processes information in parallel. To do this, it organizes its own structure, that is to say, the neurons, so that they perform the processing as needed. This is done at an extremely high speed and no computer in the world is capable of doing what the human brain does.

\footnotetext{
${ }^{5}$ POERSCH , José Marcelino. Simulações conexionistas: a inteligência artificial moderna. Linguagem em (Dis) curso, Tubarão, v. 4, n. 2, 2003.
} 
Nas redes neurais artificiais, a ideia é realizar o processamento de informações tendo como princípio a organização de neurônios do cérebro. Como o cérebro humano é capaz de aprender e tomar decisões baseadas na aprendizagem, as redes neurais artificiais devem fazer o mesmo. Assim, uma rede neural pode ser interpretada como um esquema de processamento capaz de armazenar conhecimento baseado em aprendizagem (experiência) e disponibilizar este conhecimento para a aplicação em questão.

Segundo Poersch (2004, p.02), a proposta de simulação conexionista procura reproduzir o cérebro humano em dois aspectos:

\section{O conhecimento é adquirido pela rede, por intermédio de um processo de aprendizagem;}

As forças de conexão interneuronal, conhecidas como pesos sinápticos, são utilizadas para armazenar conhecimento. Os sistemas conexionistas aprendem por intermédio do processamento estatístico do input, construindo, assim, suas próprias regras implícitas no funcionamento das redes.

Segundo esse paradigma, o conhecimento está armazenado em "engramas", ou seja, a atividade sináptica do neurônio gera o aprendizado quando são formadas novas conexões, e gera a lembrança quando são reforçadas as conexões entre os neurônios (CHIELE, 2004) $)^{6}$.

\footnotetext{
${ }^{6}$ CHIELE, L. K. A compreensão em leitura sob a perspectiva do conexionismo. In: ROSSA, A.; ROSSA, C. (Org.) Rumo à psicolinguística conexionista. Porto Alegre: EDIPUCRS, 2004.
}

The idea is to process information in the artificial neural networks based on the principle of the organization of the neurons in the brain. As the human brain is able to learn and make decisions based on learning, the artificial neural networks should do the same. Thus, a neural network can be interpreted as a processing schema capable of storing knowledge based on learning (experience) and delivering this knowledge to the particular application.

According to Poersch (2004, p.02), the connectivist simulation seeks to reproduce the human brain in two aspects:

\section{Knowledge is acquired by the network through a learning process;}

Interneuron connection strengths, known as synaptic weights, are used to store knowledge. Connectivist systems learn through the statistical processing of the input, thus building their own implicit rules to the operation of the networks.

According to this paradigm, knowledge is stored in "engrams", that is to say, the synaptic activity of the neuron generates learning whenever new connections are formed, and generates memory whenever the connections among neurons are reinforced (CHIELE, $2004)^{6}$.

\footnotetext{
${ }^{6}$ CHIELE, L. K. A compreensão em leitura sob a perspectiva do conexionismo. In: ROSSA, A.; ROSSA, C. (Org.) Rumo à psicolinguística conexionista. Porto Alegre: EDIPUCRS, 2004.
} 
Nesse processo cognitivo, cérebro e meio interagem, tendo o meio externo a função de fornecer o input e o cérebro humano de processá-lo conforme sua frequência e regularidade. Por outro lado, o conhecimento não fica armazenado em bloco, em um único neurônio, seus traços são distribuídos pela rede e sua recuperação ocorre de forma ad hoc, o que significa que não temos todos os conhecimentos prontos e disponíveis a todo o momento. Assim sendo, para a sua recuperação é necessário que os neurônios refaçam o caminho das conexões na rede em que estão "engramados". Quanto mais acessado for esse caminho, mais facilmente o conhecimento será recordado. Nesse sentido, Seidenberg e MacDonald (1999) ${ }^{7}$, acreditam que a aprendizagem envolve informação cumulativa e aspectos estatísticos e probabilísticos da linguagem.

Apesar de alguns autores argumentarem que o conexionismo não deva ser considerado uma nova teoria da aprendizagem (Kerr, 20078; Kop \& Hill, $\left.2008^{9}\right)$, é possível posicioná-lo como uma abordagem, desenvolvida a partir do construtivismo, para o cenário atual do uso da tecnologia na educação, embora funcionando como uma filosofia da educação.

\footnotetext{
$\overline{7}$ SEIDENBERG, Mark; MACDONALD, Maryellen C. A probabilistic constraints approach to language acquisition and processing. Cognitive Science, $v$. 23, n. 4, 1999.

${ }^{8}$ Kerr, B. (2007, February). A Challenge to Connectivism. Transcript of Keynote Speech, Online Connectivism Conference. University of Manitboa. Disponível em http://ltc.umanitoba.ca/wiki/index.php?title=Kerr_ Presentation. Acesso em 04 de junho de 2015.

${ }^{9}$ Kop, R., \& Hill, A. (2008). Connectivism: Learning theory of the future or vestige of the past? The International Review of Research in Open and Distance Learning, 9(3). Disponível em http://www. irrodl.org/index.php/irrodl/article/view/523/1137. Acesso em 04 de junho de 2015.
}

In this cognitive process, brain and environment interact, the latter providing the input and the former processing it, according to its frequency and regularity. On the other hand, knowledge is not stored en bloc in a single neuron. Its traces are distributed along the network and its recovery happens in an ad hoc manner, which means that not all knowledge is ready and available at all times. Therefore, the recovery of knowledge requires the neurons to retrace the connections in the network they are "engrammed" in. The more accessed the way, the easier the recollection of knowledge. In this respect, Seidenberg and MacDonald $(1999)^{7}$ believe that learning involves cumulative information and statistical and probabilistic aspects of language.

Although some authors argue that connectivism should not be considered a new theory of learning (Kerr, 20078; Kop \& Hill, 2008), it is possible to position it as an approach - developed from constructivism - for the current scenario of using technology in education, though it has been used as an education philosophy.

\footnotetext{
${ }_{7}^{7}$ SEIDENBERG, Mark; MACDONALD, Maryellen C. A probabilistic constraints approach to language acquisition and processing. Cognitive Science, v. 23, n. 4, 1999.

${ }^{8}$ Kerr, B. (2007, February). A Challenge to Connectivism. Transcript of Keynote Speech, Online Connectivism Conference. University of Manitoba. Available from http://ltc.umanitoba.ca/wiki/index.php?title=Kerr Presentation. Cited on June 4, 2015.

${ }^{9}$ Kop, R., \& Hill, A. (2008). Connectivism: Learning theory of the future or vestige of the past? The International Review of Research in Open and Distance Learning, 9(3). Available from http://www. irrodl.org/index.php/irrodl/article/view/523/1137. Cited on June 4, 2015.
} 
Siemens $(2004)^{10}$, discute as limitações do behaviorismo, cognitivismo e construtivismo como teorias de aprendizagem, porque elas não abordam a aprendizagem que ocorre fora dos indivíduos (ou seja, a aprendizagem que é armazenada e manipulada pela tecnologia), mas no contexto organizacional.

Nesse sentido, o conexionismo é proposto, então, como uma teoria de aprendizagem mais adequada para a realidade atual, para a era digital, onde é necessária uma ação humana na utilização de artefatos baseados em tecnologias digitais (por exemplo, utilização de um smartphone), sem que haja necessariamente uma aprendizagem pessoal sobre essas tecnologias.

Assim sendo, as teorias de aprendizagem devem ser ajustadas a era digital, na qual o conhecimento não é adquirido de maneira linear, pois a tecnologia realiza muitas das operações cognitivas anteriormente realizadas pelos aprendizes (por exemplo, armazenagem e recuperação de informações) e, em muitos casos, é necessário a interação humana, mesmo na ausência de um entendimento completo. De fato, atualmente, a aprendizagem é um processo cognitivo que não ocorre somente sob o controle total de um único indivíduo (uma atividade individualizada internamente), mas uma atividade que ocorre também no contexto, fora do indivíduo, na interação com outros indivíduos e com grupos de indivíduos, ou mesmo na interação com uma organização e seus artefatos. Justamente, essas conexões externas, que

\footnotetext{
$\overline{10}$ Siemens, G. (2004, December). Connectivism: A theory for the digital age. Disponível em http://www. elearnspace.org/Articles/connectivism.htm. Acesso em 04 de junho de 2015.
}

Siemens (2004) ${ }^{10}$ discusses the limitations of behaviorism, cognitivism and constructivism as learning theories, since they do not cover the learning process that occurs outside the individuals (that is to say, learning that is stored and manipulated by technology), but rather within the organizational context.

In this regard, connectivism is proposed as a learning theory more suitable to the current reality - the digital era -, in which human actions are needed for the use artifacts based on digital technologies (for example, the use of a smartphone), without the need of personally learning these technologies.

Therefore, learning theories should be adjusted to the digital era, in which knowledge is not acquired in a linear way, since the technology performs most cognitive operations previously performed by learners (for example, information storage and recovery) and, in many cases, the human interaction is needed, even in the absence of a full understanding. Indeed, learning is a cognitive process that not only happens under the total control of a single individual (an internally individualized activity), but an activity that also happens in the context, outside the individual, in the interaction with other individuals and groups of individuals, or even in the interaction with an organization and its artifacts. Those very external connections themselves, which

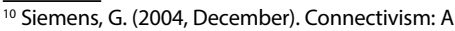
theory for the digital age. Available from http:// www.elearnspace.org/Articles/connectivism.htm. Cited on June 4, 2015.
} 
potencializam o que podemos aprender, são mais importantes do que o nosso atual estágio de conhecimento.

Esta abordagem nos permite repensar a educação e a aprendizagem, no contexto da era digital, objetivando a definição de políticas públicas, sem o viés ideológico que predominou na área nos últimos anos, mas, como salienta a referida publicação da UNESCO, numa visão humanista da educação e do desenvolvimento, com base nos princípios de respeito pela vida e dignidade humanas, igualdade de direitos, justiça social, diversidade cultural, solidariedade internacional e responsabilidade compartilhada, com vistas a construir um futuro sustentável para o Brasil. Essas políticas, devidamente implementadas, nos permitirá avançar mais rapidamente para alcançarmos os objetivos do Plano Nacional de Educação 2014-2024.

De fato, os dados que analisaremos a seguir mostram que o País tem evoluído, mas não na velocidade e na dimensão que, potencialmente, poderíamos estar avançando.

\section{Análise descritiva dos dados sobre analfabetismo e escolarização no Brasil}

Os dados descritos na Tabela 6.1 (Taxa de analfabetismo das pessoas de 10 anos ou mais de idade, por sexo, segundo os grupos de idade) demonstram que, no período de 2008 a 2014, as taxas de analfabetismo no Brasil diminuíram entre homens e mulheres de diferentes grupos de idade. De fato, em termos percentuais, em todos os grupos etários, caiu o número de pessoas analfabetas em relação ao total de pessoas do boost what we can learn, are more important than our current stage of knowledge.

This approach allows us to rethink education and learning in the context of the digital era, aiming at establishing public policies without the ideological bias that prevailed in this area in the last years, but rather, according to the above-mentioned UNESCO publication, in a humanist view of education and development, based on the principles of respect to human life and dignity, equal rights, social justice, cultural diversity, international solidarity and shared responsibility, with a view to building a sustainable future for Brazil. Once implemented, these policies will allow us to advance faster to reach the objectives of the National Plan of Education 2014-2024.

Actually, the data that will be further analyzed show that Brazil has evolved, but not at the potential speed and rate that we should be advancing.

\section{Descriptive analysis of data on illiteracy and schooling in Brazil}

The data described on Table 6.1 (Illiteracy rate of persons aged 10 years and over, by sex, according to age groups) show that the illiteracy rates in Brazil decreased among men and women of different age groups in the period from 2008 to 2014. Indeed, in every age group, the number of illiterate persons in relation to the total number of persons in the same group decreased in percentage 
mesmo grupo. Todavia, no geral, a queda no período foi mais significativa em relação às mulheres $(1,4 \%)$ do que em relação aos homens $(1,1 \%)$. Da mesma forma, a redução não ocorreu na mesma intensidade nos diferentes grupos etários. Pode-se observar, por exemplo, que na faixa etária de 10 a 14 anos, a redução foi mais significativa para os homens $(0,9 \%)$ do que para as mulheres $(0,5 \%)$, o que evidencia que a universalização do ensino fundamental está levando os meninos desta faixa etária a terem o mesmo nível de escolarização que as meninas. Já na faixa etária de 15 anos ou mais, ao contrário, a queda é mais significativa para as mulheres $(1,7 \%)$ do que para os homens $(1,2 \%)$, o que pode evidenciar que, além da evasão escolar, o não ingresso no ensino básico atinge de forma mais intensa os meninos do que as meninas.

Em todo caso, a taxa de analfabetismo no Brasil ainda continua muito elevada. Considerando-se em números absolutos a quantidade de pessoas analfabetas em 2014 era de 15,5 milhões, para uma população residente estimada em 202.768.562 milhões de habitantes. Em comparação com 2009, a população residente estimada era de 193.543.969 milhões de habitantes, dos quais cerca de 17,2 milhões eram analfabetos. Portanto, houve uma redução de mais de 1,6 milhões de analfabetos no País, no referido período. Todavia, como ser observado na Tabela 6.1, a taxa de analfabetismo é mais significativa para os grupos de idade mais elevada. Por exemplo, ela é de $2,5 \%$ para o grupo de idade de 10 a 14 anos e de $27,7 \%$ para as pessoas de mais de 60 anos. Assim sendo, o envelhecimento da população brasileira, naturalmente, provocará uma redução do número de analfabetos no terms. Nevertheless, in general figures, the drop in relation to women (1.4\%) was more significant than that related to men (1.1\%). Likewise, the reduction did not happen with the same intensity in the different age groups. For example, it can be noticed that the reduction was more significant for men $(0.9 \%)$ than for women $(0.5 \%)$ in the age group between 10 and 14 years, which leads to the conclusion that the universalization of the primary school is taking boys in this age bracket to the same level of schooling of girls. Conversely, the drop is more significant for women (1.7\%) than for men (1.2\%) in the age group of 15 years and over, which could lead to the conclusion that, besides school evasion, non-admission in the secondary school hits boys more significantly than girls.

In any case, the illiteracy rate in Brazil is still very high. In absolute figures, the number of illiterate persons in 2014 was of 15.5 million for an estimated resident population of $202,768,562$ inhabitants. Compared with 2009 , the estimated resident population was of $193,543,969$ inhabitants, of which about 17.2 million were illiterate. Therefore, the number of illiterate persons in Brazil reduced by more than 1.6 million inhabitants in the period above. Nonetheless, the illiteracy rate is more significant for the older age groups, as it can be seen on Table 6.1. For example, the rate is $2.5 \%$ for the group aged between 10 and 14 years and $27.7 \%$ for persons aged over 60 years. Therefore, the aging of the Brazilian population will naturally reduce the number of 
Brasil. Mas, são necessárias ações mais efetivas que a simples evolução da pirâmide etária. São necessárias a continuação e uma maior ênfase nas políticas de Educação de Jovens e Adultos (EJA), bem como programas que estimulem uma maior colaboração entre entes federados, com a finalidade de erradicar, de uma vez por todas, esta mancha social, que é o analfabetismo no País.

O gráfico 6.1, relativo a taxa de analfabetismo da população brasileira, de 15 anos ou mais de idade, por situação do domicílio, evidencia que, no período de 2005/2014, a população rural teve uma redução na taxa de analfabetismo (de $25 \%$ para $20,1 \%$ ), mais significativa que a população urbana (de $8,4 \%$ para $6,3 \%$ ). No total, a população brasileira, teve uma redução na taxa de analfabetismo de $11,1 \%$ para $8,3 \%$, no referido período.

Outro dado relevante a ser destacado é a média de anos de estudo das pessoas de 10 anos ou mais de idade, por sexo, segundo os grupos de idade 2009/2014, conforme mostra a tabela 6.2. Em todas os grupos etários houve um aumento significativo na média de anos de estudo. No total, o País avançou de 7,2 para 8,0 anos na média de anos de estudos, mas ainda muito longe da média de 12 anos de estudos dos países da OCDE. Todavia, merece destaque a média de anos de estudos dos jovens da faixa etária de 25 a 29 anos, que passou de 9,4 para 10,5 anos, bem mais próxima da média dos países da OCDE.

Entretanto, observa-se no Gráfico 6.2, que ainda há uma forte disparidade entre as diferentes regiões do Brasil, no que diz respeito à taxa de analfabetismo das pessoas de 15 anos ou mais de idade, no illiterate persons in Brazil. However, more effective actions than the mere evolution of the age pyramid are needed. In order to eradicate illiteracy in Brazil once and for all, Education of Youngsters and Adults (EJA) policies, as well as programs that stimulate a bigger cooperation among Federation Units should be enhanced.

Graph 6.1 (Illiteracy rate of the Brazilian population aged 15 years and over, by urban/rural housing unit) shows that the rural population reduced the illiteracy rate (from $25 \%$ to $20.1 \%$ ) more significantly than the urban population (from $8.4 \%$ to $6.3 \%$ ) in the 2005-2014 period. As a whole, the Brazilian population reduced the illiteracy rate from $11.1 \%$ to $8.3 \%$ in the period.

Other relevant data to be highlighted is the length of schooling of persons aged 10 years and over, by sex, according to age groups in 2009-2014, as shown on Table 6.2. Every age group recorded a significant increase in the length of schooling. As a whole, Brazil advanced from 7.2 to 8.0 years in the length of schooling, though it is still very far from the average of 12 years of schooling in the OECD countries. Nevertheless, the length of schooling of youngsters in the age group between 25 and 29 years should be highlighted, as it changed from 9.4 to 10.5 years, being much closer to the average in the OECD countries.

However, Graph 6.2 shows a strong gap in the illiteracy rate of persons aged 15 years and over among the Brazilian regions in the 2009-2014 period. While this rate dropped 
período de 2009 a 2014. Enquanto na Região Sul esta taxa caiu de 5,5\% em 2009 para 4,4\% em 2014, na Região Nordeste a taxa caiu de $18,7 \%$ em 2009 para $16,6 \%$ em 2014.

Da mesma forma, na Tabela 6.3, relativa a distribuição das pessoas de 25 anos ou mais de idade, por Grandes Regiões, segundo o sexo e o nível de instrução, mostra que em 2014, há ainda uma forte disparidade entre as diferentes regiões do Brasil e entre mulheres e homens. Enquanto na Região Sudeste, em 2014, o percentual de pessoas sem instrução era de 7,3\%, na Região Nordeste era praticamente três vezes mais elevada, alcançando o percentual de $20,9 \%$. Em relação a formação de nível superior completo, em 2014, na Região Sudeste alcançava 16,1\%, na Região Nordeste este percentual era praticamente a metade, $8,1 \%$. Em relação ao sexo, também uma forte disparidade, pois enquanto $o$ percentual de mulheres sem instrução da Região Nordeste alcança 20,9\%, na Região Sudeste este percentual cai para 7,3\%. Em contrapartida, o percentual de homens sem instrução na Região Nordeste alcança $23 \%$, na Região Sudeste este percentual cai para $6,9 \%$. Mas, a maior diferença entre os sexos se evidencia na educação superior completa, na qual o percentual de homens da Região Nordeste, o menor do País, é apenas 6,1\%, enquanto na Região Sudeste, o maior do País, alcança $14,7 \%$. Em contrapartida, em relação as mulheres, a Região Nordeste este percentual fica em 9,8\% e na Região Sudeste alcança $17,8 \%$.

Outro dado relevante, relativo à média de anos de estudo da população de 10 anos ou mais, por grupos de idade, em 2014, pode ser observado no Gráfico 6.3. from $5.5 \%$ in 2009 to $4.4 \%$

in 2014 in the South Region, it fell from $18.7 \%$ in 2009 to $16.6 \%$ in 2014 in the Northeast Region.

Likewise, Table 6.3 (Distribution of persons aged 25 years and over, by Major Regions, according to sex and level of schooling) still shows a huge gap among the different Brazilian regions and between women and men in 2014. While the percentage of illiterate persons was $7.3 \%$ in the Southeast Region in 2014, this percentage was nearly three times higher in the Northeast Region, reaching $20.9 \%$. Concerning complete higher education in 2014, the Southeast Region hit 16.1\%, whereas this percentage was nearly half in the Northeast Region (8.1\%). Sex also shows a big gap, for while the percentage of illiterate women in the Northeast Region reached $20.9 \%$, this percentage dropped to $7.3 \%$ in the Southeast Region. In contrast, while the percentage of illiterate men in the Northeast Region hit 23\%, this percentage dropped to $6.9 \%$ in the Southeast Region. Complete higher education shows the biggest difference among sexes, in which the percentage of men in the Northeast Region, the lowest in Brazil, was only 6.1\%, whereas the Southeast Region, the highest in Brazil, it hit 14,7\%. Concerning women, percentages stood at $9.8 \%$ in the Northeast Region and reached $17.8 \%$ in the Southeast Region.

Other relevant data, related to the length of schooling of the population aged 10 years and over, by age groups in 2014, can be seen in Graph 6.3. 
Constata-se que na faixa etária de 20 a 24 anos e de 25 a 29 anos, encontrase a maior média de anos de estudo da população brasileira, alcançando 10,1 anos. Acima da faixa etária dos 29 anos há uma queda significativa, de maneira tal que o grupo de idade acima dos 60 anos não passa dos 4,8 anos de estudo, o que revela que a população brasileira mais idosa tem um nível de escolaridade, em média, menor que a população mais jovem.

Outro dado que merece destaque e a distribuição das pessoas que frequentavam escola ou creche, por Grandes Regiões, segundo o nível e a rede de ensino que frequentavam em 2014, conforme mostra a Tabela 6.4. Constata-se que, grosso modo, não há grandes diferenças percentuais entre as Grandes Regiões, no que diz respeito a Educação Pública e Privada, nos diferentes níveis e redes de ensino. Todavia, observa-se uma maior concentração percentual no ensino superior, de instituições privadas, nas Regiões Sul $(77,4 \%)$ e Sudeste $(80,2 \%)$, em relação às Regiões Norte $(67,1 \%)$ e Nordeste $(67,8 \%)$.

Em relação a taxa de frequência a creche no Brasil, das crianças de 0 a 3 anos de idade, no período de $2009 / 2014$, pode- se observar na Tabela 6.5 que houve um aumento significativo no período, passando de $18,4 \%$ para $24,6 \%$, mas ainda muito aquém das necessidades dos jovens casais brasileiros. Observa-se, também, nesta tabela que não diferenças significativas por sexo. Em contrapartida, há uma diferença mais significativa por Região. Enquanto na Região Sul a taxa de frequência a creche passou de $24,3 \%$ para $32,2 \%$, na Região Norte a taxa de frequência passou apenas de $8,2 \%$ para $8,3 \%$.
The highest length of schooling of the Brazilian population were registered in the age groups between 20 and 24 years and between 25 and 29 years, reaching 10.1 years. A significant drop was observed in the age group above 29 years of age, so that the age group over 60 years did not surpass 4.8 years of schooling, revealing that the older Brazilian population, on average, has a lower level of schooling than the younger population.

Other data that should be highlighted is the distribution of persons attending school or daycare, by Major Regions, according to the level of education and network attended in 2014, as shown in Table 6.4. Roughly, no big percentage differences were recorded among the Major Regions concerning Public and Private Education, no matter the level of education and education network. Nevertheless, a greater percentage concentration of private higher education institutions was noticed in the South (77.4\%) and Southeast Regions (80.2\%) in relation to the North (67.1\%) and Northeast Regions (67.8\%).

As it can be seen in Table 6.5, the daycare attendance rate of children aged between 0 and 3 years in the 2009-2014 period in Brazil increased significantly, changing from $18.4 \%$ to $24.6 \%$, though it is still far from the needs of the Brazilian young couples. This table also shows no significant differences by sex. In contrast, it presents a more significant difference per Region. While daycare attendance rate changed from $24.3 \%$ to $32.2 \%$ in the South Region, it changed only from $8.2 \%$ to $8.3 \%$ in the North Region. 
Enfim, em relação a taxa de escolarização das pessoas de 4 anos ou mais de idade, por Grandes Regiões, segundo os grupos de idade e o sexo, no ano de 2014, observa-se alguns dados significativos na Tabela 6.6. Primeiramente, na faixa etária de 4 ou 5 anos ainda há esforço a ser feito para que o País possa garantir a escolaridade dessas crianças, pois apenas $82,7 \%$ delas estão sendo atendidas pela pré-escola. Por região, observa-se uma certa desigualdade, enquanto na Região Nordeste $87,7 \%$ das crianças são atendidas, na Região Norte apenas $70 \%$. Por sexo, a diferença não é significativa. No que diz respeito a escolaridade da faixa etária de 06 a 14 anos, observa-se que praticamente o País já alcançou a universalização do ensino fundamental, pois $98,7 \%$ das crianças são atendidas, não havendo grandes diferenças por região e nem por sexo. Todavia, no que diz respeito a faixa etária dos 15 aos 17 anos a escolaridade cai de forma significativa (84,3\%), evidenciando dois problemas crônicos da educação brasileira, o primeiro que um número significativo de jovens termina o ensino fundamental, mas não ingressa no ensino básico e, também, a evasão considerável daqueles que ingressam, mas que efetivamente não terminam esse nível de ensino. Não há diferenças significativas nem por região e nem por sexo. Entretanto, no que diz respeito a faixa etária dos 18 ou 19 e dos 20 aos 24 anos e, sobretudo, dos 25 anos ou mais, os problemas na educação brasileira deixam de ser crônicos e passam a ser agudos, pois a queda na taxa de escolarização das pessoas é extremamente elevada $(42,4 \%, 24,5 \%$ e $4,0 \%$ respectivamente). Essa queda está diretamente associada ao percentual importante de jovens que não
Table 6.6 shows some significant data in relation to the schooling rate of persons aged 4 years and over, by Major Regions, according to age groups and sex, in 2014. Firstly, some effort should be made to ensure the schooling of children in the age group between 4 and 5 years of age, since only $82.7 \%$ of them are attending preschool. Some inequality can be noticed by Region, since $87.7 \%$ of children in the Northeast Region are attended to, whereas only $70 \%$ are attended to in the North Region. The difference by sex is not significant. Having included $98.7 \%$ of children in the age group between 6 and 14 years, Brazil virtually reached the universalization of the primary school, showing no great differences either by Region or by sex. Nonetheless, schooling drops significantly (84.3\%) in the age group between 15 and 17 years, highlighting two chronic problems of the Brazilian education: firstly, a significant number of youngsters finish primary school, but are not admitted in secondary school; secondly, there is substantial evasion of those admitted, but who ultimately do not complete this level. No significant differences were registered either by Region or by sex. In regard to the age group between 18 and 19 years, between 20 and 24 years and especially those aged 25 years and over, the education problems are no longer chronic but acute, since the drop in the schooling rate of persons is extremely high (42.4\%, $24.5 \%$ and $4.0 \%$, respectively). This drop is directly associated with the important percentage of youngsters who do not finish 
terminam o ensino básico e, portanto, não ingressam no ensino superior, fazendo com que o Brasil tenha o menor percentual de jovens, na faixa etária dos 17 aos 24, no ensino superior da América Latina. Observa-se também que, na faixa etária dos 20 aos 24 anos, este fenômeno atinge mais profundamente os jovens do sexo masculino $(22,2 \%)$ do que as jovens do sexo feminino $(26,9 \%)$.

\section{Considerações finais}

A análise descritiva dos dados, anteriormente realizados, mostra que, no período de 2009 a 2014, houve uma melhoria dos índices de alfabetização e de escolarização e, em consequência, um decréscimo na taxa de analfabetismo no Brasil. Todavia, a velocidade dessa melhoria ainda muito lenta, face aos desafios que o País tem que enfrentar neste novo Século, em termos de competitividade e de inovação. De fato, a globalização da economia e o advento das novas tecnologias de comunicação e de informação exigem uma mudança significativa nas políticas públicas educacionais do País, de forma a nos alinhar estrategicamente aos países mais desenvolvidos, que estão implementando uma verdadeira revolução na educação, pois não é possível gerenciar o novo com as ferramentas do velho. Nesse sentido, uma das ações mais urgentes, a serem desenvolvidas, é o combate e encaminhamento para a erradicação definitiva do analfabetismo. A análise descritiva dos dados, acima apresentados, evidenciam que o nível de escolarização entre as mulheres na maioria dos grupos etários, para o período considerado 20092014, é maior do que entre os homens e se projeta para uma maior ampliação num futuro próximo. De uma maneira geral, secondary school and, therefore, are not admitted in higher education, causing Brazil to be the country with the lowest percentage of youngsters in the age group between 17 and 24 years in higher education in Latin America. In the age group between 20 and 24 years, this phenomenon hits male youngsters (22.2\%) deeper than female youngsters (26.9\%).

\section{Final remarks}

The descriptive analysis of the data above shows an improvement in the literacy and schooling rates in the period between 2009 and 2014 and, as a consequence, a decrease in the illiteracy rate in Brazil. Nevertheless, the speed of this improvement is still slow, in face of the challenges in terms of competition and innovation that Brazil has to face in this new century. Indeed, the globalization of the economy and the arrival of new communication and information technologies require a significant change in the public educational policies in Brazil. We need to aim at the strategic alignment with the policies of more developed countries, which are implementing a real revolution in education, since it is not possible to manage new concepts with old tools. In this respect, one of the most urgent actions to take is to fight and definitely eradicate illiteracy. The descriptive analysis of the data above points out that the schooling level of women is greater than men's for the majority of the age groups in the 2009-2014 period, and it is meant to widen up 
pode-se constatar também que, para ambos os sexos, o nível de escolarização ainda precisa avançar de maneira significativa, não apenas numericamente, mas, sobretudo, qualitativamente. De fato, não somente a oferta numérica de vagas, nos diferentes níveis de ensino, deve ser objeto de preocupação das políticas públicas na área de educação, para reverter o quadro de escolarização e erradicação do analfabetismo no País, como foi implementado nos últimos anos. É necessário, também, repensar a educação e a aprendizagem, no contexto da era digital, para se construir uma escola pública de qualidade, que privilegie $o$ mérito e a criatividade, além da valorização dos profissionais da educação, que são os maiores desafios das políticas públicas educacionais. É necessário, também, incorporar todos os meios tecnológicos para que todos tenham acesso e condições para avançar em cada uma das etapas acadêmicas, de forma equitativa, conforme previsto nas diretrizes do Plano Nacional de Educação 2014-2024.

Neri dos Santos

Engenheiro Mecânico pela Universidade Federal de Santa Catarina - UFSC Mestre em Ergonomia pela "Université de Paris XIII', Paris - França

Doutor em "Ergonomie de l'Ingenierie" pelo "Conservatoire National des Arts et Métiers" CNAM, Paris - França

Pós-Doutorado em "Ingenierie Cognitive" pela École Polytechnique de Montréal - Canadá Professor Sênior do Departamento de Engenharia do Conhecimento da Universidade Federal de Santa Catarina - UFSC Decano da Escola Politécnica da Pontifícia Universidade Católica do Paraná - PUCPR in the near future. Broadly speaking, it can be noticed that the schooling level still needs to significantly advance for both sexes, not only numerically, but qualitatively above all. To revert the schooling and illiteracy issues in Brazil, public policies in the education area should address not only the numeric supply of vacancies in the different levels of education as it was implemented in the last years. It is also important to rethink education and learning in the context of the digital era to build a quality public education that favors merit and creativity, as well as to value education professionals: these are the greatest challenges of public educational policies. Every technological means should be incorporated, so as to give everybody equal opportunities and conditions to advance in each academic step, in compliance with the National Plan of Education 2014-2024.

Mechanical Engineer from the Federal University of Santa Catarina - UFSC Master in Ergonomics from the "Université de Paris XIII", Paris - France $P h D$ in "Ergonomie de I'Ingenierie" from the "Conservatoire National des Arts et Métiers" CNAM, Paris - France Post-Doctorate in "Ingenierie Cognitive" from the École Polytechnique de Montréal - Canada Senior Professor at the Knowledge Engineering Department of the Federal University of Santa Catarina-UFSC

Dean of the Polytechnic School of the Catholic Pontificial University of Paraná - PUCPR 


\section{Alvino Moser}

Bacharel e Licenciado em Química pela PUCPR. Bacharel, Licenciado, Mestre em Filosofia pela

Université Catholique de Louvain- Bélgica.

Doutor em Filosofia (Ética e Filosofia das ciências) pela pela Université Catholique de Louvain- Bélgica.

Pós-doutor pela mesma Universidade com a pesquisa sobre Lógica Normativa, Deôntica e Jurídica.

Professor Decano da Centro Universitário Internacional Uninter. Professor do programa de Mestrado em Formação Docente e Novas Tecnologias. Vice-Reitor Acadêmico do Centro Universitário Internacional Uninter.
Bachelor and Graduate in Chemistry

from the PUCPR

Bachelor, Graduate, Master in Philosophy from the Université Catholique de Louvain - Belgium

PhD in Philosophy (Ethics and Philosophy of

Sciences) form the Université Catholique de

Louvain-Belgium

Post-Doctorate from the Université Catholique de

Louvain-Belgium, researching on Legal, Deontic and Normative Logic

Dean Professor of the Centro Universitário Internacional - UNINTER

Professor at the Master Program in Teacher

Training and New Technologies

Academic Vice-Rector of the Centro Universitário

Internacional - UNINTER

Translated by: La-Fayette Côrtes Neto 
Tabela 6.1 - Taxa de analfabetismo das pessoas de 10 anos ou mais de idade, por sexo, segundo os grupos de idade - 2009/2014

Table 6.1 - Illiteracy rate of persons 10 years old and over,

by sex and age groups - 2009/2014

\begin{tabular}{|c|c|c|c|c|c|c|}
\hline \multirow{3}{*}{$\begin{array}{c}\text { Grupos de idade/ } \\
\text { Age groups }\end{array}$} & \multicolumn{6}{|c|}{$\begin{array}{l}\text { Taxa de analfabetismo das pessoas de } 10 \text { anos } \\
\text { ou mais de idade/ } \\
\text { Illiteracy rate of persons } 10 \text { years and over (\%) }\end{array}$} \\
\hline & \multicolumn{3}{|c|}{2009} & \multicolumn{3}{|c|}{2014} \\
\hline & $\begin{array}{l}\text { Total/ } \\
\text { Total }\end{array}$ & $\begin{array}{l}\text { Homens/ } \\
\text { Male }\end{array}$ & $\begin{array}{l}\text { Mulheres/ } \\
\text { Female }\end{array}$ & $\begin{array}{l}\text { Total/ } \\
\text { Total }\end{array}$ & $\begin{array}{l}\text { Homens/ } \\
\text { Male }\end{array}$ & $\begin{array}{c}\text { Mulheres/ } \\
\text { Female }\end{array}$ \\
\hline Total/Total & 8,9 & 9,1 & 8,8 & 7,7 & 8,0 & 7,4 \\
\hline 10 a 14 anos/ 10 to 14 years old & 2,5 & 3,3 & 1,6 & 1,8 & 2,4 & 1,1 \\
\hline $\begin{array}{l}15 \text { anos ou mais/ } 15 \text { years old and } \\
\text { over }\end{array}$ & 9,7 & 9,8 & 9,6 & 8,3 & 8,6 & 7,9 \\
\hline 15 a 19 anos/ 15 to 19 years old & 1,5 & 2,1 & 1,0 & 0,9 & 1,3 & 0,6 \\
\hline 20 a 24 anos/ 20 to 24 years old & 2,4 & 3,0 & 1,7 & 1,4 & 1,9 & 0,9 \\
\hline 25 a 29 anos/ 25 to 29 years old & 3,6 & 4,7 & 2,6 & 2,1 & 2,8 & 1,4 \\
\hline 30 a 39 anos/ 30 to 39 years old & 6,4 & 7,9 & 5,1 & 4,3 & 5,6 & 3,1 \\
\hline 40 a 49 anos/ 40 to 49 years old & 9,3 & 10,3 & 8,3 & 7,8 & 9,3 & 6,4 \\
\hline 50 a 59 anos/ 50 to 59 years old & 13,5 & 13,3 & 13,7 & 10,9 & 11,7 & 10,1 \\
\hline $\begin{array}{l}60 \text { anos ou mais/ } 60 \text { years old and } \\
\text { over }\end{array}$ & 27,7 & 25,7 & 29,2 & 23,1 & 22,1 & 23,8 \\
\hline
\end{tabular}

Fonte/Source: IBGE, Diretoria de Pesquisas, Coordenação de Trabalho e Rendimento, Pesquisa Nacional por Amostra de Domicílios 2009/2014. 
Tabela 6.2 - Média de anos de estudo das pessoas de 10 anos ou mais de idade, por sexo, segundo os grupos de idade - 2009/2014

Table 6.2 - Average of years of schooling of persons 10 years old and over, by sex and age groups - 2009/2014

\begin{tabular}{|c|c|c|c|c|c|c|}
\hline \multirow{3}{*}{$\begin{array}{l}\text { Grupos de idade/ } \\
\text { Age groups }\end{array}$} & \multicolumn{6}{|c|}{$\begin{array}{c}\text { Média de anos de estudo das pessoas de } 10 \text { anos } \\
\text { ou mais de idade/ } \\
\text { Average of years of schooling of persons } \\
10 \text { years old and over }\end{array}$} \\
\hline & \multicolumn{3}{|c|}{2009} & \multicolumn{3}{|c|}{2014} \\
\hline & $\begin{array}{l}\text { Total/ } \\
\text { Total }\end{array}$ & $\begin{array}{l}\text { Homens/ } \\
\text { Male }\end{array}$ & $\begin{array}{l}\text { Mulheres/ } \\
\text { Female }\end{array}$ & $\begin{array}{l}\text { Total/ } \\
\text { Total }\end{array}$ & $\begin{array}{l}\text { Homens/ } \\
\text { Male }\end{array}$ & $\begin{array}{l}\text { Mulheres/ } \\
\text { Female }\end{array}$ \\
\hline Total/Total & 7,2 & 7,0 & 7,3 & 7,7 & 7,5 & 8,0 \\
\hline 10 a 14 anos/ 10 to 14 years old & 4,1 & 3,9 & 4,3 & 4,4 & 4,2 & 4,5 \\
\hline 15 anos ou mais/ 15 years old and over & 7,5 & 7,4 & 7,7 & 8,1 & 7,9 & 8,3 \\
\hline 15 a 17 anos/15 to 17 years old & 7,3 & 7,0 & 7,6 & 7,8 & 7,5 & 8,1 \\
\hline 18 anos ou mais/ 18 years old and over & 7,6 & 7,4 & 7,7 & 8,1 & 7,9 & 8,3 \\
\hline $\begin{array}{l}18 \text { ou } 19 \text { anos/18 or } 19 \text { years old } \\
\text { and over }\end{array}$ & 9,0 & 8,6 & 9,4 & 9,4 & 9,1 & 9,7 \\
\hline $\begin{array}{l}20 \text { anos ou mais/ } 20 \text { years old and } \\
\text { over }\end{array}$ & 7,5 & 7,3 & 7,6 & 8,0 & 7,8 & 8,2 \\
\hline 20 a 24 anos/20 to 24 years old & 9,6 & 9,3 & 9,9 & 10,1 & 9,7 & 10,5 \\
\hline $\begin{array}{l}25 \text { anos ou mais/ } 25 \text { years old and } \\
\text { over }\end{array}$ & 7,2 & 7,1 & 7,3 & 7,8 & 7,6 & 8,0 \\
\hline 25 a 29 anos/25 to 29 years old & 9,4 & 9,1 & 9,7 & 10,1 & 9,8 & 10,5 \\
\hline 30 a 39 anos/30 to 39 years old & 8,2 & 7,8 & 8,6 & 9,3 & 8,8 & 9,7 \\
\hline 40 a 49 anos $/ 40$ to 49 years old & 7,6 & 7,4 & 7,9 & 8,2 & 7,8 & 8,6 \\
\hline 50 a 59 anos/50 to 59 years old & 6,4 & 6,4 & 6,5 & 7,1 & 6,9 & 7,2 \\
\hline $\begin{array}{l}60 \text { anos ou mais/ } 60 \text { years old and } \\
\text { over }\end{array}$ & 4,2 & 4,4 & 4,1 & 4,8 & 4,9 & 4,7 \\
\hline
\end{tabular}

Fonte/Source: IBGE, Diretoria de Pesquisas, Coordenação de Trabalho e Rendimento, Pesquisa Nacional por Amostra de Domicílios 2009/2014. 
Tabela 6.3 - Distribuição das pessoas de 25 anos ou mais de idade, por Grandes Regiões, segundo o sexo e o nível de instrução - 2014

Table 6.3 - Distribution of persons 25 years old and over,

by Major Regions, sex and level of schooling - 2014

(continua/to be continued)

Distribuição das pessoas de 25 anos ou mais de idade/

Distribution of persons 25 years old and over

Sexo e

nível de instrução/

Sex and

level of schooling
Grandes Regiões/Major Regions

\begin{tabular}{|c|c|c|c|c|c|}
\hline \multirow[b]{2}{*}{$\begin{array}{l}\text { Brasil/ } \\
\text { Brazil }\end{array}$} & \multicolumn{5}{|c|}{ Grandes Regiões/Major Regions } \\
\hline & $\begin{array}{l}\text { Norte/ } \\
\text { North }\end{array}$ & $\begin{array}{l}\text { Nordeste/ } \\
\text { Northeast }\end{array}$ & $\begin{array}{l}\text { Sudeste/ } \\
\text { Southeast }\end{array}$ & $\begin{array}{l}\text { Sul/ } \\
\text { South }\end{array}$ & $\begin{array}{c}\text { Centro- } \\
\text { Oeste/ } \\
\text { Central- } \\
\text { West }\end{array}$ \\
\hline
\end{tabular}

Total (1)/

Total (1)

Sem instrução/

Without instruction

Fundamental incompleto/

Incomplete elementary school

Fundamental completo/

Complete elementary school

Médio incompleto/

Incomplete high school

Médio completo/

Complete high school

Superior incompleto/

Incomplete college/university

Superior completo/

Complete college/university

Homens (1)/

Male (1)

Sem instrução/

Without instruction

Fundamental incompleto/

Incomplete elementary school

Fundamental completo/

Complete elementary school

Médio incompleto/

Incomplete high school

Médio completo/

Complete high school

Superior incompleto/

Incomplete college/university

Superior completo/

Complete college/university

$\begin{array}{llllll}100,0 & 100,0 & 100,0 & 100,0 & 100,0 & 100,0\end{array}$

$\begin{array}{llllll}11,7 & 14,0 & 20,9 & 7,3 & 7,6 & 11,0\end{array}$

$\begin{array}{llllll}32,0 & 33,1 & 33,3 & 30,3 & 34,7 & 30,2\end{array}$

$\begin{array}{llllll}9,5 & 8,5 & 7,8 & 10,2 & 10,9 & 9,5\end{array}$

$\begin{array}{llllll}4,2 & 5,1 & 4,1 & 4,0 & 4,0 & 4,8\end{array}$

$\begin{array}{llllll}25,5 & 26,0 & 22,6 & 27,8 & 24,2 & 24,4\end{array}$

$\begin{array}{llllll}3,9 & 3,8 & 3,1 & 4,1 & 4,4 & 4,4\end{array}$

$\begin{array}{llllll}13,1 & 9,2 & 8,1 & 16,1 & 14,0 & 15,5\end{array}$

$\begin{array}{llllll}100,0 & 100,0 & 100,0 & 100,0 & 100,0 & 100,0\end{array}$

$\begin{array}{llllll}12,1 & 15,2 & 23,0 & 6,9 & 7,0 & 11,4\end{array}$

$\begin{array}{llllll}33,2 & 36,1 & 34,9 & 31,1 & 35,3 & 32,7\end{array}$

$\begin{array}{llllll}10,0 & 8,7 & 7,9 & 10,7 & 11,9 & 10,3\end{array}$

$\begin{array}{llllll}4,4 & 5,2 & 4,1 & 4,2 & 4,4 & 5,1\end{array}$

$\begin{array}{llllll}25,0 & 23,8 & 21,0 & 28,0 & 24,6 & 22,9\end{array}$

$\begin{array}{llllll}3,9 & 3,4 & 2,8 & 4,4 & 4,6 & 4,3\end{array}$

$\begin{array}{llllll}11,4 & 7,5 & 6,1 & 14,7 & 12,0 & 13,1\end{array}$


Tabela 6.3 - Distribuição das pessoas de 25 anos ou mais de idade, por Grandes Regiões e nível de instrução - 2014

Table 6.3 - Distribution of persons 25 years old and over, by Major Regions and level of schooling- 2014

(conclusão/concluded)

Distribuição das pessoas de 25 anos ou mais de idade/ Distribution of persons 25 years old and over

\begin{tabular}{|c|c|c|c|c|c|c|}
\hline \multirow{2}{*}{$\begin{array}{l}\text { Nível de instrução/ } \\
\text { Level of schooling }\end{array}$} & \multirow[b]{2}{*}{$\begin{array}{l}\text { Brasil/ } \\
\text { Brazil }\end{array}$} & \multicolumn{5}{|c|}{ Grandes Regiões/Major Regions } \\
\hline & & $\begin{array}{l}\text { Norte/ } \\
\text { North }\end{array}$ & $\begin{array}{l}\text { Nordeste/ } \\
\text { Northeast }\end{array}$ & $\begin{array}{l}\text { Sudeste/ } \\
\text { Southeast }\end{array}$ & $\begin{array}{l}\text { Sul/ } \\
\text { South }\end{array}$ & $\begin{array}{c}\text { Centro- } \\
\text { Oeste/ } \\
\text { Central- } \\
\text { West }\end{array}$ \\
\hline $\begin{array}{l}\text { Mulheres (1)/ } \\
\text { Female (1) }\end{array}$ & 100,0 & 100,0 & 100,0 & 100,0 & 100,0 & 100,0 \\
\hline $\begin{array}{l}\text { Sem instrução/ } \\
\text { Without instruction }\end{array}$ & 11,3 & 12,8 & 19,0 & 7,8 & 8,1 & 10,6 \\
\hline $\begin{array}{l}\text { Fundamental incompleto/ } \\
\text { Incomplete elementary school }\end{array}$ & 30,8 & 30,3 & 31,9 & 29,7 & 34,1 & 27,9 \\
\hline $\begin{array}{l}\text { Fundamental completo/ } \\
\text { Complete elementary school }\end{array}$ & 9,1 & 8,3 & 7,6 & 9,8 & 10,0 & 8,8 \\
\hline $\begin{array}{l}\text { Médio incompleto/ } \\
\text { Incomplete high school }\end{array}$ & 4,0 & 5,1 & 4,1 & 3,7 & 3,7 & 4,6 \\
\hline $\begin{array}{l}\text { Médio completo/ } \\
\text { Complete high school }\end{array}$ & 26,0 & 28,1 & 23,9 & 27,7 & 23,8 & 25,9 \\
\hline $\begin{array}{l}\text { Superior incompleto/ } \\
\text { Incomplete college/university }\end{array}$ & 3,8 & 4,2 & 3,3 & 3,9 & 4,1 & 4,4 \\
\hline $\begin{array}{l}\text { Superior completo/ } \\
\text { Complete college/university }\end{array}$ & 14,7 & 10,8 & 9,8 & 17,4 & 15,8 & 17,6 \\
\hline
\end{tabular}

Fonte/Source: IBGE, Diretoria de Pesquisas, Coordenação de Trabalho e Rendimento, Pesquisa Nacional por Amostra de Domicílios 2014.

(1) Inclusive as pessoas com nível de instrução não determinado. /(1) Including persons with non determined level of schooling. 
Tabela 6.4 - Distribuição das pessoas que frequentavam escola ou creche, por Grandes Regióes, segundo o nível e a rede de ensino que frequentavam - 2014

Table 6.4 - Distribution of persons attendeding school or nursery school, by Major Regions, level of education and type of network attended - 2014

\begin{tabular}{|c|c|c|c|c|c|c|}
\hline \multirow{3}{*}{$\begin{array}{l}\text { Nível e rede de ensino } \\
\text { que frequentavam/ } \\
\text { Level of education and } \\
\text { network attended }\end{array}$} & \multicolumn{6}{|c|}{$\begin{array}{l}\text { Distribuição das pessoas que frequentavam escola ou creche/ } \\
\text { Distribution of persons attending school or nursery school }\end{array}$} \\
\hline & \multirow[b]{2}{*}{$\begin{array}{l}\text { Brasil/ } \\
\text { Brazil }\end{array}$} & \multicolumn{5}{|c|}{ Grandes Regiões/Major Regions } \\
\hline & & $\begin{array}{l}\text { Norte/ } \\
\text { North }\end{array}$ & $\begin{array}{l}\text { Nordeste/ } \\
\text { Northeast }\end{array}$ & $\begin{array}{l}\text { Sudeste/ } \\
\text { Southeast }\end{array}$ & $\begin{array}{l}\text { Sul/ } \\
\text { South }\end{array}$ & $\begin{array}{c}\text { Centro- } \\
\text { Oeste/ } \\
\text { Central- } \\
\text { West }\end{array}$ \\
\hline Creche/Earlychilhood education & 100,0 & 100,0 & 100,0 & 100,0 & 100,0 & 100,0 \\
\hline Pública/Public & 69,9 & 69,3 & 66,3 & 71,6 & 72,3 & 65,5 \\
\hline Particular/Private & 30,1 & 30,7 & 33,7 & 28,4 & 27,7 & 34,5 \\
\hline Pré-escolar/Pre-primary & 100,0 & 100,0 & 100,0 & 100,0 & 100,0 & 100,0 \\
\hline Pública/Public & 71,5 & 82,1 & 65,0 & 73,4 & 77,2 & 67,8 \\
\hline Particular/Private & 28,5 & 17,9 & 35,0 & 26,6 & 22,8 & 32,2 \\
\hline Fundamental (1)/Primary and & & & & & & \\
\hline lower secondary & 100,0 & 100,0 & 100,0 & 100,0 & 100,0 & 100,0 \\
\hline Pública (1)/Public (1) & 85,5 & 92,4 & 84,3 & 83,7 & 88,6 & 84,3 \\
\hline Particular (1)/Private (1) & 14,5 & 7,6 & 15,7 & 16,3 & 11,4 & 15,7 \\
\hline Médio/Upper secondary & 100,0 & 100,0 & 100,0 & 100,0 & 100,0 & 100,0 \\
\hline Pública/Public & 87,3 & 91,5 & 89,8 & 85,2 & 85,6 & 85,7 \\
\hline Particular/Private & 12,8 & 8,5 & 10,2 & 14,8 & 14,4 & 14,3 \\
\hline Superior (2)/Tertiary education (2) & 100,0 & 100,0 & 100,0 & 100,0 & 100,0 & 100,0 \\
\hline Pública (2)/Public (2) & 24,6 & 32,9 & 32,2 & 19,8 & 22,6 & 25,0 \\
\hline Particular (2)/Private (2) & 75,4 & 67,1 & 67,9 & 80,2 & 77,4 & 75,0 \\
\hline
\end{tabular}

Fonte/Source: IBGE, Diretoria de Pesquisas, Coordenação de Trabalho e Rendimento, Pesquisa Nacional por Amostra de Domicílios 2014.

(1) Inclusive os estudantes de classe de alfabetização. (2) Inclusive os estudantes de curso de mestrado ou doutorado./(1) Including the students of literacy classes. (2) Including the students of master's Ph.D. programs. 
Tabela 6.5 - Taxa de frequência a creche das crianças de 0 a 3 anos de idade, por sexo, segundo as Grandes Regiões - 2009/2014

Table 6.5 - Attendance rate to nursery school of children 0 to 3 years old, by sex and Major Regions - 2009/2014

\begin{tabular}{|c|c|c|c|c|c|c|}
\hline \multirow{3}{*}{$\begin{array}{l}\text { Grandes Regiões/ } \\
\text { Major Regions }\end{array}$} & \multicolumn{6}{|c|}{$\begin{array}{l}\text { Taxa de frequência a creche das crianças de } 0 \text { a } 3 \text { anos de idade/ } \\
\text { Attendance rate to nursery school of children } 0 \text { to } 3 \text { years old (\%) }\end{array}$} \\
\hline & \multicolumn{3}{|c|}{2009} & \multicolumn{3}{|c|}{2014} \\
\hline & $\begin{array}{l}\text { Total/ } \\
\text { Total }\end{array}$ & $\begin{array}{l}\text { Homens/ } \\
\text { Male }\end{array}$ & $\begin{array}{l}\text { Mulheres/ } \\
\text { Female }\end{array}$ & $\begin{array}{l}\text { Total/ } \\
\text { Total }\end{array}$ & $\begin{array}{l}\text { Homens/ } \\
\text { Male }\end{array}$ & $\begin{array}{l}\text { Mulheres/ } \\
\text { Female }\end{array}$ \\
\hline Brasil/Brazil & 18,4 & 18,4 & 18,3 & 24,6 & 24,4 & 24,8 \\
\hline Norte/North & 8,2 & 8,6 & 7,8 & 8,3 & 7,9 & 8,7 \\
\hline Nordeste/Northeast & 15,5 & 15,4 & 15,6 & 20,0 & 18,9 & 21,1 \\
\hline Sudeste/Southeast & 22,8 & 22,5 & 23,2 & 31,3 & 31,1 & 31,4 \\
\hline Sul/South & 24,3 & 25,2 & 23,3 & 32,2 & 33,2 & 31,1 \\
\hline Centro-Oeste/Central-West & 14,7 & 16,0 & 13,3 & 19,3 & 20,2 & 18,3 \\
\hline
\end{tabular}

Fonte/Source: IBGE, Diretoria de Pesquisas, Coordenação de Trabalho e Rendimento, Pesquisa Nacional por Amostra de Domicílios 2009/2014.

\section{Gráfico 6.1 - Taxa de analfabetismo da população de 15 anos ou mais de idade, por situação do domicílio - Brasil - 2005/2014 Graph 6.1 - Illiteracy rate of the population 15 years and over, by urban/rural - Brazil - 2005/2014}

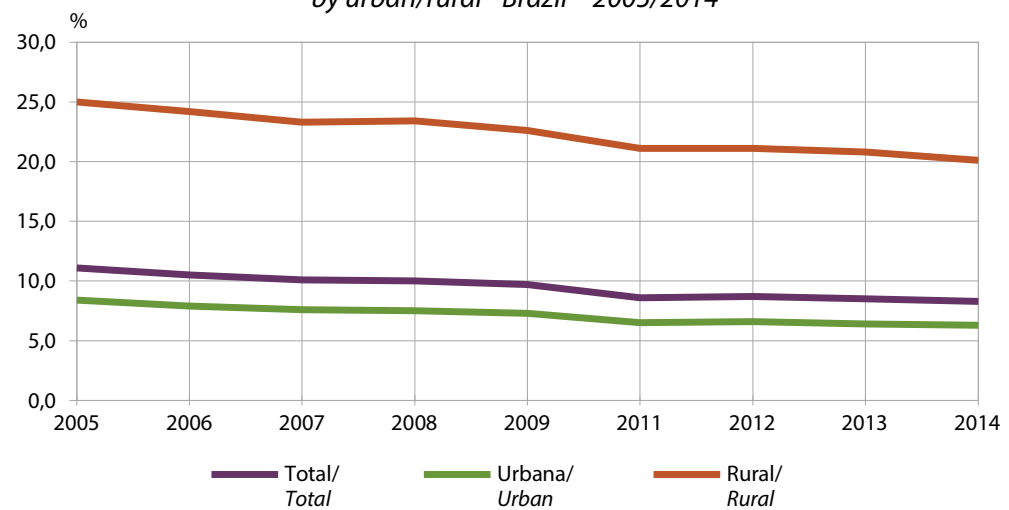

Fonte/Source: IBGE, Diretoria de Pesquisas, Coordenação de Trabalho e Rendimento, Pesquisa Nacional por Amostra de Domicílios 2004/2014. 
Tabela 6.6 - Taxa de escolarização das pessoas de 4 anos ou mais de idade, por Grandes Regiões, segundo os grupos de idade e o sexo - 2014

Table 6.6 - Schooling rate of persons 4 years old and over, by Major Regions, age groups and sex - 2014

\begin{tabular}{|c|c|c|c|c|c|c|}
\hline \multirow{3}{*}{$\begin{array}{l}\text { Grupos de idade } \\
\text { e sexo/ } \\
\text { Age groups and sex }\end{array}$} & \multicolumn{6}{|c|}{$\begin{array}{l}\text { Taxa de escolarização das pessoas de } 4 \text { anos } \\
\text { ou mais de idade/ } \\
\text { Schooling rate of persons } 4 \text { years old and over (\%) }\end{array}$} \\
\hline & \multirow[b]{2}{*}{$\begin{array}{l}\text { Brasil/ } \\
\text { Brazil }\end{array}$} & \multicolumn{5}{|c|}{ Grandes Regiões/Major Regions } \\
\hline & & $\begin{array}{l}\text { Norte/ } \\
\text { North }\end{array}$ & $\begin{array}{l}\text { Nordeste/ } \\
\text { Northeast }\end{array}$ & $\begin{array}{l}\text { Sudeste/ } \\
\text { Southeast }\end{array}$ & $\begin{array}{l}\text { Sul/ } \\
\text { South }\end{array}$ & $\begin{array}{l}\text { Centro- } \\
\text { Oeste/ } \\
\text { Central- } \\
\text { West }\end{array}$ \\
\hline 4 ou 5 anos $/ 4$ to 5 years old & 82,7 & 70,0 & 87,7 & 85,9 & 78,4 & 73,4 \\
\hline Homens/Male & 81,9 & 68,8 & 86,9 & 85,1 & 78,2 & 73,3 \\
\hline Mulheres/Female & 83,5 & 71,3 & 88,5 & 86,7 & 78,7 & 73,5 \\
\hline 6 a 14 anos/ 6 to 14 years old & 98,5 & 97,8 & 98,2 & 98,9 & 98,7 & 98,7 \\
\hline Homens/Male & 98,4 & 97,8 & 98,0 & 98,8 & 98,5 & 98,8 \\
\hline Mulheres/Female & 98,6 & 97,8 & 98,4 & 99,0 & 98,8 & 98,6 \\
\hline 7 a 14 anos/7 a 14 years old & 98,7 & 98,3 & 98,3 & 99,0 & 98,8 & 99,1 \\
\hline Homens/Male & 98,5 & 98,2 & 98,0 & 98,9 & 98,7 & 99,1 \\
\hline Mulheres/Female & 98,8 & 98,3 & 98,5 & 99,2 & 99,0 & 99,1 \\
\hline 15 a 17 anos/15 to 17 years old & 84,3 & 82,9 & 83,2 & 86,5 & 82,8 & 81,9 \\
\hline Homens/Male & 84,1 & 83,3 & 84,0 & 85,9 & 81,5 & 81,2 \\
\hline Mulheres/Female & 84,4 & 82,5 & 82,2 & 87,0 & 84,1 & 82,8 \\
\hline 18 ou 19 anos/ 18 to 19 years old & 42,4 & 48,6 & 43,4 & 40,9 & 38,6 & 45,2 \\
\hline Homens/Male & 42,0 & 49,4 & 43,9 & 40,7 & 34,8 & 43,2 \\
\hline Mulheres/Female & 42,9 & 47,6 & 43,0 & 41,0 & 42,4 & 47,5 \\
\hline 20 a 24 anos/20 to 24 years old & 24,5 & 25,6 & 22,2 & 24,9 & 26,2 & 27,0 \\
\hline Homens/Male & 22,2 & 24,1 & 19,3 & 22,6 & 24,1 & 24,9 \\
\hline Mulheres/Female & 26,9 & 27,0 & 25,0 & 27,2 & 28,4 & 29,0 \\
\hline 25 anos ou mais/ 25 years old and over & 4,0 & 5,2 & 4,4 & 3,5 & 3,7 & 4,8 \\
\hline Homens/Male & 3,5 & 4,0 & 3,5 & 3,3 & 3,4 & 4,0 \\
\hline Mulheres/Female & 4,4 & 6,3 & 5,2 & 3,7 & 4,0 & 5,5 \\
\hline
\end{tabular}

Fonte/Source: IBGE, Diretoria de Pesquisas, Coordenação de Trabalho e Rendimento, Pesquisa Nacional por Amostra de Domicílios 2014. 


\section{Gráfico 6.2 - Taxa de analfabetismo das pessoas de \\ 15 anos ou mais de idade - Brasil - 2009/2014}

Graph 6.2 - Illiteracy rate of the population

15 years old and over - Brazil - 2009/2014

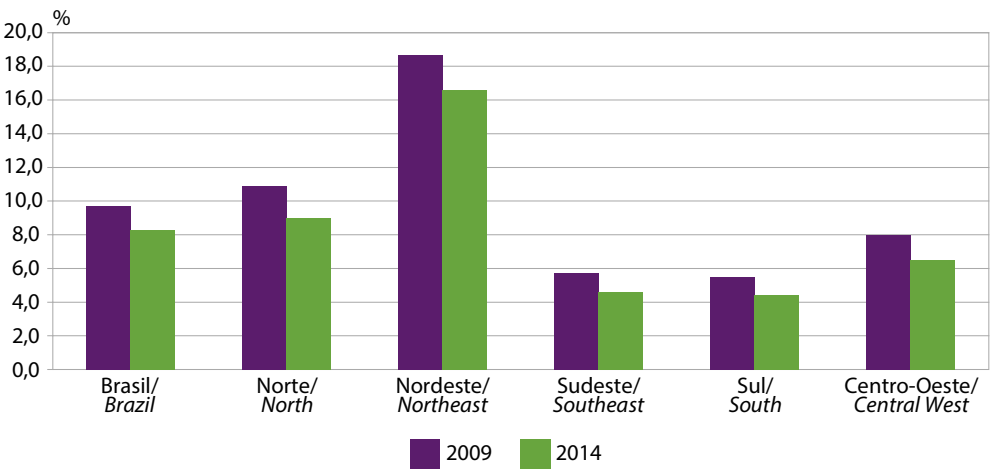

Fonte/Source: IBGE, Diretoria de Pesquisas, Coordenação de Trabalho e Rendimento, Pesquisa Nacional por Amostra de Domicílios 2009/2014.

\section{Gráfico 6.3 - Média de anos de estudo da população de 10 anos ou mais de idade, por grupos de idade - 2014 Graph 6.3 - Average of years of schooling of the population 10 years old and over, by age groups - 2014}

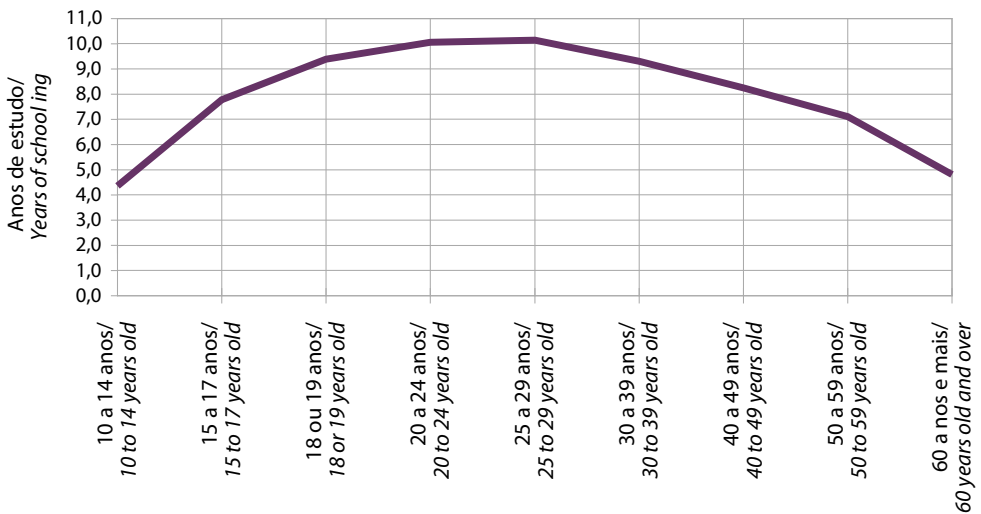

Fonte/Source: IBGE, Diretoria de Pesquisas, Coordenação de Trabalho e Rendimento, Pesquisa Nacional por Amostra de Domicílios 2014. 


\section{Trabalho}

\section{Labor}

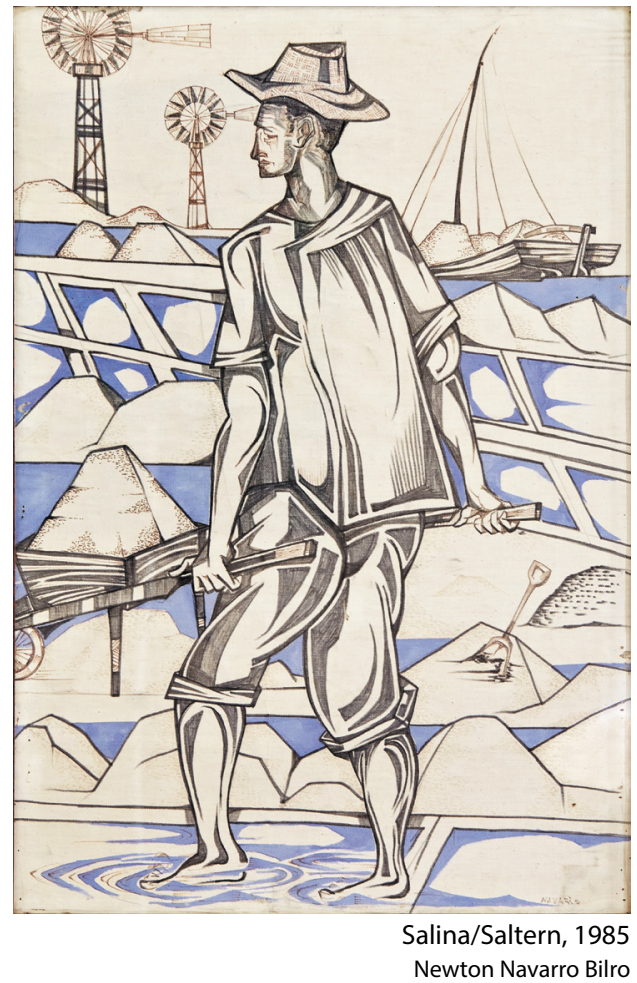





\section{Trabalho}

\section{Trabalho em 2015: fim do ciclo de prosperidade no mercado de trabalho?}

A análise do mercado de trabalho de um país conjuga aspectos relacionados ao comportamento dos empregadores, que demandam trabalhadores, e da força de trabalho disponível e apta a se inserir na atividade laboral. A partir da interação entre estes dois atores, e, considerando o contexto macroeconômico e o aparato institucional que regulamenta as relações de trabalho, podemos entender os resultados dos indicadores de trabalho.

Em termos macroeconômicos, o período de 2007 a 2015 foi marcado por três etapas: (i) o primeiro biênio 2007-2008 apresentou uma trajetória de crescimento expressivo do PIB, estancado a partir de 2009 devido à deflagração da crise mundial no final de 2008; (ii) nos anos seguintes, apesar da crise de 2008, a política macroeconômica adotada impediu uma queda expressiva da atividade econômica, o ano de 2010 foi marcado pela recuperação da atividade econômica, registrando crescimento do PIB de 7,5\% e (iii) nos anos de 2011 para frente, $o$

\section{Labor}

\section{Labor in 2015: end of a prosperity cycle in labor market?}

The analysis of the labor market in any country couples aspects related to the behavior of employers - who demand workers - with that of the available workforce which can join the labor activity. Taking into account the interaction between those two actors and considering the macroeconomic context and the institutional framework that regulates labor relations, one can understand labor indicator results.

In macroeconomic terms, the period from 2007 to 2015 can be split into three stages: (i) the 2007-2008 biennium showed a significant increase of the GDP, halted in 2009 due to the world crisis in the end of 2008; (ii) despite the 2008 crisis, the adopted macroeconomic policy prevented an expressive drop of the economic activity in the following years; 2010 was marked by the recovery of the economic activity, registering an increase of $7.5 \%$ in the GDP; and (iii) from 2011 
crescimento econômico permaneceu, mas a taxas relativamente menores. Esta evolução da taxa de atividade econômica afetou o comportamento dos principais indicadores de mercado de trabalho, tais como a taxa de desocupação, o nível de rendimentos e a estrutura setorial/ocupacional.

De acordo com a Tabela 7.3, de 2007 a 2014, com exceção de 2008 para 2009, a média anual da taxa de desocupação para o total das seis principais regiões metropolitanas investigadas pela PME/ IBGE, caiu de forma contínua. No ano de $2007,9,3 \%$, em média, das pessoas economicamente ativas de 10 anos ou mais estavam desocupadas, enquanto que no ano de 2014, esta taxa decresceu para $4,8 \%$.

Esta média anual é influenciada pelo comportamento mensal da taxa de desocupação. O Gráfico 7.3 apresenta para cada ano, as taxas mensais de desocupação para as pessoas com mais de 10 anos de idade das seis regiões metropolitanas investigadas pela PME. O ano de 2007 é o que apresenta o quadro mais desfavorável. Para todos os meses do ano de 2007, a taxa de desocupação foi a mais alta da série de 2007 a 2015 . O biênio 2008-2009 claramente foi melhor que o ano de 2007, dado o registro de menores taxas mensais de desocupação. Destaca-se que de 2008 para 2009 não houve mudança no patamar das taxas mensais de ocupação. Somente a partir de 2010, as taxas mensais de desocupação apresentam diminuição progressiva comparativamente aos meses dos anos anteriores. Este movimento, entretanto, fica menos intenso quando comparamos 2012 com 2013. De 2013 para 2014, as taxas mensais de desocupação caíram, registrando a menor média anual $(4,8 \%)$. onwards, economic growth persisted, but at relatively lower rates. The evolution of the economic activity rate affected the behavior of the main labor market indicators, like unemployment rate, level of earnings and sectorial/job structure.

According to Table 7.3, the annual average of the unemployment rate for all the six main metropolitan areas surveyed by the PME/IBGE fell almost continuously between 2007 and 2014, except from 2008 to 2009. In $2007,9.3 \%$, on average, of the persons in the workforce aged 10 years and over were unemployed, whereas this rate decreased to $4.8 \%$ in 2014.

The annual average is influenced by the monthly behavior of the unemployment rate. Graph 7.3 shows, for each year, monthly unemployment rates for persons aged 10 years and over for the six metropolitan areas surveyed by the PME. The year of 2007 shows the most unfavorable picture. For every month of 2007, the unemployment rate was the highest in the time series from 2007 to 2015. The 2008-2009 biennium was clearly better than 2007, since the monthly unemployment rates were lower. The level of the monthly unemployment rates did not change between 2008 and 2009. Only after 2010, did they decrease progressively compared with the same months in the previous years. Nevertheless, this movement decelerates when 2012 is compared with 2013. From 2013 to 2014, the monthly unemployment rates dropped, recording their lowest annual average (4.8\%). 
A partir de 2015, o cenário muda drasticamente. A taxa mensal de desocupação das pessoas de 10 anos ou mais subiu continuamente. Em janeiro de $2015,5,3 \%$ das pessoas economicamente ativas de 10 anos ou mais das seis regiões metropolitanas investigadas pela PME estavam desocupadas. $\mathrm{O}$ ano de 2015 fecha com uma taxa mensal de desocupação de 6,9\% em dezembro, aproximando-se rapidamente dos valores registrados para o ano de 2007. Em termos de média anual, a taxa de desocupação para o ano de 2015 ficou em 6,8\%.

Em termos regionais, as trajetórias das médias anuais das taxas de desocupação seguem a tendência nacional. Nas regiões metropolitanas do Nordeste (Recife e Salvador), a taxa de desocupação começou a subir a partir de 2013. Em Recife, chegou a $6,4 \%$ e em Salvador, a $8,1 \%$ em 2013. Nas demais regiões metropolitanas, as taxas de desocupação começaram a se elevar apenas a partir do ano de 2014.

É importante destacar que o mercado de trabalho brasileiro, mesmo quando nos restringimos à análise das seis regiões metropolitanas investigadas pela PME/IBGE, é marcado por uma grande heterogeneidade regional. Apesar da trajetória da taxa de desocupação para as diferentes regiões metropolitanas ser praticamente similar, várias peculiaridades são constatadas no nível da desocupação. As regiões metropolitanas do Nordeste possuem taxas mais altas. No ano de 2015, as médias anuais das taxas de desocupação eram 8,9\% e 11,8\%, respectivamente, para Recife e Salvador. Já no Rio de Janeiro, a média anual da taxa de desocupação de 2015 era de
The picture changes drastically from 2015 onwards. The monthly unemployment rate of persons aged 10 years and over rose continuously. In January 2015, 5.3\% of the persons in the workforce aged 10 years and over in the six metropolitan areas surveyed by the PME were unemployed. The year of 2015 closes with a monthly unemployment rate of $6.9 \%$ in December, quickly approaching the figures registered in 2007. In terms of the annual average, the unemployment rate for 2015 stayed at $6.8 \%$.

In regional terms, the unemployment rate annual averages follow the national trend. In the metropolitan areas in the Northeast (Recife and Salvador), the unemployment rate started to increase from 2013 onwards. It reached $6.4 \%$ in Recife and, in Salvador, $8.1 \%$ in 2013. In the other metropolitan areas, the unemployment rates started to increase only after 2014.

It is important to highlight that the Brazilian labor market is marked by a great regional heterogeneity, even when the analysis is restricted to the six metropolitan areas surveyed by the PME/IBGE. Although the path of the unemployment rate for different metropolitan areas is virtually similar, a number of peculiarities are noticed in the employment population ratio. The metropolitan areas in the Northeast have higher rates. In 2015, the annual averages of the unemployment rates were $8.9 \%$ and $11.8 \%$ for Recife and Salvador, respectively. In Rio de Janeiro, the annual average of the unemployment 
$5,2 \%$, a mais baixa de todas as seis regiões metropolitanas. Belo Horizonte e Porto Alegre registraram taxas de desocupação iguais a 5,6\% e São Paulo, mesmo sendo um centro mais dinâmico economicamente, teve $7 \%$ de pessoas economicamente ativas desocupadas.

São vários os aspectos que podem influenciar estas diferenças, tais como:

(i) o próprio dinamismo do mercado de trabalho em termos de geração e manutenção dos postos de trabalho, (ii) as flutuações no comportamento da força de trabalho (movimentos da taxa de participação no mercado de trabalho) e a (iii) a estrutura do mercado de trabalho da região, tanto em termos de tipo (setor e ocupação) de posto de trabalho e características da força de trabalho.

O Gráfico 7.2 nos dá alguns indicativos das características da força de trabalho brasileira com mais de 15 anos de idade no ano de 2014 segundo dados da PNAD para o total do Brasil (não apenas metropolitano). Primeiro, observamos que os homens, independente do grupo etário ao qual pertencem, sempre estão mais presentes no mercado de trabalho que as mulheres. A taxa de atividade dos homens com mais de 15 anos de idade é de $77,9 \%$, enquanto que para as mulheres da mesma idade, registra-se uma taxa de $56,1 \%$. A taxa de atividade é menor para os mais jovens, de 15 a 17 anos, e para a faixa etária mais velha, formada por pessoas de 60 anos ou mais de idade. $O$ grupo etário com maior participação no mercado de trabalho, seja para homens e mulheres, é o de 30 a 39 anos. Para os homens de 30 a 39 anos, a taxa de atividade chega a $94,1 \%$, enquanto que para as mulheres fica em $74,7 \%$. rate was $5.2 \%$ in 2015 , the lowest among the six metropolitan areas. Belo Horizonte and Porto Alegre posted an unemployment rate of $5.6 \%$ and São Paulo, an even more dynamic economic center, had $7 \%$ of unemployed persons in the workforce.

A number of aspects can influence these differences, like: (i) the dynamism of the labor market in terms of generation and maintenance of job positions; (ii) the fluctuations in the behavior of the workforce - movements of the rate of participation in the labor market; and (iii) the structure of the labor market in the area, both in terms of type - sector and employment - of job position and characteristics of the workforce.

Graph 7.2 points out some signs of the characteristics of the Brazilian workforce aged 15 years and over in 2014, according to PNAD data for the whole country - not only metropolitan areas. Regardless of the age group they belong to, men are always more present in the labor market than women. The activity rate for men aged over 15 years is $77.9 \%$, whereas that for women of the same age group it is $56.1 \%$. The activity rate is lower for youngsters, between 15 and 17 years, and for the oldest age group, formed by persons aged 60 years and over. The age group between 30 and 39 years has the biggest participation in the labor market, for either men or women. For men between 30 and 39 years old, the activity rate reaches $94.1 \%$, while for women, it stays at $74.7 \%$. 
Como as pessoas de 30 a 39 anos são as que mais participam do mercado de trabalho, elas acabam sendo mais representativas no total de ocupados. Isto pode ser visualizado na Tabela 7.1. A porcentagem de $25,5 \%$ da população de 15 anos ou mais ocupadas no total do Brasil está na faixa etária de 30 a 39 anos. Em todas as regiões do Brasil, o perfil etário das pessoas de 15 anos ou mais de idade ocupadas é muito parecido: os menos representativos entre os ocupados são os mais jovens de 15 a 17 e de 18 a 19 anos de idade e os mais representativos são os que tem entre 30 e 39 anos de idade.

As diferenças regionais são mais gritantes quando olhamos a distribuição dos trabalhadores ocupados de mais de 15 anos de idade por nível de escolaridade. Apesar da expansão recente das políticas de incentivo ao ingresso no nível superior e término do nível médio, para o Brasil como um todo, apenas $50 \%$ do total de pessoas ocupadas com mais de 15 anos de idade tem mais de 11 anos de estudos completos. Nas regiões Norte e Nordeste, estes percentuais são iguais a 43,1\% e 40,0\%, respectivamente. As regiões Sul e CentroOeste possuem percentuais próximos ao encontrado para o Brasil como um todo $(50,5 \%)$ enquanto que no Sudeste, mais da metade $(56,9 \%)$ da população ocupada com mais de 15 anos de idade possui mais de 11 anos de estudos.

O cenário se deteriora quando focamos o limite inferior da distribuição educacional, ou seja, o percentual dos que não tem escolaridade ou que nem completaram a parte inicial do ensino Fundamental (possuem menos de 4 anos de estudos completos). No Brasil, $6,6 \%$ e $6,8 \%$ do total de ocupados com mais de
Since persons aged between 30 and 39 years are those who participate the most in the labor market, they become the most representative in the total number of employed persons. This can be seen in Table 7.1. The percentage of $25.5 \%$ of the employed population aged 15 years and over is found in the age group between 30 and 39 years. In every region of Brazil, the age profile of employed persons aged 15 years and over is quite the same: those less representative among the employed persons are the youngsters between 15 and 17 and between 18 and 19 years old, and the most representative are those between 30 and 39 years old.

The most striking regional differences can be seen in the distribution of employed workers aged over 15 years by level of schooling. Despite the recent expansion of incentive policies for conclusion of secondary education and access to higher education in the whole country, only $50 \%$ of the total number of employed persons aged over 15 years have more than 11 complete years of schooling. These percentages are $43.1 \%$ and $40.0 \%$ in the North and Northeast Regions, respectively. The South and Central-West Regions register percentages closer to those found in the whole country $(50.5 \%)$, whereas more than half $(56.9 \%)$ of the employed population aged over 15 years have more than 11 years of schooling.

This picture worsens as we focus on the lower limit of the educational distribution, that is to say, the percentage of those with either no education or who did not complete the initial part of the primary school (and have less than four complete years of schooling). In Brazil, $6.6 \%$ and $6.8 \%$ of the total number of employed 
15 anos de idade não tem escolaridade ou tem de 1 a 3 anos de estudos apenas. Nas regiões Norte e Nordeste, estes percentuais são bem superiores. $O$ percentual sem escolaridade é $8,7 \%$ e $13,3 \%$ para o Norte e Nordeste. A população ocupada com mais de 15 anos de idade destas duas regiões que tinha entre 1 e 3 anos de estudos representava uma parcela de $9,2 \%$ e $10,5 \%$ do total de ocupados, respectivamente. $\mathrm{Na}$ região Centro-Oeste, 5,8\% da população ocupada de 15 anos ou mais não tinham escolaridade e $5,9 \%$ tinham menos de 3 anos de estudos completos. Já no Sul e no Sudeste, há uma menor proporção de trabalhadores ocupados com escolaridade inferior a 3 anos de estudos (menos de 10\%).

As políticas educacionais de universalização do Ensino Fundamental se mostraram eficazes para gerações mais novas, pois a frequência escolar aumentou consideravelmente nos grupos etários em idade escolar. Contudo, para aqueles que já se inserem no mercado de trabalho, sobretudo os mais velhos e moradores de regiões menos desenvolvidas, ainda há um percentual não desprezível de trabalhadores que não finalizaram nem 4 anos de Ensino Fundamental ou que nem passaram pelos bancos escolares. O resultado para esta parcela da população será uma inserção mais precária e instável no mercado de trabalho (menores rendimentos do trabalho).

Em termos de distribuição das pessoas ocupadas com mais de 15 anos de idade por posição na ocupação do trabalho principal, a Tabela 7.1 mostra que no ano de 2014 mais da metade se insere na atividade econômica como empregada. No Brasil, esta proporção é de $61,3 \%$. Nas regiões Norte e Nordeste, este percentual persons aged over 15 years have either no education or only 1 to 3 years of schooling. These percentages are quite higher in the North and Northeast Regions. The percentage of those without schooling is $8.7 \%$ and $13.3 \%$ for the North and Northeast. The employed population aged over 15 years in these two regions with 1 to 3 years of schooling represented a parcel of $9.2 \%$ and $10.5 \%$ of the total number of employed persons, respectively. In the CentralWest Region, $5.8 \%$ of the employed population aged 15 years and over had no education and $5.9 \%$ had less than 3 complete years of schooling. In the South and Southeast, the proportion of employed workers with less than 3 years of schooling is lower (less than 10\%).

The educational policies of universalization of primary education have been efficient for the younger generations, since the school attendance significantly increased in those groups at school age. However, a non-insignificant percentage of workers in the labor market, especially those older and living in less developed regions, did not even complete 4 years of the primary school or never attended school. As a result, this parcel of the population will be precariously and unstably employed (with lower earnings from work).

In terms of the distribution of employed persons aged over 15 years by employment type in the main job, Table 7.1 shows that more than half of them enter the economic activity as employees in 2014. In Brazil, this proportion was $61.3 \%$. This percentage is lower in the North and 
é mais baixo, $53,2 \%$ para ambas. Por outro lado, na região Sudeste, a parcela de empregados chega a $66,2 \%$. Nas regiões Norte e Nordeste, apenas $25,6 \%$ e $26,4 \%$ do total de pessoas ocupadas com mais de 15 anos de idade eram empregados com carteira de trabalho assinada. Para o Brasil, este percentual é de $39,5 \%$ enquanto que para as regiões Sudeste, Sul e Centro-Oeste, os percentuais encontrados são $47,2 \%$, $45,7 \%$ e $40,9 \%$, respectivamente.

Por outro lado, há um percentual não desprezível de pessoas empregadas sem carteira de trabalho. Para o Brasil como um todo, no ano de $2014,14,6 \%$ das pessoas ocupadas com mais de 15 anos de idade estavam nesta situação. Nas regiões Norte e Nordeste, estes valores foram $18,6 \%$ e $19,8 \%$, respectivamente, bem acima do registrado para as demais regiões Sudeste $(12,1 \%)$, Sul $(10,6 \%)$ e Centro-Oeste $(15,1 \%)$. Do total de pessoas ocupadas nas regiões Norte e Nordeste em 2014, 27,8\% e 25,2\% estavam inseridas como trabalhadores por conta própria, bem superior as demais regiões e ao total do Brasil (21,4\%).

Com relação à forma de inserção no mercado de trabalho, portanto, a heterogeneidade regional fica bem clara. Norte e Nordeste apresentam as maiores parcelas de trabalhadores inseridos em ocupações precárias: emprego sem carteira de trabalho assinada, trabalho por conta própria, trabalho não remunerado e trabalho na produção para o próprio consumo. Na região Nordeste, 9,3\% das pessoas ocupadas exercem trabalho na produção para o próprio consumo, bem acima do valor de 4,3\% registrado para o Brasil e para o Sudeste $(1,7 \%)$.

Esta inserção mais precária é reflexo da importância de determinados setores
Northeast Regions (53.2\% for both). On the other hand, the parcel of employees reaches $66.2 \%$ in the Southeast Region. Only $25.6 \%$ and $26.4 \%$ of the total number of persons aged over 15 years were employed with a formal contract in the North and Northeast Regions. This percentage is $39.5 \%$ for Brazil, while those for the Southeast, South and Central-West Regions are 47.2\%, 45.7\% and $40.9 \%$, respectively.

Conversely, a non-insignificant percentage of persons are employed without a formal contract. For the whole country, $14.6 \%$ of the employed persons aged over 15 years were in this situation in 2014. These figures were $18.6 \%$ and $19.8 \%$ for the North and Northeast Regions, respectively, well above those registered in the Southeast (12.1\%), South (10.6\%) and Central-West (15.1\%) Regions. Out of the total number of employed persons in the North and Northeast Regions in 2014, $27.8 \%$ and $25.2 \%$ were self-employed workers, well above the other regions and Brazil (21.4\%).

Therefore, the regional heterogeneity is quite clear when it comes to the way of entry in the labor market. The North and Northeast record the biggest parcels of workers in precarious jobs: employed without a formal contract, self-employed, employed in unpaid jobs and subsistence workers. In the Northeast Region, $9.3 \%$ of the employed persons work in the production for their own consumption, well above the figures for Brazil (4.3\%) and the Southeast (1.7\%).

These more precarious forms of entry reflect the importance of particular 
de atividade econômica nestas regiões, conforme pode ser visto na Tabela 7.2. A distribuição das pessoas ocupadas por grupamentos de atividade do trabalho principal indica que a Agricultura na região Nordeste absorve uma parcela não desprezível da mão-de-obra (25\%). Usualmente, no setor agrícola as relações de trabalho são mais informais e o trabalho é exercido mais por conta própria ou para subsistência. Conforme já destacado acima, dentre as pessoas ocupadas na região, há uma parcela importante nestas formas de inserção. Na região Norte, a Agricultura também tem um papel importante, pois $20,8 \%$ das pessoas ocupadas desta região encontram-se neste setor.

Já em termos de participação do setor industrial, tradicionalmente o que gera ocupações de melhor qualidade (formais e com melhores níveis de rendimentos), na absorção de trabalhadores, as regiões Norte, Nordeste e Centro-Oeste estão bem atrás do Sul, do Sudeste e do total do Brasil no ano de 2014. Na Região Norte, de acordo com o Gráfico 7.5, o percentual de pessoas ocupadas na indústria foi de $9,7 \%$, na Região Nordeste, este valor ficou em $8,7 \%$ e na região Centro-Oeste, foi de $10,8 \%$. No Brasil, o percentual de trabalhadores ocupados na indústria foi de $13,1 \%$. Nas regiões Sul e Sudeste, os percentuais foram $18,7 \%$ e $14,8 \%$, respectivamente.

Vale destacar que o agregado dos grupamentos de atividade referentes ao chamado Setor de Serviços (educação, saúde, serviços pessoais, domésticos, coletivos e sociais) tanto para o total do Brasil como para cada uma das regiões separadamente absorve $21,1 \%$ das pessoas ocupadas. Na Região Sudeste, sectors of the economic activity in those regions, as shown in Table 7.2. The distribution of employed persons along activity groups of the main job points out that Agriculture absorbs a non-insignificant parcel of the workforce in the Northeast Region (25\%). In the agricultural sector, the labor relations are usually more informal and there are more self-employed or subsistence workers. As already highlighted above, an important parcel of the employed persons in that region belong to these categories. Agriculture also plays an important role in the North Region, since $20.8 \%$ of the persons there are employed in this sector.

As a producer of better quality jobs - formal and with higher earnings -, the industrial sector employs fewer workers in the North, Northeast and Central-West Regions, well behind the South, Southeast and the whole country in 2014. According to Graph 7.5 , the percentage of persons employed in industry was $9.7 \%$ in the North Region, $8.7 \%$ in the Northeast Region and $10.8 \%$ in the Central-West Region. In Brazil, the percentage of workers employed in industry was $13.1 \%$. In the South and Southeast Regions, the percentages were $18.7 \%$ and $14.8 \%$, respectively.

It is worth highlighting that the set of activity groups related to the Services Sector-education, health, personal, domestic, collective and social services - for both Brazil and every separate region holds $21.1 \%$ of the employed persons. In the Southeast Region, $23 \%$ of the employed 
$23 \%$ das pessoas ocupadas estão neste setor. Já nas regiões Norte e Nordeste, estes valores são 19,3\% e 19,7\%.

Claramente é um setor muito heterogêneo que comporta relações de trabalho diferenciadas e rendimentos do trabalho inferiores comparativamente ao setor industrial. Entretanto, sabe-se que sua representatividade na atividade econômica é crescente e que nele encontram-se os serviços ligados à educação e saúde fundamentais para qualidade da força de trabalho futura e para as condições de vida da população em geral de um país. O grupamento que agrega mais pessoas ocupadas é o de Educação, Saúde e Serviços Sociais, em torno de 10\% para todas as regiões do Brasil.

Quando olhamos a distribuição do total de ocupados no ano de 2014 pela participação na previdência social (Gráfico 7.4), as diferenças do Sudeste e do Sul em relação às demais são bem expressivas. No Sul e no Sudeste, $72,9 \%$ e $70,6 \%$ das pessoas ocupadas com mais de 15 anos de idade, contribuem para a Previdência Social. No Norte, este percentual decresce para $44,8 \%$, no Nordeste, para $43,9 \%$ e no Centro-Oeste para $65,1 \%$. Os dados sugerem que a precariedade da inserção no mercado de trabalho também se reflete nas dificuldades de participação no esquema de previdência social.

Outra forma de analisar a qualidade da inserção no mercado de trabalho é olhar os dados de rendimento do trabalho. O Gráfico 7.1 mostra que $58,6 \%$ das pessoas ocupadas tinham rendimento mensal de todos os trabalhos inferior a 2 salários mínimos no ano de 2014. Outro dado alarmante é que $7,1 \%$ não tinham rendimento. persons are in this sector. In the North and Northeast Region, the figures are $19.3 \%$ and $19.7 \%$. It is clearly a very heterogeneous sector, which has differentiated labor relations and earnings comparatively lower than those in the industrial sector. Nonetheless, its representativeness is increasing in the economic activity, as well as including services related to education and health, which are key to the quality of the future workforce and to the living conditions of the population as a whole. Having posted about $10 \%$ for every region, the group of Education, Health and Social Services is the one that employs most persons.

When looking into the distribution of the total number of employed persons in 2014 by the contribution to social security (Graph 7.4), the differences between the Southeast and South and the other regions are quite significant. In the South and Southeast, $72.9 \%$ and $70.6 \%$ of the employed persons aged over 15 years contribute to the Social Security. In the North, that percentage decreases to $44.8 \%$, in the Northeast, to $43.9 \%$ and in the Central-West, to $65.1 \%$. The data suggest that low quality jobs are also directly related to difficulties in the contribution to social security.

Another way to assess the quality of the entry in the labor market is looking into the earnings data. Graph 7.1 shows that $58.6 \%$ of the employed persons had monthly earnings from all jobs lower than 2 minimum wages in 2014. Another alarming piece of information is that $7.1 \%$ of them had no earnings at all. 
Com relação à evolução, a Tabela 7.4 nos mostra que de 2014 para 2015, a situação em termos de rendimento médio mensal habitual do trabalho principal piorou. Para o total das seis regiões metropolitanas investigadas pela PME/IBGE, o rendimento caiu $3,7 \%$. Esta redução foi generalizada para todas as seis regiões, sendo mais marcante nas regiões de Belo Horizonte $(4,6 \%)$, São Paulo (4\%) e Rio de Janeiro (4\%). No total das seis regiões metropolitanas, as perdas de rendimento foram maiores para os empregadores $(6,2 \%)$, os empregados sem carteira de trabalho assinada $(5,1 \%)$ e para os trabalhadores por conta própria $(4,1 \%)$. Na maior parte das regiões, são estas posições na ocupação que tiveram maiores reduções, com destaque para os empregadores em Belo Horizonte (10,3\%) e Rio de Janeiro (11,5\%).

Em resumo, em todas as regiões metropolitanas e para todas as posições na ocupação houve perda de rendimento do trabalho. Comparando os meses de 2015 com os mesmos meses de 2014, conforme mostra o Gráfico 7.6, as variações sempre foram negativas depois de janeiro e mais intensas no final do ano. A partir de outubro, os trabalhadores por conta própria, por exemplo, tiveram variações negativas superiores a $8 \%$.

A breve análise dos dados de mercado de trabalho nos mostra algumas peculiaridades do Brasil que persistem no cenário atual e que tendem a se aprofundar nos próximos anos tendo em vista o quadro de baixo crescimento econômico que se delineia. Destacamos a heterogeneidade regional em termos de inserção no mercado de trabalho. A distribuição da população ocupada em termos setoriais e de posição na ocupação é diferenciada nas regiões do Brasil.
Concerning its evolution, Table 7.4 shows that the situation in terms of the usual average monthly earnings from the main job worsened between 2014 and 2015 . Earnings dropped $3.7 \%$ for all the six metropolitan areas surveyed by the PME/IBGE. That reduction was widespread along all the six areas, standing out in the areas of Belo Horizonte (4.6\%), São Paulo (4\%) and Rio de Janeiro (4\%). Considering all the six metropolitan areas, the losses in earnings were higher for employers (6.2\%), employees without a formal contract (5.1\%) and self-employed workers (4.1\%). Those employment types registered the biggest reductions in most regions, highlighted by the employers in Belo Horizonte (10.3\%) and Rio de Janeiro (11.5\%).

In summary, earnings from work recorded losses in every metropolitan area and for every employment type. Comparing the 2015 months with the same months of 2014, as shown in Graph 7.6, we see the changes were always negative after January and more intense by the end of the year. For example, self-employed workers recorded negative changes above $8 \%$ from October onwards.
A brief analysis of the data on the labor market points out a number of Brazilian peculiarities that remain in the current scenario and tend to deepen in the coming years, given the expected low economic growth. The regional heterogeneity in relation to the entry in the labor market is quite relevant, with a differentiated distribution of the employed population in sectorial and employment type terms among the Brazilian regions. On the other 
Por outro lado, há espaço para maior escolarização da força de trabalho tendo em vista a existência de pessoas pouco escolarizadas no mercado de trabalho. Por fim, salientamos que o avanço constatado em meados dos anos 2000, em termos de formalização e melhora dos principais indicadores de mercado de trabalho (redução do desemprego e aumento dos rendimentos) parece ter estagnado. $\mathrm{O}$ ano de 2015 foi marcado por redução do nível de rendimentos e por aumento do desemprego. hand, investment in education would be very welcome since there are still very poorly educated persons in the labor market. Finally, we highlight that the advance recorded in the mid2000s in terms of formalization and improvement of the major labor market indicators - reduction of unemployment and increase of earnings - seems to have stagnated. The year of 2015 was marked by a reduction in the level of earnings and by an increase in the unemployment.

Danielle Carusi Machado

Possui graduação em Economia pela Universidade Federal do Rio de Janeiro (1993), mestrado em Economia da Industria e da Tecnologia pela Universidade Federal do Rio de Janeiro (1997) e doutorado em Economia pelo Departamento de Economia da PUC-Rio (2006). Tem experiência na área de Economia, com ênfase em Economia Aplicada, atuando principalmente nos seguintes temas: mercado de trabalho, educação, pobreza e bem estar social. Atualmente é professora adjunta da Faculdade de Economia da Universidade Federal Fluminense.
Bachelor in Economics from the Federal University of Rio de Janeiro (1993), Master in Economics of Industry and Technology from the Federal University of Rio de Janeiro (1997) and PhD in Economics from the School of Economics of the Pontifical Catholic University of Rio de Janeiro (2006). She has expertise in Economics, especially in the following subjects of Applied Economics: labor market, education, poverty and social welfare. She is currently Assistant Professor at the School of Economics of the Fluminense Federal University. 
Tabela 7.1 - Distribuição das pessoas de 15 anos ou mais de idade, ocupadas, por Grandes Regiões, segundo algumas

características - 2014

Table 7.1 - Percent distribution of employed persons 15 years old and over, by Major Regions and some characteristics - 2014

(continua/to be continued)

Distribuição das pessoas de 15 anos ou mais

de idade, ocupadas/

Percent distribuition of employed persons

Características/

15 years old and over

Characteristics

\begin{tabular}{|c|c|c|c|c|}
\hline \multirow[b]{2}{*}{$\begin{array}{l}\text { Brasil/ } \\
\text { Brazil }\end{array}$} & \multicolumn{4}{|c|}{ Grandes Regiões/Major Regions } \\
\hline & $\begin{array}{ll}\text { Norte / Nordeste/ } \\
\text { North Northeast }\end{array}$ & $\begin{array}{l}\text { Sudeste/ } \\
\text { Southeast }\end{array}$ & $\begin{array}{l}\text { Sul/ } \\
\text { South }\end{array}$ & $\begin{array}{c}\text { Centro- } \\
\text { Oeste/ } \\
\text { Central West }\end{array}$ \\
\hline
\end{tabular}

\section{Grupos de idade/}

Age groups

15 a 17 anos/
15 to 17 years old
18 ou 19 anos/
18 to 19 years old
20 a 24 anos/
20 a 24 years old
25 a 29 anos/
25 a 29 years old
30 a 39 anos/
30 to 39 years old
40 a 49 anos/
40 to 49 years old
50 a 59 anos/
50 to 59 years old
60 anos ou mais/
60 years old and over
Grupos de anos de estudo/
Years of school completed
Sem instrução e menos de 1 ano/
No schooling and less than 1 year
1 a 3 anos/
1 to 3 years
4 a 7 anos/
4 to 7 years
8 a 10 anos/
8 to 10 years
11 anos ou mais/
11 years and over

$100,0100,0$

100,0

$100,0 \quad 100,0$

100,0

$\begin{array}{rrrrrr}2,5 & 3,2 & 2,8 & 2,0 & 2,7 & 2,5 \\ 3,5 & 3,7 & 3,7 & 3,2 & 3,7 & 3,6 \\ 10,6 & 11,3 & 10,6 & 10,4 & 10,4 & 10,9 \\ 12,1 & 13,1 & 12,4 & 11,9 & 11,1 & 12,6 \\ 25,5 & 27,1 & 25,5 & 25,6 & 24,2 & 26,5 \\ 21,9 & 21,1 & 21,2 & 22,5 & 21,8 & 22,3 \\ 15,8 & 13,6 & 14,9 & 16,5 & 16,8 & 14,5 \\ 8,2 & 6,9 & 8,9 & 7,9 & 9,2 & 7,1\end{array}$

$\begin{array}{llllll}100,0 & 100,0 & 100,0 & 100,0 & 100,0 & 100,0\end{array}$

$\begin{array}{llllll}6,6 & 8,7 & 13,3 & 3,5 & 3,6 & 5,8\end{array}$

$\begin{array}{llllll}6,8 & 9,2 & 10,5 & 4,9 & 5,4 & 5,9\end{array}$

$\begin{array}{llllll}19,5 & 21,5 & 20,7 & 17,8 & 21,2 & 19,7\end{array}$

$\begin{array}{llllll}17,0 & 17,2 & 15,3 & 16,9 & 19,2 & 18,1\end{array}$

$\begin{array}{llllll}50,0 & 43,1 & 40,0 & 56,9 & 50,5 & 50,5\end{array}$ 


\section{Tabela 7.1 - Distribuição das pessoas de 15 anos ou mais de idade, ocupadas, por Grandes Regiões, segundo algumas \\ características - 2014}

Table 7.1 - Percent distribution of employed persons 15 years old and over, by Major Regions and some characteristics - 2014

(conclusão/concluded)

\begin{tabular}{|c|c|c|c|c|c|c|}
\hline \multirow{3}{*}{$\begin{array}{l}\text { Características/ } \\
\text { Characteristics }\end{array}$} & \multicolumn{6}{|c|}{$\begin{array}{c}\text { Distribuição das pessoas de } 15 \text { anos ou mais } \\
\text { de idade, ocupadas/ } \\
\text { Percent distribuition of employed persons } \\
15 \text { years old and over }\end{array}$} \\
\hline & \multirow[b]{2}{*}{$\begin{array}{l}\text { Brasil/ } \\
\text { Brazil }\end{array}$} & \multicolumn{5}{|c|}{ Grandes Regiões/Major Regions } \\
\hline & & $\begin{array}{l}\text { Norte / } \\
\text { North }\end{array}$ & $\begin{array}{l}\text { Nordeste/ } \\
\text { Northeast }\end{array}$ & $\begin{array}{l}\text { Sudeste/ } \\
\text { Southeast }\end{array}$ & $\begin{array}{l}\text { Sul/ } \\
\text { South }\end{array}$ & $\begin{array}{c}\text { Centro- } \\
\text { Oeste/ } \\
\text { Central West }\end{array}$ \\
\hline $\begin{array}{l}\text { Posição na ocupação no trabalho } \\
\text { principal/ } \\
\text { Status in employment in main work }\end{array}$ & 100,0 & 100,0 & 100,0 & 100,0 & 100,0 & 100,0 \\
\hline $\begin{array}{l}\text { Empregado/ } \\
\text { Employee }\end{array}$ & 61,3 & 53,2 & 53,2 & 66,2 & 63,0 & 65,4 \\
\hline $\begin{array}{l}\text { Com carteira de trabalho assinada/ } \\
\text { With a formal contract }\end{array}$ & 39,5 & 25,6 & 26,4 & 47,2 & 45,7 & 40,9 \\
\hline $\begin{array}{l}\text { Militares e funcionários públicos } \\
\text { estatutários/ } \\
\text { Military and statutory public officers }\end{array}$ & 7,3 & 8,9 & 7,1 & 6,9 & 6,7 & 9,4 \\
\hline $\begin{array}{l}\text { Sem carteira de trabalho assinada/ } \\
\text { Without a format contract }\end{array}$ & 14,6 & 18,6 & 19,8 & 12,1 & 10,6 & 15,1 \\
\hline $\begin{array}{l}\text { Trabalhador doméstico/ } \\
\text { Private household worker }\end{array}$ & 6,5 & 5,7 & 6,0 & 7,3 & 5,6 & 6,9 \\
\hline $\begin{array}{l}\text { Com carteira de trabalho assinada/ } \\
\text { With a formal contract }\end{array}$ & 2,1 & 1,2 & 1,1 & 2,8 & 2,0 & 2,4 \\
\hline $\begin{array}{l}\text { Sem carteira de trabalho assinada/ } \\
\text { Without a format contract }\end{array}$ & 4,4 & 4,5 & 4,9 & 4,5 & 3,6 & 4,5 \\
\hline $\begin{array}{l}\text { Conta própria/ } \\
\text { Own account }\end{array}$ & 21,4 & 27,8 & 25,2 & 19,1 & 19,4 & 19,6 \\
\hline $\begin{array}{l}\text { Empregador/ } \\
\text { Employer }\end{array}$ & 3,8 & 2,7 & 2,5 & 4,3 & 4,9 & 4,2 \\
\hline $\begin{array}{l}\text { Não remunerado/ } \\
\text { Unpaid worker }\end{array}$ & 2,6 & 4,6 & 3,7 & 1,4 & 3,4 & 1,4 \\
\hline $\begin{array}{l}\text { Trabalhador na produção para o } \\
\text { próprio consumo/ } \\
\text { Worker in production for own } \\
\text { consumption }\end{array}$ & 4,3 & 5,8 & 9,3 & 1,7 & 3,5 & 2,3 \\
\hline $\begin{array}{l}\text { Trabalhador na construção para o } \\
\text { próprio uso/ } \\
\text { Worker in construction for own use }\end{array}$ & 0,1 & 0,1 & 0,1 & 0,1 & 0,1 & 0,2 \\
\hline
\end{tabular}

Fonte/Source: IBGE, Diretoria de Pesquisas, Coordenação de Trabalho e Rendimento, Pesquisa Nacional por Amostra de Domicílios 2014. 


\section{Tabela 7.2 - Distribuição das pessoas de 15 anos ou mais de idade, ocupadas, por Grandes Regiões, segundo os grupamentos de atividade do trabalho principal - 2014}

Table 7.2 - Percent distribution of employed persons 15 years old and over, by Major Regions and groups of activity of the main job - 2014

\begin{tabular}{|c|c|c|c|c|c|c|}
\hline \multirow{3}{*}{$\begin{array}{l}\text { Grupamentos de atividade } \\
\text { do trabalho principal/ } \\
\text { Groups of activity } \\
\text { in the main work }\end{array}$} & \multicolumn{6}{|c|}{$\begin{array}{c}\text { Distribuição das pessoas de } 15 \text { anos ou mais de idade, } \\
\text { ocupadas/ } \\
\text { Percent distribution of employed persons } 15 \text { years } \\
\text { old and over }\end{array}$} \\
\hline & \multirow[b]{2}{*}{$\begin{array}{l}\text { Brasil/ } \\
\text { Brazil }\end{array}$} & \multicolumn{5}{|c|}{ Grandes Regiões/Major Regions } \\
\hline & & $\begin{array}{l}\text { Norte/ } \\
\text { North }\end{array}$ & $\begin{array}{l}\text { Nordeste/ } \\
\text { Northeast }\end{array}$ & $\begin{array}{l}\text { Sudeste/ } \\
\text { Southeast }\end{array}$ & $\begin{array}{l}\text { Sul/ } \\
\text { South }\end{array}$ & $\begin{array}{l}\text { Centro- } \\
\text { Oeste/ } \\
\text { Central } \\
\text { West }\end{array}$ \\
\hline $\begin{array}{l}\text { Total/ } \\
\text { Total }\end{array}$ & 100,0 & 100,0 & 100,0 & 100,0 & 100,0 & 100,0 \\
\hline $\begin{array}{l}\text { Agricultura/ } \\
\text { Agriculture, hunting, forestry and fishing }\end{array}$ & 14,2 & 20,8 & 25,0 & 7,1 & 14,1 & 11,8 \\
\hline $\begin{array}{l}\text { Indústria/ } \\
\text { Industry }\end{array}$ & 13,1 & 9,7 & 8,7 & 14,8 & 18,7 & 10,8 \\
\hline $\begin{array}{l}\text { Indústria de transformação/ } \\
\text { Manufacturing }\end{array}$ & 12,3 & 8,7 & 8,1 & 13,9 & 18,1 & 10,0 \\
\hline $\begin{array}{l}\text { Construção/ } \\
\text { Construction }\end{array}$ & 9,2 & 10,1 & 9,1 & 9,2 & 8,4 & 10,4 \\
\hline $\begin{array}{l}\text { Comércio e reparação/ } \\
\text { Trade and reparation }\end{array}$ & 18,2 & 18,5 & 17,5 & 18,4 & 18,0 & 18,9 \\
\hline $\begin{array}{l}\text { Alojamento e alimentação/ } \\
\text { Hotels and restaurants }\end{array}$ & 4,7 & 4,6 & 4,6 & 5,0 & 3,7 & 5,3 \\
\hline $\begin{array}{l}\text { Transporte, armazenagem e comuni- } \\
\text { cação/ } \\
\text { Transport, storage and communication }\end{array}$ & 5,5 & 5,0 & 4,6 & 6,3 & 5,5 & 5,2 \\
\hline $\begin{array}{l}\text { Administração pública/ } \\
\text { Public administration }\end{array}$ & 5,2 & 6,7 & 5,3 & 4,7 & 4,5 & 7,6 \\
\hline $\begin{array}{l}\text { Educação, saúde e serviços pessoais/ } \\
\text { Education, health and social services }\end{array}$ & 10,3 & 10,0 & 9,9 & 10,9 & 9,8 & 10,0 \\
\hline $\begin{array}{l}\text { Serviços domésticos/ } \\
\text { Domestic services }\end{array}$ & 6,5 & 5,7 & 6,0 & 7,3 & 5,6 & 6,9 \\
\hline $\begin{array}{l}\text { Outros serviços coletivos, sociais e } \\
\text { pessoais/ } \\
\text { Other collective, social and personal } \\
\text { services }\end{array}$ & 4,2 & 3,6 & 3,7 & 4,8 & 3,8 & 4,4 \\
\hline $\begin{array}{l}\text { Outras atividades/ } \\
\text { Other activities }\end{array}$ & 8,7 & 5,2 & 5,4 & 11,5 & 8,2 & 8,9 \\
\hline $\begin{array}{l}\text { Atividades maldefinidas } \\
\text { Not adequately defined activities }\end{array}$ & 0,1 & 0,1 & 0,1 & 0,1 & 0,0 & 0,0 \\
\hline
\end{tabular}

Fonte/Source: IBGE, Diretoria de Pesquisas, Coordenação de Trabalho e Rendimento, Pesquisa Nacional por Amostra de Domicílios 2014. 
Tabela 7.3 - Média anual da taxa de desocupação das pessoas de 10 anos ou mais de idade, por principais regiões metropolitanas - 2006-2015 Table 7.3 - Annual average of unemployment rate of persons 10 years and over, by main Metropolitan Areas - 2006-2015

\begin{tabular}{|c|c|c|c|c|c|c|c|c|c|c|}
\hline \multirow{2}{*}{$\begin{array}{l}\text { Principais } \\
\text { regiões metropolitanas/ } \\
\text { Metropolitan Areas }\end{array}$} & \multicolumn{10}{|c|}{$\begin{array}{l}\text { Média anual da taxa de desocupação (\%)/ } \\
\text { Annual average of unemployment rate (\%) }\end{array}$} \\
\hline & 2006 & 2007 & 2008 & 2009 & 2010 & 2011 & 2012 & 2013 & 2014 & 2015 \\
\hline Total/Total & 10,0 & 9,3 & 7,9 & 8,1 & 6,7 & 5,9 & 5,5 & 5,4 & 4,8 & 6,8 \\
\hline Recife & 14,6 & 12,0 & 9,3 & 9,9 & 8,7 & 6,5 & 6,0 & 6,4 & 6,5 & 8,9 \\
\hline Salvador & 13,7 & 13,7 & 11,5 & 11,3 & 11,0 & 9,6 & 7,2 & 8,1 & 9,0 & 11,8 \\
\hline Belo Horizonte & 8,5 & 7,6 & 6,5 & 6,4 & 5,5 & 4,9 & 4,4 & 4,2 & 3,7 & 5,6 \\
\hline Rio de Janeiro & 7,9 & 7,2 & 6,8 & 6,1 & 5,6 & 5,2 & 5,0 & 4,5 & 3,5 & 5,2 \\
\hline São Paulo & 10,5 & 10,1 & 8,4 & 9,2 & 7,0 & 6,2 & 6,0 & 5,9 & 5,0 & 7,0 \\
\hline Porto Alegre & 8,0 & 7,3 & 5,9 & 5,6 & 4,5 & 4,5 & 4,0 & 3,5 & 3,8 & 5,6 \\
\hline
\end{tabular}

Fonte/Source: IBGE, Diretoria de Pesquisas, Coordenação de Trabalho e Rendimento, Pesquisa Mensal de Emprego 2006-2015.

\section{Gráfico 7.1 - Distribuição percentual das pessoas de 15 anos ou mais de} idade, ocupadas, por classes de rendimento mensal de trabalho - 2014 Graph 7.1 - Percentage distribution of employed persons 15 years old and over, by ranges of monthly earnings - 2014

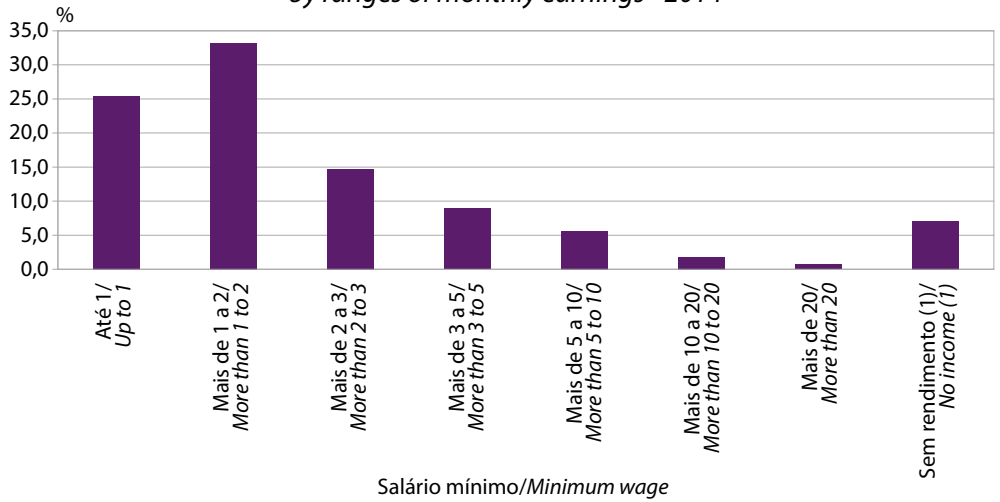

Fonte/Source: IBGE, Diretoria de Pesquisas, Coordenação de Trabalho e Rendimento, Pesquisa Nacional por Amostra de Domicílios 2014.

(1) Inclusive as pessoas que recebiam somente em benefícios./ (1) Including persons who received only benefits. 
Tabela 7.4 - Variação anual do rendimento médio mensal real habitual do trabalho principal de categorias selecionadas das pessoas ocupadas, por principais regiões metropolitanas - período 2014-2015

Table 7.4 - Annual variation of average monthly real income usually received from the main job of selected categories of employed persons, by main metropolitan areas - 2014-2015

\begin{tabular}{|c|c|c|c|c|c|c|c|}
\hline \multirow{2}{*}{$\begin{array}{l}\text { Categorias selecionadas/ } \\
\text { Selected categories }\end{array}$} & \multicolumn{7}{|c|}{$\begin{array}{l}\text { Variação anual do rendimento médio mensal real habitual } \\
\text { do trabalho principal/ } \\
\text { Annual variation of average monthly real income usually } \\
\text { received from the main job }\end{array}$} \\
\hline & $\begin{array}{l}\text { Total/ } \\
\text { Total }\end{array}$ & Recife & $\begin{array}{l}\text { Salva- } \\
\text { dor }\end{array}$ & $\begin{array}{l}\text { Belo } \\
\text { Hori- } \\
\text { zonte }\end{array}$ & $\begin{array}{l}\text { Rio } \\
\text { de } \\
\text { Janeiro }\end{array}$ & $\begin{array}{l}\text { São } \\
\text { Paulo }\end{array}$ & $\begin{array}{l}\text { Porto } \\
\text { Alegre }\end{array}$ \\
\hline $\begin{array}{l}\text { Pessoas ocupadas/ } \\
\text { Occupied person }\end{array}$ & $(-) 3,7$ & $(-) 2,8$ & $(-) 3,0$ & $(-) 4,6$ & (-) 4,0 & $(-) 4,0$ & (-) 2,8 \\
\hline $\begin{array}{l}\text { Conta própria/ } \\
\text { Own account }\end{array}$ & (-) 4,1 & (-) 4,0 & (-) 5,7 & $(-) 9,1$ & (-) 1,3 & (-) 4,0 & (-) 7,8 \\
\hline $\begin{array}{l}\text { Empregadores/ } \\
\text { Employer }\end{array}$ & (-) 6,2 & (-) 5,7 & $(-) 2,5$ & (-) 10,3 & (-) 11,5 & (-) 4,4 & (-) 1,0 \\
\hline $\begin{array}{l}\text { Empregados/ } \\
\text { Employee }\end{array}$ & (-) 3,0 & (-) 1,3 & (-) 0,6 & (-) 1,9 & (-) 3,1 & (-) 4,0 & (-) 1,9 \\
\hline $\begin{array}{l}\text { Com carteira de } \\
\text { trabalho assinada no } \\
\text { setor privado/ } \\
\text { With a formal contrac } \\
\text { in private setor }\end{array}$ & $(-)$ 3,3 & (-) 3,5 & (-) 1,3 & $(-) 2,8$ & (-) 4,4 & (-) 3,5 & (-) 2,6 \\
\hline $\begin{array}{l}\text { trabalho assinada no } \\
\text { setor privado/ } \\
\text { Without a formal } \\
\text { contractin private setor }\end{array}$ & (-) 5,1 & (-) 1,8 & (-) 3,4 & $(-) 4,1$ & (-) 2,3 & (-) 7,4 & (-) 3,8 \\
\hline $\begin{array}{l}\text { Militares e funcionários } \\
\text { públicos estatutários/ } \\
\text { Military and statutory } \\
\text { public servants }\end{array}$ & (-) 1,8 & (-) 1,5 & 1,8 & $(-) 0,0$ & (-) 2,7 & $(-)$ 3,9 & (-) 0,3 \\
\hline
\end{tabular}

Fonte/Source: IBGE, Diretoria de Pesquisas, Coordenação de Trabalho e Rendimento, Pesquisa Mensal de Emprego 2014-2015.

Nota: A preços de dezembro de 2015./Note: At prices of December 15.

(1) Rendimento inflacionado pelo Índice de Preços ao Consumidor - INPC, a preços de dezembro de 2015./(1) Income inflated by National Index of Consumer Prices, at prices of December 2015. 


\section{Gráfico 7.2 - Taxa de atividade das pessoas de 15 anos ou mais de idade, por grupos de idade, segundo o sexo - Brasil - 2014}

Graph 7.2 - Labor force participation rate of persons 15 years old and over,

by age, groups and sex - Brazil - 2014

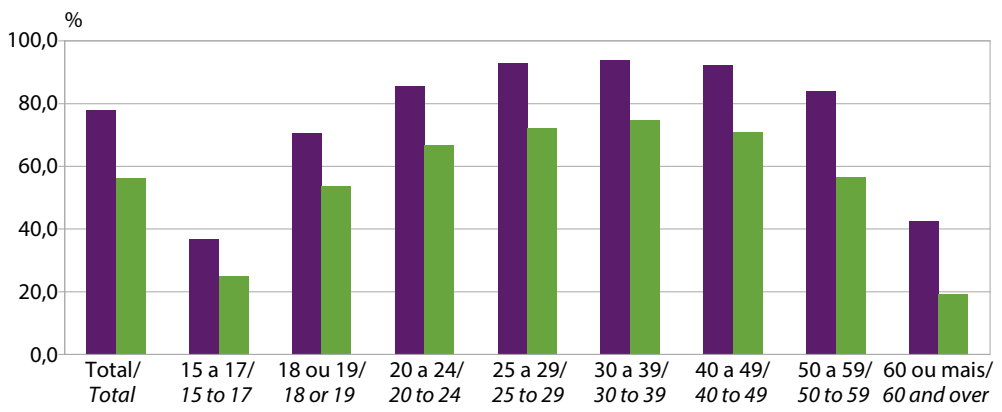

Anos/Years

$\begin{array}{ll}\text { Homens/ } & \text { Mulheres/ } \\ \text { Male } & \text { Female }\end{array}$

Fonte/Source: IBGE, Diretoria de Pesquisas, Coordenação de Trabalho e Rendimento, Pesquisa Nacional por Amostra de Domicílios 2014.

\section{Gráfico 7.3 - Taxa de desocupação das pessoas de 10 anos} ou mais de idade - total das principais Regiões Metropolitanas - 2007-2015 Graph 7.3 - Unemployment rate of persons 10 years old and over - total of main Metropolitan Areas - 2007-2015

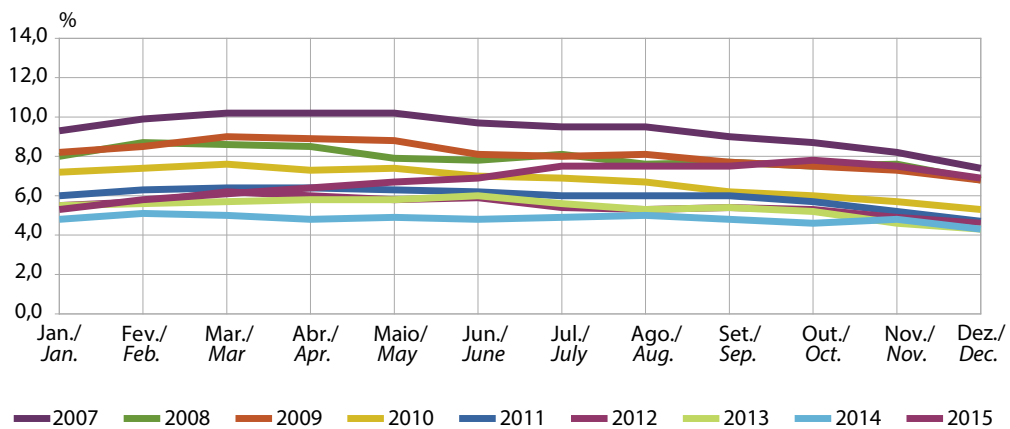

Fonte/Source: IBGE, Diretoria de Pesquisas, Coordenação de Trabalho e Rendimento, Pesquisa Mensal de Emprego 2007-2015.

Nota: Média das Regiões Metropolitanas de Recife, Salvador, Belo Horizonte, Rio de Janeiro, São Paulo e Porto Alegre. /Note: Average of Metropolitan Areas of Recife, Salvador, Belo Horizonte, Rio de Janeiro, São Paulo and Porto Alegre. 


\section{Gráfico 7.4 - Distribuição das pessoas de 15 anos ou mais de idade, ocupadas, por Grandes Regiões, segundo a contribuição para instituto de previdência - 2014}

Graph 7.4 - Distribution of employed persons 15 years old and over, by Major Regions, according to the participation in social security - 2014

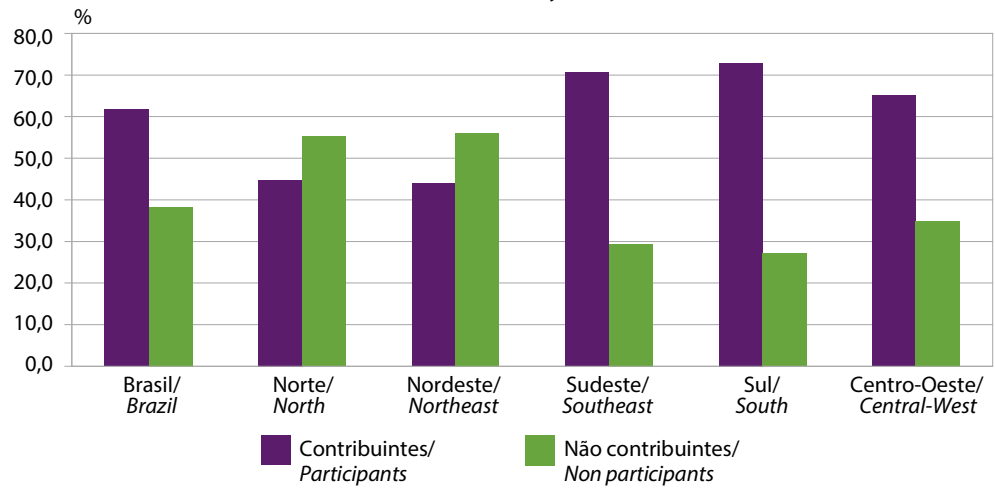

Fonte/Source: IBGE, Diretoria de Pesquisas, Pesquisa Nacional por Amostra de Domicílios 2014.

Gráfico 7.5 - Percentual de pessoas ocupadas na indústria no total da população de 15 anos ou mais de idade, ocupada, por Grandes Regiões - 2014

Graph 7.5 - Percentage of employed persons in industry in the total employed population 15 years old and over, by Major Regions - 2014

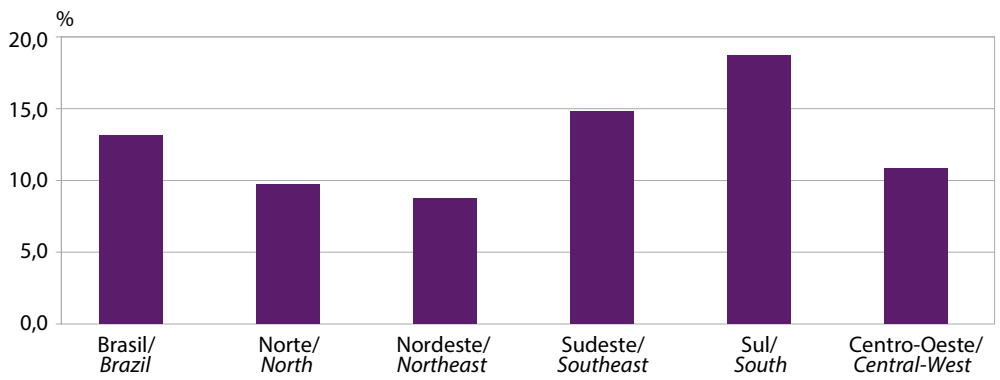

Fonte/Source: IBGE, Diretoria de Pesquisas, Coordenação de Trabalho e Rendimento, Pesquisa Nacional por Amostra de Domicílios 2014. 


\section{Gráfico 7.6 - Variação anual do rendimento médio mensal real habitual de categorias selecionadas de pessoas ocupadas - período 2014-2015}

Graph 7.6 - Annual percent variation of average real monthly income by selected categories of employed persons - period 2014-2015

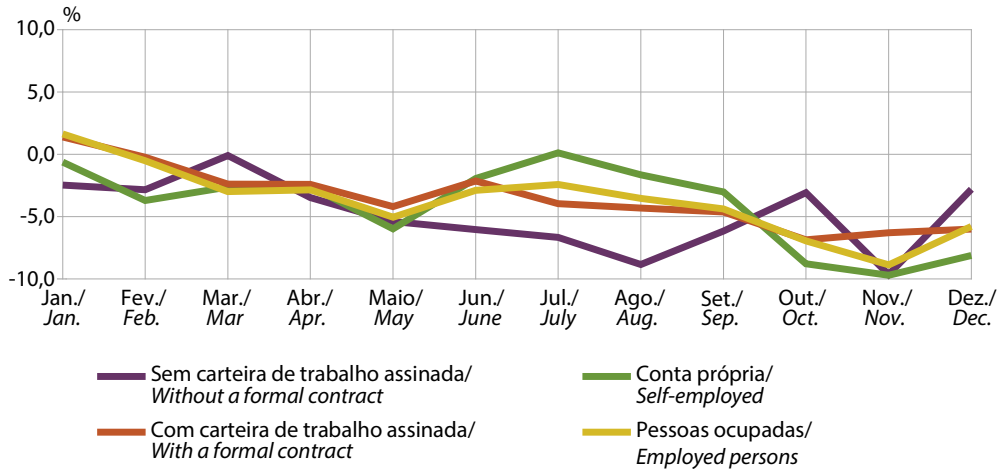

Fonte/Source: IBGE, Diretoria de Pesquisas, Coordenação de Trabalho e Rendimento, Pesquisa Mensal de Emprego 2014-2015.

Notas: 1. Rendimento inflacionado pela média ponderada do Índice Nacional de Preços ao Consumidor INPC das seis regiões metropolitanas.

2. A preços de novembro de 2015.

3. Média das Regiões Metropolitanas de Recife, Salvador, Belo Horizonte, Rio de Janeiro, São Paulo e Porto Alegre./

Notes: 1. Income inflated by weighted average of National Index of Consumer Pices of the six metropolitan areas.

2. Prices of November 2015.

3. Average of Metropolitan Areas of Recife, Salvador, Belo Horizonte, Rio de Janeiro, São Paulo and Porto Alegre. 



\section{Participação Política Political Participation}

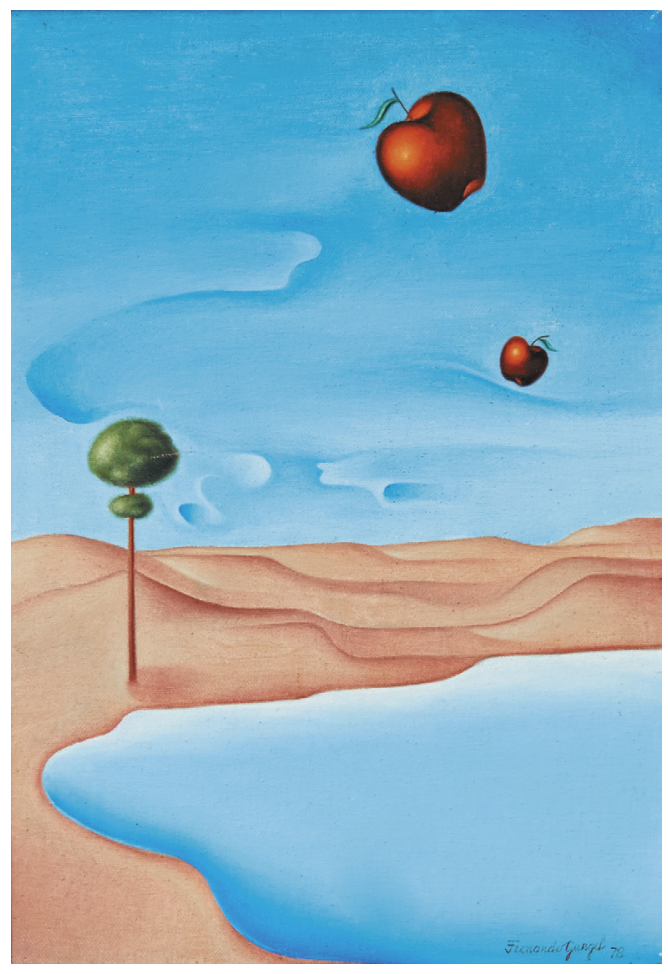

Natureza I/Nature I, 1978

Fernando Henrique de Oliveira Gurgel 



\section{Participação Política Political Participation}

A democracia é um regime político onde governantes e governados encontramse em uma relação de dependência recíproca. De um lado, os governantes dependem do voto popular a fim de legitimar sua ação governamental. De outro lado, os governados são afetados pelas decisões tomadas no âmbito do governo. $O$ que nutre essa relação de dependência mútua é a crença nos valores democráticos e o respeito às suas regras de funcionamento. Nesse sentido, é possível dizer que a participação política é condição para que a democracia funcione adequadamente.

A participação política, para uma importante parte da teoria democrática, restringe-se ou deve restringir-se à participação eleitoral. Para os teóricos da democracia elitista, as massas seriam potencialmente perigosas, antidemocráticas e susceptíveis a tiranos. Joseph Schumpeter e Giovanni Sartori seriam alguns dos expoentes desse argumento. Os estudos que analisam o impacto de uma participação política ampliada como virtude democrática ainda são minoritários no conjunto das teorias da democracia, embora sejam
Democracy is a political regime in which governors and governed ones establish a relationship of mutual dependence. On the one hand, governors depend on the people's vote to legitimate their governmental actions. On the other hand, the governed ones are affected by the decisions made in the governmental scope. What nourishes this interdependent relationship is the belief in the democratic values and the respect dedicated to their operational rules. Therefore, it is possible to say that political participation is a condition for democracy to succeed.

Political participation, for an important part of the democratic theorists, is restricted or must be restricted to electoral participation. For theorists of elite democracy, the masses would be potentially dangerous, anti-democratic and susceptible to tyrants. Joseph Schumpeter and Giovanni Sartori are some of the experts supporting this view. Studies that analyze the impact of an expanded political participation as a democratic virtue, although very relevant, are still a minority among all the democracy theories. In favor of this view 
muito relevantes, como os de Carole Pateman, Benjamin Barber e David Held.

De qualquer forma, o quantitativo de eleitores pode nos dar uma ideia da influência potencial que cada região brasileira tem sobre o resultado da eleição presidencial. No Brasil, o maior contingente eleitoral concentra-se na Região Sudeste, a mais desenvolvida e industrializada, reunindo os maiores centros urbanos. É também a região mais heterogênea do ponto de vista sociodemográfico, mas certamente é a que concentra maior parte da riqueza do país. Nesta região encontra-se $43,3 \%$ do eleitorado nacional. A segunda região com maior concentração de eleitores é a Nordeste, congregando quase $27 \%$ do total, uma das regiões com pior desempenho econômico e amplas desigualdades, situação que vem se alterando nos últimos anos. As outras três regiões somadas não chegam a $30 \%$ do eleitorado: $14,8 \%$ na Sul, $7,7 \%$ na Norte e $7,2 \%$.

Curiosamente, o Brasil "rico" (Sudeste, Sul e Centro-Oeste) votou, em sua maioria no candidato do PSDB, enquanto o Brasil "pobre" (Norte e Nordeste) votou na candidata à reeleição pelo PT. Obviamente as expressões Brasil "rico" e Brasil "pobre" não dão conta da diversidade socioeconômica de cada região; esta seria uma definição mais impressionista do que real. Entretanto, chama a atenção da disparidade da vantagem eleitoral de cada candidato nas duas principais regiões: enquanto a vantagem numérica em votos do candidato do PSDB no Sudeste foi mais estreita, $12,4 \%$, a vantagem eleitoral da candidata do PT no Nordeste foi significativamente alta, pois ela recebeu $43,4 \%$ de votos a mais que seu adversário are Carole Pateman, Benjamin Barber and David Held.

One way or another, the amount of voters can give us an idea of the potential influence that each Brazilian region has on the outcome of the presidential election. In Brazil, the largest electoral college is concentrated in the Southeast, the most developed and industrialized Region, bringing together the largest urban centers. It is also the most heterogeneous region in terms of socio-demographic aspects, but it is certainly the one that concentrates most of the country's wealth. In this Region there are $43.3 \%$ of the national electorate. The second Region with the highest concentration of voters is the Northeast, accounting for nearly $27 \%$ of the total. It records the worst economic performance and great inequality, a situation that has been changing in recent years. The other three Regions combined do not reach $30 \%$ of the electorate: $14.8 \%$ in the South, $7.7 \%$ in the North and $7.2 \%$ in the Central-West.

Surprisingly enough, "rich" Brazil (Southeast, South and Central-West) voted mostly for the PSDB candidate, while "poor" Brazil (North and Northeast) voted for the reelection of the PT candidate. Obviously, the expressions "rich" and "poor" Brazil do not account for the socioeconomic diversity of each Region; this would be a more impressionistic than a real definition. However, the disparity of the electoral advantage of each candidate in the two main regions draws one's attention: while the numerical advantage of the PSDB candidate's votes in the Southeast was small, $12.4 \%$, the electoral advantage of the PT candidate in the Northeast was significantly high because it received 
no segundo turno. Esses números nos dão alguns indícios do perfil eleitoral de cada um dos candidatos, mas são insuficientes para gerar uma conclusão mais robusta.

\section{A distribuição de votos nas eleições} para deputado federal, que se traduz na formação das bancadas partidárias, revela como a política eleitoral reflete e molda alguns componentes básicos do sistema político, produzindo consequências significativas para o governo representativo. Isso é verdadeiro, especialmente quando se trata de um sistema partidário hiperfragmentado como é o caso brasileiro. Em 2014, 34 partidos receberam votos dos eleitores brasileiros. O primeiro impacto que se pode perceber é na governabilidade. A fim de aprovar suas medidas, o governo precisa de apoio majoritário no Congresso e, para tanto, a bancada governista deve ser tanto ampla quanto confiável. Em 2014, nada menos do que 28 partidos elegeram representantes para a Câmara dos Deputados; apenas 12 deles obtiveram um número igual ou maior de 15 cadeiras, em um universo de 513 vagas. Os partidos que obtiveram mais cadeiras foram o PT (68), o PMDB (65) e o PSDB (54), coerente com o resultado da eleição presidencial que foi resolvida no segundo turno entre o PT (Presidente) coligado ao PMDB (Vice-Presidente) e o PSDB (derrotado).

Os partidos vitoriosos obtiveram juntos apenas 133 cadeiras parlamentares, quando precisavam de pelo menos 257 para atingir a maioria simples, o que significa que a chapa vencedora precisou compor aliança com outros partidos, como tradicionalmente ocorre no Brasil,
$43.4 \%$ more votes than her opponent in the second round. These figures give us some indications of the electoral profile of each of the candidates, but are not enough for drawing a firm conclusion.

The distribution of votes in the elections for Federal Congressmen, which results in the formation of party benches, reveals how the electoral politics reflects and shapes some basic components of the political system, producing significant consequences for the representative government. That is true, especially when it refers to an extremely fragmented party system, as is the case in Brazil. In 2014, 34 parties received votes from Brazilian voters. The first impact that can be seen is in governance. In order to approve its measures, the government needs majority support in Congress and, therefore, the government allies should be both reliable and in a large number. In 2014, no fewer than 28 parties elected representatives to the Congress; only 12 of them have obtained a number of seats equal or greater than 15 , out of a total of 513 seats. The parties that had more seats were PT (68), PMDB (65) and PSDB (54). The distribution was consistent with the outcome of the presidential election that was taken to the second round, in which the PT (President) and the PMDB (Vice-President) run against the PSDB (defeated).

The winning parties together accounted for only 133 congress seats, while they needed at least 257 to reach a simple majority, which means that the winning coalition had to make an alliance with other parties, as traditionally occurs in Brazil, 
caracterizando o chamado Presidencialismo de Coalizão (Abranches, 1988).

A questão numérica não é apenas um dos problemas da formação da base governista. Um segundo aspecto, ainda mais problemático, é o da coerência ideológica das coalizões a serem formadas. Segundo a teoria das coalizões (Sartori, 1994), uma coligação ideologicamente coerente deveria ser formada pela aliança entre partidos de um mesmo ponto da escala ideológica (Esquerda, centro-esquerda, centro, centro-direita, direita) ou entre partidos vizinhos nessa mesma escala. Ou seja, um partido de centro-esquerda pode se unir a partidos de esquerda, centro-esquerda e de centro, sem ferir a coerência ideológica da coalizão. Para formar uma aliança com um partido de centro-direita, deveria ter um partido de centro na coalizão, que funcionaria como um mediador entre as distintas perspectivas acerca da condução governamental. Mesmo assim, essa última, seria uma coalizão mais frágil ideologicamente, ocasionando problemas tanto de governabilidade quanto de responsividade (accountability) com relação ao eleitor. Uma coalizão governamental frágil ideologicamente é menos confiável, pois dependerá de maiores incentivos para votar, muitas vezes, de forma contrária às suas convicções.

O resultado das eleições para governadores de Estado naquele ano, revelou, uma vez mais, a preferência dos eleitores por candidatos vinculados aos partidos que encabeçaram as chapas presidenciais que disputaram o segundo turno: PT, PMDB e PSDB (chapa pura). Isso significa que os presidenciáveis de cada partido fizeram campanha para os candidatos a governador de seus próprios partidos. featuring the so-called Coalitional Presidentialism (Abranches, 1988).

The numerical issue is not the only problem faced in the formation of the governing coalition. A second aspect, even more complex, is the ideological consistency of the coalitions to be formed. According to the theory of coalitions (Sartori, 1994), an ideologically consistent coalition should be formed by the alliance between parties with the perspective in the ideological scale (left, centerleft, center, center-right, right) or between neighboring parties in that same scale. Thus, a center-left party can join the leftist, the center-left and center parties without compromising the ideological coherence of the coalition. An alliance with a centerright party should have a center party in the coalition, which would act as a mediator between the different views on how to govern. Yet, the latter would be a more fragile coalition in terms of ideology, causing problems both in terms of governance and responsiveness (accountability) to the voter. An ideologically fragile government coalition is less reliable as it will depend on greater incentives to vote, oftentimes contrary to its convictions.

The outcome of the elections for State governors that year showed, once again, the voters' preference for candidates linked to parties of the presidential candidates that ran in the second round: PT, PMDB and PSDB. This means that the presidential candidates of each party supported the State government candidates of their own parties. It is worth highlighting 
Importa ressaltar o PMDB já possuía bases eleitorais sólidas em alguns Estados, o que explica que sozinho tenha conquistado $26 \%$ dos governos estaduais, mais de um quarto do total. Entretanto, o apoio da candidata do PT à presidência, de quem o PMDB tornou-se vice-presidente, nos Estados em que este partido concorria, mesmo que apoiasse simultaneamente $o$ candidato de seu próprio partido, pode ter sido determinante na escolha dos eleitores, especialmente em disputas de segundo turno. PT e PSDB conquistam o governo de 5 Estados: cada um venceu em 18,5\% do total.

Um dado comprobatório deste argumento é o fato de que o quarto partido que mais recebeu governos estaduais nessa eleição foi o PSB (11\%), partido que chegou em terceiro lugar na disputa do primeiro turno das eleições presidenciais. Sua bancada parlamentar também foi sustentada pelo desempenho eleitoral de sua candidatura presidencial, tornando-se a quinta maior bancada da Câmara dos Deputados.

Chama atenção, finalmente, o baixo índice de votos brancos e nulos na eleição presidencial de 2014. Na maioria dos Estados brasileiros, os votos inválidos não chegaram a $10 \%$ do eleitorado; apenas no Rio de Janeiro (13,3\%) e no Rio Grande do Norte (10,34\%) os votos brancos e nulos ultrapassaram a marca dos $10 \%$. Isso é um importante indicativo da mobilização do eleitorado para o voto naquela eleição. Especialmente os votos brancos tiveram a maior queda já registrada.

Estudiosos demonstraram que determinados aspectos do contexto político podem tornar as pessoas mais propensas ao voto. De acordo com esses that the PMDB already had solid electoral ground in some states, which explains why the party alone has $26 \%$ of the state governments, more than a quarter of the total. However, the support of the PT's presidential candidate - to whom the PMDB is vice president - in the States where the latter was running (even when simultaneously given to PT's candidates) may have been decisive in the choice of the voters, especially in the second round. PT and PSDB won the elections in five states: each one got $18.5 \%$ of the total.

An evidence that support this view is the fact that the fourth party to get State governments in this election was the PSB (11\%) - a party that came in the third position in the first round of the presidential elections. Its parliamentary bench was also leveraged by the electoral performance of its presidential candidate, making it the fifth largest party in the National Congress.

Noteworthy, finally, is the low rate of blank and null votes in the presidential election of 2014. In most Brazilian states, the invalid votes did not reach $10 \%$ of the electorate; only in Rio de Janeiro (13.3\%) and Rio Grande do Norte $(10.34 \%)$ did blank and null votes exceed the mark of $10 \%$. This is an important indication of voter mobilization in the 2014 election. Blank votes, in particular, had the biggest drop ever.

Studies have shown that certain aspects of the political context can make people more likely to vote. According to these studies, factors such as compulsory 
estudos, fatores como o voto obrigatório, o sistema eleitoral proporcional e eleições altamente competitivas aumentam o percentual de participação eleitoral.

A eleição presidencial de 2014 foi a mais competitiva e polarizada desde a redemocratização. Prova disso foi o resultado do segundo turno que deu à candidatura vitoriosa (PT) uma vantagem de apenas $1,64 \%$ de vantagem sobre seu adversário (PSDB). A polarização eleitoral intensificou-se ao longo da campanha e levou ao acirramento das discussões políticas, especialmente durante $o$ período que antecedeu a votação em segundo turno. Há relatos de que a disputa política se radicalizou de tal forma que foi responsável pela ruptura de laços familiares e de amizade em função da discordância ideológica. Essa seria, portanto, a primeira razão identificada para o baixo índice de votos inválidos acima identificado.

Outra razão possível para a elevada participação eleitoral no segundo turno da eleição presidencial de 2014 poderia estar associada ao fato de que o voto no Brasil é obrigatório. Quanto a este ponto, gostaria de fazer uma ressalva: o que é obrigatório no Brasil é o comparecimento eleitoral e, mesmo assim, a abstenção pode ser abonada com o pagamento uma multa de valor irrisório. O eleitor tem à sua disposição a opção do voto em branco, viabilizado na urna eletrônica em uma tecla de fácil acesso. $O$ voto nulo, índice sempre maior do que o voto em branco em todos os Estados, resulta, na maioria das vezes de um erro do eleitor na escolha da opção eleitoral, embora alguns eleitores deliberadamente optem pela anulação do seu voto, temendo que o voto branco favoreça a uma das voting, the proportional electoral system and highly competitive elections increase the percentage of voter turnout.

The presidential election of 2014 was the most competitive and polarized one since the re-democratization. Proof of this was the result of the second round that gave the winning candidate (PT) an advantage of only $1.64 \%$ over her opponent (PSDB). The electoral polarization grew intense throughout the campaign and led to heated political discussion, especially during the run-up to the vote in the second round. There are reports that the political debate was so fierce that caused misunderstanding between family and friends because of ideological disagreement. It would be, therefore, the first identified reason for the low rate of invalid votes as mentioned above.

Another possible reason for the high turnout in the second round of the presidential election in 2014 could be linked to the fact that voting in Brazil is mandatory. Concerning this point, there is a clarification to be made: what is mandatory in Brazil is the voter turnout and, even so, abstention is allowed since the voter pays an insignificant fine. Blank vote is also an option - made possible in the electronic ballot box by pressing an easy access button. The null vote, always more frequent than the blank vote in all States, mostly results from voter errors when choosing the electoral option - although some voters make deliberate mistakes in order to cancel their vote, fearing that blank votes could favor one of the 
candidaturas, o que não procede.

Rejeito, portanto, a influência do voto obrigatório na alta participação eleitoral em 2014. candidates, which is not true. Therefore, I definitely disagree that the compulsory voting exerted influence on the high turnout in the 2014 elections.

Marcia Ribeiro Dias

Professora adjunta na Escola de Ciência Política da Unirio e pesquisadora do CNPq, doutora em Ciência Política pelo luperj (2000), tendo realizado pós-doutorado na Universidade de Oxford/UK (2010-2011)
Assistant Professor in the School of Political Sciences of Unirio and CNPq Researcher, PhD in Political Sciences from luperj (2000), with a Postdoctoral degree from the Oxford University/UK

(2010-2011)

Translated by: Gisele Flores Caldas Manhães 


\section{Tabela 8.1 - Média de eleitores por seção, seções e eleitores existentes - 2014}

Table 8.1 - Average voters by polling sections, zones and voters - 2014

\begin{tabular}{|c|c|c|c|}
\hline $\begin{array}{l}\text { Grandes Regiões e } \\
\text { Unidades da Federação/ } \\
\text { Major Regions and } \\
\text { Federation Units }\end{array}$ & $\begin{array}{l}\text { Média de eleitores } \\
\text { por seção/ } \\
\text { Average voters by } \\
\text { polling sections }\end{array}$ & $\begin{array}{l}\text { Seções/ } \\
\text { Polling } \\
\text { sections }\end{array}$ & $\begin{array}{l}\text { Eleitores } \\
\text { existentes/ } \\
\text { Voters }\end{array}$ \\
\hline Brasil/Brazil & 336 & 427724 & 143610341 \\
\hline Norte/North & 327 & 33826 & 11060902 \\
\hline Rondônia & 315 & 3629 & 1144095 \\
\hline Acre & 316 & 1636 & 516232 \\
\hline Amazonas & 352 & 6528 & 2297666 \\
\hline Roraima & 290 & 1059 & 306840 \\
\hline Pará & 333 & 15999 & 5319882 \\
\hline Amapá & 320 & 1461 & 467666 \\
\hline Tocantins & 287 & 3514 & 1008521 \\
\hline Nordeste/Northeast & 321 & 120735 & 38711921 \\
\hline Maranhão & 295 & 15463 & 4559374 \\
\hline Piauí & 299 & 7937 & 2374656 \\
\hline Ceará & 317 & 19921 & 6310782 \\
\hline Rio Grande do Norte & 340 & 6961 & 2366006 \\
\hline Paraíba & 301 & 9523 & 2867527 \\
\hline Pernambuco & 344 & 18791 & 6471266 \\
\hline Alagoas & 335 & 6111 & 2045052 \\
\hline Sergipe & 311 & 4760 & 1478246 \\
\hline Bahia & 327 & 31268 & 10239012 \\
\hline Sudeste/Southeast & 355 & 175134 & 62175326 \\
\hline Minas Gerais & 334 & 45762 & 15302264 \\
\hline Espírito Santo & 335 & 7944 & 2663807 \\
\hline Rio de Janeiro & 371 & 32675 & 12136861 \\
\hline São Paulo & 361 & 88753 & 32072394 \\
\hline Sul/South & 322 & 66120 & 21294239 \\
\hline Paraná & 317 & 25090 & 7965506 \\
\hline Santa Catarina & 338 & 14593 & 4932811 \\
\hline Rio Grande do Sul & 318 & 26437 & 8395922 \\
\hline Centro-Oeste/Central-West & 325 & 31909 & 10367953 \\
\hline Mato Grosso do Sul & 327 & 5564 & 1820447 \\
\hline Mato Grosso & 327 & 6748 & 2204123 \\
\hline Goiás & 335 & 13145 & 4406870 \\
\hline Distrito Federal/Federal District & 300 & 6452 & 1936513 \\
\hline Exterior/Abroad & 374 & 954 & 357186 \\
\hline
\end{tabular}

Fonte/Source: Estatísticas eleitorais 2014. Estatísticas de eleitorado. Comparecimento e votação. In: Eleições. Brasília, DF: Tribunal Superior Eleitoral - TSE, 2014. Disponível em/Available from: <http:// www.tse.jus.br/hotSites/estatistica_2014/resultados/comparecimento.html>. Acesso em: abr. 2016/ Cited: Apr. 2016.

Nota: Dados de outubro de 2014./Note: Data of October 2014. 
Tabela 8.2 - Distribuição percentual dos resultados da apuração para presidente - 20 turno - 2014

Table 8.2 - Percentage distribution of vote cast for president 2nd round - 2014

\begin{tabular}{|c|c|c|c|}
\hline $\begin{array}{c}\text { Unidades da Federação } \\
\text { e exterior/Federation Units } \\
\text { and abroad }\end{array}$ & $\begin{array}{l}\text { Votos válidos/ } \\
\text { Valid votes }\end{array}$ & $\begin{array}{l}\text { Votos brancos/ } \\
\text { Blank votes }\end{array}$ & $\begin{array}{l}\text { Votos nulos/ } \\
\text { Void votes }\end{array}$ \\
\hline Rondônia & 94,63 & 1,17 & 4,20 \\
\hline Acre & 97,03 & 0,88 & 2,09 \\
\hline Amazonas & 92,26 & 1,58 & 6,17 \\
\hline Roraima & 94,64 & 0,91 & 4,46 \\
\hline Pará & 94,49 & 1,10 & 4,41 \\
\hline Amapá & 95,12 & 0,82 & 4,07 \\
\hline Tocantins & 96,07 & 0,91 & 3,02 \\
\hline Maranhão & 96,26 & 1,05 & 2,68 \\
\hline Piauí & 95,82 & 0,86 & 3,32 \\
\hline Ceará & 93,56 & 1,41 & 5,03 \\
\hline Rio Grande do Norte & 89,66 & 1,82 & 8,52 \\
\hline Paraíba & 92,44 & 1,88 & 5,68 \\
\hline Pernambuco & 93,73 & 1,95 & 4,32 \\
\hline Alagoas & 94,49 & 1,56 & 3,95 \\
\hline Sergipe & 94,28 & 1,56 & 4,16 \\
\hline Bahia & 94,21 & 1,43 & 4,36 \\
\hline Minas Gerais & 94,97 & 1,46 & 3,56 \\
\hline Espírito Santo & 95,19 & 1,75 & 3,05 \\
\hline Rio de Janeiro & 86,72 & 2,78 & 10,51 \\
\hline São Paulo & 93,56 & 1,97 & 4,47 \\
\hline Paraná & 96,28 & 1,27 & 2,45 \\
\hline Santa Catarina & 95,84 & 1,28 & 2,88 \\
\hline Rio Grande do Sul & 94,02 & 2,14 & 3,85 \\
\hline Mato Grosso do Sul & 96,84 & 1,12 & 2,04 \\
\hline Mato Grosso & 96,96 & 0,90 & 2,14 \\
\hline Goiás & 93,71 & 1,65 & 4,64 \\
\hline Distrito Federal/Federal District & 92,02 & 1,76 & 6,23 \\
\hline Exterior/Abroad & 95,28 & 2,23 & 2,49 \\
\hline
\end{tabular}

Fonte/Source: Repositório de dados eleitorais. In: Eleições 2014. Tribunal Superior Eleitoral - TSE. Disponível em/Available from: <http://agencia.tse.jus.br/estatistica/sead/odsele/detalhe_votacao_munzona/ detalhe_votacao_munzona_2014.zip>.Acesso em: abr. 2016/Cited:Apr. 2016. 
Tabela 8.3 - Candidatos eleitos, por partido político - 2014

Table 8.3 - Candidates elected by political parties - 2014

\begin{tabular}{|c|c|c|}
\hline $\begin{array}{c}\text { Partido político/ } \\
\text { Political party }\end{array}$ & $\begin{array}{l}\text { Governador/ } \\
\text { Governor }\end{array}$ & $\begin{array}{c}\text { Deputado Federal/ } \\
\text { Congressman }\end{array}$ \\
\hline DEM & - & 21 \\
\hline PC do B & 1 & 10 \\
\hline PDT & 2 & 19 \\
\hline PEN & - & 2 \\
\hline PHS & - & 5 \\
\hline PMDB & 7 & 66 \\
\hline PMN & - & 3 \\
\hline PP & 1 & 38 \\
\hline PPS & - & 10 \\
\hline$P R$ & - & 34 \\
\hline PRB & - & 21 \\
\hline PROS & 1 & 11 \\
\hline PRP & - & 3 \\
\hline PSB & 3 & 34 \\
\hline PSC & - & 13 \\
\hline PSD & 2 & 36 \\
\hline PSDB & 5 & 54 \\
\hline PSDC & - & 2 \\
\hline PSL & - & 1 \\
\hline PSOL & - & 5 \\
\hline PT & 5 & 68 \\
\hline PT do B & - & 2 \\
\hline РTB & - & 25 \\
\hline PTC & - & 2 \\
\hline PTN & - & 4 \\
\hline PV & - & 8 \\
\hline SD & - & 15 \\
\hline PRTB & - & 1 \\
\hline
\end{tabular}

Fonte/Source: Repositório de dados eleitorais. In: Eleições 2014. Brasília, DF: Tribunal Superior Eleitoral TSE, 2014. Disponível em/Available from: <http://agencia.tse.jus.br/estatistica/sead/odsele/detalhe_ munzona_2014.zip >. Acesso em: abr. 


\section{Gráfico 8.1 - Deputados Federais eleitos, por partido político - 2014}

Graph 8.1 - Congressmen elected, by political parties - 2014

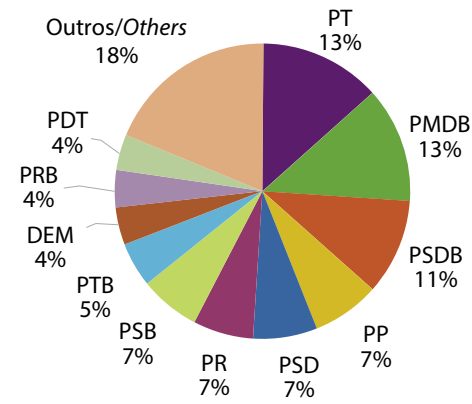

Fonte/Source: Repositório de dados eleitorais. In: Eleições. Brasília, DF: Tribunal Superior Eleitoral - TSE, 2014. Disponível em/Available from: http://agencia.tse.jus.br/estatistica/sead/odsele/consulta_cand/ consulta_cand_2014.zip>. Acesso em: abr. 2016/Cited: Apr. 2016.

Gráfico 8.2 - Governadores eleitos, por partido político - 2014 Graph 8.2 - Governors elected, by political parties - 2014

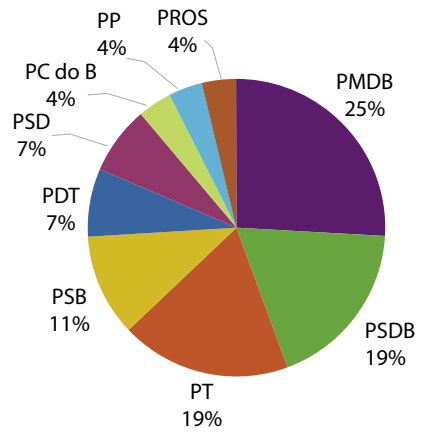

Fonte/Source: Repositório de dados eleitorais. In: Eleições 2014. Tribunal Superior Eleitoral - TSE. Disponível em/Available from: <http://agencia.tse.jus.br/estatistica/sead/odsele/consulta_cand/consulta_cand_ 2014.zip>. Acesso em: abr. 2016/Cited:Apr. 2016. 


\section{Quadro 8.1 - Partidos políticos com votação - 2014}

Figure 8.1 - Political parties with votes - 2014

\begin{tabular}{|c|c|}
\hline Sigla & Partido \\
\hline DEM & Partido Democrata \\
\hline PC do B & Partido Comunista do Brasil \\
\hline PCB & Partido Comunista Brasileiro \\
\hline PCO & Partido da Causa Operaria \\
\hline PDT & Partido Democrático Trabalhista \\
\hline PEN & Partido Ecológico Nacional \\
\hline PHS & Partido Humanista da Solidariedade \\
\hline PMB & Partido da Mulher Brasileira \\
\hline PMDB & Partido do Movimento Democrático Brasileiro \\
\hline PMN & Partido da Mobilização Nacional \\
\hline NOVO & Partido Novo \\
\hline PP & Partido Progressista \\
\hline PPL & Partido Patria Livre \\
\hline PPS & Partido Popular Socialista \\
\hline PR & Partido da República \\
\hline PRB & Partido Republicano Brasileiro \\
\hline PROS & Partido Republicano da Ordem Social \\
\hline PRP & Partido Republicano Progressista \\
\hline PRTB & Partido Renovador Trabalhista Brasileiro \\
\hline PSB & Partido Socialista Brasileiro \\
\hline PSC & Partido Social Cristão \\
\hline PSD & Partido Social Democratico \\
\hline PSDB & Partido da Social Democracia Brasileira \\
\hline PSDC & Partido Social Democrata Cristão \\
\hline PSL & Partido Social Liberal \\
\hline PSOL & Partido do Socialismo e Liberdade \\
\hline PSTU & Partido Socialista dos Trabalhadores Unificado \\
\hline PT & Partido dos Trabalhadores \\
\hline PT do B & Partido Trabalhista do Brasil \\
\hline PTB & Partido Trabalhista Brasileiro \\
\hline PTC & Partido Trabalhista Cristão \\
\hline PTN & Partido Trabalhista Nacional \\
\hline PV & Partido Verde \\
\hline SD & Solidariedade \\
\hline
\end{tabular}

Fonte/Source: Estatísticas eleitorais 2014. In: Eleições. Brasília, DF: Tribunal Superior Eleitoral - TSE, 2014. Disponível em/Available from: <http://www.tse.jus.br/eleicoes/estatisticas/estatisticas-eleitorais-2014eleitorado>. Acesso em/Cited: fev. 2015. Acesso em: abr. 2016/Cited: Apr. 2016.

Nota: Dados de outubro de 2014./Note: Data of October 2014. 


\section{Preços Prices}

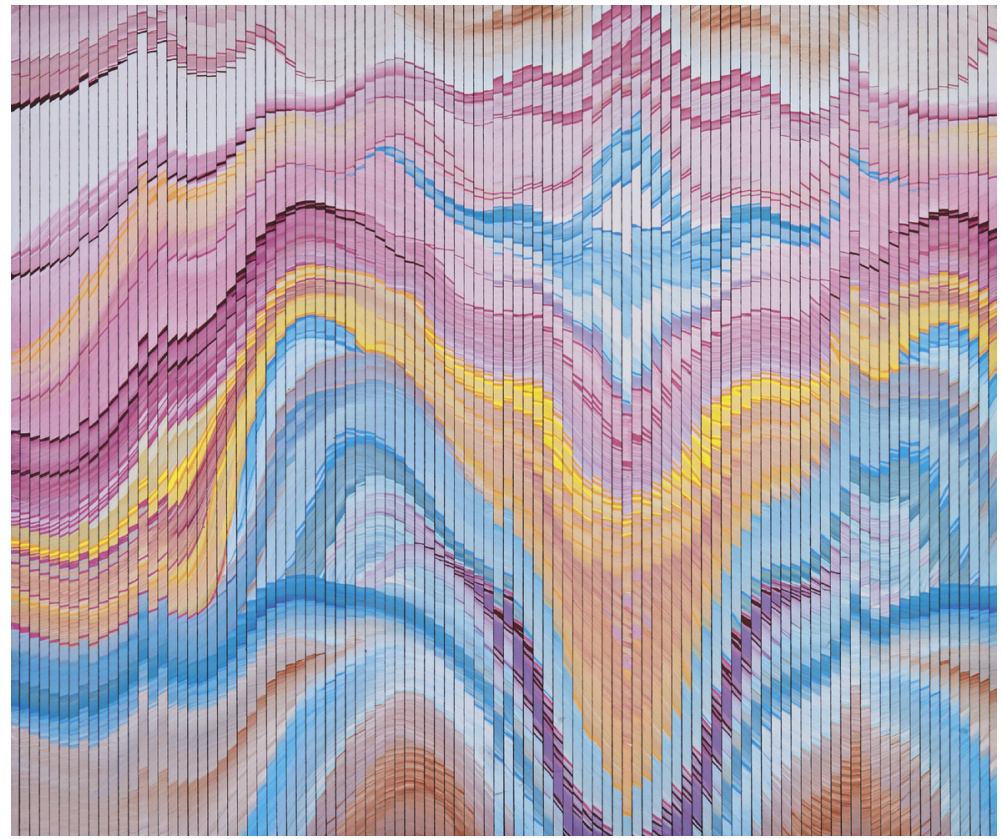

Progressão de ripas de madeira, sem título/Wooden slat, untitled, 2004 Abraham Palatnik 



\section{Preços}

\section{Prices}

Em 2015, a taxa de inflação medida pela variação do Índice Nacional de Preços ao Consumidor Amplo (IPCA) atingiu $10,67 \%$, ultrapassando, pela primeira vez desde 2003, o limite superior do intervalo de tolerância em torno da meta central de inflação estabelecido pelo Conselho Monetário Nacional (CMN) que, para 2015, estabelecia uma inflação máxima de $6,5 \%$ (2 pontos percentuais acima da meta central de $4,5 \%$ ao ano).

Conforme mostra a Tabela 9.1, os grupos de produtos com aumentos mais significativos de preços no ano foram os grupos de habitação (aumento de $18,31 \%$ ), alimentação e bebidas $(12,03 \%)$ e transportes (10,16\%). Parcela considerável dos aumentos nos grupos de habitação e transportes se deveu à forte elevação dos chamados "preços administrados ou monitorados" - preços estabelecidos por contratos, por órgão público ou agência reguladora - no período, particularmente no primeiro trimestre. Com efeito, após terem permanecido artificialmente represados ao longo de 2013 e 2014, muitos desses preços sofreram fortes correções ao longo de 2015, com destaque para os preços da energia elétrica residencial (aumento
In 2015, the interest rate measured by the change of the Extended National Consumer Price Index (IPCA) reached $10.67 \%$, surpassing, for the first time since 2003, the upper limit of the tolerance interval over the core inflation target established by the National Monetary Council (CMN) - which, for 2015 , set a maximum inflation of $6.5 \%$ ( 2 percentage points above the central target of $4.5 \%$ per year).

As shown in Table 9.1, the groups of products with the most significant price increases in the year were housing (a rise of $18.31 \%$ ), food and beverages (12.03\%) and transportation (10.16\%). A considerable share of the increases in the groups of housing and transportation was due to the strong rise in the so-called "administered and regulated prices" - prices set by contracts, by public bodies or regulatory agencies - in the period, especially in the first quarter. In fact, after having remained artificially stagnated over 2013 and 2014, many of these prices suffered sharp corrections over 2015, with a highlight to the prices of residential electricity (cumulative increase of $51 \%$ in the year), bottled gas 
acumulado de $51 \%$ no ano), gás de botijão (22,55\%), etanol $(28,63 \%)$, gasolina $(20,1 \%)$, gás veicular (15,4\%), óleo diesel $(13,34 \%)$ e ônibus urbano (15,09\%). Os preços do grupo alimentação e bebidas, por sua vez, foram impactados pela elevada desvalorização da moeda nacional no período - desvalorização de $41,6 \%$ em relação ao dólar norte-americano, na média de 2015 em relação a 2014 -, resultante da crise política e econômica que marcou o país em 2015. Esta maxidesvalorização do real contribuiu para aumentar os preços dos produtos alimentícios por meio de três efeitos principais: (i) elevação dos custos de adubos, fertilizantes, defensivos agrícolas e demais insumos importados utilizados nas atividades agropecuárias; (ii) aumento dos preços dos produtos alimentícios importados; e (iii) redução da oferta doméstica de produtos agropecuários, dado o maior incentivo para que o setor exportador deslocasse parte de sua produção ao mercado externo. Dentre os itens do grupo com aumentos de preços mais significativos, pode-se destacar aqueles referentes a tubérculos, raízes e legumes (aumento de $40,91 \%$ no ano), sal e condimentos $(21,2 \%)$, açúcares e derivados $(20,48 \%)$, hortaliças e verduras $(19,65 \%)$ e frutas $(15,23 \%)$.

Não obstante a taxa de inflação de 2015 ser parcialmente explicada por tais eventos atípicos - fortes aumentos corretivos de preços administrados e maxidesvalorização cambial -, é importante notar que a inflação no país já vinha em patamar elevado, e flertando com a possibilidade de ultrapassar o limite superior do intervalo de tolerância em torno da meta de inflação, desde 2010. Conforme mostra a Tabela 9.2, entre 2010 e 2014 a mais baixa taxa de inflação registrada, tomando-se
(22.55\%), ethanol (28.63\%), gasoline (20.1\%), LPG/autogas (15.4\%), diesel fuel (13.34\%) and urban bus (15.09\%). The prices of food and beverages, on their turn, were affected by the sharp depreciation of the national currency in the period $-41.6 \%$ in relation to the US dollar in the average of 2015 over $2014-$, resulting from the political and economic crisis that hit the country in 2015. This maxidepreciation of the real contributed to the price increase of food products by means of three main effects: (i) increase in the costs of fertilizers, pesticides and other imported inputs used in agricultural activities; (ii) increase in the prices of imported food products; and (iii) reduction in the domestic supply of agricultural products due to the greater incentive for the export sector to allocate part of its production to the foreign market. Among the items of the group with the most significant price rises, the highlights are those related to tubers, roots and vegetables (increase of $40.91 \%$ ), salt and spices $(21.2 \%)$, sugars and derivatives $(20.48 \%)$, green vegetables and leaves (19.65\%) and fruit (15.23\%).

Despite the fact the 2015 inflation rate is partially explained by such non-typical events - sharp corrective increases in administered prices and currency maxi-devaluation -, it is important to notice that the country's inflation, since 2010, has been high and on the edge of trespassing the upper limit of the tolerance interval around the inflation target. As shown in Table 9.2, between 2010 and 2014, the lowest inflation rate, taking the IPCA as a basis, was $5.84 \%$ - more 
por base o IPCA, foi de 5,84\%, mais de um ponto percentual acima da meta central de inflação fixada para estes anos (4,5\%); e, em pelo menos duas ocasiões, chegou-se muito próximo de superar a taxa de inflação máxima tolerada $(6,5 \%)$. Além disso, desde 2012 a inflação vinha em aceleração, aproximando-se gradativamente do limite superior do intervalo de tolerância em torno da meta e indicando, de um lado, a persistência de desequilíbrios importantes na economia brasileira - em particular, a elevação dos salários reais acima dos índices de produtividade e a incerteza relativa à sustentabilidade das contas públicas -; e, de outro lado, a necessidade de medidas corretivas de política econômica destinadas a reverter a tendência altista dos preços. O ciclo de alta da taxa de juros básica da economia implementado pelo Banco Central do Brasil (BCB) entre outubro de 2014 e julho de 2015, que levou a meta da Taxa Selic - taxa média dos financiamentos diários, com lastro em títulos federais, apurados no Sistema Especial de Liquidação e Custódia - de 11\%a.a. a 14,25\%a.a., teve por objetivo justamente estabilizar as expectativas de mercado e retomar o controle inflacionário. Em um contexto de profunda incerteza política e econômica e persistência do desequilíbrio fiscal, agravado pelos choques de preços administrados e cambial, o aperto da política monetária não foi capaz de evitar a alta da inflação em 2015. Contudo, ao final do ano as expectativas de mercado já apontavam para a desaceleração do ritmo de elevação dos preços e para a gradual recondução da taxa de inflação para níveis mais próximos da meta de $4,5 \%$ a.a. ao longo de 2016 e 2017; segundo o Boletim Focus, relatório de mercado divulgado semanalmente pelo $B C B$ com previsões than one percentage points above the core inflation target set for those years ( $4.5 \%)$; and, at least in two occasions, figures were very close to surpassing the maximum tolerated inflation rate $(6.5 \%)$. Besides, since 2012, the inflation has been on a rise, getting gradually closer to the upper limit of the tolerance interval around the target and indicating, on the one hand, a persistence of significant imbalances in the Brazilian economy - in particular, the increase of the real salaries above the productivity indexes and the uncertainties in relation to the sustainability of the public accounts; and, on the other hand, the need for corrective measures of economic policy to offset the upward trend in prices. The upward trend of the basic interest rate of the economy implemented by the Central Bank of Brazil (BCB) between October 2014 and July 2015, which increased the target of the Selic rate average rate of daily financing, backed by government bonds, calculated the Special System for Settlement and Custody - from $11 \%$ p.a. to $14.25 \%$ p.a., aimed precisely at stabilizing market expectations and regaining control over inflation. In a context of deep political and economic uncertainty and persistent fiscal imbalances, aggravated by the gap between administered prices and exchange rates, the tightening of the monetary policy was unable to prevent high inflation in 2015. However, at the end of the year, market expectations already pointed to the slowdown in the pace of price increases and to the gradual renewal of the inflation rate to levels closer to the target of $4.5 \%$ p.a. along 2016 and 2017; according to the Boletim Focus (Focus Bulletin) - a market report released weekly by the $B C B$ with 
macroeconômicas de diversos analistas financeiros, as previsões medianas de inflação formadas em 31 de dezembro de 2015 para os anos de 2016 e 2017 eram de $6,87 \%$ e $5,2 \%$, respectivamente.

A Tabela 9.2 também revela que, em 2015, a taxa de inflação calculada com base na variação do Índice Nacional de Preços ao Consumidor (INPC) - que mede o custo de vida para famílias com renda entre 1 e 5 salários mínimos, com chefes assalariados e residentes nas regiões urbanas das 11 áreas metropolitanas consideradas pelo IBGE - foi 0,6 ponto percentual superior à inflação medida pelo IPCA - cuja população-objetivo é formada por famílias com renda entre 1 e 40 salários mínimos (também residentes nas regiões urbanas das áreas metropolitanas).

Pode-se afirmar, portanto, que neste ano o custo de vida aumentou relativamente mais para as famílias de menor renda invertendo o resultado observado nos dois anos anteriores. Isto decorreu, em grande medida, dos fortes aumentos de preços registrados em 2015 nos grupos de habitação e alimentação e bebidas, que compõem uma maior fração do orçamento das famílias de menor renda.

O Gráfico 9.2 mostra a evolução mensal, ao longo dos anos de 2014 e 2015, do Índice Nacional da Construção Civil calculado pelo IBGE no âmbito do Sistema Nacional de Pesquisa de Custos e Índices da Construção Civil (SINAPI). Além da variação acumulada do índice em 2015 ter sido apenas um pouco inferior à registrada em 2014 (5,5\% contra $6,2 \%)$, observa-se padrão de evolução dos custos dentro de cada ano bastante semelhante, caracterizado por um pico em maio, taxas de variação relativamente macroeconomic forecasts by many financial analysts -, the average inflation forecasted on December 31, 2015 for the years 2016 and 2017 was of $6.87 \%$ and $5.2 \%$ respectively.

Table 9.2 also shows that in 2015 , the inflation rate based on the change of the National Consumer Price Index (INPC) - which measures the cost of living for families with income between 1 and 5 minimum wages, with a salaried household head and encompasses rural residents of the 11 metropolitan areas considered by the IBGE - was 0.6 percentage points higher than inflation measured by the IPCA, whose target population consists of families with income between 1 and 40 minimum wages (also residents in urban metropolitan areas). It can be said, therefore, that in 2015 the cost of living increased relatively more for lower-income families - reversing the results observed in the previous two years. That was due, to a large extent, to the sharp price increases recorded in 2015 in housing and food and beverages, which constitute a larger amount of the budget of low-income families.

Graph 9.2 shows the monthly evolution, over the years 2014 and 2015, of the National Index of Civil Construction calculated by the IBGE in the scope of the National System of Costs Survey and Indexes of Construction (SINAPI). Besides the fact that the cumulative change of the index in 2015 was just slightly below that of 2014 (5.5\% against $6.2 \%)$, the cost evolution pattern within each year is quite similar, characterized by a peak in May, relatively high change rates until August and relatively low ones between 
altas até agosto e relativamente baixas entre setembro e novembro. Este padrão decorre, em grande medida, das pressões sobre os custos de mão de obra no setor decorrentes de acordos salariais coletivos, que se concentram principalmente no meio do ano. É interessante notar que este padrão se manteve razoavelmente constante ao longo destes dois anos, mesmo sob circunstâncias bastante distintas no que diz respeito à atividade do setor. De acordo com as Contas Nacionais Trimestrais calculadas pelo IBGE, o valor adicionado do setor de construção ficou relativamente estagnado em 2014, tendo caído 0,9\% na média do ano, ao passo que apresentou forte retração em 2015, com queda de 7,6\%.

A Tabela 9.3 apresenta para o ano de 2015, por grandes regiões e unidades da Federação, o custo médio na construção civil em $\mathrm{R} \$ \mathrm{~m}^{2}$ e em número-índice (com base em dezembro de $1998=100$ ), além da variação deste custo acumulada no ano. Observa-se que o maior custo em termos absolutos ocorreu na região Sudeste, com valor de $\mathrm{R} \$ 1.001,61 / \mathrm{m}^{2}$, mas que nesta região a variação do custo observada em 2015 foi uma das menores do país $(4,95 \%$, contra uma variação de $5,50 \%$ para o país como um todo), assim como a variação acumulada desde 1998 (379,35\%, contra $382,28 \%$ para o país). A região Nordeste apresentou o mais baixo custo médio de construção do país em 2015, com valor de $\mathrm{R} \$ 889,98 /$ $\mathrm{m}^{2}$. As maiores variações acumuladas de custos entre 1998 e 2015 ocorreram nas regiões Centro-Oeste (398,11\%) e Norte $(395,89 \%)$. As maiores variações de custos ao longo de 2015 foram registradas nas regiões Norte $(7,92 \%$, com destaque para os estados do Amapá e Pará, com aumentos de 10,69\% e
September and November. This pattern stems largely from the pressures on workforce costs in the sector resulting from collective wage agreements, which are mainly concentrated in the middle of the year. It is interesting to notice that this pattern remained fairly constant throughout these two years, even under very different circumstances related to the sector activity. According to the Quarterly National Accounts calculated by the IBGE, the value added of the construction sector was relatively stagnant in 2014, having fallen $0.9 \%$ in the annual average, while showing a strong decline in 2015 , with a $7.6 \%$ drop.

Table 9.3 shows the average cost in civil construction for 2015 by Major Regions and Federation Units - $\mathrm{R} \$ / \mathrm{m}^{2}$ and index-number (based on

December $1998=100$ ), as well as the cumulative change of the cost in the year. The highest cost in absolute terms occurred in the Southeast, amounting to $R \$ 1,001.61 / \mathrm{m}^{2}$, but in that same region the cost change seen in 2015 was one of the lowest in the country $(4.95 \%$, against a change of $5.50 \%$ for the country as a whole). The cumulative change since 1998 also fell (379.35\%, against $382.28 \%$ for the country). The Northeast had the smallest average cost of construction in the country in 2015, with $\mathrm{R} \$ 889.98 / \mathrm{m}^{2}$. The largest cumulative changes in costs between 1998 and 2015 occurred in the CentralWest (398.11\%) and North Regions (395.89\%). The greatest changes of costs in 2015 were recorded in the North $(7.92 \%$, especially in the states of Amapá and Pará, with increases of 
$8,85 \%$, respectivamente) e Sul $(7,81 \%$, com destaque para o aumento de $9,21 \%$ em Santa Catarina). O Gráfico 9.5 permite visualizar com mais clareza as diferenças regionais nas variações dos custos médios de construção em 2015.

A Tabela 9.4 mostra a série histórica da variação acumulada no ano do Índice Nacional da Construção Civil entre 2006 e 2015. A série se caracteriza por relativamente baixa variância, apresentando valores entre $5,13 \%$ e $7,36 \%$ em 8 dos 10 anos analisados; as exceções são os anos de 2008 (11,73\%) e 2013 (0,52\%). A variação atipicamente baixa dos custos em 2013 deveu-se aos efeitos da desoneração da folha de pagamento de empresas do setor da construção civil prevista na lei 12.844 e sancionada em 19 de julho de 2013.

O Gráfico 9.3 apresenta a decomposição do custo médio da construção civil no país $\left(\mathrm{R} \$ 963,39 / \mathrm{m}^{2}\right)$ nas parcelas referentes ao custo de materiais $\left(\mathrm{R} \$ 516,06 / \mathrm{m}^{2}\right) \mathrm{e}$ ao custo de mão de obra (R\$447,33/ $\left.\mathrm{m}^{2}\right)$. As variações mensais destas parcelas do custo da construção civil ao longo de 2015 são ilustradas no Gráfico 9.4. Observa-se que o custo da mão de obra aumentou de forma mais intensa do que o custo dos materiais de construção na maioria dos meses do ano, especialmente entre maio e agosto - meses em que ocorreram reajustes salariais decorrentes de acordos coletivos em diversos estados. No acumulado do ano, o custo de mão de obra registrou alta de $7,55 \%$, enquanto que o custo de material de construção aumentou apenas $3,78 \%$.

Vale ressaltar que, apesar de superior à meta oficial de inflação, a variação de 5,5\% do Índice Nacional da Construção
$10.69 \%$ and $8.85 \%$, respectively) and South $(7.81 \%$, with a highlight to the increase of $9.21 \%$ in Santa Catarina). Graph 9.5 allows a more clear view of the regional differences in average cost changes in construction in 2015.

Table 9.4 shows the time series of the cumulative change in the year of the National Index of Civil Construction between 2006 and 2015. The series is characterized by relatively low variance, with values between $5.13 \%$ and $7.36 \%$ in 8 of the 10 years analyzed; exceptions are the years $2008(11.73 \%)$ and 2013 $(0.52 \%)$. The unusually low change in costs in 2013 was due to the effects of the exemption of the payroll of the construction sector companies established by law 12,844 and enacted on July 19, 2013.

Graph 9.3 shows the breakdown of the average cost of construction in Brazil $\left(R \$ 963.39 / \mathrm{m}^{2}\right)$ in shares for the cost of materials $\left(R \$ 516.06 / \mathrm{m}^{2}\right)$ and of workforce $\left(\mathrm{R} \$ 447.33 / \mathrm{m}^{2}\right)$. Monthly of the shares of the construction cost over 2015 are presented in Graph 9.4. It is observed that the cost of workforce increased more than the cost of construction materials in most of the months of the year, especially between May and August - months in which there were salary increases resulting from collective agreements in several states. In the cumulative rates in the year, the cost of workforce recorded a rise of $7.55 \%$, while the cost of construction materials increased by only $3.78 \%$.

It is worthy mentioning that, although higher than the official inflation target, the $5.5 \%$ change of the National Index 
Civil em 2015 foi substancialmente mais baixa do que a inflação geral medida pelo IPCA, indicando que o custo da construção foi relativamente pouco afetado pelos fatores que fizeram a inflação geral ultrapassar o limite superior do intervalo de tolerância em torno da meta.

\section{Marco A.F.H. Cavalcanti}

Doutor em Economia pela Pontifícia Universidade Católica do Rio de Janeiro (PUC-

Rio), Pesquisador do Instituto de Pesquisa Econômica Aplicada (IPEA) e Professor do Departamento de Economia da PUC-Rio na área de Métodos Econométricos. Editor da revista Pesquisa e Planejamento Econômico entre 2008 e 2015. Diretor Adjunto de Estudos e Políticas Macroeconômicas do IPEA de 2012 a 2016. Principais áreas de interesse: Modelos Macroeconométricos, Política Monetária, Política Fiscal. of Civil Construction in 2015 was substantially lower than the general inflation measured by the IPCA, indicating that the cost of construction was relatively little affected by the factors that made the general inflation exceed the upper limit of the tolerance interval of the target.

PhD in Economics from the Pontifical Catholic University of Rio de Janeiro (PUC-Rio), Researcher at the Institute for Applied Economic Research (IPEA) and Professor of Economics at PUC-Rio in the field of Econometric Methods. Editor of the journal Research and Economic Planning between 2008 and 2015. Associate Director of Macroeconomic Studies and Policies of IPEA 2012-2016. Main areas of interest: Macroeconometric Models, Monetary Policy, Fiscal Policy.

Translated by: Gisele Flores Caldas Manhães 
Tabela 9.1 - Índice Nacional de Preços ao Consumidor

Amplo - IPCA - 2015

Table 9.1 - Extended National Consumer Price Index - IPCA - 2015

(continua/to be continued)

Variação mensal, por grupos de produtos (\%)/

Monthly change by groups of products (\%)

\begin{tabular}{|c|c|c|c|c|c|}
\hline $\begin{array}{c}\text { Mês/ } \\
\text { Month }\end{array}$ & $\begin{array}{l}\text { IPCA/ } \\
\text { IPCA }\end{array}$ & $\begin{array}{l}\text { Alimentação } \\
\text { e bebidas/ } \\
\text { Food and } \\
\text { beverages }\end{array}$ & $\begin{array}{l}\text { Habitação/ } \\
\text { Housing }\end{array}$ & $\begin{array}{l}\text { Artigos de } \\
\text { residência/ } \\
\text { Household } \\
\text { articles }\end{array}$ & $\begin{array}{l}\text { Vestuário/ } \\
\text { Wearing } \\
\text { apparel }\end{array}$ \\
\hline Janeiro/January & 1,24 & 1,48 & 2,42 & (-) 0,28 & (-) 0,69 \\
\hline Fevereiro/February & 1,22 & 0,81 & 1,22 & 0,87 & (-) 0,60 \\
\hline Março/March & 1,32 & 1,17 & 5,29 & 0,35 & 0,59 \\
\hline Abril/April & 0,71 & 0,97 & 0,93 & 0,66 & 0,91 \\
\hline Maio/May & 0,74 & 1,37 & 1,22 & 0,36 & 0,61 \\
\hline Junho/June & 0,79 & 0,63 & 0,86 & 0,72 & 0,58 \\
\hline Julho/July & 0,62 & 0,65 & 1,52 & 0,86 & (-) 0,31 \\
\hline Agosto/August & 0,22 & (-) 0,01 & 0,29 & 0,37 & 0,20 \\
\hline Setembro/September & 0,54 & 0,24 & 1,30 & 0,19 & 0,50 \\
\hline Outubro/October & 0,82 & 0,77 & 0,75 & 0,39 & 0,67 \\
\hline Novembro/November & 1,01 & 1,83 & 0,76 & 0,31 & 0,79 \\
\hline Dezembro/December & 0,96 & 1,50 & 0,49 & 0,46 & 1,15 \\
\hline $\begin{array}{l}\text { Acumulado no ano/ } \\
\text { Cumulative in the year }\end{array}$ & 10,67 & 12,03 & 18,31 & 5,36 & 4,46 \\
\hline
\end{tabular}


Tabela 9.1 - Índice Nacional de Preços ao Consumidor

Amplo - IPCA - 2015

Table 9.1 - Extended National Consumer Price Index - IPCA - 2015

(conclusão/concluded)

\begin{tabular}{|c|c|c|c|c|c|}
\hline \multirow[b]{2}{*}{$\begin{array}{l}\text { Mês/ } \\
\text { Month }\end{array}$} & \multicolumn{5}{|c|}{$\begin{array}{c}\text { Variação mensal, por grupos de produtos (\%)/ } \\
\text { Monthly change by groups of products (\%) }\end{array}$} \\
\hline & $\begin{array}{l}\text { Transportes/ } \\
\text { Transportation }\end{array}$ & $\begin{array}{c}\text { Saúde e Cui- } \\
\text { dados pessoais/ } \\
\text { Health and } \\
\text { personal care }\end{array}$ & $\begin{array}{l}\text { Despesas } \\
\text { pessoais/ } \\
\text { Personal } \\
\text { expenses }\end{array}$ & $\begin{array}{l}\text { Educação/ } \\
\text { Education }\end{array}$ & $\begin{array}{l}\text { Comunicação/ } \\
\text { Communication }\end{array}$ \\
\hline Janeiro/January & 1,83 & 0,32 & 1,68 & 0,31 & 0,15 \\
\hline Fevereiro/February & 2,20 & 0,60 & 0,86 & 5,88 & (-) 0,02 \\
\hline Março/March & 0,46 & 0,69 & 0,36 & 0,75 & (-) 1,16 \\
\hline Abril/April & 0,11 & 1,32 & 0,51 & 0,21 & 0,31 \\
\hline Maio/May & (-) 0,29 & 1,10 & 0,74 & 0,06 & 0,17 \\
\hline Junho/June & 0,70 & 0,91 & 1,63 & 0,20 & 0,34 \\
\hline Julho/July & 0,15 & 0,84 & 0,61 & 0,00 & 0,30 \\
\hline Agosto/August & (-) 0,27 & 0,62 & 0,75 & 0,82 & 0,14 \\
\hline Setembro/September & 0,71 & 0,55 & 0,33 & 0,25 & 0,01 \\
\hline Outubro/October & 1,72 & 0,55 & 0,57 & 0,10 & 0,39 \\
\hline Novembro/November & 1,08 & 0,64 & 0,52 & 0,22 & 1,03 \\
\hline Dezembro/December & 1,36 & 0,70 & 0,57 & 0,22 & 0,43 \\
\hline Acumulado no ano/ & 10,16 & 9,23 & 9,50 & 9,25 & 2,11 \\
\hline Cumulative in the year & & & & & \\
\hline
\end{tabular}

Fonte/Source: Índice nacional de preços ao consumidor amplo - IPCA 2015. In: IBGE. Sidra: sistema IBGE de recuperação automática. Rio de Janeiro, [2015]. Disponível em/Available from: <http:// www.sidra.ibge.gov.br>. Acesso em: abr. 2016/Cited: Apr. 2016. 
Tabela 9.2 - Variação acumulada no ano do Índice Nacional de Preços ao Consumidor - INPC e do Índice Nacional de Preços ao

Consumidor Amplo - IPCA - 2002-2015

Table 9.2 - Cumulative change in the year of the Extended National Consumer Price Index - IPCA and of the National Consumer Price Index - INPC - 2002-2015

\begin{tabular}{|c|c|c|c|c|c|}
\hline \multirow[t]{2}{*}{$\begin{array}{l}\text { Ano/ } \\
\text { Year }\end{array}$} & \multicolumn{2}{|c|}{$\begin{array}{l}\text { Variação acumulada } \\
\text { no ano/ } \\
\text { Cumulative change in the year }\end{array}$} & \multirow[t]{2}{*}{$\begin{array}{l}\text { Ano/ } \\
\text { Year }\end{array}$} & \multicolumn{2}{|c|}{$\begin{array}{c}\text { Variação acumulada } \\
\text { no ano/ } \\
\text { Cumulative change in the year }\end{array}$} \\
\hline & IPCA & INPC & & IPCA & INPC \\
\hline 2002 & 12,53 & 14,74 & 2009 & 4,31 & 4,11 \\
\hline 2003 & 9,30 & 10,38 & 2010 & 5,91 & 6,46 \\
\hline 2004 & 7,60 & 6,13 & 2011 & 6,50 & 6,08 \\
\hline 2005 & 5,69 & 5,05 & 2012 & 5,84 & 6,20 \\
\hline 2006 & 3,14 & 2,81 & 2013 & 5,91 & 5,56 \\
\hline 2007 & 4,46 & 5,16 & 2014 & 6,41 & 6,23 \\
\hline 2008 & 5,90 & 6,48 & 2015 & 10,67 & 11,28 \\
\hline
\end{tabular}

Fonte/Source: Índice nacional de preços ao consumidor amplo - IPCA 2002-2015. In: IBGE. Sidra: sistema IBGE de recuperação automática. Rio de Janeiro, [2015]. Disponível em/Available from: <http://www.sidra.ibge.gov.br>. Acesso em: abr. 2016/Cited: Apr. 2016.

\section{Gráfico 9.1 - Variação mensal do Índice Nacional de Preços ao Consumidor Amplo - IPCA - 2015}

Graph 9.1 - Monthly change of the Extended National Consumer Price

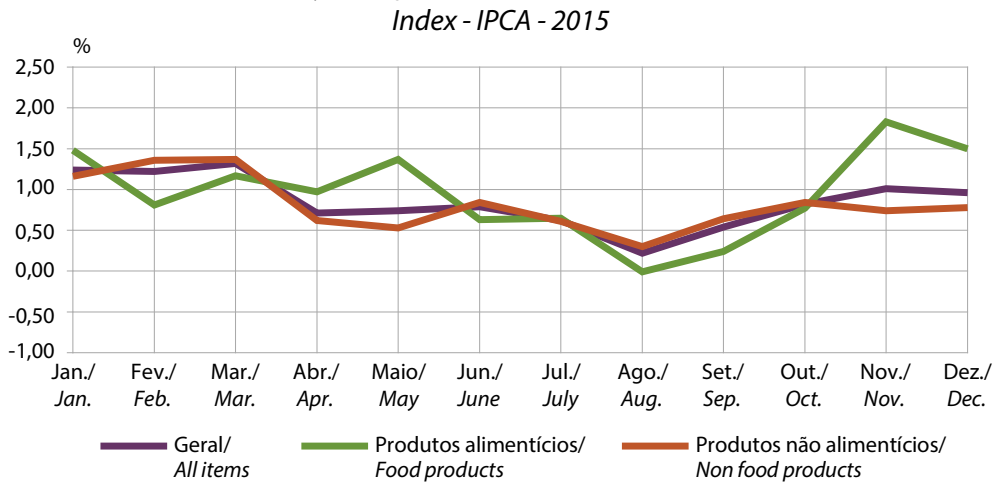

Fonte/Source: Índice nacional de preços ao consumidor amplo - IPCA 2015. In: IBGE. Sidra: sistema IBGE de recuperação automática. Rio de Janeiro, [2015]. Disponível em/Available from: <http://www.sidra.ibge. gov.br>. Acesso em: abr. 2016/Cited: Apr. 2016. 
Tabela 9.3 - Custo médio, número-índice e variação acumulada no ano, na construção civil, segundo as Grandes Regiões

e Unidades da Federação - 2015

Table 9.3 - Average cost, index-number and cumulative change in the year in civil construction, by Major Regions and Federation Units - 2015

\begin{tabular}{|c|c|c|c|}
\hline $\begin{array}{l}\text { Grandes Regiões e } \\
\text { Unidades da Federação/ } \\
\text { Major Regions and } \\
\text { Federation Units }\end{array}$ & $\begin{array}{l}\text { Custo médio } \\
\left(\mathrm{R} \$ / \mathrm{m}^{2}\right) / \\
\text { Average } \\
\text { cost }\left(R \$ / \mathrm{m}^{2}\right)\end{array}$ & $\begin{array}{l}\text { Número-índice } \\
(\text { dez./98 = 100 }) / \\
\text { Index number } \\
(\text { Dec. } / 98=100)\end{array}$ & $\begin{array}{c}\text { Variação acumulada } \\
\text { no ano (\%)/ } \\
\text { Cumulative change } \\
\text { in the year (\%) }\end{array}$ \\
\hline Brasil/Brazil & 963,39 & 482,28 & 5,50 \\
\hline Norte /North & 995,18 & 495,89 & 7,92 \\
\hline Rondônia & 1032,30 & 575,46 & 5,96 \\
\hline Acre & 1068,72 & 567,33 & 4,94 \\
\hline Amazonas & 995,46 & 487,29 & 8,69 \\
\hline Roraima & 1025,80 & 426,11 & 2,75 \\
\hline Pará & 973,02 & 466,27 & 8,85 \\
\hline Amapá & 988,34 & 480,02 & 10,69 \\
\hline Tocantins & 1009,13 & 530,46 & 5,82 \\
\hline Nordeste/Northeast & 889,98 & 480,74 & 4,51 \\
\hline Maranhão & 912,49 & 480,69 & 4,92 \\
\hline Piauí & 905,00 & 601,36 & 2,92 \\
\hline Ceará & 895,69 & 517,25 & 6,00 \\
\hline Rio Grande do Norte & 868,88 & 437,92 & 6,11 \\
\hline Paraíba & 934,24 & 516,53 & 3,84 \\
\hline Pernambuco & 858,40 & 458,96 & 0,41 \\
\hline Alagoas & 891,27 & 445,36 & 6,70 \\
\hline Sergipe & 864,05 & 459,13 & 7,40 \\
\hline Bahia & 887,50 & 469,57 & 5,49 \\
\hline Sudeste/Southeast & 1001,61 & 479,35 & 4,95 \\
\hline Minas Gerais & 891,55 & 490,66 & 2,40 \\
\hline Espírito Santo & 881,99 & 489,22 & 5,43 \\
\hline Rio de Janeiro & 1081,69 & 492,90 & 3,54 \\
\hline São Paulo & 1045,34 & 472,07 & 6,87 \\
\hline Sul/South & 999,77 & 478,19 & 7,81 \\
\hline Paraná & 996,29 & 476,48 & 6,75 \\
\hline Santa Catarina & 1055,41 & 571,74 & 9,21 \\
\hline Rio Grande do Sul & 952,01 & 432,17 & 8,29 \\
\hline Centro-Oeste/Central-West & 975,71 & 498,11 & 5,25 \\
\hline Mato Grosso do Sul & 957,68 & 450,32 & 5,76 \\
\hline Mato Grosso & 980,45 & 559,42 & 5,01 \\
\hline Goiás & 958,47 & 506,23 & 5,79 \\
\hline Distrito Federal/Federal District & 1005,84 & 444,29 & 4,51 \\
\hline
\end{tabular}

Fonte/Source: IBGE, Diretoria de Pesquisas, Coordenação de Índices de Preços. 
Tabela 9.4 - Variação acumulada no ano do Índice Nacional da Construção Civil - 2006-2015

Table 9.4- Cumulative change in the year of the National Index of Civil Construction - 2006-2015

\begin{tabular}{|c|c|c|c|c|}
\hline & $\begin{array}{l}\text { Ano/ } \\
\text { Year }\end{array}$ & $\begin{array}{l}\text { Variação acumulada } \\
\text { no ano (\%)/ } \\
\text { Cumulative change } \\
\text { in the year (\%) }\end{array}$ & $\begin{array}{l}\text { Ano/ } \\
\text { Year }\end{array}$ & $\begin{array}{c}\text { Variação acumulada } \\
\text { no ano (\%)/ } \\
\text { Cumulative change } \\
\text { in the year (\%) }\end{array}$ \\
\hline 2006 & & 5,13 & 2011 & 5,65 \\
\hline 2007 & & 6,08 & 2012 & 5,68 \\
\hline 2008 & & 11,73 & 2013 & 0,52 \\
\hline 2009 & & 5,85 & 2014 & 6,20 \\
\hline 2010 & & 7,36 & 2015 & 5,50 \\
\hline
\end{tabular}

Fonte/Source: IBGE, Diretoria de Pesquisas, Coordenação de Índices de Preços.

\section{Gráfico 9.2 - Variação mensal do Índice Nacional da Construção Civil - 2014-2015}

Graph 9.2 - Monthly change of the National Index of Civil Construction 2014-2015

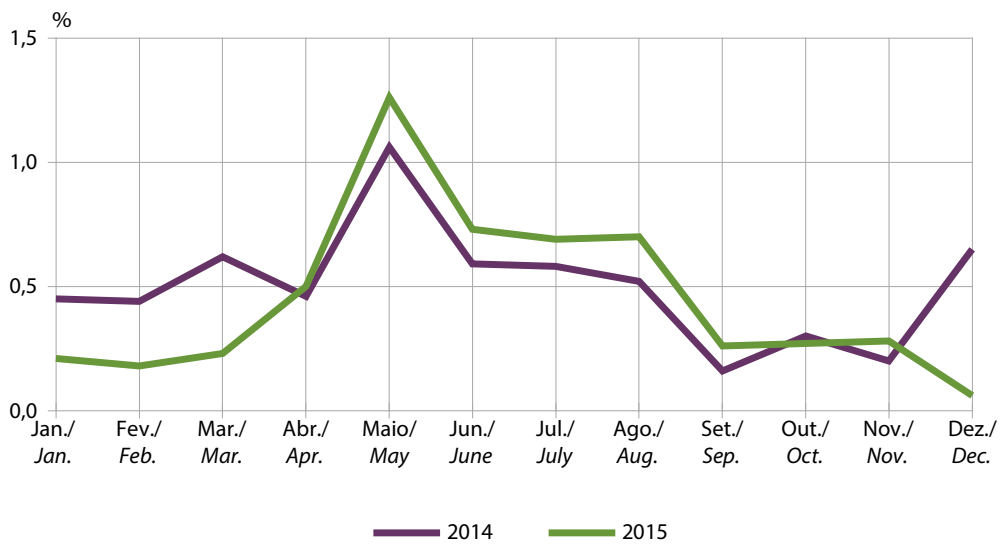

Fonte/Source: IBGE, Diretoria de Pesquisas, Coordenação de Índices de Preços. 


\section{Gráfico 9.3 - Custo total por metro quadrado, parcela de materiais e mão-de-obra - dez. 2015}

Graph 9.3 - Total cost per square meter, portion of material and labor force - Dec. 2015

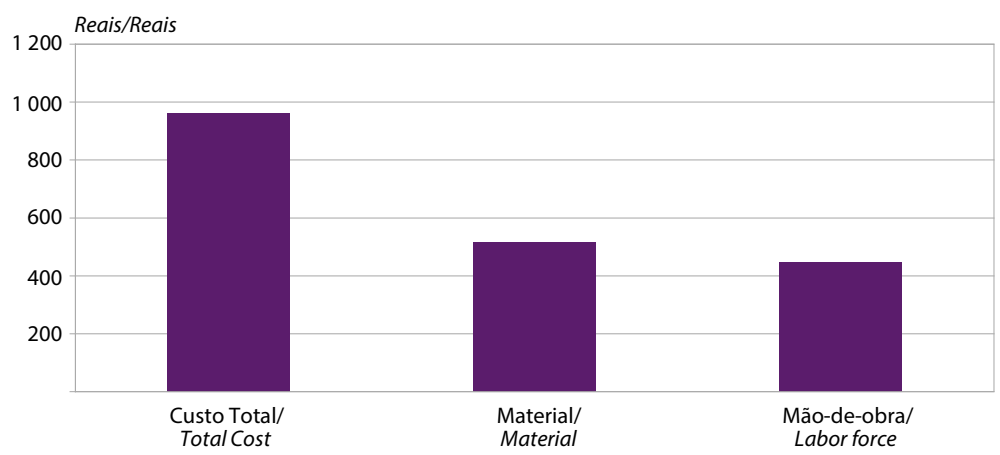

Fonte/Source: IBGE, Diretoria de Pesquisas, Coordenação de Índices de Preços.

Gráfico 9.4 - Variação mensal das parcelas de materiais e de mão-de-obra na composição do custo Nacional da Construção Civil - 2015

Graph 9.4-Monthly change of the Portion of material and labor force in the composition of the National Cost of the Civil Construction - 2015

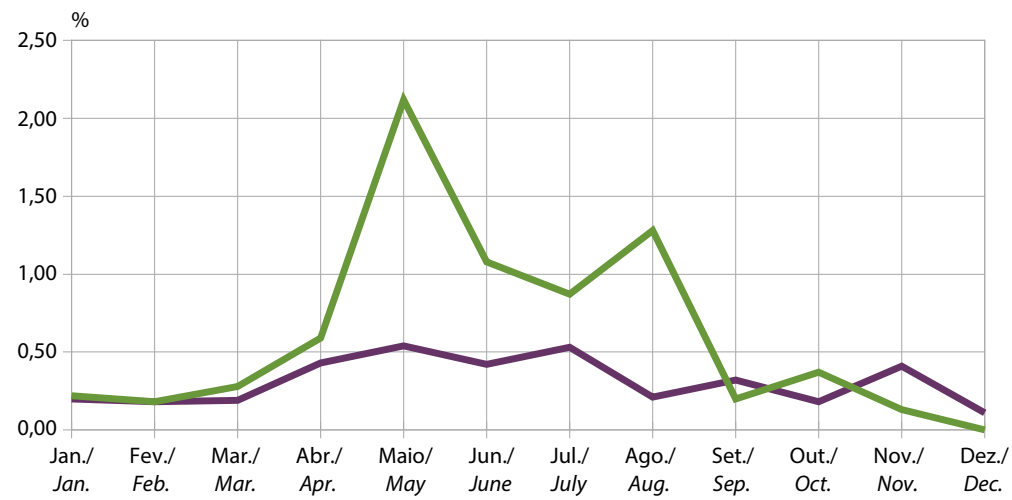

Material/Material Mão-de-obra/Labor force

Fonte/Source: IBGE, Diretoria de Pesquisas, Coordenação de Índices de Preços. 


\section{Gráfico 9.5 - Variação acumulada do Custo Nacional e Custos Regionais da}

Construção Civil - 2015

Graph 9.5 - Cumulative change of the National and Regional Costs of

Civil Construction - 2015

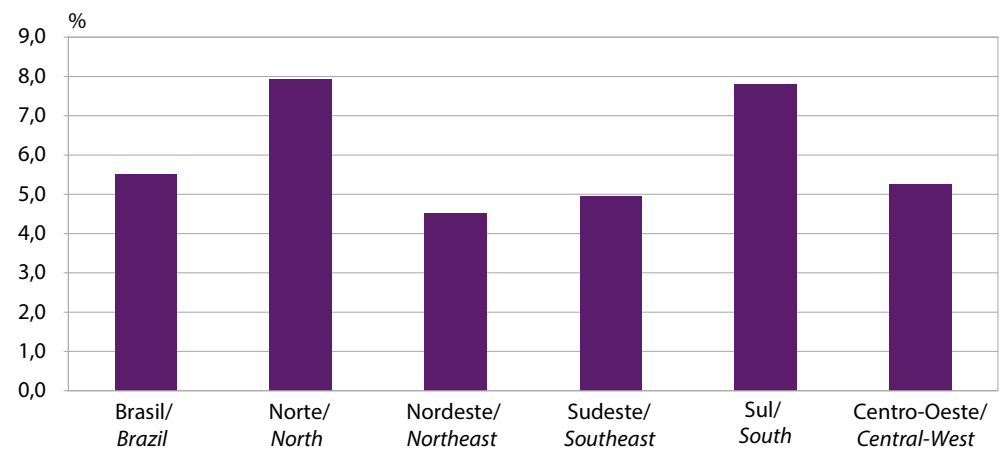

Fonte/Source: IBGE, Diretoria de Pesquisas, Coordenação de Índices de Preços. 


\section{Contas Nacionais}

\section{National Accounts}

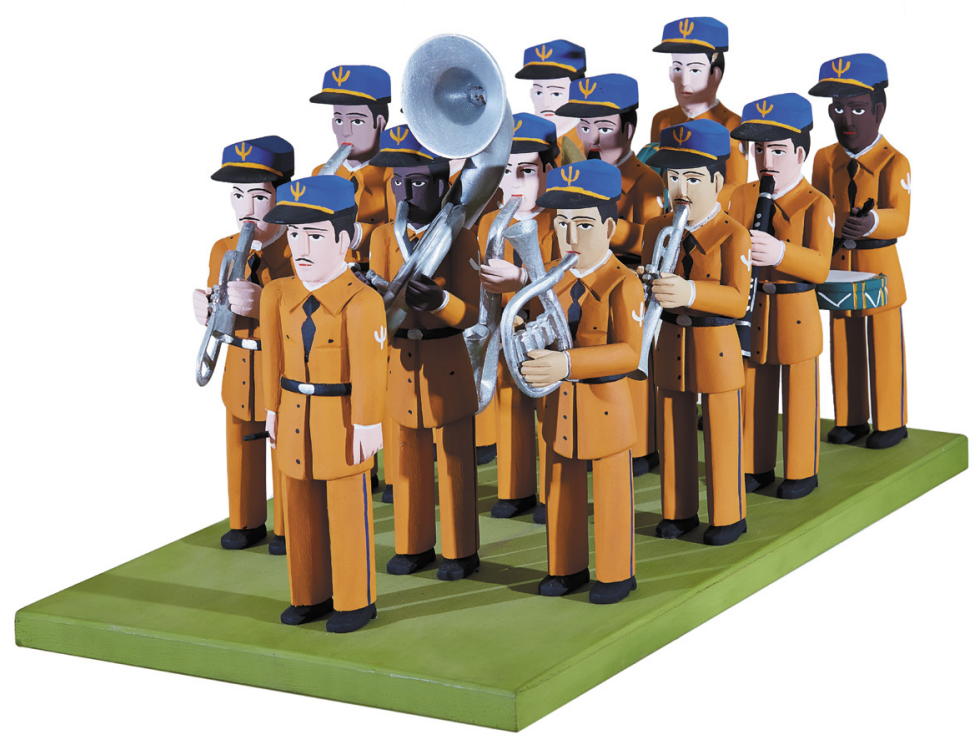

Banda/ Marching band, 2014

Geicifran Francisco de Assis Azevedo 



\section{Contas Nacionais}

Em 2015, a economia brasileira vivenciou um agravamento da conjuntura recessiva iniciada no ano anterior, registrando uma variação anual de $-3,8 \%$ no Produto Interno Bruto (PIB), o que veio a se constituir no pior resultado da produção de bens e serviços na era do Real. Com isso, o PIB a preços de mercado totalizou $\mathrm{R} \$$ 5.904.331 milhões (Tabela 10.1) $\mathrm{A}$ deterioração da atividade produtiva tornou-se evidente ao longo do ano, porquanto a comparação do PIB em cada trimestre com o período correspondente no ano anterior mostrou taxas crescentemente negativas, evoluindo de uma queda de $-2,0 \%$ no primeiro trimestre para uma variação de $-5,9 \%$ no último trimestre e completando, assim, sete períodos ininterruptos com taxas trimestrais negativas (Tabela 10.4). Esta piora nas condições de produção impactou no mercado de trabalho - o nível geral de emprego diminuiu e a taxa de desemprego sofreu rápida elevação -, bem como se fez acompanhar por um aumento na taxa de inflação que fechou o ano acima do limite superior da meta. Neste contexto, o PIB per capita a preços correntes oscilou de R\$28.046 em 2014 para R\$ 28.846 em 2015 (Tabela 10.1), numa variação de apenas $2,8 \%$, bem

\section{National Accounts}

In 2015, the Brazilian economy experienced a deterioration of the recessive conditions observed since the previous year. The Gross Domestic Product (GDP) recorded a negative change of $3.8 \%$ in real terms, which was the worst outcome regarding the production of goods and services throughout the period of Real. As a consequence, GDP reached $R \$$ $5,904,331$ million. The slump in the productive activity became evident during the year, for quarterly rate changes showed increasing negative results from $-2.0 \%$ in the 1 st quarter down to $-5.9 \%$ in the 4 th quarter. Thus, the Brazilian economy completed seven uninterrupted periods of negative quarterly rate changes (Table 10.4). This downward trend in the conditions of production affected the labor market - the general level of employment decreased and the rate of unemployment showed a rapid increase - as well as went along with an increase in the rate of inflation to a level beyond the upper limit of the target. Under these circumstances, the GDP per capita fluctuated from $\mathrm{R} \$ 28,046$ in 2014 to $R \$$ 28,846 in 2015 at current prices (Table 10.1 ), changing by only $2.8 \%$, a rate well 
inferior ao crescimento dos preços em geral.

O recuo na atividade produtiva tem origem em múltiplos fatores, incluindo aqueles presentes há mais tempo como a continuidade da política monetária contracionista. Em 2015, delineou-se também uma política fiscal de mesmo tipo, baseada em contenção nos gastos e reversão em benefícios fiscais, que afetou diretamente a demanda agregada. Abandonou-se, assim, a combinação de políticas observada nos anos imediatamente anteriores, quando a condução da política fiscal procurou reduzir os efeitos contracionistas da política monetária e da instabilidade no cenário externo. Além disso, do lado da oferta, o efeito de uma severa seca sobre as condições de geração de energia mostrou-se mais intenso do que fora previsto, ao passo que, no setor externo, a economia brasileira veio a ser negativamente impactada pela redução nos preços das commodities.

O conjunto de condições desfavoráveis à atividade produtiva pesou sobre $o$ já precário estado de confiança dos setores institucionais, atingindo as decisões de consumo e investimento. A despesa de consumo das famílias cresceu apenas 5,5\% em valores correntes, chegando a $\mathrm{R} \$ 3.741 .855$ milhões em 2015 (Tabela 10.3). Dado que os preços ao consumidor aumentaram em ritmo bastante superior, conclui-se que houve uma redução real no consumo familiar em conformidade com a piora generalizada no mercado de trabalho e na confiança do consumidor. $\mathrm{O}$ aumento de um ponto percentual sobre o PIB resultou meramente de uma menor diminuição below the increase in the general level of prices.

This decline in the production activity stemmed from multiple factors, including those in progress for a longer period such as the continuity of a restrictive monetary policy. In 2015, a fiscal policy of similar nature was adopted through measures for both slowing expenditures down and reversing tax benefits, which exerted a direct impact on the aggregate demand. By so proceeding, the government has abandoned the combination of policies carried on in previous years, according to which fiscal policy was set to minimize the contraction effects of both the monetary policy and the instability of the external sector. Moreover, on the supply side of the economy, the effects of a severe drought over the conditions to generate power proved stronger than they had been speculated, whereas, as for the external sector, the Brazilian economy was negatively affected by a drop in the price of commodities.

All these objective conditions detrimental to the production activity put more pressure over an already precarious degree of confidence of the institutional sectors, having an adverse effect on the decisions of consumption and investment. Thus, household consumption grew by only $5.5 \%$ at current prices, reaching $R \$ 3,741,855$ million in 2015 (Table 10.3). Since the increase of consumer prices was greater, this brought about a real contraction in household consumption, which conforms to the overall worsening of both the labor market and the consumer confidence. An increase of household consumption by one percentage point over the GDP was a result of a lower 
relativa do consumo vis-à-vis o produto, a preços constantes.

As consequências do quadro econômico adverso evidenciaram-se de modo ainda mais agudo no desempenho da formação bruta de capital, que declinou de R\$ 1.186.631 milhões em 2014 para R\$ 1.045.771 milhões em 2015, em valores correntes (Tabela 10.3). Isto levou a taxa de investimento para a casa dos $18,2 \%$ do PIB, fixando-a num patamar entre dois e três pontos percentuais abaixo do que se observara em anos recentes (Tabela 10.5). A par das consequências negativas sob a ótica do dispêndio, a redução no volume e na taxa de investimento também ocasiona uma piora nas condições de crescimento da capacidade produtiva e dos níveis gerais de produtividade da economia brasileira.

A dinâmica recessiva da economia conduziu ao aprofundamento do processo de desindustrialização do país, evidenciado por uma menor participação da indústria na formação do produto. As taxas trimestrais correspondentes ao produto da indústria mostraram-se sistemática e crescentemente negativas, passando de $-4,4 \%$ no primeiro trimestre até atingir $-8,0 \%$ no quarto trimestre (Tabela 10.4). Com isso, a participação do setor industrial no produto recuou 1,3 pontos percentuais e fechou o ano em nível correspondente a $22,7 \%$ do valor adicionado a preços básicos. Já o setor de serviços aumentou sua participação para $72,1 \%$, pois, dentre outros motivos, a redução no seu produto foi menos pronunciada do que a do produto em geral. O setor da agropecuária manteve estável sua participação em torno a 5,2\% do valor adicionado a preços correntes (Tabela 10.2). negative change vis-à-vis the GDP at constant prices.

The consequences of this adverse economic context were sharply felt in an appalling performance of gross capital formation, which shrank from $\mathrm{R} \$ 1,186,631$ million in 2014 to $\mathrm{R} \$$ $1,045,771$ million in 2015 at current prices (Table 10.3). This lessened the investment rate to $18.2 \%$ of the GDP, a level between two and three percentage points below the previous years (Table 10.5). In addition to its negative consequences over aggregate demand, the reduction in investment also aggravated the conditions for both the expansion of productive capacity and the increase in the general productivity levels of the Brazilian economy.

The recessive economic performance has deepened the process of deindustrialization of the country, which may be evidenced in an even smaller proportion of the manufacturing product over the general economy added value. Quarterly rates regarding manufacturing, mining and quarrying showed increasingly negative changes, oscillating from $-4.4 \%$ in the 1 st quarter down to $-8.0 \%$ in the 4 th quarter (Table 10.4). As a consequence, the proportion of manufacturing over the product curtailed by 1.3 percentage points, closing the year in a level equal to $22.7 \%$ of added value at basic prices. As for the services sector, it accounted for $72.1 \%$ of added value in 2015 , rising from a lower figure in the previous year in spite of displaying negative changes in every quarterly rates. Agriculture, forestry and fishing kept unchanged its proportion in $5.2 \%$ of added value at current prices (Table 10.2). 
De modo diverso ao que se verificou no front interno, houve uma melhora no desempenho de bens e serviços no setor externo. Conforme exposto na Tabela 10.3 , enquanto as importações oscilaram $6,8 \%$ em valores correntes, atingindo $\mathrm{R} \$$ 845.779 milhões em 2015, as exportações cresceram do modo mais vigoroso $(+21,0 \%)$ e chegaram $\mathrm{R} \$ 770.084$ milhões, reduzindo o saldo negativo das exportações líquidas para menos de metade do que se observara em 2014. O grau de abertura da economia, medido pelo fluxo total de comércio, passou de $25,1 \%$ para $27,4 \%$ (Tabela 10.5 ). Estes resultados refletem, de um lado, o desaquecimento observado na absorção interna, em que a soma do consumo final e do investimento aumentou tão somente $2,3 \%$ em valores correntes (Tabela 10.1), freando o crescimento mais rápido das importações, e, de outro lado, as consequências do movimento de depreciação real da moeda doméstica que se sustentou durante o ano de 2015.

A melhora no saldo das operações com bens e serviços levou a uma expressiva redução na necessidade líquida de financiamento da economia brasileira, que passou de $\mathrm{R} \$ 263.600$ milhões em 2014 para R\$ 194.708 milhões em 2015, a preços correntes (Tabela 10.1). Conforme se verifica no comportamento dos agregados de renda da Tabela 10.1, a economia nacional manteve seu padrão estrutural em que a Renda Nacional Bruta é menor do que o PIB em face do saldo negativo na renda primária, mormente devido a pagamentos líquidos de juros e lucros. Esta renda líquida enviada ao exterior, no entanto, não se ampliou de modo significativo entre 2014 e 2015, de modo que a dinâmica das exportações líquidas foi a principal responsável pela
Transactions with goods and services have showed a better performance in the external sector vis-à-vis the domestic front. According to Table 10.3, whereas imports increased by $6.8 \%$ at current prices, reaching $\mathrm{R} \$ 845,779$ million in 2015, exports enjoyed a greater rise $(21.0 \%)$ to $\mathrm{R} \$ 770,084$ million, cutting down the negative balance of net exports by more than $50 \%$ in contrast to the 2014 value. The degree of openness of the economy, as measured by to total flow of trade, grew larger from $25.1 \%$ to $27.4 \%$ (Table 10.5 ). These outcomes reflected, on the one hand, the slowdown in the domestic demand, according to which the sum of final consumption and investment increased by no more than $2.3 \%$ at current prices (Table 10.1), curbing a more vigorous rise in imports, and, on the other hand, the consequences of a process of real depreciation of the domestic currency that lasted for the whole year of 2015 .

The improvement in the net balance of goods and services brought about a sizeable reduction in net borrowing, which changed from $R \$ 263,600$ million in 2014 to R\$ 194,708 million in 2015 at current prices (Table 10.1). By looking at the aggregates of income in Table 10.1 , one can observe that the Brazilian economy kept unchanged a structural pattern according to which the Gross National Income is lower than GDP due to a negative balance in primary income, especially concerning net payments of interest and transfers of profits. However, the increase in the net income sent to the rest of the world cannot be considered expressive in 2015 , so that it was mainly the performance of the trade balance that 
contração da necessidade líquida de financiamento em mais de $25 \%$ do valor registrado no ano anterior.

A redução no investimento em R\$ 140.860 milhões fez-se acompanhar não apenas pela menor necessidade líquida de financiamento, como por uma diminuição na poupança bruta, que passou de R\$ 922.476 milhões em 2014 para R\$ 859.535 milhões em 2015 em valores correntes (Tabela 10.1). caused a decrease in net borrowing by more than $25 \%$ in comparison to the previous year.

The reduction in investment by R\$ 140,860 million was followed not only by a lower net borrowing, but also by a decrease in gross saving, which oscillated from $\mathrm{R} \$ 922.476$ million in 2014 to $\mathrm{R} \$ 859.535$ million in 2015 at current prices (Table 10.1).

\section{Carlos Henrique Horn}

Mestre em economia pela Universidade Federal do Rio Grande do Sul - UFRGS Doutor em relações de trabalho pela London School of Economics and Political Science - LSE, da Universidade de Londres Professor Associado da Faculdade de Ciências Econômicas da Universidade Federal do Rio Grande do Sul - UFRGS
M.Sc. Economics, Federal University of Rio Grande do Sul - UFRGS

Ph.D. Industrial Relations, London School of Economics and Political Science - LSE, University of London

Associate Professor, School of Economic Sciences, Federal University of Rio Grande do Sul - UFRGS 
Tabela 10.1 - Principais agregados macroeconômicos - 2013-2015

Table 10.1 - Main macroeconomic aggregates - 2013-2015

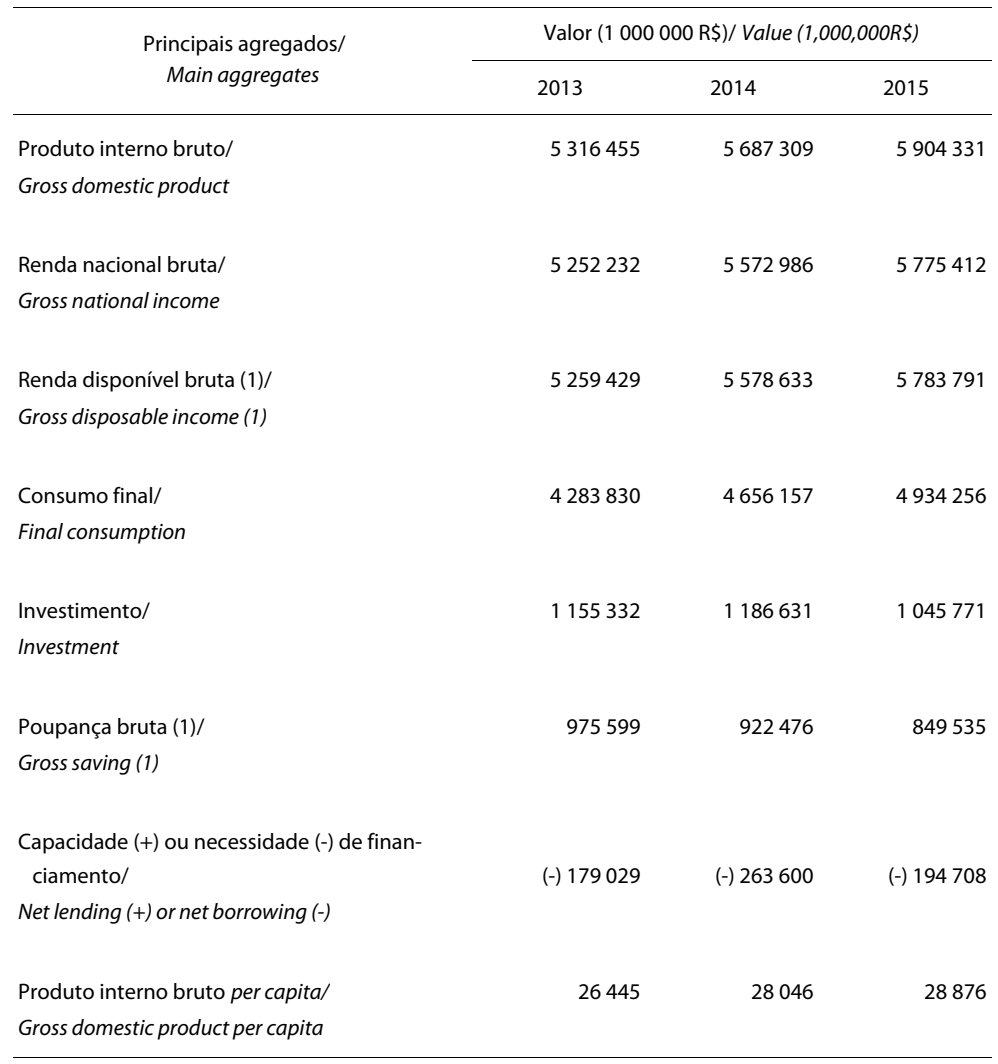

Fontes/Sources: 1. Sistema de contas nacionais: Brasil: 2010-2013. Rio de Janeiro: IBGE, 2015. (Contas nacionais, n. 46). Disponível em/Available from: <http://www.ibge.gov.br/home/estatistica/economia/ contasnacionais/2013/default.shtm>. Acesso em: mar. 2016/Cited: Mar. 2016. 2. Indicadores IBGE: contas nacionais trimestrais: indicadores de volume e valores correntes out./dez. 2015. Rio de Janeiro: IBGE, 2016. Disponível em/Available from: <http://www.ibge.gov.br/home/estatistica/indicadores/ pib/defaultcnt.shtm>. Acesso em: mar. 2016/Cited: Mar. 2016.

Nota: Os dados de 2014 e 2015 são preliminares baseados em Contas Nacionais Trimestrais./ Note: Preliminary data for 2014 and 2015 based on the Quarterly National Accounts.

(1) Inclui as transferências de capital por impossibilidade de identificá-las, até o momento./ (1) Includes capital transfers due to the impossibility of identifying them up to now. 
Tabela 10.2 - Participação percentual dos impostos e do valor adicionado, a preços básicos no Produto Interno Bruto - PIB, e dos setores de atividade, no valor adicionado a preços básicos - 2013-2015

Table 10.2 - Percentage participation of the taxes and of the value added, at basic prices in the Gross Domestic Product - GDP, and of the sectores of activity, in value added at basic prices - 2013-2015

\begin{tabular}{|c|c|c|c|}
\hline \multirow{2}{*}{$\begin{array}{l}\text { Especificação/ } \\
\text { Item }\end{array}$} & \multicolumn{3}{|c|}{ Participação percentual (\%)/ Percent participation (\%) } \\
\hline & 2013 & 2014 & 2015 \\
\hline $\begin{array}{l}\text { Produto interno bruto/ } \\
\text { Gross domestic product }\end{array}$ & 100,0 & 100,0 & 100,0 \\
\hline $\begin{array}{l}\text { Impostos/ } \\
\text { Taxes }\end{array}$ & 14,6 & 14,2 & 14,4 \\
\hline $\begin{array}{l}\text { Valor adicionado a preços básicos / } \\
\text { Value added at basic price }\end{array}$ & 85,4 & 85,8 & 85,6 \\
\hline $\begin{array}{l}\text { Valor adicionado a preços básicos / } \\
\text { Value added at basic prices }\end{array}$ & 100,0 & 100,0 & 100,0 \\
\hline $\begin{array}{l}\text { Agropecuária/ } \\
\text { Agriculture, forestry and fishing }\end{array}$ & 5,3 & 5,2 & 5,2 \\
\hline $\begin{array}{l}\text { Indústria (1)/ } \\
\text { Manufacturing, mining and quarrying }\end{array}$ & 24,9 & 24,0 & 22,7 \\
\hline $\begin{array}{l}\text { Serviços/ } \\
\text { Services }\end{array}$ & 69,8 & 70,8 & 72,1 \\
\hline
\end{tabular}

Fontes/Sources: 1. Sistema de contas nacionais: Brasil: 2010-2013. Rio de Janeiro: IBGE, 2015. (Contas nacionais, n. 46). Disponível em/Available from: <http://www.ibge.gov.br/home/estatistica/economia/ contasnacionais/2013/default.shtm>. Acesso em: mar. 2016/Cited: Mar. 2016. 2. Indicadores IBGE: contas nacionais trimestrais: indicadores de volume e valores correntes out./dez. 2015. Rio de Janeiro: IBGE, 2016. Disponível em/Available from: <http://www.ibge.gov.br/home/estatistica/indicadores/ pib/defaultcnt.shtm>. Acesso em: mar. 2016/Cited:Mar. 2016.

Nota: Os dados de 2014 e 2015 são preliminares baseados em Contas Nacionais Trimestrais./ Note: Preliminary data for 2014 and 2015 based on the Quarterly National Accounts.

(1) Inclusive eletricidade, gás, água e construção./ (1) Includes electricity, gas, water and construction. 


\section{Tabela 10.3 - Composição do Produto Interno Bruto - PIB, sob a ótica da despesa - 2013-2015 \\ Table 10.3 - Composition of Gross Domestic Product - GDP, considering expenditures - 2013-2015}

\begin{tabular}{|c|c|c|c|c|c|c|}
\hline \multirow{2}{*}{$\begin{array}{l}\text { Composição/ } \\
\text { Composition }\end{array}$} & \multicolumn{3}{|c|}{$\begin{array}{l}\text { Valor (1 } 000000 \mathrm{R} \$) / \\
\text { Value }(1,000,000 \mathrm{R} \$)\end{array}$} & \multicolumn{3}{|c|}{$\begin{array}{l}\text { Percentual do PIB (\%)/ } \\
\text { Percent of GDP (\%) }\end{array}$} \\
\hline & 2013 & 2014 & 2015 & 2013 & 2014 & 2015 \\
\hline $\begin{array}{l}\text { Produto interno bruto/ } \\
\text { Gross domestic product }\end{array}$ & 5316455 & 5687309 & 5904331 & 100,00 & 100,00 & 100,00 \\
\hline $\begin{array}{l}\text { Consumo final/ } \\
\text { Final consumption }\end{array}$ & 4283830 & 4656157 & 4934256 & 80,58 & 81,87 & 83,57 \\
\hline $\begin{array}{l}\text { Despesa de consumo das } \\
\text { famílias (1)/ }\end{array}$ & 3276050 & 3547428 & 3741855 & 61,62 & 62,37 & 63,37 \\
\hline $\begin{array}{l}\text { Final consumption expenditure of } \\
\text { households (1) }\end{array}$ & & & & & & \\
\hline $\begin{array}{l}\text { Despesa de consumo da administ } \\
\text { ção pública/ }\end{array}$ & 1007780 & 1108729 & 1192401 & 18,96 & 19,49 & 20,20 \\
\hline $\begin{array}{l}\text { General Government final consump } \\
\text { expenditure }\end{array}$ & & & & & & \\
\hline $\begin{array}{l}\text { Formação bruta de capital/ } \\
\text { Gross capital formation }\end{array}$ & 1155332 & 1186631 & 1045771 & 21,73 & 20,86 & 17,71 \\
\hline $\begin{array}{l}\text { Exportação de bens e serviços/ } \\
\text { Exports of goods and services }\end{array}$ & 620077 & 636230 & 770084 & 11,66 & 11,19 & 13,04 \\
\hline $\begin{array}{l}\text { Importação de bens e serviços (-)/ } \\
\text { Imports of goods and services (-) }\end{array}$ & (-) 742784 & (-) 791709 & (-) 845779 & (-) 13,97 & (-) 13,92 & (-) 14,32 \\
\hline
\end{tabular}

Fontes/Sources: 1. Sistema de contas nacionais: Brasil: 2010-2013. Rio de Janeiro: IBGE, 2015. (Contas nacionais, n. 46). Disponível em/Available from: <http://www.ibge.gov.br/home/estatistica/economia/ contasnacionais/2013/default.shtm>. Acesso em: mar. 2016/Cited: Mar. 2016. 2. Indicadores IBGE: contas nacionais trimestrais: indicadores de volume e valores correntes out./dez. 2015. Rio de Janeiro: IBGE, 2016. Disponível em/Available from:<http://www.ibge.gov.br/home/estatistica/indicadores/pib/ defaultcnt.shtm>. Acesso em: mar. 2016/Cited: Mar. 2016.

Nota: Os dados de 2014 e 2015 são preliminares baseados em Contas Nacionais Trimestrais./ Note: Preliminary data for 2014 and 2015 based on the Quarterly National Accounts.

(1) Os dados de consumo das famílias incluem o consumo das famílias + despesa de consumo das instituições sem fins de lucro a serviço das famílias./ (1) Data for household consumption include the household final consumption expenditure + consumption non profit institutions serving households final consumption expenditure. 
Tabela 10.4 - Variação da taxa trimestral do Produto Interno Bruto - PIB, por setor de atividade - 2014-2015

Table 10.4 - Quarterly rate change of the Gross Domestic Product - GDP,

by sector of activity - 2014-2015

\begin{tabular}{|c|c|c|c|c|c|c|c|c|}
\hline \multirow[b]{3}{*}{$\begin{array}{l}\text { Setor de atividade/ } \\
\text { Sector of activity }\end{array}$} & \multicolumn{8}{|c|}{ Taxa trimestral (\%)/Quarterly rate (\%) } \\
\hline & \multicolumn{4}{|c|}{2014} & \multicolumn{4}{|c|}{2015} \\
\hline & $\begin{array}{c}1 \stackrel{0}{\text { tri- }} \\
\text { mestre/ } \\
1 s t \\
\text { quarter }\end{array}$ & $\begin{array}{l}2^{\circ} \text { tri- } \\
\text { mestre/ } \\
\text { 2nd } \\
\text { quarter }\end{array}$ & $\begin{array}{l}3^{\circ} \text { tri- } \\
\text { mestre/ } \\
3 r d \\
\text { quarter }\end{array}$ & $\begin{array}{l}4^{\circ} \text { tri- } \\
\text { mestre/ } \\
4 \text { th } \\
\text { quarter }\end{array}$ & $\begin{array}{l}1 \stackrel{0}{ } \text { tri- } \\
\text { mestre/ } \\
1 s t \\
\text { quarter }\end{array}$ & $\begin{array}{l}2{ }^{\circ} \text { tri- } \\
\text { mestre/ } \\
\text { 2nd } \\
\text { quarter }\end{array}$ & $\begin{array}{l}\text { 3o tri- } \\
\text { mestre/ } \\
\text { 3rd } \\
\text { quarter }\end{array}$ & $\begin{array}{l}4^{\circ} \text { tri- } \\
\text { mestre/ } \\
4 \text { th } \\
\text { quarter }\end{array}$ \\
\hline $\begin{array}{l}\text { Produto interno bruto a } \\
\text { preço de mercado / } \\
\text { Gross domestic product at } \\
\text { market prices }\end{array}$ & 3,2 & $(-) 0,8$ & (-) 1,1 & $(-) 0,7$ & (-) 2,0 & $(-) 3,0$ & $(-)$ 4,5 & $(-) 5,9$ \\
\hline $\begin{array}{l}\text { Agropecuária / } \\
\text { Agriculture, forestry and } \\
\text { fishing }\end{array}$ & 6,2 & $(-) 0,6$ & 0,3 & 2,2 & 5,4 & 2,2 & $(-) 2,0$ & 0,6 \\
\hline $\begin{array}{l}\text { Indústria / } \\
\text { Manufacturing, mining and } \\
\text { quarrying }\end{array}$ & 4,6 & (-) 2,7 & (-) 2,9 & (-) 2,1 & (-) 4,4 & (-) 5,7 & $(-) 6,7$ & (-) 8,0 \\
\hline $\begin{array}{l}\text { Serviços / } \\
\text { Services }\end{array}$ & 2,2 & $(-) 0,0$ & $(-) 0,3$ & $(-) 0,3$ & $(-) 1,4$ & $(-) 1,8$ & $(-) 2,9$ & (-) 4,4 \\
\hline $\begin{array}{l}\text { Valor adicionado a preços } \\
\text { básicos / } \\
\text { Value added at basic prices }\end{array}$ & 3,1 & $(-) 0,7$ & $(-) 1,0$ & $(-) 0,7$ & $(-) 1,7$ & $(-) 2,5$ & $(-) 3,8$ & $(-) 5,0$ \\
\hline
\end{tabular}

Fonte/Source: Indicadores IBGE: contas nacionais trimestrais: indicadores de volume e valores correntes: out./dez. 2015. Rio de Janeiro: IBGE, 2016. Disponível em/Available from: < http://www.ibge.gov.br/ home/estatistica/indicadores/pib/defaultcnt.shtm>.Acesso em: mar. 2016/Cited:Mar. 2016.

Notas: 1. Dados preliminares.

2. Variação percentual em relação ao mesmo trimestre do ano anterior./

Notes: 1. Preliminary data.

2. Percent change vis-à-vis the same quarter of previous year. 
Tabela 10.5 - Principais relações macroeconômicas - 2013-2015

Table 10.5 - Main macroeconomic relationships - 2013-2015

\begin{tabular}{lccr}
\hline \multicolumn{1}{c}{$\begin{array}{c}\text { Principais relações/ } \\
\text { Main relationships }\end{array}$} & \multicolumn{2}{c}{ Em percentual (\%)/ Percent (\%) } \\
\cline { 2 - 4 } & 2013 & 2014 & 2015 \\
\hline $\begin{array}{l}\text { Taxa de investimento/ } \\
\text { Investment rate }\end{array}$ & 20,9 & 20,2 & 18,2 \\
Carga tributária bruta/ (1) & 33,6 & - & - \\
Tax burden (1) & & 25,1 & \\
$\begin{array}{l}\text { Grau de abertura da economia/ } \\
\text { Degree of opening of the economy }\end{array}$ & 25,6 & \\
\hline
\end{tabular}

Fonte/Source: IBGE, Diretoria de Pesquisas, Coordenação de Contas Nacionais.

Nota: Os dados de 2014 e 2015 são preliminares baseados em Contas Nacionais Trimestrais./ Note: Preliminary data for 2014 and 2015 based on the Quarterly National Accounts.

(1) Não inclui as contribuições sociais imputadas./ (1) Does not include imputed social. 


\section{Agropecuária Agriculture}

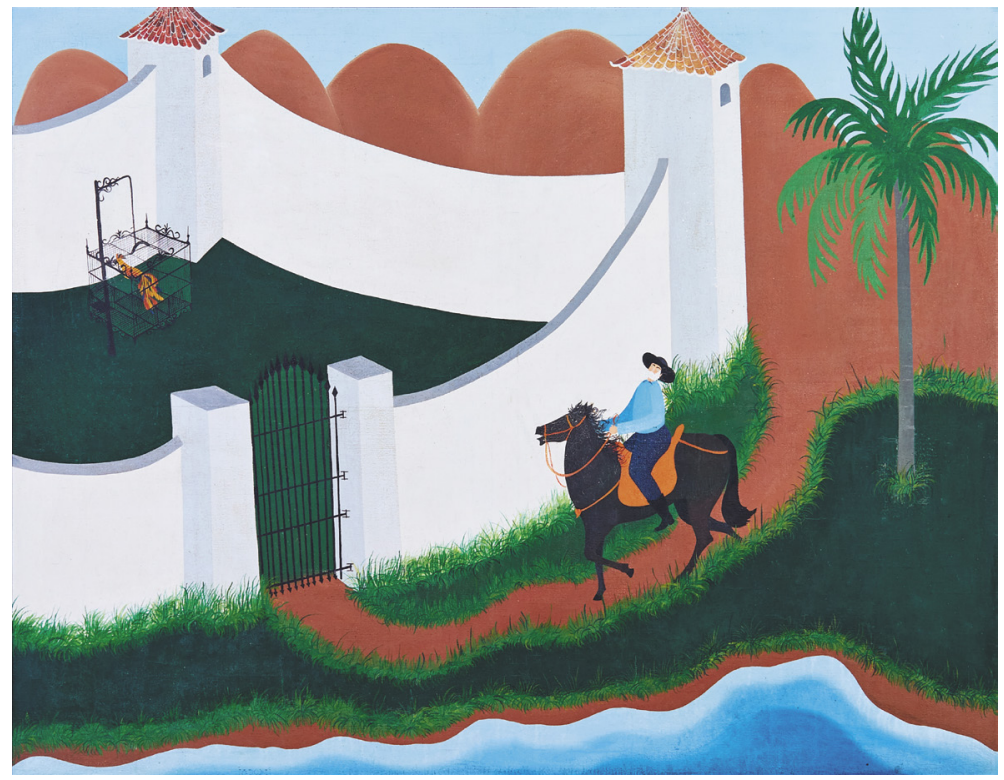

Sem título, s.d/Untitled, n.d. Iaponi Araújo 



\section{Agropecuária}

\section{Agriculture}

As mudanças ocorridas no conjunto do setor agropecuário brasileiro nos últimos 20 anos vão consolidando o País como uma referência global em agricultura tropical. Os índices de modernização do setor evoluem a cada safra tornando os estabelecimentos agropecuários nacionais capitalintensivos. Não obstante, se consolida um modelo dual de agropecuária, pois os ganhos de produtividade não alcançam a maioria dos produtores. Pelo contrário, há sinais muito claros de uma concentração econômica e produtiva em que poucos estabelecimentos respondem por quantidades cada vez maiores da riqueza gerada na atividade. Este processo afeta tanto os grandes como os pequenos produtores, chamados de agricultores familiares.

Em termos conjunturais, as evidências disponíveis dão conta de que a agropecuária brasileira tem mantido sua trajetória de crescimento mesmo diante do cenário desfavorável vivenciado pela economia doméstica, que mergulha em uma fase recessiva a partir de 2014. O desempenho do segmento varia de produto para produto e de região
The changes occurred in the Brazilian agricultural sector as a whole in the last 20 years have been confirming the Country as a global reference in tropical agriculture. The modernization indexes of the sector evolve at each crop, turning national agriculture establishments into capital-intensive businesses. Nevertheless, a dual model of agriculture has been consolidated, since the productivity gains do not reach most producers. On the contrary, there are very clear signs of economic and productive concentration in which few establishments account for the highest levels of wealth generated by the activity. This process affects both big and small producers, called family farmers.

In the short-term, the available evidences show that the Brazilian agriculture has been keeping the upward trend even in the midst of the difficulties faced by the domestic economy, which has dived into recession since 2014. The performance of the sector varies from product to product and from region to region. 
para região. Mas, de maneira geral, o comportamento dos indicadores de desempenho das lavouras, da pecuária e do ramo florestal é extremamente positivo.

Conforme demonstra o Gráfico 11.1, a quantidade produzida nas lavouras de cereais, leguminosas e oleaginosas vem crescendo de forma ininterrupta, atingindo um novo recorde de 194,6 milhões de toneladas ao final da série analisada. Em termos de valor da produção, no intervalo de apenas uma década, a cifra evoluiu de $R \$ 63,4$ bilhões para R\$136,7 bilhões. Notese que, de 2013 a 2014, enquanto a economia brasileira registrou uma taxa de crescimento do PIB de apenas $0,1 \%$, a riqueza gerada pelas plantações avançou $11,6 \%$, sinalizando a força do setor relativamente às demais atividades produtivas do País.

O expressivo avanço da produção agrícola no Brasil tem sido possível devido à elevação dos níveis de produtividade das lavouras. Como podese observar no Gráfico 11.1, embora se registre uma expansão absoluta da área plantada, que alcançou o patamar histórico de 57,4 milhões de hectares em 2014, esse indicador permaneceu constante na maior parte do período. Isso significa que os ganhos de produção obtidos no campo decorrem, principalmente, da incorporação de inovações tecnológicas nos processos produtivos, via o uso crescente de insumos químicos, máquinas agrícolas e variedades de plantas geneticamente modificadas.

Vale assinalar que o aumento da utilização de agroquímicos e de plantas transgênicas tem sido um aspecto bastante sensível
But, as a whole, the behavior of the performance indicators of crops, livestock farming and forestry is quite positive.

As indicated in Graph 11.1, the amount produced in the crops of cereals, legumes and oilseeds have been growing uninterruptedly, reaching a new record of 194.6 million metric tons at the end of the analyzed series. In terms of production value, within a decade only, the figure evolved from R\$63.4 billion to R\$136.7 billion. It should be noted that, between 2013 and 2014, whereas the Brazilian economy recorded a GDP growth rate of only $0.1 \%$, the wealth generated by the crops increased $11.6 \%$, proving the power of the sector in relation to the other productive activities of the Country.

The significant advance of the agricultural production in Brazil has been made possible by the rise in the productivity level of crops. As shown in Graph 11.1, although there is a record of an absolute expansion of the planted area, which registered a historical mark of 57.4 million hectares in 2014, this indicator remained constant most of the period. That means that the productivity gains obtained in the fields result mainly from the incorporation of technological innovations in the productive processes, by means of the increased use of chemical inputs, agricultural machinery and a variety of genetically modified plants.

It is worth highlighting that the use of pesticides and transgenic plants has been a very sensitive issue for the 
para a agropecuária brasileira. É inequívoco que essas tecnologias contribuem para o avanço do setor. Porém, há estudos que alertam para os seus potenciais efeitos negativos sobre a sanidade dos produtos e a saúde humana. Além disso, o uso excessivo de agrotóxicos nas grandes lavouras comerciais tem contribuído para o surgimento de pragas, doenças e plantas invasoras resistentes aos métodos de controle químicos, o que implica na elevação dos custos de produção e gera riscos à biodiversidade.

Grosso modo, a agricultura brasileira é formada por uma ampla variedade de plantações e culturas distribuídas em todas as regiões do País. Apesar de sua diversidade, a Tabela 11.1 indica que o dinamismo econômico do setor está assentado em um leque reduzido de produtos destinados à exportação e ao abastecimento interno, com destaque para a soja, cana-de-açúcar, milho, café, mandioca, arroz, algodão, fumo, banana, laranja, tomate, feijão, batata-inglesa, trigo e uva.

Na lista de produtos elencados, a soja e o milho despontam como as duas culturas mais importantes. Em 2014, a área colhida nas fazendas de soja foi de 30,3 milhões de hectares e a produção da oleaginosa alcançou 86,8 milhões de toneladas. Já o milho ocupava uma área de 15,4 milhões de hectares nos quais foram colhidas 79,9 milhões de toneladas de grãos. Juntas, essas commodities respondem por $79,6 \%$ da área colhida e $85,7 \%$ do volume da produção de cereais, leguminosas e oleaginosas do País, o que denota uma significativa concentração no setor.

Outra lavoura de destaque na economia agrícola brasileira é a cana-de-açúcar, cuja
Brazilian agriculture. The contribution of such technologies to the sector's advance is undeniable. However, there are studies warning of the potential negative effects of this use in relation to product safety and human health. Besides, the excessive use of pesticides in big cash crops have contributed to the emerging of pests, diseases and invasive species resistant to chemical control methods, which implies increase in the production costs and puts biodiversity at risk.

Generally speaking, the Brazilian agriculture comprises a wide variety of crops and cultures spread in all regions of the Country. Despite its diversity, Table 11.1 indicates that the economic dynamism of the sector lies on a reduced set of products destined for exportation and domestic supply, with a highlight to soybeans, sugarcane, corn, coffee, cassava, rice, cotton, tobacco, bananas, oranges, tomatoes, beans, potatoes, wheat and grapes.

In the list above, soybeans and corn stand out as the most important cultures. In 2014, the harvested area in the soybean farms was of 30.3 million hectares and the oilseed production reached 86.8 million metric tons. Corn had an area of 15.4 million hectares in which 79.9 million metric tons of grains were harvested. Together, these commodities account for $79.6 \%$ of the volume of the production of cereals, legumes and oilseeds of the country, which means a significant concentration in the sector.

Another crop standing out in the Brazilian agricultural economy is 
área colhida de 10,4 milhões de hectares ocupa a terceira posição no ranking nacional, apresentando um rendimento de 70.625 quilogramas por hectare $(\mathrm{kg} /$ ha). No campo brasileiro, figuram ainda importantes produtos de exportação, tais como o café, o algodão, a laranja e o fumo, que apresentam altos índices de produtividade por área ocupada.

Além das culturas de exportação, que abrangem as maiores áreas de terras ocupadas, a relação apresentada na Tabela 11.1 reúne também um grupo de produtos que garante o abastecimento doméstico e contribui decisivamente para a segurança alimentar do País. Entre eles, destacam-se alimentos como o feijão e a mandioca, produzidos predominantemente pela agricultura familiar, além do arroz, do trigo, do tomate, da batata-inglesa, da banana e da uva.

No conjunto dos 15 principais cultivos, os estados de São Paulo e Mato Grosso figuram como os maiores produtores das lavouras de exportação. São Paulo se destaca na produção de cana-de-açúcar e laranja. Mato Grosso, por sua vez, lidera a produção nacional de soja, milho e algodão. Outras unidades federativas que aparecem no topo do ranking das principais lavouras do Brasil, especialmente naquelas voltadas ao mercado interno, são: Minas Gerais, Paraná, Rio Grande do Sul, Goiás, Pará e Bahia (Tabela 11.1).

Os números apresentados sinalizam que há uma grande disparidade entre a capacidade produtiva dos diferentes estados brasileiros. Essa realidade pode ser constatada de forma bastante clara no Gráfico 11.2. Nele, observa-se que o valor da produção agrícola nacional em 2014 estava fortemente concentrado sugarcane, whose harvested area is of 10.4 million hectares: the third position in the national ranking, presenting a yield of 70,625 kilograms by hectare (kg/ ha). In the Brazilian rural context, there are still important export products, such as coffee, cotton, oranges and tobacco, which present high indexes of productivity by planted area.

Besides the export crops, which encompass the largest areas of planted land, the list presented on Table 11.1 also gathers a group of products that guarantee the domestic supply and contribute decisively to food security in the Country. Among them, there are food products such as beans and cassava - produced mostly by family farms - as well as rice, wheat, tomatoes, potatoes, bananas and grapes.

Concerning the 15 main crops, the states of São Paulo and Mato Grosso are the biggest producers of export crops. São Paulo stands out in the production of sugarcane and oranges. Mato Grosso, on its turn, leads the national production of soybeans, corn and cotton. Other Federation Units ranking at the top of the main crops in Brazil, especially those targeted to the domestic market, are: Minas Gerais, Paraná, Rio Grande do Sul, Goiás, Pará and Bahia (Table 11.1).

The figures presented register a great disparity in the productive capacity of the Brazilian states - which can be seen quite clearly in Graph 11.2. There, it is possible to observe that the national agricultural production in 2014 was strongly concentrated in the states of the South, Southeast and 
nos estados das regiões Sul, Sudeste e Centro-Oeste, os quais sediam as principais lavouras de exportação. Note-se que apenas cinco estados, com São Paulo e Mato Grosso na dianteira, responderam por $63,7 \%$ da riqueza gerada no setor. As demais unidades da federação (UFs), com exceção de algumas áreas de expansão agrícola nas regiões Norte e Nordeste, apresentam uma baixa participação no total.

A expansão da produção agrícola em espaços territoriais que até recentemente eram pouco produtivos ou que não apareciam com posições destacadas no ranking das principais regiões produtoras do Brasil, reflete o deslocamento das lavouras comerciais para novas fronteiras de produção. O território chamado de MATOPIBA, que é a abreviatura dos estados do Maranhão, Tocantins, Piauí e Bahia, é um claro exemplo deste processo. Outra rota de expansão da agricultura situa-se na Amazônia Ocidental, particularmente no estado do Pará. Nessa região, o avanço das plantações e das fazendas de gado não raro tem provocado conflitos fundiários e disputas por terra, assim como em relação aos recursos naturais, uma vez que ainda se verificam problemas de desmatamento e abertura de áreas mediante queimadas.

A distribuição geográfica desigual do valor da produção agrícola do Brasil é reflexo de vários fatores, tanto históricos como conjunturais. Os estados que lideram o ranking nacional possuem características naturais favoráveis e detém as melhores condições de infraestrutura produtiva. Ademais, nas últimas décadas, a política agrícola do governo brasileiro tem apoiado majoritariamente às culturas de
Central-West Regions, which hold the main export crops. It should be noted that only five states, led by São Paulo and Mato Grosso, accounted for $63.7 \%$ of the wealth generated in the sector. The other Federation Units (FUs), except for some areas of agricultural expansion in the North and Northeast Regions, present a low contribution to the total.

The expansion of the agricultural production in areas which, up to recent times, were either little productive or did not stand out in the ranking of the major producing regions of Brazil reflects the dislocation of cash crops to the new production frontiers. The area called MATOPIBA, which stands for the states of Maranhão, Tocantins, Piauí and Bahia, is a clear example of this process. Another route of expansion for agriculture is located in the Western Amazon, particularly in the state of Pará. In this area, the advance of crops and livestock farming has many times provoked land conflicts and disputes, as well as natural resources issues, since there are still problems related to deforestation and fires to clear land.

The uneven geographic distribution of the agricultural production is a reflex of several factors from the past and present as well. The states which lead the national ranking have favorable natural characteristics and gather the best conditions of productive infrastructure. Moreover, in the last decades, the agricultural policies of the Brazilian government have given support mainly to the export 
exportação localizadas nesses espaços.

Esse apoio, porém, tem sido insuficiente em algumas áreas estratégicas, a exemplo da logística de transporte e da estrutura de armazenamento. A capacidade instalada dos armazéns nacionais, apesar do desempenho geral positivo verificado no período de 2005 a 2015 (Gráfico 11.3), ainda está aquém das necessidades das safras recordes geradas pelo setor que prometem superar em um curto intervalo de tempo a barreira de 200 milhões de toneladas.

Quanto à atividade pecuária desenvolvida no território brasileiro, a Tabela 11.2 mostra que a maioria dos rebanhos de animais criados no País avançou em termos quantitativos. Embora com taxas de variação inferiores às da produção agrícola, a tendência geral do setor é de crescimento. No período de 2013 a 2014, o único tipo de rebanho que registrou queda foi o de bubalinos, que reduziu seu efetivo em torno de 1,0\%. O rebanho bovino contabilizou um ligeiro aumento, passando de 211,8 milhões no ano de 2013 para 212,3 milhões de cabeças em 2014. As taxas de crescimento do efetivo de suínos e aves, contudo, foram bem mais expressivas, tendo o segmento de galináceos (galos, frangos e pintos de um dia) e de galinhas superado a marca de 1,5 bilhão de cabeças.

À exceção dos rebanhos de maior valor agregado, que respondem pelo abastecimento interno e contribuem para a geração de divisas em moeda estrangeira, o segmento de pequenos animais (caprinos, ovinos e codornas) também manteve o seu ritmo de expansão estimulado pelos investimentos nas pequenas e médias propriedades da agricultura familiar, com destaque para cultures located in those areas. Such support, however, has not been enough in some strategic fields, as transportation logistics and storage structure, for example. The installed capacity of the national warehouses, despite the positive performance as a whole in the period from 2005 to 2015 (Graph 11.3), still falls short of the needs of the record crops harvested by the sector, which soon enough are to surpass the 200-million-metric-ton barrier.

As to the livestock farming in the Brazilian territory, Table 11.2 shows that most of the animal herds in the Country increased in quantitative terms. Although with change rates below those of the agricultural production, the sector is on the rise. In the period from 2013 to 2014 , the herd of buffaloes was the only one to register decrease: nearly $1.0 \%$ of reduction in the inventory. Cattle herds accounted for a slight increase, going from 211.8 million in 2013 to 212.3 million head in 2014. The growth rates of the inventory of hogs and pigs and birds, however, were much more significant, having the segment of poultry (roosters, pullets and chicks) and of hens surpassed the mark of 1.5 billion head.

Except for the highest value-added herds, which meet the domestic demands and contribute to the generation of foreign currency, the segment of small-sized animals (goats, sheep and quails) has also kept the pace of increase due to the investments in the small and medium-sized family farms, with a highlight to the increase in the quail 
aumento do efetivo de codornas. Em relação aos caprinos e ovinos, se não fosse a seca registrada na região Nordeste no período, onde se encontra a maior parte dos criatórios, provavelmente a expansão do segmento teria sido bem maior haja vista que a demanda doméstica pela carne dessas espécies de animais tem aumentado anualmente.

$\mathrm{Na}$ esteira da expansão dos principais rebanhos comerciais, verifica-se também o crescimento da produção de carnes, especialmente de aves e suínos (Gráfico 11.4). Puxado pelo aumento da demanda doméstica e pela escalada das vendas externas, a avicultura nacional avança ano a ano. Um prova disso é que, de 2009 a 2014, o peso médio das carcaças de aves saltou de 9,9 milhões para 12,5 milhões de toneladas. A produção de carne suína, depois de registrar leves quedas em alguns anos, voltou a crescer, alcançando 3,2 milhões de toneladas em 2014. Já no que se refere a oferta de carne bovina, nota-se uma pequena redução no período recente fruto da queda do consumo interno, situação que tende a ser revertida no curto prazo pela recuperação das exportações do setor.

O bom desempenho da pecuária brasileira pode ser observado, ainda, ao se analisar a variação da quantidade e do valor dos produtos de origem animal produzidos nas fazendas e sítios do País (Tabela 11.3). Nesse aspecto, o destaque recai sobre a produção de leite, ovos, mel de abelha, peixes e camarão.

O leite é o principal produto de origem animal produzido no Brasil. A produção nacional desse alimento, majoritariamente desenvolvida em propriedades familiares, cresceu 2,7\% de 2013 a 2014, atingindo inventory. In relation to goats and sheep, had it not been for the droughts recorded in the Northeast Region in the period, where most of the raising farms are concentrated, the expansion of the segment would have probably been greater, given that the domestic demand for this kind of meat has increased annually.

In the wake of the expansion of the main commercial herds, it is also possible to see the increase of meat production, especially poultry and pork (Graph 11.4). Leveraged by the rise of the domestic demand and by the foreign sales boost, the national aviculture grows year after year. Proof of that is the increase in the average weight of poultry carcasses from 9.9 million to 12.5 million metric tons between 2009 and 2014. The production of pork, after recording slight drops in some years, has been growing again, reaching 3.2 million metric tons in 2014. In relation to beef, there is a subtle reduction in the period due to the decreased domestic consumption - a situation that tends to be offset in the short run by the recovery of the sector's exports.

The good performance of the Brazilian livestock farming can also be assessed in terms of the variety in the value and quantity of animal products from the farms in the Country (Table 11.3). In this context, the highlight is the production of milk, eggs, honey, fish and shrimp.

Milk is the main animal product of Brazil. The national output of milk, mostly produced in family farms, grew $2.7 \%$, from 2013 to 2014 , reaching the mark of 35.2 billion liters. As 
a marca de 35,2 bilhões de litros. Como resultado da melhoria da produtividade e da alta dos preços praticados no mercado interno, o valor da produção leiteira passou de $\mathrm{R} \$ 32,4$ bilhões para $\mathrm{R} \$ 33,8$ bilhões, 0 que representou uma variação positiva de $4,2 \%$ no período.

O setor dedicado à produção de ovos de galinha, que representa uma importante parcela da riqueza gerada pela avicultura nacional, ampliou em $12,7 \%$ o seu valor da produção. A Tabela 11.3 mostra que outras atividades não tradicionais também registraram taxas de expansão significativas, a exemplo da produção de ovos de codorna, mel de abelha e camarões. No entanto, entre os todos os segmentos listados, o que apresentou as maiores taxas de crescimento no período foi a produção de peixes, cuja quantidade e o valor aumentaram $20,9 \%$ e $34,4 \%$, respectivamente.

A produção pesqueira de água doce no Brasil provém, historicamente, do extrativismo realizado nos lagos e rios, especialmente na região Norte. Mas, recentemente, a criação de peixes em cativeiro tem atraído cada vez mais produtores familiares e não familiares, convertendo-se em uma alternativa para diversificar e ampliar a renda de propriedades rurais em diferentes áreas do território nacional. $O$ resultado desse movimento, como foi visto, tem sido o avanço vertiginoso da atividade. Entre as principais espécies responsáveis pelos ganhos de produção da piscicultura brasileira, conforme consta no Gráfico 11.5, destacam-se: a tilápia (41,9\%), o tambaqui $(29,3 \%)$, o tambacu e a tambatinga (8,5\%), a carpa $(4,4 \%)$ e outros peixes $(15,9 \%)$. a result of the productivity improvement and price rise in the domestic market, the value of dairy production went from $\mathrm{R} \$ 32.4$ billion to $\mathrm{R} \$ 33.8$ billion, corresponding to a positive change of $4.2 \%$ in the period.

The producing sector of hen eggs, which represents an important part of the wealth generated by the national aviculture, expanded its production value by $12.7 \%$. Table 11.3 shows that other nontraditional activities have also recorded significant expansion rates, as the production of quail eggs, honey and shrimp. However, of all listed segments, the one with the highest growth rates in the period is fish production, whose quantity and value increased $20.9 \%$ and $34.4 \%$, respectively.

Freshwater fish production in Brazil derives historically from the extractive fishing in lakes and rivers, especially in the North Region. Recently, though, fish farming in tanks has attracted even more family and nonfamily producers, becoming an opportunity to diversify and improve the income of rural properties in different areas of the national territory. As a result, the activity has grown immensely, as already observed. Among the main species responsible for the production gains of the Brazilian fish farming are: tilapia (41.9\%), tambaqui (29.3\%), tambacu and tambatinga (8.5\%), carp (4.4\%) and other fish (15.9\%). 
Outro ramo que busca substituir práticas extrativas por cultivos racionais e que tem demonstrado notável avanço no rural brasileiro é a silvicultura. Um dos fatores responsáveis pala ampliação da silvicultura nacional é o crescimento do consumo das mineradoras, que tem utilizado cada vez mais as florestas plantadas de eucalipto como fonte de energia para suas demandas de aquecimento dos alto-fornos. Mas a utilização da madeira para produção de papel e mesmo a sua exportação como matéria-prima para a indústria de celulose também vem estimulando o crescimento desta atividade.

Atualmente, o setor florestal responde pela maior parte da oferta de produtos madeireiros do País (Tabela 11.4). Entre os subprodutos do segmento, merece destaque a produção de carvão vegetal, cuja quantidade gerada passou de 5,6 para 6,2 milhões de toneladas, de 2013 a 2014. A produção de madeira em tora, porém, registrou uma queda no período, refletindo o desaquecimento da indústria de papel e celulose e, principalmente, a retração do setor moveleiro nacional.

As áreas de florestas plantadas se distribuem em todas as regiões brasileiras (Gráfico 11.6). Contudo, a maior parte do setor encontra-se concentrada nas regiões Sul $(40,1 \%)$, Sudeste $(32,6 \%)$ e Centro-Oeste (13,9\%). De forma agregada, esses espaços detêm $86,6 \%$ da silvicultora nacional. As regiões Norte e Nordeste, por seu turno, ainda ocupam uma pequena participação na atividade, que tende a crescer impulsionada também pelos compromissos internacionais recentes assumidos pelo Brasil no sentido de reduzir as emissões de carbono e minimizar a devastação das matas nativas.
Another segment that aims at the substitution of the extractive activities for rational farming and that has demonstrated a notable advance in the Brazilian rural production is silviculture. One of the factors accounting for the expansion of the national silviculture is the growth in the consumption of mining companies, which have gradually been using more eucalyptus planted forests as energy sources to heat their blast furnaces. Moreover, the use of wood for paper production and even its exportation as raw material for the pulp industry have also been stimulating the growth of this activity.

Nowadays, forestry accounts for the greatest part of the wood products supply in the Country (Table 11.4). Among the sub-products in the sector, the highlight is charcoal, whose quantity went from 5.6 to 6.2 million metric tons, from 2013 to 2014 . The production of logwood, though, recorded a decrease in the period, reflecting the paper and pulp industry slowdown and, mainly, the decrease in the national furniture sector.

The areas of planted forests are spread along the Brazilian Regions (Graph 11.6). However, most of them are concentrated in the South $(40.1 \%)$, Southeast (32.6\%) and Central-West

Regions (13.9\%). Together, those areas account for $86.6 \%$ of the national silviculture. The North and Northeast Regions, on the other hand, still hold a timid participation in the activity, which tends to grow due to the recent international commitments taken by Brazil so as to reduce carbon emissions and minimize the devastation of native forests. 
Percebe-se, portanto, que a agropecuária brasileira se constitui em um setor pujante que desempenha um papel estratégico na produção de alimentos, fibras e energia renovável. É bem verdade que os dados destacados aqui não refletem os efeitos da crise econômica e política que vêm abalando a nação mais fortemente desde 2015. A recessão e o desemprego podem afetar os níveis de consumo do mercado doméstico, inibir os investimentos e mudar parcialmente o comportamento de alguns produtos. Apesar disso, tudo indica que o setor deverá manter seu ritmo de expansão nos anos vindouros. Isso porque se no plano interno o cenário não é favorável, a recente desvalorização do real aumenta a competividade dos produtos brasileiros no mercado internacional, favorecendo a recuperação da rentabilidade dos segmentos de exportação que caminham para transformar o País no maior fornecedor mundial de produtos agrícolas nas próximas décadas.

Nessa trajetória, além das questões estritamente produtivas, persistem alguns desafios que necessitam ser enfrentados. Não resta dúvida de que o perfil da agropecuária do Brasil é de um setor bem-sucedido em termos técnicos e econômicos. Contudo, seu processo continuado de crescimento tem provocado contradições e resistências em face da elevada concentração em poucos produtos e regiões específicas, suscitando um problema distributivo que necessita ser equacionado pela ação planejada do Estado e dos atores privados. Ademais, a imagem de um setor moderno e em expansão precisa focalizar o uso de tecnologias harmoniosas com a natureza, pois no
Therefore, there is a strategic role played by the thriving Brazilian agriculture sector in the production of food, fibers and renewable energy. Obviously, the data portrayed herein do not reflect the economic and political crisis Brazil has undergone, especially since 2015 . Recession and unemployment can affect the consumption levels of the domestic market, discourage investments and partially change the behavior of some products. Nevertheless, the sector has everything to keep its pace of expansion in the years to come. That happens because, if from the domestic perspective, the scenario is not favorable, the recent depreciation of the real increased the competitiveness of the Brazilian products in the foreign market, favoring the recovery of the profitability of the export segments headed to transform the Country into the biggest world supplier of agricultural products in the next decades.

In order to get there, besides dealing with issues strictly related to production, we still have to face some challenges. There is no doubt that the profile of the Brazilian agriculture is of a well-succeeded sector in technical and economic terms. However, its continuous growth has been arousing been provoking contradictions and resistance before the high concentration of few products and specific regions, causing a distribution problem that needs to the figured out by the planned action of the State and private actors. Besides, the image of a modern sector, which is on the rise, needs to be focused on the use 
século XXI será cada vez mais importante combinar o crescimento econômico com a sustentabilidade ambiental. E, nessa perspectiva, nossos agricultores certamente poderão ensinar ao mundo algumas lições de como é possível compatibilizar produção de riqueza no campo com preservação e cuidado com a natureza, algo fundamental para a prática da própria agricultura. of nature-friendly technologies, since in the 21 st century it will be even more important to combine economic growth and environmental sustainability. In this sense, our farmers will certainly be able to teach the world some lessons on how to match production wealth in the fields with nature preservation and care - after all, these aspects are vital for agriculture itself.

\section{Joacir Rufino de Aquino}

Economista pela Universidade do Estado do Rio Grande do Norte (2000) e Mestre em Economia Rural e Regional pela Universidade Federal de Campina Grande. Atualmente é Professor Adjunto IV e Pesquisador na Universidade do Estado do Rio Grande do Norte (Campus de Assú).
Economist from the State University of Rio Grande do Norte (2000) and Master in Rural and Regional Economics from the Federal University of Campina Grande. Currently, he is Assistant Professor IV and Researcher at the State University of Rio Grande do Norte (Assu Campus)

\section{Sergio Schneider}

Possui graduação em Ciências Sociais pela Universidade Federal do Rio Grande do

Sul (UFRGS), mestrado em Sociologia pela Universidade Estadual de Campinas (UNICAMP) e doutorado em Sociologia (UFRGS/Université Paris X). Atualmente é Professor Associado IV e Pesquisador dos Programas de Pós-Graduação em Sociologia e Desenvolvimento Rural da Universidade Federal do Rio Grande do Sul (UFRGS).
Bachelor in Social Sciences from the Federal University of Rio Grande do Sul (UFRGS), Master in Sociology from the State University of Campinas (UNICAMP) and PhD in Sociology (UFRGS/University of Paris X). Currently, he is Associate Professor IV and Researcher of the Postgraduate Programs in Sociology and Rural Development at the Federal University of Rio Grande do Sul (UFRGS). 


\section{Tabela 11.1 - Principais produtos agrícolas, segundo valor da produção e principal Unidade da Federação produtora - 2014}

Table 11.1 - Major agricultural crops, according to the value of production and main producer Federation Unit - 2014

\begin{tabular}{|c|c|c|c|c|c|}
\hline \multirow[b]{2}{*}{$\begin{array}{l}\text { Principais produtos/ } \\
\text { Main products }\end{array}$} & \multirow{2}{*}{$\begin{array}{c}\text { Área } \\
\text { colhida } \\
\text { (ha)/ } \\
\text { Harvested } \\
\text { area } \\
\text { (ha) }\end{array}$} & \multirow{2}{*}{$\begin{array}{l}\text { Quanti- } \\
\text { dade } \\
\text { produ- } \\
\text { zida } \\
\text { (t)/ } \\
\text { Total } \\
\text { production } \\
\text { (tons) }\end{array}$} & \multirow{2}{*}{$\begin{array}{c}\text { Rendi- } \\
\text { mento } \\
\text { médio } \\
\text { (kg/ha)/ } \\
\text { Average } \\
\text { yield } \\
(\mathrm{kg} / \mathrm{ha})\end{array}$} & \multicolumn{2}{|c|}{$\begin{array}{l}\text { Principal produtor/ } \\
\text { Major producer }\end{array}$} \\
\hline & & & & $\begin{array}{l}\text { Unidades } \\
\text { da } \\
\text { Federação/ } \\
\text { Federation } \\
\text { Units }\end{array}$ & $\begin{array}{l}\text { Quantidade } \\
\text { produzida } \\
\text { (t)/ } \\
\text { Production } \\
\text { (tons) }\end{array}$ \\
\hline $\begin{array}{l}\text { Soja (em grão)/ } \\
\text { Soybeans (grain) }\end{array}$ & 30273763 & 86760520 & 2866 & Mato Grosso & 26495884 \\
\hline $\begin{array}{l}\text { Cana-de-açúcar/ } \\
\text { Sugarcane }\end{array}$ & 10437567 & 737155724 & 70625 & São Paulo & 401332100 \\
\hline $\begin{array}{l}\text { Milho (em grão)/ } \\
\text { Corn (grain) }\end{array}$ & 15431709 & 79877714 & 5176 & Mato Grosso & 18071316 \\
\hline $\begin{array}{l}\text { Café (em grão)/ } \\
\text { Coffee beans }\end{array}$ & 1997827 & 2804070 & 1404 & Minas Gerais & 1364409 \\
\hline $\begin{array}{l}\text { Mandioca/ } \\
\text { Cassava }\end{array}$ & 1567683 & 23242064 & 14826 & Pará & 4914831 \\
\hline $\begin{array}{l}\text { Arroz (em casca)/ } \\
\text { Paddy rice }\end{array}$ & 2340878 & 12175602 & 5201 & Rio Grande do Sul & 8241840 \\
\hline $\begin{array}{l}\text { Algodão herbáceo (em caroço)/ } \\
\text { Upland cotton seed }\end{array}$ & 1129399 & 4236763 & 3751 & Mato Grosso & 2384448 \\
\hline $\begin{array}{l}\text { Fumo (em folha)/ } \\
\text { Tobacco (leaves) }\end{array}$ & 415842 & 862396 & 2074 & Rio Grande do Sul & 412618 \\
\hline $\begin{array}{l}\text { Bananal } \\
\text { Bananas }\end{array}$ & 478060 & 6946567 & 14531 & Bahia & 1088647 \\
\hline $\begin{array}{l}\text { Laranjal } \\
\text { Oranges }\end{array}$ & 680268 & 16927637 & 24884 & São Paulo & 12290107 \\
\hline $\begin{array}{l}\text { Tomate/ } \\
\text { Tomatoes }\end{array}$ & 64363 & 4302777 & 66852 & Goiás & 1055337 \\
\hline $\begin{array}{l}\text { Feijão (em grão)/ } \\
\text { Beans seed }\end{array}$ & 3185745 & 3294586 & 1034 & Paraná & 813623 \\
\hline $\begin{array}{l}\text { Batata-inglesa/ } \\
\text { Potatoes }\end{array}$ & 132058 & 3689836 & 27941 & Minas Gerais & 1200359 \\
\hline $\begin{array}{l}\text { Trigo (em grão)/ } \\
\text { Wheat (grain) }\end{array}$ & 2834945 & 6261895 & 2209 & Paraná & 3816201 \\
\hline $\begin{array}{l}\text { Uva/ } \\
\text { Grapes }\end{array}$ & 78753 & 1453889 & 18461 & Rio Grande do Sul & 812517 \\
\hline
\end{tabular}

Fonte/Source: Produção agrícola municipal 2014. In: IBGE. Sidra: sistema IBGE de recuperação automática. Rio de Janeiro, [2015]. Disponível em/Available from :<http://www.sidra.ibge.gov.br/bda/>. Acesso em: jan. 2016/Cited: Jan. 2016. 
Tabela 11.2 - Efetivo dos rebanhos e das aves - 2013-2014

Table 11.2 - Number of livestock and poultry on farms - 2013-2014

\begin{tabular}{|c|c|c|}
\hline \multirow{2}{*}{$\begin{array}{l}\text { Tipos/ } \\
\text { Type }\end{array}$} & \multicolumn{2}{|c|}{ Efetivos ( $1000 /$ cabeças)/Number ( 1,000 heads) } \\
\hline & 2013 & 2014 \\
\hline $\begin{array}{l}\text { Bovinos/ } \\
\text { Cattle }\end{array}$ & 211764 & 212344 \\
\hline $\begin{array}{l}\text { Bubalinos/ } \\
\text { Buffaloes }\end{array}$ & 1332 & 1319 \\
\hline $\begin{array}{l}\text { Equinos/ } \\
\text { Horses }\end{array}$ & 5312 & 5451 \\
\hline $\begin{array}{l}\text { Suínos - total (1)/ } \\
\text { Hogs and pigs - total (1) }\end{array}$ & 36744 & 37929 \\
\hline $\begin{array}{l}\text { Suínos - matrizes de suínos/ } \\
\text { Hogs and pigs - breeding sows }\end{array}$ & 4615 & 4753 \\
\hline $\begin{array}{l}\text { Caprinos/ } \\
\text { Goats }\end{array}$ & 8779 & 8852 \\
\hline $\begin{array}{l}\text { Ovinos/ } \\
\text { Sheep }\end{array}$ & 17291 & 17614 \\
\hline $\begin{array}{l}\text { Galináceos (2)/ } \\
\text { Poultry }\end{array}$ & 1248786 & 1331054 \\
\hline $\begin{array}{l}\text { Galinhas/ } \\
\text { Hens }\end{array}$ & 221862 & 226616 \\
\hline $\begin{array}{l}\text { Codornas/ } \\
\text { Quails }\end{array}$ & 18172 & 20339 \\
\hline
\end{tabular}

Fonte/Source: Pesquisa da pecuária municipal 2013-2014. In: IBGE. Sidra: sistema IBGE de recuperação automática. Rio de Janeiro, [2015]. tab. 73, 3939. Disponível em/Availabe from: <http://www.sidra.ibge. gov.br/bda/>. Acesso em: jan. 2016/Cited: Jan. 2016.

(1) Inclui matrizes de suínos. (2) Inclui galinhas./(1) Including breeding sows; (2) Include hens. 


\section{Tabela 11.3 - Quantidade e valor dos produtos de origem animal e}

variação anual - 2013-2014

Table 11.3 - Amount and value of products of animal origin and annual variation - 2013-2014

\begin{tabular}{|c|c|c|c|c|c|c|}
\hline \multirow[t]{2}{*}{$\begin{array}{l}\text { Produtos/ } \\
\text { Products }\end{array}$} & \multicolumn{2}{|c|}{$\begin{array}{l}\text { Quantidade produzida/ } \\
\text { Total production }\end{array}$} & \multirow{2}{*}{$\begin{array}{c}\text { Variação/ } \\
\text { Variation } \\
2014 / \\
2013\end{array}$} & \multicolumn{2}{|c|}{$\begin{array}{l}\text { Valor da produção/ } \\
\text { Value of production } \\
\qquad(1000 \mathrm{R} \$)\end{array}$} & \multirow{2}{*}{$\begin{array}{c}\text { Variação/ } \\
\text { Variation } \\
2014 / \\
2013\end{array}$} \\
\hline & 2013 & 2014 & & 2013 & 2014 & \\
\hline $\begin{array}{l}\text { Leite ( } 1000 \text { litros }) / \\
\text { Milk (1,000 liters) }\end{array}$ & 34255236 & 35174271 & 2,7 & 32417960 & 33785670 & 4,2 \\
\hline $\begin{array}{l}\text { Ovos de galinha ( } 1000 \text { dúzias)/ } \\
\text { Hen eggs ( } 1,000 \text { dozens) }\end{array}$ & 3619217 & 3734257 & 3,2 & 8302291 & 9354322 & 12,7 \\
\hline $\begin{array}{l}\text { Ovos de codorna ( } 1000 \text { dúzias)/ } \\
\text { Quail eggs ( } 1,000 \text { dozens) }\end{array}$ & 342503 & 392725 & 14,7 & 281411 & 312219 & 10,9 \\
\hline $\begin{array}{l}\text { Mel de abelha }(\mathrm{t}) / \\
\text { Honey (tons) }\end{array}$ & 35365 & 38472 & 8,8 & 263195 & 316229 & 20,2 \\
\hline $\begin{array}{l}\text { Casulos de bicho-da-seda (t)/ } \\
\text { Silkworn cocoons (tons) }\end{array}$ & 2709 & 2682 & $-1,0$ & 34818 & 41246 & 18,5 \\
\hline $\begin{array}{l}\text { Lã (t)/ } \\
\text { Wool (tons) }\end{array}$ & 12041 & 11851 & $-1,6$ & 88122 & 84264 & $-4,4$ \\
\hline $\begin{array}{l}\text { Peixes (t)/ } \\
\text { Fish (tons) }\end{array}$ & 392493 & 474329 & 20,9 & 2020922 & 2714553 & 34,4 \\
\hline $\begin{array}{l}\text { Camarão (t)/ } \\
\text { Shrimp (tons) }\end{array}$ & 64669 & 65018 & 0,5 & 765014 & 793567 & 3,7 \\
\hline
\end{tabular}

Fonte/Source: Pesquisa da pecuária municipal 2013-2014. In: IBGE. Sidra sistema IBGE de recuperação automática. Rio de Janeiro, [2015]. tab. 74. Disponível em/Available from: <http://www.sidra.ibge.gov. br/bda/>. Acesso em: jan. 2016/Cited: Jan. 2016. 
Tabela 11.4 - Produção madeireira da extração vegetal e da

silvicultura - 2013-2014

Table 11.4 - Production from wood wild crop harvesting and silviculture

2013-2014

\begin{tabular}{ccc}
\hline Produtos/ & \multicolumn{2}{c}{ Quantidade obtida/ Total production } \\
\cline { 2 - 3 } Products & 2013 & 2014 \\
\hline
\end{tabular}

Extração vegetal/Wild crop harvesting

Carvão vegetal (t)/

1006254

1021062

Charcoal (tons)

Lenha $\left(\mathrm{m}^{3}\right) /$

30955224

28907314

Firewood (cubic meters)

Madeira em tora $\left(\mathrm{m}^{3}\right) /$

13519281

13807066

Logwood (cubic meters)

Silvicultura/ Silviculture

Carvão vegetal (t)/

5583166

6219325

Charcoal (tons)

Lenha $\left(\mathrm{m}^{3}\right) /$

55392485

56167873

Firewood (cubic meters)

Madeira em tora $\left(\mathrm{m}^{3}\right)$ /

129641245

124239602

Logwood (cubic meters)

Para papel e celulose $\left(\mathrm{m}^{3}\right) /$

72470855

72389306

For paper and pulp $\left(\mathrm{m}^{3}\right)$

Para outras finalidades $\left(\mathrm{m}^{3}\right)$ /

57170390

51850296

For other uses $\left(\mathrm{m}^{3}\right)$

Fonte/Source: Produção da extração vegetal e da silvicultura 2013-2014. In: IBGE. Sidra sistema IBGE de recuperação automática. Rio de Janeiro, [2015]. tab. 291. Disponível em/Available from: <http://www. sidra.ibge.gov.br/bda/>. Acesso em: jan. 2016/Cited: Jan. 2016. 


\section{Gráfico 11.1 - Área plantada, quantidade produzida e valor da produção de cereais, leguminosas e oleaginosas - 2004-2014}

Graph 11.1 - Planted area, amount produced and value of production of cereals, legumes and oilseeds - 2004-2014

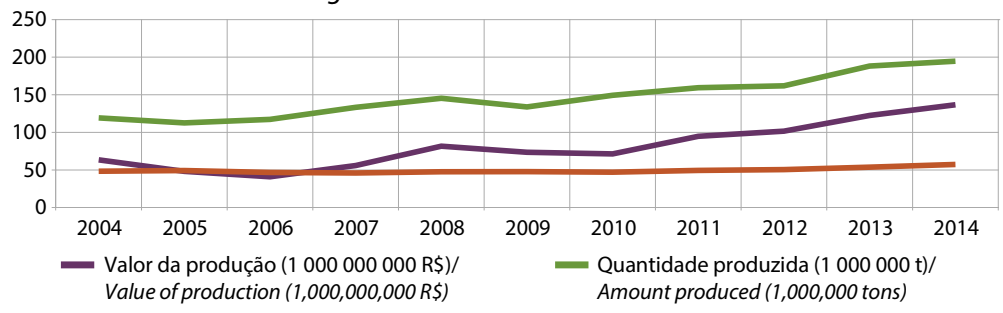

- Área plantada (1 000000 ha)/

Planted area $(1,000,000$ ha)

Fonte/Source: Produção Agrícola Municipal 2004-2014. In: IBGE. Sidra: sistema IBGE de recuperação automática. Rio de Janeiro, [2015]. Disponível em/Available from:<http://www.sidra.ibge.gov.br/bda/>. Acesso em: jan. 2016/Cited: Jan 2016.

Nota: Compreende a produção de algodão arbóreo (em caroço), algodão herbáceo (em caroço), amendoim em casca, arroz em casca, aveia em grão, centeio em grão, cevada em grão, feijão em grão, girassol em grão, mamona, milho em grão, soja em grão, sorgo em grão, trigo em grão e triticale em grão./Note: Comprises the production of tree cotton (in seed), upland cotton (in seed), peanuts (in shell), rice (in the husk), oat (grain), rye (grain), barley (grain), beans (grain), and sunflower (grain), castor beans, corn (grain), soybeans (grain), sorghum (grain), wheat (grain) and triticale (grain).

\section{Gráfico 11. 2 - Participação das Unidades da Federação no valor da produção agrícola - 2014}

Graph 11.2 - Brazilian states participation in the value of agricultural production - 2014

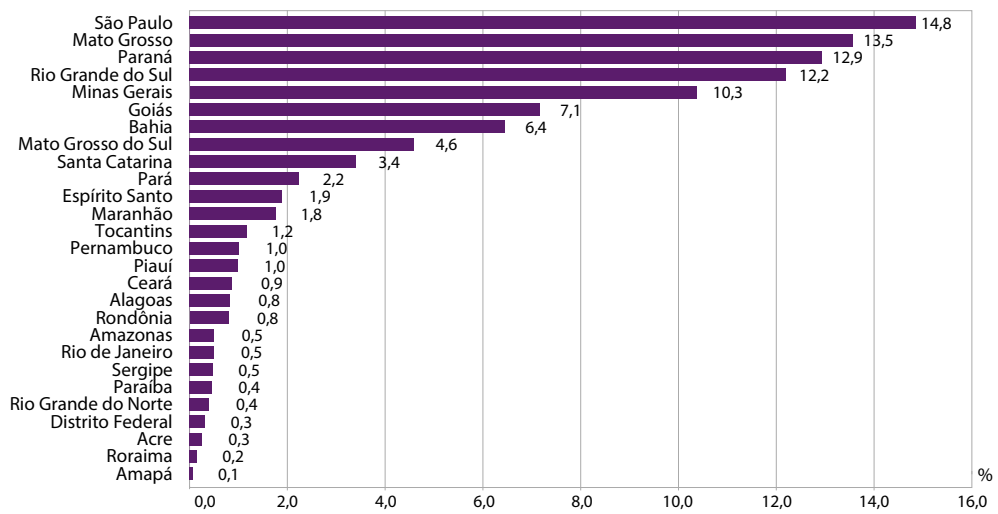

Fonte/Source: Produção Agrícola Municipal 2014. In: IBGE. Sidra: sistema IBGE de recuperação automática. Rio de Janeiro, [2015]. Disponível em/Available from:<http://www.sidra.ibge.gov.br/bda/>. Acesso em: jan 2016/Cited: Jan 2016. 


\section{Gráfico 11.3 - Evolução da capacidade útil de armazenagem - 2005-2015 \\ Graph 11.3 - Evolution of storage capacity - 2005-2015}

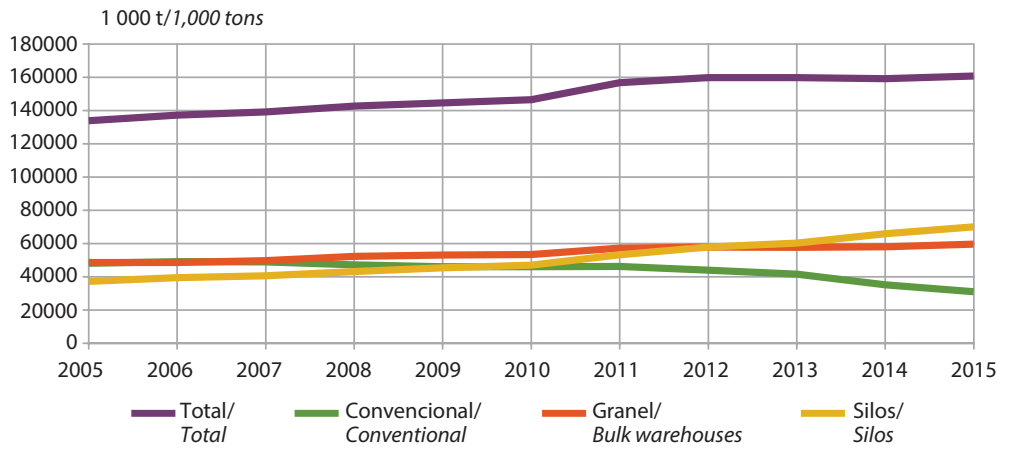

Fonte/Source: Pesquisa de Estoques 2005-2015. Brasil. Rio de Janeiro: IBGE, n. 1, pt.1, jan./jun. 2005-2015. Disponível em/Available from: <ftp://ftp.ibge.gov.br/Estoque/>. Acesso em: jan. 2016/Cited: Jan. 2016.

Gráfico 11.4 - Peso das carcaças - 2009-2014

Graph 11.4 - Weight of carcasses - 2009-2014

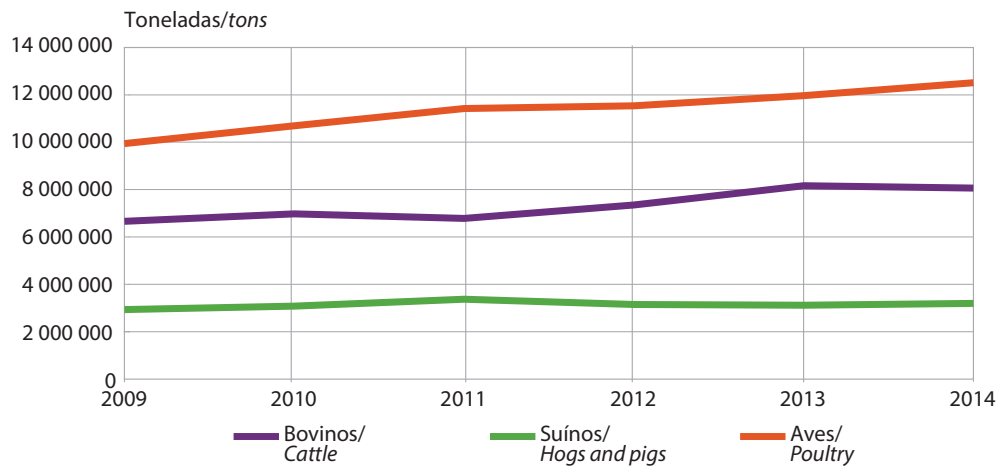

Fonte/Source: Pesquisa trimestral do abate de animais 2009-2014. In: IBGE. Sidra: sistema IBGE de recuperação automática. Rio de Janeiro, [2009-2015]. tab. 1092. Disponível em/Availabe from: <http://www.sidra. ibge.gov.br/bda/>. Acesso em: jan. 2016/Cited: Jan. 2016. 


\section{Gráfico 11.5 - Participação das principais espécies \\ na produção da piscicultura - 2014 \\ Graph 11.5 - Participation of the main species \\ in the production of fish farming - 2014}

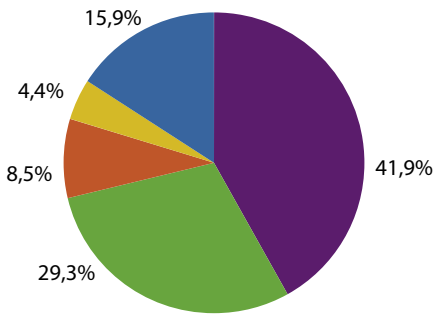

Tilápia/Tilapia

Tambaqui/Tambaqui

Tambacu e Tambatinga/

Tambacu and Tambatinga

\section{Carpa/Carp}

Outros peixes/Other fishes

Fonte/Source: Pesquisa da pecuária municipal 2014. In: IBGE. Sidra: sistema IBGE de recuperação automática. Rio de Janeiro, [2015]. tab. 3940. Disponível em/Availabe from: <http://www.sidra.ibge.gov.br/bda/>. Acesso em: jan. 2016/Cited: Jan. 2016.

\section{Gráfico 11.6 - Área total existente em 31.12 dos efetivos da silvicultura, por Grandes Regiões - 2014}

Graph 11.6 - Total area existing in 31.12 of silviculture effective, by Major Regions - 2014

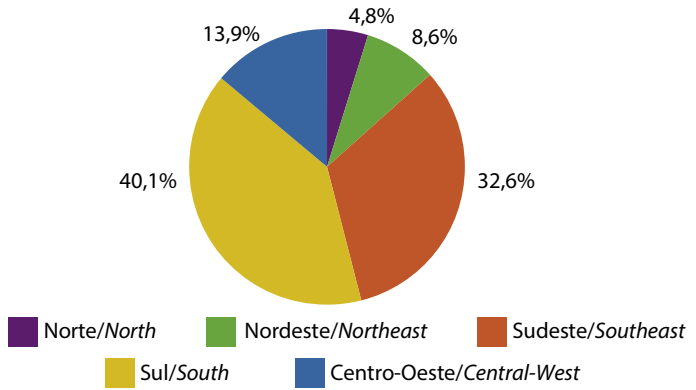

Fonte/Source: Produção da Extração Vegetal e da Silvicultura 2014. In: IBGE. Sidra sistema IBGE de recuperação automática. Rio de Janeiro, [2015]. Disponível em/Available from: <http://www.sidra.ibge. gov.br/bda/>. Acesso em: jan. 2016/Cited: Jan. 2016.

Nota: Compreende a área total dos cultivos florestais de eucalipto, pinus e outras espécies./ Note: Comprises the total area of eucalyptus, pine and other species forest cultivation. 


\section{Indústria Industry}

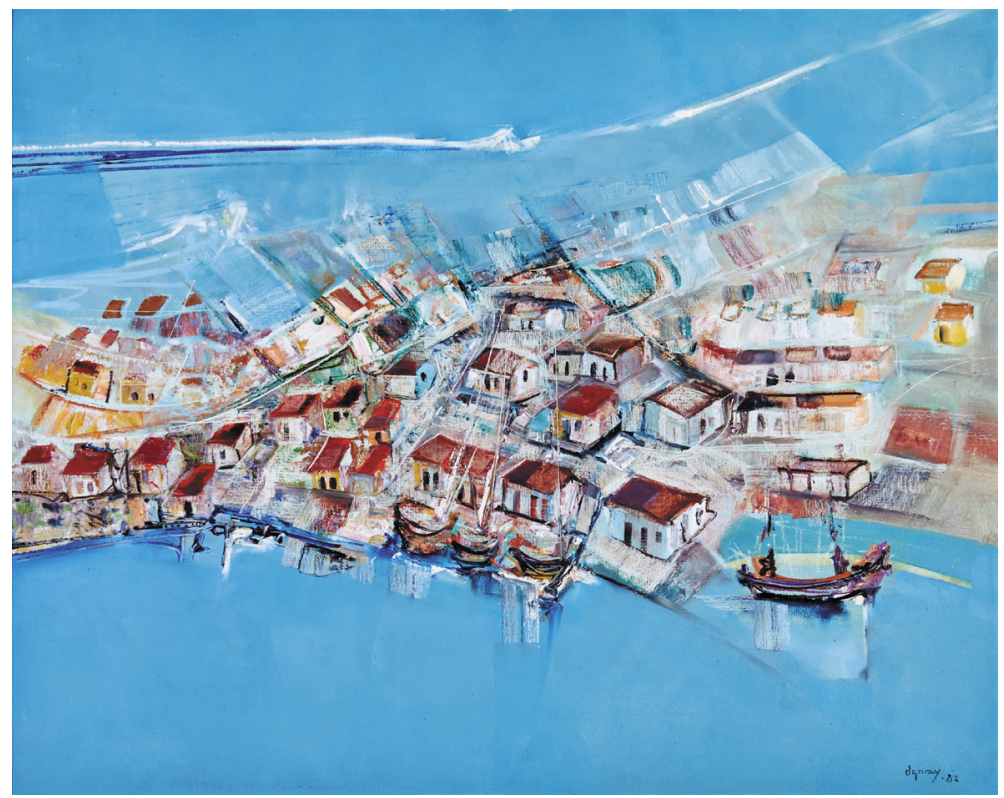

Casario rio/Row houses river flow, 1982 Dorian Gray Caldas 



\section{Indústria}

A Indústria brasileira apresentou em 2015 seu pior desempenho histórico, superando até mesmo a evolução negativa de 2009 em função dos desdobramentos da crise financeira internacional iniciada em 2007-2008 nos EUA e propagada para o resto do mundo. Em 2015, o produto industrial teve uma brutal queda de $-8,3 \%$, acelerando a tendência de queda verificada no ano anterior $(-3 \%)$. Cabe destacar também a sensível piora nos indicadores do segundo semestre com relação aos do primeiro (tabela 2.1.). Os determinantes da forte contração industrial em 2015 são de ordem econômica e política.

No plano econômico, o padrão de crescimento sustentado nas maiores exportações de commodities desde o início dos anos 2000, em função da vigorosa demanda chinesa, e, posteriormente, na expansão do consumo doméstico, puxado pela ampliação da massa salarial e do crédito; e, em menor medida, nos gastos públicos foi perdendo força e não foi acompanhado pela necessária expansão dos investimentos industriais e em infraestrutura.

\section{Industry}

The Brazilian industry experienced in 2015 its weakest performance ever, bad enough to overshadow the negative evolution of 2009, as a consequence of the world financial crisis that began in 2007-2008 in the USA and which spread to all parts of the world. Industrial production faced a drastic decrease, $-8.3 \%$, which accelerated the downward trend as observed in the previous year $(-3 \%)$. Also worth of mention is the significant worsening of second quarter indicators versus first quarter ones (table 2.1). Economic and political reasons accounted for the severe contraction of industry in 2015.

At the economic level, the pattern of increase - sustained by the major commodity exports since the beginning of the 2000s, due to the strong demand from China, and later, by the expansion of domestic consumption, due to the increase of the wage bill and the expansion of credit, and, to a lower extent, due to public expenditure - gradually lost relevance and was not followed by any increase of investments in either industry or infrastructure. 
O breve ciclo de investimento de 2005-2008 foi interrompido pela crise financeira internacional. As políticas de incentivos à recuperação dos investimentos e da rentabilidade da atividade produtiva, a partir de 2009, não foram capazes de mudarem as expectativas negativas dos empresários, que optaram por estratégias defensivas de suprir com maiores importações o consumo interno, dado que os produtos importados foram favorecidos pela valorização cambial; e de ampliar o grau de liquidez da sua estrutura de ativos. A busca por maior liquidez, não ampliando nem renovando o capital fixo depreciado, e um maior grau de endividamento externo visavam compensar com ganhos financeiros a retração dos ganhos operacionais, através da arbitragem do diferencial de juros doméstico e internacional. Essas estratégias defensivas postergaram e/ou reduziram a ampliação dos investimentos produtivos.

O aumento da dívida pública em relação ao PIB, resultante sobretudo das políticas anticíclicas e dos juros elevados, contribuiu para desacelerar os investimentos públicos e para ampliar as incertezas e a desconfiança empresarial com relação à condução da política econômica, ampliando as pressões por um ajuste fiscal. A política de austeridade reduziu ainda mais a demanda agregada e, consequentemente, as receitas tributárias, ampliou o endividamento público e lançou o país numa recessão econômica sem precedentes, com fortes impactos negativos sobre a indústria.

No plano político, a concentração das atividades do Executivo e do Congresso em torno do processo de
The brief cycle of investment of 20052008 was interrupted by the world financial crisis. Policies of incentive to the recovery of investments and of production profitability from 2009 on were not capable of changing the negative expectations of businessmen, who made an option for defensive strategies: to supply the domestic market with more imports, since these products had benefited from currency appreciation; and to expand the liquidity of their asset structure. The search for increased liquidity, without expansion or renewal of the depreciated fixed capital, and the higher foreign indebtedness were aimed at the compensation of losses in operational gains by means of financial gains and the arbitrage of domestic and international interest differences. Such defensive strategies delayed and/ or reduced the expansion of productive investments.

The increase of public debt in relation to GDP, resulting mainly from anticyclical policies and high interests, has contributed to the deceleration of public investments and to the increase of uncertainty and distrust in relation to the economic policy adopted, thus intensifying the pressure for a fiscal adjustment. Austerity measures reduced the aggregated demand even more - and, as a consequence, tax revenues -, increased public indebtedness and threw the country into an unprecedented economic recession, with strong impacts on industry.

At the political level the concentration of activities related to the impeachment of President Dilma in the House of 
impedimento da presidenta Dilma, bem como as ações da justiça no combate à corrupção e às relações espúrias entre os setores público e privado, envolvendo as principais estatais e empreiteiras do país, contribuíram para desacelerar ainda mais os investimentos, sobretudo na Petrobras. Importante destacar que a Petrobras foi responsável, nos últimos dez anos, desde a descoberta do Pré-sal, por aproximadamente $10 \%$ do investimento total no país.

Assim como em 2014, o desempenho da indústria extrativa $(3,9 \%)$ impediu uma queda ainda maior da produção industrial. A contribuição positiva deveuse, primeiro, ao aumento do volume de produção de petróleo, sobretudo na área do Pré-sal, colhendo frutos de investimentos anteriores, em que pese toda a crise financeira e política envolvendo a Petrobras. Segundo, ao aumento do volume de produção de minério de ferro pela empresa Vale, apesar da desaceleração da demanda chinesa. Embora a produção tenha se expandido em volume para os dois produtos, atingindo recordes históricos de produção em 2015, a evolução decrescente dos preços teve impacto negativo no valor da produção e na rentabilidade, sobretudo no segundo semestre.

Foi na indústria de transformação que a crise mostrou sua face mais perversa com uma expressiva queda de $-9,9 \%$, que foi generalizada para todos os setores industriais, sem uma única exceção (tabela 12.1). Como essa queda se deu a partir de um patamar já bastante baixo, dado que o produto manufatureiro havia encolhido $-4,2 \%$ em 2014, o nível de produção no final de 2015 ficou abaixo daquele
Representatives and the Executive Power, as well as the legal measures taken to combat corruption and the illegitimate relationships between private and public sectors including the main state companies and construction contractors in the country, have contributed to the deceleration of investments, especially at Petrobras. It is worth highlighting that, in the last ten years, since the discovery of the pre-salt layer, Petrobras has been responsible for $10 \%$ of the overall investment in the country.

As in 2014, the performance of mining and quarrying industry (3.9\%) prevented an even greater decrease of the industrial output. The main positive contribution came, firstly, from the increased production of oil, mainly in the area of the pre-salt layer, which reaped the benefits from previous investments in spite of the financial and political crisis in Petrobras. Secondly, from the increased volume of iron ore produced by Vale, in spite of the deceleration of Chinese demand. Although both products have recorded increase of production volume, reaching record output figures in 2015, the decreasing evolution of prices had a negative impact on the volume of production and on the profitability, mainly in the second semester.

It was in manufacturing industry that the crisis presented its darkest side, with significant decrease of $-9.9 \%$ which spread to all the sectors of industry, without an exception (table 12.1). The level of industrial production was already low before this result, because of the contraction of manufacturing by $-4.2 \%$ in 2014 , so the production in the end of 2015 fell to a level below that of 
verificado no auge da crise em 2009 e recuou para um patamar muito próximo do início da década dos anos 2000, ou seja, um retrocesso de quase 15 anos.

Os dois setores industriais com piores desempenhos em 2015 foram os de equipamentos de informática e produtos eletrônicos (-30\%) e automobilístico $(-26 \%)$. Cabe destacar que esses dois setores já haviam apresentado um desempenho negativo em 2014 $(-3,1 \%$ e $-16,8 \%$, respectivamente), que contrasta com o forte dinamismo apresentado nos anos de expansão do consumo. A capacidade de arrasto do setor automobilístico na economia é muito elevada. Portanto, uma crise nesse setor tem impactos negativos sobre a produção e emprego de vários setores industriais e de serviços. Outros setores industriais com peso importante na produção e/ou no emprego com expressivo desempenho negativo em 2015 e 2014 foram os de máquinas e equipamentos e máquinas, aparelhos e materiais elétricos, refletindo, sobretudo, a contração dos investimentos, mas também a elevação do coeficiente importado no segmento. Dentre os setores mais tradicionais, aqueles com pior desempenho foram os de têxteis e de vestuário. $O$ desempenho negativo do setor de produtos de metal deveu-se à desaceleração dos investimentos em geral e na construção civil em particular. O impacto negativo sobre o emprego industrial só não foi maior porque o setor de alimentos, bastante heterogêneo e diversificado, e maior empregador na indústria de transformação, teve uma das menores quedas de produção $(-2,3 \%)$, atrás apenas do setor de celulose e papel $(-0,7 \%)$, que passou recentemente por um ciclo de investimento e foi favorecido the height of the crisis, in 2009 and matched figures similar to those in the 2000 s, which meant a step fifteen years backwards.

Among the industrial sectors, computer equipment and electronic products $(-30 \%)$ and cars $(-26 \%)$ had the weakest performances in 2015. It is worth highlighting that these two sectors had already recorded negative results in $2014(-3.1 \%$ and $-16.8 \%$, respectively), opposite to the dynamism observed in the years of consumption expansion. The car sector exerts strong influence on the economy. Therefore, a crisis in this sector brings negative impacts to production and employment in many industrial and services sectors. Other relevant sectors in terms of output and employment with a significantly negative performance in 2015 and 2014 were machinery and equipment and electrical machinery and apparatuses. They reflected the contraction of investments, but also the rise of the import coefficient in this segment. Among traditional sectors, the weakest performances were those of textiles and wearing apparel. The negative performance of metal products resulted from the deceleration of investments in general and in civil construction, in particular. The negative impact on industrial employment could have been bigger, but the food sector, which, besides being very heterogeneous and diverse, is a major employer in manufacturing industry, recorded one of the lowest output decreases $(-2.3 \%)$, only second to pulp and paper $(-0.7 \%)$, which has lately been part of an investment cycle and was favored by the 
pela recente desvalorização cambial e a consequente expansão das exportações.

Como visto, a recessão econômica de 2015 afetou e foi afetada pela contração dos gastos em consumo e em investimento. A queda acentuada nos investimentos industriais, em infraestrutura e na construção civil tanto públicos quanto privados teve impactos bastante negativos sobre a produção no segmento de bens de capital $(-25,5 \%)$, cujo desempenho em 2014 já tinha sido bastante negativo (-9,3\%). A segunda pior queda foi no segmento de bens de consumo duráveis $(-18,7 \%)$, acentuando a queda de $-9,1 \%$ em 2014. Dentro dessa categoria de uso encontram-se os produtos de maior valor que dependem muito da evolução da renda e das condições de crédito (juro e prazo), como é o caso de automóveis. O segmento de bens de consumo semiduráveis e nãoduráveis é fortemente condicionado pela evolução do poder aquisitivo e da massa salarial (emprego e salário). $O$ aumento das taxas de desemprego, a redução dos salários reais e o acirramento da inflação ao longo de 2015 contribuíram para a redução da demanda e da produção nesse segmento $(-6,7 \%)$. Finalmente, a categoria de bens intermediários teve uma redução de $-5,2 \%$, mais que dobrando a taxa de contração em 2014 $(-2,4 \%)$, refletindo a retração da demanda dos demais setores e também o aumento do conteúdo importado dos bens de consumo e de capital (gráfico 12.1).

A tabela 12.3 ilustra o desempenho de alguns produtos com elevado peso na estrutura industrial. Enquanto aço bruto, máquinas agrícolas e automóveis tiveram uma forte redução no volume de produção entre 2013-2015; outros produtos como recent currency depreciation and the subsequent expansion of exports.

As seen above, the economic recession of 2015 affected and was affected by the contraction of expenditure and investment. The sharp decrease in investments in industry, in infrastructure and in civil construction, either in public or private sectors, exerted some negative impacts on the output of the capital goods segment (-25.5\%), whose performance in 2014 had been significantly negative (-9.3\%). The second negative decrease was recorded by durable consumer goods (-18.7\%), even worse than in 2014 (-9.1\%). Within this category of use, we can find the most expensive products which depend a lot on the evolution of income and on credit conditions (interest rate and maturity date), as it is the case of cars. The segment of semi-durable and non-durable goods is conditioned by the evolution of the purchasing power and of the wage bill (employment and wages). The increase of unemployment rates, the decline of real wages and the worsening of inflation throughout 2015 have contributed to the reduction of demand and of production in this segment (-6.7\%). Finally, the category of intermediate goods had a decrease of $-5.2 \%$, more than twice the rate of decrease in $2014(-2.4 \%)$, reflecting the retraction of demand of the other sectors and also the increase of the imported content of capital and consumer goods (graph 12.1).

Table 12.3 illustrates the performance of some relevant products in the industrial structure. Whereas crude steel, agricultural machinery and cars faced significant decrease in volume of production between 2013 and 2015, 
petróleo, gás natural e celulose ampliaram seu volume de produção. De fato, a queda mais pronunciada foi na produção de automóveis de passageiros. Em 2013, o Brasil atingiu o patamar de 2,9 milhões de unidades produzidas, o que posicionava o país como o $7^{\circ}$ maior produtor mundial, atrás apenas de China, Japão, Alemanha, EUA, Coreia do Sul e Índia. O Brasil reduziu sua produção de automóveis para 2,5 milhões em 2014 e para 2 milhões em 2015, perdendo sua posição para a Espanha (2,2 milhões). No mesmo período, a produção mundial saltou de 65,7 milhões em 2013 para 68,5 milhões em 2015. A China expandiu sua produção de 18 milhões para 21 milhões de unidades. Importante destacar que dentre os 10 maiores produtores globais, apenas EUA (4,4 milhões para 4,2 milhões), Japão ( 8,2 milhões para 7,8 milhões) e Brasil (2,9 milhões para 2 milhões) reduziram sua produção entre 2013 e 2015, ainda assim as retrações para EUA e Japão foram bem inferiores à brasileira.

O setor de siderurgia no Brasil vem sendo negativamente afetado nos últimos dois anos pelo fraco desempenho do setor da construção residencial e pesada e pela crise nos setores automobilístico e de bens de capital. Além disso, a estagnação da demanda internacional e a agressiva concorrência chinesa contribuem para agravar ainda mais o quadro do setor. Ainda assim, o Brasil segue líder absoluto na América Latina na produção de aço (33,9 milhões de toneladas) em 2014 (gráfico 12.3). A rigor, a soma da produção de todos os países latinoamericanos é inferior à produção brasileira.

A produção de aço nos três maiores produtores na América Latina (Brasil, other products such as oil, natural gas and pulp recorded increases. Passenger automobiles recorded the biggest output decline. In 2013, Brazil reached a total 2.9 million units produced, and was placed as the 7 th biggest manufacturer in the world, behind China, Japan, Germany, the USA, South Korea and India. Brazil reduced its car output to 2.5 million in 2014 and to 2 million in 2015, and lost its position to Spain (2.2 million). In the same period, the world production rose from 65.7 million in 2013 to 68.5 million in 2015. China expanded its output from 18 to 21 million units. It is important to highlight that among the 10 biggest producers in the world, only the USA (from 4.4 million to 4.2 million), Japan (from 8.2 million to 7.8 million) and Brazil (from 2.9 million to 2 million) recorded output decrease between 2013 and 2015. Even so, the decreases recorded by the USA and Japan were not as low as those of Brazil.

The steel sector in Brazil has been negatively affected, in the last two years, by the weak performance of the residential and heavy construction sectors and by the crisis in the sectors of cars and capital goods. Besides, the stagnation of the international demand and the strong Chinese competition have contributed to aggravate this scenario. Even so, Brazil kept on leading the steel production in Latin America (with 33.9 million metric tons) in 2014 (graph 12.3). In fact, the Brazilian output surpasses the amount produced by all the other Latin American countries together.

The production of steel in the three main Latin American producers 
México e Argentina) segue estagnada nos últimos anos, em torno de 58 a 59 milhões de toneladas. Não é o caso da produção na China, de longe a maior produtora mundial de aço. De 2011 para 2014, a produção chinesa saltou de 702 milhões de toneladas para 822,7 milhões (gráfico 12.2.). Ou seja, apenas o acréscimo de produção em quatro anos corresponde ao dobro da produção total dos três líderes latino-americanos. Além disso, estima-se que a China tenha uma elevada capacidade ociosa no setor siderúrgico. $O$ resultado tem sido uma política muito agressiva de exportação, impondo um enorme desafio aos demais produtores. A produção de aço no Japão ( 110 milhões de toneladas) e nos EUA (88 milhões), respectivamente o segundo e terceiro maiores produtores, também segue relativamente estagnada. A Índia, quarto maior produtor, é o único país, além da China, dentro do grupo dos dez maiores produtores, que vem ampliando de forma significativa sua produção, que saltou de 73,5 milhões para 86,5 milhões de toneladas em 2014. Nos casos da China e da Índia, além das exportações, os investimentos associados ao processo de urbanização, sobretudo em infraestrutura, explicam em grande parte a demanda por aço, e, em menor medida, o dinamismo do segmento metal-mecânico.

A produção industrial é ainda bastante concentrada na região Sudeste $57,5 \%$ do valor da produção, $59,2 \%$ do valor da transformação industrial, 51,8\% do emprego e $62,6 \%$ do total de salários em 2013) que contempla os três Estados com os maiores parques industriais do país: São Paulo (36\% do valor da produção), Minas Gerais (11\%) e Rio de Janeiro $(8,4 \%)$. Além do maior
(Brazil, Mexico and Argentina) has remained stagnant in the last few years, around 58 to 59 million metric tons. That is not the case in China, by far the main steel producer in the world: from 2011 to 2014, the Chinese output jumped from 702 million metric tons to 822.7 million metric tons (graph 12.2). That means the increase of production in four years alone corresponds to twice the overall production of three Latin American leaders. Besides, China is estimated to have high spare capacity in the steel sector. The result has been a very strict exports policy, which poses a big challenge on other producers. Steel production in Japan (110 million metric tons) and in the USA (88 million), respectively the second and third major producers, is also relatively stagnant. India, the fourth main producer, is the only country other than China, in a group of three major producers, to have expanded the output significantly, from 73.5 million to 86.5 million metric tons in 2014. In China and India, besides exports, investments associated to the urbanization process, mainly regarding infrastructure, account for most of the demand for steel; and partly explain the dynamism of the metal-mechanic sector.

The Southeast Region still concentrates most industrial output (57.5\% of the value of output, $59.2 \%$ of value of manufacturing, $51.8 \%$ of employment and $62.6 \%$ of total salaries in 2013), covering three states that house the biggest industrial parks in the country: São Paulo ( $36 \%$ of the value of output), Minas Gerais (11\%) and Rio de Janeiro (8.4\%). Besides their 
contingente de assalariados industriais, cabe destacar também que o salário industrial médio de São Paulo (24\%) e Rio de Janeiro (61\%) são bem superiores à média brasileira (tabela 12.4).

A região Sul vem a seguir com $21,2 \%$ do valor da produção, $21,3 \%$ do total de salários e $25,6 \%$ do emprego industrial. O salário médio da região é $30 \%$ inferior ao da região Sudeste. Enquanto Rio Grande do Sul $(8,1 \%$ do valor da produção) e Santa Catarina (5\%) apresentaram na última década um baixo dinamismo industrial e perderam participação no produto industrial brasileiro, Paraná ( $8 \%$ do valor da produção e $8,4 \%$ do emprego) apresentou a evolução mais positiva do país, atrás apenas do Estado de Goiás ( $2,9 \%$ do valor da produção e $3 \%$ do emprego), que foi beneficiado pelo dinamismo do agronegócio (tabela 12.4).

O bom desempenho da indústria extrativa e dos setores de commodities agrícolas e minerais explicam o maior dinamismo de algumas regiões e Estados (Paraná, Pará, Goiás e Minas Gerais). Já a crise da indústria de transformação, sobretudo nos setores de bens de capital e bens de consumo duráveis, cuja produção é proporcionalmente mais concentrada nos Estados mais industrializados do Sul e Sudeste, explica essa desconcentração industrial pouco virtuosa. A rigor, a desconcentração industrial desejada seria aquela provocada por um dinamismo mais que proporcional nos Estados menos industrializados do Nordeste, Norte e Centro-oeste vis-à-vis os mais industrializados do Sul e Sudeste e não pela desindustrialização dos Estados mais industrializados e do país como um todo. greater number of salaried persons in industry, São Paulo and Rio de Janeiro also pay salaries that surpass the national average by $24 \%$ and $61 \%$, respectively (table 12.4).

The South Region comes in second place with $21.2 \%$ of the value of output, $21.3 \%$ of the total salaries and $25.6 \%$ of employment in industry. Its average salary is 30\% lower than in the Southeast Region. Whereas Rio Grande do Sul ( $8.1 \%$ of the value of output) and Santa Catarina (5\%) had low dynamism and lower participation in the Brazilian industrial product in the last decade, Paraná ( $8 \%$ of output value and $8.4 \%$ of employment) recorded the most positive evolution in the country, only second to the state of Goiás (2.9\% of the output value and $3 \%$ of employment), which benefited from its dynamic agricultural industry (table 12.4).

The positive performance of mining and quarrying industry and of the sectors of agricultural and mineral commodities accounts for the increased dynamism of some Major Regions and states (Paraná, Pará, Goiás and Minas Gerais). The crisis of manufacturing industry, especially in the sectors of capital goods and durable consumer goods, whose production is proportionally more concentrated in the most industrialized states of the South and Southeast, accounts for this deconcentration of industry. In fact, the desired movement would be a more proportional participation of less industrialized states in the Northeast, North and Central West Regions versus the more industrialized one in the South and Southeast. The deindustrialization of the latter is definitely not a desired effect. 
A saída da profunda crise atual

e uma paulatina retomada do

desenvolvimento industrial, abrangendo

uma gama diversificada de setores,

depende incialmente da expansão

dos investimentos, sobretudo em

infraestrutura (transporte, energia, telecomunicações e saneamento), que

apresentam uma forte demanda reprimida e são menos condicionados pelo nível de atividade corrente. Importante destacar que o investimento cumpre o duplo papel de ser, de um lado, um vetor de aumento de eficiência (inovação e produtividade) e de capacidade de produção (escala) e, de outro, um vetor de demanda por um leque amplo de bens e serviços industriais, embora com maior intensidade pelo segmento de bens de capital.

Os efeitos multiplicadores do investimento sobre a renda e o emprego da economia podem contribuir para uma recuperação sustentada do consumo doméstico e seus ganhos de eficiência para uma inserção exportadora mais virtuosa. No caso das exportações industriais, o acirramento da concorrência internacional, sobretudo por parte dos países asiáticos, impõe importantes desafios às estratégias empresariais e às políticas públicas. Mas um fator é e será sempre crucial: a manutenção por um longo período de uma taxa de câmbio competitiva para a indústria. Com relação ao consumo, sua expansão dependerá da recuperação do nível de emprego e da elevação dos salários reais, sobretudo nos salários de base, acompanhando os ganhos de produtividade da indústria e da economia. A expansão da massa salarial e do poder aquisitivo facilitará um novo ciclo de crédito, desde que
The recovery from the current deep crisis and the gradual restart of industrial development in a variety of sectors depends firstly on the expansion of investments, especially in infrastructure (transportation, energy, telecommunications and sanitation), which present a strong unmet demand and are less affected by the level of the current activity. It is important to highlight that investments play a double part. On the one hand, as a vector to increase efficiency (innovation and productivity) and production capacity (scale). On the other, as a vector of demand for a variety of industrial goods and services, though mainly for capital goods.

The effects that multiply investments in income and employment can contribute to a sustained recovery of domestic consumption and to increased efficiency aimed at a more profitable participation in exports. In the case of industrial exports, the aggravation of international competition, mainly from Asian countries, poses important challenges on business strategies and public policies. One aspect remains crucial: the long-term maintenance of a competitive currency rate for industry. In relation to consumption, its expansion will depend on the recovery of employment and on the rise of real wages, especially regarding base salaries, according to the gains of productivity in industry and in the economy. The expansion of wage bills and the purchasing power will facilitate a new cycle of credit, as long as it is followed by more 
acompanhado por prazos e taxas de juros mais compatíveis com os níveis internacionais. compatible maturity dates and interest rates when compared with international levels.

\section{Fernando Sarti}

Professor do Instituto de Economia da UNICAMP, pesquisador do Núcleo de Economia Industrial e da Tecnologia (NEIT) do IEUNICAMP e Diretor Executivo da Fundação de Desenvolvimento da UNICAMP - FUNCAMP.
Professor at the Institute of Economics of UNICAMP, researcher at the Center for Industrial Economics and Technology (NEIT) of IE-UNICAMP and Executive Director of the Foundation for Development of UNICAM-FUNICAMP.

Translated by: Aline Milani Romeiro Pereira 
Tabela 12.1 - Produção industrial, segundo as seções e atividades

de indústria - 2013-2015

Table 12.1 - Industrial output, according to industry sectors

and activities - 2013-2015

(continua/to be continued)

\begin{tabular}{|c|c|c|c|c|c|}
\hline \multirow{3}{*}{$\begin{array}{l}\text { Seções e atividades } \\
\text { de indústria/ } \\
\text { Sections and industry } \\
\text { activities }\end{array}$} & \multicolumn{5}{|c|}{ Produção industrial/ Industrial Output } \\
\hline & \multirow[b]{2}{*}{2013} & \multicolumn{2}{|c|}{2014} & \multicolumn{2}{|c|}{2015} \\
\hline & & $\begin{array}{l}\text { Total } \\
\text { no ano/ } \\
\text { Total in } \\
\text { the year }\end{array}$ & $\begin{array}{c}\text { 10 semes- } \\
\text { tre/ } \\
1 s t \\
\text { semester }\end{array}$ & $\begin{array}{l}\text { Total } \\
\text { no ano/ } \\
\text { Total in } \\
\text { the year }\end{array}$ & $\begin{array}{c}\text { 10 semes- } \\
\text { tre/ } \\
1 s t \\
\text { semester }\end{array}$ \\
\hline $\begin{array}{l}\text { Indústria geral/ } \\
\text { Overall industry }\end{array}$ & 2,10 & $(-) 3,00$ & $(-) 2,40$ & $(-) 8,30$ & (-) 5,90 \\
\hline $\begin{array}{l}\text { Indústria extrativa/ } \\
\text { Mining and quarrying }\end{array}$ & $(-) 3,60$ & 6,80 & 5,00 & 3,90 & 10,40 \\
\hline $\begin{array}{l}\text { Indústrias de transformação/ } \\
\text { Manufacturing }\end{array}$ & 2,80 & $(-) 4,20$ & $(-) 3,20$ & $(-) 9,90$ & (-) 8,00 \\
\hline $\begin{array}{l}\text { Produtos alimentícios/ } \\
\text { Food products }\end{array}$ & 0,60 & (-) 1,00 & 2,70 & (-) 2,30 & (-) 3,00 \\
\hline $\begin{array}{l}\text { Bebidas/ } \\
\text { Beverages }\end{array}$ & (-) 2,10 & 1,30 & 3,70 & (-) 5,40 & (-) 7,10 \\
\hline $\begin{array}{l}\text { Produtos do fumo/ } \\
\text { Tobacco products }\end{array}$ & (-) 8,20 & (-) 1,50 & (-) 6,90 & (-) 9,30 & $(-) 10,70$ \\
\hline $\begin{array}{l}\text { Produtos têxteis/ } \\
\text { Textiles }\end{array}$ & 0,20 & (-) 6,60 & (-) 7,30 & $(-) 14,60$ & (-) 8,60 \\
\hline $\begin{array}{l}\text { Confecção de artigos do vestuário e acessórios/ } \\
\text { Apparel and accessories }\end{array}$ & (-) 0,50 & (-) 3,00 & (-) 2,60 & $(-) 10,80$ & $(-) 10,30$ \\
\hline $\begin{array}{l}\text { Couros, artigos para viagem e calçados/ } \\
\text { Leather, travel articles and footwer }\end{array}$ & 4,30 & (-) 4,20 & (-) 6,20 & (-) 7,50 & (-) 4,30 \\
\hline $\begin{array}{l}\text { Produtos de madeira/ } \\
\text { Wood products }\end{array}$ & 2,00 & (-) 2,60 & (-) 1,30 & (-) 4,60 & (-) 1,80 \\
\hline $\begin{array}{l}\text { Celulose, papel e produtos de papel/ } \\
\text { Pulp, paper and paper products }\end{array}$ & (-) 0,60 & (-) 1,00 & (-) 2,00 & (-) 0,70 & (-) 0,90 \\
\hline $\begin{array}{l}\text { Impressão e reprodução de gravações/ } \\
\text { Printing and reproduction of recorded media } \\
\text { Coque, produtos derivados do petróleo e bio- }\end{array}$ & (-) 4,50 & (-) 3,80 & (-) 8,10 & (-) 17,20 & (-) 10,90 \\
\hline $\begin{array}{l}\text { combustíveis/ } \\
\text { Coke, petroleum products and biofuels } \\
\text { Perfumaria, sabões, detergentes, produtos de }\end{array}$ & 6,50 & 2,30 & 1,70 & (-) 5,90 & (-) 5,20 \\
\hline $\begin{array}{l}\text { limpeza e de higiene pessoal/ } \\
\text { Toiletries, soaps, detergents, cleaning and } \\
\text { personal hygiene products }\end{array}$ & 5,60 & 2,70 & 4,00 & (-) 3,80 & (-) 3,90 \\
\hline $\begin{array}{l}\text { Outros produtos químicos/ } \\
\text { Other chemicals }\end{array}$ & 4,70 & (-) 3,90 & (-) 4,20 & (-) 4,90 & (-) 2,90 \\
\hline $\begin{array}{l}\text { Produtos farmoquímicos e farmacêuticos/ } \\
\text { Pharmaceuticals and chemicals }\end{array}$ & (-) 0,60 & 2,50 & 3,50 & $(-) 12,20$ & (-) 15,70 \\
\hline $\begin{array}{l}\text { Produtos de borracha e de material plástico/ } \\
\text { Rubber and plastics products }\end{array}$ & 0,70 & (-) 3,60 & (-) 2,30 & (-) 9,10 & (-) 6,20 \\
\hline
\end{tabular}


Tabela 12.1 - Produção industrial, segundo as seções e atividades

de indústria - 2013-2015

Table 12.1 - Industrial output, according to industry sectors

and activities - 2013-2015

(conclusão/concluded)

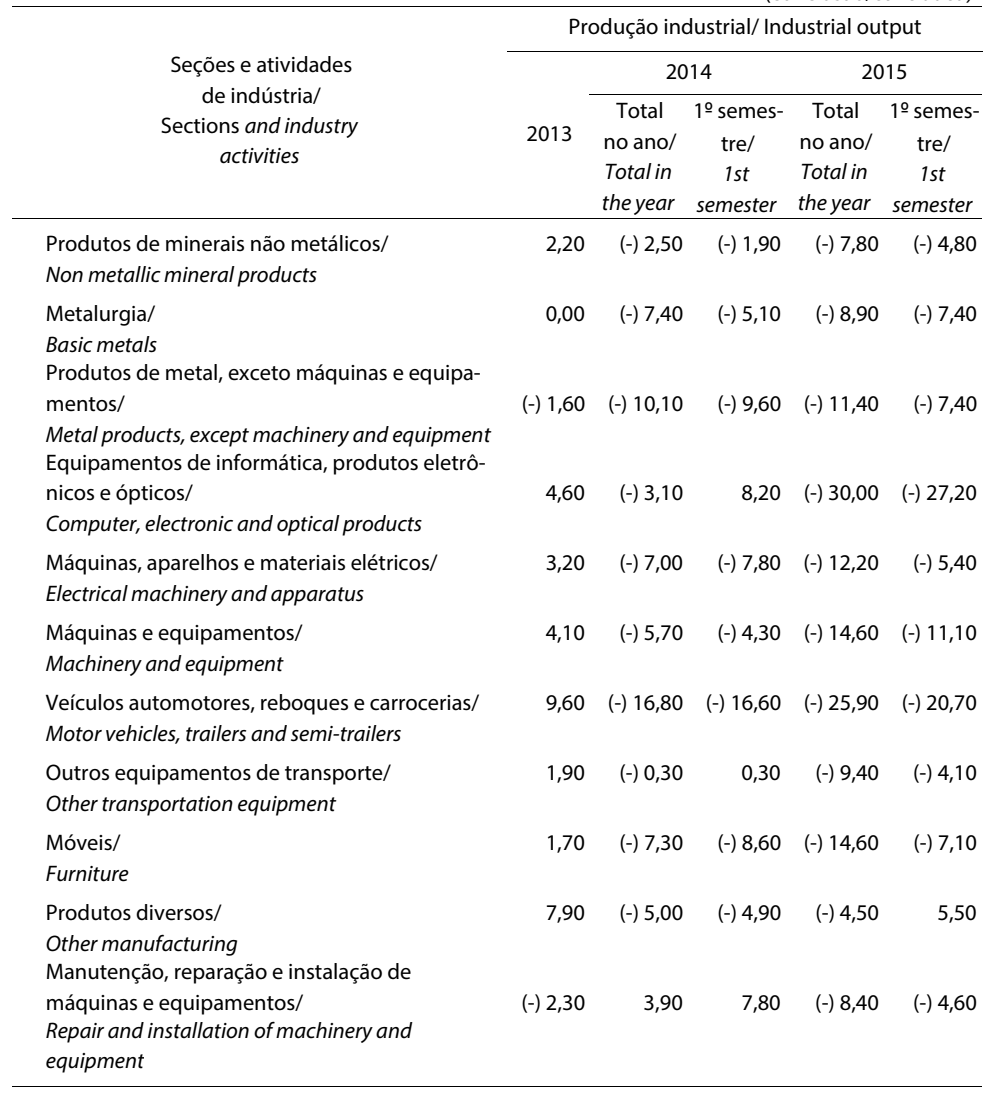

Fonte/Source: IBGE, Diretoria de Pesquisas, Coordenação de Indústria, Pesquisa Industrial Mensal: Produção Física Brasil 2013-2015.

Notas: 1. Taxas de crescimento da produção industrial (Base: igual período do ano anterior).

2. As taxas anuais de crescimento de Impressão e reprodução de gravações e Manutenção, reparação e instalação de máquinas e equipamentos têm início em 2013.

Notes: 1. Growth rates of industrial output (Base: same period of previous year).

2. The annual growth rate of printing and reproduction of recorded media and repair and installation of machinery and equipment began in 2013. 
Tabela 12.2 - Produção industrial e grau de intensidade de energia

elétrica - 2013-2015

Table 12.2 - Industrial output and degree of intensity

of eletricity consumption - 2013-2015

\begin{tabular}{|c|c|c|c|c|c|c|}
\hline \multirow[b]{2}{*}{$\begin{array}{l}\text { Especificação/ } \\
\text { Item }\end{array}$} & \multicolumn{2}{|c|}{2013} & \multicolumn{2}{|c|}{2014} & \multicolumn{2}{|c|}{2015} \\
\hline & $\begin{array}{l}\text { Total } \\
\text { no ano/ } \\
\text { Total in } \\
\text { the year }\end{array}$ & $\begin{array}{l}\text { 10 semes- } \\
\text { tre/ } \\
1 s t \\
\text { semester }\end{array}$ & $\begin{array}{l}\text { Total } \\
\text { no ano/ } \\
\text { Total in } \\
\text { the year }\end{array}$ & $\begin{array}{l}\text { 10 semes- } \\
\text { tre/ } \\
1 s t \\
\text { semester }\end{array}$ & $\begin{array}{l}\text { Total } \\
\text { no ano/ } \\
\text { Total in } \\
\text { the year }\end{array}$ & $\begin{array}{c}\text { 10 semes- } \\
\text { tre/ } \\
1 \text { st } \\
\text { semester }\end{array}$ \\
\hline Indústria geral/ Overall industry & 2,10 & 3,00 & $(-) 3,00$ & $(-) 2,40$ & (-) 8,30 & (-) 5,90 \\
\hline $\begin{array}{l}\text { Grau de intensidade de } \\
\text { energia elétrica/ } \\
\text { Degree of intensity of electrity } \\
\text { consumption }\end{array}$ & & & & & & \\
\hline Alto/High & 1,10 & 1,40 & (-) 3,50 & (-) 2,90 & (-) 6,80 & (-) 4,70 \\
\hline Médio/Medium & (-) 0,20 & (-) 0,80 & (-) 2,60 & (-) 3,10 & (-) 4,70 & $(-) 1,20$ \\
\hline Baixo/Low & 3,90 & 6,50 & (-) 2,90 & (-) 1,50 & (-) 11,20 & (-) 9,60 \\
\hline
\end{tabular}

Fonte/Source: IBGE, Diretoria de Pesquisas, Coordenação de Indústria, Pesquisa Industrial Mensal: Produção Física Brasil 2013-2015.

Nota: Taxas de crescimento da produção industrial (Base: igual período do ano anterior)./ Note: Growth rates of industrial output (Base: same period of previous year)

\section{Gráfico 12.1 - Taxas anuais de crescimento da produção industrial, por grandes categorias econômicas - 2012-2015 \\ Graph 12.1 - Annual growth rates of industrial output, by major economic categories - 2012-2015}

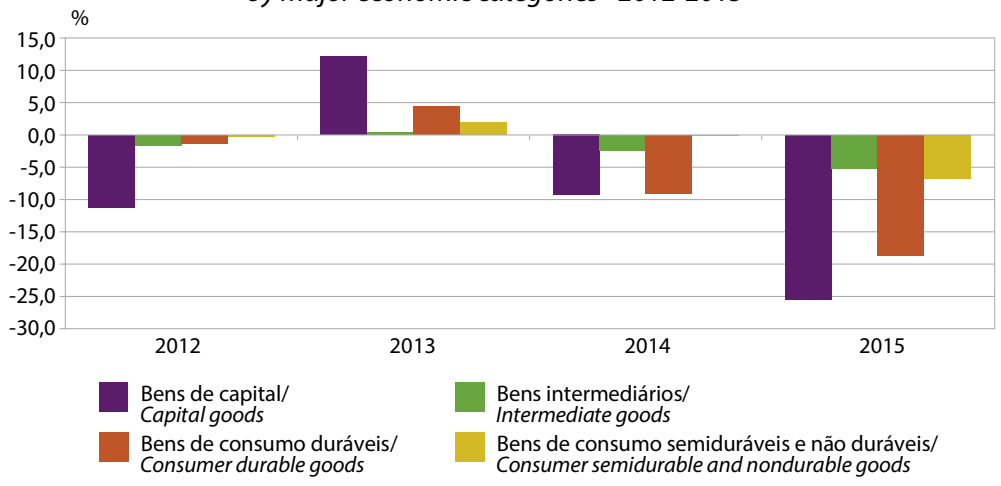

Fonte/Source: IBGE, Diretoria de Pesquisas, Coordenação de Indústria, Pesquisa Industrial Mensal: Produção Física Brasil 2012-2015. 
Tabela 12.3 - Produção industrial - 2012-2015

Table 12.3 - Mining and manufacturing production - 2012-2015

\begin{tabular}{|c|c|c|c|c|c|}
\hline $\begin{array}{l}\text { Produtos selecionados/ } \\
\text { Selected products }\end{array}$ & $\begin{array}{l}\text { Unidade de } \\
\text { medida/ } \\
\text { Unit }\end{array}$ & 2012 & 2013 & 2014 & 2015 \\
\hline Aço bruto/ & $1000 t$ & 34524 & 34163 & 33897 & - \\
\hline Crude steel & 1,000 tons & & & & \\
\hline Petróleo/ & $1000 \mathrm{~m}^{3}$ & 116654 & 117446 & 130835 & - \\
\hline Petroleum & 1,000 cu.meters & & & & \\
\hline Gás natural/ & $1000000 \mathrm{~m}^{3}$ & 25739 & 28174 & 31895 & - \\
\hline Natural gas & $1,000,000$ cu.meters & & & & \\
\hline Máquinas agrícolas automotrizes/ & Unidade & 83704 & 100400 & 82318 & 55262 \\
\hline Self-propelled agricultural machines & Unit & & & & \\
\hline Automóveis/ & Unidade & 2623704 & 2954279 & 2502293 & 2017639 \\
\hline Automobiles & Unit & & & & \\
\hline Papel/ & $1000 t$ & 10260 & 10444 & 10397 & (1) 10343 \\
\hline Paper & 1,000 tons & & & & \\
\hline Celulose/ & $1000 t$ & 13977 & 15129 & 16465 & (1) 17214 \\
\hline Cellulose & 1,000 tons & & & & \\
\hline
\end{tabular}

Fonte/Source: Anuário estatístico do Brasil 2015. Rio de Janeiro: IBGE, v. 75, 2016. Disponível em/ Available from: $<$ http://biblioteca.ibge.gov.br/index.php/biblioteca-catalogo?view=detalhes\&id= 720 >. Acesso em: abr. 2016/Cited: Apr. 2016.

(1) Dados preliminares. /(1) Preliminary data. 
Tabela 12.4 - Variáveis selecionadas das unidades locais industriais de empresas industriais com 5 ou mais pessoas ocupadas, segundo as Grandes Regiões e as Unidades da Federação - 2013

Table 12.4 - Selected variables from industrial local units with 5 or more employed persons, by Major Regions and Federtion Units - 2013

\begin{tabular}{|c|c|c|c|}
\hline \multirow[t]{2}{*}{$\begin{array}{l}\text { Grandes Regiões e } \\
\text { Unidades da Federação/ } \\
\text { Major Regions and } \\
\text { Federation Units }\end{array}$} & $\begin{array}{l}\text { Número de } \\
\text { unidades } \\
\text { locais/ } \\
\text { Number } \\
\text { of local } \\
\text { units }\end{array}$ & $\begin{array}{l}\text { Pessoal } \\
\text { ocupado } \\
\text { em 31.12/ } \\
\text { Employed } \\
\text { persons } \\
\text { on } 31.12\end{array}$ & $\begin{array}{c}\text { Salários, } \\
\text { retiradas e outras } \\
\text { remunerações/ } \\
\text { Salaries, } \\
\text { withdrawals } \\
\text { and other } \\
\text { compensation }\end{array}$ \\
\hline & \multicolumn{3}{|c|}{$1000 \mathrm{R} \$ / 1,000 R \$$} \\
\hline Brasil/Brazil & 204020 & 8441503 & 258001343 \\
\hline Norte/North & 5959 & 309358 & 8235134 \\
\hline Rondônia & 1311 & 38233 & 590284 \\
\hline Acre & 271 & 6207 & 91415 \\
\hline Amazonas & 1267 & 138404 & 4083626 \\
\hline Roraima & 124 & 2181 & 30621 \\
\hline Pará & 2193 & 101278 & 2995398 \\
\hline Amapá & 180 & 6079 & 136097 \\
\hline Tocantins & 613 & 16976 & 307693 \\
\hline Nordeste/Northeast & 24775 & 1107808 & 22469132 \\
\hline Maranhão & 1139 & 48246 & 1115810 \\
\hline Piauí & 1162 & 30875 & 382932 \\
\hline Ceará & 5698 & 251096 & 3682097 \\
\hline Rio Grande do Norte & 1905 & 71649 & 1624440 \\
\hline Paraíba & 1760 & 78972 & 1172120 \\
\hline Pernambuco & 5407 & 239975 & 4537396 \\
\hline Alagoas & 808 & 89079 & 1190048 \\
\hline Sergipe & 1128 & 50403 & 1336570 \\
\hline Bahia & 5768 & 247513 & 7427719 \\
\hline
\end{tabular}


Tabela 12.4 - Variáveis selecionadas das unidades locais industriais de empresas industriais com 5 ou mais pessoas ocupadas, segundo as Grandes Regiões e as Unidades da Federação - 2013

Table 12.4 - Selected variables from industrial local units with 5 or more employed persons, by Major Regions and Federtion Units - 2013

\begin{tabular}{|c|c|c|c|}
\hline \multirow{3}{*}{$\begin{array}{l}\text { Grandes Regiões e } \\
\text { Unidades da Federação/ } \\
\text { Major Regions and } \\
\text { Federation Units }\end{array}$} & & & \\
\hline & $\begin{array}{l}\text { Número de } \\
\text { unidades } \\
\text { locais/ } \\
\text { Number } \\
\text { of local } \\
\text { units }\end{array}$ & $\begin{array}{l}\text { Pessoal } \\
\text { ocupado } \\
\text { em 31.12/ } \\
\text { Employed } \\
\text { persons } \\
\text { on } 31.12\end{array}$ & $\begin{array}{l}\text { Salarıos, } \\
\text { retiradas e outras } \\
\text { remunerações/ } \\
\text { Salaries, } \\
\text { withdrawals } \\
\text { and other } \\
\text { compensation }\end{array}$ \\
\hline & \multicolumn{3}{|c|}{$1000 \mathrm{R} \$ / 1,000 R \$$} \\
\hline Sudeste/Southeast & 100633 & 4369396 & 161422314 \\
\hline Minas Gerais & 24634 & 905889 & 23794898 \\
\hline Espírito Santo & 4660 & 140022 & 4158158 \\
\hline Rio de Janeiro & 11131 & 497602 & 26035766 \\
\hline São Paulo & 60208 & 2825883 & 107433492 \\
\hline Sul/South & 59168 & 2160725 & 54975412 \\
\hline Paraná & 18716 & 710322 & 18108249 \\
\hline Santa Catarina & 18993 & 692283 & 16554816 \\
\hline Rio Grande do Sul & 21459 & 758120 & 20312347 \\
\hline Centro-Oeste/Central-West & 13485 & 494216 & 10899351 \\
\hline Mato Grosso do Sul & 1756 & 91781 & 2172888 \\
\hline Mato Grosso & 3272 & 113334 & 2266763 \\
\hline Goiás & 7209 & 257078 & 5716368 \\
\hline Distrito Federal/Federal District & 1248 & 32023 & 743332 \\
\hline
\end{tabular}


Tabela 12.4 - Variáveis selecionadas das unidades locais industriais de empresas industriais com 5 ou mais pessoas ocupadas, segundo as Grandes Regiões e as Unidades da Federação - 2013

Table 12.4 - Selected variables from industrial local units with 5 or more employed persons, by Major Regions and Federtion Units - 2013

(continuação/to be continued)

\begin{tabular}{|c|c|c|c|c|}
\hline $\begin{array}{l}\text { Grandes Regiões e } \\
\text { Unidades da Federação/ } \\
\text { Major Regions and } \\
\text { Federation Units }\end{array}$ & $\begin{array}{l}\text { Receita } \\
\text { líquida de } \\
\text { vendas/ } \\
\text { Net revenue } \\
\text { of sales }\end{array}$ & $\begin{array}{c}\text { Valor bruto } \\
\text { da produção } \\
\text { industrial/ } \\
\text { Gross value } \\
\text { of industrial } \\
\text { production }\end{array}$ & $\begin{array}{c}\text { Custos das } \\
\text { operações } \\
\text { industriais/ } \\
\text { Costs of } \\
\text { industrial } \\
\text { operations }\end{array}$ & $\begin{array}{c}\text { Valor da } \\
\text { transformação } \\
\text { industrial/ } \\
\text { Gross value } \\
\text { added }\end{array}$ \\
\hline & \multicolumn{4}{|c|}{$1000 \mathrm{R} \$ / 1,000 \mathrm{R} \$$} \\
\hline Brasil/Brazil & 2498389163 & 2384181531 & 1314959669 & 1069221867 \\
\hline Norte/North & 146825079 & 140635096 & 70979575 & 69655521 \\
\hline Rondônia & 8060896 & 8528977 & 5628773 & 2900204 \\
\hline Acre & 870140 & 859226 & 529704 & 329522 \\
\hline Amazonas & 86267200 & 79460242 & 45549138 & 33911104 \\
\hline Roraima & 179514 & 169105 & 83927 & 85178 \\
\hline Pará & 46012180 & 46185229 & 15828244 & 30356985 \\
\hline Amapá & 1770236 & 1788485 & 893391 & 895095 \\
\hline Tocantins & 3664913 & 3643832 & 2466398 & 1177433 \\
\hline Nordeste/Northeast & 240797600 & 221519991 & 121298958 & 100221035 \\
\hline Maranhão & 11189473 & 10871036 & 6990252 & 3880785 \\
\hline Piauí & 4009858 & 3948035 & 2347318 & 1600717 \\
\hline Ceará & 36622201 & 29786858 & 15470199 & 14316659 \\
\hline Rio Grande do Norte & 10313947 & 11931261 & 4690098 & 7241163 \\
\hline Paraíba & 10081048 & 9374384 & 4690512 & 4683873 \\
\hline Pernambuco & 42175870 & 40941316 & 22351498 & 18589817 \\
\hline Alagoas & 6696768 & 7191814 & 3392041 & 3799773 \\
\hline Sergipe & 7752909 & 9266687 & 4601868 & 4664819 \\
\hline Bahia & 111955526 & 98208600 & 56765172 & 41443429 \\
\hline
\end{tabular}


Tabela 12.4 - Variáveis selecionadas das unidades locais industriais de empresas industriais com 5 ou mais pessoas ocupadas, segundo as Grandes Regiões e as Unidades da Federação - 2013

Table 12.4 - Selected variables from industrial local units with 5 or more employed persons, by Major Regions and Federtion Units - 2013

\begin{tabular}{|c|c|c|c|c|}
\hline \multirow[t]{2}{*}{$\begin{array}{l}\text { Grandes Regiões e } \\
\text { Unidades da Federação/ } \\
\text { Major Regions and } \\
\text { Federation Units }\end{array}$} & $\begin{array}{l}\text { Receita } \\
\text { líquida de } \\
\text { vendas/ } \\
\text { Net revenue } \\
\text { of sales }\end{array}$ & $\begin{array}{c}\text { Valor bruto } \\
\text { da produção } \\
\text { industrial/ } \\
\text { Gross value } \\
\text { of industrial } \\
\text { production }\end{array}$ & $\begin{array}{c}\text { Custos das } \\
\text { operações } \\
\text { industriais/ } \\
\text { Costs of } \\
\text { industrial } \\
\text { operations }\end{array}$ & $\begin{array}{c}\text { Valor da } \\
\text { transformação } \\
\text { industrial/ } \\
\text { Gross value } \\
\text { added }\end{array}$ \\
\hline & \multicolumn{4}{|c|}{$1000 \mathrm{R} \$ / 1,000 R \$$} \\
\hline Sudeste/Southeast & 1404110873 & 1370588715 & 737272180 & 633316536 \\
\hline Minas Gerais & 270656010 & 262079664 & 137073680 & 125005984 \\
\hline Espírito Santo & 44768262 & 49378243 & 20866418 & 28511825 \\
\hline Rio de Janeiro & 190728416 & 201377677 & 88853343 & 112524335 \\
\hline São Paulo & 897958185 & 857753131 & 490478739 & 367274392 \\
\hline Sul/South & 536524734 & 504609837 & 295420070 & 209189767 \\
\hline Paraná & 203228478 & 190690997 & 112671383 & 78019614 \\
\hline Santa Catarina & 124404250 & 120185826 & 66017059 & 54168767 \\
\hline Rio Grande do Sul & 208892006 & 193733014 & 116731628 & 77001386 \\
\hline Centro-Oeste/Central-West & 170130877 & 146827892 & 89988886 & 56839008 \\
\hline Mato Grosso do Sul & 29947330 & 30687400 & 18553725 & 12133675 \\
\hline Mato Grosso & 43469565 & 40884942 & 25604765 & 15280177 \\
\hline Goiás & 91435303 & 70058184 & 43261880 & 26796305 \\
\hline Distrito Federal/Federal District & 5278679 & 5197366 & 2568516 & 2628851 \\
\hline
\end{tabular}

Fonte/Source: Pesquisa industrial Anual. Empresa 2013. Rio de Janeiro: IBGE, v. 32, n. 1, 2013. Disponível em/Available from: <http://www.ibge.gov.br/home/estatistica/economia/industria/pia/empresas/2013/ defaultempresa.shtm>.Acesso em: abr. 2016/Cited: Apr.2016. 


\section{Gráfico 12.2 - Produção de aço, por países selecionados - 2014}

Graph 12.2 - Production of steel, by selected countries - 2014

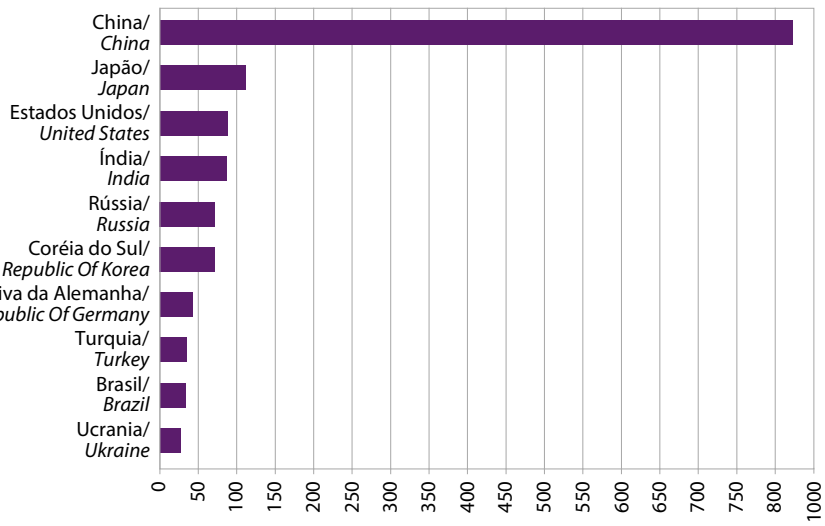

Milhões de toneladas/Millions tons

Fonte/Source: Instituto Aço Brasil, Departamento de Economia.

Nota: Dados preliminares./Note: Preliminary figures.

\section{Gráfico 12.3 - Produção de aço, da América Latina - 2014}

Graph 12.3 - Production of steel, in Latin America - 2014

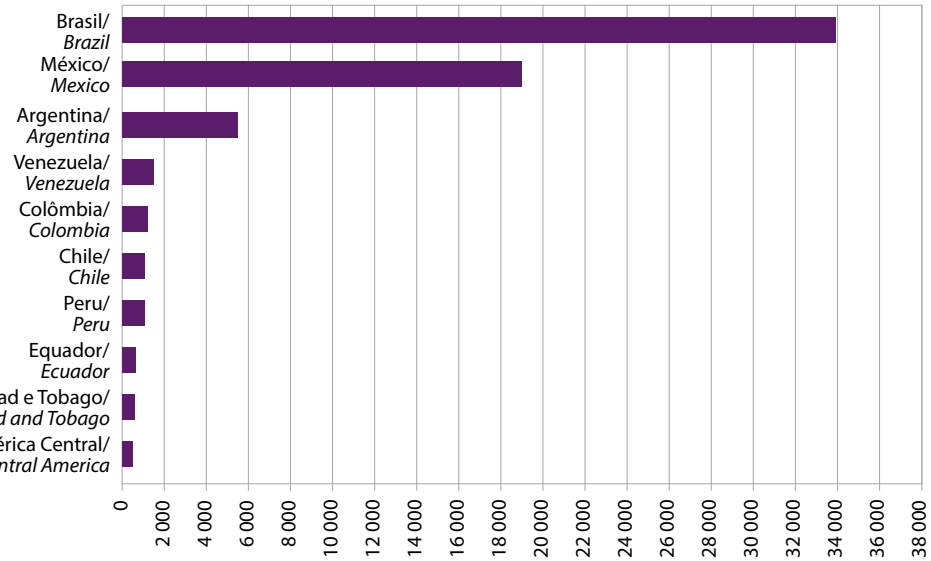

Mil toneladas/Thousand of tons

Fonte/Source: Instituto Aço Brasil, Departamento de Economia.

Nota: Dados preliminares./Note: Preliminary figures. 



\section{Energia}

\section{Energy}

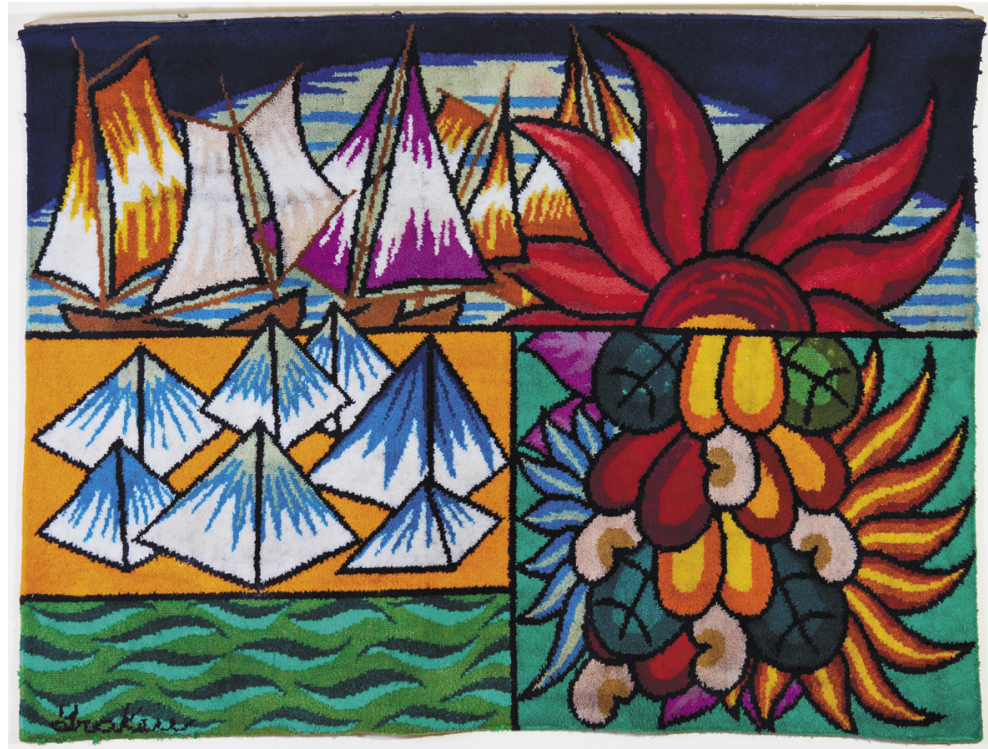

Salinas/Cajus, s.d/Salterns/cashews, n.d. Iraken Marques de Lima 



\section{Energia}

\section{Introdução}

As dimensões geográficas e

populacionais do Brasil impõem ao país grandes desafios para assegurar fontes e geração de energia. Dentre as nações de maior território e população, o Brasil apresenta a matriz energética com maior preponderância de fontes renováveis.

A disponibilidade de energia em todo o território nacional é fundamental para assegurar condições adequadas para o desempenho das atividades econômicas de um país e para proporcionar conforto e qualidade de vida para sua população.

O Brasil é atendido eletricamente por meio de uma rede interligada denominada Sistema Interligado Nacional (SIN). O SIN é composto de quatro zonas geográficas a saber: a) Sul; b) Sudeste/ Centro-Oeste; c) Nordeste, e d) Norte. Algumas poucas áreas do amplo território brasileiro que ainda não se encontram interligadas ao SIN são chamadas de "sistemas isolados" (algumas comunidades no interior dos estados do Amazonas, Acre e Roraima). Do ponto de vista gerencial, a estrutura do sistema elétrico brasileira é bastante complexa,

\section{Energy}

\section{Introduction}

The geographic extent and the population size of Brazil pose major challenges to assure energy sources and generation. Brazil shows the greatest predominance of renewable sources in the energy matrix among the countries with large territory and population.

The availability of energy in the whole national territory is key to assure appropriate conditions for the performance of economic activities and to provide the population with comfort and quality of life.

Brazil is served in terms of energy by an interconnected network called Interconnected Electric System - SIN. SIN comprises four geographic zones: a) South; b) Southeast/Central-West; c) Northeast, and d) North. Few areas of the wide Brazilian territory that are still not connected to SIN are called "isolated systems" - some communities in the states of Amazonas, Acre and Roraima. From the management point of view, the structure of the Brazilian electric system is quite complex, both legally and geographically. The 
tanto jurídica quanto geograficamente. Os principais órgãos de chefia do setor foram criados há relativamente pouco tempo: Aneel (Agência Nacional de Energia Elétrica) - 1996, ONS (Operador Nacional do Sistema) - 1998, EPE (Empresa de Pesquisa Energética) - 2004 e CCEE (Câmara de Comercialização de Energia Elétrica) 2004. Isso essencialmente tem a ver com a gradual mudança de modelo: daquele diretamente operado por um monopólio estatal para um, mais complexo e regulado, que mescla a participação estatal com investimento privado e estrangeiro.

O setor de petróleo e gás, por sua vez, tem regulação e fiscalização conduzidas pela ANP (Agência Nacional de Petróleo, Gás e Biocombustíveis), enquanto o calendário dos leilões de áreas exploratórias é elaborado em conjunto com o Ministério de Minas e Energia e a EPE, e aprovado pelo CNPE Conselho Nacional de Política Energética.

\section{Histórico recente}

O Brasil tem mudado sensivelmente o seu perfil de produção de eletricidade nos últimos 30 anos, partindo de uma matriz predominantemente hídrica (vigente até a década de 1990), para uma matriz um pouco mais diversificada, onde o ponto de partida foi o ano de 2001, quando ocorreu grande crise nos reservatórios hidráulicos do País.

Naquele contexto, em 2002, surgiu o PROINFA (Programa de Incentivo às Fontes Alternativas de Energia Elétrica), que tinha como meta principal diversificar a matriz elétrica brasileira e fomentar o desenvolvimento das fontes alternativas às hidrelétricas. O programa ambicionava contratar, gradualmente, 3,3 GW em main bodies heading this sector were created quite recently: Aneel (Brazilian Electricity Regulatory Agency) - 1996, ONS (Electric System National Operator) - 1998, EPE (Energy Research Company) - 2004 and CCEE (Electric Energy Trading Chamber) - 2004. This is due to the gradual change from a model directly operated by a state monopoly to a more complex and regulated one, which mixes the state participation with private and foreign investments.

On its turn, the sector of oil and gas is regulated and supervised by the ANP (National Agency of Petroleum, Natural Gas and Biofuels), while the schedule of auctions of oil blocks is developed together with the Ministry of Mines and Energy and the EPE and approved by the CNPE (National Council of Energy Policy).

\section{Recent background}

Brazil has been significantly changing the profile of electricity generation along the last 30 years, from a predominantly hydric matrix - in effect until the 1990s - to a slightly more diversified matrix, launched in 2001, when a big crisis affected the hydraulic reservoirs in Brazil.

In that context, PROINFA (Alternative Electricity Source Incentive Program) was created in 2002, aiming at diversifying the Brazilian electricity matrix and fostering the development of sources other than hydroelectric plants. The program strove to 
potência instalada de fontes eólicas, solar, biomassa e das chamadas PCH's (Pequenas Centrais Hidrelétricas). $O$ PROINFA representou o marco inicial de uma nova política energética brasileira, $\mathrm{e}$ contou com o apoio essencial do BNDES (Banco Nacional de Desenvolvimento Econômico e Social) - que destinou financiamentos federais para até $80 \%$ de cada um dos projetos - bem como da holding energética estatal Eletrobrás, provedora da garantia de compra da energia.

Mais recentemente, uma nova crise no reabastecimento dos reservatórios hídricos evoluiu nos últimos 5 anos, tendo chegado a posições críticas em 2014/2015. Em janeiro de 2015 , $98 \%$ dos 21 principais reservatórios de água do Brasil estavam com níveis mais baixos do que os indicados em junho de 2001, época em que chegou a ser decretado um racionamento de energia elétrica no Brasil. É bem verdade que, comparado ao cenário pós-2014, o parque de geração térmico no Brasil, em 2001, era bem menor e o número de linhas de transmissão também era bem inferior. No entanto, em 2001, aconteceu uma queda de aproximadamente $25 \%$ no consumo de energia da típica família brasileira, fato que não ocorreu em 2015, quando a demanda na verdade aumentou, chegando inclusive a picos de energia que resultaram em um apagão que atingiu 10 estados brasileiros.

A situação hídrica se complicou sensivelmente quando o reservatório (um dos maiores do Brasil e o maior do Nordeste) da hidrelétrica de Sobradinho chegou a $1 \%$ (em 2001, o volume registrado foi de $5.3 \%$ ) de seu volume útil (o menor valor registrado) em Dezembro de 2015 fazendo com que sua operadora (CHESF) chegasse a anunciar gradually hire an installed power of 3.3 GW from wind, solar and biomass sources and from the SHPs (Small Hydroelectric Plants). PROINFA represented the initial milestone of a new Brazilian energy policy and was supported by the BNDES (Brazilian Development Bank), which allocated federal funds for up to $80 \%$ of every project, as well as by the state-run energy holding Eletrobrás, which guaranteed the acquisition of energy.

More recently, a new crisis of replenishment of the hydraulic reservoirs has increased in the last five years, having reached a critical position in 2014-2015. In January $2015,98 \%$ of the 21 main water reservoirs in Brazil were below the level of June 2001, when energy rationing was established in Brazil. It is quite true that the thermal generation park and the number of transmission lines were much smaller in 2001 when compared with the post-2014 scenario. Nevertheless, the consumption of energy by typical Brazilian families dropped about $25 \%$ in 2001. In 2015, the demand actually increased, even reaching energy peaks that resulted in blackouts in 10 Brazilian states.

The water situation significantly worsened when the reservoir of the Sobradinho hydroelectric plant one of the largest in Brazil and the largest in the Northeast - hit $1 \%$ - in 2001 , the lowest volume registered had been $5.3 \%$ - of its usable volume - the lowest level ever recorded - in December 2015, causing CHESF to 
que poderia desligar a hidrelétrica. Atualmente (Junho/2016), o reservatório da hidrelétrica de Sobradinho, responsável por cerca de $58 \%$ do abastecimento da região Nordeste, instalado na Bacia do Rio São Francisco trabalha a $24 \%$ de sua capacidade de abastecimento. A partir do inicio de 2016, o ONS (Operador Nacional do Sistema) já não precisou despachar continuamente, especialmente no Nordeste, as termelétricas mais caras - aquelas com custo de geração maior que $\mathrm{R} \$ 600 / \mathrm{MWh}$.

A gigante da geração elétrica brasileira é certamente a hidrelétrica binacional de Itaipu (localizada na fronteira entre Brasil e Paraguai). Em 2015, Itaipu bateu novo recorde de geração e assumiu o posto de maior geradora de energia elétrica no planeta: 89,2 milhões de MWh, superando a hidrelétrica chinesa de Três Gargantas (que produziu 87 milhões de $M W h$ no mesmo ano). $O$ mérito não é pequeno, pois o recorde da hidrelétrica de Itaipu partiu de seus $14 \mathrm{GW}$ de capacidade instalada, bem inferiores aos $22 \mathrm{GW}$ de capacidade instalada da hidrelétrica de Três Gargantas. Recentemente (12/06/2016), a vazão da hidrelétrica de Itaipu chegou a 6 vezes o volume das cataratas do Iguaçu: foi registrado um escoamento de 9,3 milhões de litros por segundo. Já no inicio do ano de 2016, Itaipu bateu diversos recordes de produção: os melhores meses de janeiro, fevereiro, bimestre, trimestre, quadrimestre, maio e período de 5 meses de todos os 32 anos de operação da usina.

Dados da tabela 13.2 indicam que a geração de eletricidade no Brasil entre 2013 e 2014 cresceu de 570.025 GWh eventually announce the power down of the plant. Today, June 2016, the reservoir of Sobradinho, installed in the São Francisco River Basin and responsible for about $58 \%$ of the supply in the Northeast Region, operates at $24 \%$ of its replenishment capacity. From the beginning of 2016 onwards, the ONS did not need to continually deliver the most expensive thermoelectric energy plants - those with generation cost higher than $\mathrm{R} \$ 600 / \mathrm{MWh}-$, particularly in the Northeast.

The largest hydroelectric plant in Brazil is definitely the Itaipu binational plant, located in the border between Brazil and Paraguay. In 2015, Itaipu hit a new generation record and ranked as the largest electricity generator on the planet: 89.2 million MWh, surpassing the Chinese Three Gorges Dam, which produced 87 million MWh that year. It was not a small achievement, since the record of the Itaipu plant was based on an installed capacity of $14 \mathrm{GW}$, well below the installed capacity of $22 \mathrm{GW}$ of the Three Gorges Dam plant. On June 12, 2016, the outflow of the Itaipu plant reached six times the volume of the Iguazu Falls: 9.3 million liters per second. In the beginning of 2016, Itaipu hit a number of production records: the best months of January, February, bimester, quarter, fourmonth period, May and 5-month period of all the 32 years of operation.

Data from table 13.2 point out that electricity generation in Brazil increased from $570,025 \mathrm{GWh}$ to 590 
para $590.479 \mathrm{GWh}$, o que representa um avanço de $3,6 \%$, sendo que a maior crescimento $(136,4 \%)$ ocorreu no estado de Rondônia, que partiu de $6.407 \mathrm{GWh}$ em 2013 para 15.148 GWh em 2014.

Dados de junho de 2016 da ANEEL (Agência Nacional de Energia Elétrica) mostram que o Brasil tem 4535 usinas elétricas com 144,9 GW em capacidade instalada e cerca de 116 mil km em linhas de transmissão, com tensões a partir de $230 \mathrm{kV}$. Deste quantitativo de usinas, a maioria são de termelétricas (2896) que representam $27,69 \%$ da potência instalada do país; $61,02 \%$ de sua potência instalada no Brasil é de hidrelétricas. Aqui, cabe ressaltar que apesar da participação das hidrelétricas em capacidade instalada ser de aproximadamente $60,97 \%$, a participação delas na produção efetiva de energia é em geral superior a $80 \%$ (podendo variar sazonalmente em função da disponibilidade das águas). O CMSE (Comitê de Monitoramento do Setor Elétrico) aprovou em maio de 2016 o desligamento de 12 termelétricas que estavam operacionais a um custo a partir de R\$ 150/MWh. O ONS (Operador Nacional do Sistema) estimou que o desligamento de $2.011 \mathrm{MW}$ médios provocaria uma redução prevista de $\mathrm{R} \$$ 288 milhões/mês. Para o futuro, o PDE 2024 (Plano Decenal de Expansão) da EPE indica uma previsão de 10,5 GW até 2024.

Apesar da predominância em fontes limpas, o Brasil ainda opera duas usinas nucleares Angra I e Angra II, estando Angra III em construção (67\% das obras civis já estão concluídas, de acordo com a Eletronuclear) e prevista para entrar em operação em 2018. As três usinas estão instaladas em Angra dos Reis no estado do Rio de Janeiro, com Angra I tendo
GWh between 2013 and 2014, which represented an advance of $3.6 \%$, the highest one (136.4\%) taking place in the state of Rondônia, which changed from 6.407 GWh in 2013 to 15.148 GWh in 2014.

Data of June 2016 from Aneel show that Brazil has 4,535 electricity plants with an installed capacity of 144.9 GW and about 116 thousand $\mathrm{km}$ of transmission lines, with voltages from $230 \mathrm{kV}$ upwards. Most of these plants are thermoelectric plants $(2,896)$, which accounted for $27.69 \%$ of the installed power in Brazil; $61.02 \%$ of the installed power in Brazil is from hydroelectric plants. Although the contribution of hydroelectric plants in installed capacity is about $60.97 \%$, their contribution to the effective production of energy is usually higher than $80 \%$, seasonally changing depending on the availability of water. The CMSE (Committee for Monitoring the Electricity Sector) approved the power down of 12 thermoelectric plants that were in operation in May 2016 at a cost higher than $\mathrm{R} \$ 150 / \mathrm{MWh}$. The ONS estimated that the power down of 2,011 MW, on average, would cause a reduction of $R \$ 288$ million/ month. For the future, the EPE's 2024 PDE (Decennial Expansion Plan for Electricity) forecasts $10.5 \mathrm{GW}$ until 2024.

Despite the predominance of clean sources, Brazil still operates two nuclear plants: Angra I and Angra II. Angra III is under construction - $67 \%$ of civil works completed, according to Eletronuclear - and scheduled to become operational in 2018. The three plants are installed in Angra dos Reis in the state of Rio de Janeiro. The authorized power of 
uma potência outorgada de $640.000 \mathrm{~kW}$ e Angra II de $1.350 .000 \mathrm{~kW}$.

A desvantagem de uma termelétrica é claramente o impacto ambiental e o custo com combustível. Sua vantagem, em contrapartida, é a possibilidade de sua construção poder acontecer em locais próximos aos centros urbanos consumidores, economizando assim com as redes de transmissão e evitando perdas de energia elétrica. Talvez o ponto mais relevante, no entanto, seja o fato das termelétricas terem uma produção constante, já que independe de fatores externos, como às relativas ao clima.

\section{Uma nova era energética}

Nos últimos anos, o mundo tem empreendido esforços em prol do crescimento acentuado do uso de fontes renováveis para geração de energia elétrica. Em 2015, foram 329 bilhões de dólares investidos em empreendimentos energia limpa no mundo, e a capacidade instalada em renováveis foi $30 \%$ maior do que a de 2014. Em 2015, apenas em usinas eólicas foram instalados $64 \mathrm{GW}$, e 57 GWs em usinas fotovoltaicas. A líder em instalação de renováveis, em 2015, foi a China que investiu US\$110,5 bilhões (17\% a mais que o registrado em 2014).

No Brasil, a hidrologia desfavorável resultante de causas climáticas - mas também do aumento da escala de uso da água para usos agro-industriais e outros - também tem provocado a necessidade de diversificação da nossa matriz de geração, mesmo apesar desta ser a mais renovável dentre os grandes consumidores energéticos do mundo.
Angra I is $640,000 \mathrm{~kW}$ and of Angra II, $1,350,000 \mathrm{~kW}$.

Clearly speaking, the disadvantage of thermoelectric plants is the environmental impact and the cost with fuel. Conversely, the possibility of building them near the consuming urban centers is an advantage, as they save transmission lines and avoid electricity losses. Nonetheless, the fact that the output of thermoelectric plants is constant might be the most relevant point, as they do not depend on external factors like climate.

\section{A new energy era}

In the last years, the world has been fostering the sharp increase of renewable sources to generate electricity. In 2015, US\$329 billion were invested in clean-energy projects in the world and the installed capacity of renewable energy was $30 \%$ bigger than in 2014. 64 GW of wind power plants and $57 \mathrm{GW}$ of photovoltaic plants were installed in 2015. Having invested US\$110.5 billion, 17\% more than in 2014, China led the installation of renewable energy in 2015.

Regardless of being the most renewable among the major energy consumers in the world, an unfavorable hydrology resulting from climate conditions in Brazil - as well as the escalating use of water for agribusiness and others - has been causing the diversification of our generation matrix. 
Nos últimos anos, o Brasil promoveu uma grande mudança em relação à participação das fontes de energia na capacidade instalada e em operação comercial da matriz elétrica nacional. Em 2016, a biomassa alcançou 9,4\% enquanto a geração eólica alcançou $6,6 \%$, e as PCHs $3,4 \%$. A liderança ainda permanece com a fonte hidrelétrica, nossa principal fonte de oferta de energia elétrica, com $61,1 \%$ de toda a capacidade instalada em operação comercial da matriz elétrica brasileira.

Dados do GWEC (Global Wind Energy Council) informam que, em 2015, o Brasil ampliou em $46 \%$ a sua capacidade eólica instalada, adicionando $2,75 \mathrm{GW}$ e alcançando 8,72 GW. Mantém, assim, a 10a posição entre os maiores em potência eólica instalada no planeta, conquistada em 2014.

As usinas eólicas vêm aumentado cada vez mais o seu papel na matriz elétrica nacional, chegando a 375 usinas e um montante de $9,2 \mathrm{GW}$ em potência instalada. Isso representa $6,32 \%$ da matriz nacional, com cerca de $80 \%$ destas concentradas na Região Nordeste, onde o estado do Rio Grande do Norte assume destaque com seus mais de 1400 aerogeradores e $2,86 \mathrm{GW}$ em potência instalada eólica - mais de $30 \%$ de toda a potência eólica instalada no país, sendo ainda o estado com a maior matriz eólica do Brasil.

O país deverá alcançar $10 \mathrm{GW}$ em potência eólica instalada entre $o$ primeiro e o segundo semestres de 2016.

Quanto à energia solar, o Brasil apresenta um dos melhores índices de insolação do planeta, chegando a superar as 3.000
In the last years, Brazil promoted a big change in the contribution of energy sources to the installed capacity in commercial operation of the national electricity matrix. In 2016, biomass reached 9.4\%, while wind generation hit $6.6 \%$ and SHPs, 3.4\%. Our main electricity source, hydroelectric, remains the leader with $61.1 \%$ of all the installed capacity in the commercial operation of the Brazilian electricity matrix.

Data from the GWEC (Global Wind Energy Council) report that Brazil increased by $46 \%$ its installed wind power capacity in 2015 , adding 2.75 GW and reaching $8.72 \mathrm{GW}$. Thereby, Brazil has remained in the tenth position among the leaders in installed wind power on the planet since 2014 .

Wind power plants have been increasing their role in the national electricity matrix, reaching 375 plants and an amount of $9.2 \mathrm{GW}$ of installed power. This power accounts for $6.32 \%$ of the national matrix, $80 \%$ of which is concentrated in the Northeast Region. The state of Rio Grande do Norte stands out with more than 1,400 windmills and $2.86 \mathrm{GW}$ of installed wind power, representing more than $30 \%$ of all the wind power installed in Brazil and being the state with the biggest wind power matrix in Brazil.

Brazil should reach $10 \mathrm{GW}$ of installed wind power between the first and second semesters of 2016.

Concerning solar energy, Brazil has one of the best sunlight rates on the planet, even surpassing 3,000 hours 
horas por ano. Os melhores máximos de irradiação solar são observados no interior da Bahia e no noroeste de Minas Gerais.

O Nordeste, em especial, possui os maiores valores de irradiação solar do planeta, com a maior média e a menor variabilidade anual. No Nordeste, por exemplo, temos uma incidência média diária na faixa de 4,5 a $6 \mathrm{kWh}$. Estes números por si já indicam que o Brasil é uma potência solar. Apesar de todo este potencial para geração de eletricidade, a fonte ainda é pouco explorada no Brasil. O país realizou o primeiro leilão federal admitindo a solar em outubro de 2014, o que resultou na contratação de 890 MW a um preço final $\mathrm{R} \$ 215,12 / \mathrm{MWh}$. Em 2015, tivemos dois leilões realizados, contratando 2,653 GW de energia de reserva para início de suprimento em 2017 e 2018.

A quantidade de painéis fotovoltaicos instalados no Brasil ainda é pequena quando comparamos, por exemplo, com a Alemanha, país que tem mais de um milhão de sistemas solares instalados nos telhados das residências. Claro que as razões e o histórico de geração e demanda energética de cada pais é diferente, e houve países que aceleraram o desenvolvimento tecnológico e operacional de fontes alternativas bem precocemente, diante da existência de poucas opções efetivamente confiáveis para suprimento de suas necessidades energéticas.

No Brasil, no sentido de alavancar a instalação dos sistemas fotovoltaicos, é uma tendência irreversível que se tenha gradualmente mais políticas públicas focadas no incentivo da energia solar. per year. The maximum rates of solar irradiation are noticed in the interior of Bahia and in the northwest of Minas Gerais.

The Northeast, in particular, has the highest figures of solar irradiation on the planet, as well as the highest average and the lowest annual variability. For example, the Northeast shows an average daily incidence between 4.5 and $6 \mathrm{kWh}$. These figures on their own indicate that Brazil is a solar superpower. Despite all this potential to generate electricity, this source is still underexploited in Brazil. Brazil carried out the first federal auction admitting solar energy in October 2014, which resulted in the hiring of $890 \mathrm{MW}$ at a final price of $\mathrm{R} \$ 215.12 / \mathrm{MWh}$. Two auctions were carried out in 2015, hiring $2.653 \mathrm{GW}$ of backup power supply in 2017 and 2018.

The number of photovoltaic panels installed in Brazil is still small when we compare, for example, with Germany, which has more than one million solar systems installed on the rooftop of houses. Of course, the reasons and background of energy generation and demand differ from country to country. Some countries accelerated the technological and operational development of alternative sources much earlier, in face of few reliable options to supply their energy needs.

Brazil irreversibly tends to gradually implement more public policies fostering solar energy, leveraging the installation of photovoltaic systems. The approval and 
Grande evolução regulatória ocorreu nos últimos anos, com a aprovação e implementação de um marco regulatório para a micro e mini geração. Os próximos desafios se referem às linhas de crédito para financiamento e a redução dos impostos em duas frentes: para os equipamentos e para a energia gerada.

Dados da EPE (Empresa de Pesquisa Energética) mostram em seu PDE 2024 (Plano Decenal de Expansão de Energia) uma estimativa em que alcançamos uma capacidade instalada de geração solar de 8,3 GW (7 GW de geração descentralizada e 1,3 GW de geração distribuída), em 2024.

Dados do relatório "Energia Solar no Brasil e no Mundo - Ano de Referência - 2014", publicado pelo MME (Ministério de Minas e Energia) informam que, em 2018, o Brasil deverá figurar entre os 20 países com maior geração de energia solar. Há ainda estimativas de que, em 2050, tenhamos geração fotovoltaica em $18 \%$ dos domicílios brasileiros (ou 13\% da demanda residencial total).

\section{Petróleo e gás}

A Lei 9.478/1997, também denominada Lei do Petróleo, promoveu a abertura do setor por meio da concessão de áreas exploratórias, criou um ambiente em que a estatal monopolista Petrobras pudesse ter ações negociadas e captar financiamento e também criou a um órgão regulador e fiscalizador: a ANP (Agência Nacional do Petróleo, Gás e Biocombustíveis). Sob a égide deste novo marco regulatório, as atividades de exploração e produção de petróleo e gás natural passaram a ser regidas pelo regime de concessão, envolvendo tanto a Petrobrás como empresas privadas nacionais $\mathrm{e}$ implementation of a regulatory mark for micro and mini-generation were a great regulatory evolution in the last years. The next challenges will be the credit lines for funding and the tax reduction in two fronts: for equipment and for generated energy.

EPE data in the 2024 PDE estimate that Brazil will reach an installed capacity of solar generation of 8.3 GW in 2024: 7 GW of decentralized generation and $1.3 \mathrm{GW}$ of distributed generation.

Data from the report "Energia Solar no Brasil e no Mundo - Ano de Referência - 2014", published by the MME (Ministry of Mines and Energy) inform that Brazil should stay among the 20 countries with higher generation of solar energy in 2018. Other estimates show that we will have photovoltaic generation in $18 \%$ of the Brazilian housing units in 2050 , or $13 \%$ of the total residential demand.

\section{Oil and gas}

Law no. 9,478/1997, also called Petroleum Act, opened this sector through the granting of oil blocks and created an environment in which the monopolist state-run Petrobras could trade its stocks and be funded, as well as created a regulating and supervising body, the ANP. Under the auspices of this new regulatory mark, the activities of exploitation and production of petroleum and natural gas began to be ruled by the concession regime, involving either Petrobras or private national companies and foreign private and 
conglomerados privados e estatais estrangeiros. Refino, processamento de gás natural, dutos e terminais, por sua vez, ficaram sujeitos a um regime de autorizações federais, e igualmente abertos à pluralidade de investidores interessados.

O advento da anunciada auto-suficiência nacional em petróleo em 2005 (ver Gráfico 13.5) e o anúncio das descobertas na camada pré-sal das bacias marítimas de Campos, Santos e Espírito Santo, em 2006, provocaram a discussão e a implantação de um marco regulatório paralelo, especialmente destinado a regular a exploração e produção de hidrocarbonetos no Pré-Sal brasileiro. A Lei 12.351/10 instituiu o regime de partilha de produção sobre tais áreas reforçando a participação direta do Estado brasileiro nos seus resultados. Além da regulação e fiscalização das atividades pela ANP, foi criada uma empresa $100 \%$ estatal, a Pré-Sal Petróleo S.A. (PPSA) para representar os interesses da União nos empreendimentos do présal, onde ela passou a ter direito a parte do óleo e/ou gás produzidos.

O Brasil tem hoje, portanto, dois regimes legais em vigor quanto à exploração e produção de petróleo: o de concessões - que continua valendo para todas as áreas como regra geral, e o de partilha de produção - que vale para as áreas do polígono do Pré-Sal (área de $800 \times 200$ quilômetros ao longo da projeção marítima dos Estados do Espírito Santo a Santa Catarina, a 300-500 quilômetros da costa brasileira). O primeiro prevê que os riscos exploratórios e operacionais, bem como os resultados da produção de petróleo e gás sejam integralmente do concessionário (empresa ou consórcio), que, em retorno, paga royalties, state-run conglomerates. Natural gas refining, processing, ducts and terminals, on their turn, were subject to federal authorizations and were equally open to any interested investor.

The announcements of the petroleum self-sufficiency in 2005 (see Graph 13.5) and of the petroleum discovery in the pre-salt layer in the offshore basins of Campos, Santos and Espírito Santo in 2006 raised the discussion for the implementation of a parallel regulatory mark, especially destined to regulate the exploitation and production of hydrocarbons in the Brazilian Pre-Salt. Law no. 12,351/2010 established the sharing regime for the production in these areas, reinforcing the direct participation of the Union in their output. Besides the regulation and supervision of the activities by the ANP, a $100 \%$ state-run company, Pré-Sal Petróleo S.A. - PPSA, was created to represent the interests of the Union in the pre-salt ventures, whose rights on the oil and/or gas produced were partly for the Union.

Therefore, Brazil has today two legal regimes in force concerning the exploitation and production of petroleum: the concession regime which is still valid for every area as a general rule - and the sharing regime valid for the areas of the Pre-Salt polygon (800x200 kilometer areas along the maritime projection from the states of Espírito Santo to Santa Catarina, at a 300500 kilometer distance from the Brazilian coastline). The first regime states that operational and exploitation risks, as well as the output of the production of oil and gas are under the entire responsibility of the operator - either a company 
participação especial e impostos ao Estado brasileiro. O segundo prevê não apenas que a União (através da PPSA) receba parte do resultado da produção mas também que o único operador admitido (na qualidade de empresalíder de consórcio ou sozinha) seja a Petrobras e sua participação mínima em qualquer empreendimento de partilha seja de $30 \%$. Estes dois últimos pontos estão sendo objeto de acirrada discussão parlamentar, com vistas a serem eliminados do regime de partilha brasileiro.

O Brasil contabiliza hoje 347 blocos exploratórios em atividade, sendo 123 localizados no mar (incluindo a área de Libra) e 224 em terra. Estes números se alteram ocasionalmente por conta das devoluções de áreas, mas hoje a atividade exploratória se desenvolve por 24 bacias brasileiras. A Petrobras é a maior detentora de áreas: 62 marítimas e 50 terrestres. Mais de 40 empresas já atuam na exploração de hidrocarbonetos no Brasil, mas a atividade se ressente da ausência de novos leilões de áreas, da crise financeira da Petrobras e, ultimamente, da queda do preço do petróleo no mercado internacional.

Há quase 12 anos não ocorrem leilões de blocos exploratórios em águas profundas para as áreas convencionais (não Pré-Sal) das bacias de Campos e Santos, principais produtoras do país. Ejá se decorrem seis anos sem a realização de qualquer leilão de área exploratória. Em 2015, a maior parte da atividade de perfuração exploratória foi concentrada em trabalhos de avaliação no pré-sal da bacia de Santos. A atividade de perfuração exploratória encontra-se extremamente reduzida: em 2015, foram apenas 77 poços sendo $53 \mathrm{em}$ or a joint venture - which, in return, pay royalties, special participation and taxes to the Union. The second regime states that not only the Union through the PPSA - receives part of the output, but also that Petrobras should be the only operator - either as the leader of the joint venture or by itself - and that its minimal participation in any sharing venture should be $30 \%$. These two last points are now the subject of a heated debate in the Congress, so as to being eliminated from the Brazilian sharing regime.

Brazil counts today with 347 exploitation blocks in activity, being 123 located offshore - including the Libra area - and 224 onshore. Although changing at times due to the returning areas, the exploitation activity is in operation today in 24 Brazilian basins. Petrobras is the biggest holder of areas: 62 offshore and 50 onshore. More than 40 companies explore hydrocarbons in Brazil, yet this activity has been suffering from the lack of new block auctions, the financial crisis of Petrobras, and, lately, the drop in the petroleum prices in the international market.

As the major producers in Brazil, the basins of Campos and Santos have not auctioned off exploitation blocks in deep waters for conventional areas - not Pre-Salt - for nearly 12 years. None auction of exploitation area has been carried out for six years. In 2015, most of the activity of exploratory drilling was concentrated on the assessment of yet-to-find oil in the pre-salt area in the Santos basin. The activity of exploratory drilling has significantly reduced: 77 oil wells 
terra e 24 no mar, praticamente um terço do que se realizou em 2011. É esperado que as campanhas exploratórias em águas profundas na Margem Equatorial (objeto da 11a Rodada da ANP), comecem a mostrar resultados ao longo de 2016 e 2017: são 14 empresas operando em 39 blocos desta nova fronteira exploratória.

Os anos de 2013 e 2014 foram caracterizados pelos esforços para cumprir os prazos de entrada em operação dos campos, principalmente no Pré-Sal. Por parte da Petrobrás, iniciaram-se as atividades de nove novos sistemas de produção e registrou-se um significativo aumento da eficiência operacional. Além da Petrobrás, outras concessionárias também atuaram no mesmo sentido. Isso assegurou ao país uma capacidade adicional instalada de cerca de $660 \mathrm{mil} \mathrm{b} / \mathrm{d}$ de petróleo e outros 26 milhões de m3/d de gás e projetou para o biênio 2015/2016 a interligação dos poços dos sistemas já em operação em Santos e Campos, o que fará a produção destas áreas atingir o nível a ser mantido nos próximos anos ("plateau de produção"). Em 2014, a produção média de petróleo nacional registrou crescimento e ultrapassou a marca dos 2.300 barris/dia e a de gás superou a marca dos 88 milhões de $\mathrm{m} 3 /$ dia. Houve aumento significativo da produção dos campos de gás, tanto marítimos quanto terrestres.

A grande fonte do aumento da produção de petróleo nacional tem sido as áreas marítimas da bacia de Santos, que apresentou crescimento de $134 \%$ em 2014. Os maiores campos produtores do Brasil continuam sendo das áreas convencionais da Bacia de Campos: Roncador, Marlim Sul e Marlim. Os campos do pré-sal vem evoluindo em in 2015, being 53 onshore and 24 offshore, nearly one third of what was accomplished in 2011. Exploratory campaigns in deep water in the Equatorial Margin - object of the 11th Round of the ANP - are expected to show results along 2016 and 2017: 14 companies operating 39 blocks of this new exploratory frontier.

The years of 2013 and 2014 marked the efforts to fulfill the field operation deadlines, mainly in the Pre-Salt. Petrobras, on its turn, began to operate nine new production systems, registering a significant increase in the operational efficiency. Besides Petrobras, other concessionaires also worked in the same way. That effort provided Brazil with an additional installed capacity of about 660 thousand bpd of oil and 26 million $\mathrm{m} 3 / \mathrm{d}$ of gas and set for the 2015-2016 biennium the interconnection of the wells of the systems in operation in Santos and Campos. This will cause the output in these areas to hit the level to be maintained in the years to come ("production plateau"). In 2014, the average production of the national oil surpassed 2,300 bpd and that of gas, 88 million $\mathrm{m} 3 / \mathrm{d}$. The production of gas fields increased significantly, both offshore and onshore.

Having increased $134 \%$ in 2014 , the offshore areas of the Santos basin accounted for most of the increase in the production of the national oil. The conventional areas of the Campos basin remain as the biggest producers in Brazil: Roncador, Marlim Sul and Marlim. The presalt fields have been evolving at an 
ritmo acelerado, em especial Lula e Sapinhoá. No biênio 2015/2016 os destaques deverão ser os campos de Lula Central, Lula Alto, Iracema, Lapa e Libra (que inicia teste de longa duração) todos operados pela Petrobras. No grupo das demais operadoras, os destaques são o início das operação do campo de óleo ultra-pesado de Atlanta (Bacia de Santos), pelo consórcio Queiroz GalvãoPetrobras-Barra Energia, e a estabilidade dos níveis de produção de petróleo pesado em Pelegrino (Bacia de Campos) pela operadora estatal norueguesa Statoil, que tem como parceira a chinesa Sinochem. No Parque das Conchas, operado pela Shell, novos poços e interconexões visam incrementar a produtividade deste conjunto de campos da bacia de Campos.

As perspectivas para 2016 são de crescimento contínuo da produção nacional, embora provavelmente em níveis inferiores aos de 2014 e 2015. Com isso, o nível de 2,5 milhões de barris/dia ainda não deverá ser alcançado. Projetase também avanço na produção e na reinjeção de gás natural em detrimento da sua queima.

A gradual aceleração do crescimento econômico do país resultou em aumento consistente do consumo de petróleo e derivados por uma década 2004-2014 (Gráfico 13.3). Isso também fez com que se planejasse o incremento da capacidade de refino brasileira, em especial após ser constatada a qualidade do óleo que adviria do Pré-Sal.

A Petrobras é a empresa hegemônica do parque de refino nacional, e a sua má fase financeira combinada com accelerated pace, especially Lula and Sapinhoá. In the 2015-2016 biennium, the highlights should be the fields of Central Lula, High Lula, Iracema, Lapa and Libra - which is beginning the long-term tests, all of them operated by Petrobras. Concerning the other operators, the highlights were the beginning of the operation of the ultraheavy oil field of Atlanta (Santos basin) by the joint venture Queiroz GalvãoPetrobras-Barra Energia, and the stabilization of the production levels of heavy oil in Pelegrino (Campos basin) by Norwegian state-run Statoil, partnered with Sinochem from China. Operated by Shell, new wells and interconnections in the Conchas Park aim at increasing the productivity of this set of fields in the Campos basin.

The perspectives for 2016 point out to the continuous increase of the national production, though probably at lower levels than in 2014 and 2015. As a result, the level of 2.5 million barrels per day is yet to be reached. The advance in the production and in the re-injection of natural gas rather than burning it is also in the agenda.

The gradual acceleration of the economic growth of Brazil resulted in the consistent increase in the consumption of petroleum and its derivatives along the 2004-2014 period (Graph 13.3). This also caused the country to plan the increase in its refining capacity, especially after determining the quality of the oil coming from the Pre-Salt.

As Petrobras is the hegemonic company in the national refining park, its bad financial situation coupled 
a baixa do mercado internacional de petróleo e derivados certamente atinge drasticamente os planos de expansão da capacidade de refino brasileira. A maior parte do plano qüinqüenal da Petrobras para o refino será destinada à manutenção das refinarias em operação.

Além da confirmação do cancelamento dos projetos das refinarias Premium le II (no Nordeste) até 2019, a finalização da RNEST (Refinaria Abreu e Lima), em Pernambuco, e do COMPERJ, no Rio, serão pauta de intensos debates internos e externos à Petrobras. Inicialmente projetado para ser um moderno complexo petroquímico, o COMPERJ foi reconfigurado para ser uma refinaria de $465 \mathrm{mil}$ barris/dia mas teve suas obras interrompidas com mais de $80 \%$ concluído. Já a RNEST, já chega a processar mais de 90 mil barris/dia de petróleo, produzindo diesel e coque. É esperado que a instalação de uma unidade de abatimento de emissões (SNOX) permita que a nova refinaria passe processar até $130 \mathrm{mil}$ barris/dia. No entanto, a construção do chamado "segundo trem" da RNEST deve ser adiada, e sua entrada em operação deverá ficar para 2023, ampliando a capacidade total da refinaria para $260 \mathrm{mil}$ barris/dia.

A RNEST foi configurada para contribuir sobremaneira com a produção de diesel $\mathrm{S} 10$. Com teor mais baixo de enxofre, o diesel S-10 já alcançou $30 \%$ do mercado nacional e é menos poluente, obedecendo às exigências do Conselho Nacional de Meio Ambiente (CONAMA). Mais de 5.500 postos de combustíveis, ao longo das principais rodovias brasileiras, já contam com o novo combustível para o abastecimento da frota de transporte de carga. with the drop in the international market of petroleum and its derivatives severely hits the expansion plans of the Brazilian refining capacity. Most of the Petrobras' five-year plan for refining will be destined to the maintenance of the refineries in operation.

The cancellation of the projects of the Premium I and II refineries (in the Northeast) up to 2019, the finishing of RNEST (Abreu e Lima Refinery) in Pernambuco and COMPERJ in Rio de Janeiro will be subject to intense debates inside and outside Petrobras. Initially designed to be a state-of-theart petrochemical complex, COMPERJ was redesigned to be a 465 thousand bpd refinery and was $80 \%$ completed, when it had its civil works interrupted. RNEST, on its turn, processes more than 90 thousand barrels per day, producing diesel and coke. The installation of a unit for reducing emissions (SNOX) is expected to allow the new refinery to process up to 130 thousand barrels per day. Nonetheless, the construction of the "second train" of RNEST should be delayed until 2023, when it will improve the total capacity of the refinery to 260 thousand barrels per day.

RNEST was designed to deeply contribute to the production of S10 diesel. Containing lower levels of sulfur, S10 diesel has already reached $30 \%$ of the national market and is less polluting, complying with the requirements of the National Council of Environment - CONAMA. Along the major Brazilian highways, more than 5,500 gas stations count with S10 diesel to refuel the freight transportation fleet. 


\section{Biocombustíveis}

Dados divulgados pela Agência Nacional do Petróleo, Gás Natural e Biocombustíveis (ANP) e compilados pela União da Indústria de Cana-de-Açúcar (UNICA) indicam que o consumo total de combustíveis utilizados pela frota de veículos leves (ciclo Otto) permaneceu estável em 2015 em relação a 2014 (aumento de 0,34\%). No entanto, a participação do etanol hidratado carburante na matriz energética aumentou, atingindo $33,3 \%$ do total consumido. Houve ampliação de $37,47 \%$ do consumo, alcançando quase 18 bilhões de litros (13 bilhões registrados em 2014), contra queda de $7,27 \%$ no consumo de gasolina $\mathrm{C}$ (onde está presente o etanol anidro): o aumento do nível de mistura de $25 \%$ para $27 \%$ do etanol anidro na gasolina a partir de março de 2015 levou a uma redução no consumo de gasolina $A$ (-9,22\% em relação a 2014).

Quanto ao biodiesel consumido no Brasil, a soja permanece responsável por mais de $82 \%$ da produção de biodiesel no Brasil, seguida pela gordura bovina, com $16 \%$. A principal destinação do biodiesel é o abastecimento dos veículos a diesel. Para se tornar um combustível compatível com os motores a diesel, a gordura vegetal ou animal passa por um processo químico chamado transesterificação. Este processo é realizado por mais de 60 usinas produtoras de biodiesel autorizadas pela ANP em território nacional. Depois, o biodiesel produzido na usina segue para a base de distribuição, onde é misturado ao diesel de petróleo, em percentuais regulamentados pela ANP. Em seguida, é transportado para os postos de combustíveis.

\section{Biofuels}

Data released by the ANP and compiled by the Brazilian Sugarcane Industry Association - UNICA point out that the total consumption of fuels used in light vehicles (Otto cycle) remained stable in 2015 in relation to 2014 (increase of $0.34 \%$ ). Nevertheless, the contribution of hydrated ethanol fuel to the energy matrix increased, hitting $33.3 \%$ of the total consumed. The consumption increased $37.47 \%$, reaching nearly 18 billion liters ( 13 billion recorded in 2014), against a drop of $7.27 \%$ in the consumption of $C$ gasoline (which includes anhydrous ethanol): the increase in the level of anhydrous ethanol in gasoline from $25 \%$ to $27 \%$ since March 2015 led to a reduction in the consumption of A gasoline $(-9.22 \%$ in relation to 2014).

Regarding the biofuel consumed in Brazil, soybeans remain responsible for more than $82 \%$ of the production of biofuels in Brazil, followed by beef tallow $(16 \%)$. Biofuel is mainly destined to refuel diesel vehicles. To become a fuel compatible with diesel engines, vegetable or animal fat is submitted to a chemical process called transesterification. This process is carried out by more than 60 biodiesel plants authorized by the ANP in the national territory. After that, the biodiesel produced in the plant is delivered to the distribution, where it is mixed with petroleum diesel in percentages regulated by the ANP. It is then transported to the gas stations. 


\section{Análise dos Dados das Tabelas e Gráficos}

A tabela 13.1 apresenta os dados gerais de energia referentes a oferta interna de energia, consumo final de energia, geração de eletricidade, produção de petróleo, importação e exportação de energia. Nos últimos três anos em análise (2012 a 2014), há um aumento na oferta interna de energia, muito justificado pelo aumento de $15,92 \%$ da produção de petróleo, 2.351 (103b/d) em 2014 contra 2.028 em 2013 (103b/d /) e pelo aumento da geração de eletricidade quando comparados os anos de 2013 (570,025 TWh) e de 2014 (590,479 TWh) - registrando um aumento percentual de $3,59 \%$.

O aumento de eletricidade, apresentado na tabela 13.2, é justificado na sua maior parte pelo crescimento na geração dos estados que se destacam com o surgimento e implementação de novas fontes de energias, alternativas às hidroelétricas e termelétricas existentes. É o caso principalmente da fonte eólica, das pequenas centrais hidrelétricas, da biomassa e, em menor numero mas com tendência de crescimento consistente no futuro, da fonte solar.

Estados da região Nordeste como Rio Grande do Norte, Bahia, Ceará, Pernambuco, Piauí, Paraíba e, na região sul, os estados de Santa Catarina e Rio Grande do Sul, destacam-se pelo desenvolvimento recente do aproveitamento da energia dos ventos: a energia eólica. Esse tipo de fonte renovável de energia teve um crescimento exponencial desde o ano de 2009, quando passou a participar do sistema de contratação dos leilões

\section{Analysis of Table and Graph Data}

Table 13.1 shows general data on energy, like primary supply of energy, final consumption of energy, electricity generation, petroleum production, and energy import and export. In the last three years (2012 to 2014), the primary supply of energy increased, mostly due to the increase of $15.92 \%$ in the petroleum production, 2,351 (103 bpd) in 2014 against 2,028 in 2013 (103 bpd) and to the increase in electricity generation when compared with 2013 (570.025 TWh) and 2014 (590.479 TWh), posting a percentage increase of $3.59 \%$.

The increase in electricity, showed in table 13.2, is mostly explained by the increase in the generation in states that stood out in terms of new energy sources, replacing existing hydroelectric and thermoelectric plants. It is mainly the case of wind power, small hydroelectric plants, biomass and, at a lower level but tending to consistently increase in the future, solar energy.

The states of Rio Grande do Norte, Bahia, Ceará, Pernambuco, Piauí and Paraíba in the Northeast Region and the states of Santa Catarina and Rio Grande do Sul in the South Region stood out as they recently took advantage of the wind energy: wind power. This type of renewable source of energy exponentially increased since 2009, when it started to participate in the federal auctions of energy. It is expected 
federais para compra de energia. Esperase que, em 2016, a fonte eólica atingirá a marca de $10 \mathrm{GW}$ de potência instalada operando comercialmente no Brasil.

A Tabela 13.3 apresenta os dados de oferta interna de energia no ano de 2014 de países selecionados. Verificase que o Brasil, saiu de um patamar intermediário ao final do ano de 2013 para um nível de liderança quando comparado com os demais países no que diz respeito à oferta interna absoluta de energia primária. Em 2014, o Brasil alcançou a marca de 306 milhões de toneladas equivalentes de petróleo (tep), ultrapassando a Alemanha (304 milhões de tep) e ficando atrás apenas dos EUA (2.206 milhões de tep) e Japão (441 milhões de tep). Em relação à América Latina, o Brasil apresenta uma oferta interna de energia bastante superior à de países como a Argentina ( 80 milhões de tep) e Chile (39 milhões de tep).

Todavia, com uma população estimada em um pouco mais 203 milhões de habitantes (IBGE, 2014) os dados da oferta de energia per capita, no entanto, mostram que ela permanece baixa em relação a estes e outros países comparáveis. Isso significa que o nível de conforto energético oferecido a cada habitante ainda é mais baixo no Brasil do que em outras economias. Merecem destaque quanto a este indicador: 0 Canadá (7,2 tep/per capita) e os Estados Unidos (6,9 tep/per capita). Na América Latina, Argentina (1,9 tep/per capita) e Chile (2,2 tep/per capita) superam o Brasil neste quesito. $O$ desejável aumento da oferta interna de energia per capita deverá associar uma prudência na escolha das fontes sob o ponto de vista econômico e ambiental, associada ao that wind power hit the mark of $10 \mathrm{GW}$ of installed power under commercial operation in Brazil in 2016.

Table 13.3 shows data on primary energy supplied by selected countries in 2014. It can be noticed that Brazil left the intermediate level by the end of 2013 to a leadership position, when compared with other countries in terms of the absolute primary energy supply. In 2014, Brazil reached the mark of 306 million tonnes of oil equivalent (toe), surpassing Germany (304 million toe) and staying just behind the USA (2,206 million toe) and Japan (441 million toe). Compared with Latin America, Brazil supplies much more primary energy than countries like Argentina (80 million toe) and Chile (39 million toe).

Considering an estimated population of slightly more than 203 million inhabitants (IBGE, 2014), per capita energy supply data remained low in relation to these and other comparable countries. This means that the energy comfort level provided to each inhabitant is still lower in Brazil than in other economies. Canada (7.2 toe per capita) and the United States (6.9 toe per capita) stood out in this indicator. In Latin America, Argentina (1.9 toe per capita) and Chile (2.2 toe per capita) surpass Brazil in this regard. The desirable increase of the per capita supply of primary energy should select the sources with caution, either from the environmental or economic 
esforço na melhoria da distribuição de renda do país, de forma a assegurar as possibilidades de aumento da qualidade de vida do conjunto da população brasileira.

O aumento da geração de eletricidade (GWh), apresentado na tabela 13.2, é resultado do incentivo e desenvolvimento de novas fontes de geração de energia elétrica. A baixa operacionalização do potencial hidrelétrico brasileiro é apresentada na tabela 13.4 para cada bacia hidrográfica separadamente. $O$ potencial em operação, de apenas 38,04\% em relação ao potencial total que poderia ser utilizado, é devido ao fato de mais de $60 \%$ do potencial remanescente estar localizado na Região Amazônica que impõem grandes desafios de caráter sócio-ambiental para novos empreendimentos.

O gráfico 13.1 apresenta a evolução da produção de energia primária no Brasil para o período 2002-2014. No período analisado, a fonte de produção de energia primária com maior crescimento foi o gás natural com $105,46 \%$, seguido pelos produtos cana-de-açúcar com $94,81 \%$, petróleo com $55,35 \%$, energia hidráulica com $30,58 \%$ e por último a lenha com 4,62\%. Cabe destacar que, de acordo com o estudo "Logística de energia: redes e fluxos do território" do Instituto Brasileiro de Geografia e Estatística (IBGE), publicado em junho de 2016, compilando dados de agências reguladoras e outros órgãos do governo ligados ao setor de energia a geração eólica cresceu aproximadamente $460,9 \%$, de 2010 a 2014 , saltando de 2.177 gigawatts/hora (GWh) para 12.210 GWh anuais no período. Rio Grande point of view, and should couple with the effort to improve income distribution in Brazil, so as to ensure the increase of life quality of the Brazilian population as a whole.

The increase in the electricity generation (GWh), shown in table 13.2, resulted from the incentives to develop new sources of electricity generation. The low operationalization of the hydroelectric potential in Brazil is shown in table 13.4 for the individual river basins. The potential in operation, of only $38.04 \%$ in relation to the total potential that could be used, is due to the fact that more than $60 \%$ of the remaining potential is located in the Amazon Region, which imposes major socioenvironmental challenges for new endeavors.

Graph 13.1 shows the evolution of the production of primary energy in Brazil for the 2002-2014 period. In the period under analysis, the source of production of primary energy that mostly increased was natural gas $(105.46 \%)$, followed by sugarcane (94.81\%), petroleum (55.35\%), hydropower (30.58\%) and, lastly, firewood (4.62\%). According to the study "Logística de energia: redes e fluxos do território" (Energy Logistics: Territorial Networks and Flows) from the Brazilian Institute of Geography and Statistics - IBGE, published in June 2016, compiling data from regulatory agencies and other government bodies of the energy sector, wind power increased about $460.9 \%$ between 2010 and 2014, jumping from 2,177 annual GWh to 12,210 annual GWh 
do Norte, Bahia, Ceará e Rio Grande do Sul, nessa ordem, são os estados que dominam a geração de energia elétrica com a força do vento.

O Gráfico 13.2 indica a evolução da oferta interna de energia no Brasil para o período 2002-2014. Verifica-se que o gás natural apresentou o maior crescimento na oferta, com 179,49\%, seguido dos derivados da cana-deaçúcar (89,25\%), petróleo e derivados (40,94\%), hidráulica e eletricidade $(26,25 \%)$, e lenha e carvão vegetal (4,61\%).

O Gráfico 13.3 apresenta a evolução do consumo final de energia no Brasil por fonte para o período 2002-2014. Observa-se que acompanhando o perfil da evolução da oferta de energia interna, o gás natural apresentou o maior crescimento no consumo final de energia, com $88,11 \%$, seguido por eletricidade $(63,73 \%)$, bagaço de cana (63,54\%), derivados de petróleo $(42,99 \%)$ e lenha (15,21\%).

Ao se comparar os dados da evolução da oferta interna com os da evolução do consumo final observa-se uma pressão de demanda quanto à eletricidade, cujo crescimento do consumo no período, da ordem de $63,73 \%$, bastante superior ao crescimento da oferta de energia hidráulica e eletricidade, de $26,25 \%$. Esses números revelam um aumento do consumo (demanda) maior que o aumento da oferta de eletricidade principalmente proveniente das hidrelétricas, resultando em um aumento da produção de energia oriunda de termelétricas, com custo bem mais elevado. Além de refletir em uma energia mais cara para consumidor final, ocorrem in this period. Rio Grande do Norte, Bahia, Ceará and Rio Grande do Sul, in this order, are the states that master electricity generation with wind power.

Graph 13.2 shows the evolution of the supply of primary energy in Brazil for the 2002-2014 period. It can be seen that natural gas recorded the highest increase in the supply (179.49\%), followed by sugarcane products $(89.25 \%)$, petroleum and derivatives (40.94\%), hydropower and electricity $(26.25 \%)$, and firewood and charcoal (4.61\%).

Graph 13.3 shows the evolution of the final consumption of energy in Brazil, by source, for the 2002-2014 period. The profile of the evolution of the supply of primary energy showed that natural gas registered the biggest increase in the final consumption of energy ( $88.11 \%)$, followed by electricity (63.73\%), sugarcane bagasse $(63.54 \%)$, petroleum derivatives (42.99\%) and firewood (15.21\%).

The comparison between the evolution of primary supply and the evolution of final consumption pointed out a pressure on the demand concerning electricity, whose increase in the consumption in this period (about $63.73 \%$,) is considerably higher than the increase of hydropower and electricity (26.25\%). These figures unveil a higher increase in the consumption (demand) than the increase of the electricity supply mainly from hydroelectric plants, resulting in an increase in the production of energy from thermoelectric plants, at a much higher cost. Besides representing a more expensive energy for final 
consequências ambientais pela utilização de fontes que geram maiores impactos ao meio ambiente - normalmente óleo diesel e óleo combustível, derivados de petróleo.

É neste contexto que se revela a importância das medidas que $o$ Governo Federal tomou para incentivar fontes renováveis de energia como eólica, solar e a biomassa, que podem contribuir para complementar significativamente a oferta de energia elétrica gerada pelas hidrelétricas. No caso da biomassa, isso se observa no crescimento acentuado dos produtos da cana de açúcar no gráficos 13.1 referente à oferta primária de energia, e no gráfico 13.2 que indica a evolução da oferta interna de energia.

Outro aspecto importante a ser analisado é o menor crescimento do consumo final do gás natural $(88,11 \%)$ em relação ao crescimento da oferta (179,49\%). Esses números comprovam as dificuldades de se consolidar o consumo de gás natural no mercado interno. Além de um reforço na política energética brasileira voltada para estimular o consumo do gás natural como substituto de alguns derivados do petróleo em processos industriais ou no uso automotivo, caberiam investimentos em uma maior extensão da rede de canalização do gás natural nos principais centros urbanos do país para uso em cocção e aquecimento direto, trazendo benefícios ambientais e de eficiência energética.

O Gráfico 13.5 traz a evolução da dependência externa de energia, e confirma, com dados de 2002-2014, o aumento da necessidade de importação consumers, it causes environmental consequences as the sources used greatly impact the environment - usually diesel fuel and fuel oil, petroleum derivatives.

This context reveals the importance of the measures that the Federal Administration took to encourage renewable sources of energy like wind power, solar power and biomass, which may contribute to significantly complement the supply of electricity generated in hydroelectric plants. The case of biomass can be noticed through the sharp increase of sugarcane products in graph 13.1, related to primary energy supply, and in graph 13.2 , which shows the evolution of the supply of primary energy.

Another important aspect to be analyzed is the lower increase of the final consumption of natural gas $(88.11 \%)$ in relation to the increase of the supply (179.49\%). These figures prove the difficulties to consolidate the consumption of natural gas in the domestic market. In addition to a reinforcement in the Brazilian energy policy to encourage the consumption of natural gas as a replacement for some petroleum derivatives in industrial processes and in vehicles, investments to extend the pipeline network of natural gas in the major urban centers in Brazil should be made in order to use it in cooking and direct heating, bringing environmental and energy efficiency benefits.

Graph 13.5 brings the evolution of the dependency on foreign energy and validates, with the 20022014 data, the increasing need to 
do óleo diesel e gás liquefeito de petróleo (GLP) para satisfazer a demanda doméstica, mesmo com uma queda de 13,7 em 2013 para 6,3 em 2014. Este indicador é representativo do quanto o consumo final de derivados de petróleo não foi acompanhada pelo aumento da oferta, principalmente devido às dificuldades encontradas para a expansão da capacidade de refino no país. Saliente-se, entretanto, que tal descompasso não é tão incomum em outras economias. O investimento em refinarias é normalmente considerado de alto risco e representa grandes montantes de capital a ser empenhado. Por isso, é normal que economias que apresentem taxas variáveis de crescimento passem por períodos de maior ou menor dependência de importação de derivados, mesmo aquelas com grande capacidade de produção de petróleo, como o Brasil. Uma vez consolidados novos patamares de consumo, acima das oscilações periódicas relacionadas com a economia, os investimentos em refino passam a ser efetivamente prioritários.

O Gráfico 13.4 mostra a evolução do consumo final de energia no Brasil por setor, para o período 2002-2014. Percebe-se que o setor de transportes apresenta a maior taxa de crescimento (com $75,56 \%$ ), seguido do setor comercial (com $74,84 \%)$, setor industrial $(33,85 \%)$ e setor residencial (19,85\%). Estes dados seguem reforçando o caráter ineficiente do transporte de bens e pessoas no país, principalmente o modal rodoviário que consome óleo diesel a preços subsidiados, e pela precariedade do transporte coletivo, além de depender do estado de conservação das rodovias e das medidas em favor da mobilidade nos grandes centros urbanos. import diesel fuel and liquefied petroleum gas - LPG to meet the domestic demand, even after the drop from 13.7 in 2013 to 6.3 in 2014. This indicator represents how much the final consumption of petroleum derivatives was not followed by the increase in the supply, mainly due to the difficulties to expand the refining capacity in Brazil. Yet, this gap is not so unusual in other economies. The investment in refineries is usually considered high risk, involving a huge amount of capital. For this reason, economies with varying growth rates usually pass through periods of more or less dependency on the import of derivatives, even those with great production of petroleum, like Brazil. Once consolidating new levels of consumption, above the periodic fluctuations of the economy, the investments in refining become an effective priority.

Graph 13.4 shows the evolution of the final consumption of energy in Brazil, by sector, for the 2002-2014 period. The sector of transportation posts the highest growth rate $(75.56 \%)$, followed by the trade sector $(74.84 \%)$, industrial sector (33.85\%) and residential sector (19.85\%). These data reinforce, once more, the inefficient character of the transportation of goods and persons in Brazil, particularly the road mode. It consumes diesel fuel at subsidized prices, offers precarious public transportation, and depends on the conservation of roads and on the mobility measures in the large urban centers. 
O cenário para o setor de transportes pode ser modificado com estudos e incentivos quanto a medidas alternativas como a cabotagem (navegação costeira interna), sistemas urbanos de transporte coletivo e a adoção de veículos elétricos, que de fato proporcionariam uma redução de custos a médio e longo prazo, permitindo que a cadeia ganhasse em mão dupla - com a redução de custos de transporte, impactando positivamente nos custos de produtos e pessoas transportadas e, também, com a economia de combustíveis derivados do petróleo, que poderiam ser utilizados em outras aplicações, além de proporcionar menores impactos ambientais com a redução de emissão de gases poluentes para a atmosfera.

A predominância do consumo de energia permanece com o setor industrial, seguido dos setores residencial e comercial, os quais podem ser impactados positivamente com o surgimento e a evolução das fontes alternativas de energia que permitem a adesão de estratégias para geração/ produção de sua própria energia, seja por fonte eólica, pela fonte solar fotovoltaica e por sistemas híbridos de aplicação de ambas tecnologias. $O$ grande incentivo recente para aplicação destes mecanismos foi a implementação da resolução 482 da ANEEL que estabelece os critérios para geração e compensação de energia na conta de energia elétrica, favorecendo os consumidores inclusive na forma de condomínios ou consórcios.

Finalmente o Gráfico 13.7 ilustra a situação do Brasil como um dos países com maior participação de fontes renováveis em sua matriz energética,
The picture of the transportation sector can be changed with studies and incentives on alternative measurements like cabotage (inland cabotage), urban systems of public transportation and the use of electric vehicles. The measurements would reduce the costs in the medium and long terms, providing the chain with a two-way gain: reducing the transportation costs, positively impacting in the costs of products and persons transported, as well as saving fuels derived from petroleum, which could be used in other applications, besides causing less environmental impacts with the reduced emission of pollutant gases to the atmosphere.

Energy is predominantly consumed by the industrial sector, followed by the residential and trade sectors. These sectors can be positively impacted with the emerging alternative sources of energy, which allow strategies to generate/ produce their own energies, either through wind power, photovoltaic solar source or hybrid systems mixing both technologies. A recent major incentive for the application of these mechanisms was the implementation of the ANEEL's resolution no. 482, which establishes the criteria for energy generation and compensation in the electricity bills, benefiting the consumers, either as condominiums or joint ventures.

Finally, graph 13.7 portrays the situation of Brazil as one of the countries with the biggest participation of renewable sources 
comparado à média mundial. $O$ país apresenta cerca de $41 \%$ de sua matriz energética proveniente de renováveis. Grande parte disso deve-se à energia hidráulica. Todavia, deve-se considerar que a inserção de novas fontes renováveis como a eólica, solar e a biomassa tende a manter a nossa matriz diversificada e limpa.

Jean-Paul Prates

Mestre em Gestão de Energia e Recursos Renováveis pela Universidade da Pennsylvania, e em Economia da Energia pelo Instituto Francês do Petróleo. Tem mais de 20 anos de experiência na concepção e execução de centenas de projetos nas áreas de petróleo, gás natural, biocombustíveis e energia renovável. Participou da elaboração do marco regulatório da atual indústria do petróleo nacional. Exerceu o cargo de Secretário de Estado de Energia do Rio Grande do Norte onde atualmente dirige o primeiro think-tank do Nordeste: o CERNE - Centro de Estratégias em Recursos Naturais e Energia, voltado para estratégias públicas e privadas nestes setores.

Preside o Sindicato das Empresas do Setor Energético do RN, além da representação regional do Instituto Brasileiro de Petróleo (IBP). Fundador e sócio-sênior da Expetro (primeira consultoria de petróleo e gás do Brasil), da CRNBio (consultoria ambiental atuante na Região Nordeste) e da TradeWinds (aliança de investimentos em energia). in its energy matrix, compared with the world average. About $41 \%$ of the Brazilian energy matrix come from renewable sources. Great part of it is due to hydropower. It should be taken into account that the inclusion of new renewable sources like wind power, solar power and biomass tends to keep our matrix diversified and clean.

Master in Management of Energy and Renewable Resources from the University of Pennsylvania, and in Energy Economics from the French Institute of Petroleum. He has more than 20 years of experience in conceiving and implementing hundreds of projects in the petroleum, natural gas, biofuels and renewable energy areas. He participated in the creation of the regulatory mark of the current petroleum industry in Brazil.

He held office of State Secretary of Energy

of Rio Grande do Norte, where he currently manages the first think tank in the Northeast:

CERNE (Center for Strategies in Natural Resources and Energy), oriented to public and private strategies in these sectors. He chairs the Union of Enterprises in the Energy Sector of Rio Grande do Norte-SEERN, as well as the regional representation of the Brazilian Institute of Petroleum - IBP. Founder and senior partner of Expetro - the first advisor in oil and gas in Brazil -, CRNBio - environmental advisor in the Northeast Region - and TradeWinds - investment partnership in energy.

\section{Milton Pinto}

Especialista em energia eólica e engenheiro eletricista, com ambas formações pela Universidade Federal do Rio Grande do Norte. Autor de "Fundamentos de Energia Eólica" e Energia Elétrica - Geração, Transmissão e Sistemas Interligados". Mestrando em Energias

Renováveis pelo Departamento de Energia

Mecânica da Universidade Federal do Rio Grande do Norte e Diretor de Infra Estrutura Elétrica do CERNE - Centro de Estratégias em Recursos Naturais e Energia.
Expert in wind power energy and electrical engineer, both from the Federal University of Rio Grande do Norte. Author of "Fundamentos de Energia Eólica" and "Energia Elétrica - Geração, Transmissão e Sistemas Interligados". Attending the Master's Program in Renewable Energies from the Department of Mechanical Energy of the Federal University of Rio Grande do Norte and Director of Electric Infrastructure at CERNE. 


\section{João Agra Neto}

Graduado e Mestre em Engenharia de Produção pela Universidade Federal do Rio

Bachelor and Master in Production Engineering from the Federal University of Rio Grande do

Grande do Norte - UFRN. Coordenador de

Norte - UFRN. Coordinator of Management of Gestão de Dados e Estatísticas Setoriais do do

CERNE - Centro de Estratégias em Recursos

Naturais e Energia e do SEERN - Sindicato das

Empresas do Setor Energético do Rio Grande

do Norte. 
Tabela 13.1 - Dados gerais de energia - 2012-2014

Table 13.1 - General data of energy - 2012-2014

\begin{tabular}{|c|c|c|c|c|}
\hline $\begin{array}{c}\text { Especificação/ } \\
\text { Item }\end{array}$ & $\begin{array}{c}\text { Unidade/ } \\
\text { Unit }\end{array}$ & 2012 & 2013 & 2014 \\
\hline Oferta interna de energia/ & $10^{6}$ tep/ & 285 & 296 & 306 \\
\hline Total primary energy supply & $10^{6}$ toe & & & \\
\hline Per capital & tep/hab & 1,42 & 1,47 & 1,51 \\
\hline Per capita & toe/inhab & & & \\
\hline Por PIB/ & tep/1 000 US\$ & 0,096 & 0,097 & 0,100 \\
\hline Per GDP & toe $/ 1,000$ US\$ & & & \\
\hline Consumo final de energia/ & $10^{6}$ tep/ & 253 & 260 & 266 \\
\hline Final energy consumption & $10^{6}$ toe & & & \\
\hline Geração de eletricidade/ & TWh & 552 & 571 & 590 \\
\hline Electricity generation & $T W h$ & & & \\
\hline Produção de petróleo/ & $10^{3} \mathrm{~b} / \mathrm{d} /$ & 2072 & 2028 & 2351 \\
\hline Petroleum production & $10^{3} \mathrm{~b} / \mathrm{d}$ & & & \\
\hline Importação total de energia/ & $10^{3} \mathrm{bep} / \mathrm{d} /$ & 1.548 & 1.537 & 1.581 \\
\hline Total energy imports & $10^{3} \mathrm{boe} / \mathrm{d}$ & & & \\
\hline Exportação total de energia/ & $10^{3} \mathrm{bep} / \mathrm{d} /$ & 801 & 673 & 776 \\
\hline Total energy exports & $10^{3} \mathrm{boe} / \mathrm{d}$ & & & \\
\hline
\end{tabular}

Fonte/Source: Balanço energético nacional 2015: ano base 2014. Brasília, DF: Ministério de Minas e Energia, Empresa de Pesquisa Energética-EPE, 2015. Disponível em/Available from: <https://ben. epe.gov.br/default.aspx>. Acesso em: abr. 2016/Cited: Apr. 2016.

Nota: tep - tonelada equivalente de petróleo; bep - barril equivalente de petróleo; b/d - barril por dia; produção de petróleo inclui óleo de xisto e líquidos de gás natural./Note: toe - ton of oil equivalent; boe - barril of oil equivalent; b/d - barril per day. 
Tabela 13.2 - Geração de energia elétrica - 2013-2014

Table 13.2 - Generation of electric energy - 2013-2014

\begin{tabular}{|c|c|c|c|}
\hline \multirow{3}{*}{$\begin{array}{l}\text { Unidades da Federação/ } \\
\text { Federation Units }\end{array}$} & \multicolumn{3}{|c|}{$\begin{array}{l}\text { Geração de energia elétrica/ } \\
\text { Generation of eletric energy }\end{array}$} \\
\hline & 2013 & 2014 & $\begin{array}{l}\text { Percentual de } \\
\text { crescimento } \\
\text { 2014/2013/ }\end{array}$ \\
\hline & \multicolumn{2}{|c|}{ GWh/ GWh } & $2014 / 2013$ \\
\hline Brasil/ Brazil & 570025 & 590479 & 3,6 \\
\hline Rondônia & 6407 & 15148 & 136,4 \\
\hline Acre & 234 & 250 & 7,1 \\
\hline Amazonas & 9970 & 8946 & (-) 10,3 \\
\hline Roraima & 169 & 245 & 44,5 \\
\hline Pará & 41191 & 41951 & 1,8 \\
\hline Amapá & 1816 & 1933 & 6,5 \\
\hline Tocantins & 11881 & 12227 & 2,9 \\
\hline Maranhão & 11181 & 15972 & 42,9 \\
\hline Piauí & 731 & 990 & 35,5 \\
\hline Ceará & 10396 & 15957 & 53,5 \\
\hline Rio Grande do Norte & 3756 & 7011 & 86,7 \\
\hline Paraíba & 1854 & 3434 & 85,2 \\
\hline Pernambuco & 9733 & 12712 & 30,6 \\
\hline Alagoas & 13029 & 11374 & (-) 12,7 \\
\hline Sergipe & 6760 & 5896 & (-) 12,8 \\
\hline Bahia & 22416 & 23103 & 3,1 \\
\hline Minas Gerais & 54013 & 46127 & (-) 14,6 \\
\hline Espírito Santo & 8464 & 10368 & 22,5 \\
\hline Rio de Janeiro & 55112 & 59298 & 7,6 \\
\hline São Paulo & 75517 & 65409 & (-) 13,4 \\
\hline Paraná & 103447 & 98834 & $(-) 4,5$ \\
\hline Santa Catarina & 25660 & 29416 & 14,6 \\
\hline Rio Grande do Sul & 27306 & 34042 & 24,7 \\
\hline Mato Grosso do Sul & 25281 & 24339 & (-) 3,7 \\
\hline Mato Grosso & 12361 & 14260 & 15,4 \\
\hline Goiás & 31212 & 31110 & (-) 0,3 \\
\hline Distrito Federal/Federal District & 129 & 127 & (-) 1,7 \\
\hline
\end{tabular}

Fonte/Source: Balanço energético nacional 2015: ano base 2014. Brasília, DF: Ministério de Minas e Energia, Empresa de Pesquisa Energética - EPE, 2015. Disponível em/Available from : $<$ https://ben. epe.gov.br/default.aspx>. Acesso em: abr. 2016/Cited: Apr. 2016.

Nota: Inclusive geração de autoprodutores./Note: Including generation from auto-producers. 


\section{Tabela 13.3 - Produção de petróleo e oferta interna de energia,}

por países selecionados - 2014

Table 13.3 - Petroleum production and total primary energy supply, by selected countries - 2014

\begin{tabular}{|c|c|c|c|}
\hline \multirow{2}{*}{$\begin{array}{l}\text { Países selecionados/ } \\
\text { Selected countries }\end{array}$} & \multirow{2}{*}{$\begin{array}{c}\text { Produção } \\
\text { de petróleo } \\
1000 \text { bbl/dia (1) / } \\
\text { Petroleum } \\
\text { production } \\
\text { 1,000 barrels } \\
\text { per day (1) }\end{array}$} & \multicolumn{2}{|c|}{$\begin{array}{l}\text { Oferta interna de energia/ } \\
\text { Total primary energy supply }\end{array}$} \\
\hline & & $\begin{array}{c}\text { Total } \\
10^{6} \text { tep } \\
\text { Total } \\
10^{6} \text { toe }\end{array}$ & $\begin{array}{c}\text { tep } \\
\text { per capital } \\
\text { toe } \\
\text { per capita }\end{array}$ \\
\hline Alemanha/ Germany & 142 & 304 & 3,8 \\
\hline Argentina/Argentina & 668 & 80 & 1,9 \\
\hline Brasil/ Brazil & 2028 & 306 & 1,5 \\
\hline Canadá/Canada & 4251 & 258 & 7,2 \\
\hline Chile/Chile & 9 & 39 & 2,2 \\
\hline Estados Unidos/United States & 12734 & 2206 & 6,9 \\
\hline França/France & 86 & 242 & 3,8 \\
\hline Itália/Italy & 140 & 146 & 2,4 \\
\hline Japão/Japan & 13 & 441 & 3,5 \\
\hline Reino Unido/United Kingdom & 882 & 178 & 2,8 \\
\hline
\end{tabular}

Fonte/Source : International Energy Agency - IEA.

(1) Inclusive NGL - Líquidos de Gás Natural. / (1) Includes NGL - Natural Gas Liquified.

Tabela 13.4 - Potencial hidrelétrico, segundo as bacias hidrográficas - 2015 Table 13.4 - Hydroelectric potential, according to river basins - 2015

\begin{tabular}{|c|c|c|c|c|c|}
\hline \multirow[b]{2}{*}{$\begin{array}{l}\text { Bacias hidrográficas/ } \\
\text { Hydrographic basins }\end{array}$} & \multicolumn{5}{|c|}{$\begin{array}{l}\text { Potencial hidrelétrico (Potência instalada-MW)/ } \\
\text { Hydroelectric potential (Installed power-MW) }\end{array}$} \\
\hline & $\begin{array}{l}\text { Total/ } \\
\text { Total }\end{array}$ & $\begin{array}{c}\text { Em } \\
\text { operação/ } \\
\text { In } \\
\text { operation }\end{array}$ & $\begin{array}{l}\text { Inventário/ } \\
\text { Inventory }\end{array}$ & $\begin{array}{l}\text { Estimado/ } \\
\text { Estimated }\end{array}$ & $\begin{array}{l}\text { Outros/ } \\
\text { Others }\end{array}$ \\
\hline Total Total & 247465 & 94125 & 71939 & 46132 & 35268 \\
\hline Amazônica/Amazon & 96638 & 10742 & 38362 & 32976 & 14558 \\
\hline Tocantins/Tocantins & 26895 & 13229 & 7889 & 1908 & 3869 \\
\hline $\begin{array}{l}\text { Atlântico Norte e Nordeste/North } \\
\text { and Northeast Atlantic }\end{array}$ & 2889 & 587 & 855 & 707 & 740 \\
\hline São Francisco/São Francisco & 22615 & 10724 & 3896 & 1561 & 6433 \\
\hline Atlântico Leste/ Eastern Atlantic & 14170 & 5394 & 5796 & 1423 & 1557 \\
\hline Paraná/Paraná & 62336 & 43371 & 9251 & 5112 & 4602 \\
\hline Uruguai/Uruguay & 11718 & 6354 & 4054 & 416 & 895 \\
\hline Atlântico Sudeste/ Southeast Atlantic & 10205 & 3724 & 1837 & 2031 & 2613 \\
\hline
\end{tabular}

Fonte/Source: Potencial hidrelétrico brasileiro por bacia hidrográfica. Rio de Janeiro: Centrais Elétricas Brasileiras - ELETROBRAS, [2015]. Disponível em/Available from: <https://www.eletrobras.com/elb/ data/Pages/LUMIS21D128D3PTBRIE.htm>. Acesso em: abr. 2016./Cited: Apr. 2016.

Nota: Dados de dezembro 2015./Note: Data for December 2015. 


\section{Gráfico 13.1 - Produção de energia primária - 2002-2014 \\ Graph 13.1 - Primary energy production - 2002-2014}

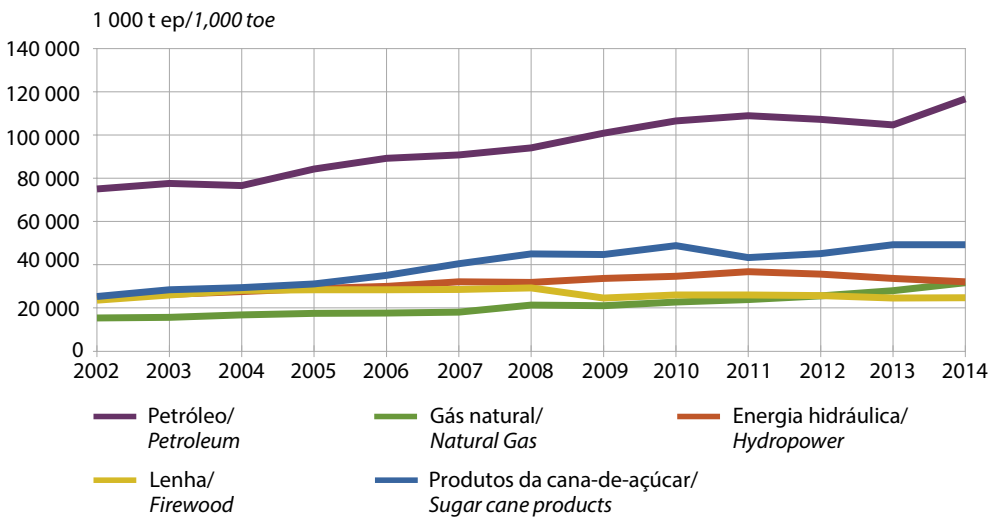

Fonte/Source: Balanço energético nacional 2015: ano base 2014. Brasília, DF: Ministério de Minas e Energia, Empresa de Pesquisa Energética - EPE, 2015. Disponível em/Available from: <https://ben.epe.gov.br/ default.aspx>. Acesso em: abr. 2016/Cited:Apr. 2016.

\section{Gráfico 13.2 - Evolução da oferta interna de energia - 2002-2014 Graph 13.2 - Evolution of primary energy supply - 2002-2014}

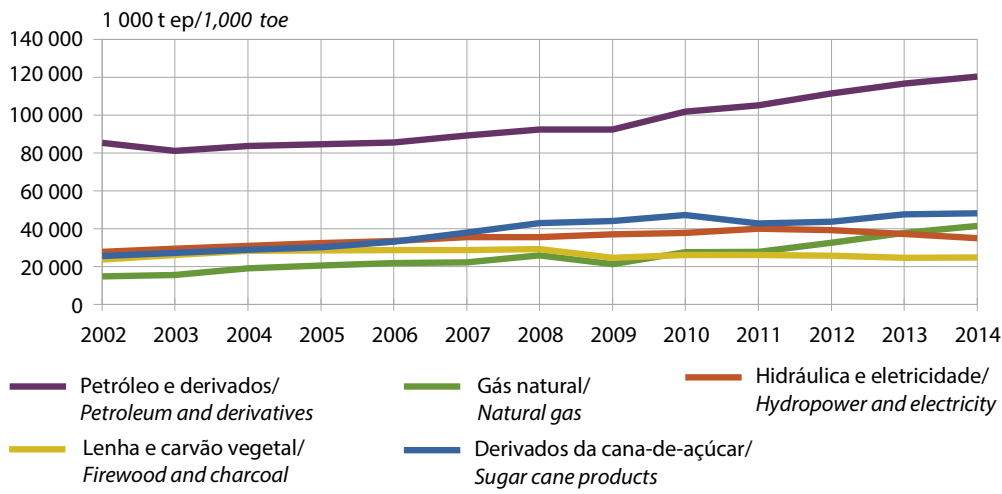

Fonte/Source: Balanço energético nacional 2015: ano base 2014. Brasília, DF: Ministério de Minas e Energia, Empresa de Pesquisa Energética - EPE, 2015. Disponível em/Available from: <https://ben.epe.gov.br/ default.aspx>. Acesso em: abr. 2016/Cited:Apr. 2016. 
Gráfico 13.3 - Evolução do consumo final de energia, por fonte - 2002-2014 Graph 13.3 - Evolution of final energy consumption, by source - 2002-2014

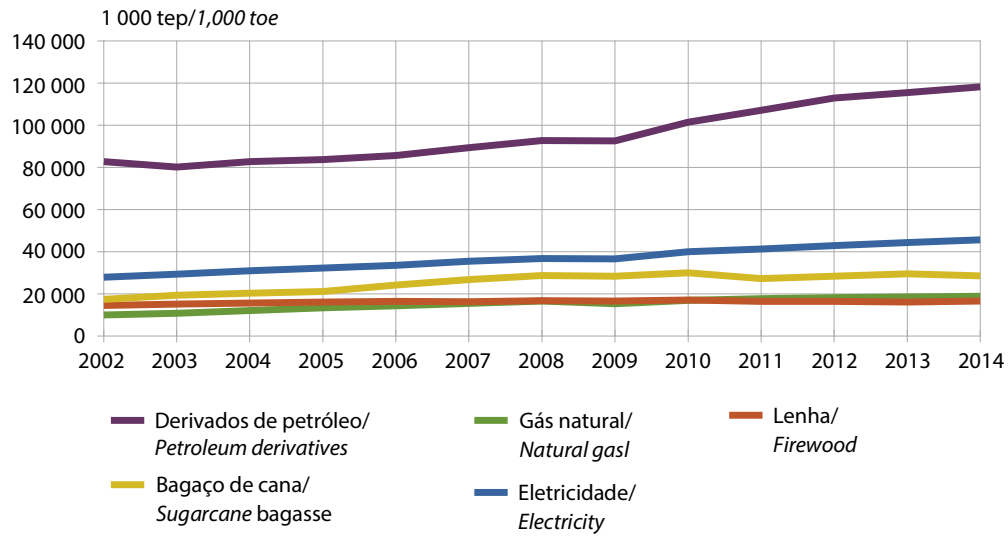

Fonte/Source: Balanço energético nacional 2015: ano base 2014. Brasília, DF: Ministério de Minas e Energia, Empresa de Pesquisa Energética - EPE, 2015. Disponível em/Available from: <https://ben.epe.gov.br/ default.aspx>. Acesso em: abr. 2016/Cited:Apr. 2016.

\section{Gráfico 13.4 - Evolução do consumo final de energia, por setor - 2002-2014} Graph 13.4 - Evolution of final energy consumption, by sector - $2002-2014$

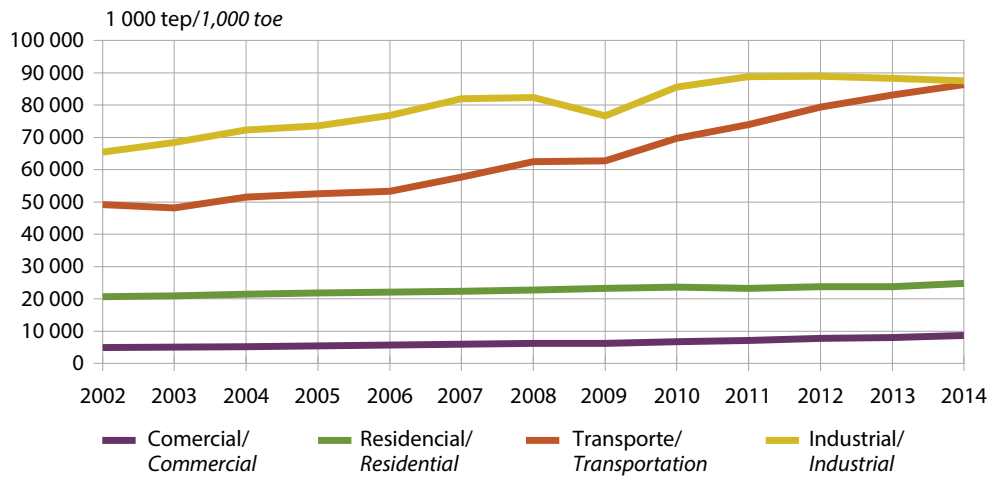

Fonte/Source: Balanço energético nacional 2015: ano base 2014. Brasília, DF: Ministério de Minas e Energia, Empresa de Pesquisa Energética - EPE, 2015. Disponível em/Available from: <https://ben.epe.gov.br/ default.aspx>. Acesso em: abr. 2016/Cited: Apr. 2016. 


\section{Gráfico 13.5 - Dependência externa de energia - 2002-2014 Graph 13.5 - Dependence on foreign energy - 2002-2014}

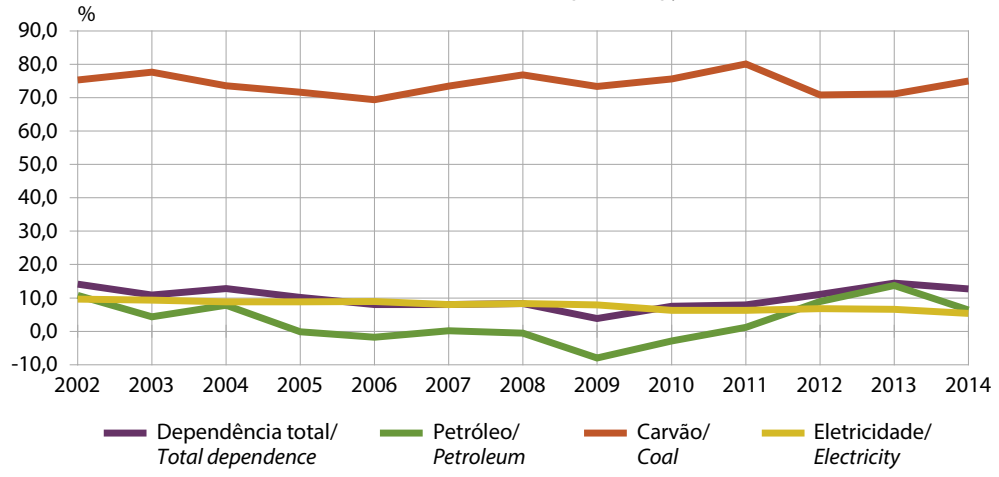

Fonte/Source: Balanço energético nacional 2015: ano base 2014. Brasília, DF: Ministério de Minas e Energia, Empresa de Pesquisa Energética - EPE, 2015. Disponível em/Available from: <https://ben.epe.gov.br/ default.aspx>. Acesso em: abr. 2016/Cited: Apr. 2016.

\section{Gráfico 13.6 - Evolução do consumo final de energia em relação ao valor agregado, por setor - 2003-2014}

Graph 13.6 - Evolution of final energy consumption in relation to the added value, by sector - 2003-2014

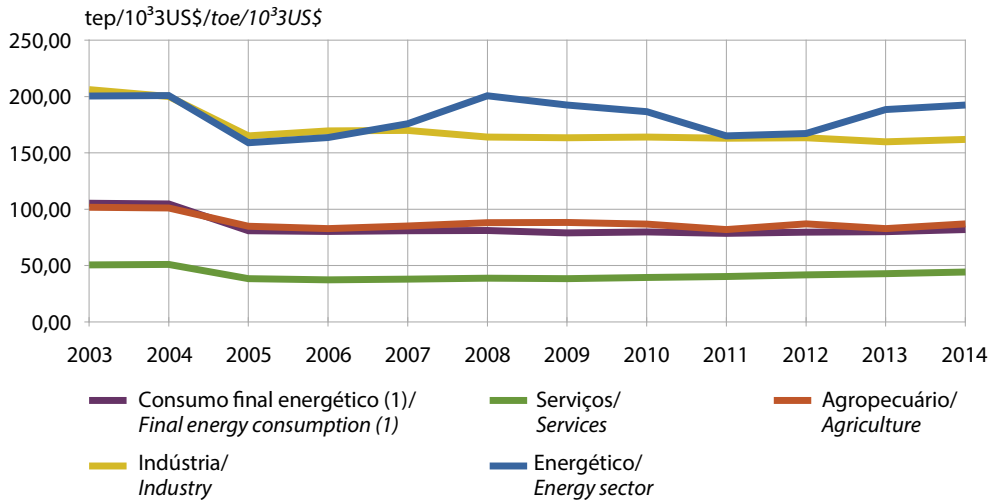

Fonte/Source: Balanço energético nacional 2015: ano base 2014. Brasília, DF: Ministério de Minas e Energia, Empresa de Pesquisa Energética - EPE, 2015. Disponível em/Available from: <https://ben.epe.gov.br/ default.aspx>. Acesso em: abr. 2016/Cited:Apr. 2016.

Nota: Dólar constante PPC de 2014./Note: Constant PPP dollar of 2014.

(1) Inclusive consumo residencial./ (1) Includes residential consumption. 


\section{Gráfico 13.7 - Participação de fontes renováveis na matriz energética 2013-2014}

Graph 13.7- Share of renewables sources in the energy matrix - 2013-2014

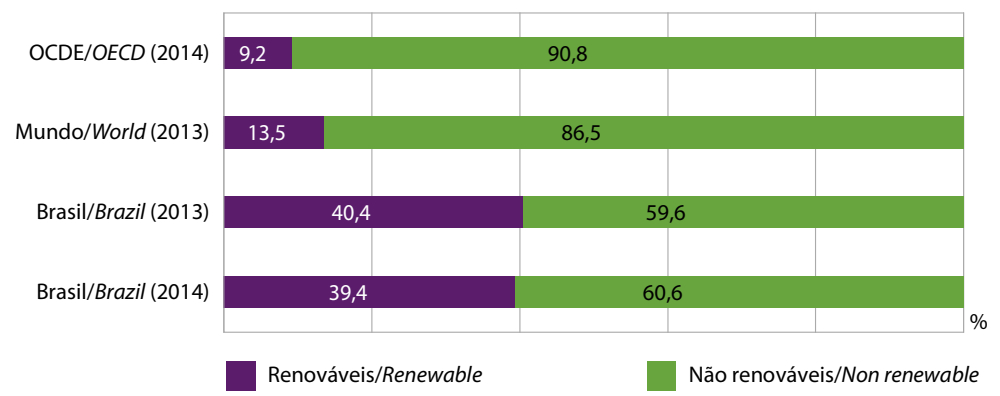

Fonte/Source: Balanço energético nacional 2015: ano base 2014. Brasília, DF: Ministério de Minas e Energia, Empresa de Pesquisa Energética - EPE, 2015. Disponível em/Available from: <https://ben.epe.gov.br/ default.aspx>. Acesso em: dez. 2015/Cited:Dec. 2015. 



\section{Comércio Trade}

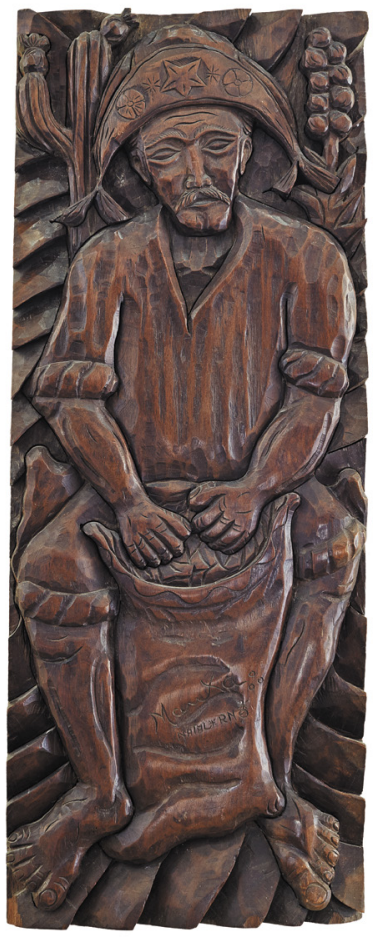

Lavrador, s.d./Farmer, n.d. Ziltamir Sebastião Soares de Maria 'Manxa' 



\section{Comércio}

\section{Breve contextualização}

Os diferentes papéis desempenhados pelos elementos que configuram $o$ espaço variam no tempo de acordo com suas características econômicas, sociais, políticas e culturais. Nesse contexto, as atividades comerciais, sejam estas atacadistas ou varejistas, possuem uma importância incontestável para compreender a dinâmica socioeconômica do território nacional, uma vez que ocupa posição de destaque no crescimento econômico, principalmente quando se trata do reordenamento territorial que se espalha por todas as escalas geográficas.

Nesse cenário, analisar o desenvolvimento e a evolução do setor comercial no Brasil se coloca como uma tarefa essencial no sentido de compreender que $o$ comércio se constitui como um elemento fundamental à produção do espaço. Essa produção se dá em razão da relação direta do setor comercial com a sociedade e a sua economia, que se constitui por dois fatores principais: a geração de emprego e de renda, notadamente por ser um setor que emprega um montante expressivo de trabalhadores o que, consequentemente, reflete na dinâmica socioeconômica do país.

\section{Trade}

\section{A brief background}

The different roles played by the space-forming elements change in time according to their economic, social and cultural characteristics. In this context, trade activities, either retail or wholesale, are of key importance to the socioeconomic dynamics of the national territory, due to their privileged position in economic growth, mainly regarding the territory reorganization observed at all geographic scales.

In this scenario, analyzing the development and evolution of the trade sector in Brazil is essential to understand how it holds a key position in the production of space. Production comes from the direct relationship between the trade sector and the society and its economy, in respect with two main factors: the generation of employment and the generation of income. As a sector that employs a significant number of workers, trade consequently reflects the socioeconomic structure of the country. 
Além disso, a atividade comercial nas cidades tem um papel fundamental no sentido das relações socioespaciais e econômicas, destacando-se como elemento marcante do cotidiano da sociedade brasileira, de forma geral. Essa dinâmica que envolve a cidade e o comércio promove um conjunto de processos, formas, estruturas e funções (SANTOS, 1985) que recorrentemente são alteradas e redefinem o espaço enquanto um lugar de transformações cotidianas.

Dentro dessa lógica socioeconômica, o comércio se sobressai como um elemento de essencial importância em relação a fonte de arrecadação de impostos para as contas públicas, participa da geração de renda e de postos de trabalho e define eixos de circulação, de valorização e desvalorização imobiliária (CAMILO PEREIRA; LAMOSO, 2005), que ocorre em uma dinâmica rede aonde por vezes locais de comércio atuam como relevantes nós neste intenso circuito.

Desse modo, compreendemos ainda que o comércio produz e consome espaço. Para Pintaudi (1996), analisar o comércio nos permite uma melhor compreensão do espaço, pois comércio e a cidade são elementos indissociáveis. Não podemos nos esquecer que muitas cidades nasceram e cresceram a partir da concentração de algumas atividades comerciais, no litoral ou as margens de importantes corredores fluviais ou terrestres.

Mesmo, em alguns casos, com o crescimento das cidades provocado pela industrialização, o comércio ainda assim desempenhou um importante papel, se adaptando e se tornando ainda mais ativo e influente nestes espaços, complementando por vezes os circuitos
Besides, trade activity in cities is fundamental to socio-spatial and economic relations, being a distinguishing element in the lives of the Brazilian society. This dynamics - that encompasses the city and trade - promotes a group of processes, forms, structures and functions (SANTOS, 1985) which change recurrently, thus redefining the space as a place for everyday transformations.

Considering this socioeconomic approach, trade stands out as a very important item in terms of tax collection for public accounts; it participates in the generation of income and employment and determines axes of circulation and the valuation and devaluation of real estate (CAMILO PEREIRA; LAMOSO, 2005). That takes place into a dynamic network of which trade is a relevant piece.

We see that trade produces and consumes space. For Pintaudi (1996), analyzing trade allows better understanding of space, since the city and trade are inseparable. We should remember that many cities have grown due to the concentration of some commercial activities, either on the coast or near some important river or land corridors.

Even in cases in which the growth of cities resulted mainly from industrialization, trade played a very important part by adapting itself and becoming even more active and influential. At times trade would complement the productive 
produtivos dos setores agropecuário e industrial, pois o comércio tem a capacidade de transformar a forma de vida e os hábitos de consumo da sociedade, promovendo uma relação dinâmica entre oferta e demanda.

Por fim, é patente que a atividade comercial desempenha papel de destaque na produção do espaço porque ao longo da história humana, relações comerciais interferiram preponderantemente na forma como o homem atuava sobre meio. Na atualidade a sociedade está subordinada aos mecanismos da publicidade, aos espaços urbanos comerciais, à concorrência, que estimula o consumismo, fazendo com que o comércio mais antigo se adapte a essa nova linhagem de modernidade (CAMILO PEREIRA; LAMOSO, 2005), em um dinâmica incessante de (re)estabelecimento e renovação das relações socioeconômicas.

\section{Analisando o comércio no Brasil: entre dados estatísticos e os desafios}

É incontestável a importância do setor comercial historicamente no Brasil no sentido de sua contribuição seja em relação a geração de renda, emprego e desenvolvimento econômico, mas sobretudo no que diz respeito aos números dessa evolução que diametralmente estão relacionados ao crescimento econômico do país e suas relações globais. Podemos dizer que a dinâmica econômica comercial está suscetível às intempéries do processo de globalização e aos ajustes e desajustes político-econômicos que ocorrem no Brasil e no Mundo. networks of the agricultural and industrial sectors, since it is capable of transforming the life and the consumer habits of society, thus promoting a dynamic relationship between supply and demand.

The significance of commercial activity to the production of space is unquestionable, since, throughout human history, commercial relations have interfered preponderantly in the way man works on the environment. Society is currently subject to publicity mechanisms, commercial urban spaces, competition, which stimulates consumerism, causing the oldest trade to adapt to this new line of modernity (CAMILO PEREIRA; LAMOSO, 2005), in a continuous movement of reestablishment and renewal of socioeconomic relations.

\section{An analysis of trade in Brazil: statistical data and challenges}

The historical relevance of the trade sector in Brazil regarding its contribution to the generation of income, of employment and to economic development is undeniable. Above all, the importance of the sector lies in its connection to the figures that are related to the economic growth of the country and to its global relations. One can say the economiccommercial dynamics is susceptible to the drawbacks of the globalization process and to the political and economic oscillations seen in Brazil and all over the world. 
A observação de alguns dados da

Pesquisa Anual de Comércio (PAC), produzida pelo Instituto Brasileiro de Estatística e Geografia (IBGE), nos permite fazer algumas análises que refletem a complexa dinâmica do setor comercial como um todo.

Os dados gerais do comércio, em 2013, revelam que há aproximadamente 1,6 milhão de empresas que atuam neste setor que conjuntamente obtiveram uma receita que ultrapassou os $R \$$ 2,67 trilhões, com um forte impacto na geração de emprego e renda ao ocupar 10,4 milhões de pessoas e com gastos de R\$ 168,2 bilhões em salários, retiradas e outras remunerações (Tabela 14.1)

Analisando estes dados de forma mais detalhadas, podemos dividir o setor em três grandes setores: comércio varejista, comércio atacadista e comércio de veículos, peças e motocicletas.

O comércio varejista ocupa posição de destaque, pois representa $79 \%$ (aproximadamente 1,2 milhão de estabelecimentos) do total das empresas do setor, ocupa $73 \%$ dos empregados (algo em torno de 7,6 milhões de pessoas), contribuiu com $62 \%$ dos gastos com salários e remunerações ( $R \$ 105$ bilhões) e atinge uma receita de $43 \%$ do total (R\$ 1,1 trilhão) (Tabelas 14.2 e 14.3)

Já o comércio atacadista ocupa uma posição de menor destaque, com apenas $12 \%$ das empresas ( $188 \mathrm{mil}$ ), ocupando $17 \%$ dos trabalhadores (1,8 milhão), contribuindo com $27 \%$ dos salários ( 45 bilhões) e detendo $44 \%$ das receitas (R\$ 1,2 trilhão) (Tabelas 14.2 e 14.3). Nessa breve análise já é possível observar que apesar de possuir muito menos
A look at some data from the Annual Survey of Trade (PAC), produced by the Brazilian Institute of Geography and Statistics (IBGE), allows us to produce analyses which reflect the complex dynamics of the trade sector as a whole.

General trade data for year 2013 show that approximately 1.6 million companies in this sector generated, together, revenue above $R \$ 2.67$ trillion. They exerted a strong impact on the generation of income and employment by hiring 10.4 million persons and accounting for an expenditure of $\mathrm{R} \$ 168.2$ billion in salaries, withdrawals and other compensation (Table 14.1)

By analyzing these data in a more detailed way we can subdivide the sector into three major areas: retail trade, wholesale trade and trade of vehicles, pieces and motorcycles.

Retail trade holds a privileged position, since it represents 79\% (approximately 1.2 million establishments) out of the total companies in the sector, employs $73 \%$ of the workers (about 7.6 million persons), contributes with $62 \%$ of the expenditure with salaries and compensation ( $\mathrm{R} \$ 105$ billion) and reaches a revenue of $43 \%$ of the total (R\$1.1 trillion) (Tables 14.2 and 14.3)

Wholesale trade does not hold such a good position, and has only $12 \%$ of the companies (188 thousand), employing $17 \%$ of the workers (1.8 million), contributing with $27 \%$ of the salaries (45 billion) and holding $44 \%$ of the revenue (R\$1.2 trillion) (Tables 14.2 and 14.3). In this brief analysis it is already possible to observe that, in spite 
estabelecimentos e ocupar quatro vezes menos empregados, o setor atacadista consegue atingir uma receita equivalente do setor varejista, sem dúvida alguma, por ser um segmento marcado pelas vendas em grande quantidade que diminuem os custos e ampliam a margem de lucro.

Por fim, o comércio de veículos, peças e motocicletas, representa somente $9 \%$ das empresas (149 mil), ocupando também $9 \%$ dos trabalhadores ( $960 \mathrm{mil})$, contribui com $11 \%$ dos salários (18 bilhões) e detém 13\% das receitas (R\$ 357 bilhões) (Tabelas 14.2 e 14.3), revelando uma maior coesão entre os dados e deixando claro sua importância em menor grau em comparação com os outros dois segmentos abordados.

Partindo para a participação dos setores do comércio elencados, considerando primeiramente em conjunto o varejo e o comércio de veículos, peças e motocicletas, observamos que as vendas nos super/ hipermercados, além da venda de veículo e peças representou em conjunto mais de $42 \%$ do total da receita em 2013 . O restante da participação se divide dentre todos os sete outros segmentos sem grandes destaques com percentuais que variam de 7\% a $11 \%$ (Gráfico 14.1)

Na mesma análise para o setor atacadista, nota-se claramente uma mudança no perfil, dado que a receita oriunda do comércio de combustíveis representa $23 \%$ e produtos alimentícios, bebidas e fumo $17 \%$. Infelizmente não é possível detalhar outros $23 \%$ atrelados a "outros" produtos, pois sua representatividade é relevante. $O$ residual das receitas se divide dentre os outros quatro segmentos sem grandes variações (Gráfico 14.2). of having fewer establishments and employing four times fewer workers, the wholesale sector can reach equivalent revenue to that of retail trade, because it is a segment characterized by sales in great amount, which reduces costs and expands profits.

Finally, the trade of vehicles, pieces and motorcycles represents only $9 \%$ of the companies (149 thousand), also employing $9 \%$ of the workers (960 thousand), accounting for $11 \%$ of the salaries ( $\mathrm{R} \$ 18$ billion) and for $13 \%$ of the revenue ( $R \$ 357$ billion (Tables 14.2 and 14.3). Data for this segment present more cohesion, evidencing its lesser importance in comparison with the other two.

Regarding the participation of the aforementioned commercial segments, first considering retail and the trade of vehicles, pieces and motorcycles, we see that sales in super/hypermarkets, besides sales of vehicles and pieces, accounted, together, for more than $42 \%$ of the total revenue in 2013 . The remainder is shared among all the other seven segments without much highlight, with percentages ranging from $7 \%$ to $11 \%$ (Graph 14.1 ).

In an analogue analysis for the wholesale segment, we clearly see a change in profile, since the revenue from trade of fuels represents $23 \%$, and from trade of food products, beverages and tobacco, $17 \%$. Unfortunately it is not possible to provide detailed information on the $23 \%$ relative to other products, because their participation is not relevant. The remainder is shared among the other four segments without much difference (Graph 14.2). 
Partindo para a questão que envolve a geração de empregos, a Pesquisa nos revela a importância dos extremos (pequenas e grandes empresas). São nas pequenas empresas, com até 19 funcionários, que estão ocupados mais de $53 \%$ dos trabalhadores do comércio, estando outros $18 \%$ nas grandes empresas, com mais de 500 funcionários (Gráfico 14.6).

Mas é curioso notar que a mesma análise considerando o porte das empresas em relação a receita operacional, as pequenas detêm $26 \%$ do total enquanto que as grandes empresas $32 \%$ (Gráfico 14.3). Isso destaca uma questão fundamental de produtividade, pois apesar de ocupar menos trabalhadores, as grandes empresas possuem a maior fatia das receitas nominais.

Por fim, em uma breve análise histórica entre os anos de 2007 e 2014, considerando a receita nominal e o volume de vendas observamos que o setor do comércio sempre cresceu, mas com variações constantes e uma tendência de queda a partir de 2012, algo que condiz com a recente desaceleração econômica que perpassa o país, em decorrência, dentre vários fatores, da instabilidade política existente desde o final de 2014.

Em suma, o setor comercial é suscetível aos diferentes desdobramentos de outros setores da economia e, ao mesmo tempo, seu desempenho também influencia diretamente e/ou indiretamente os demais setores. Definitivamente o que se pode afirmar é que o comércio tem seu desempenho configurado pela multiplicação de novas tecnologias de informação e comunicação, pela inserção
With regard to employment generation, the survey shows the importance of both extremes (small and big companies). Small companies, with up to 19 employees, employ more than $53 \%$ of the trade workers, and big companies, with more than 500 workers, another $18 \%$ (Graph 14.6).

It is interesting to observe that the same analysis, considering the size of companies in relation to the operating revenue, shows that the small ones hold $26 \%$ of the total, whereas big companies, $32 \%$ (Graph 14.3). That calls attention to a fundamental aspect in terms of productivity: in spite of employing fewer workers, big companies have a bigger participation in nominal revenue.

Finally, in a brief historical analysis between the years 2007 and 2014, considering nominal revenue and the volume of sales, we see the commercial sector has always grown, but with constant changes and a trend to decrease from 2012 on, something that is in accordance with the recent economic deceleration observed in the country, as a result, among many factors, of the existing political instability since the end of 2014.

In summary, the commercial sector is susceptible to different transformations effected in other economic sectors and, at the same time, its performance also influences the other sectors directly and indirectly. It is possible to ensure the development of trade has been based on the multiplication of new information and communication technologies, by the inclusion of new 
de novos competidores, sobretudo globais, por uma dinâmica econômica que se redesenha constantemente, mediante os sabores e dissabores do movimento político-econômico nacional e, por vezes, mundial.

\section{Perspectivas e desafios ao setor comercial}

Diante da conjuntura político-econômico em que o Brasil está inserido atualmente, observa-se que alguns setores da economia têm se deparado com inúmeros obstáculos para manter o nível de crescimento.

Os desajustes face a ingovernabilidade a que o país está inserido tem comprometido o desempenho econômico do comércio atacadista e varejista, considerando principalmente os impactos na geração de emprego e renda no país, uma vez que esta crise impele diretamente no encarecimento do crédito, na alta dos preços dos produtos e, consequentemente afeta o consumo da população, provocando dessa maneira reflexos em cadeia que atingem vários setores, sobretudo o agropecuário e a indústria de bens de consumo familiares.

Somados esses fatores, as perspectivas para o comércio denotam uma projeção de desaceleração gradual, que carece de planos estratégicos que sejam consoantes ao seu desenvolvimento, a ponto de promover, manter e continuar contribuindo com o crescimento econômico brasileiro.

A necessidade de se criar e executar políticas públicas de incentivo ao crescimento deste setor é fundamental para se vislumbrar uma retomada do desenvolvimento econômico no país, mas isso não ocorrerá de forma competitors, mainly global ones, an economic dynamics that is constantly redefined, given the tastes and disappointments of the political and economic movements in Brazil and sometimes, abroad.

\section{Prospects and challenges for the commercial sector}

Given the political and economic status of Brazil, some economic sectors have faced many obstacles to keep their level of growth.

The maladjustment resulting from the difficult situation the country finds itself has affected the economic performance of both retail and wholesale trade, mainly the generation of labor and income in the country. The current crisis leads to more expensive credit, price rises, and it also interferes with the population's consumption, causing a series of chained effects that reaches many sectors, especially agriculture and the industry of household consumer goods.

Considering all the aforementioned factors, prospects for the trade sector point to gradual deceleration, and a need for strategic plans which are in accordance with its development, in order to promote, keep and remain contributing to the economic growth of Brazil.

The need to create and implement public policies to foster this sector is necessary to restart economic development in the country, but that will not occur in a satisfactory 
satisfatória até que a atual conjuntura de instabilidade econômica e, sobretudo política seja superada.

Diante de um contexto econômico adverso, em que a democracia nacional é subterfugiada, promover a sustentabilidade do crescimento econômico, considerando a importância que o setor comercial tem na dinâmica socioeconômica do país, não se configura como uma saída de fácil acesso. Contudo, sair dessa crise políticoeconômica requer mais compromisso político com vistas a uma mudança estrutural no setor, que possibilite seu crescimento, a geração de emprego e renda compatíveis com a magnitude de sua representatividade para a economia do país. way unless the current scenario of economic and political instability is overcome.

Before an adverse economic scenario, in which national democracy has been undermined, promoting the sustainability of economic growth - taking into consideration the relevance of the commercial sector to the socioeconomic dynamics of the country - does not seem to be an easy task. Nonetheless, escaping from this political and economic crisis requires more political commitment towards a structural change in the trade sector, leading to growth, generation of employment and of income at levels that match its significance to the country's economy.

\section{Referências / References}

CAMILO PEREIRA, Ana Paula; LAMOSO, Lisandra Pereira. O comércio varejista na cidade de Dourado/MS. In: Geografia Revista do Departamento de Geociências. V. 14, n. 1, jan/jun. 2005.

PINTAUDI, Silvana Maria. A cidade e as formas de comércio. In: Novos caminhos da Geografia, São Paulo, 2002, p. 143-159.

SANTOS, Milton. Espaço e método. São Paulo: Nobel, 1985

\section{Ana Paula Camilo Pereira}

Doutora em Geografia Humana pela Universidade de São Paulo, Faculdade de Filosofia, Letras e Ciências Humanas (USP/ FFLCH), com estágio de Doutorado Sanduíche na Université Sorbonne Nouvelle Paris III, junto ao Centre National de la Recherche

Scientifique (CNRS).

Docente da Universidade Estadual de Mato Grosso do Sul (UEMS).
PhD in Human Geography from the University of São Paulo, School of Philosophy, Letters and Human Sciences (USP/FFLCH), with a PhD scholarship at the New Sorbonne University Paris III, at the National Center for Scientific Research (CNRS).

Professor at the State University of Mato Grosso do Sul (UEMS). 


\section{Rafael Oliveira Fonseca}

Geógrafo e Doutorando em Geografia Humana pela Universidade de São Paulo, Faculdade de Filosofia, Letras e Ciências

Humanas (USP/FFLCH).

Docente da Universidade Estadual de Mato Grosso do Sul (UEMS).
Geographer and student at the PhD program in Human Geography at the University of São Paulo, School of Philosophy, Letters and Human Sciences (USP/FFLCH). Professor at the State University of Mato Grosso do Sul (UEMS).

Translated by: Aline Milani Romeiro Pereira 
Tabela 14.1 - Dados gerais do comércio - 2013

Table 14.1 - General data of trade - 2013

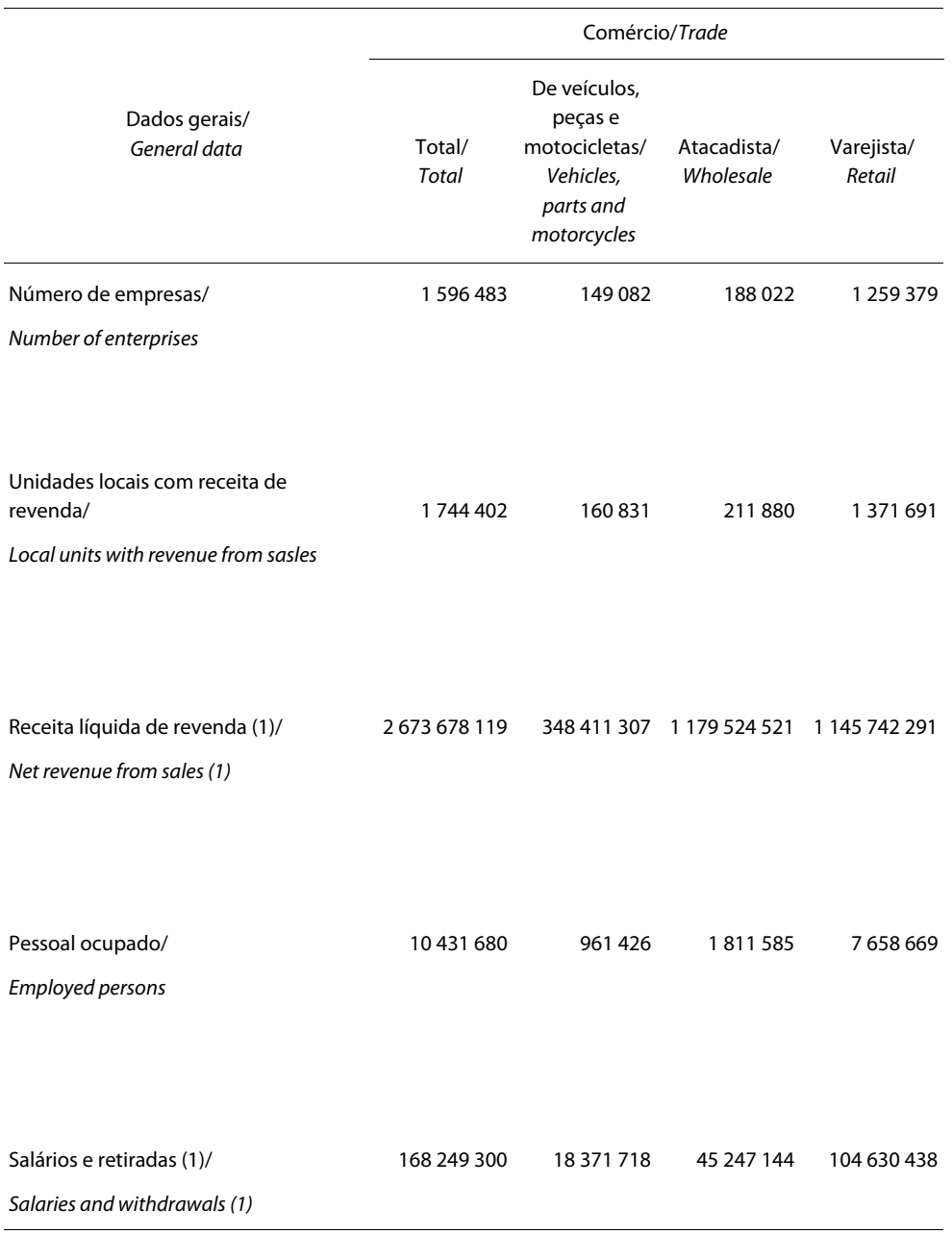

Fonte/Source: Pesquisa anual de comércio 2013. Rio de Janeiro: IBGE, v. 25, 2013. Disponível em/ Available from: <http://biblioteca.ibge.gov.br/index.php/biblioteca-catalogo?view=detalhes\&id= 755 >. Acesso em: abr. 2016/Cited: Apr. 2015.

(1) Valores expressos em mil reais./(1) Figures in thousands of $R \$$. 
Tabela 14.2 - Número de empresas, pessoal ocupado, salários

e receita total, segundo as divisões do comércio - 2013

Table 14.2-Number of enterprises, employed persons, salaries and total revenue, according to trade divisions - 2013

\begin{tabular}{|c|c|c|c|c|}
\hline $\begin{array}{l}\text { Divisões do comércio/ } \\
\text { Divisions of trade }\end{array}$ & $\begin{array}{l}\text { Número } \\
\text { de } \\
\text { empresas/ } \\
\text { Number } \\
\text { of } \\
\text { enterprises }\end{array}$ & $\begin{array}{l}\text { Pessoal } \\
\text { ocupado } \\
\text { (1)/ } \\
\text { Employed } \\
\text { persons } \\
\text { (1) }\end{array}$ & $\begin{array}{c}\text { Salários, } \\
\text { retiradas e outras } \\
\text { remunerações (2)/ } \\
\text { Salaries, } \\
\text { withdrawals } \\
\text { and other } \\
\text { compensation (2) }\end{array}$ & $\begin{array}{l}\text { Receita } \\
\text { total } \\
(2) / \\
\text { Total } \\
\text { revenue } \\
\text { (2) }\end{array}$ \\
\hline $\begin{array}{l}\text { Total/ Total } \\
\text { Comércio de veículos, peças e }\end{array}$ & 1596483 & 10432 & 168 & 2746 \\
\hline $\begin{array}{l}\text { motocicletas/ } \\
\text { Sale of vehicles, parts, pieces and } \\
\text { motorcycles }\end{array}$ & 149082 & 961 & 18 & 357 \\
\hline $\begin{array}{l}\text { Comércio atacadista/ } \\
\text { Wholesale trade }\end{array}$ & 188022 & 1812 & 45 & 1218 \\
\hline $\begin{array}{l}\text { Comércio varejista } \\
\text { Retail trade }\end{array}$ & 1259379 & 7659 & 105 & 1172 \\
\hline
\end{tabular}

Fonte/Source: Pesquisa anual de comércio 2013. Rio de Janeiro: IBGE, v. 25, 2013. Disponível em:/ Available from: $<$ http://biblioteca.ibge.gov.br/index.php/biblioteca-catalogo?view=detalhes\&id= 755>. Acesso em: abr. 2016/Cited: Apr. 2016.

(1) Em mil pessoas. (2) Valores expressos em bilhões de reais./ (1) In thousand persons. (2) Figures in

\section{Tabela 14.3 - Participação dos segmentos do comércio - 2013}

Table 14.3 - Participation of trade segments - 2013

\begin{tabular}{|c|c|c|c|c|}
\hline $\begin{array}{l}\text { Divisões do comércio/ } \\
\text { Divisions of trade }\end{array}$ & $\begin{array}{c}\text { Número } \\
\text { de } \\
\text { empresas/ } \\
\text { Number } \\
\text { of } \\
\text { enterprises }\end{array}$ & $\begin{array}{l}\text { Pessoal } \\
\text { ocupado/ } \\
\text { Employed } \\
\text { persons }\end{array}$ & $\begin{array}{l}\text { Salários e outras } \\
\text { remunerações/ } \\
\text { Salaries } \\
\text { and other } \\
\text { compensation }\end{array}$ & $\begin{array}{l}\text { Receita } \\
\text { líquida de } \\
\text { revenda/ } \\
\text { Net revenue } \\
\text { from sales }\end{array}$ \\
\hline $\begin{array}{l}\text { Total/ Total } \\
\text { Comércio de veículos, peças e }\end{array}$ & 100,0 & 100,0 & 100,0 & 100,0 \\
\hline $\begin{array}{l}\text { motocicletas/ } \\
\text { Sale of vehicles, parts, pieces and } \\
\text { motorcycles }\end{array}$ & 9,3 & 9,2 & 10,7 & 13,0 \\
\hline $\begin{array}{l}\text { Comércio atacadista/ } \\
\text { Wholesale trade }\end{array}$ & 11,8 & 17,4 & 26,8 & 44,3 \\
\hline $\begin{array}{l}\text { Comércio varejista } \\
\text { Retail trade }\end{array}$ & 78,9 & 73,4 & 62,5 & 42,7 \\
\hline
\end{tabular}

Fonte/Source: IBGE, Diretoria de Pesquisas, Coordenação de Serviços e Comércio, Pesquisa Anual de Comércio 2013. 


\section{Gráfico 14.1 - Participação dos segmentos na receita total do comércio varejista e de veículos - 2013}

Graph 14.1 - Participation of segments in total revenue of retail and vehicles trade - 2013

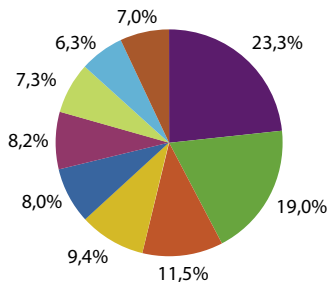

Veículos e peças/

Vehicles and pieces

Super/Hipermercados/

Supermarkets/hypermarkets

Combustíveis/

Fuels

Lojas de Departamento, eletrodomésticos e móveis/

Department and stores, household appliances and furniture stores

Material de construção/ Construction material

Tecidos e artigos do vestuário/

Textiles and wearing apparel

Armazéns, mercearias e produtos alimentícios/

Stores, grocery stores and food products

Produtos farmacêuticos/

Pharmaceuticals

Outros/

Others

Fonte/Soure: IBGE, Diretoria de Pesquisas, Coordenação de Serviços e Comércio, Pesquisa Anual de Comércio 2013.

\section{Gráfico 14.2 - Participação dos segmentos na receita total do comércio atacadista - 2013}

Graph 14.2 - Participation of segments in total revenue of whosale trade - 2013

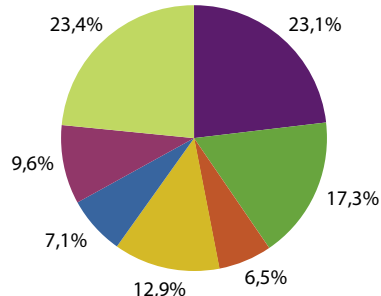

Combustíveis/

Fuels

Produtos agropecuários in natura/

Raw agricultural products

Máquinas e equipamentos industriais/

Industrial machinery and equipment

$12,9 \%$

Produtos alimentícios, bebidas e fumo/

Food products, beverages and tobacco

Mercadorias em geral/

Miscellaneous goods

Produtos farmacêuticos/

Pharmaceuticals

Outros/

Others

Fonte/Source: IBGE, Diretoria de Pesquisas, Coordenação de Serviços e Comércio, Pesquisa Anual de Comércio 2013. 


\section{Gráfico 14.3 - Participação das empresas, por faixas de pessoal ocupado,}

na receita operacional líquida do comércio - 2013

Graph 14.3 - Participation of companies, by ranges of employed persons in net operacional revenue of trade - 2013

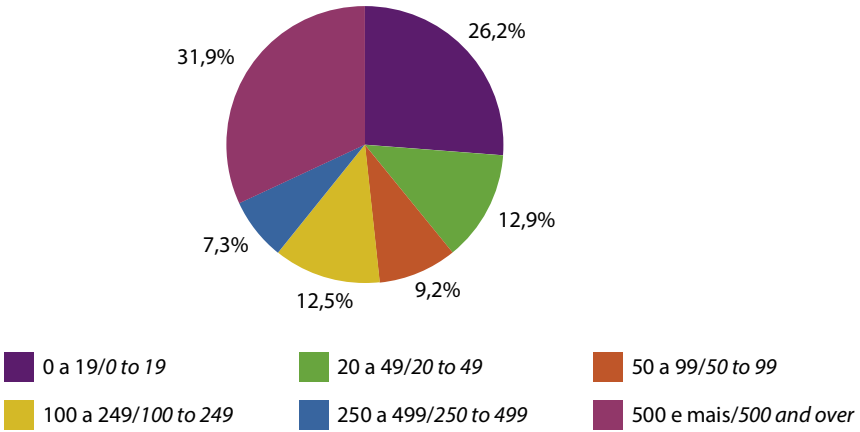

Fonte/Source: IBGE, Diretoria de Pesquisas, Coordenação de Serviços e Comércio, Pesquisa Anual de Comércio 2013.

\section{Gráfico 14.4 - Evolução da receita operacional líquida,} por faixas de pessoal ocupado - 2012-2013

Graph 14.4 - Evolution of net operating revenue, by ranges of employed persons - 2012-2013

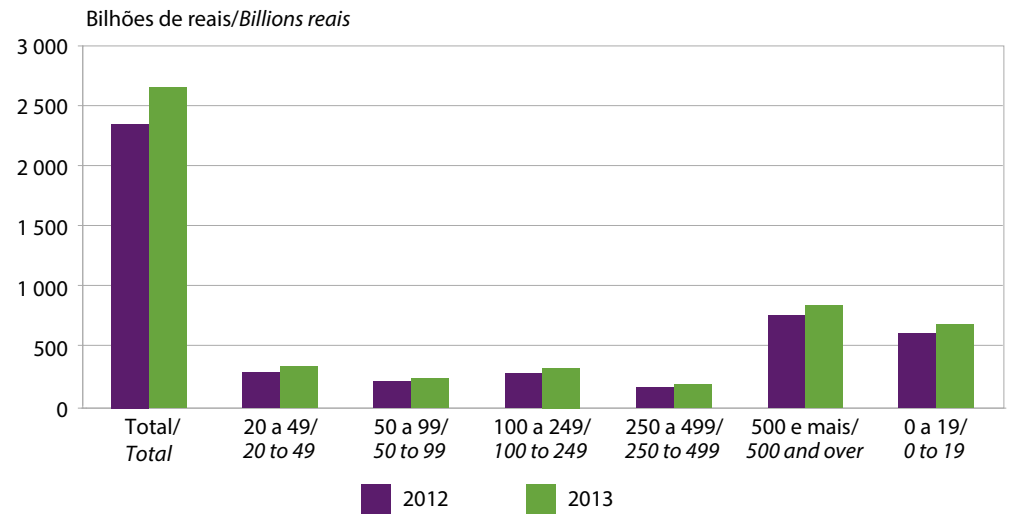

Fonte/Source: IBGE, Diretoria de Pesquisas, Coordenação de Serviços e Comércio, Pesquisa Anual de Comércio 2012-2013. 


\section{Gráfico 14.5 - Taxa acumulada de desempenho no comércio varejista 2007-2014}

Graph 14.5 - Accumulated performance rate in retail trade - 2007-2014

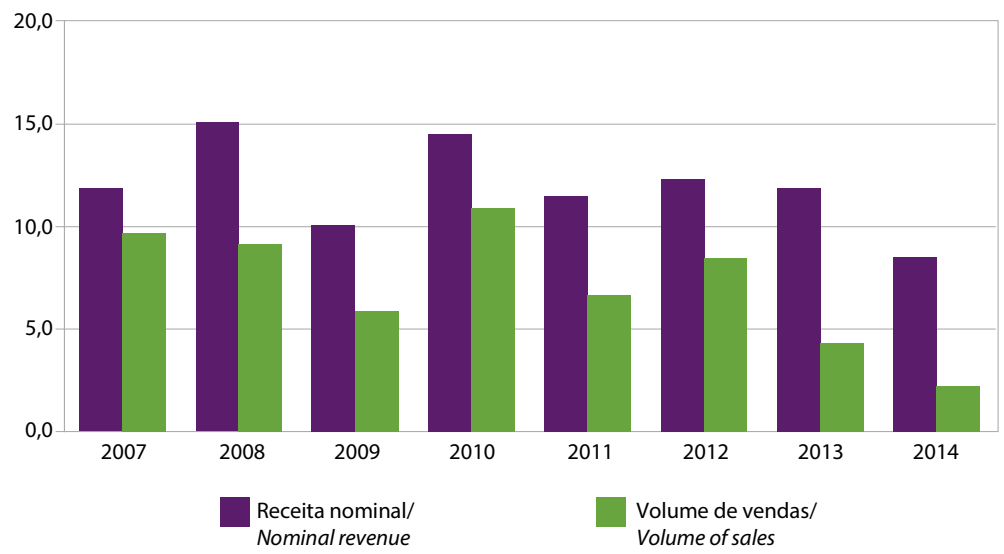

Fonte/Source: IBGE, Diretoria de Pesquisas, Coordenação de Serviços e Comércio, Pesquisa Mensal de Comércio 2007-2014.

Nota: Base: ano anterior $=100 . /$ Note: Base: previous year $=100$.

\section{Gráfico 14.6 - Participação das empresas, por faixas de pessoal ocupado, no total de pessoal ocupado do comércio - 2013}

Graph 14.6 - Participation of companies in total employed person in trade, by ranges of employed persons - 2013

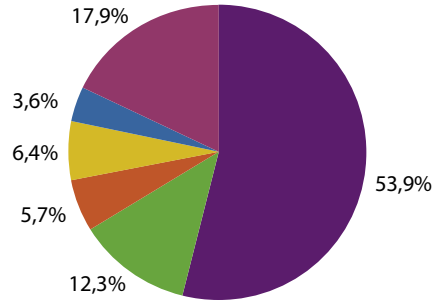
0 a $19 / 0$ to 19
20 a $49 / 20$ to 49
50 a $99 / 50$ to 99
100 a $249 / 100$ to 249
250 a $499 / 250$ to 499
500 e mais/500 and over

Fonte/Source: IBGE, Diretoria de Pesquisas, Coordenação de Serviços e Comércio, Pesquisa Anual de Comércio 2013. 


\section{Transportes \\ Transportation}

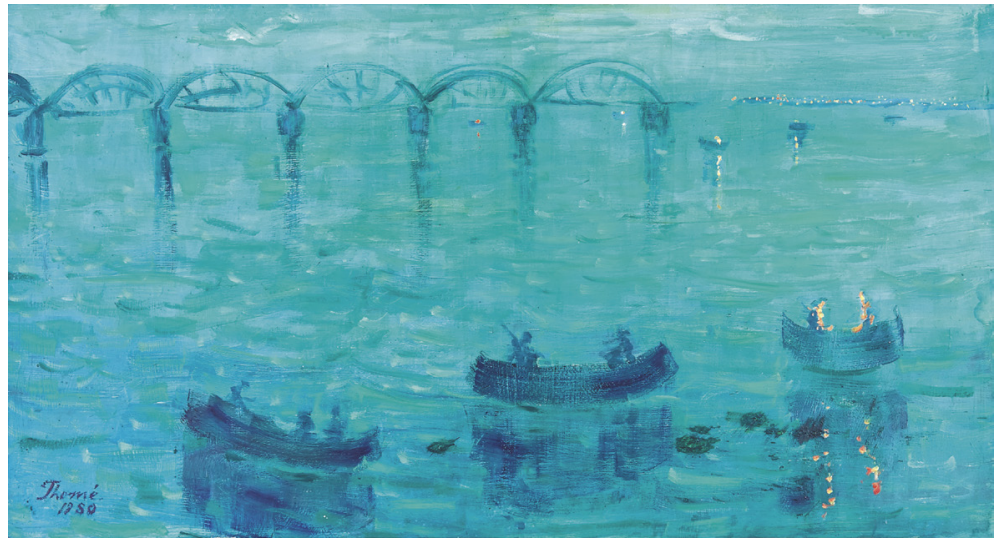

A Ponte de lgapó/The lgapó's bridge, 1980

Thomé Soares Filgueira 



\section{Transportes}

\section{Introdução}

É sabido que dentre as funções sociais, a função transportes é uma das mais importantes, em qualquer sociedade, desde o nível local até o internacional, dadas as relações sociais e econômicas as quais ela dá suporte, considerando o serviço por ela prestado. Do ponto de vista das relações sociais, o turismo é o exemplo mais cabal; e das relações econômicas, o transporte de passageiros e cargas, em suas diversas modalidades, são os meios essenciais para qualquer sociedade desenvolver as suas atividades. Partindo desta premissa, este artigo tem por objetivo analisar a evolução deste setor de transportes, tendo como espaço temporal o período de 2005 a 2014, partindo das informações dadas pelo IBGE - Instituto Brasileiro de Geografia e Estatística consubstanciadas nas tabelas e gráficos disseminadas ao longo do texto.

Este capítulo foi elaborado com comentários e discussões sobre cada modal de transporte, precedidos por uma visão geral da matriz de transporte brasileira, em termos de cargas e de passageiros. A análise realizada abrange

\section{Transportation}

\section{Introduction}

Transportation is certainly one of the most important social functions in any society, from local to international level, a service that provides support to several social and economic relations. From the perspective of social relations, tourism is the most significant example, and, with regard to economic relations, the several modalities of cargo and passenger transportation are essential means for the development of activities by society. Following this premise, the objective of this article is to analyze the evolution of the transportation sector, with a focus on the period from 2005 to 2014, considering the information provided by the IBGE Brazilian Institute of Geography and Statistics - and presented as tables and graphs throughout the text.

This chapter brings comments and discussions about each mode of transportation, preceded by an overall view of the Brazilian transportation matrix, in terms of cargo and passengers. The analysis 
alguns aspectos do setor de transportes, a saber: extensão das malhas viárias do país, número de habitantes por autoveículo em circulação em alguns países, movimentação de carga dos transportes ferroviário e hidroviário, evolução do número de voos do modal aéreo e sua movimentação de cargas e de passageiros. Por fim, são apresentadas considerações finais de fechamento deste capítulo.

\section{Panorama geral da matriz de transportes brasileira}

O setor de transportes brasileiro é fortemente caracterizado por uma matriz de transporte bastante desequilibrada em relação ao transporte de cargas. $\mathrm{O}$ Gráfico 15.1 ilustra esse aspecto, com a apresentação da matriz de transporte de cargas referentes a 2015, em valores percentuais de cada modal em relação ao total transportado, em toneladas por quilômetro útil (TKU).

Percebe-se a participação expressiva do transporte rodoviário na movimentação de cargas pelo país, correspondente a mais de $60 \%$ do total. Em seguida, encontram-se o transporte ferroviário e aquaviário, que somam quase $35 \%$ do total de cargas transportadas. Essa concentração de transporte de cargas no modal rodoviário, num país de extensão continental como o Brasil, conduz a elevação dos custos de transporte e ao consequente aumento dos preços finais dos produtos. É notável a necessidade de equilíbrio dessa matriz, com investimentos na infraestrutura necessária à ampliação dos modais ferroviário e aquaviário. Esses modos de transporte possuem, como característica fundamental, um encompasses some aspects of the sector: the extension of the road network in the country, the number of residents per vehicle in circulation in some countries, the handling of cargo by means of rail and waterway transportation, the evolution of the number of flights as for and the respective transit of cargo and passengers. Some final considerations conclude the chapter.

\section{Overall view of the Brazilian transportation matrix}

A distinguishing characteristic of the transportation sector in Brazil is the unbalanced transportation matrix regarding cargo distribution, as shown in Graph 15.1. It illustrates this aspect by presenting the cargo transportation matrix relative to year 2015, in percentage figures for each transportation mode with the amount transported, in tonne-kilometer (TKM).

As seen in the Graph 15.1, road transportation has an active participation in cargo handling across the country, accounting for more than $60 \%$ of it. Rail and waterway transportation, together, are responsible for almost all of the remaining $35 \%$. The concentration of cargo handling in the road transportation modality, in a country of continental dimensions like ours, leads to the rise of transportation costs and, subsequently, of final prices as well. This matrix requires urgent balancing, with investments in the necessary infrastructure in order to expand rail and waterway transportation. Such modes have, as a fundamental characteristic, high 
alto custo de implantação; porém estão associados a menores custos de operação e manutenção quando são considerados os volumes transportados, se comparados ao modal rodoviário.

\section{Comentários sobre o transporte rodoviário no Brasil}

A Tabela 15.1 apresenta a extensão da malha rodoviária brasileira. Nela, percebese claramente a predominância das rodovias municipais $(80,7 \%)$, seguida pelas rodovias estaduais $(14,4 \%$ ) e federais (4,9\%). Em relação a extensão pavimentada dessas rodovias, verifica-se que as vias federais possuem maior porcentagem de trechos pavimentados $(85,0 \%)$. Contudo, considerando a extensão territorial do país de $8.513 .644 \mathrm{~km}^{2}$, tem-se uma densidade baixíssima de rodovias pavimentas, de apenas $0,02 \mathrm{~km}$ de rodovias pavimentadas por $\mathrm{km}^{2}$ de extensão territorial. As vias pavimentadas de administração estadual somam $53,1 \%$ do total de rodovias estaduais. Por fim, na jurisdição municipal as rodovias municipais são apenas $2,1 \%$ de vias pavimentadas. É importante lembrar que esses números poderiam ser mais generosos se a CIDE - Contribuição Para a Intervenção do Domínio Econômico - não estivessem com os seus recursos contingenciados.

Outro aspecto relevante quanto a avaliação do transporte rodoviário no Brasil diz respeito ao aumento da frota de automóveis. Esse crescimento tem sido maior que o crescimento populacional do país, conduzindo a uma relação do número de habitantes por veículo decrescente. Em 2001, essa relação era de 8,6 e passou a 5,1 em 2013. Países desenvolvidos com extensão territorial bem menor que o Brasil (e.g. Alemanha, Itália, Japão e Reino Unido) costs of implementation, but are nonetheless associated with smaller costs of operation and maintenance considering the volume transported, in comparison with road transportation.

\section{Some comments on road transportation in Brazil}

Table 15.1 presents the extension of the Brazilian road network. There is a clear predominance of municipal roads $(80.7 \%)$, followed by state $(14.4 \%)$ and federal ones (4.9\%). In relation to the extension of paved roads, it is observed that the federal ones have a bigger percentage of paved areas (85.0\%). However, considering the size of the Brazilian territory, $8,513,644 \mathrm{~km}^{2}$, the density of paved roads is still very low, of only $0.02 \mathrm{~km}$ per $\mathrm{km}^{2}$ of the territory. Considering the roads administered by the state, $53.1 \%$ are paved. Finally, among the roads under municipal jurisdiction, only $2.1 \%$ are paved. It is worth mentioning these figures could be higher if the resources from CIDE - Contribution of Intervention in the Economic Domain - were not subject to contingency.

Another relevant aspect in the evaluation of road transportation refers to the expansion of the car fleet. Its increase has surpassed the population growth in the country, and led to a decreasing ratio of the number of inhabitants per vehicle. In 2001, this ratio was 8.6 , and it changed to 5.1 in 2013. Developed countries with much smaller territories than Brazil (e.g. Germany, Italy, Japan and Great Britain) 
apresentam índices entre 1,0 e 2,0. Nesses países, além da renda há um transporte público bem mais eficiente baseado no transporte ferroviário.

O Gráfico 15.2 apresenta a relação entre o número de habitantes e o de veículos para diversos países. No caso brasileiro, esta expansão da frota deveu-se, de um lado, ao incentivo dado pelo governo federal à indústria automobilística entre 2012 e dezembro de 2014, através da redução da alíquota do IPI - Imposto Sobre Produtos Industrializados - e de outro, ao programas de distribuição de renda às famílias de baixa renda, o que elevou a massa salarial no país inteiro. Ademais, a absurda diferença na relação veículos/habitantes, quando se compara o Brasil com os demais países listados, deve-se à baixa renda per capta para a grande maioria da sua população, apesar dos programas de distribuição de renda já referidos.

O cenário rodoviário exposto indica a forte necessidade de investimentos neste modal, com ampliação da malha e melhoria da qualidade da malha existente e a necessidade de implantação e otimização dos meios transportes de massa para melhor atender as populações urbanas (ônibus, metrô, trens suburbanos).

\section{Transporte Ferroviário de carga}

A Tabela 15.1 apresenta a distribuição da malha ferroviária brasileira. Nesse modal, percebe-se tratar-se de uma malha pouco extensa, diante das dimensões continentais do Brasil. A malha ferroviária soma apenas $59741 \mathrm{~km}$ de extensão, valor corresponde a apenas 3,8\% da extensão da malha viária recorded indexes between 1.0 and 2.0. Besides higher income, these countries have more efficient rail transportation systems.

Graph 15.2 presents the ratio between the number of inhabitants and vehicles in several countries. In Brazil, the increase of the fleet resulted, firstly, from the incentive given by the Federal government to the car industry between 2012 and December 2014, by means of the reduction of the IPI - Tax on Industrialized Goods - and, also, from the income distribution programs directed to low-income families, which led to an increase of the wage bill all over the country. Furthermore, the enormous difference in the vehicle/inhabitant ratio, in a comparison between Brazil and the aforementioned countries, is a consequence of the low per capita income of most of our population, despite the distribution programs listed above.

The current scenario of road transportation points to a strong need for investments in this modality, with expansion and improvement of the existing network, and implementation and optimization of mass transportation, in order to provide the urban population with better services (buses, subway, trains).

\section{Freight transportation by rail}

Table 15.1 presents the distribution of the Brazilian railway network - which is not very extensive in relation to the continental dimensions of Brazil. Our railway network has $59,741 \mathrm{~km}$ of extension, corresponding to only $3.8 \%$ of the road network. There is a very 
brasileira. A distribuição entre os trechos concedidos e aqueles sob administração pública federal é praticamente igualitária, com cerca de $48,8 \%$ de ferrovias concedidas.

Em relação ao transporte de cargas pelo modal ferroviário, destaca-se a concentração em determinados trechos e regiões do país. A Tabela 15.2 indica os dados deste transporte no Brasil. Verifica-se que, ao considerarem-se as três concessionárias de maior quantidade transportada por quilômetro útil (em TKU, definida pelo produto da carga líquida transportada, em toneladas, pela distância percorrida, em km), obtém-se mais de três quartos do total transportado por essas concessionárias $(78,1 \%)$. A MRS Logística S.A. administra uma malha ferroviária de $1.643 \mathrm{~km}$ nos Estados de Minas Gerais, Rio de Janeiro e São Paulo. A carga que transporta é bastante diversificada, incluindo contêineres, produtos siderúrgicos, cimento, bauxita, produtos agrícolas, coque, carvão e minério de ferro (<http://www.mrs. com.br/empresa/ quem-somos $>$, acessado em 10/06/2016).

A Estrada de Ferro Carajás estende-se por $892 \mathrm{~km}$ e interliga a maior mina de minério de ferro a céu aberto do mundo, em Carajás (PA), ao Porto de Ponta da Madeira, em São Luís (MA). Realiza o transporte de cargas (principalmente minério de ferro, ferro-gusa, manganês, cobre, combustíveis e carvão) e de passageiros (http://www.vale.com/brasil/ PT/business /logistics/railways/Paginas/ default.aspx, acessado 10/06/2016). A Estrada de Ferro Vitória a Minas, por sua vez, possui $905 \mathrm{~km}$ de extensão e interliga a região metropolitana de Vitória (ES) a Belo Horizonte (MG). É responsável pelo escoamento de diversas cargas (minério, aço, carvão, calcário, granito, contêineres, egalitarian distribution of stretches granted by the railway system, nearly $48.8 \%$, and of stretches under public administration.

In relation to rail freight transportation, a highlight is the concentration in some areas and regions of the country. Table 15.32 presents data on this mode of transportation in Brazil. The three main concessionaries in terms of the biggest amount transported by tonnekilometer (TKM, which is the product of the net freight transported, in metric tons, by the distance covered, in $\mathrm{km}$ ) account for more than three quarters of the total freight (78.1\%). MRS Logística S.A. manages a railroad network of $1,643 \mathrm{~km}$ in the states of Minas Gerais, Rio de Janeiro and São Paulo. It handles very diverse freight, including containers, steel products, cement, bauxite, agricultural products, coke, coal and iron ore $(<\mathrm{http}: / / \mathrm{www}$. mrs.com.br/empresa/quem-somos>, cited on June 10, 2016).

The Carajás Railroad is $892 \mathrm{~km}$ long and connects the biggest open-pit iron ore mine in the world, in Carajás (PA), to the Ponta da Madeira Port, in São Luís (MA). It is used for freight (mainly iron ore, pig iron, manganese, copper, fuels and coal) and passenger transportation (http://www.vale. com/brasil/PT/business/logistics/ railways/Paginas/default.aspx, cited on June 10, 2016). The Vitória-Minas railroad, on its turn, is $905 \mathrm{~km}$ long and connects the metropolitan area of Vitória (ES) to Belo Horizonte (MG). It is responsible for the handling of several types of freight (ore, steel, coal, limestone, granite, containers, 
ferro-gusa, produtos agrícolas, madeira, celulose e combustíveis) de Minas Gerais até o Complexo de Tubarão, ao Terminal de Vila Velha, ao Cais de Paul, Codesa e ao Porto de Barra do Riacho, na cidade de Aracruz (ES). Caracteriza-se, ainda por ser a única ferrovia do Brasil com transporte de passageiros com saídas diárias entre duas regiões metropolitanas (Cariacica/ES e Belo Horizonte/MG) (<http://www.vale. com/brasil/PT/business/logistics/railways/ Passenger-Train-Vitoria-Minas/Pagina/ default. aspx $>$, acessado em 10/06/2016).

\section{Transporte Aquaviário}

O transporte aquaviário representa apenas $13,6 \%$ das cargas transportadas no Brasil (Gráfico 15.1), apesar da grande quantidade e extensão dos rios brasileiros e da sua longa linha de costa com quase $8.500 \mathrm{~km}$. A extensão dessa malha hidroviária corresponde a $4,1 \%$ da malha viária do Brasil, com um total de 63672 $\mathrm{km}$, da qual apenas $34,6 \%$ são utilizadas economicamente (Tabela 15.1). Isto se dá ora porque o trajeto dos rios passa por áreas com baixo potencial produtivo, ora devido à baixa profundidade do leito do rio e traçado extenso, mas muito sinuoso. Segundo o levantamento das vias economicamente navegadas, realizado pela ANTAQ - Agência Nacional de Transporte Aquaviário - em 2014, as principais hidrovias do país são: Amazônica (17.651 km), TocantinsAraguaia $(1.360 \mathrm{~km})$, Paraná-Tietê (1.359 $\mathrm{km})$, Paraguai (591 km), São Francisco (576 km), Sul (500 km).

A navegação de longo curso representa $74,2 \%$ das cargas transportadas em 2015. A navegação de cabotagem e outras formas de navegação (navegação interior e cabotagem em vias interiores) pig iron, agricultural products, wood, pulp and fuels) from Minas Gerais up to the Tubarão Complex, to the Vila Velha Terminal, to Cais de Paul, Codesa and the Barra do Riacho Port, in the city of Aracruz (ES). It is the only passenger transportation railroad in Brazil with daily departures between two metropolitan areas (Cariacica/ES and Belo Horizonte/ MG) (<http://www.vale. com/brasil/ PT/business/logistics/railways/ Passenger-Train-Vitoria-Minas/Pagina/ default. aspx>, cited on June 10, 2016).

\section{Waterway transportation}

Waterway transportation is responsible for $13.6 \%$ of the cargo transported in Brazil (Graph 15.1), in spite of the big number of rivers in the country, and of a coast that is almost $8,500 \mathrm{~km}$ long. The extension of the water transportation network corresponds to $4.1 \%$ of the total road network in Brazil, with a total $63,672 \mathrm{~km}$, out of which only $34.6 \%$ is used for economic purposes (Table 15.1). That takes place either because of rivers that flow in areas with low productive potential or because of the low depth of riverbeds in long, but sinuous, rivers. According to a survey about economically viable waterways, conducted by ANTAQ - National Agency of Waterway Transportation - in 2014, the main waterways in the country are: Amazônica $(17,651 \mathrm{~km})$, Tocantins-Araguaia $(1,360 \mathrm{~km})$, ParanáTietê $(1,359 \mathrm{~km})$, Paraguai $(591 \mathrm{~km})$, São Francisco (576km), Sul (500km).

Long-haul navigation accounts for $74.2 \%$ of the cargo transported in 2015. Cabotage and other navigation forms (inland navigation and inland cabotage) are responsible for, 
representam, respectivamente, 20,8 $\%$ e $5,0 \%$ das cargas transportadas no país pelo modal aquaviário. Apesar de ainda haver grande necessidade de investimento, o transporte de cargas pelo modal aquaviário apresentou, na última década, um crescimento de cerca de $56 \%$, conforme ilustrado no Gráfico 15.3, que apresenta as informações gerais sobre o transporte aquaviário no Brasil entre 2005 e 2015.

\section{Transporte Aéreo}

O transporte aéreo tem apresentado uma pequena participação na movimentação de cargas no Brasil (Gráfico 15.1), correspondendo a $0,4 \%$ do total transportado em 2015. Por outro lado, tem apresentado uma participação cada vez maior no transporte de passageiros. Em 2015, a participação do modal aéreo no transporte de passageiros corresponde a $45,4 \%$ do total brasileiro. O Gráfico 15.4 indica o quantitativo de voos domésticos e internacionais entre os anos de 2005 e 2014. Destaca-se que os dados referentes a 2015 ainda não foram publicados.

Do Gráfico 15.4, percebe-se claramente o aumento expressivo no número de voos domésticos entre 2005 e 2011 (71,4\%). Em 2012, houve uma redução nessa tendência de crescimento, confirmada por dois anos consecutivos (2013 e 2014) de decréscimo do número de voos domésticos no Brasil. Por outro lado, o número de voos internacionais apresentou um crescimento praticamente constante no período analisado, exceto pelo decréscimo apresentado em 2009. Em termos gerais, o aumento no número de voos domésticos e internacionais foi igual a $68,6 \%$ e $62,3 \%$, respectivamente. respectively, $20.8 \%$ and $5.0 \%$ of the cargo transported in the country by waterway transportation mode. Although there is still a great need for investment, this mode of cargo transportation recorded an increase of about $56 \%$ in the last decade, as illustrated in Graph 15.3 which gathers general information on waterway transportation in Brazil between 2005 and 2015.

\section{Air transportation}

There has been small participation of air transportation in the transit of cargo in Brazil (Graph 15.1), corresponding to a $0.4 \%$ of the total amount transported in 2015. On the other hand, its participation in the transit of passengers has increased day-by-day. In 2015, the air modality accounted for $45.4 \%$ of the total passenger transportation in Brazil. Graph 15.4 shows the number of domestic and international flights between the years of 2005 and 2014. Data relative to 2015 have not yet been released.

Graph 15.4 evidences the significant increase of domestic flights between 2005 and 2011 (71.4\%). In 2012, there was a decrease of this trend, confirmed in the two following years (2013 and 2014) with reduction of domestic flights in Brazil. The number of international flights recorded an almost constant increase in the period analyzed, except for the decline observed in 2009. In general terms, the number of domestic and international flights rose by $68.6 \%$ and $62.3 \%$, respectively. 
Avaliar apenas o número de voos torna-se superficial para determinar a evolução do modal aeroviário. É preciso verificar também a quantidade de passageiros e de cargas transportadas no período. O Gráfico 15.5 apresenta esses dados e permite verificar o crescimento expressivo no número de passageiros transportados pelo modal aéreo no período analisado, tanto em voos domésticos (147,7\%) como em voos internacionais (104,6\%). O transporte de cargas, por sua vez, apresentou crescimento menos expressivo na quantidade de cargas transportadas no período $(37,2 \%$ em voos domésticos e $40,2 \%$ em voos internacionais). Destaca-se, ainda, a preponderância do transporte de passageiros nos voos domésticos $(81,8 \%$ dos passageiros transportados em voos domésticos em 2014) e do transporte de cargas nos voos internacionais $65,9 \%$ das cargas transportadas em voos internacionais em 2014).

\section{Considerações finais}

O setor de transportes de uma nação possui grande influência na sua economia, promovendo o deslocamento de insumos, mercadorias e pessoas, atividades para uma produção competitiva no mercado atual. No Brasil, a grande presença do modal rodoviário na matriz de transportes conduz a uma situação de maiores custos de transportes. Outros modais apresentam-se menos onerosos (ferroviário e aquaviário), principalmente em países de dimensões continentais como o Brasil. Os investimentos na ampliação das malhas ferroviária e aquaviária, bem como a melhoria na qualidade da malha rodoviária se mostram essenciais para o crescimento econômico do país.
Assessing only the number of flights is a superficial method to determine the evolution of the air transportation mode. It is also necessary to consider the number of passengers and cargo transported in the period. Graph 15.5 presents these data and makes it possible to measure the significant increase in the number of passengers transported by air in the period analyzed, both in domestic $(147.7 \%)$ and in international flights (104.6\%). The amount of cargo transported, on its turn, recorded a less significant increase in the period (37.2\% in domestic flights and $40.2 \%$ in international ones). Also relevant is the predominance of passenger transportation in domestic flights (81.8\% of the domestic flights in 2014) and of cargo transportation in international flights $(65.9 \%$ of the international flights in 2014).

\section{Final remarks}

The transportation sector of a nation exerts strong influence on its economy, and promotes the transit of inputs, goods and persons, providing support for competitive production in the current market. In Brazil, the massive presence of the road modality in the transportation matrix leads to higher transportation costs, whereas there are other options of less expensive modes (rail and waterway transportation), especially in countries of continental dimensions such as Brazil. Investments in the expansion of rail and ship transport networks, and the improvement in the quality of the road network are essential for the economic growth of the country. 
A malha rodoviária brasileira tem predominância de vias sob administração municipal, com pequena proporção pavimentada. As rodovias federais e estaduais apresentam maiores porcentagem de trechos pavimentados (cerca de $85 \%$ e $53 \%$, respectivamente). Um fator que vem gerando grande impacto na circulação de veículos é a evolução da relação entre o número de habitantes e o número veículos. Essa relação teve uma redução de $40,7 \%$ entre 2001 e 2013, indicando a sobrecarga do sistema viário urbano e interurbano, principalmente próximo aos grandes centros populacionais. Esse fato é difícil de ser contornado pelos municípios devido à grande dependência deles em relação às políticas e investimentos federais. Por exemplo, a política de redução do IPI Imposto sobre Produtos Industrializados - para automóveis de 2012 a 2015 levou a uma sobrecarga das vias urbanas sem a contrapartida de investimentos no transporte público.

A avaliação do transporte ferroviário indica uma divisão equilibrada entre vias nacionais e concedidas. Existe uma concentração no volume de carga transportado por quilômetro útil em três ferrovias, situadas regiões Norte e Sudeste do país. Entre as cargas mais transportadas encontram-se o minério de ferro, produtos siderúrgicos, ferro-gusa, produtos agrícolas, carvão, combustíveis e contêineres.

O transporte aquaviário apresentou crescimento entre os anos de 2005 e $2015 \mathrm{em}$ todas as suas modalidades. Entre elas, a navegação de longo curso apresenta-se como preponderante em 2015 (74,2\%), seguida pela navegação de cabotagem (20,8\%) e outras modalidades (navegação interior e cabotagem em vias
Roads under municipal jurisdiction, with a small proportion of paved stretches, predominate in the Brazilian network. Federal and state roads have a bigger percentage of paved stretches (about $85 \%$ and $53 \%$, respectively). The circulation of vehicles has been greatly affected by the falling ratio of inhabitants per vehicle. This ratio fell by $40.7 \%$ between 2001 and 2013 , indicating a burden on the urban and interurban road system, principally near big population centers. That is a difficult problem to solve, for municipalities depend a great deal on federal policies and investments. For example, the reduced IPI - Tax on Industrialized Goods - for the purchase of cars between 2012 to 2015 led to a overburden of urban roads without equivalent investments in public transportation.

The analysis of railroad transportation indicates a balanced distribution between national roads and granted ones. The volume of freight transported by useful kilometer is concentrated in three railroads, located in the North and Southeast of the country. Among the most commonly handled products are iron ore, steel products, pig iron, agricultural products, coal, fuels and containers.

Waterway transportation recorded increased between the years 2005 and 2015 in all its modalities. Among these, long-haul navigation prevailed in 2015 (74.2\%), followed by cabotage (20.8\%) and other modes (inland navigation and inland cabotage) (5.0\%). In spite of this increase, it is important to highlight 
interiores) (5,0\%). Apesar do crescimento, é importante destacar a pequena extensão da malha aquaviária brasileira, possuindo apenas $3,7 \%$ da malha viária total.

Por fim, relatou-se o comportamento do transporte aeroviário no Brasil, com crescimento no número de voos, no número de passageiros transportados e nas cargas transportadas entre 2005 e 2014. O mercado doméstico é predominante em relação ao transporte de passageiros, enquanto que $o$ transporte de cargas é mais expressivo nos voos internacionais. the small extension of the Brazilian waterway transportation network, which represents only $3.7 \%$ of the total.

At the end we have provided an overall view of air transportation in Brazil, reporting increases in the number of flights, in the number of passengers and of the cargo transported between 2005 and 2014. The domestic market prevails in terms of passenger transportation, whereas cargo transportation is more significant in international flights.

Moacir Guilhermino da Silva

Engenheiro Civil pela Universidade Federal de Alagoas - UFAL (1975)

Mestre em Geotecnia e Planejamento de Sistemas de Transportes pela Universidade Federal de Campina Grande - UFCG (1979) Doutor em Ciências e Engenharia dos Materiais pela Universidade Federal do Rio Grande do Norte - UFRN (2012) Professor Associado II do Departamento de Engenharia Civil da Universidade Federal do Rio Grande do Norte - UFRN
Civil Engineer from the Federal University of Alagoas - UFAL (1975)

Master in Geotechnics and Planning of Transportation Systems from the Federal University of Campina Grande - UFCG (1979) $\mathrm{PhD}$ in Material Engineering and Sciences from the Federal University of Rio Grande do NorteUFRN (2012)

Associate Professor Il at the Civil Engineering Department of the Federal University of Rio Grande do Norte - UFRN (2012)

Fagner Alexandre Nunes de França

Engenheiro Civil pela Universidade Federal do Rio Grande do Norte - UFRN (2004) Mestre em Geotecnia pela Escola de Engenharia de São Carlos da Universidade de São Paulo - EESC/USP (2007) Doutor em Geotecnia pela Escola de Engenharia de São Carlos da Universidade de São Paulo - EESC/USP (2012) Professor Adjunto A do Departamento de Engenharia Civil da Universidade Federal do Rio Grande do Norte - UFRN
Civil Engineer from the Federal University of Rio Grande do Norte - UFRN (2004)

Master in Geotechnics from the School of Engineering of São Carlos, University of São Paulo-EESC/USP (2007)

$\mathrm{PhD}$ in Geotechnics from the School of Engineering of São Carlos, University of São Paulo - EESC/USP (2012)

Assistant Professor A at the Civil Engineering Department of the Federal University of Rio Grande do Norte-UFRN 
Tabela 15.1 - Extensão das malhas viárias do País - 2015

Table 15.1 - Roads network extention of Brazil - 2015

\begin{tabular}{ccc}
\hline & Extensão das malhas & \\
Item & viárias $(\mathrm{km}) /$ & Porcentagem(\%)/ \\
& Roads network extension & Percentage $(\%)$
\end{tabular}

Malha rodoviária/Roads extension

Federal/Federal
Pavimentada/Paved
Não pavimentada/Unpaved
Estadual/State
Pavimentada/Paved
Não pavimentada/Unpaved
Municipal/Municipality
Pavimentada/Paved
Não pavimentada/Unpaved
Total/Total
Pavimentada/Paved
Não pavimentada/Unpaved

Malha ferroviária/Railways extension

Nacional/National

Concedida/Concessionaire

Total/Total

$\begin{array}{rr}76353,8 & 4,9 \\ 64894,6 & 85,0 \\ 11459,2 & 15,0 \\ 225347,6 & 14,4 \\ 119747,0 & 53,1 \\ 105600,6 & 46,9 \\ 1261745,0 & 80,7 \\ 26826,7 & 2,1 \\ 1234918,3 & 97,9 \\ 1563446,4 & 100,0 \\ 211468,3 & 13,5 \\ 1351978,1 & 86,5\end{array}$

$30576,0 \quad 51,2$

$29165,0 \quad 48,8$

$59741,0 \quad 100,0$

Malha hidroviária/Waterways extension

Rede fluvial nacional/National waterways

$41635,0 \quad 65,4$

Vias utilizadas economicamente/Economic

exploited

22037,0

34,6

Total/Total

63672,0

100,0

Fonte/Source: Boletim estatístico [da Confederação Nacional dos Transportes]. Brasília, DF: CNT, dez. 2015. Disponível em/Available from: <http://www.cnt.org.br/boletim/boletim-estatistico-cnt $>$. Acesso em: abr. 2016/Cited: Apr. 2016. 
Tabela 15.2 - Dados gerais do transporte ferroviário - 2015

Table 15.2 - General data of railway transportation - 2015

\begin{tabular}{|c|c|c|c|c|}
\hline $\begin{array}{c}\text { Concessionárias/ } \\
\text { Concessionary railways }\end{array}$ & $\begin{array}{c}\text { Carga } \\
\text { transportada } \\
\text { (mil toneladas) } \\
\text { (tku)/ } \\
\text { Transported } \\
\text { cargo } \\
\text { (thousands } \\
\text { of tons)(tku) }\end{array}$ & $\begin{array}{l}\text { Locomo- } \\
\text { tivas/ } \\
\text { Locomotives }\end{array}$ & $\begin{array}{c}\text { Vagões/ } \\
\text { Freight } \\
\text { cars }\end{array}$ & $\begin{array}{c}\text { Carga } \\
\text { Transportada x } \\
\text { KM útil } \\
\text { (mil toneladas) } \\
\text { (tku)/ } \\
\text { Transported } \\
\text { cargo } x \mathrm{~km} \\
\text { (thousands } \\
\text { of tons) (tku) }\end{array}$ \\
\hline América Latina Logística Malha Oeste S.A & 4100 & 51 & 1165 & 927 \\
\hline Ferrovia Centro-Atlântica S.A. & 21677 & 899 & 15900 & 17319 \\
\hline MRS Logística S.A. & 115981 & 767 & 19231 & 53642 \\
\hline Ferrovia Tereza Cristina S.A. & 2911 & 15 & 424 & 225 \\
\hline América Latina Logística Malha Sul S.A & 17757 & 447 & 11812 & 11912 \\
\hline Transnordestina Logística S.A & 1026 & 91 & 1698 & 475 \\
\hline América Logística Malha Paulista S.A & 3953 & 286 & 3466 & 3037 \\
\hline Estrada de Ferro Vitória a Minas & 107027 & 313 & 20748 & 61974 \\
\hline Estrada de Ferro Carajás & 108586 & 285 & 17803 & 95498 \\
\hline Ferrovia Paraná Oeste S.A & 340 & 10 & 696 & 126 \\
\hline América Latina Logística Malha Norte S.A & 13965 & 194 & 5355 & 21288 \\
\hline Ferrovia Norte-Sul Tramo Norte & 4632 & 66 & 1217 & 3724 \\
\hline
\end{tabular}

Fonte/Source: Anuário da indústria automobilística brasileira 2016. São Paulo: Associação Nacional dos Fabricantes de Veículos Automotores - ANFAVEA, 2016. Disponível em/Available from: <http://www. anfavea.com.br>. Acesso em: abr. 2016/Cited:Apr. 2016.

\section{Gráfico 15.1 - Matriz de transporte de cargas, por modalidade - Brasil - 2015 Graph 15.1 - Cargo transportation matrix - Brazil - 2015}

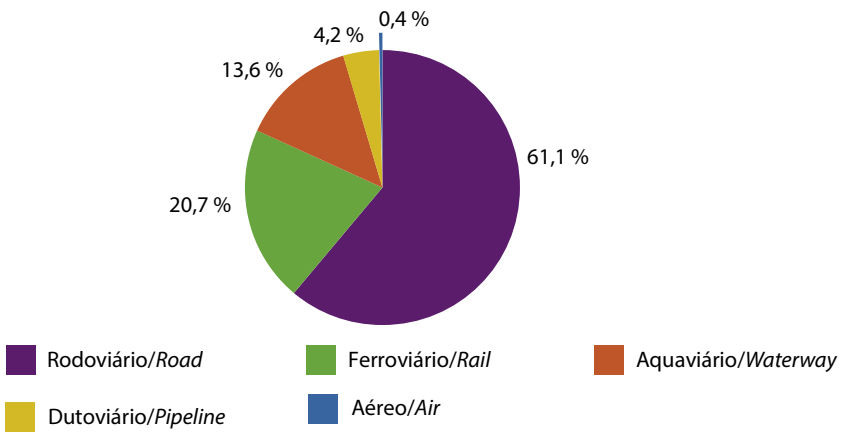

Fonte/Source: Boletim estatístico [da Confederação Nacional dos Transportes]. Brasília, DF: CNT, dez. 2015. Disponível em/Available from: <http://www.cnt.org.br/boletim/boletim-estatistico-cnt>. Acesso em: abr. 2016/Cited: Apr. 2016. 


\section{Gráfico 15.2 - Habitantes por autoveículo em alguns países - 2001/2013}

Graph 15.2 - Inhabitants per vehicle in selected countries - 2001/2013

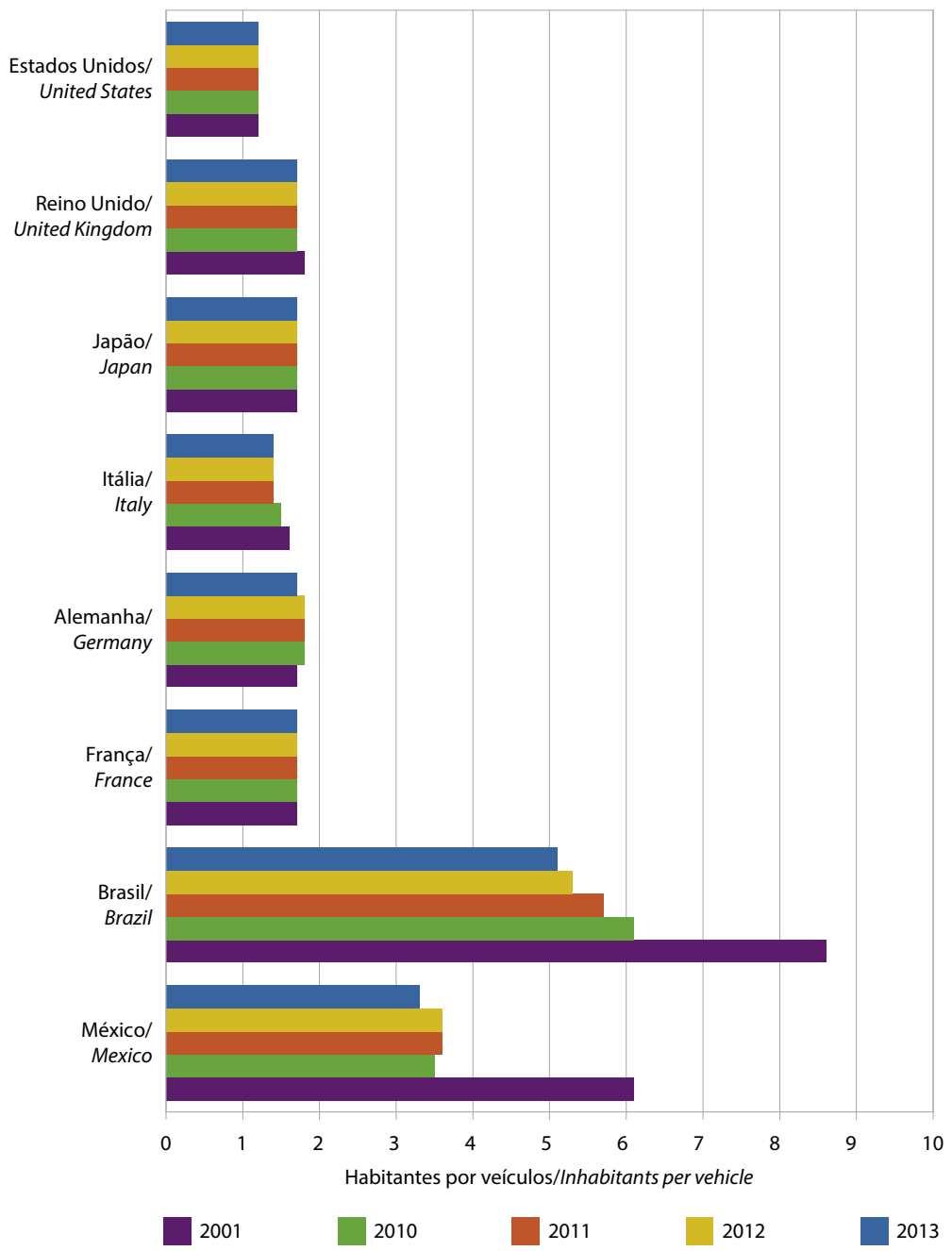

Fonte/Source: Anuário da indústria automobilística brasileira 2016. São Paulo: Associação Nacional dos Fabricantes de Veículos Automotores - ANFAVEA, 2016. Disponível em/Available from: http://www.virapagina. com.br/anfavea2015>. Acesso em: jan. 2016/Cited: Jan 2016. 


\section{Gráfico 15.3 - Movimento de carga, por tipo de navegação - 2005-2013}

Graph 15.3 - Cargo transportation by modal of navigation - 2005-2013

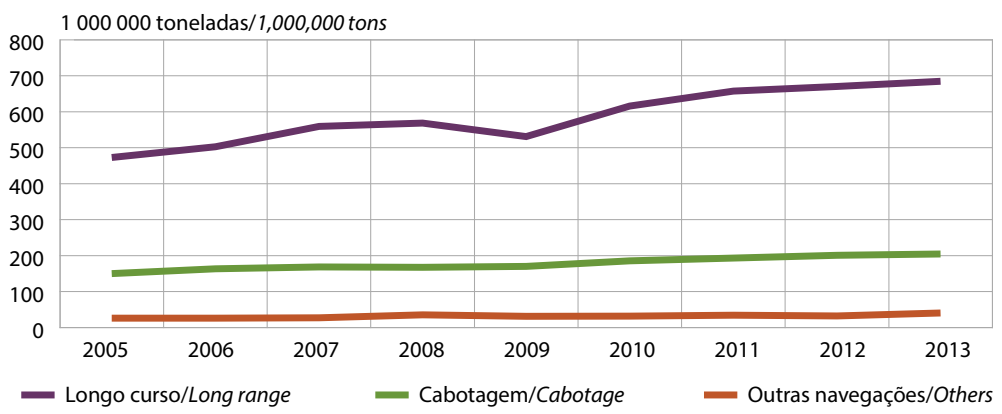

Fonte/Source: Anuário estatístico aquaviário 2014. Brasília, DF: Agência Nacional de Transportes Aquaviários - ANTAQ, [2016]. Disponível em/Available from: <http://www.antaq.gov.br/Portal/Estatisticas_Anuarios .asp>. Acesso em: abr. 2016/Cited: Apr. 2016.

\section{Gráfico 15.4 Evolução da quantidade de voos - 2005-2014 Graph 15.4 - Evolution of the number of flights - 2005-2014}

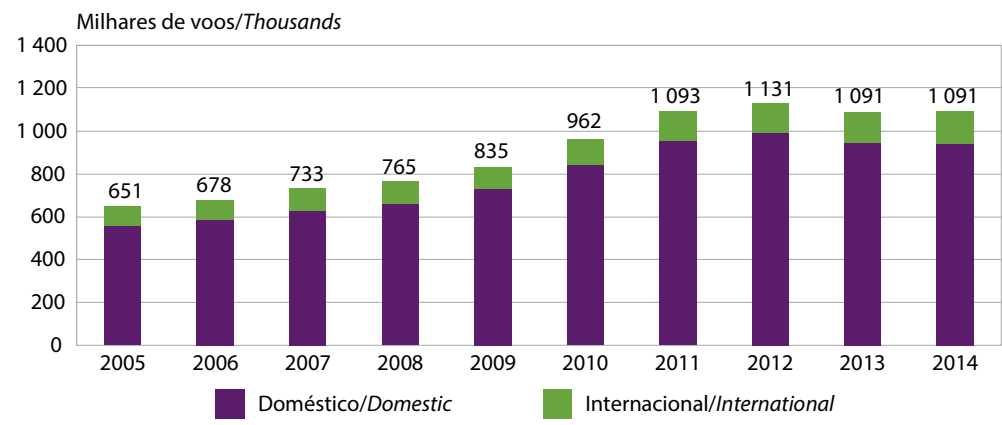

Fonte/Source: Agência Nacional de Aviação Civil - ANAC. 


\section{Gráfico 15.5 - Evolução da quantidade de passageiros transportada 2005-2014}

Graph 15.5 - Evolution of the number of passengers transported - 2005-2014

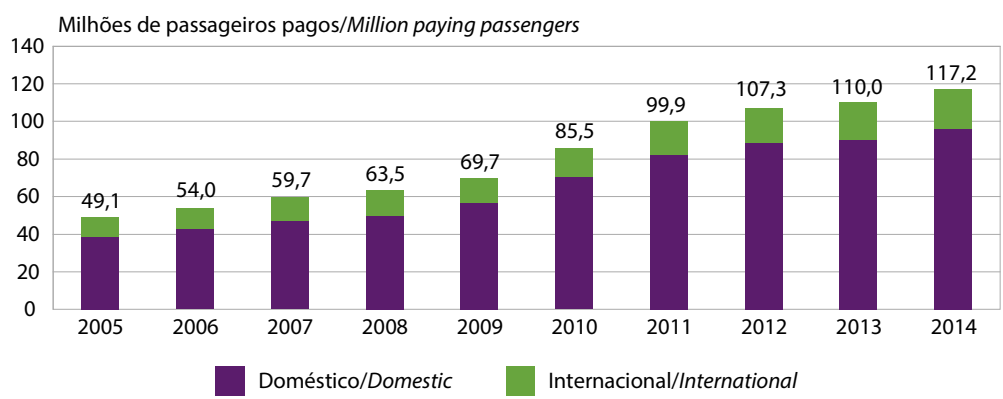

Fonte/Source: Agência Nacional de Aviação Civil - ANAC.

Gráfico 15.6 - Carga aérea transportada - 2005-2014

Graph 15.6 - Air Cargo transported - 2005-2014

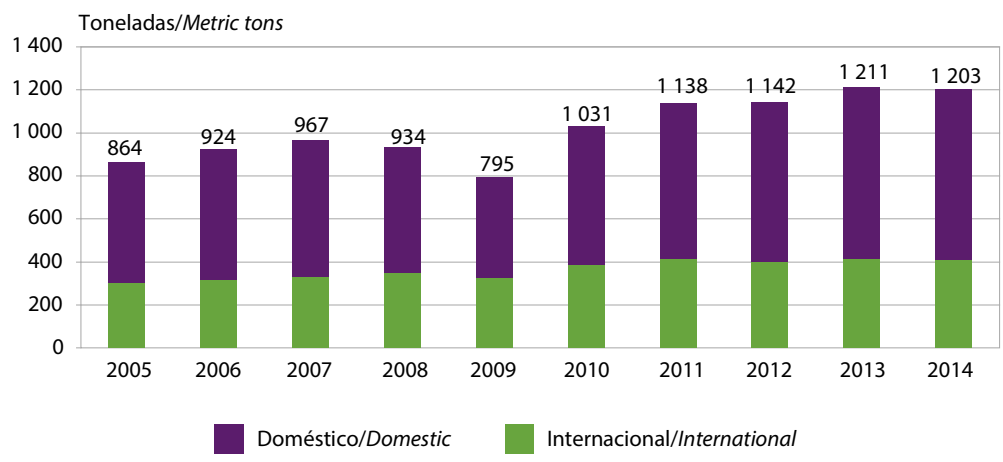

Fonte/Source: Agência Nacional de Aviação Civil - ANAC. 



\section{Turismo}

\section{Tourism}

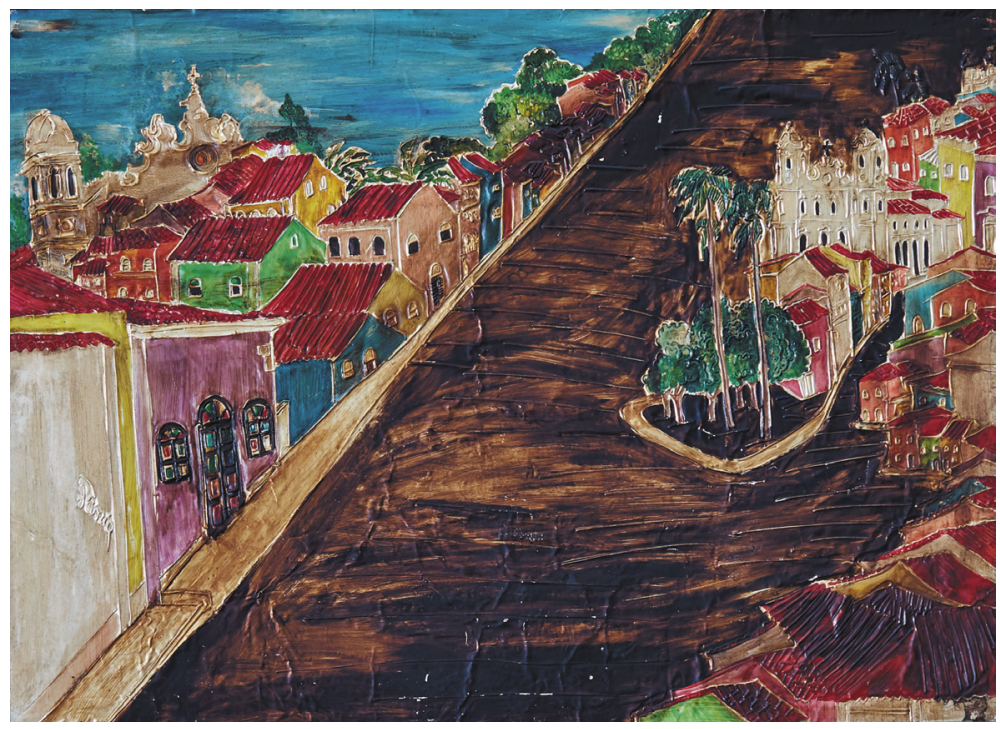

Igreja Casario, s.d./Church row houses, n.d. Tânia Maria de Oliveira Monte Melo 



\section{Turismo}

\section{Tourism}

\section{Turismo brasileiro - uma abordagem a partir dos eventos esportivos}

Nos anos de 2013 e 2014 a atividade turística mundial e, sobretudo a brasileira vivenciou os preparativos e a realização de dois eventos esportivos dos mais expressivos em âmbito mundial, a Copa das Confederações e a Copa do Mundo de Futebol. Na ocasião da realização dos dois eventos, o Brasil se voltou a investimentos de diferentes naturezas, como a qualificação profissional e infraestrutura básica e turística voltadas para amplificar a experiência positiva dos turistas motivados em visitar o território brasileiro.

A tabela 16.1 aponta que entre 2012 e 2014 houve um crescimento sistemático relacionado ao número de chegada dos turistas no Brasil, atingindo um ápice na chegada destes visitantes ao Brasil na ordem de 6.429.852.em 2014 , isto é, um aumento de $2,40 \%$ entre 2012 e 2013, e 10,60\% entre os anos de 2013 e 2014. Este fato ocorreu sem dúvida, pela realização da Copa do Mundo em 2014 realizada em 12 sedes municipais distribuídas entre
Tourism in brazil - an analysis based on sporting events

In the years 2013 and 2014 global tourism and especially tourism in Brazil underwent the preparations of and held two of the most significant sporting events in the world: FIFA Confederations Cup and the FIFA World Cup. In preparation for these two big events, Brazil made investments in various areas, such as professional qualification, and basic and tourism infrastructure, with the intention to improve the positive experiences of tourists in Brazil.

Table 16.1 shows that between 2012 and 2014 there was a systematic increase in the number of arrivals of tourists in Brazil, reaching a peak of $6,429,852$ tourists, which corresponds to a $2.4 \%$ increase between 2012 and 2013 and a 10.6\% increase between 2013 and 2014. The latter increase can most certainly be attributed to the 2014 FIFA World Cup which took place in 12 municipal capitals spread 
as 5 regiões das unidades federativas brasileiras. Dentre os países que mais visitaram o Brasil destacaram-se os Estados Unidos, na América do Norte com 844.969; a Argentina, na América do Sul com 1.743.930; e a França e a Alemanha, na Europa, com 282.375 e 265.498 respectivamente. Países como Itália e Espanha tiveram uma retração no número de desembarques no Brasil nos anos de 2012 e 2014. Já Portugal, manteve-se instável no mesmo período.

Já em relação à chegada de turistas por Unidade Federativa (ver tabela 16.2), foi possível aferir que na Região Norte houve um crescimento expressivo na cidade de Manaus, registrando um crescimento de $44,3 \%$ quando comparamos os anos de 2012 e 2014. Na região Nordeste, ocorreu um pequeno crescimento na cidade de Salvador, registrando 2,0\% quando comparamos os anos de 2012 e 2014. Por outro lado, considerando o mesmo período, observamos uma redução de $(-6,1 \%)$ na cidade de Natal. Na região sudeste, os destaques foram para as cidades do Rio de Janeiro com crescimento de 37,2\%, e São Paulo com 5,4\%. Apesar de São Paulo não apresentar um crescimento expressivo em percentuais, vale registrar que foi o destino que recebeu o maior número de turistas, atingindo 2.219.917 em 2014, representando $34,52 \%$ de todos os turistas que visitaram o Brasil, muito embora, acreditamos que parte deste montante foi motivada por visitar São Paulo em função da realização de negócios.

Diante desse cenário, é possível aferir em sua grande maioria, que as cidades que sediaram jogos da Copa do Mundo tiveram um crescimento em 2014 quando comparado ao ano de 2012. across Brazil's five regions. Among the countries that most visited Brazil were the United States of America with 844,969 visitors, Argentina with 1.743 .930 visitors, and France and Germany with 282,375 and 265,498 visitors, respectively. In the case of Italy and Spain, the number of visitors to Brazil in 2012 and 2014 decreased. Meanwhile, in Portugal, the number of nationals visiting Brazil remained stable during that same period.

With respect to the arrival of foreign tourists to Brazil by Federation Unit (see Table 16.2), in the North Region, we observe a significant increase of $44.3 \%$ in the city of Manaus from 2012 to 2014. In the Northeast, we can see a slight increase of $2 \%$ in the city of Salvador between 2012 and 2014; however, in contrast, during the same period, we observe a reduction of $6.1 \%$ in the city of Natal. In the Southeast region, the cities of Rio de Janeiro and São Paulo stand out, both showing an increase of foreign tourists of $37.2 \%$ and $5.4 \%$, respectively. Although São Paulo did not have a large increase in foreign visitors in percentage terms, it is important to note that, in numerical terms, it was the city that most welcomed foreign tourists, reaching 2,219,917 tourists in 2014, representing $34.52 \%$ of all tourists who visited Brazil that year, most likely due to people visiting São Paulo for business.

Given this scenario, it is possible to observe that, for the most part, the cities that hosted World Cup games had an increase of foreign visitors in 2014, when compared to 2012. 
Contudo, durante o evento Copa das Confederações, realizada em 2013, houve uma redução no quantitativo em boa parte dos estados que sediariam os turistas em 2014, tais como: Rio Grande do Norte, Rio Grande do Sul, Ceará, Bahia e Minas Gerais, apenas para explicitar algumas. Este fato ocorreu, talvez por se tratar de um evento com a participação reduzida de Nações que participaram do evento. Os números apontam que talvez os turistas preferiram visitar o território brasileiro durante a Copa do Mundo de 2014, por se tratar de um evento mais expressivo no setor esportivo, com exceção de poucos países como, por exemplo, Japão e Uruguai que tiveram números mais expressivos em 2013 quando comparados aos do ano de 2014. Também vale considerar que países como os Estados Unidos da América, apenas para explicitar, foi contemplado com a isenção da apresentação de vistos, fato este que sem dúvida impulsionou as visitas durante a Copa do Mundo de 2014.

Vale registrar que apesar da cidade de Campo Grande, no Estado do Mato Grosso do Sul, não ter sediado o evento, houve um crescimento significativo de turistas na ordem de $41,3 \%$ quando comparado os anos de 2012 e 2014. Acreditamos que este fato pode ter ocorrido pela motivação dos turistas internacionais em conhecer o Bioma do Pantanal.

Com No que diz respeito às 10 cidades brasileiras mais visitadas pelos turistas estrangeiros, que buscavam lazer nos anos de 2013 e 2014 (gráfico 16.1), foi possível observar que as cidades do Rio de Janeiro e São Paulo, além de Foz do Iguaçu e Florianópolis foram os destinos que mais receberam turistas nos anos de
However, during the Confederations Cup, in 2013, the number of international tourists to Brazil decreased for the most part in the states that hosted tourists in 2014, such as: Rio Grande do Norte, Rio Grande do Sul, Ceará, Bahia and Minas Gerais, to name a few. This could possibly be attributed to the fact that the Confederations Cup is an event with a smaller number of participating nations, and also to the economic crisis in Europe and North America. The numbers show that perhaps tourists preferred to visit Brazil during the 2014 World Cup, because it is the most significant event in the sports industry. However, there are exceptions: for example, certain countries, such as Japan and Uruguay, had more visitors to Brazil in 2013 than in 2014. It is also important to consider that countries such as the United States of America, among others, were granted a temporary visa waiver, which most certainly encouraged visitors during the 2014 World Cup.

Interestingly, the city of Campo Grande, in the state of Mato Grosso do Sul, which did not host any 2014 World Cup games, had a significant increase of tourists of $41.3 \%$ from 2012 to 2014 . We believe that this is possibly due to international tourists' interest in visiting the Pantanal.

With respect to the 10 most visited Brazilian cities by foreigners for tourism purposes between 2013 and 2014 (Figure 16.1), Rio de Janeiro, São Paulo, Iguaçu Falls and Florianopolis were the destinations that most welcomed international tourists between 2012 and 2014 . 
2012 e 2014. As duas primeiras cidades são consideradas portões de entrada no território brasileiro, considerando principalmente a emissão do continente norte americano, asiático e europeu. Diante disso, é fato que estas cidades tenham recebido o maior número de visitantes, inclusive porque foram as sedes que iniciaram e finalizaram os eventos supracitados. Já em relação aos destinos de Foz de Iguaçu e Florianópolis, apesar de não terem sediado jogos da Copa do Mundo em 2014, estes recebem regularmente um volume expressivo de turistas provenientes de países do Mercosul. Acreditamos que a América do Sul, continente menos afetado pela crise econômica neste período, de maneira geral, apresentavam tendência de crescimento. Podemos afirmar que a instabilidade econômica sentida na Europa nos anos de 2012 e 2014 afetou sobremaneira, principalmente os países mais frágeis economicamente no continente europeu, como por exemplo, Espanha e Portugal.

Em resumo, o Brasil depende cerca de $50 \%$ do mercado da América do Sul, tendo os argentinos os maiores consumidores de produtos e serviços prestados por empresas brasileiras. Já os Franceses e Alemães lideram o ranking no continente europeu, e os Estadunidenses ocupam o primeiro lugar em número dos que visitam o território nacional brasileiro proveniente da América do Norte.

Grande parte dos serviços prestados, tanto nos destinos emissores como nos receptores é oferecido por agências de viagens. No Brasil, de acordo com os dados do MTUR 2015, destacadas na (tabela 16.3) tem 18.227 agências distribuídas nas 5 regiões do território
The first two cities are considered entry points into the country, particularly for tourists from North America, Asia and Europe. Thus, it is no surprise that São Paulo and Rio de Janeiro welcomed a larger number of foreign tourists. Add to that the fact that they hosted the opening and closing ceremonies of the World Cup, respectively. As to Iguaçu Falls and Florianopolis, although neither hosted any 2014 World Cup games, both cities have a regular influx of tourists from Mercosur. We believe that South America, a continent less affected by the global economic crisis during the period 2012 to 2014, in general, showed a tendency for growth, whereas the economic instability in Europe between 2012 and 2014 impacted primarily the most economically fragile countries in Europe such as, for example, Spain and Portugal.

In summary, Brazil depends on nearly $50 \%$ of the South American Market for foreign tourists.

Whereas Argentines are the largest consumers of Brazilian products and services, the French and the Germans lead the ranking when it comes to the European continent. Americans, on their turn, lead the ranking of number of visitors to Brazil from North America.

Many of the services offered, in both outbound and inbound tourism, are offered by travel agencies. According to 2015 data from the Ministry of Tourism (MTUR), displayed in Table 16.3, there are 18,227 travel agencies 
brasileiro. Esses números estão presentes no CADASTUR, que é um sistema de cadastro de pessoas jurídicas e físicas que atuam na cadeia produtiva do turismo, executado pelo MTur em parceria com os Órgãos Oficiais de Turismo das Unidades da Federação. Desse total, São Paulo, Rio de Janeiro, Minas Gerais, Paraná e Rio Grande do Sul são as 5 estados que lideram em quantidade de agências cadastradas junto ao MTUR, atingindo $62,01 \%$ de todos os estabelecimentos cadastrados junto ao MTUR. A região Norte aparece como a região com o menor quantitativo de agências cadastradas, ou seja, apenas $4,71 \%$ do universo total. O restante, $33,28 \%$ está distribuído entre as regiões Nordeste (18,87\%), Centro-Oeste (8,53\%), além dos estados de Santa Catarina e Espírito Santo com (4,44\%) e (1,35\%) respectivamente.

Em resumo, podemos aferir que a constituição e a inserção das agências no CADASTUR tem uma relação direta com a oferta de atrativos naturais e culturais de cada Unidade Federativa, associada à demanda real e potencial que é mais expressiva na região sudeste, com destaque aos Estados de São Paulo e Rio de Janeiro. As demais regiões, como por exemplo, a região Norte é a mais afetada pela reduzida malha rodoviária e aeroviária, o que consequentemente impacta de forma negativa na captação de um maior fluxo turístico naquela região.

No que concerne às despesas e receitas na balança de pagamentos, podemos aferir que o aumento do fluxo turístico no Brasil entre 2012 e 2014 , tem forte impacto nas contas, o que reflete diretamente no saldo distributed throughout Brazil's five regions. These numbers come from CADASTUR, a registry system for individuals and businesses working in the tourism industry and which is issued by MTUR in partnership with the Official Tourism Bodies in each of Brazil's Federation Units. Of these agencies, São Paulo, Rio de Janeiro, Minas Gerais, Paraná and Rio Grande do Sul were the five states that led the ranking of number of agencies registered with MTUR, together representing $62.01 \%$ of all registered businesses with MTUR. The North region has the least number of agencies registered, just $4.71 \%$ of the total. The other $33.28 \%$ are distributed among the Northeast (18.87\%) and Center-West (8.53\%) Regions, and the states of Santa Catarina and Espírito Santo, with $4.44 \%$ and $1.35 \%$, respectively.

We can, therefore, infer that the creation and insertion of agencies in the CADASTUR system has a direct relationship with the supply of natural and cultural attractions in each Federation Unit, associated with real and potential demand, which is more significant in the Southeast Region, especially in the states of São Paulo and Rio de Janeiro. As for the other regions, if we look at, for example, the North, it suffers from reduced road and air networks, which generate a negative impact on its ability to attract a larger influx of tourists to the region.

With respect to expenditures and revenues on the balance of payments, we can infer that the increase of tourists to Brazil between 2012 and 2014 had a strong impact on public accounts, which is reflected directly 
da Conta Viagens Internacionais da balança de pagamentos. Analisando o comportamento das receitas e das despesas com viagens internacionais apresentado nos (gráficos 16.2 e 16.3) as receitas representam os gastos dos turistas estrangeiros no Brasil, enquanto as despesas representam os gastos dos turistas brasileiros no exterior. Os anos de 2007 e 2014 apresentam um saldo da Conta Viagens Internacionais deficitário. Ao mesmo tempo em que observamos um aumento entre 2011 e 2014 de 11,83\% nas receitas da balança de pagamento na Conta Turismo, por outro lado, observamos um aumento nas despesas no mesmo período, atingindo $21,65 \%$, fato este que gerou um déficit de $(-9,82 \%)$. Essa diferença se dá pelo volume superior de brasileiros que viajam ao exterior, quando comparado com a entrada de estrangeiros no território brasileiro. Também é relevante destacar o papel da taxa de câmbio no desempenho do saldo da Conta Viagens Internacionais. Uma análise do gráfico 16.4 aponta a correlação existente entre a taxa de Câmbio e o saldo da Conta Viagens Internacionais. Nesse sentido, houve uma valorização cambial da moeda americana nos anos de 2012, 2013 e 2014, quando comparado aos anos de 2010 e 2011. Esse fato traduziu num aumento do déficit da conta viagens internacionais, principalmente pelo aumento das despesas dos turistas brasileiros no exterior.

Em resumo, podemos sinalizar que as crises na Europa e América do Norte interferiram no mercado brasileiro entre 2012 e 2014. Numa análise macro, é possível observar uma tendência de crescimento tímida no mercado internacional, afinal, com o advento da Copa do Mundo houve um crescimento in the international travel balance of payments. Figures 16.2 and 16.3 show the trends of revenues and expenditures on international travel, where revenues represent foreign tourists' spending in Brazil and expenditures represent Brazilian tourists' spending abroad. The years 2007 and 2014 show a deficit in the balance of payments of the tourism account. While we see an increase in revenues between 2011 and 2014 of $11.83 \%$ in the Balance of Payments of Tourism Account, we also observe an increase in expenditures during that same period, which reached $21.65 \%$ and generated an overall deficit of $9.82 \%$. This difference is due to the greater volume of Brazilians who traveled abroad compared to the volume of foreigners who entered Brazil. It is also important to highlight the role of the exchange rate on the performance of the International Travel Account. An analysis of Figure 16.4 shows a correlation between the exchange rate and the balance of the International Travel Account. In the years 2012, 2013 and 2014 there was a trend of currency appreciation for the US dollar against the Brazilian Real, when compared to the years 2010 and 2011. This led to an increase in the International Travel Account deficit, particularly due to an increase in Brazilian expenditures abroad.

In summary, we can infer that the economic crisis in Europe and North America had an impact on the Brazilian tourism market between 2012 and 2014. On the whole, a small, albeit increasing trend can be observed in the international market, after all, with the advent of the FIFA World 
da ordem de 10,60\% entre 2013 e 2014. Este cenário não pode ser associado diretamente à influência de variáveis macroeconômicas, como, por exemplo, a taxa de câmbio ou até mesmo a inflação, por ter uma influência direta relacionada à realização das Copas do Mundo e das Confederações. Sugerimos estudos mais profundos acerca do posicionamento do país numa escala global, bem como uma análise da qualidade dos produtos e serviços prestados no território nacional.

Diante do cenário atual, sobretudo ao desempenho do Brasil face ao número de turistas internacionais, e elementos relacionados à taxa de câmbio, por exemplo, sugerimos uma maior atenção na definição de estratégias no âmbito nacional que possa otimizar e maximizar o turismo doméstico, ou seja, que estimulem o fluxo turístico dos residentes no território nacional como forma de equacionar o déficit da conta de viagens internacionais, ou seja, evitar a evasão de dinheiro do país.
Cup, there was an increase of $10.6 \%$ between 2013 and 2014. This scenario cannot be directly attributed to macroeconomic factors, such as, for example, the exchange rate or inflation, given the direct influence of hosting the Confederations Cup and the World Cup. We suggest further studies to examine how the country fits into the global market, in addition to an analysis of the quality of products and services offered in Brazil.

Given the current state of affairs, particularly the performance of Brazil with respect to the number of international tourists, and elements related to the exchange rate, for example, we suggest that greater attention be paid to national strategies that optimize and maximize domestic tourism, i.e., that encourage residents in Brazil to explore the country as a way to counterbalance the International Tourism Account deficit.

\section{Wilker Ricardo de Mendonça Nóbrega}

Doutor em Ciências Socio Ambientais pela Universidade Federal do Pará (NAEA / UFPA). Professor e pesquisador do Departamento de Turismo da Universidade Federal do Rio Grande do Norte. Coordenador do Programa de Pós Graduação em Turismo da UFRN (Mestrado e Doutorado)
PhD em Socio-Environmental Sciences from the Federal University of Pará (NAEA/UFPA).

Professor and researcher of the Department of Tourism at the Federal University of Grande do Norte (UFRN). Coordinator of the Graduate Program in Tourism at UFRN (Master's and PhD programs). 
Tabela 16.1 - Chegadas de turistas no Brasil - 2012-2014

Table 16.1 - Tourist arrivals to Brazil - 2012-2014

\begin{tabular}{|c|c|c|c|}
\hline \multirow{2}{*}{$\begin{array}{l}\text { País de residência } \\
\text { permanente/ } \\
\text { Country of } \\
\text { permanent residence }\end{array}$} & \multicolumn{3}{|c|}{$\begin{array}{c}\text { Chegadas de turistas/ } \\
\text { Tourist arrivals }\end{array}$} \\
\hline & 2012 & 2013 & 2014 \\
\hline Total/Total & 5676843 & 5813342 & 6429852 \\
\hline África/Africa & 92349 & 94832 & 128252 \\
\hline América Central/Central America & 43154 & 52520 & 61968 \\
\hline América do Norte/North America & 716583 & 737175 & 844969 \\
\hline Canadá/Canada & 68462 & 67610 & 78531 \\
\hline Estados Unidos/United States & 586463 & 592827 & 656801 \\
\hline México/Mexico & 61658 & 76738 & 109637 \\
\hline América do Sul/South America & 2822519 & 2936753 & 3133629 \\
\hline Argentina/Argentina & 1671604 & 1711491 & 1743930 \\
\hline Bolívia/Bolivia & 112639 & 95028 & 95300 \\
\hline Chile/Chile & 250586 & 268203 & 336950 \\
\hline Colômbia/Colombia & 100324 & 116461 & 158886 \\
\hline Paraguai/Paraguay & 246401 & 268932 & 293841 \\
\hline Peru/Peru & 91996 & 98602 & 117230 \\
\hline Uruguai/Uruguay & 253864 & 262512 & 223508 \\
\hline Venezuela/Venezuela & 51106 & 68309 & 108170 \\
\hline Outros/Other countries & 43999 & 47215 & 55814 \\
\hline Ásia/Asia & 297032 & 300998 & 332585 \\
\hline Japão/Japan & 73102 & 87225 & 84636 \\
\hline Outros/Other countries & 223930 & 213773 & 247949 \\
\hline
\end{tabular}


Tabela 16.1 - Chegadas de turistas no Brasil - 2012-2014

Table 16.1 - Tourist arrivals to Brazil - 2012-2014

(conclusão/concluded)

\begin{tabular}{|c|c|c|c|}
\hline \multirow{2}{*}{$\begin{array}{l}\text { País de residência } \\
\text { permanente/ } \\
\text { Country of } \\
\text { permanent residence }\end{array}$} & \multicolumn{3}{|c|}{$\begin{array}{c}\text { Chegadas de turistas/ } \\
\text { Tourist arrivals }\end{array}$} \\
\hline & 2012 & 2013 & 2014 \\
\hline Europa/Europe & 1652205 & 1636569 & 1847834 \\
\hline Alemanha/Germany & 258437 & 236505 & 265498 \\
\hline Áustria/Austria & 28035 & 25929 & 28532 \\
\hline Bélgica/Belgium & 34169 & 33356 & 42964 \\
\hline Espanha/Spain & 180406 & 169751 & 166759 \\
\hline França/France & 218626 & 224078 & 282375 \\
\hline Holanda/Netherlands & 73133 & 69187 & 81655 \\
\hline Inglaterra/England & 155548 & 169732 & 217003 \\
\hline Itália/Italy & 230114 & 233243 & 228734 \\
\hline Portugal/Portugal & 168649 & 168250 & 170066 \\
\hline Suíça/Switzerland & 69571 & 68390 & 80277 \\
\hline Outros/Other countries & 235517 & 238148 & 283971 \\
\hline Oceânia/Oceania & 52815 & 54281 & 80447 \\
\hline Não especificado/Unspecified & 186 & 214 & 168 \\
\hline
\end{tabular}

Fonte/Source: Anuário estatístico de turismo 2015. Brasília, DF: Ministério do Turismo, v. 42, 2015. Ano base 2014. Disponível em/Available from: <http://www.dadosefatos.turismo.gov.br/dadosefatos/ home.html>. Acesso em: abr. 2016/Cited:Apr. 2016. 
Tabela 16.2 - Chegadas de turistas no Brasil, por Unidades da Federação de acesso - 2012-2014

Table 16.2 - Tourist arrivals to Brazil, by Federation Unit of access - 2012-2014

\begin{tabular}{|c|c|c|c|}
\hline \multirow{2}{*}{$\begin{array}{l}\text { Unidades da Federação de acesso/ } \\
\text { Federation Unit of access }\end{array}$} & \multicolumn{3}{|c|}{$\begin{array}{c}\text { Chegadas de turistas/ } \\
\text { Tourist arrivals }\end{array}$} \\
\hline & 2012 & 2013 & 2014 \\
\hline Total/Total & 5676843 & 5813342 & 6429852 \\
\hline Amazonas & 34720 & 32993 & 50032 \\
\hline Pará & 16877 & 13269 & 14813 \\
\hline Pernambuco & 70259 & 75174 & 78075 \\
\hline Bahia & 142803 & 128838 & 145660 \\
\hline Ceará & 91648 & 84119 & 85025 \\
\hline Rio de Janeiro & 1164187 & 1207800 & 1597153 \\
\hline Minas Gerais & 54480 & 46639 & 50916 \\
\hline São Paulo & 2110427 & 2219513 & 2219917 \\
\hline Paraná & 791396 & 839728 & 837046 \\
\hline Rio Grande do Norte & 40488 & 35888 & 38014 \\
\hline Rio Grande do Sul & 810670 & 782887 & 907669 \\
\hline Mato Grosso do Sul & 43891 & 41523 & 61999 \\
\hline Santa Catarina & 195708 & 175023 & 156976 \\
\hline Distrito Federal/Federal District & 68540 & 74287 & 100063 \\
\hline Outros/Others & 40749 & 55661 & 86494 \\
\hline
\end{tabular}

Fonte/Source: Anuário estatístico de turismo 2015. Brasília, DF: Ministério do Turismo, v. 42, 2015. Ano base 2014. Disponível em/Available from: <http://www.dadosefatos.turismo.gov.br/dadosefatos/ home.html>. Acesso em: abr. 2016/Cited:Apr. 2016. 
Tabela 16.3 - Agências de turismo cadastradas no CADASTUR - 2014

Table 16.3 - Travel agencies registered in CADASTUR - 2014

\begin{tabular}{|c|c|c|c|}
\hline $\begin{array}{l}\text { Unidades da Federação/ } \\
\text { Federation Units }\end{array}$ & $\begin{array}{l}\text { Agências/ } \\
\text { Agencies }\end{array}$ & $\begin{array}{l}\text { Unidades da Federação/ } \\
\text { Federation Units }\end{array}$ & $\begin{array}{l}\text { Agências/ } \\
\text { Agencies }\end{array}$ \\
\hline Brasil/Brazil & 18227 & Alagoas & 265 \\
\hline Rondônia & 133 & Sergipe & 157 \\
\hline Acre & 71 & Bahia & 925 \\
\hline Amazonas & 235 & Minas Gerais & 1535 \\
\hline Roraima & 40 & Espírito Santo & 247 \\
\hline Pará & 254 & Rio de Janeiro & 2464 \\
\hline Amapá & 70 & São Paulo & 4988 \\
\hline Tocantins & 57 & Paraná & 1172 \\
\hline Maranhão & 203 & Santa Catarina & 821 \\
\hline Piauí & 99 & Rio Grande do Sul & 1144 \\
\hline Ceará & 427 & Mato Grosso do Sul & 307 \\
\hline Rio Grande do Norte & 226 & Mato Grosso & 285 \\
\hline Paraíba & 328 & Goiás & 451 \\
\hline Pernambuco & 811 & Distrito Federal/Federal District & 512 \\
\hline
\end{tabular}

Fonte/Source: Anuário estatístico de turismo 2015. Brasília, DF: Ministério do Turismo, v. 42, 2015. Ano base 2014. Disponível em/Available from: <http://www.dadosefatos.turismo.gov.br/dadosefatos/ home.html>. Acesso em: abr. 2016/Cited:Apr. 2016.

\section{Gráfico 16.1 - Dez cidades brasileiras mais visitadas, pelos turistas estrangeiros para lazer - 2013-2014}

Graph 16.1 - Top ten Brazilian cities visited cities by foreign tourists in Brazil for leisure - 2013-2014

Armação dos Búzios

Rio de Janeiro

São Paulo

Florianópolis

Foz do Iguaçu

Salvador
S

Belo Horizonte

Porto Alegre

Balneário Camboriú

Brasília

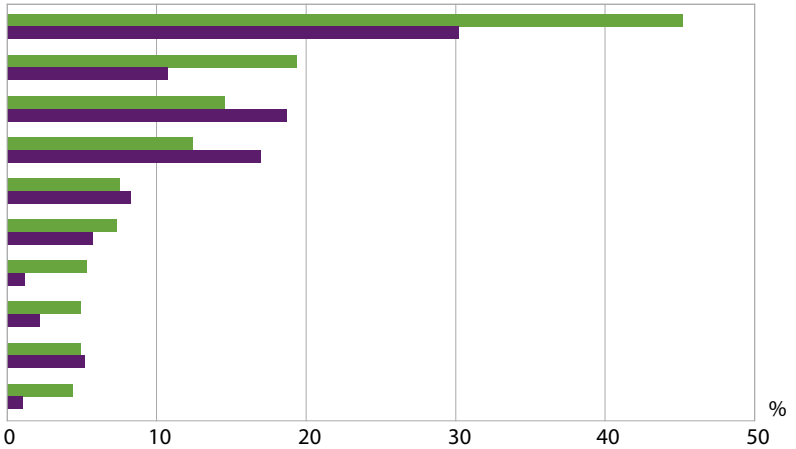

2013

2014

Fonte/Source: Estudo da demanda turística internacional 2010-2014. Brasília, DF: Ministério do Turismo, 2015. Disponível em/Available from: <http://www.dadosefatos.turismo.gov.br/dadosefatos/demanda turistica/internacional/>. Acesso em: abr. 2016/Cited:Apr.2016. 


\section{Gráfico 16.2 - Despesa da balança de pagamentos \\ da conta turismo - 2007-2014}

Graph16.2 - Expenditure of the balance of payments of tourism account - 2007-2014

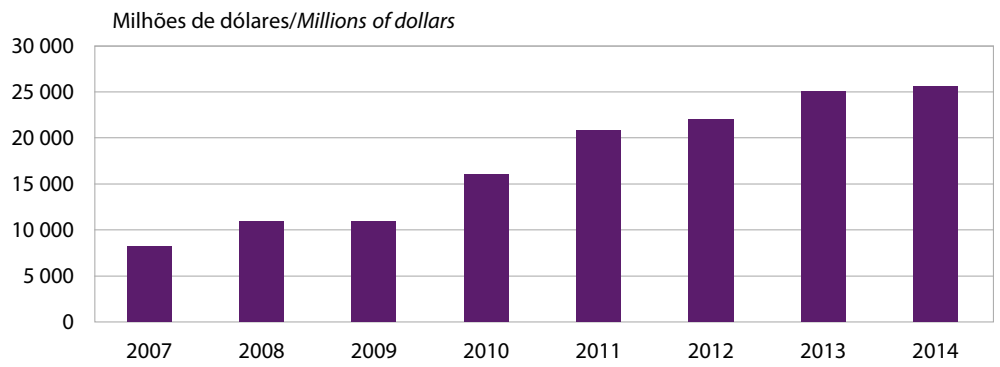

Fonte/Source: Receita e despesa cambial turística. Dados e fatos: estudos, pesquisas e dados sobre o setor de turismo. Brasília, DF: Ministério do Turismo, [2015]. Disponível em/Available from: <http://www.dadosefatos. turismo.gov.br/dadosefatos/estatisticas_indicadores/receita_cambial/>. Acesso em: abr. 2016/Cited:Apr. 2016.

\section{Gráfico 16.3 - Receita da balança de pagamentos da conta-turismo 2007-2014}

Graph 16.3 - Receipt of the balance of payments of tourism account - 2007-2014

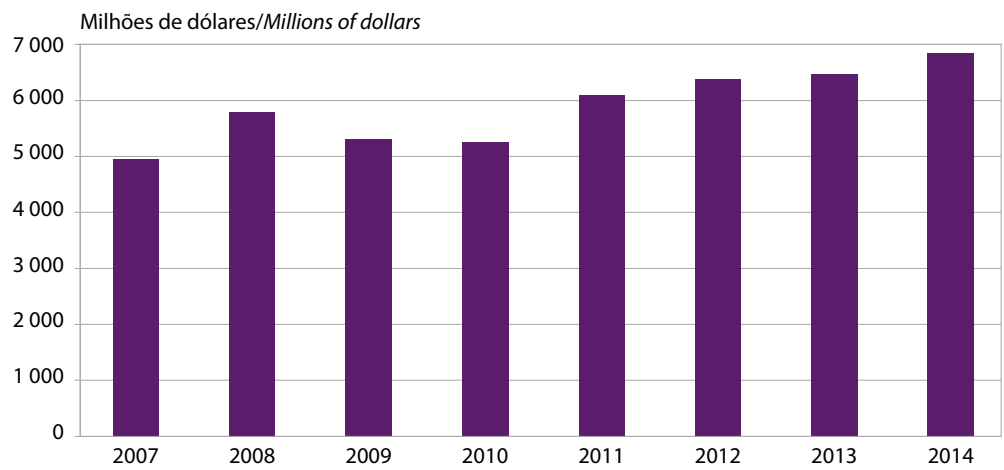

Fonte/Source: Receita e despesa cambial turística. Dados e fatos: estudos, pesquisas e dados sobre o setor de turismo. Brasília, DF: Ministério do Turismo, [2015]. Disponível em/Available from: <http://www.dadosefatos. turismo.gov.br/dadosefatos/estatisticas_indicadores/receita_cambial/>. Acesso em: abr. 2016/Cited:Apr. 2016. 


\section{Gráfico 16.4 - Taxa de câmbio e saldo da balança de pagamentos da conta-turismo - 2005-2014}

Graph 16.4 - Exchange rate and balance of tourism account - 2005-2014

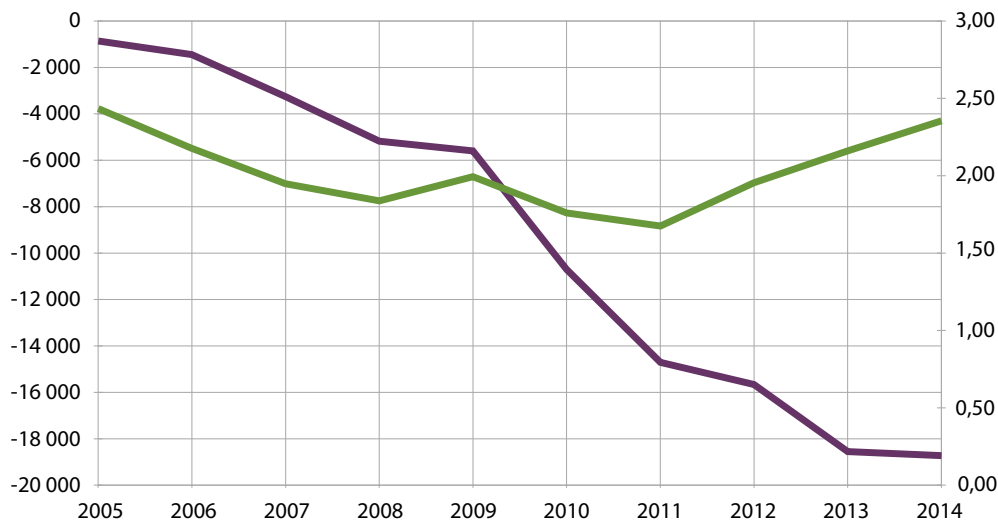

Saldo da conta-turismo/ Taxa de câmbio - Livre - Dólar americano (venda) -

Ballance of the tourism account

Média de período - anual/Exchange rate - Free - USD (sale) - Annual average

Fonte/Source: Receita e despesa cambial turística. Dados e fatos: estudos, pesquisas e dados sobre o setor de turismo. Brasília, DF: Ministério do Turismo, [2015]. Disponível em/Available from: $<$ http://www. dadosefatos.turismo.gov.br/dadosefatos/estatisticas_indicadores/receita_cambial/>. Acesso em: abr. 2016/Cited: Apr. 2016. 



\section{Comunicações \\ Communications}

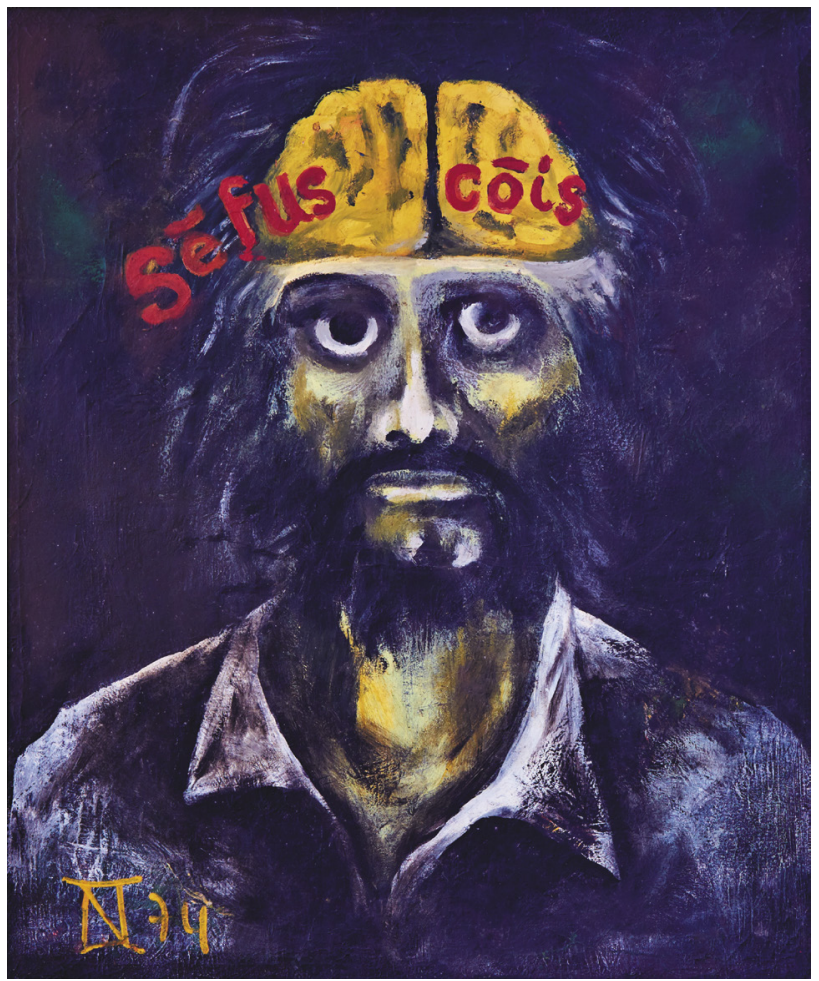

Sefus Coisa/Sefus Gõis, 1974 Leopoldo Nelson de Sousa Leite 



\section{Comunicações}

\section{Communications}

Setor essencial para o desenvolvimento social, econômico e político, as comunicações vivem, nas últimas décadas, um vácuo institucional. Desde a dispersão normativa, entre distintas autoridades regulatórias e sem contar com um marco regulatório próprio, até a ausência de transparência sobre o conjunto de dados sobre o setor. No cerne desta questão encontra-se a confusão conceitual acerca da sua função pública. A concepção plural deste setor - Comunicações porque converge Telecomunicações, Comunicação Social e os Serviços Postais - reune histórias e concepções díspares. Se, por um lado, há relativa clareza de que os serviços de postais e de telecomunicações são serviços públicos e o Estado pode optar pela exploração direta ou indireta. No caso dos serviços de comunicação, que englobam o cenário midiático, em especial o segmento audiovisual, a natureza pública dos serviços tem parca visibilidade. A ausência de um conjunto de diretrizes, normas e leis regulando o funcionamento dos serviços se complexifica no contexto da digitalização e da chamada Sociedade Informacional.
A key sector for social, economic and political development, communications has experienced, in the last few decades, an institutional vacuum: dealing with the separation of norms among different regulation authorities, not having its own regulatory benchmark and facing the lack of transparency of data on the sector. At the heart of this issue lies the misconception of the public role of communications. The plural composition of this sector - named Communications because it includes Telecommunications, Journalism and Postal Services - gathers distinct stories and concepts. On the one hand, it is relatively clear that postal and telecommunication services are public services that can be explored by the state directly or indirectly. On the other hand, it is in the case of services which encompass media segments - notably the audiovisual one - that public nature ends up overshadowed. The absence of a set of directives, norms and laws to regulate these services makes the context of digitization and of the socalled Information Society even more complex. 
A confusão conceitual transparece na própria demarcação institucional dos serviços: as telecomunicações brasileiras são regidas pela Lei Geral de Telecomunicações -LGT, N. 9.472, de 1997, que criou a Agência Nacional de Telecomunicações-ANATEL e orientou o processo de abertura dos serviços telefônicos à iniciativa privada. No entanto, a norma exclui da jurisdição da Anatel os serviços de radiodifusão sonora e de sons e imagens, popularmente conhecidos como rádio e televisão aberta (Art. 221, Lei N. 9.472), cabendo à Agência um papel meramente fiscalizador, ficando a formulação de diretrizes e a concessão dos serviços no âmbito do Ministério das Comunicações. Desta forma, um serviço como o de televisão, é separado institucionalmente pelas formas de acesso: a TV aberta segue as regras de uma Lei de 1962, o Código Brasileiro de Telecomunicações (Lei N. 4.117) e a TV por assinatura segue as premissas da LGT, supracitada. As comunicações brasileiras vivem, na época da convergência tecnológica, uma convergência divergente: de um lado, os serviços de telecomunicações - incluindo TV por assinatura e comunicação de dados, do outro os serviços de rádio e televisão aberta e os serviços postais, que têm legislação própria de 1978 (Lei N. 6.538).

Seguindo a lógica da dispersão regulatória, os dados do setor também são fragmentados, informações relevantes são de acesso difícil e as fontes oficiais muitas vezes se contradizem com relação aos dados do setor. Se, por um lado, os dados relativos às telecomunicações são fartos e consistentes, os dados relativos aos serviços postais e de radiodifusão carecem de sistematização e visibilidade.
The very institutional framework of services makes visible this confusion of concepts: Brazilian telecommunications is regulated by the General Law of Telecomunications-LGT, no. 9,472 , of 1997 , which created the National Telecommunications Agency-Anatel and led the process of opening to private companies the supply of telephone services. Therefore, Anatel is limited to inspecting, whereas the formulation of directives and granting of services are duties of the Ministry of Telecommunications. So, a service as that of television is institutionally split according to its forms of access: broadcast TV service is defined by the Brazilian Telecommunications Code (Law no. 4,117 of 1962) and subscription TV service by the aforementioned LGT. At times of technological convergence, Brazilian communications experiences a divergent convergence: telecommunication services including subscription TV and data communication on one side and broadcast television and postal services, which follow a regulation of their own, Law no 6.538 of 1978, on the other.

Similarly, the data of the sector are also fragmented: access to relevant information is difficult and many times there are contradictions between the information obtained from the sector itself and the data provided by official channels. Whereas data on telecommunications are widely available and consistent, what stands out in postal and broadcast services is the lack of systematization and the poor availability. 
No Brasil, a televisão aberta se constitui na principal fonte de informação e, muitas vezes, entretenimento e educação da sociedade brasileira. Segundo o PNAD de 2014, os domicílios brasileiros têm $97,1 \%$ dos domicílios brasileiros possuem aparelho de televisão enquanto $93,5 \%$ possuem telefone fixo ou celular e apenas $42,1 \%$ possuem acesso a microcomputador com acesso à Internet. Apenas 32,1\% dos domicílios no país tinham acesso à TV por assinatura' .

A centralidade da televisão aberta se torna mais clara quando observamos as tabelas da Pesquisa Suplementar Acesso à Internet e à Televisão do PNAD-2014. A tabela Moradores em domicílios particulares permanentes, por existência de utilização da Internet no domicílio mostra que $40,8 \%$ dos domicílios não tinham utilização de Internet, nas regiões Norte $(52,6 \%)$ e Nordeste $(54,2 \%)$ mais da metade dos domicílios não têm acesso à Internet. $O$ índice de exclusão informacional é grave se considerarmos que, dentre os que utilizam Internet no domicílio, ainda há 2,8\% deles utilizando conexão discada em vez de banda larga ${ }^{2}$. Neste contexto, a observação do setor pelas lógicas do acesso, da livre competição e da pluralidade da oferta, deve levar em conta também os contextos das grandes regiões do país.

A televisão não é central apenas no setor das comunicações. Nos índices nacionais, a televisão tem o mesmo

\footnotetext{
1 Tabelas disponíveis em: http://www.ibge.gov.br/ home/estatistica/populacao/trabalhoerendimento/ pnad2014/default.shtm. Acesso em ago. 2016.

2 Tabelas disponíveis em: http://www.ibge.gov.br/ home/estatistica/populacao/acessoainternet2014/ default_ods.shtm. Acesso em ago. 2016.
}

In Brazil, broadcast television is the main source of information, and, many times, also of entertainment and education for the Brazilian society. According to PNAD 2014, $97.1 \%$ of the Brazilian housing units had a television set, while $93.5 \%$ had a landline or mobile telephone and only $42.1 \%$ had a personal computer with Internet access. Only $32.1 \%$ of the housing units in the country had access to subscription television'.

The centrality of broadcast television is evidenced in the tables of the Supplementary Survey of Access to the Internet and Television of PNAD 2014. The table "Residents in permanent private housing units, by occurrence of Internet use in the housing unit" shows there was no Internet use in $40.8 \%$ of the housing units, and that in the North (52.6\%) and Northeast (54.2\%) more than half of the housing units did not have access to the Internet. The informational exclusion index is very high considering that, among those who used the Internet in the housing unit, $2.8 \%$ still had dial up Internet access, instead of broadband ${ }^{2}$. In this context, the analysis of the sector from the perspectives of access, free competition and diversity of supply should take into consideration the realities of Brazil's Major Regions.

Television is not central in the communications sector only. In national indexes, television is as

\footnotetext{
1 Tables available from: http://www.ibge.gov.br/ home/estatistica/populacao/trabalhoerendimento/ pnad2014/default.shtm. Cited: Aug. 2016.

2 Tables available from: http://www.ibge.gov.br/ home/estatistica/populacao/acessoainternet2014/ default_ods.shtm. Cited: Aug. 2016.
} 
índice de presença nas residências que a geladeira, 97,1\%. Ela supera de longe a máquina de lavar roupa, presente em $58,7 \%$ dos municípios. Supera até a presença de rede de água, $93,9 \%$, e esgoto, $72,6 \%$, na totalidade dos domicílios brasileiros. Em termos regionais, os domicílios tem mais TV que banheiro ou sanitário de uso exclusivo dos moradores nas regiões Norte e Nordeste. $\mathrm{Na}$ região Norte chega a ter mais TV do que iluminação elétrica. $E$ isso não é nenhuma novidade. Há mais de três décadas que as pesquisas do campo da comunicação usam estas referências dos indicadores domiciliares para falar da centralidade da TV aberta na vida domiciliar brasileira. Há mais de três décadas, também, se diz que a TV aberta está morrendo, cedendo lugar a uma nova tecnologia. $E$, mesmo com a efervescência das redes sociais e dos serviços de comunicação pela Internet, a TV aberta demonstra sua importância no acesso à informação, na elaboração de sentidos e na própria cultura nacional.

Apontar a centralidade televisiva tem caráter elucidativo para a compreensão da dimensão estratégica e sociopolítica dos serviços. Não se trata, no entanto, de uma espécie de hierarquização da sua essencialidade. Embora o ambiente de convergência tecnológica tenda à convergência dos serviços e, consequentemente, das lógicas de regulação, a comunicação postal, a comunicação por voz e a comunicação social cumprem papeis complementares no sistema de comunicações. Tratemos, então, destes serviços por ordem. present in housing units as the refrigerator, with $97.1 \%$. It surpasses by far the washing machine, seen in $58.7 \%$ of the municipalities, and even the availability of water supply (93.9\%) and sewage disposal (72.6\%) services in the Brazilian municipalities. Results by Major Region show that the housing units have more television sets than a bathroom or a toilet for the exclusive use of residents, in the North and Northeast. In the North Region the number of television sets surpasses the supply of electricity in some areas. That is nothing new. Communications surveys have used these references to illustrate the central role of broadcast television in Brazilian households. For more than three decades it has been said that broadcast TV is dying and will be replaced by a new technology. Nonetheless, even with the explosion of social media and Internet communication services, broadcast TV remains relevant to the access to information, to the creation of meanings and to the national culture itself.

Acknowledging the central position of television is elucidating when it comes to understanding the sociopolitical and strategic dimension of services. It is not, however, an attempt at the hierarchization of its essential nature. Although the environment of technological convergence leads to the convergence of services and, consequently, of regulation directives, postal communication, voice communication and social communication play complementary roles in the communication system. These services are presented below: 


\section{Comunicação Postal}

Os Correios têm uma tradição sólida de reconhecimento como serviço público de qualidade. Em 2015, a empresa estava como $79^{a}$ empresa no ranking das mil melhores empresas brasileiras, elaborado pela Editora Três. Embora ainda seja o serviço de comunicações melhor qualificado em consultas populares, os Correios têm enfrentado diversos problemas com roubos de cargas e atrasos nos prazos de entregas na prestação dos serviços postais básicos. Na estrutura organizacional, a tabela Organização dos Correios e Telégrafos - 2011-2015 - demonstra outro aspecto dos serviços a ser problematizado: a diminuição das unidades e das caixas de coleta.

Nos últimos cinco anos, o total de unidades caiu de 17.133, em 2011, para 15.661, em 2015, uma queda de $8,6 \%$. Observa-se que esta queda está diretamente relacionada às unidades terceirizadas, pois houve ligeiro crescimento das unidades próprias. Também houve queda de $19,5 \%$ no total de caixas de coleta do serviço. Por outro lado, houve aumento do efetivo de pessoal e das unidades de tratamento e distribuição, o que pode sinalizar para melhoras na prestação dos serviços.

Com relação ao Tráfego Postal - Tabela 17.2 -, observa-se a mesma tendência de diminuição da participação do setor privado no segmento. Entre 2011 e 2015, enquanto o serviço postal próprio aos Correios diminuiu $7,85 \%$, o serviço postal concorrente diminuiu 48,61\%. Em decorrência da diminuição da participação do setor privado, majoritariamente composto por

\section{Postal Communication}

The Brazilian Postal Company has a solid history as a provider of high-quality public service. In 2015, the company was at the 79th position in the ranking of the 1,000 best companies in Brazil, elaborated by Três publishing house. Although it still presents the best evaluations among communications companies according to public opinion, the Brazilian Postal Company has faced difficulties related to cargo theft and delay in delivery dates even for very basic services. The table "Organization of Postal and Telegraph Services" shows another problem to be dealt with in the company's organizational structure: the decrease of branches and of mailing boxes.

In the last five years, the number of total units fell by $8.6 \%$, from 17,133 , in 2011 to 15,661, in 2015. It is observed that this decrease is directly related to postal service franchises, since there was slight increase of state-owned branches. There was also decrease of $19.5 \%$ in the total mailing boxes. On the other hand, there was increase in the staff and in mail treatment and distribution offices, which can indicate improvement in the rendering of services.

In relation to Postal Traffic - Table 17.2 - we see the same trend to decrease as in the participation of the private sector in the segment. Between 2011 and 2015, whereas the postal service provided by the Brazilian Postal Company fell by $7.85 \%$, that of its competitor decreased by $48.61 \%$. As a result of the reduced participation of the private sector, formed mostly by 
empresas internacionais, a distribuição de objetos internacionais teve um acréscimo de $68 \%$. Os serviços postais confirmam, assim, o predomínio histórico do Estado na atividade.

\section{Telefonia}

Desde o processo de privatização do sistema de telecomunicações brasileiro, ocorrido em 1998, o segmento de transporte de dados e voz tornouse aquele com maior detalhamento dos índices relevantes no setor das comunicações. Isto não quer dizer, necessariamente, que apresente maior qualidade de serviços ou um ambiente mais plural na oferta. Ao contrário, as companhias de telecomunicações, em especial o segmento telefônico são campeãs de reclamações sobre os serviços. No ranking elaborado pelo Procon de São Paulo, o maior mercado no Brasil com 80.959 linhas telefônicas instaladas ( $26,8 \%$ do total), os quatro primeiros lugares entre as empresas que receberam mais reclamações fundamentadas são de companhias de serviços de telecomunicações. Três delas companhias telefônicas, uma de TV por assinatura. A prevalência das companhias de telecomunicações nas queixas do serviço de proteção ao consumidor paulista ocorre desde o início do ranking, em $2012^{3}$. Já na Anatel, as queixas referentes aos serviços de celular prépago, celular pós-pago e telefonia fixa totalizaram $69,14 \%$ das queixas referentes ao setor de telecomunicações em 2015. Com um crescimento de $65,08 \%$ das queixas desde $2012^{4}$.

\footnotetext{
${ }^{3}$ Disponível em: http://www.procon.sp.gov.br/ reclamacoes.asp. Acesso em: jun. de 2016.

${ }^{4}$ Disponível em: http://www.anatel.gov.br/ consumidor/index.php/reclamacoes-na-anatel2
}

international companies, the distribution of international objects had an increase of $68 \%$. Postal services confirm, this way, the historical predominance of the State in this activity.

\section{Telephony}

Since the privatization of the Brazilian telecommunications system, in 1998, the segment of transport of voice and data has held the best detailed indexes in the communications sector. That does not necessarily mean it has higher quality services or more diversity in supply. Quite the opposite: telecommunications companies, mainly the telephone segment, receive a record number of complaints about their services. In the ranking elaborated by Procon in São Paulo, the biggest market in Brazil, with 80,959 telephone lines ( $26.8 \%$ of the total), the first four positions among companies that receive grounded complaints belong to telecommunication services. Three of them are telephone companies, one is a cable TV company. The prevalence of telecommunications in complaints to the consumer protection system of São Paulo has taken place since the start of the ranking, in $2012^{3}$. Complaints to Anatel, relative to pre-paid mobile telephony, post-paid mobile telephony and landline telephony amounted to $69.14 \%$ of all complaints about telecommunications in 2015. Complaints have been $65.08 \%$ up since $2012^{4}$.

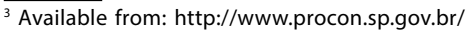
reclamacoes.asp. Cited: Jun.2016.

${ }^{4}$ Available from: http://www.anatel.gov.br/ consumidor/index.php/reclamacoes-na-anatel2
} 
A tabela e o gráfico Evolução dos

Terminais Telefônicos - 2007 a 2015 demonstram relativa estabilidade na evolução das linhas telefônicas fixas em funcionamento no país enquanto as linhas de telefonia celular aumentaram $53,07 \%$. Mesmo observando uma queda de $8,17 \%$ entre a quantidade de linhas telefônicas celulares entre os anos de 2014 e 2015. Em termos de distribuição, a tabela Telefones em Serviço - 2015 aponta a concentração das linhas na região Sudeste $(47,44 \%$ do total) e as regiões Norte e Sudeste com menor concentração, 6,4\% e 8,24\%, respectivamente.

Em termos de competição e pluralidade da oferta, o setor demonstra algumas deficiências. O Relatório Alternativas para a Revisão do Modelo de Prestação de Serviços e Telecomunicações, elaborado conjuntamente pelo Ministério das Comunicações e pela Anatel (2016) demonstra que há variedade de oferta das prestadoras de serviços apenas em grandes cidades. Em 3.699 municípios, $66,51 \%$ do total, há apenas uma prestadora de telefonia fixa, configurando monopólio na prestação do serviço (2016, p. 44).

\section{Banda Larga Fixa}

Conforme mencionado anteriormente, a presença dos serviços de dados nos domicílios brasileiros é bastante precária. Segundo os índices de Acesso à Internet e à Televisão do PNAD - 2014, 46,6\% das pessoas acima de 10 anos não utilizaram Internet no período de referência de três meses ${ }^{5}$. Mesmo considerando o

Acesso em jul. 2016.

${ }^{5}$ Tabela 1.1.4.2 - Distribuição das pessoas de 10 anos ou mais de idade, por Grandes Regiões, segundo a situação do domicílio, o sexo e a utilização da
The table and the graph "Telephone lines in service - 2007 to 2015 " show relative stability of landline telephone lines in service in the country whereas mobile telephone lines recorded an increase of $53.07 \%$, despite the $8.17 \%$ decrease of the number of mobile telephone lines between 2014 to 2015. In terms of distribution, the table "Telephones in Service - 2015" indicates the concentration of lines in the Southeast Region $(47.44 \%$ of the total) and the smaller concentration in the North and Southeast, $6.4 \%$ and $8.24 \%$, respectively.

The sector has some deficiencies regarding competition and the diversity of supply. The report Alternatives for Reviewing the Telecommunications Services Model elaborated in partnership by the Ministry of Communications and Anatel (2016) shows that only in big cities can one find a variety of telephone companies. In 3,699 municipalities, $66.51 \%$ of the total, a single landline telephony company is in the market, which characterizes a monopoly in the services (2016, p 44).

\section{Fixed broadband}

As mentioned before, the presence of data services in Brazilian households is precarious. According to the indexes of Access to the Internet and Television of PNAD - 2014, $46.6 \%$ of the persons aged 10 and over did not use the Internet in the period of reference of three months ${ }^{5}$. Even considering an

Cited: Jul. 2016.

${ }^{5}$ Table 1.1.4.2 - Distribution of persons 10 years of age and over, by Major Region, by urban or rural housing unit, by sex and use of Internet, in the period 
crescimento do acesso em $22,4 \%$, desde 2012, conforme a Tabela 17.5 - Banda Larga Fixa, por Grandes Regiões e Unidades da Federação, os acessos à banda larga fixa refletem a tendência do segmento em concentrar os serviços de acordo com critérios de concentração econômica em detrimento da universalização do acesso. Esta tendência mercantil na exploração dos serviços públicos pode ser mais facilmente observada se compararmos a distribuição dos acessos ao serviço de banda larga fixa à distribuição estimada da população brasileira, em 2015. increase by $22.4 \%$ since 2012 , according to "Table 17.5 - Fixed Broadband, by Major Regions and Federation Units", the access to fixed broadband reflects the trend of this segment to concentration of services according to criteria of economic concentration instead of the universalization of access. This market trend in the exploration of public services can be more easily observed when we compare the distribution of access to fixed broadband services and the estimated distribution of the Brazilian population, in 2015.

\section{Quadro comparativo Distribuição da Banda Larga e da População por Regiões em 2015 Comparative Chart of Broadband and Population Distribution by Major Region in 2015}

\begin{tabular}{|l|c|c|}
\hline & $\begin{array}{c}\text { Distribuição de Acessos à Banda Larga } \\
\text { Fixa'/ Distribution of Access to Fixed } \\
\text { Broadband }\end{array}$ & $\begin{array}{c}\text { Distribuição Estimada da População }{ }^{2} / \\
\text { Estimated Population Distribution }\end{array}$ \\
\hline $\begin{array}{l}\text { Norte/ } \\
\text { North }\end{array}$ & $3,47 \%$ & $8,55 \%$ \\
\hline $\begin{array}{l}\text { Nordeste/ } \\
\text { Northeast }\end{array}$ & $11,76 \%$ & $27,66 \%$ \\
\hline $\begin{array}{l}\text { Sudeste/ } \\
\text { Southeast }\end{array}$ & $59,29 \%$ & $41,94 \%$ \\
\hline $\begin{array}{l}\text { Sul/ } \\
\text { South }\end{array}$ & $17,38 \%$ & $14,3 \%$ \\
\hline $\begin{array}{l}\text { Centro-Oeste/ } \\
\text { Central West }\end{array}$ & $8,11 \%$ & $7,55 \%$ \\
\hline
\end{tabular}

Nota/ Notes

${ }^{1}$ Porcentagem elaborada com base na Tabela 17.5 - Banda Larga Fixa, por Grandes Regiões e Unidades da Federação, 2015./1 Percentage based on Table 17.5 - Fixed broadband access, by Major Regions and Federation Units, 2015.

2 Porcentagem elaborada com base nas Estimativas de População para 1o de julho de 2015. Disponível em: http://www.ibge.gov.br/home/estatistica/populacao/estimativa2015/estimativa_tcu.shtm Acesso em: ago. 2016./ 2 Percentage based on Population Estimates for July 1,2015. Available from: http://www.ibge.gov.br/home/ estatistica/populacao/estimativa2015/estimativa_tcu.shtm Cited:Aug. 2016

Internet, no período de referência dos últimos três meses - 2014. Disponível em http://www.ibge.gov. br/home/estatistica/populacao/acessoainternet2014/ default_xls.shtm acesso em jul. 2016. of reference of the last monthss - 2014. Available from: http://www.ibge.gov.br/home/estatistica/ populacao/acessoainternet2014/default_xls.shtm Cited: Jul. 2016. 
Observa-se, assim, a concentração dos serviços nas regiões Sudeste e Sul, bem como a disparidade entre população e quantidade de acessos à Internet em Banda Larga Fixa.

\section{Comunicação Social - Radiodifusão}

O serviço de Comunicação Social, se apresenta em tabela agrupada separando Televisão por Assinatura que, como dissemos anteriormente, está institucionalmente alocada entre os serviços de telecomunicações, e Radiodifusão, serviços de acesso gratuito.

Em termos de segmento comunicacional, os seviços se dividem em Televisão e Rádio. A recente unificação das tecnologias de acesso à televisão por assinatura, sob a Lei N. 12.485, de 12 de setembro de 2011, que, além de agrupar os serviços de TV a Cabo, por microondas - MMDS e por satétlite DTH, estabeleceu normas de acesso, distribuição da programação, conteúdo brasileiro e de estímulo à produção audiovisual, atualizando a legislação anterior que abarcava apenas o serviço de televisão por cabos. Contraditoriamente, o serviço de televisão por assinatura é, ao mesmo tempo, o mais democrático entre os serviços de comunicações do ponto de vista da regulação da qualidade do serviço voltada à pluralidade e às condições mais equilibradas de oferta dos serviços, e o serviço de TV por assinatura é, também, o mais elitista dos serviços de comunicações. E não se trata apenas do fato de que há que se pagar para se ter acesso ao conteúdo. Embora o número de assinaturas tenha praticamente dobrado na última década, ele decresceu no último ano, chegando, em 2015, a 19 milhões
Here we see the concentration of services in the Southeast and South Regions, and the disparity between the population and the number of accesses to the Internet.

\section{Social Communications - Radio Broadcasting}

The service of Social Communications is presented in a table that separates Subscription Television, which, as mentioned before, institutionally belongs to Telecommunications services, from Radio broadcasting, or free access services.

In terms of communication segments, services are divided into Television and Radio. The recent unification of technologies of access to subscription TV, under Law no. 12,845 of September 12,2011 , which, besides grouping cable TV services, microwave - MMDS and satellite - DTH, established rules for access, distribution of schedules, Brazilian material and content to stimulate audiovisual production, updated the previous regulation that used to encompass only cable TV services. Contradictorily, subscription TV is, at the same time, the most democratic communication service - from the perspective of quality regulation in terms of diversity and balanced conditions of offer, and aimed at the plural nature and more balanced conditions of service supply -, and the most elitist of all communication services. That is not only related to the necessity of paying in order to access the service content. Although the number of subscriptions has almost doubled in the last decade, it decreased last 
de assinantes. A distribuição do serviço no país é bastante precária, tendo uma taxa de densidade de 28,8 por 100 domicílios. Apesar de contar com normatização mais completa que todos os outros serviços do setor, o segmento por assinatura também tem crescido as reclamações sobre os serviços. Nos registros da Anatel, a taxa de queixas da TV por Assinatura subiu $83 \%$ em 2015, em relação ao ano anterior ${ }^{6}$. A maior empresa do segmento também figura como a terceira empresa com maior número de queixas registradas no Procon de São Paulo em 2015.

Avalia-se o segmento de Radiodifusão pela quantidade de outorgas existente nos registros da Anatel, através do sistema SISCOM - Sistema de Informação dos Serviços de Comunicação de Massa. Este sistema separa os serviços - Rádio e Televisão - em segmentos que são divididos por:

a) ocupação do espectro eletromagnético: Rádios em Ondas Médias - OM, Ondas Curtas - OC, Ondas Tropicais - OT, Frequência Modulada-FM e Frequência Modulada Comunitária; e,

b) por função das estações: Geradoras de $\mathrm{TV}$, que podem gerar programação e publicidade, e Retransmissoras de TV, que não podem gerar nenhum conteúdo.

Estes serviços podem, ainda, serem divididos por sua finalidade - educativa/

\footnotetext{
${ }^{6}$ Reclamações registradas na Anatel. Disponível em http://www.anatel.gov.br/consumidor/index.php/ reclamacoes-na-anatel2. Acesso em ago. 2016.

${ }^{7}$ Top 10 empresas com mais reclamações. Disponível em http://www.anatel.gov.br/consumidor/index.php/ reclamacoes-na-anatel2. Acesso em ago. 2016.
}

year, and reached a total 19 million in 2015. The distribution of this service in the country is very irregular, having a rate of density of 28.8 per 100 housing units. In spite of having more complete regulation than the other services of the sector, subscription TV services have recorded increase in complaints. In the Anatel records, the rate of complaints about subscription TV increased $83 \%$ in relation to the previous year ${ }^{6}$. The biggest company of this segment is also the third one in terms of complaints registered in the Procon of São Paulo in 20157.

The segment of broadcasting is evaluated according to the number of grants registered in Anatel records through the SISCOM - System of Information on Mass Communication Services. This system separates Radio and Television services into segments divided by:

a) use of the electromagnetic spectrum: Medium Wave Radios - MW, Short Waves - SW, Tropical Waves - TW, Frequency Modulation - FM and Community Frequency Modulation; and

b) by function: TV Generators which can create a schedule and publicity, and TV Relay transmitters, which cannot generate content.

These services can also be divided by purpose - educational/non-profit or

\footnotetext{
${ }^{6}$ Complaints registered in Anatel. Available from: http://www.anatel.gov.br/consumidor/index.php/ reclamacoes-na-anatel2. Cited: Aug. 2016.

${ }^{7}$ Top 10 companies in complaints. Available from: http://www.anatel.gov.br/consumidor/index.php/ reclamacoes-na-anatel2. Cited: Aug. 2016.
} 
sem fins lucrativos ou comercial - , por sua fase no processo de digitalização - Geradoras de TV Digital - e por sua excepcionalidade: as retransmissoras da Amazônia Legal têm caráter misto e podem gerar programação local.

Parece confuso? Pode piorar um pouco mais. A consistência dos dados é historicamente questionada pelos analistas do setor. Analisando a totalidade dos dados de concessões dos serviços de TV - Retransmissoras, 12.595; GeradorasTV, 541 - e de rádio - FM; 3.222, OM, 1.781; OC, 61; OT, 73 - poderíamos imaginar que trata-se da quantidade de emissoras existentes no país. No entanto, os dados gerados pelo SISCOM consideram a totalidade das outorgas e não a quantidade das emissoras em funcionamento. Quando se acompanha in loco a existência das emissoras, é muito frequente perceber que há uma parcela significativa delas que estão inativas há mais de uma década ou que jamais entraram em atividade. Esta é mais uma decorrência da falta de investimento em políticas de transparência para o setor. É muito difícil precisar, por exemplo, quais são os canais retransmitidos pelas RTVs brasileiras. São muito comuns, também, alterações de quadro societário sem as devidas notificações ao agente regulador. Os índices oficiais de radiodifusão são bastante precários e servem para se ter uma ideia bastante generalista do segmento. $O$ conjunto de dados das concessões não permite, por exemplo, a análise da concentração da oferta dos serviços, que são fortemente condicionados em redes de afiliação altamente horizontalizadas com a produção e a renda concentrados no eixo Rio-São Paulo. commercial -, by status of digitization process - Digital TV Generators - and by their unusual nature: the relay transmitters of the Legal Amazon have a mixed character and generate local programs.

Does it seem confusing? It can get even worse. The consistency of data is historically questioned by sector analysts. By analyzing the total data on grants of television services - Relay Transmitters, 12,595; TV Generators, 541 - and Radio Generators - FM; 3,222, MW, , 1,781; SW, 61; TW, 73 we could imagine that they show the number of stations in the country. However, data generated by the SISCOM takes into consideration the number of grants and not the number of stations in operation. When following in loco the existence of stations, it is often noticed that a significant parcel of them has been inactive for more than a decade or have not even started operating. That results from the lack of investment in transparency policies for the sector. It is difficult to know precisely which channels are transmitted by Brazilian Relay Transmitters. Also very common are changes in the corporate structure without proper report to the regulatory agency. The official indexes of broadcast are very poor and only provide a very general view of this segment. The set of data on grants does not allow, for example, the analysis of the concentration of service supply, which is strongly dependent on highly horizontal affiliation networks with production and revenue concentrated in the RioSão Paulo area. 
Na exploração dos serviços, temos um longo histórico de conivência dos governos aos interesses dos grupos privados, contrários ao avanço de regramentos para o setor voltados para a democratização das comunicações. O Brasil pouco avançou desde a Constituição de 1988, quando os serviços de radiodifusão passaram a ser compreendidos como um sistema de complementaridade entre atores públicos, privados e estatais. Nosso sistema de radiodifusão permanece majoritariamente privado, sustentado pela receita publicitária e de pouca participação comunitária, educativa e popular, cujas outorgas ainda sofrem com distorções das suas finalidades a partir da instrumentalização política e empresarial. As comunicações no Brasil ainda não conseguiram romper as barreiras ideológicas que separam a participação popular dos debates que são decididas as principais políticas da área.

De um ponto de vista mais amplo, o caráter essencial das comunicações na sociedade é ainda subvalorizado, em especial, falta observação sistemática e global sobre o setor, observando as premissas de acesso, representatividade social, características qualitativas dos serviços. No caso da comunicação social, por exemplo, não há indicadores econômicos mais amplos sobre o setor, nem dados sobre os serviços que oferecem. Se no setor agropecuário temos indicadores da produção e do comércio dos principais segmentos, tais como arroz, feijão, milho, soja e trigo, por que não temos indicadores da produção jornalística, infantil, ficcional, musical, religiosa e publicitária no rádio e na televisão brasileira? Quanto há, por exemplo, de programação televisiva de cunho religioso nas grades de
Concerning the exploration of services, we have a long history of governmental connivance with interests of private groups which object to the advance of regulation for the sector towards the democratization of communications. Brazil has advanced little since the 1988 Constitution, when broadcast services started to be seen as a complementary system formed by public, private and state actors. Our broadcast system remains mostly private, being funded by the revenue from publicity, with little participation of the community, or of educational and popular segments. The grants obtained still have their original purpose distorted due to political and business instrumentalization. Communications in Brazil has not yet been able to break the ideological barriers which separate popular participation from the debates which decide upon the main policies for the segment.

From a broader perspective, the essential character of communications in society is still undermined, especially due to the lack of a systematic and global look at the sector, given the premises of access, social representation, and characteristics related to service quality. In the case of social communication, for example, there are no comprehensive economic indicators on the sector, or data about the services provided. If there are output and trade indicators for the main segments in agricultural production, rice, beans, corn, soybeans and wheat, why not having indicators to measure the production of news, TV shows for kids, fiction, music, religion-related shows and publicity in the Brazilian radio and television? For example, what is the amount of 
programação das redes televisivas, abertas e por assinatura, nacionais? Quais grandes redes são retransmitidas pelas concessões por municípios? De toda a programação religiosa disponível na programação das concessionárias, quais são as denominações religiosas representadas? Como tem evoluído o investimento público (incluindo dados municipais, estaduais e federais) no setor? Qual a distribuição de prestadoras dos serviços entre os municípios? Estes indicadores comunicacionais poderiam ser de grande valia numa avaliação das condições de cidadania, mais ainda, quando se leva em conta que a Declaração Universal dos Diretos do Homem, declara, em seu Art. 19, que "todo indivíduo tem direito à liberdade de opinião e expressão; este direito inclui a liberdade de, sem interferência, ter opiniões e de procurar, receber e transmitir inormações e ideias por quaisquer meios, independentemente de fronteiras" (Assembleia Geral das Nações Unidas, 1948, Art. 19). A grande maioria da população brasileira sequer sabe que deveria ter esse direito à comunicação nas mesmas bases estruturantes que são a saúde, a educação, a moradia, dentre outros direitos fundamentais. religious production in the schedules of our broadcast and subscription television channels? What major networks per municipality receive grants to be rebroadcast? From all the religious programs available in TV concessionaries, what segments are represented? How has public investment (including municipal, state and federal data) advanced in the sector? What is the distribution of service providers among municipalities? These communication indicators might be of great help to evaluate citizenship conditions, even because the Universal Declaration of Human Rights states, in Article 19, states that "Everyone has the right to freedom of opinion and expression; this right includes freedom to hold opinions without interference and to seek, receive and impart information and ideas through any media and regardless of frontiers." (General Assembly of the United Nations, 1948, Art 19). Most of the Brazilian population is not even aware they should be granted these rights as they are entitled to health, education, housing, among other fundamental ones.

\section{Referências/References}

ANATEL; Ministério das Comunicações. Alternativas para a revisão do modelo de prestação de serviços e telecomunicações: Relatório final do grupo de trabalho entre o Ministério das Comunicações e a Anatel. Brasília: 2016. Disponível em: http://www.mc.gov.br/publicacoes/doc_download/2797-relatorio-gt-revisao-do-modelo Acesso em 05 ago. 2016. Available from: http://www.mc.gov.br/publicacoes/doc_download/2797-relatorio-gtrevisao-do-modelo Cited: Aug. 05, 2016. 
Fernando Salis

Possui Pós-Doutorado pela Universidad Carlos III de Madrid, Espanha,2009, Doutorado em Comunicação pela Universidade Federal do Rio de Janeiro, Brasil, 2003 (com pesquisa complementar na New York University,

EUA) e Mestrado em Filosofia pela Pontifícia Universidade Católica do Rio de janeiro, Brasil, 1996. Atualmente é Professor Associado e Superintendente de Comunicação do Fórum de Ciência e Cultura da Universidade Federal do Rio de Janeiro, Brasil. Tem experiência nas áreas de Comunicação, Filosofia e Artes, com ênfase em Cinema e Performance, atuando principalmente nos seguintes temas: comunicação audiovisual, performance audiovisual, cinema documentário e educação à distância.
Has a Post-doctoral degree from Universidad

Carlos III of Madrid, Spain, 2009, a PhD in

Communication from the Federal University of Rio de Janeiro, Brazil, 2003 (with a complementary research at the New York University, USA) and a master's degree in Philosophy from the Pontifical

Catholic University of Rio de Janeiro, Brazil, 1996. He is currently an Associate Professor and Communication Superintendent at the Science and Culture Forum of the Federal University of Rio de Janeiro, and has experience in the fields of Communication, Philosophy and Arts, with a focus on Cinema and Performance, having dealt mainly with the following themes: audiovisual communication, audiovisual performance, documentary cinema and distance learning.

\section{Suzy dos Santos}

Professora da Escola e do Programa de PósGraduação de Comunicação da Universidade Federal do Rio de Janeiro. Em 2005, recebeu o Prêmio Intercom (melhor tese de doutorado) da Sociedade Brasileira de Estudos Interdisciplinares da Comunicação e, em 1996, recebeu Menção Honrosa (melhor monografia modalidade Rádio e Televisão) como finalista na categoria Graduação da Sociedade

Brasileira de Estudos Interdisciplinares da Comunicação. Líder do Grupo de Pesquisa Políticas e Economia da Informação e da Comunicação - PEIC, em atividade desde 1995.

Coordenadora da pesquisa Metodologia de análise das políticas de comunicação no Brasil: dinâmicas assimétricas de poder e negociação no contexto pós-ditatorial, com apoio do CNPq e da Fundação Ford.
Professor of the Communication School and of the Graduate Program in Communication of the Federal University of Rio de Janeiro. In 2005, she won the Intercom Award (best PhD dissertation) from the Brazilian Society of Interdisciplinary Studies and, in 1996, an Honorable Mention (best monograph in Radio and Television) as a finalist for the category Undergraduate Studies of the Brazilian Society of Interdisciplinary Studies in Communication. Leader of the Research Group Politics and Information and Communication Economics - PEIC, at work since 1995. Coordinator of the survey Methodology of analysis of communication policies in Brazil: asymmetric dynamics of power and negotiation in the post-dictatorial context, with support from $\mathrm{CNPq}$ and Ford Foundation. 
Tabela 17.1 - Organização dos Correios e Telégrafos - 2011-2015

Table 17.1 - Organization of the Postal and Telegraph

Services - 2011-2015

\begin{tabular}{llllll}
\hline $\begin{array}{c}\text { Sistema postal/ } \\
\text { Postal system }\end{array}$ & 2011 & 2012 & 2013 & 2014 & 2015 \\
\hline Unidades próprias/ & 6379 & 6407 & 6440 & 6480 & 6511
\end{tabular}

State-owned units

Unidades terceirizadas/

$10754 \quad 10611$

9485

7274

7135

Outsourced units

Caixas de coleta/

22190

20399

20196

18424

17865

Mail collection boxes

Unidades de tratamento e distribuição/

1149

1190

1244

1296

1292

Treatment and distribution units

Pessoal/

114976

117204

125420

120461

118220

Personnel

Receita total (1 $000000 \mathrm{R} \$) /$

$14638,12 \quad 16554,56 \quad 16666,11 \quad 17693,08 \quad 18838,73$

Total revenue $(1,000,000 R \$)$

Despesa total (1 $000000 \mathrm{R} \$) /$

$12682,10 \quad 13959,83 \quad 16547,65 \quad 17683,17 \quad 20959,96$

Total expenditure $(1,000,000 R \$)$

Fonte/Source: Empresa Brasileira de Correios e Telégrafos - Correios, Departamento de Planejamento Estratégico. 
Tabela 17.2 - Tráfego postal - 2011-2015

Table 17.2 - Postal traffic - 2011-2015

\begin{tabular}{lrrrrr}
\hline \multicolumn{1}{c}{$\begin{array}{c}\text { Sistema postal/ } \\
\text { Postal system }\end{array}$} & 2011 & 2012 & 2013 & 2014 & 2015 \\
\hline $\begin{array}{l}\text { Serviço postal próprio - (C) e (RM) (1)(2) } \\
\text { State-owned postal service (C) and (RM) (1)(2) } \\
\begin{array}{l}\text { Objetos internacionais distribuidos } \\
\text { International distributed objects }\end{array}\end{array}$ & 8929 & 8919 & 8683 & 8524 & 8227 \\
$\begin{array}{l}\text { Serviço postal concorrente - (C) } \\
\text { Competing postal service (C) }\end{array}$ & 2315 & 2242 & 1703 & 1506 & 1190 \\
$\begin{array}{l}\text { Objetos distribuídos no Brasil/ } \\
\text { Objects distributed in Brazil }\end{array}$ & 8929 & 8919 & 8683 & 8524 & 8227 \\
\hline
\end{tabular}

Fonte/Source: Empresa Brasileira de Correios e Telégrafos - Correios, Departamento de Planejamento Estratégico.

(1) $\mathrm{C}=$ Concorrencial. (2) $\mathrm{RM}=$ Reserva de Mercado./(1) $C=$ Competitive. (2) $R M=$ Reserve Market.

\section{Gráfico 17.1 - Evolução dos terminais telefônicos - 2007-2015}

Graph 17.1 - Telephone lines in service - 2007-2015

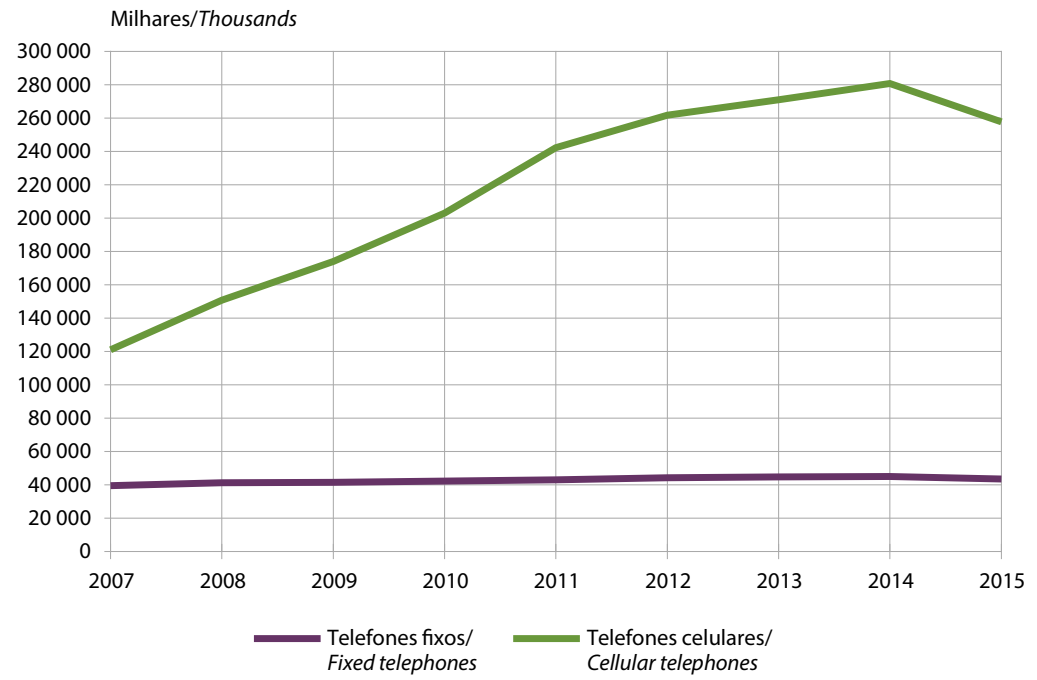

Fonte/Source: Agência Nacional de Telecomunicações - ANATEL.

Nota: Dados de 2015 relativos a dezembro./Note: Data to December 2015. 
Tabela 17.3 - Telefones em serviço - 2015

Table 17.3 - Telephones in service - 2015

\begin{tabular}{|c|c|c|c|}
\hline \multirow{3}{*}{$\begin{array}{l}\text { Unidades da Federação/ } \\
\text { Federation Units }\end{array}$} & \multicolumn{3}{|c|}{ Telefones em serviço/Telephones in service } \\
\hline & $\begin{array}{l}\text { Total/ } \\
\text { Total }\end{array}$ & $\begin{array}{c}\text { Telefones } \\
\text { celulares/ } \\
\text { Cellular } \\
\text { phones }\end{array}$ & $\begin{array}{l}\text { Telefones } \\
\text { fixos/ } \\
\text { Landline } \\
\text { phones }\end{array}$ \\
\hline & \multicolumn{3}{|c|}{ Milhares/Thousands } \\
\hline $\begin{array}{l}\text { Brasil/ } \\
\text { Brazil }\end{array}$ & 301374 & 257795 & 43580 \\
\hline Rondônia & 2.310 & 2133 & 177 \\
\hline Acre & 893 & 805 & 88 \\
\hline Amazonas & 4.054 & 3710 & 344 \\
\hline Roraima & 534 & 480 & 53 \\
\hline Pará & 8.792 & 8291 & 501 \\
\hline Amapá & 852 & 803 & 49 \\
\hline Tocantins & 1.991 & 1841 & 150 \\
\hline Maranhão & 6.408 & 6058 & 350 \\
\hline Piauí & 4.137 & 3924 & 212 \\
\hline Ceará & 11.613 & 10819 & 794 \\
\hline Rio Grande do Norte & 4.590 & 4281 & 309 \\
\hline Paraíba & 5.050 & 4712 & 339 \\
\hline Pernambuco & 12.982 & 11859 & 1123 \\
\hline Alagoas & 3.995 & 3739 & 256 \\
\hline Sergipe & 2.487 & 2270 & 217 \\
\hline Bahia & 18.253 & 16637 & 1616 \\
\hline Minas Gerais & 28.783 & 24710 & 4073 \\
\hline Espírito Santo & 4.703 & 3912 & 791 \\
\hline Rio de Janeiro & 28.531 & 23068 & 5463 \\
\hline São Paulo & 80.959 & 64541 & 16418 \\
\hline Paraná & 17.146 & 14253 & 2893 \\
\hline Santa Catarina & 10.189 & 8506 & 1684 \\
\hline Rio Grande do Sul & 17.261 & 14775 & 2487 \\
\hline Mato Grosso do Sul & 3.845 & 3360 & 485 \\
\hline Mato Grosso & 4.659 & 4161 & 498 \\
\hline Goiás & 9.769 & 8533 & 1236 \\
\hline $\begin{array}{l}\text { Distrito Federal/ } \\
\text { Federal District }\end{array}$ & 6.590 & 5615 & 975 \\
\hline
\end{tabular}

Fonte/Source: Agência Nacional de Telecomunicações - Anatel.

Nota: Dados até dezembro./Note: Data up to december. 
Tabela 17.4 - Televisão e radiodifusão - 2011-2015

Table 17.4 - Television and broadcasting - 2011-2015

\begin{tabular}{llllll}
\hline $\begin{array}{c}\text { Indicadores/ } \\
\text { Indicators }\end{array}$ & 2011 & 2012 & 2013 & 2014 & 2015 \\
\hline
\end{tabular}

Televisão por assinatura/Pay TV

Serviço de televisão por assinatura

12744

16189

18020

19574

19050

(1 000 assinaturas)/

Pay TV services (Thousands

subscriptios)

Densidade serviço TV por assinatura

21,2

27,2

28,9

29,8

28,8

(assinaturas/100 domicílios)/

Pay TV services density

(Subscriptions / hundreds housing)

Radiodifusão/Broadcasting

Geradores de TV (estação)/

514

519

541

542

541

TV Generatiors (stations)

Retransmissora de TV (estação)/

10506

10471

10513

11308

12595

$T V$ re-transmissing (stations)

Rádios FM (estação)/

3125

3162

3180

3208

3222

FM radios (stations)

Rádios OM (estação)/

1785

1783

1.781

1.781

1.781

MW radios (stations)

Rádios OC (estação)/

66

66

66

62

61

SW radios (stations)

Rádios OT (estação)/

74

74

73

73

TW radios (stations)

Rádios comunitárias (estação)/

4409

4514

4613

4650

4727

Community radios (stations)

Fonte/Source: Agência Nacional de Telecomunicações - AnAtel. 
Tabela 17.5 - Banda larga fixa, por Grandes Regiões e Unidades da Federação - 2012-2015

Table 17.5 - Fixed broadband access, by Major Regions and Federation Units 2012-2015

\begin{tabular}{|c|c|c|c|c|}
\hline \multirow{2}{*}{$\begin{array}{c}\text { Grandes Regiões e } \\
\text { Unidades da Federação/ } \\
\text { Major Regions and Federation Units }\end{array}$} & \multicolumn{4}{|c|}{$\begin{array}{l}\text { Acessos a banda larga fixa (1 } 000 \text { acessos)/ } \\
\text { Fixed broadband access (1 } 000 \text { access) }\end{array}$} \\
\hline & 2012 & 2013 & 2014 & 2015 \\
\hline Brasil/Brazil & 19828,7 & 22185,7 & 23968,4 & 25574,1 \\
\hline Norte/North & 541,1 & 622,9 & 739,4 & 887,2 \\
\hline Rondônia & 78,7 & 87,8 & 104,5 & 131,2 \\
\hline Acre & 31,1 & 36,6 & 46,6 & 51,1 \\
\hline Amazonas & 136,3 & 157,0 & 192,6 & 243,6 \\
\hline Roraima & 20,6 & 27,9 & 32,4 & 35,1 \\
\hline Pará & 194,6 & 224,3 & 255,9 & 279,8 \\
\hline Amapá & 11,7 & 14,9 & 20,6 & 48,7 \\
\hline Tocantins & 68,1 & 74,4 & 86,8 & 97,7 \\
\hline Nordeste/Northeast & 2204,4 & 2530,8 & 2864,6 & 3006,9 \\
\hline Maranhão & 130,1 & 155,0 & 178,6 & 194,7 \\
\hline Piauí & 94,1 & 109,5 & 128,8 & 143,1 \\
\hline Ceará & 404,3 & 461,4 & 557,3 & 582,2 \\
\hline Rio Grande do Norte & 172,7 & 205,3 & 232,0 & 253,4 \\
\hline Paraíba & 168,5 & 193,1 & 224,5 & 246,3 \\
\hline Pernambuco & 398,4 & 452,3 & 498,1 & 504,9 \\
\hline Alagoas & 107,9 & 134,0 & 146,3 & 147,8 \\
\hline Sergipe & 93,5 & 110,9 & 128,8 & 145,4 \\
\hline Bahia & 634,8 & 709,3 & 770,1 & 789,1 \\
\hline Sudeste/Southeast & 12130,3 & 13503,9 & 14281,2 & 15162,9 \\
\hline Minas Gerais & 1871,9 & 2063,8 & 2258,1 & 2408,5 \\
\hline Espírito Santo & 346,9 & 394,3 & 441,3 & 470,2 \\
\hline Rio de Janeiro & 2241,9 & 2518,1 & 2687,8 & 2888,8 \\
\hline São Paulo & 7669,7 & 8527,6 & 8894,1 & 9395,5 \\
\hline Sul/South & 3437,9 & 3835,8 & 4173,2 & 4443,9 \\
\hline Paraná & 1415,8 & 1559,8 & 1701,7 & 1770,6 \\
\hline Santa Catarina & 794,2 & 896,2 & 987,6 & 1098,5 \\
\hline Rio Grande do Sul & 1227,8 & 1379,9 & 1484,0 & 1574,9 \\
\hline Centro-Oeste/Central West & 1515,1 & 1692,3 & 1909,8 & 2073,1 \\
\hline Mato Grosso do Sul & 231,5 & 259,1 & 299,6 & 328,8 \\
\hline Mato Grosso & 237,6 & 261,0 & 296,5 & 327,1 \\
\hline Goiás & 560,1 & 630,7 & 715,6 & 785,1 \\
\hline Distrito Federal/Federal District & 485,9 & 541,5 & 598,1 & 632,2 \\
\hline
\end{tabular}

Fonte/Source: Agência Nacional de Telecomunicações - AnATEL. 
Tabela 17.6 - Banda larga móvel, por Grandes Regiões e Unidades da Federação - 2012-2015

Table 17.6 - Mobile broadband access, by Major Regions and Federation Units 2012-2015

\begin{tabular}{|c|c|c|c|c|}
\hline \multirow{2}{*}{$\begin{array}{c}\text { Grandes Regiões e } \\
\text { Unidades da Federação/ } \\
\text { Major Regions and Federation Units }\end{array}$} & \multicolumn{4}{|c|}{$\begin{array}{l}\text { Acessos a banda larga móvel (1 } 000 \text { acessos)/ } \\
\text { Mobile broadband access (1 } 000 \text { access) }\end{array}$} \\
\hline & 2012 & 2013 & 2014 & 2015 \\
\hline Brasil/Brazil & 59185,1 & 103107,6 & 157896,7 & 180481,8 \\
\hline Norte/North & 3797,5 & 7027,1 & 11172,6 & 12698,4 \\
\hline Rondônia & 531,3 & 932,9 & 1415,6 & 1579,3 \\
\hline Acre & 207,4 & 355,8 & 567,9 & 597,6 \\
\hline Amazonas & 929,4 & 1728,1 & 2608,1 & 2715,7 \\
\hline Roraima & 108,9 & 208,8 & 326,1 & 366,8 \\
\hline Pará & 1460,7 & 2814,4 & 4720,4 & 5624,5 \\
\hline Amapá & 194,3 & 338,4 & 541,0 & 580,6 \\
\hline Tocantins & 365,6 & 648,7 & 993,7 & 1233,9 \\
\hline Nordeste/Northeast & 12206,7 & 22837,5 & 35916,5 & 42893,8 \\
\hline Maranhão & 922,1 & 1863,5 & 3191,2 & 3974,1 \\
\hline Piauí & 728,1 & 1307,2 & 2130,9 & 2538,1 \\
\hline Ceará & 1937,8 & 3710,1 & 5896,0 & 7097,3 \\
\hline Rio Grande do Norte & 868,1 & 1599,5 & 2330,7 & 2877,7 \\
\hline Paraíba & 825,2 & 1649,7 & 2539,3 & 3047,3 \\
\hline Pernambuco & 2338,8 & 4338,8 & 6402,4 & 7975,3 \\
\hline Alagoas & 722,0 & 1372,5 & 2061,1 & 2397,9 \\
\hline Sergipe & 536,4 & 1083,8 & 1622,8 & 1707,5 \\
\hline Bahia & 3328,3 & 5912,5 & 9742,2 & 11278,5 \\
\hline Sudeste/Southeast & 28745,0 & 48890,7 & 74263,5 & 84006,3 \\
\hline Minas Gerais & 5072,1 & 9015,5 & 14000,5 & 16727,2 \\
\hline Espírito Santo & 1054,9 & 1685,9 & 2490,2 & 2785,1 \\
\hline Rio de Janeiro & 6246,1 & 10942,7 & 16648,3 & 18490,0 \\
\hline São Paulo & 16371,9 & 27246,6 & 41124,4 & 46004,0 \\
\hline Sul/South & 8640,9 & 14636,7 & 21981,9 & 25070,7 \\
\hline Paraná & 2936,3 & 5171,2 & 7977,3 & 9235,5 \\
\hline Santa Catarina & 1856,9 & 3283,1 & 5064,0 & 6012,9 \\
\hline Rio Grande do Sul & 3847,8 & 6182,4 & 8940,7 & 9822,4 \\
\hline Centro-Oeste/Central-West & 5794,8 & 9715,6 & 14562,1 & 15812,7 \\
\hline Mato Grosso do Sul & 905,8 & 1499,8 & 2215,2 & 2351,6 \\
\hline Mato Grosso & 981,6 & 1721,9 & 2684,0 & 2986,1 \\
\hline Goiás & 2266,1 & 3839,6 & 5775,7 & 6076,0 \\
\hline Distrito Federal/Federal District & 1641,3 & 2654,3 & 3887,2 & 4399,0 \\
\hline
\end{tabular}

Fonte/Source: Agência Nacional de Telecomunicações - AnATEL. 


\section{Finanças Públicas \\ Public Finance}

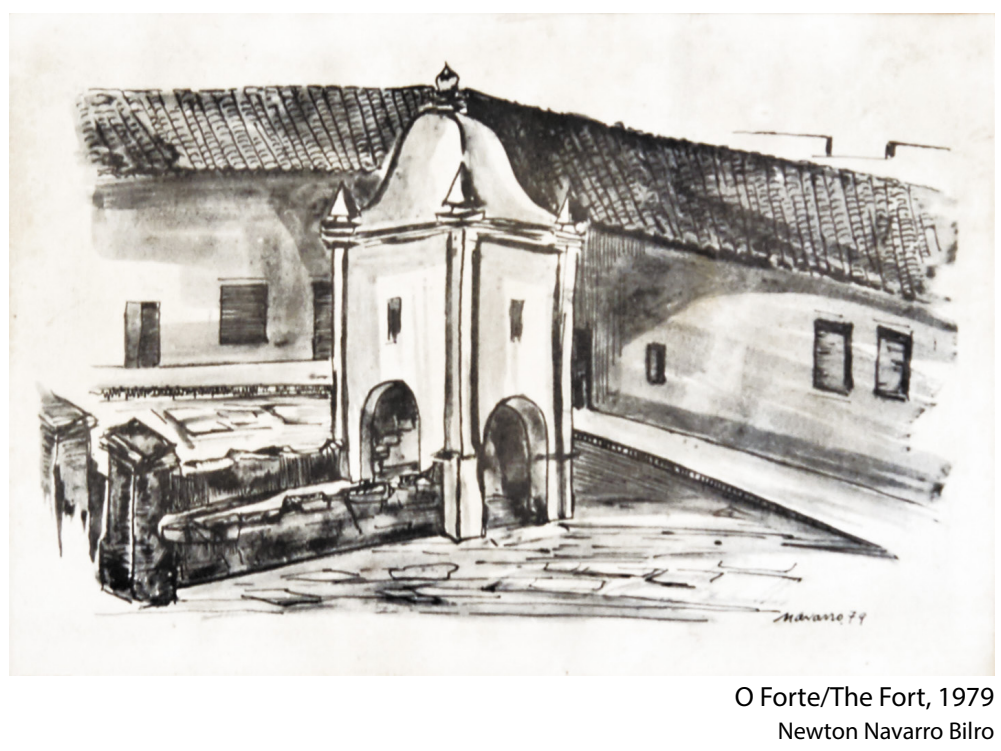





\section{Finanças Públicas}

\section{Public Finance}

\section{Políticas Tributária e Fiscal no Brasil}

As receitas de tributos e demais entradas de recursos nos cofres públicos dependem em grande extensão do comportamento dos fluxos dos fatores (faturamento, circulação de mercadorias, rendas derivadas do capital e do trabalho, por exemplo), os quais tendem a apresentar maior variabilidade do que a arrecadação decorrente da tributação sobre patrimônio, caracterizada como estoque. A manutenção de níveis de receita compatíveis com o gasto exige, portanto, que os governos se dediquem de forma concentrada nas atividades de planejamento, acompanhamento e gestão das contas públicas, especialmente quanto às tarefas de lançamento de tributos, cobrança de débitos, redução da sonegação, dentre outros itens, como forma de garantir recursos em volume capazes de reduzir as carências no atendimento das demandas sociais de toda ordem.

A estrita observância dos limites consignados no orçamento anual, ainda que este seja apenas indicativo - não impositivo - dos gastos, é insuficiente para que se atinja o equilíbrio financeiro das contas públicas. Em outras palavras,

\section{Tax and Fiscal Policies in Brazil}

Tax revenues and other resource entries in the government coffers rely heavily on the behavior of the flows of factors (income, movement of goods, revenue derived from capital and labor, for example), which tend to create greater variability than the collection resulting from tax on capital stock. Maintaining revenue levels compatible with spending demands Governments to firmly engage in planning, monitoring and managing public accounts; especially in terms of tax entries, debt collection and reduction of tax evasion, among other items. This could ensure an adequate volume of resources to reduce the shortcomings in meeting social demands of all kinds.

The strict observance of the limits laid down in the annual budget, given its indicative - not prescriptive - character, is by itself insufficient to achieve financial equilibrium in public accounts. 
as disponibilidades de caixa é que verdadeiramente condicionam a execução do gasto, não obstante o fato do orçamento constituir-se em lei aprovada pelo Poder Legislativo.

Por esse motivo, a concentração da atenção sobre o gasto público, a variável que permite maior controle, constitui a ênfase das autoridades do governo central responsáveis sobre as finanças públicas em nível nacional. Ou seja, já que o que é relevante é o equilíbrio financeiro, "não se gasta mais do que se arrecada".

Ocorre que a delimitação do gasto fixada em lei obedece a um processo longo, de natureza não apenas econômicofinanceira, mas também política, social e institucional, e envolve considerações de alocação e (re)distribuição de recursos,com objetivos de redução das disparidades pessoais, setoriais e regionais de renda e de oportunidades.

No caso brasileiro, o instrumental desenvolvido para o acompanhamento e controle da execução orçamentária dos órgãos federais - da administração direta e indireta, incluindo as empresas estatais - atinge níveis de alta complexidade e sofisticação. $O$ conjunto de atos normativos e de programas e metas de redução do tamanho da máquina pública federal, introduzidos durante a década de 1990, se traduziu nas políticas de desestatização e concessão ou, mesmo, na simples supressão de determinados serviços, tendo sido transferida quase que totalmente às administrações estaduais e municipais a responsabilidade pelo atendimento dessas demandas. Em muitas situações, o processo de absorção das responsabilidades ainda não se consolidou - em várias administrações locais ele
In other words, it is the availability of cash that truly influences spending, notwithstanding the fact that the budget is actually a law passed by the Legislative Power.

For this reason, public spending, which is a variable that allows greater control, has become the focal point of the central government authorities responsible for public finances at national level. That is to say: since financial balance is what matters, "one must not spend more than one earns".

However, ceiling spending set by law undergoes a long financial, economic, social, institutional and political process, and involves allocation considerations and (re) distribution of resources, with goals aiming at the reduction of sectorial, regional and personal income disparities and opportunities.

In the Brazilian case, the set of instruments developed for monitoring and controlling the budgetary execution of federal agencies - direct and indirect administration, including State-owned enterprises - reaches high levels of complexity and sophistication. A set of normative acts and programs for the reduction in the size of the public sector were introduced during the 1990s in the form of privatization and concession policies, or by the discontinuation of certain social services, whose demands were transferred almost entirely to State and local governments. In many situations, such transference has not yet been consolidated - in several local administrations it has not even started 
nem mesmo se iniciou - o que provoca verdadeiros vazios institucionais, gerando consequências negativas graves para a população.

Esse quadro espelha o conflito existente em países com estrutura federativa. Tal conflito assume várias dimensões, todas elas associadas à autonomia dos níveis de governo em relação aos tributos e ao gasto público.

\section{O federalismo e as estruturas tributária e fiscal brasileiras}

No caso brasileiro, é comum dizer-se que as modificações introduzidas na Constituição de 1988 impuseram aos estados e municípios, principalmente a estes últimos, uma carga de responsabilidades não condizente com sua capacidade gerencial, muito embora o volume de recursos disponíveis tenha aumentado após a promulgação da nova Constituição. Na prática, entretanto, a divisão das competências não se traduz automaticamente em uma divisão dos tributos em montante equivalente. Pela ótica constitucional, observa-se que a supremacia do poder federal, no caso brasileiro, é estabelecida de forma indireta, pois na Constituição, embora se mantenha a equivalência formal entre União e estados membros, a distribuição de competências espelha o maior poder da esfera federal, decorrente de duas causas. A primeira é que a enumeração de competências federais é ampla, abrangendo quase tudo o que é essencial em termos de direitos e deveres fundamentais, economia e finanças, bem como a organização e ação política. A segunda causa é o fato de a União ter competência para fixar regras gerais em matérias caracteristicamente de foro subnacional.
- which causes huge institutional gaps, leading to serious adverse consequences for the population.

This situation mirrors the conflict in countries with a federal structure. Such conflict comes up in many dimensions, all of them associated with the autonomy of the levels of Government in relation to taxes and public spending.

\section{Federalism and tax and fiscal structures in Brazil}

In the case of Brazil, it is common to say that the changes in the Constitution of 1988 imposed on States and municipalities, mainly on the latter, a set of responsibilities which mismatch their managerial capacity, although the volume of resources available has increased after the promulgation of the new Constitution. In practice, however, the division of powers does not necessarily imply a division of taxes of an equivalent amount. Under a constitutional approach, it appears that in Brazil the supremacy of the Federal Government is established indirectly, given that in the Constitution - although the formal equivalence between Union and Member States is maintained - the division of competences reflects the greater power of the federal sphere. This is due to two factors: firstly, the federal jurisdiction is broad, covering almost everything that is essential in terms of fundamental rights and obligations, economy and finance, as well as the organization and political action; secondly, the Federal Government has jurisdiction to lay down general rules in matters of subnational domain. 
A Tabela 18.3. Necessidades de financiamento do setor público, entre 2012 e 2015, por exemplo, evidencia dois aspectos inerentes a este conflito: o excesso de gastos em relação às receitas e a manutenção de níveis reduzidos de pagamento de juros nominais pelos municípios, quando comparados ao observado pelo Governo Central.

Com efeito, no período apresentado o resultado primário do Governo Central variou entre -1,79 e 1,97, enquanto que o mesmo indicador, no agregado, se manteve negativo com relação aos governos municipais.

Do ponto de vista das relações entre os governos, o tipo de federalismo vigente no Brasil pode ser caracterizado como do tipo centrípeto, em que há uma predominância de poder por parte da União, oposto ao federalismo centrífugo, aquele que procura se afastar do centro, permitindo que os entes subnacionais (estados e municípios) tenham maior autonomia financeira, administrativa, política e jurídica.

O que se busca como forma ideal é o denominado federalismo de equilíbrio, que deve assumir uma forma cooperativa, buscando priorizar a conciliação entre integração e autonomia, entre a unidade e a diversidade, como uma resposta às aspirações de independência e solidariedade dos cidadãos.

\section{Países em desenvolvimento e Federalismo econômico}

Países em desenvolvimento enfrentam em maior ou menor grau a difícil tarefa de estruturar e implementar sistemas
Table 18.3. Public sector net borrowing, between 2012 and 2015, for example, shows two inherent aspects of this conflict: the excess of expenditures in relation to revenue and the maintenance of reduced levels of nominal interest payment by the municipalities when compared to the Central Government.

Indeed, in the period presented, the primary result of the Central Government ranged from minus 1.79 to plus 1.97 , while the same indicator, in the aggregate analysis, remained negative with respect to municipal governments.

From the point of view of relations among governments, the kind of federalism in effect in Brazil can be characterized as the centripetal type, in which there is a predominance of power on the part of the Union, opposed to centrifugal federalism, which seeks to move away from the center, allowing sub-national entities (States and municipalities) to have greater financial, administrative, legal and political autonomy.

What is sought as an ideal way is the so-called balanced federalism, which must take a cooperative shape, seeking balance between integration and autonomy, between unity and diversity, as a response to the aspirations of independence and solidarity of citizens.

\section{Developing countries and economic Federalism}

Developing countries face, to a greater or lesser degree, the difficult task of structuring and implementing tax 
de tributação que tenham como base a renda pessoal e corporativa, o valor agregado e a propriedade. Muitos países ainda apresentam economias apoiadas no setor agrícola, com cultura marcadamente tradicional, e considerável contingente da população no setor informal. Grande parcela da carga tributária é representada pela arrecadação de tributos que incidem sobre a exportação de produtos primários. Sua maior participação no fluxo do comércio internacional, entretanto, está condicionada à aceitação de maior liberalização comercial, o que provoca consequências negativas do ponto de vista da arrecadação de tributos.

Outro conjunto de países ainda depende da arrecadação de tributos que incidem sobre o faturamento das empresas, muitos dos quais com características de bitributação e de tributação em cascata. Países como o Brasil ainda apresentam considerável contingente da população urbana na informalidade, com reflexos negativos tanto na arrecadação tributária, quanto na gestão do sistema de previdência oficial. No caso brasileiro, com exceção dos impostos sobre a propriedade (que no nosso país representam menos de $1 \%$ do PIB), todos os demais que incidem sobre a renda, sobre o faturamento, sobre a exportação, além da contribuição previdenciária, são impostos federais.

Desses tributos, apenas os que incidem de forma progressiva sobre a renda possuem atributos de equidade, enquanto os demais tendem a promover maior desigualdade, devido às suas características de regressividade.

O estágio atual do federalismo nos países em desenvolvimento requer, portanto, uma definição clara do modelo de systems which are based on personal and corporate incomes, value added and property. Many countries still have economies based on the agricultural sector, with typically traditional farming, and a considerable share of persons in the informal sector. A large amount of the tax burden is represented by the collection of taxes levied on the export of primary products. Their greater participation in the international trade flow, however, is subject to the acceptance of greater trade liberalization, which causes negative consequences from the point of view of tax collection.

Another set of countries still depends on the collection of taxes on company revenues. Many of these taxes are doubled and with a cascading effect. Countries like Brazil have a considerable contingent of the urban population in the informal market, with negative consequences on both tax collection and management of the official social security system. In the case of Brazil, all taxes levied on income, on gross sales and on exports, in addition to the social security contribution, are federal ones. The exception is property taxes (which represent less than $1 \%$ of the GDP).

Of these, only taxes that focus progressively on income have attributes of equity, while the others tend to promote greater inequality, due to their regressive effect.

The current stage of federalism in developing countries requires, therefore, a clear definition of the 
descentralização que se deseja no campo das finanças públicas, pois alterações no grau de descentralização da estrutura de competências têm o poder de modificar substancialmente a estrutura de poder, através do redimensionamento do quadro de vantagens e desvantagens experimentadas pelos representantes políticos, pela burocracia e por grupos de interesse localizados em cada um dos níveis de governo.

\section{Descentralização e Estabilização}

As estruturas tributária e fiscal no sistema federalista são importantes elementos formais que espelham a real dimensão da descentralização política das relações intergovernamentais. Enquanto as políticas monetária e cambial são implementadas pelo Executivo federal de forma discricionária, a formulação e a execução das políticas tributária e fiscal obedecem a um processo democrático de negociação entre o Executivo e o Legislativo. No ambiente federalista, essa negociação vai mais além, o que permite tratar de maneira eficaz as diferenças existentes entre regiões, estados e municípios, buscando atender com maior aderência aos preceitos da democracia definidos em bases territoriais.

O ambiente da estabilização, entretanto, limita as chances de negociação em termos de recursos financeiros disponíveis, pois apenas uma parcela do orçamento não está contingenciada, ao mesmo tempo em que coíbe a perspectiva de aumento da arrecadação de tributos nas esferas locais. model of decentralization of public finances, because changes in the degree of decentralization in competency models can substantially modify the power structure, through the resizing of the advantages and disadvantages experienced by political representatives, by bureaucracy and by interest groups in each level of Government.

\section{Decentralization and stabilization}

Fiscal and tax structures in the federal system are important formal elements that mirror the real dimension of the political decentralization of intergovernmental relations. Whereas monetary and exchange rate policies are implemented in a discretionary way by the central government, the formulation and implementation of tax and fiscal policies follow a democratic model of negotiation between the Executive and the Legislative branches. In a federalist environment, this negotiation goes further, making it possible to adequately deal with the differences between Regions, States and Municipalities, seeking to meet as much as possible the precepts of democracy defined in territorial bases.

The environment of stabilization, however, limits the chances of negotiation as to the availability of financial resources, because only a portion of the budget is not yet committed. In the meantime, it makes it diminish the prospects of increased tax collection at the local level. 
Estabilização, um conceito macroeconômico geral, se apóia em teorias de cunho geral e em indicadores igualmente gerais. Admitindo-se um país com estrutura unitária de Estado, no qual a descentralização se dá em função das vantagens decorrentes de princípios de Administração Pública e de Gestão do Território, a formulação e a implementação de políticas tributárias e fiscais seguem um modelo de comando centralizado, a exemplo do gerenciamento de uma organização empresarial complexa, com mercado de abrangência regional, nacional ou transnacional.

Não se pode, por conseguinte, permanecer agnóstico em relação a questões que partem de uma perspectiva eminentemente macroeconômica, mas que envolvem aspectos de natureza política e ideológica, e que se tornam explícitas ao se tomar os entes federados de maneira desagregada. Nesse sentido, falar-se em adequação dos conceitos e critérios do federalismo aos países em desenvolvimento significa buscar-se o fortalecimento das competências de tributar e de alocar recursos por parte dos entes subnacionais. Tal fortalecimento deve ser visto como decisivo para atender aos requisitos democráticos na formulação, acompanhamento da execução e avaliação das políticas públicas de forma participativa.

A descentralização tributária e fiscal pode se dar em maior ou menor intensidade quanto à delegação de competências do gasto e da receita, e qualquer uma das combinações possíveis entre essas duas competências (ou responsabilidades), variando desde a delegação de natureza administrativa ou gerencial, que ocorre no estágio da simples implementação
Stabilization, a general macroeconomic concept, relies on theories of general nature and also on general indicators. Admitting a unitary State, in which decentralization takes place on the basis of the advantages arising from the principles of Public Administration and Territorial Management, the formulation and implementation of fiscal and tax policies follow a model of centralized control, on the grounds of managing a complex business organization, covering regional, national or transnational markets.

One cannot, therefore, remain agnostic about issues that rely on a predominantly macroeconomic perspective, while involving political and ideological aspects, which become explicit when taking federated entities in a disaggregated way. In this sense, to speak of adequacy of federalism concepts and criteria means that developing countries must seek to strengthen tax collection and resource allocation at the sub-national level. Such strengthening should be seen as crucial to meet the democratic requirements when formulating, monitoring, implementing and evaluating public policies in a participatory way.

Tax and fiscal decentralization can take place at various degrees in terms of power delegation, as to expenditure and revenue responsibilities, and of any one of the possible combinations of these two, ranging from administrative or managerial delegation (which occur at the stage of simple implementation of national 
de programas nacionais, até a descentralização de natureza política, na qual se torna evidente a delegação de autoridade, expressa na Constituição.

Por esse aspecto, o princípio da uniformidade em que se baseiam a formulação e a execução de políticas públicas se revela contrário aos interesses voltados para a redução das desigualdades, uma especial característica dos países em desenvolvimento, devendo a busca da equidade estar baseada no reconhecimento das diferenças entre indivíduos, localidades e regiões.

Tomem-se, por exemplo, a forma de intervenção e os resultados da alocação das políticas de juros e de câmbio, em relação ao conjunto de instrumentos de políticas tributária e fiscal. Os primeiros são, em essência, de aplicação uniforme, com resultados bastante diferenciados nas respostas dos vários setores econômicos. Já a aplicação e os resultados decorrentes das políticas tributárias e fiscais apresentam, em sua essência, diversidade entre indivíduos, produtos, setores, estados e municípios, e buscam, contrariamente aos primeiros (juro e câmbio), a convergência entre os indicadores de renda, emprego, consumo, etc.

No limite máximo da autonomia dos governos locais, passam a surgir custos de toda a natureza (políticos, administrativos, etc.) decorrentes da fricção entre estes e entre os demais níveis de governo. Dadas as ligações existentes entre a busca pela estabilização e a execução das políticas tributária e fiscal, torna-se difícil esperar que o processo de descentralização da formulação e implementação das programs) to the decentralization of political nature - in which case the delegation of authority expressed in the Constitution becomes clear.

Under this circumstance, the principle of uniformity, on which are based the formulation and execution of public policies, has proved contrary to the interests focused on the reduction of inequality, a special characteristic of the developing countries; hence, the pursuit of equity should be based on the recognition of the differences between individuals, localities and regions.

Take, for example, the form of intervention and the results of the allocation of interest and exchange rate policies in relation to the set of tax and fiscal policy instruments. The interventions are, in essence, of uniform application, with very different results in the responses of the various economic sectors. On the other hand, the implementation and the results of tax and fiscal policies present, in their essence, the diversity among individuals, products, sectors, States and Municipalities, and, unlike the first ones (interest and exchange rate), aim at the convergence between the indicators of income, employment, consumption, etc.

As we reach the ceiling of the autonomy of local governments, a variety of costs (political, administrative, etc.) begin to emerge, resulting from the friction between them and between the other levels of Government. Given the links between the quest for stabilization and enforcement of tax and fiscal policies, it is difficult to expect that the decentralization process in the formulation and implementation of fiscal 
políticas tributária e fiscal ganhe força a partir de iniciativas do governo federal.

Não se deve descartar, entretanto, o papel desempenhado pelo governo central enquanto coordenador das políticas tributária e fiscal praticadas pelas unidades federadas. Ao tratarmos da descentralização das políticas tributária e fiscal, os governos estaduais e municipais devem ser vistos como agentes políticos do processo de desenvolvimento nacional, além de constituírem unidades de produção com atributos de natureza administrativa, econômica e institucional, com aspectos positivos e negativos peculiares, em função do contexto histórico, cultural, econômico, etc. relativas às regiões em que se inserem eà rede de cidades de que participam.

As especificidades das necessidades próprias a cada região podem ser exemplificadas pelos dados constantes da Tabela 18.5 - Dívida líquida e superávit primário por região - 2012-2015, onde ficam evidentes as diferenças em relação às Regiões Norte e Nordeste se comparadas às Regiões CentroOeste, Sudeste e Sul. As duas primeiras apresentaram variação média no percentual saldo da Dívida Líquida em relação à Receita Corrente Líquida de, respectivamente, 0,37 e 0,12, enquanto que as três últimas apresentaram variação média de -0,01; 0,06 e 0,00, respectivamente, no período considerado.

\section{Federalismo, descentralização e desenvolvimento - os próximos passos}

Contrariamente ao que argumentam os defensores da centralização da arrecadação e do gasto, a imposição de and tax policies might gain strength from federal government initiatives.

One should not rule out, however, the role played by the central Government as coordinator of tax and fiscal policies in effect in he Federation Units. Concerning tax policy and fiscal decentralization, State and Municipal governments must be seen as political agents of the national development process, besides being production units with of administrative, economic and institutional attributes, with positive and negative aspects, according to the peculiar historical, cultural and economic characteristics associated to their regional aspects and city related networks.

Specific requirements associated to each region can be exemplified by data presented in Table 18.5 - Net debt and primary surplus, by Major Region - 2012-2015, showing clear contrasts of North and Northeast Regions as compared to Central-West, Southeast and South Regions. The first two presented an average change in the percentage balance of Net Debt in relation to Net Current Revenue of 0.37 and 0.12 , respectively; while the last three Regions showed an average change of $-0.01,0.00$ and 0.06 , respectively, during the period surveyed.

\section{Federalism, decentralization and development - next steps}

In opposition to what revenue and expenditure centralization advocates may argue, the imposition of limits on the indebtedness of federated 
limites ao endividamento dos entes federados com vistas à obtenção de resultados positivos no balanço agregado das contas nacionais, a redução dos valores referentes aos Fundos de Participação de Estados e Municípios e a compensação desequilibrada da isenção dos créditos de ICMS nas operações de exportação (Lei Kandir), conjugadas ao aumento constante da necessidade de recursos destinados ao financiamento das políticas públicas em nível local, fomentam e acirram a guerra tributária e provocam a disputa fiscal.

Os indicadores de Dívida Líquida Total para o período 2012-2015, constantes da Tabela 18.4 - Dívida líquida do setor público, expressam a dominância do endividamento do Governo Federal em relação aos governos municipais. Enquanto a relação Dívida/PIB do Governo Federal se situa na casa dos $25 \%$, o indicador para o conjunto dos cerca de 5.500 governos municipais é inferior a $2 \%$.

Como os governos subnacionais apresentam reações diferenciadas em função de suas capacidades, suas ações individuais não podem ser agregadas como forma de explicar "uma estabilização macroeconômica", mas "várias desestabilizações submacroeconômicas". Tal falha se traduz na existência de um federalismo coercitivo, míope em relação, por exemplo, aos custos crescentes de congestionamento e deseconomias de aglomeração, de carência da infraestrutura física e social, aos problemas relativos ao equilíbrio das contas públicas, de pobreza e violência, visivelmente concentrados nas grandes metrópoles. entities aiming at achieving positive results in aggregated national accounts, the reduction in the participation of States and Municipal governments in Revenue Sharing Programs and the unbalanced compensation of exemption from ICMS credits in export operations (Kandir law), combined with the constant increase of the need for resources intended for the financing of public policies at the local level, encourage and exacerbate the war tax and cause the dispute for additional budget allocation.

Total Net Debt indicators for the period 2012-2015, listed in Table 18.4Public sector net debt, express the prevalence of the Federal Government's debt in relation to municipal governments. While the Debt to GDP ratio of the Federal Government stands around $25 \%$, the indicator for the set of approximately 5,500 municipal governments is less than $2 \%$.

As subnational governments present differentiated reactions according to their resource capabilities, their individual actions cannot be aggregated as a way to explain "a macroeconomic stabilization", but "several sub-macroeconomic destabilization". Such failure is reflected in the existence of a coercive federalism, myopic in relation, for example, to increasing congestion costs and diseconomies of agglomeration, to the lack of physical and social infrastructure, to problems relating to the balance of public accounts, poverty and violence, visibly concentrated in the large metropolises. 
Já o aumento da autonomia tributária dos governos locais produz resultados positivos sob os critérios da eficiência alocativa (atendimento das demandas de serviços localmente definidos) e da eficiência produtiva (aumento da accountability dos governos locais, redução da burocracia, melhor conhecimento dos custos referentes à provisão dos serviços contribuindo, assim, com impactos positivos do ponto de vista da estabilização). Tais vantagens decorrem do maior controle social, manifestado pelo efeito de "voice", das expressões da população local com referência aos governantes; regras e limites a serem observados na execução dos orçamentos locais, capazes de permitir a obtenção de equilíbrio sustentado das contas públicas em nível agregado e responsabilização dos governantes pela má utilização dos recursos públicos postos à sua disposição. E o que é mais importante: as complexas relações intergovernamentais conflituosas que tendem a exacerbar com o aprofundamento do processo de autonomia política dos governos locais devem ser vistas como um fato de natureza virtuosa e não como um entrave à busca pela estabilização e ao desenvolvimento. A gradual adoção de mecanismos voltados para a tributação e alocação mais eficiente de recursos funciona como incentivo ao "bom governo" e à consolidação do ente local como importante agente estratégico do desenvolvimento, situação expressa na máxima "fazer mais com menos".
On the contrary, an increasing tax autonomy of local governments may produce positive results under the criteria of allocative efficiency (meeting the demand of services locally defined) and productive efficiency (increasing the accountability of local governments, reducing bureaucracy, a more adequate knowledge of the costs relating to the provision of services, thus contributing to positive impacts in terms of stabilization). These advantages stem from greater social control, manifested by the "voice" effect, the expressions of the local population with reference to the rulers, norms and limits to be observed in the execution of local budgets, to allow obtaining sustained balance of public accounts in the aggregate level as well as accountability of rulers for the misuse of the public resources available. And what is more important: the complex intergovernmental conflictive relations, which tend to increase as the process of political autonomy of local governments grows, should be viewed as virtuous and not as an obstacle to the pursuit of stabilization and development. The gradual adoption of mechanisms geared to taxation and more efficient resource allocation works as an incentive to "good government" and to the consolidation of the local entity as an important strategic development agent, as the motto goes "doing more with less". 


\section{Nelson Chalfun Homsy}

Doutor em Políticas Públicas, Estratégias e Desenvolvimento (IE/UFRJ), Mestre em

Planejamento Urbano e Regional (UCLA) e Graduado em Economia (I E/UFRJ). Sua carreira profissional inclui importantes posições de administração, gerência e supervisão em departamentos de planejamento, mercado de capitais, pesquisa, sistemas de informação e finanças, nas áreas de habitação e infraestrutura. Como consultor atua nas áreas de políticas tributária e fiscal, provisão e distribuição de serviços públicos, gestão do crescimento econômico, política habitacional e análise e avaliação de projetos. É professor de Economia do Setor Público e de Matemática Financeira no Instituto de Economia da UFRJ. Seus principais temas de pesquisa, além das questões acadêmicas, são Economia Urbana e Regional e Finanças Públicas e Empresariais.
Ph.D. in Public Policy Strategies and Development (IE/UFRJ), Master in Urban and Regional Planning from UCLA, and Bachelor in Economics from the University of Rio de Janeiro. His professional career includes important supervisory positions in the fields of housing and infrastructure, planning, capital markets, research, information systems and finance departments. His main focus as a consultant includes Municipal Tax and Fiscal Policies, Provision and Distribution of Public Services, Growth Management, Housing Policy and Project Evaluation and Analysis. He is also Professor of Public Sector Economics and Mathematics of Finance at the School of Economics, in the University of Rio de Janeiro. His major research topics, besides academic issues related to his teaching, are Urban and Regional Economics and Corporate and Public Finance. 
Tabela 18.1 - Evolução da dívida líquida do setor público - 2004-2015

Table 18.1 - Public sector net debt evolution - 2004-2015

\begin{tabular}{|c|c|c|c|c|c|c|}
\hline \multirow{2}{*}{$\begin{array}{l}\text { Especificação/ } \\
\text { Item }\end{array}$} & \multicolumn{6}{|c|}{ Percentual do PIB/Percent of GDP } \\
\hline & 2004 & 2005 & 2006 & 2007 & 2008 & 2009 \\
\hline $\begin{array}{l}\text { Dívida líquida total - saldo/ } \\
\text { Total net debt-balance }\end{array}$ & 50,19 & 47,92 & 46,49 & 44,55 & 37,57 & 40,88 \\
\hline $\begin{array}{l}\text { Necessidade de financiamento do setor público/ } \\
\text { Public sector net borrowing }\end{array}$ & 2,88 & 3,54 & 3,57 & 2,74 & 1,99 & 3,19 \\
\hline $\begin{array}{l}\text { Primário/ } \\
\text { Primary }\end{array}$ & (-) 3,69 & $(-)$ 3,74 & (-) 3,15 & (-) 3,24 & (-) 3,33 & $(-) 1,94$ \\
\hline $\begin{array}{l}\text { Juros nominais/ } \\
\text { Nominal interest }\end{array}$ & 6,56 & 7,28 & 6,72 & 5,98 & 5,32 & 5,13 \\
\hline $\begin{array}{l}\text { Variação cambial e paridade de moedas/ } \\
\text { Exchange rate variation and parity of currencies }\end{array}$ & (-) 0,60 & (-) 0,99 & $(-) 0,15$ & 0,70 & (-) 3,37 & 2,75 \\
\hline $\begin{array}{l}\text { Reconhecimento de dívidas e privatizações/ } \\
\text { Recognition of debt and privatizations }\end{array}$ & 0,29 & 0,11 & (-) 0,10 & (-) 0,07 & (-) 0,02 & $(-) 0,11$ \\
\hline $\begin{array}{l}\text { Efeito crescimento PIB - dívida/ } \\
\text { GDP growth effect - debt }\end{array}$ & (-) 6,65 & (-) 4,92 & (-) 4,75 & $(-)$ 5,31 & (-) 5,58 & $(-) 2,52$ \\
\hline \multirow{2}{*}{$\begin{array}{l}\text { Especificação/ } \\
\text { Item }\end{array}$} & \multicolumn{6}{|c|}{ Percentual do PIB/Percent of GDP } \\
\hline & 2010 & 2011 & 2012 & 2013 & 2014 & 2015 \\
\hline $\begin{array}{l}\text { Dívida líquida total - saldo/ } \\
\text { Total net debt - balance }\end{array}$ & 37,98 & 34,49 & 32,25 & 30,59 & 33,11 & 36,04 \\
\hline $\begin{array}{l}\text { Necessidade de financiamento do setor público/ } \\
\text { Public sector net borrowing }\end{array}$ & 2,41 & 2,47 & 2,27 & 2,96 & 6,05 & 10,34 \\
\hline $\begin{array}{l}\text { Primário/ } \\
\text { Primary }\end{array}$ & (-) 2,62 & (-) 2,94 & (-) 2,18 & (-) 1,72 & 0,57 & 1,88 \\
\hline $\begin{array}{l}\text { Juros nominais/ } \\
\text { Nominal interest }\end{array}$ & 5,03 & 5,41 & 4,45 & 4,68 & 5,48 & 8,46 \\
\hline $\begin{array}{l}\text { Variação cambial e paridade de moedas/ } \\
\text { Exchange rate variation and parity of currencies }\end{array}$ & 0,49 & (-) 1,73 & (-) 1,28 & (-) 1,47 & (-) 1,47 & (-) 6,14 \\
\hline $\begin{array}{l}\text { Reconhecimento de dívidas e privatizações/ } \\
\text { Recognition of debt and privatizations }\end{array}$ & 0,01 & 0,01 & (-) 0,12 & (-) 0,06 & (-) 0,06 & 0,08 \\
\hline $\begin{array}{l}\text { Efeito crescimento PIB - dívida/ } \\
\text { GDP growth effect - debt }\end{array}$ & (-) 5,82 & (-) 4,24 & (-) 3,10 & (-) 3,10 & (-) 1,99 & (-) 1,35 \\
\hline
\end{tabular}

Fonte/Source: Séries temporais. Tabelas especiais. Dívida líquida e necessidades de financiamento do setor público. In: Banco Central do Brasil. SGS: sistema gerenciador de séries temporais. Brasília, DF, [2015]. Disponível em/Available from: <http://www4.bcb.gov.br/pec/series/port/aviso.asp >. Acesso em: abr. 2016/Cited:Apr. 2016. 
Tabela 18.2 - Evolução da Dívida bruta do Governo Geral - 2009-2015

Table 18.2 - General government gross debt evolution - 2009-2015

\begin{tabular}{llllllll}
\hline \multirow{2}{*}{$\begin{array}{c}\text { Especificação/ } \\
\text { Item }\end{array}$} & \multicolumn{7}{c}{ Percentual do PIB/Percent of GDP } \\
\cline { 2 - 8 } & 2009 & 2010 & 2011 & 2012 & 2013 & 2014 & 2015 \\
\hline $\begin{array}{l}\text { Dívida bruta total - saldo/ } \\
\text { Total gross debt - balance }\end{array}$ & 59,21 & 51,77 & 51,30 & 53,77 & 51,69 & 57,19 & 66,23 \\
& & & & & & &
\end{tabular}

Necessidade de financiamento/

7,97

0,92

4,88

6,80

2,68

$8,47 \quad 9,83$

Net borrowing

Emissões líquidas/

Net issues

2,23

(-) 4,65

$(-) 0,92$

1,62

$(-) 2,46$

2,97

2,29

Juros nominais/

5,74

5,57

5,80

5,18

5,14

5,51

7,54

Nominal interest

Variação cambial e paridade de moedas/

Exchange rate variation and

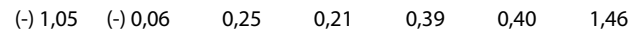

parity of currencies

Reconhecimento de dívidas e

privatizações/

0,06

0,12

0,17

0,07

0,01

0,00

0,10

Recognition of debt and

privatizations

Efeito crescimento PIB - dívida/

GDP growth effect - debt

(-) 3,75

$(-) 8,42$

(-) 5,77

$(-) 4,61$

(-) 5,16

$(-)$ 3,37

(-) 2,34

Fonte/Source: Séries temporais. Tabelas especiais. Dívida líquida e necessidades de financiamento do setor público. In: Banco Central do Brasil. SGS: sistema gerenciador de séries temporais. Brasília, DF, [2015]. Disponível em/Available from: <http://www4.bcb.gov.br/pec/series/port/aviso.asp >. Acesso em: abr. 2016/Cited: Apr. 2016. 


\section{Tabela 18.3 - Necessidades de financiamento do setor público}

2012-2015

Table 18.3 - Public sector net borrowing - 2012-2015

\begin{tabular}{|c|c|c|c|c|}
\hline \multirow{2}{*}{$\begin{array}{l}\text { Especificação/ } \\
\text { Item }\end{array}$} & \multicolumn{4}{|c|}{$\begin{array}{l}\text { Fluxo acumulado no ano (\% do PIB)/ } \\
\text { Cumulative flows in the year (\% of GDP) }\end{array}$} \\
\hline & 2012 & 2013 & 2014 & 2015 \\
\hline I. Nominal / & 2,27 & 2,96 & 6,05 & 10,34 \\
\hline \multicolumn{5}{|l|}{ I. Nominal balance } \\
\hline $\begin{array}{l}\text { Governo central/ } \\
\text { Central government }\end{array}$ & 1,27 & 2,08 & 4,77 & 8,67 \\
\hline $\begin{array}{l}\text { Governos estaduais/ } \\
\text { State government }\end{array}$ & 0,71 & 0,69 & 1,05 & 1,24 \\
\hline $\begin{array}{l}\text { Governos municipais/ } \\
\text { Municipal government }\end{array}$ & 0,17 & 0,14 & 0,09 & 0,27 \\
\hline $\begin{array}{l}\text { Empresas estatais/ } \\
\text { State-owned enterprises }\end{array}$ & 0,11 & 0,06 & 0,14 & 0,17 \\
\hline III. Primário/ & (-) 2,18 & (-) 1,72 & 0,57 & 1,88 \\
\hline \multicolumn{5}{|l|}{ III. Primary } \\
\hline $\begin{array}{l}\text { Governo central/ } \\
\text { Central government }\end{array}$ & (-) 1,79 & (-) 1,42 & 0,36 & 1,97 \\
\hline $\begin{array}{l}\text { Governos estaduais/ } \\
\text { State government }\end{array}$ & (-) 0,39 & (-) 0,24 & 0,23 & (-) 0,15 \\
\hline $\begin{array}{l}\text { Governos municipais/ } \\
\text { Municipal government }\end{array}$ & (-) 0,06 & (-) 0,06 & (-) 0,10 & (-) 0,01 \\
\hline $\begin{array}{l}\text { Empresas estatais/ } \\
\text { State-owned enterprises }\end{array}$ & 0,06 & 0,01 & 0,08 & 0,07 \\
\hline II. Juros nominais/ & 4,45 & 4,68 & 5,48 & 8,46 \\
\hline II. Nominal interest & & & & \\
\hline $\begin{array}{l}\text { Governo central/ } \\
\text { Central government }\end{array}$ & 3,06 & 3,50 & 4,41 & 6,70 \\
\hline $\begin{array}{l}\text { Governos estaduais/ } \\
\text { State government }\end{array}$ & 1,10 & 0,93 & 0,82 & 1,39 \\
\hline $\begin{array}{l}\text { Governos municipais/ } \\
\text { Municipal government }\end{array}$ & 0,22 & 0,20 & 0,18 & 0,28 \\
\hline $\begin{array}{l}\text { Empresas estatais/ } \\
\text { State-owned enterprises }\end{array}$ & 0,06 & 0,05 & 0,06 & 0,10 \\
\hline
\end{tabular}

Fonte/Source: Séries temporais. Tabelas especiais. Dívida líquida e necessidades de financiamento do setor público. In: Banco Central do Brasil. SGS: sistema gerenciador de séries temporais. Brasília, DF, [2015]. Disponível em/Available from:<http://www4.bcb.gov.br/pec/series/port/aviso.asp >. Acesso em: abr. 2016/Cited: Apr. 2016. 
Tabela 18.4 - Dívida líquida do setor público - 2012-2015

Table 18.4 - Public sector net debt - 2012-2015

\begin{tabular}{|c|c|c|c|c|}
\hline \multirow{2}{*}{$\begin{array}{l}\text { Especificação/ } \\
\text { Item }\end{array}$} & \multicolumn{4}{|c|}{$\begin{array}{l}\text { Percentual do PIB/ } \\
\text { Percent of GDP }\end{array}$} \\
\hline & 2012 & 2013 & 2014 & 2015 \\
\hline I. Dívida líquida total & 32,25 & 30,59 & 33,11 & 36,04 \\
\hline \multicolumn{5}{|l|}{ I. Total net debt } \\
\hline $\begin{array}{l}\text { Governo Federal/ } \\
\text { Federal government }\end{array}$ & 22,09 & 20,51 & 22,38 & 25,31 \\
\hline $\begin{array}{l}\text { Banco Central/ } \\
\text { Central Bank }\end{array}$ & (-) 1,24 & (-) 1,22 & (-) 1,27 & (-) 3,16 \\
\hline $\begin{array}{l}\text { Governos estaduais/ } \\
\text { State government }\end{array}$ & 9,18 & 9,09 & 9,69 & 11,13 \\
\hline $\begin{array}{l}\text { Governos municipais/ } \\
\text { Municipal government }\end{array}$ & 1,63 & 1,63 & 1,62 & 1,88 \\
\hline $\begin{array}{l}\text { Empresas estatais/ } \\
\text { State-owned enterprises }\end{array}$ & 0,59 & 0,59 & 0,69 & 0,88 \\
\hline $\begin{array}{l}\text { II. Dívida líquida interna } \\
\text { II. Net domestic debt }\end{array}$ & 45,14 & 44,03 & 46,94 & 55,47 \\
\hline $\begin{array}{l}\text { Governo Federal/ } \\
\text { Federal government }\end{array}$ & 20,29 & 18,83 & 20,50 & 23,01 \\
\hline $\begin{array}{l}\text { Banco Central/ } \\
\text { Central Bank }\end{array}$ & 14,43 & 15,13 & 16,00 & 20,84 \\
\hline $\begin{array}{l}\text { Governos estaduais/ } \\
\text { State government }\end{array}$ & 8,43 & 8,10 & 8,39 & 9,21 \\
\hline $\begin{array}{l}\text { Governos municipais/ } \\
\text { Municipal government }\end{array}$ & 1,52 & 1,50 & 1,47 & 1,68 \\
\hline $\begin{array}{l}\text { Empresas estatais/ } \\
\text { State-owned enterprises }\end{array}$ & 0,47 & 0,48 & 0,58 & 0,73 \\
\hline $\begin{array}{l}\text { III. Dívida líquida externa } \\
\text { III. Net foreign debt }\end{array}$ & (-) 12,89 & (-) 13,44 & (-) 13,83 & (-) 19,43 \\
\hline $\begin{array}{l}\text { Governo Federal/ } \\
\text { Federal government }\end{array}$ & 1,80 & 1,68 & 1,87 & 2,30 \\
\hline $\begin{array}{l}\text { Banco Central/ } \\
\text { Central Bank }\end{array}$ & (-) 15,67 & (-) 16,35 & (-) 17,27 & (-) 24,01 \\
\hline $\begin{array}{l}\text { Governos estaduais/ } \\
\text { State government }\end{array}$ & 0,75 & 0,99 & 1,30 & 1,92 \\
\hline $\begin{array}{l}\text { Governos municipais/ } \\
\text { Municipal government }\end{array}$ & 0,11 & 0,13 & 0,14 & 0,20 \\
\hline $\begin{array}{l}\text { Empresas estatais/ } \\
\text { State-owned enterprises }\end{array}$ & 0,12 & 0,11 & 0,12 & 0,15 \\
\hline
\end{tabular}

Fonte/Source: Séries temporais. Tabelas especiais. Dívida líquida e necessidades de financiamento do setor público. In: Banco Central do Brasil. SGS: sistema gerenciador de séries temporais. Brasília, DF, [2015]. Disponível em/Available from: <http://www4.bcb.gov.br/pec/series/port/aviso.asp>. Acesso em: abr. 2016/Cited: Apr. 2016 
Tabela 18.5 - Dívida líquida e superávit primário por região - 2012-2015

Table 18.5 - Net debt and primary surplus, according to Region - 2012-2015

\begin{tabular}{|c|c|c|c|c|}
\hline \multirow{2}{*}{$\begin{array}{l}\text { Especificação/ } \\
\text { Item }\end{array}$} & \multicolumn{4}{|c|}{$\begin{array}{c}\text { Receita corrente líquida (\%)/ } \\
\text { Current net revenue (\%) }\end{array}$} \\
\hline & 2012 & 2013 & 2014 & 2015 \\
\hline \multicolumn{5}{|l|}{ I. Dívida líquida } \\
\hline \multicolumn{5}{|l|}{ I. Net debt } \\
\hline $\begin{array}{l}\text { Região Norte/ } \\
\text { North Region }\end{array}$ & 7,86 & 10,09 & 13,99 & 20,10 \\
\hline $\begin{array}{l}\text { Região Nordeste/ } \\
\text { Northeast Region }\end{array}$ & 22,38 & 21,49 & 24,82 & 31,14 \\
\hline $\begin{array}{l}\text { Região Centro-Oeste/ } \\
\text { Center-West Region }\end{array}$ & 40,21 & 38,45 & 37,36 & 39,43 \\
\hline $\begin{array}{l}\text { Região Sudeste/ } \\
\text { Southeast Region }\end{array}$ & 104,14 & 102,17 & 108,75 & 123,33 \\
\hline $\begin{array}{l}\text { Região Sul/ } \\
\text { South Region }\end{array}$ & 68,07 & 63,87 & 63,28 & 68,40 \\
\hline $\begin{array}{l}\text { II. Superávit primário } \\
\text { II. Primary surplus }\end{array}$ & & & & \\
\hline $\begin{array}{l}\text { Região Norte/ } \\
\text { North Region }\end{array}$ & 2,32 & (-) 0,79 & (-) 1,72 & (-) 0,32 \\
\hline $\begin{array}{l}\text { Região Nordeste/ } \\
\text { Northeast Region }\end{array}$ & 1,28 & 1,38 & (-) 1,67 & 0,28 \\
\hline $\begin{array}{l}\text { Região Centro-Oeste/ } \\
\text { Central-West Region }\end{array}$ & 3,37 & 2,30 & 0,24 & 3,97 \\
\hline $\begin{array}{l}\text { Região Sudeste/ } \\
\text { Southeast Region }\end{array}$ & 2,35 & 2,33 & (-) 1,79 & 1,58 \\
\hline $\begin{array}{l}\text { Região Sul/ } \\
\text { South Region }\end{array}$ & 2,13 & 3,67 & 1,21 & 0,65 \\
\hline
\end{tabular}

Fonte/Source: Séries temporais. Tabelas especiais. Estatísticas Fiscais Regionalizadas. In: Banco Central do Brasil. SGS: sistema gerenciador de séries temporais. Brasília, DF, [2015]. Disponível em/Available from: <http://www4.bcb.gov.br/pec/series/port/aviso.asp>. Acesso em: abr. 2016/Cited: Apr. 2016. 
Tabela 18.6 - Despesa liquidada da União - 2010-2015

Table 18.6 - Government settled expenditure - 2010-2015

\begin{tabular}{|c|c|c|c|c|c|c|}
\hline \multirow{2}{*}{$\begin{array}{l}\text { Despesa/ } \\
\text { Expenditure }\end{array}$} & \multicolumn{6}{|c|}{$\begin{array}{l}\text { Valor nominal (1 } 000000 \mathrm{R} \$) / \\
\text { Nominal value }(1,000,000 \mathrm{R} \$)\end{array}$} \\
\hline & 2010 & 2011 & 2012 & 2013 & 2014 & 2015 \\
\hline Total/Total & 1417560 & 1574332 & 1712331 & 1780986 & 2159760 & 2255000 \\
\hline $\begin{array}{l}\text { Despesas correntes/ } \\
\text { Current expenditures }\end{array}$ & 851397 & 948736 & 1032074 & 1133182 & 1271865 & 1431877 \\
\hline $\begin{array}{l}\text { Pessoal e encargos sociais/ } \\
\text { Payroll and social levies }\end{array}$ & 168861 & 181833 & 188771 & 204500 & 220201 & 235825 \\
\hline $\begin{array}{l}\text { Juros e encargos da dívida } \\
\text { Interests and debt charges }\end{array}$ & 122018 & 131036 & 134080 & 141691 & 170351 & 208363 \\
\hline $\begin{array}{l}\text { Outras despesas correntes/ } \\
\text { Other current expenditures }\end{array}$ & 560516 & 635866 & 709223 & 786991 & 881314 & 987688 \\
\hline $\begin{array}{l}\text { Despesas de capital/ } \\
\text { Expenditure of capital }\end{array}$ & 192725 & 146287 & 381267 & 188771 & 271077 & 251225 \\
\hline $\begin{array}{l}\text { Investimentos/ } \\
\text { Investments }\end{array}$ & 23757 & 17071 & 22084 & 19524 & 21568 & 13306 \\
\hline $\begin{array}{l}\text { Inversões financeiras/ } \\
\text { Financial investment }\end{array}$ & 29096 & 31588 & 39231 & 51541 & 58757 & 55948 \\
\hline $\begin{array}{l}\text { Amortização da Dívida } \\
\text { Debt Amortization }\end{array}$ & 139872 & 97628 & 319952 & 117706 & 190752 & 181971 \\
\hline $\begin{array}{l}\text { Amortização/refinanciamento } \\
\text { da dívida/ } \\
\text { Amortization/debt refinancing }\end{array}$ & 373437 & 479368 & 298990 & 459033 & 616818 & 571898 \\
\hline
\end{tabular}

Fonte/Source: Séries históricas. Despesa da União por grupo de natureza 2009-2014. Brasília, DF: Ministério da Fazenda, Sistema Integrado de Administração Financeira do Governo Federal - SIAFI, [2015]. Disponível em/Available from : <http://www.tesouro.fazenda.gov.br/series-historicas >. Acesso em: abr. 2016/Cited: Apr. 2016. 
Tabela 18.7 - Despesa liquidada da União, por áreas

de atuação/funções - 2014-2015

Table 18.7 - Government settled expenditure, according to

practice areas - 2014-2015

\begin{tabular}{|c|c|c|}
\hline \multirow{2}{*}{$\begin{array}{c}\text { Áreas de atuação/funções/ } \\
\text { Practice areas }\end{array}$} & \multicolumn{2}{|c|}{$\begin{array}{l}\text { Valor nominal (1 } 000000 \mathrm{R} \$) / \\
\text { Nominal value }(1,000,000 \mathrm{R} \$)\end{array}$} \\
\hline & 2014 & 2015 \\
\hline Total/Total & 2159760 & 2255000 \\
\hline $\begin{array}{l}\text { Educação/ } \\
\text { Education }\end{array}$ & 73060 & 79998 \\
\hline $\begin{array}{l}\text { Cultura/ } \\
\text { Culture }\end{array}$ & 859 & 825 \\
\hline $\begin{array}{l}\text { Saúde/ } \\
\text { Health }\end{array}$ & 85131 & 92915 \\
\hline $\begin{array}{l}\text { Defesa Nacional/ } \\
\text { National Defense }\end{array}$ & 34436 & 35665 \\
\hline $\begin{array}{l}\text { Saneamento/ } \\
\text { Sanitation }\end{array}$ & 483 & 260 \\
\hline $\begin{array}{l}\text { Meio ambiente/ } \\
\text { Environment }\end{array}$ & 3618 & 2908 \\
\hline $\begin{array}{l}\text { Previdência social/ } \\
\text { Social security }\end{array}$ & 493125 & 539707 \\
\hline $\begin{array}{l}\text { Assistência social/ } \\
\text { Social assistance }\end{array}$ & 68326 & 70705 \\
\hline $\begin{array}{l}\text { Trabalho/ } \\
\text { Labor }\end{array}$ & 69420 & 65120 \\
\hline $\begin{array}{l}\text { Organização agrária/ } \\
\text { Agrarian organization }\end{array}$ & 2511 & 1626 \\
\hline $\begin{array}{l}\text { Energia/ } \\
\text { Energy }\end{array}$ & 884 & 1491 \\
\hline $\begin{array}{l}\text { Encargos especiais/ } \\
\text { Special charges }\end{array}$ & 1230919 & 1257284 \\
\hline $\begin{array}{l}\text { Outras/ } \\
\text { Others }\end{array}$ & 96991 & 106496 \\
\hline
\end{tabular}

Fonte/Source: Relatório resumido de execução orçamentária 2014-2015. Brasília, DF: Tesouro Nacional, [2015]. Disponível em/Available fom: <https://www.tesouro.fazenda.gov.br/-/relatorio-resumido-deexecucao-orcamentaria>. Acesso em: abr. 2016/Cited: Apr. 2016.

Nota: Exclusive as despesas intra-orçamentárias./Note: Excludes intra-budgetary expenditure. 
Tabela 18.8 - Despesas com o pessoal da União - 2001-2015

Table 18.8 - Federal personnel expenditures - 2001-2015

\begin{tabular}{|c|c|c|c|c|c|c|}
\hline & \multirow[b]{2}{*}{$\begin{array}{l}\text { Ano/ } \\
\text { Year }\end{array}$} & \multicolumn{5}{|c|}{$\begin{array}{l}\text { Despesas com o pessoal da União (1 } 000000 \mathrm{R} \$) / \\
\text { Federal personnel expenditures }(1,000,000 \mathrm{R} \$)\end{array}$} \\
\hline & & $\begin{array}{l}\text { Total/ } \\
\text { Total }\end{array}$ & $\begin{array}{c}\text { Executivo/ } \\
\text { Executive } \\
\text { power }\end{array}$ & $\begin{array}{l}\text { Legislativo/ } \\
\text { Legislative } \\
\text { power }\end{array}$ & $\begin{array}{c}\text { Judiciário/ } \\
\text { Judicial } \\
\text { power }\end{array}$ & $\begin{array}{c}\text { Transferências } \\
\text { intergover- } \\
\text { namentais/ } \\
\text { Intergovernmental } \\
\text { transfers }\end{array}$ \\
\hline 2001 & & 65449 & 51821 & 2426 & 8403 & 2800 \\
\hline 2002 & & 75029 & 59523 & 2890 & 9162 & 3454 \\
\hline 2003 & & 78975 & 64778 & 3488 & 10225 & 484 \\
\hline 2004 & & 89432 & 72701 & 3986 & 12374 & 370 \\
\hline 2005 & & 100287 & 76839 & 4410 & 12820 & - \\
\hline 2006 & & 115012 & 87308 & 5468 & 17400 & - \\
\hline 2007 & & 126878 & 96727 & 5621 & 18923 & - \\
\hline 2008 & & 144483 & 110286 & 5974 & 22140 & - \\
\hline 2009 & & 167066 & 127646 & 5951 & 26464 & - \\
\hline 2010 & & 183278 & 140252 & 6588 & 26547 & - \\
\hline 2011 & & 197481 & 151077 & 7336 & 28185 & - \\
\hline 2012 & & 204502 & 156849 & 7471 & 28195 & - \\
\hline 2013 & & 221981 & 170881 & 7920 & 30117 & - \\
\hline 2014 & & 239420 & 185869 & 8218 & 31193 & - \\
\hline 2015 & & 256457 & 203315 & 8950 & 34723 & - \\
\hline
\end{tabular}

Fonte/Source: Boletim estatístico de pessoal e informações organizacionais. Brasília, DF: Ministério do Planejamento, Orçamento e Gestão, v. 21, n. 237, jan. 2016. Disponível em/Available from : <http://www. servidor.gov.br/arquivos-publicacoes>. Acesso em/Available from : jan. 2016/Cited : Jan. 2016. 
Tabela 18.9 - Número de servidores públicos federais - 2001-2015

Table 18.9 - Number of federal public employees - 2001-2015

\begin{tabular}{|c|c|c|c|c|}
\hline & \multicolumn{4}{|c|}{ Servidores públicos federais/Federal public employees } \\
\hline $\begin{array}{l}\text { Ano/ } \\
\text { Year }\end{array}$ & $\begin{array}{l}\text { Total/ } \\
\text { Total }\end{array}$ & $\begin{array}{c}\text { Ativos/ } \\
\text { In activity }\end{array}$ & $\begin{array}{l}\text { Inativos/ } \\
\text { Retirees }\end{array}$ & $\begin{array}{l}\text { Pensões/ } \\
\text { Pensioners }\end{array}$ \\
\hline 2001 & 1895460 & 958071 & 541902 & 395487 \\
\hline 2002 & 1855966 & 912192 & 538537 & 405237 \\
\hline 2003 & 1922765 & 961199 & 545867 & 415699 \\
\hline 2004 & 1969174 & 990577 & 545367 & 433230 \\
\hline 2005 & 1959360 & 987403 & 537624 & 434333 \\
\hline 2006 & 2090900 & 1116002 & 532048 & 442850 \\
\hline 2007 & 2096199 & 1118260 & 529563 & 448376 \\
\hline 2008 & 2114058 & 1136468 & 529632 & 447958 \\
\hline 2009 & 1983460 & 1046727 & 534974 & 401759 \\
\hline 2010 & 2061431 & 1111583 & 543722 & 406126 \\
\hline 2011 & 2088726 & 1131178 & 548942 & 408606 \\
\hline 2012 & 2097429 & 1130460 & 557295 & 409674 \\
\hline 2013 & 2125804 & 1154512 & 560823 & 410469 \\
\hline 2014 & 2176959 & 1200094 & 565390 & 411475 \\
\hline 2015 & 2344652 & 1310715 & 613909 & 420028 \\
\hline
\end{tabular}

Fonte/Source : Boletim estatístico de pessoal e informações organizacionais. Brasília, DF: Ministério do Planejamento, Orçamento e Gestão, v. 21, n. 37, jan. 2016. Disponível em/Available from : <http://www. servidor.gov.br/arquivos-publicacoes>. Acesso em: jan. 2016/Cited: Jan. 2016.

Nota: Posição dezembro 2015./Note: 


\section{Gráfico 18.1 - Dívida do Setor Público - 2005-2015}

Graph 18.1 - Public Debt - 2005-2015

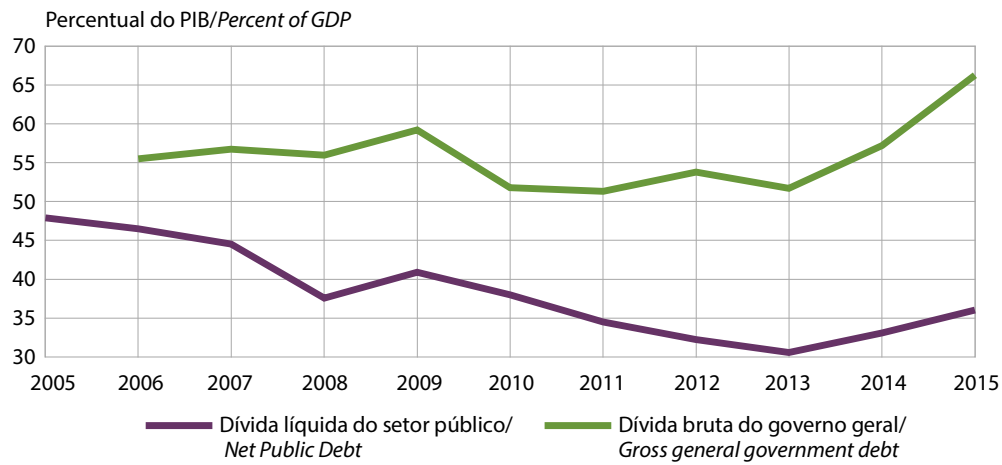

Fonte/Source: Séries temporais. Tabelas especiais. Dívida líquida e necessidades de financiamento do setor público. In: Banco Central do Brasil. SGS: sistema gerenciador de séries temporais. Brasília, DF, [2015]. Disponível em/Available from: <http://www4.bcb.gov.br/pec/series/port/aviso.asp >. Acesso em: abr. 2016/Cited:Apr. 2016.

\section{Gráfico 18.2 - Dívida Líquida do Setor Público - 2005-2015 Graph 18.2 - Public Sector Net Debt - 2005-2015}

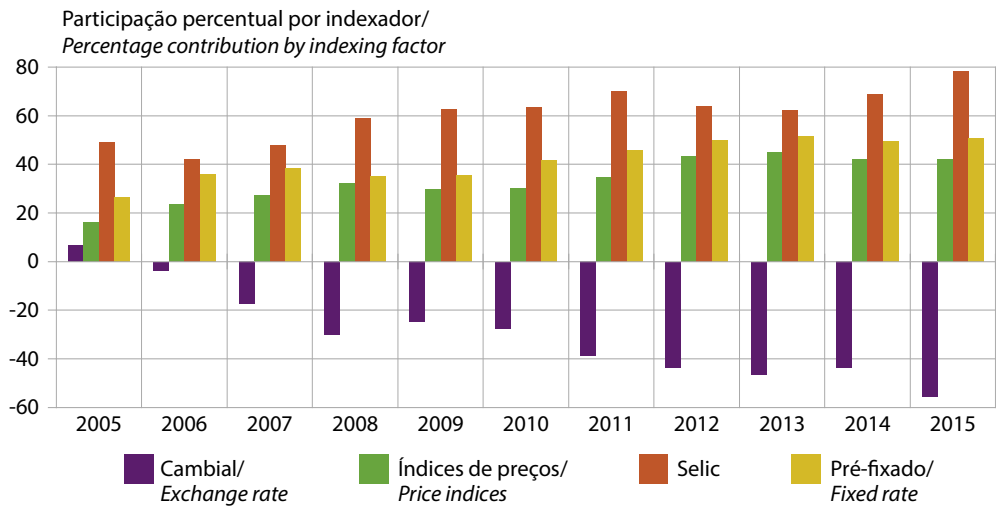

Fonte/Source: Séries temporais. Tabelas especiais. Dívida líquida e necessidades de financiamento do setor público. In: Banco Central do Brasil. SGS: sistema gerenciador de séries temporais. Brasília, DF, [2015]. Disponível em/Available from: <http://www4.bcb.gov.br/pec/series/port/aviso.asp >. Acesso em: abr. 2016/Cited: Apr. 2016. 


\section{Gráfico 18.3 - Prazo médio dos títulos federais - 2005-2015 Graph 18.3 - Average term of federal securities - 2005-2015}

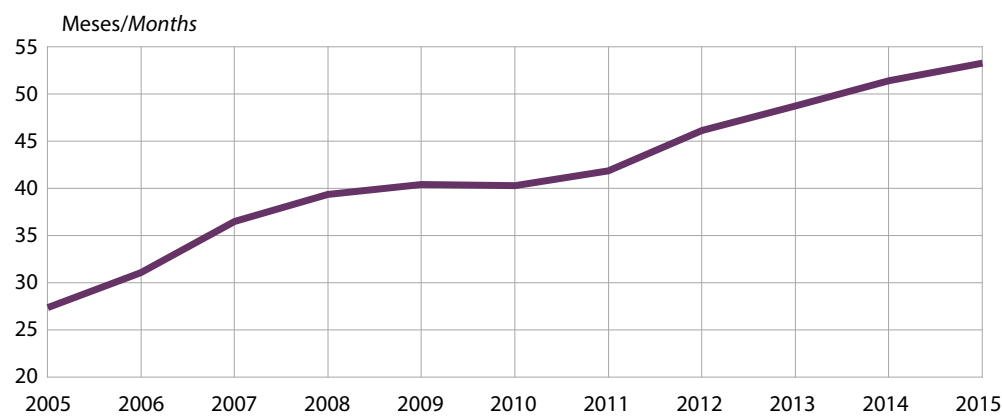

Fonte/Source: Política fiscal: nota para a imprensa [de] jan. 2016. Brasília, DF: Banco Central do Brasil - Bacen, 2016. Disponível em/Available from: $<$ http://www.bcb.gov.br/htms/infecon/notas.asp?idioma=p $>$. Acesso em: abr. 2016/Cited: Apr. 2016.

\section{Gráfico 18.4 - Dívida líquida dos governos regionais - 2005-2015}

Graph 18.4 - Net debt of regional governments - 2005-2015

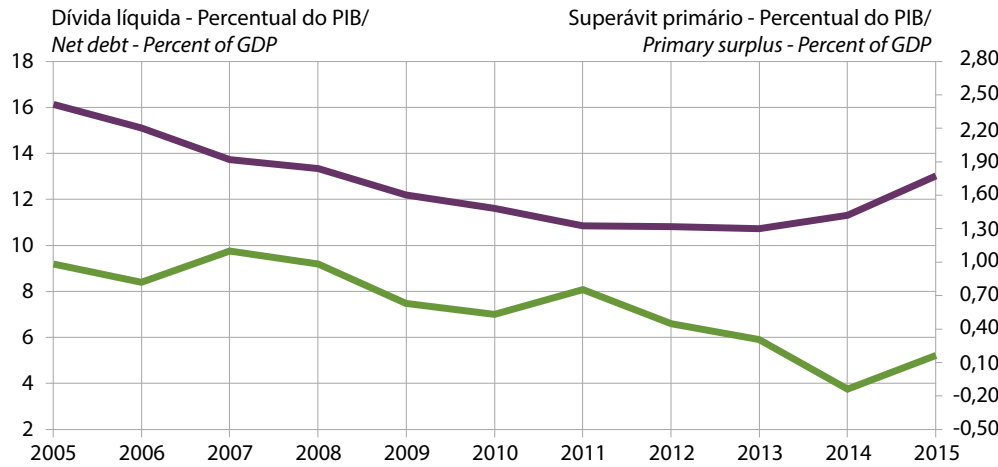

Dívida líquida - governos regionais setor público/ Net debt - regional governments

Superávit primário - governos regionais/ Primary surplus - regional governments

Fonte/Source: Séries temporais. Tabelas especiais. Dívida líquida e necessidades de financiamento do setor público. In: Banco Central do Brasil. SGS: sistema gerenciador de séries temporais. Brasília, DF, [2015]. Disponível em/Available from: <http://www4.bcb.gov.br/pec/series/port/aviso.asp >. Acesso em/Available from: abr. 2016/Cited: Apr. 2016. 



\title{
Comércio Exterior
}

\author{
Foreign Trade
}

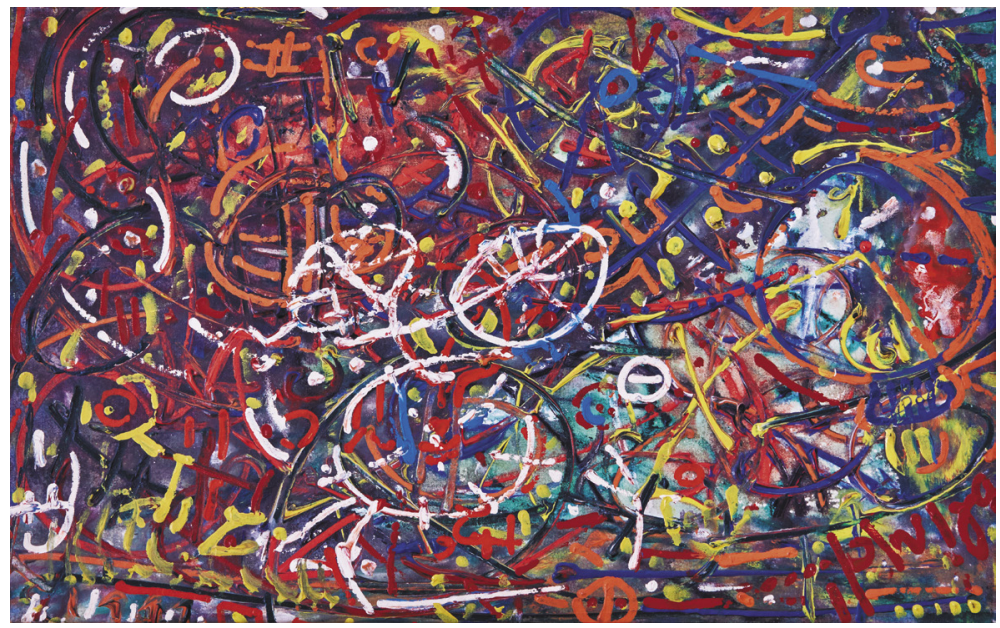

Sem Título/Untitled, 1994 J. Medeiros 



\section{Comércio Exterior}

\section{Foreign Trade}

\section{Fragilidades dos Fluxos Externos do Brasil no Período 2013-2015}

O setor externo brasileiro apresenta extrema relevância para o crescimento do país, contribuindo para o fechamento da Conta de Transações Correntes, na medida em que é um setor dinâmico e afetado pelas crises externas e internas. No período de expansão da economia mundial (2003 a 2007), o Brasil foi beneficiado pelo aumento do saldo comercial e, consequemente, pela recuperação da liquidez internacional. Contudo, a crise de 2008 afetou sobremaneira as contas externas brasileiras, tornando-se um ponto de inflexão na dinâmica do setor externo e, posteriormente, agravando a deterioração das contas externas.

De acordo com o Gráfico 19.1, as exportações brasileiras vêm oscilando ao longo do período analisado (2008 a 2015), devido a fatores externos e internos. Primeiramente, houve uma queda na demanda mundial, ocasionando em uma redução do total exportado. Segundo, após a recuperação da confiança do mercado internacional,

\section{Weaknesses of Brazil's Foreign Flows in the 2013-2015 Period}

The Brazilian foreign sector has great relevance in the country's growth, contributing to the closing of the Current Account Transactions, since it is a dynamic sector and affected by the internal and external crisis. In the expansion period of the world economy (2003 to 2007), Brazil was benefited by the increase of the trade balance and, consequently, by the recovery of the international liquidity. However, the 2008 crisis exerted a great impact on the Brazilian foreign accounts, becoming an inflection point in the dynamics of the foreign sector and, later, worsening the deterioration of the foreign accounts.

According to Graph 19.1, the Brazilian exports oscillated throughout the analyzed period (2008 to 2015) due to external and internal factors. At first, there was a decrease in the world demand, causing a reduction of the total exports. Secondly, after regaining the confidence of the international 
a apreciação do real deslocou as exportações brasileiras, o que resultou que esses dois fatores combinados reforçam a vulnerabilidade externa estrutural do país. No entanto, esse movimento contribuiu para o aumento das importações, reduzindo assim o hiato do saldo comercial, o qual registou saldo deficitário de aproximadamente US\$ 4 bilhões no ano de 2014. Assim, ainda que as exportações tenham crescido durante todo o período, as importações também o fizeram pari passu.

Contudo em 2015, fatores como retração da economia interna e a desvalorização cambial contribuíram para o saldo comercial positivo de aproximadamente US\$ 19 bilhões. Esse cenário foi alcançado pela queda superior das importações (em média 25\%) vis-àvis das exportações (em média 15\%), contribuindo para o saldo positivo.

Segundo Tabela 19.2, entre todas as categorias exportadas aquela que apresentou maior queda entre 2013 e 2015 foram os Produtos Primários com cerca de $22 \%$, seguido de Produtos Manufaturados com decréscimo de aproximadamente $21 \%$ e, por fim, os Produtos Semimanufaturados com perda de $13 \%$ em média do total exportado. Percebe-se que o padrão das exportações brasileiras ainda é intensivo em Produtos Primários, categoria essa que possui participação média de $46 \%$ entre 2013 e 2015, ou seja, em produtos pouco processados e com determinação do preço e demanda no mercado internacional (HIRATUKA E CUNHA, 2011).

Este padrão de especialização torna o país vulnerável às crises externas, na medida em que não é capaz de estabelecer market, the appreciation of the real displaced the Brazilian exports. The result of these two factors combined was the reinforcement of the external structural vulnerability of the country. However, this movement contributed to the increase of imports, narrowing down the gap of the trade balance, which registered a deficit of approximately US\$4 billion in 2014. Thus, even though the exports increased along the whole period, so did the imports hand in hand.

Nevertheless, some factors such as the contraction of the domestic economy and currency depreciation contributed to the positive trade balance of nearly US\$19 billion in 2015. This scenario was depicted by the higher drop of the imports (on average $25 \%$ ) in comparison with the exports (on average 15\%), contributing to the positive balance.

According to Table 19.2, the highest fall among all export categories between 2013 and 2015 was Primary Products, with nearly $22 \%$, followed by Manufactured Products with a decrease of around $21 \%$ and, at last, the Semi-Manufactured Products, with a $13 \%$ loss, on average, of the total exports. It can be seen that the Brazilian export pattern is still focused on Primary Products - a category which had an average contribution of $46 \%$ between 2013 and 2015 -, i.e., on lightly-processed products and with pricing and demand determined by the foreign market (HIRATUKA E CUNHA, 2011).

This pattern of specialization makes the country vulnerable to external crises because it is not able to 
o preço dos seus principais produtos exportados. Os produtos manufaturados que compõem cerca de $38 \%$ em média da pauta exportadora brasileira ainda apresentam baixa participação e concentração com relação ao destino das exportações, na medida em que seus principais mercados são ALADI, MERCOSUL E NAFTA (CUNHA, 2014).

Um dos principais fatores que contribuiu para a queda das exportações de Produtos Primários foi a retração da economia chinesa, uma vez que esse era um dos principais mercados consumidores de commodities brasileiras. No entanto, a principal motivação para a retração foi o preço das commodities que tem apresentado um declínio desde 2014 (IPEADATA, 2016). $O$ mercado internacional de produtos manufaturados brasileiros também não consegue se expandir na medida em que seu principal destino é a Argentina, país este que também passa por um período de crise econômica.

Enquanto as exportações são intensivas em produtos com baixo valor agregado e poder de negociação internacional, as importações se caracterizam pelo alto valor adicionado. A categoria de Matérias-primas e Bens Intermediários possui participação de $45 \%$ em média na pauta importadora, seguida pela categoria de Bens de Capital com $22 \%$ de participação e Combustíveis e Lubrificantes e Bens de Consumo ambos com média de $17 \%$ no período analisado. Este perfil do setor externo torna o Brasil vulnerável, na medida em que não apresenta proteção contra crises externas e internas dada sua dependência estrutural de commodities e/ou produtos pouco processados establish the price of its main exporting goods. The manufactured products that comprise nearly $38 \%$ on average of the export list of Brazil still record low contribution and concentration in relation to the destination of exports, since the main markets are LAIA, MERCOSUR and NAFTA (CUNHA, 2014).

One of the main factors that contributed to the decrease of the exports of Primary Products was the contraction of the Chinese economy, for it was one of the major consuming markets of the Brazilian commodities. However, the main motivation for the contraction was the price of the commodities that has been decreasing since 2014 (IPEADATA, 2016). Besides, the international market of the Brazilian manufactured products is not able to grow because their main destination is Argentina, a country that is also undergoing a period of economic crisis.

While the exports are intensive in products with low value added and international trading power, the imports are characterized by high value added. The category Raw Material and Intermediate Goods contributes with $45 \%$ on average to the import list, followed by the category Capital Goods, with $22 \%$ of participation and Fuel and Lubricants and Consumer Goods, both with 17\% on average in the analyzed period. Such profile of the foreign sector makes Brazil vulnerable, since there is no protection against external or internal crises given its structural dependence on commodities and/or lightly processed products coupled 
aliada a uma indústria interna débil e dependente.

A queda das importações, portanto, tem sido influenciada pela retração da atividade e investimento em capital produtivo. De acordo com a Tabela 19.3, a importações de Bens de Capital tiveram uma redução de aproximadamente $40 \%$ no período de 2013 a 2015, passando de mais de US\$ 51 milhões para apenas US\$ 37 milhões. Bem como a categoria de matérias primas e bens intermediários teve uma redução de cerca $31 \%$, o que indica uma redução da atividade industrial.

A categoria Bens de Consumo sofreu uma queda de $32 \%$ no período analisado, sendo parte significativa atribuída à redução de Bens de Consumo Duráveis (queda de 49\%), movimento esse influenciado pela queda da demanda interna devido ao aumento do custo de vida mensurado pela inflação de 10,67\% (IPEADATA, 2016) e pela taxa de desemprego que ficou em torno de 8,5\% (IBGE, 2016). No entanto, a maior redução do período foi atribuída à categoria de Combustíveis e Lubrificantes que reduziu-se em $44 \%$ desde 2014 e quase $90 \%$ desde 2013. Essa queda é atribuída, além da redução da atividade industrial, também à depreciação cambial que tornou esses produtos mais caros para o Brasil.

Apesar do saldo comercial positivo, o Saldo de Transações Correntes ainda é negativo, conforme Tabela 19.1. Esse saldo negativo nas Transações Correntes é de natureza estrutural devido ao déficit na Conta de Serviços. Este déficit está associado à saída with a fragile and dependent domestic industry.

The drop of the imports, therefore, has been influenced by the contraction of the activity and of the investment in productive capital. According to Table 19.3 , the import of capital goods had a reduction of nearly $40 \%$ in the period between 2013 and 2015, going from more than US\$51 million to just US\$37 million. The category of raw material and intermediate goods, as well, had a reduction of around $31 \%$, which indicates a decrease in the industrial activity.

The Consumer Goods category fell by $32 \%$ over the analyzed period, with a significant part attributable to the reduction of Durable Consumer Goods (down 49\%), a movement that was influenced by the fall in the domestic demand due to the increased cost of living measured by the $10.67 \%$ inflation (IPEADATA, 2016) and by the unemployment rate, which was approximately $8.5 \%$ (IBGE, 2016). However, the further reduction of the period was due to the category of Fuels and Lubricants, which has fallen by $44 \%$ since 2014 and almost $90 \%$ since 2013. Besides the reduction of the industrial activity, this decrease is also attributed to the exchange rate depreciation that made these products more expensive for Brazil.

Despite the positive trade balance, the Current Account Balance is still negative, as shown in Table 19.1. This negative balance in the Current Account is of structural nature, due to the deficit in the Services Account. This deficit is associated with the exit of Profits 
de Lucros e Dividendos das Empresas Multinacionais instaladas no país, bem como também despesas com viagens internacionais, embora tenha havido uma queda de $23 \%$ com relação ao ano de 2014. No entanto, segundo Pereira (2014), esse déficit histórico da Conta de Serviços apesar de se reduzir com a desvalorização cambial, ainda depende mais de aumentos contínuos de produtividade do que os efeitos cambiais. Isto porque a produtividade brasileira é muito baixa comparada com outros países como a própria China e mesmo assim não tem apresentado melhoria na sua posição (PEREIRA, 2014).

O déficit em Transações Correntes, juntamente com o déficit na Conta Financeira e Investimento Direto Estrangeiro, mantêm o Balanço de Pagamentos negativo nos últimos 3 anos (2013 a 2015). Isso implica uma necessidade de poupança externa elevada, o que resulta em políticas econômicas restritivas da atividade econômica como, por exemplo, desvalorização da taxa de cambio, redução do nível das atividades econômicas via aumento da taxa de juros, restrições às importações e controle de remessa de capitais ao exterior, dentre outras. Embora haja déficit no Balanço de Pagamento pode-se perceber que as Reservas Internacionais têm crescido desde 2001 a 2015, conforme Gráfico 19.2. Isto porque as reservas internacionais, que tem a função de manter a credibilidade internacional e evitar a vulnerabilidade, tem se elevado fortemente por meio do endividamento público, tornando o país ainda mais fragilizado frente a crises internacionais.

Portanto, as políticas econômicas deveriam caminhar no sentido de maior apoio ao and Dividends from Multinational Enterprises established in the country, and also with expenses with international trips, although there has been a fall of $23 \%$ in relation to 2014 . However, according to Pereira (2014), this historical deficit of the Services Account, despite being reduced with the currency devaluation, still depends more on the continued productivity increases than on the currency effects. It happens because the Brazilian productivity is very low when compared with other countries' (like China) and has not yet shown improvement (PEREIRA, 2014).

The deficit in Current Accounts along with the deficit in the Financial Account and Foreign Direct Investment kept the Balance of Payments negative in the last 3 years (2013-2015). This implies a need for high foreign savings, which results in economic policies that restrict the economic activities, as, for example, the depreciation of the exchange rate, the reduction of the level of economic activities by the increased interest rate, import restrictions and control of the remittance of capital abroad, among others. Although there is a deficit in the Balance of Payments, it is notable that the international reserves grew from 2001 to 2015, as shown in Graph 19.2. That is because the international reserves, which play the role of maintaining international credibility and avoiding vulnerability, have strongly increased by means of public debt, making the country even more exposed to the international crises.

Therefore, economic policies should provide more support to the external 
setor externo com o fortalecimento da indústria interna e, por consequência, redução da vulnerabilidade externa. Este cenário somente seria possível com uma mudança do padrão de especialização rumo a produtos mais intensivos em tecnologia e diversificação dos parceiros comerciais. sector by strengthening the domestic industry and, consequently, reducing external vulnerability. This could only be possible with a shift in the specialization pattern towards more technology-intensive products and with the diversification of trading partners.

\section{Referências}

CUNHA, S. F. (2014) Especialização Comercial Intraproduto: o desempenho exportador brasileiro e chinês em variedades verticalmente diferenciadas. Universidade Estadual de Campinas, Instituto de Economia: Campinas. Tese de Doutoramento.

HIRATUKA, C.; CUNHA, S. F. (2011) Qualidade e Diferenciação Das Exportações Brasileiras E Chinesas: Evolução Recente No Mercado Mundial E Na Aladi. Instituto de Pesquisa em Economia Aplicada - IPEA. Texto para discussão, 1622.

INSTITUTO BRASILEIRO DE GEOGRAFIA E ESTATISTICA (2016). Disponível em:<http://www.ibge.gov.br/home/ estatistica/indicadores/precos/inpc_ipca/defaultinpc.shtm>. Acesso em 4 jul. 2016.

INSTITUTO DE PESQUISA ECONÔMICA APLICADA (2016) - Ipeadata. Dados macroeconômicos e regionais. Disponível em: <http://www.ipeadata.gov.br>. Acesso em 4 jul. 2016.

PEREIRA, L. V. (2014) Déficits, uma questão estrutural. Revista Conjuntura Econômica, vol. 68, no. 03 , março.

\section{References}

CUNHA, S. F. (2014) Especialização Comercial Intraproduto: o desempenho exportador brasileiro e chinês em variedades verticalmente diferenciadas. State University of Campinas, Institute of Economics: Campinas. PhD Thesis.

HIRATUKA, C.; CUNHA, S. F. (2011) Qualidade e Diferenciação Das Exportações Brasileiras E Chinesas: Evolução Recente No Mercado Mundial E Na Aladi. Institute for Applied Economic Research - IPEA. Discussion paper, 1622.

INSTITUTO BRASILEIRO DE GEOGRAFIA E ESTATISTICA (2016). Disponível em/Available from:<http://www.ibge. gov.br/home/estatistica/indicadores/precos/inpc_ipca/defaultinpc.shtm>. Acesso em/Cited: Jul. 2016.

INSTITUTO DE PESQUISA ECONÔMICA APLICADA (2016) - Ipeadata. Dados macroeconômicos e regionais. Disponível em/Available from: <http://www.ipeadata.gov.br>. Acesso em/Cited: Jul. 2016.

PEREIRA, L. V. (2014) Déficits, uma questão estrutural. Revista Conjuntura Econômica, vol. 68, no. 03, March. 


\section{Clésio Lourenço Xavier}

Graduação em Economia pela Universidade

Federal de Uberlândia - UFU

Mestre em Economia - Universidade Estadual

de Campinas - UNICAMP

Doutor em Economia - Instituto de Economia

UNICAMP

Professor do Instituto de Economia da Universidade Federal de Uberlândia IEUFU e Pesquisador Bolsista de Produtividade do

CNPq.
Bachelor in Economics from the Federal

University of Uberlândia - UFU

Master in Economics from the State University of

Campinas - UNICAMP

PhD in Economics from the Institute of Economics of UNICAMP

Professor of the Institute of Economics of the

Federal University of Uberlândia - IEUFU and Fellow Researcher on Productivity at the CNPq.

\section{Maria Inês Cunha Miranda}

Graduação em Economia pela Universidade Federal de Uberlândia - UFU

Mestre em Economia - Universidade Federal de Uberlândia - UFU

Pesquisadora do Núcleo de Economia Aplicada do IEUFU e Doutoranda do Programa de PósGraduação em Economia.
Bachelor in Economics from the Federal University of Uberlândia - UFU

Master in Economics from the Federal University of Uberlândia - UFU

Attending a PhD Program in Economics at the Federal University of Uberlândia - UFU

Researcher of the Applied Economics Center of IEUFU and attending a PhD Program in Economics.

Translated by: Gisele Flores Caldas Manhães 
Tabela 19.1 - Balanço de pagamentos - 2013-2015

Table 19.1 - Balance of payments - 2013-2015

\begin{tabular}{|c|c|c|c|}
\hline \multirow{2}{*}{$\begin{array}{l}\text { Especificação/ } \\
\text { Item }\end{array}$} & \multicolumn{3}{|c|}{$\begin{array}{l}\text { Valor } 1000000 \text { US\$/ } \\
\text { Value } 1,000,000 \text { US\$ }\end{array}$} \\
\hline & 2013 & 2014 & 2015 \\
\hline $\begin{array}{l}\text { Balança comercial/ } \\
\text { Trade balance }\end{array}$ & 319 & $(-) 6629$ & 17670 \\
\hline $\begin{array}{l}\text { Exportações/ } \\
\text { Exports }\end{array}$ & 241507 & 224098 & 190092 \\
\hline $\begin{array}{l}\text { Importações/ } \\
\text { Imports }\end{array}$ & 241189 & 230727 & 172422 \\
\hline $\begin{array}{l}\text { Serviços/ } \\
\text { Services }\end{array}$ & (-) 46232 & (-) 48107 & (-) 36978 \\
\hline $\begin{array}{l}\text { Renda Primária/ } \\
\text { Primary income }\end{array}$ & (-) 32538 & (-) 52170 & (-) 42357 \\
\hline $\begin{array}{l}\text { Renda secundária/ } \\
\text { Secondary income }\end{array}$ & 3683 & 2725 & 2724 \\
\hline $\begin{array}{l}\text { Transações correntes/ } \\
\text { Current account }\end{array}$ & (-) 74769 & (-) 104181 & (-) 58942 \\
\hline $\begin{array}{l}\text { Conta capital/ } \\
\text { Capital account }\end{array}$ & 322 & 231 & 440 \\
\hline $\begin{array}{l}\text { Conta financeira/ } \\
\text { Financial account }\end{array}$ & (-) 72336 & (-) 100599 & (-) 56692 \\
\hline $\begin{array}{l}\text { Investimento direto (líquido)/ } \\
\text { Direct investment (net) }\end{array}$ & (-) 54240 & (-) 70855 & (-) 61576 \\
\hline $\begin{array}{l}\text { Erros e omissões/ } \\
\text { Errors and omissions }\end{array}$ & 2110 & 3351 & 1810 \\
\hline
\end{tabular}

Fonte/Source: Séries temporais. Tabelas especiais. Setor externo. In: Banco Central do Brasil. SGS: sistema gerenciador de séries temporais. Brasília, DF, [2016]. Disponível em/Available from: <http://www4.bcb.gov.br/pec/series/port/aviso.asp>. Acesso em: jan. 2016/Cited: Jan. 2016. 
Tabela 19.2 - Exportação - 2013-2015

Table 19.2 - Exports - 2013-2015

\begin{tabular}{|c|c|c|c|}
\hline \multirow{2}{*}{$\begin{array}{l}\text { Especificação/ } \\
\text { Item }\end{array}$} & \multicolumn{3}{|c|}{$\begin{array}{l}\text { Valor } 1000000 \text { US\$ Fob/ } \\
\text { Value } 1,000,000 \text { US\$ Fob }\end{array}$} \\
\hline & 2013 & 2014 & 2015 \\
\hline $\begin{array}{l}\text { Total/ } \\
\text { Total }\end{array}$ & 242034 & 225101 & 191134 \\
\hline $\begin{array}{l}\text { Produtos básicos/ } \\
\text { Primary products }\end{array}$ & 113023 & 109556 & 87188 \\
\hline $\begin{array}{l}\text { Produtos semimanufaturados/ } \\
\text { Semi-manufactured products }\end{array}$ & 30526 & 29065 & 26463 \\
\hline $\begin{array}{l}\text { Produtos manufaturados/ } \\
\text { Manufactured products }\end{array}$ & 92945 & 80211 & 72791 \\
\hline $\begin{array}{l}\text { Operações especiais/ } \\
\text { Special operations }\end{array}$ & 5540 & 6268 & 4692 \\
\hline
\end{tabular}

Fonte/Source: Séries temporais. Tabelas especiais. Setor externo. In: Banco Central do Brasil. SGS: sistema gerenciador de séries temporais. Brasília, DF, [2015]. Disponível em/Available from: <http://www4. bcb.gov.br/pec/series/port/aviso.asp>. Acesso em: jan. 2016/Cited: Jan. 2016.

Tabela 19.3 - Importação - 2013-2015

Table 19.3 - Imports - 2013-2015

\begin{tabular}{|c|c|c|c|}
\hline \multirow{2}{*}{$\begin{array}{l}\text { Especificação/ } \\
\text { Item }\end{array}$} & \multicolumn{3}{|c|}{$\begin{array}{l}\text { Valor } 1000000 \text { US\$ Fob/ } \\
\text { Value } 1,000,000 \text { US\$ Fob }\end{array}$} \\
\hline & 2013 & 2014 & 2015 \\
\hline $\begin{array}{l}\text { Total/ } \\
\text { Total }\end{array}$ & 239748 & 229145 & 171461 \\
\hline $\begin{array}{l}\text { Bens de capital/ } \\
\text { Capital goods }\end{array}$ & 51662 & 47769 & 37662 \\
\hline $\begin{array}{l}\text { Bens de consumo/ } \\
\text { Consumer goods }\end{array}$ & 40966 & 38832 & 30842 \\
\hline $\begin{array}{l}\text { Duráveis/ } \\
\text { Durable }\end{array}$ & 22229 & 20273 & 14862 \\
\hline $\begin{array}{l}\text { Não duráveis/ } \\
\text { Non-durable }\end{array}$ & 18737 & 18560 & 15980 \\
\hline $\begin{array}{l}\text { Matérias-primas e bens intermediários/ } \\
\text { Raw materials and intermediate goods }\end{array}$ & 106503 & 102999 & 81200 \\
\hline $\begin{array}{l}\text { Combustíveis e lubrificantes/ } \\
\text { Fuels and lubricants }\end{array}$ & 40616 & 39545 & 21757 \\
\hline
\end{tabular}

Fonte/Source: Séries temporais.Tabelas especiais. Setor externo. In: Banco Central do Brasil. SGS: sistema gerenciador de séries temporais. Brasília, DF, [2015]. Disponível emVAvailable from: <http://www4. bcb.gov.br/pec/series/port/aviso.asp>. Acesso em: jan. 2016/Cited: Jan. 2016. 


\section{Gráfico 19.1 - Comércio exterior - 2008-2015 \\ Graph 19.1 - Foreign trade - 2008-2015}

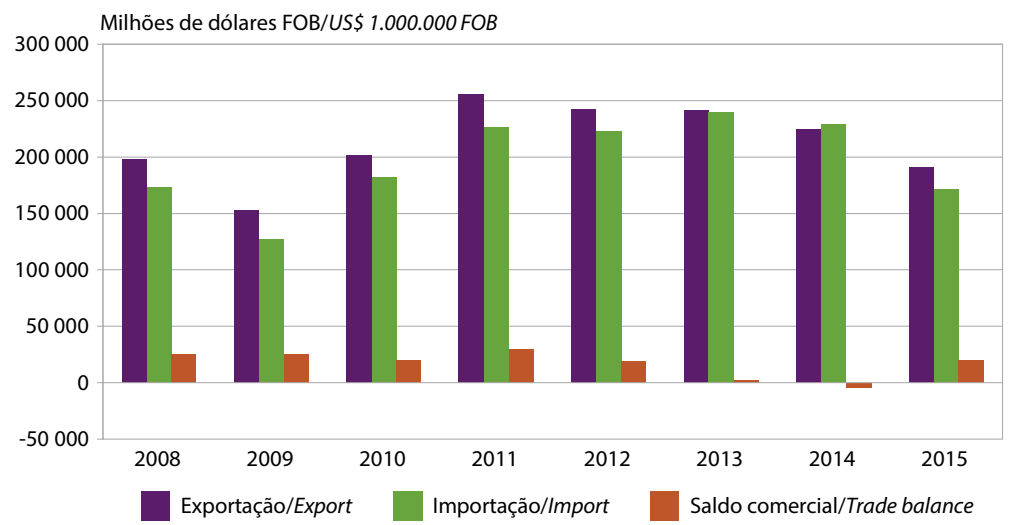

Fonte/Source: Brasil. Secretaria de Comércio Exterior. AliceWeb: sistema de análise das informações de comércio exterior. Brasília, DF: Secex, 2015. Disponível em/Available from: <http://aliceweb.mdic.gov.br/>. Acesso em: jan. 2016/Cited: Jan. 2016.

\section{Gráfico 19.2 - Reservas internacionais - 2001-2015}

Graph 19.2 - International reserves - 2001-2015

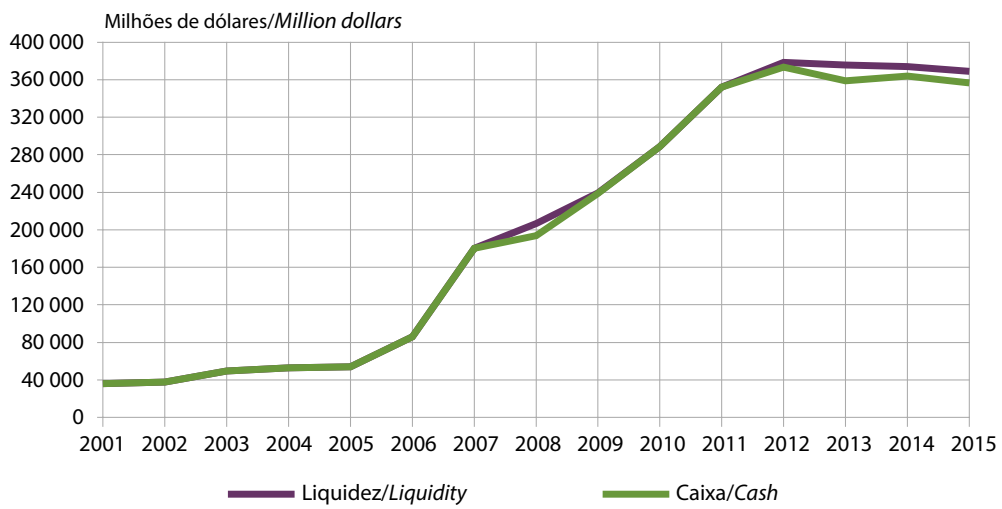

Fonte/Source: Séries temporais. Tabelas especiais. Setor externo. In: Banco Central do Brasil. SGS: sistema gerenciador de séries temporais. Brasília, DF, [2015]. Disponível em/Available from: <http://www4.bcb. gov.br/pec/series/port/aviso.asp>. Acesso em: jan. 2015/Cited: Jan. 2015. 


\section{Ciência e Tecnologia Science and Technology}

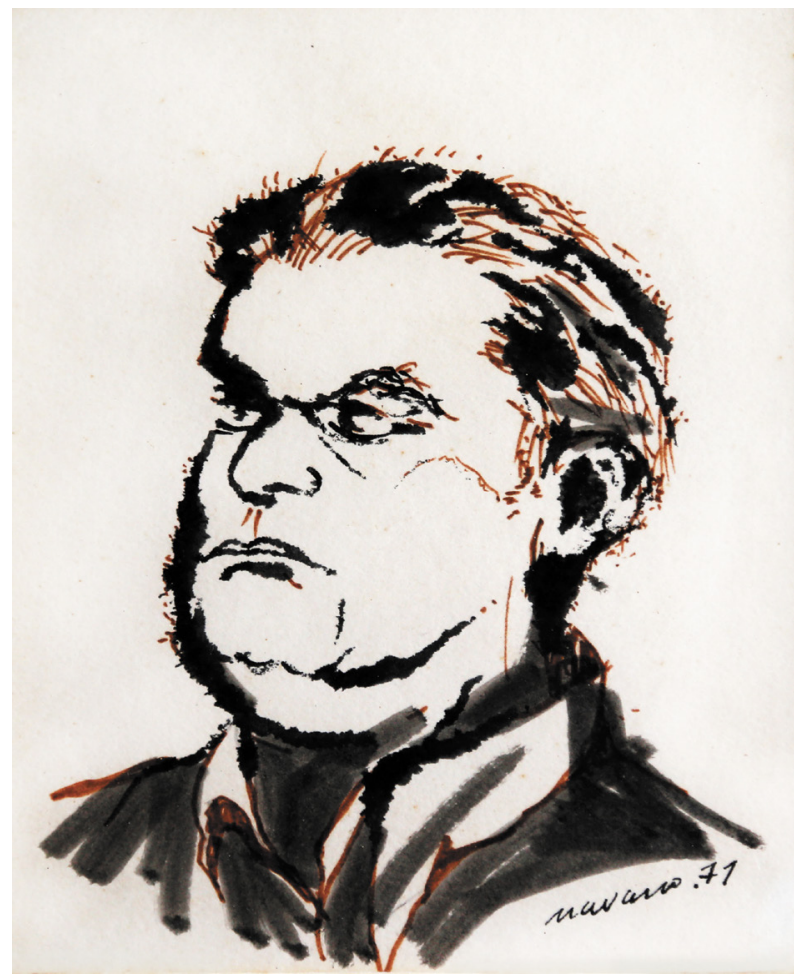

Câmara Cascudo/ Câmara Cascudo, 1971 Newton Navarro Bilro 



\section{Ciência e Tecnologia}

\section{Science and Technology}

\section{Ciência, Tecnologia e Inovação (CT\&l)}

A construção e o uso de indicadores de CT\&l é um instrumento essencial para compreender melhor e monitorar o processo de produção, difusão e utilização do conhecimento científico, das novas tecnologias e inovações. A importância de se monitorar os indicadores de CT\&l é devida à existência de uma relação forte entre o crescimento econômico e a elevação da produtividade (MACEDO;VIOTTI, 2003, p. 49) causada pela introdução e difusão de novas tecnologias nos processos de produção. Outro ponto importante é a possibilidade de usar os indicadores de CT\&I com um instrumento para formular e acompanhar as políticas públicas voltadas para os diversos setores produtivos. Por fim, medir apropriadamente o crescimento em CT\&I pode auxiliar na definição e avaliação de estratégias tecnológicas a serem adotadas pelas empresas e outros atores e agentes.

\section{Science, Technology and Innovation (ST\&l)}

The construction and use of ST\&l indicators are essential to provide better understanding and assessment of the production, dissemination and application of scientific knowledge, new technologies and innovations. The importance of monitoring the ST\&l indicators lies in the strong relationship between economic growth and increased productivity (MACEDO; VIOTTI, 2003, p 49) leveraged by the introduction and spread of new technologies in the production processes. Another important aspect is the possibility of using the ST\&I indicators as a tool to formulate and monitor public policies for the several productive sectors. Finally, measuring growth in ST\&I properly can help develop and assess technological strategies to be adopted by companies and by other actors and agents. 


\section{Produção Científica e Tecnológica no Brasil}

A produção científica no período entre 2000 a 2013 cresceu acima da média mundial como pode ser visto na Tabela 20.5 , onde se observa a crescente participação do Brasil em relação ao mundo, tanto em número de artigos produzidos quanto em citações recebidas. No entanto, o significativo crescimento observado no volume da produção científica brasileira não é acompanhado pelos indicadores de patentes visto na Tabela 20.6. O fato relevante é que, no período de 2000 a 2013, o número de artigos produzidos no País cresceu em 325 $\%$, enquanto que o do Mundo cresceu em $105 \%$, revelando um esforço significativo no Brasil na produção cientifica.

O Gráfico 20.2 mostra que na série histórica entre 2000 a 2013 há uma taxa de crescimento linear de 0,99 entre número de artigos produzidos no Brasil em relação ao Mundo.

\section{Inovação}

Na Tabela 20.6, entre 2010 e 2015, verificase que o número de pedidos depositados junto ao Instituto Nacional da Propriedade Industrial (INPI) teve um crescimento de $20,9 \%$, enquanto que o crescimento da produção cientíica para o período entre 2000 e 2013 foi de $325 \%$, mostrando a prioridade dos pesquisadores em publicar em vez de depositar uma patente.

A Tabela 20.6 chama a atenção sobre o modelo de utilidade que decresceu em 9,6\% para o mesmo período, mostrando que as empresas pouco investem para aprimorar uma nova aplicação dos seus produtos ou na melhoria destes.

\section{Scientific Production and Technology in Brazil}

The scientific production in the 20002013 period grew above the global average, as shown in Table 20.5. The growing participation of Brazil in relation to the world can be seen, both in the number of articles written and in the citations received. However, the significant growth observed in the volume of Brazilian scientific production does not match the patent indicators seen in Table 20.6. The relevant fact is that, from 2000 to 2013 , the number of papers produced in the country grew by $325 \%$, while in the world it grew by $105 \%$. This is evidence that Brazil made a great effort in terms of scientific production.

Graph 20.2 shows that in the time series between 2000 and 2013 there is a linear growth rate of 0.99 between the number of papers produced in Brazil in relation to the world.

\section{Innovation}

According to table 20.6, the number of applications filed by the National Institute of Industrial Property (INPI), between 2010 and 2015, grew $20.9 \%$, while the growth of scientific output in the period between 2000 and 2013 reached $325 \%$. This shows that researchers prioritize publishing over filing a patent.

Table 20.6 points out that the utility model decreased by $9.6 \%$ for the same period, making it clear that companies invest little to upgrade a new app for their products or to improve the products themselves. 
No período citado observa-se que em média foram concedidas 3.486 patentes para um total de solicitação de pedidos de 29.916 respectivamente, com um baixo número de pedidos indeferidos em média 2.463 e uma grande quantidade arquivada em média de 16.743 . Assim, pode-se presumir que poucos pedidos de depósito são efetivamente concedidos e que muitos acabam sendo arquivados por algum problema durante o processo de concessão. Esses dados indicam neste sentido uma baixa capacidade de inovação no País.

No período compreendido entre 2002 e 2014 observa-se na Tabela 20.4 que o número de instituições aumentou em $83,5 \%$, o grupo de pesquisas em 133,7 $\%$, o número de pesquisadores em 216,9 $\%$ e o número de doutores em $239 \%$ respectivamente. Não há uma correlação linear entre o crescimento das instituições de pesquisa e o crescimento de grupos de pesquisa, o número de pesquisadores e doutores, ou seja, o crescimento das instituições de pesquisa não acompanhou o crescimento do capital humano.

\section{Formação de Mestres e Doutores}
A Tabela 20.3 mostra que no período entre 2000 e 2014, o número de alunos matriculados ao final do ano para os cursos de Mestrado Acadêmico cresceu em $91,2 \%$. Por sua vez, para os cursos de Mestrado Profissional, o crescimento de $1.843 \%$ é particularmente significativo. Finalmente, para os de Doutorado, o crescimento no período foi de $188,3 \%$. Paralelamente, neste mesmo período, observa-se o aumento significativo da produção científica.

In the aforementioned period, on average, 3,486 patents were granted for a total of 29,916 application requests, with a low number of rejected requests (on average 2,463 ) and a great amount of filed ones (on average 16,743). Thus, it can be assumed that few applications are effectively granted and that many end up being filed due to some problem during the process. These data, therefore, indicate low innovation capacity in the country.

In the period between 2002 and 2014, Table 20.4 shows that the number of institutions increased by $83.5 \%$, research groups, by $133.7 \%$, the number of researchers, by $216.9 \%$ and the number of doctors grew $239 \%$. There was no linear correlation between the growth of research institutions and the growth of research groups, of the number of researchers and doctors, i.e., the growth of research institutions did not follow that of human capital.

\section{Master's and Doctoral Degrees}

Table 20.3 shows that between 2000 and 2014, the number of students enrolled at the end of the year in Academic Master's courses grew by 91.2\%. For the Professional Master's courses, the growth of 1,843\% is particularly significant. Finally, for Doctoral Degree programs, the advance in the period was of $188.3 \%$. In that same period, there was a relevant increase in scientific production, as well. 
A comparação do aumento do número de alunos matriculados em programas de Mestrado Acadêmico/Profissional e de Doutorado com os mais de $325 \%$ de crescimento de artigos publicados no País, bem como o incremento da participação brasileira no percentual mundial de citações (conforme Tabela 20.5, entre 2000 e 2013), sugerem uma maturação do sistema nacional de pós-graduação e refletem também o impacto da forte expansão do ensino superior no período. Entretanto, ao se comparar o crescimento do número de doutores (Tabela 20.4) com o número de pedidos de depósitos de propriedade intelectual (Tabela 20.6), porém no período entre 2002 e 2014, observa-se um crescimento de $216,9 \%$ no número de pesquisadores no Brasil (Tabela 20.4) com relativo aumento da produção de artigos científicos (Tabela 20.5) apontando que o País está mais voltado para a produção científica do que para a inovação propriamente dita.

Em relação aos Indicadores de titulados dos cursos de pós-graduação stricto sensu (Mestrado e Doutorado) entre 2000 e 2014 (conforme Tabela 20.3a), nota-se um aumento expressivo de $2.627 \%$ nos programas de Mestrado Profissional enquanto que nos programas de Mestrado Acadêmico e de doutorado, o crescimento foi de $152,7 \%$ e $214,9 \%$, respectivamente.

\section{Dispêndios em Pesquisa e Desenvolvimento (P\&D) por setores}

Na Tabela 20.1, verifica-se a forte participação do setor público na composição do P\&D nacional representando $57,7 \%$ em 2013. Em 2012 essa participação foi de $54,9 \%$
The rise in the number of students enrolled in Academic/Professional Master's and Doctoral programs, coupled with more than $325 \%$ of growth in published papers in the country, and the increase of the Brazilian participation in the global percentage of citations (as shown in Table 20.5, between 2000 and 2013) suggest a maturation of the national system of postgraduate programs and also reflect the impact of the strong expansion of higher education in the period. However, when comparing the growth in the number of PhDs (Table 20.4) with the number of applications filed for intellectual property (Table 20.6), between 2002 and 2014, it is possible to see there was a growth of $216.9 \%$ in the number researchers in Brazil (Table 20.4) with relative increase in the production of scientific papers (Table 20.5); therefore, data indicate that the country is more focused on scientific production than on innovation itself.

Regarding Indicators of researchers with a Master's or Doctoral degree between 2000 and 2014 (according to Table 20.3a), there is a remarkable increase of $2,627 \%$ in the Professional Master's Program while in the Academic Master's and Doctoral Programs, the growth was of $152.7 \%$ and $214.9 \%$, respectively.

\section{Expenditures in Research and Development (R\&D) by sectors}

In Table 20.1, it is possible to detect a strong public sector participation in the national R\&D composition, representing $57.7 \%$ in 2013. In 2012 the contribution had been $54.9 \%$, meaning that in 2013 
mostrando que houve em 2013 um aumento de $2,8 \%$ nos gastos do governo com $P \& D$, porém abaixo da inflação anual que foi de $6,20 \%$ segundo INPC (Índice Nacional de Preços ao Consumidor - dado do site do IBGE, 2016, ago.). O percentual de crescimento dos gastos de P\&D em relação ao PIB total cresceu de $1,15 \%$ para $1,24 \%$, porém este comportamento é ainda inferior ao encontrado nas economias de maior $\mathrm{PIB}$, que possuem resultados mais expressivos em termos de produção tecnológica, conforme dados da OCDE, apontando mais de $2 \%$ (OCDE, 2013). Os gastos com $P \& D$ em relação ao PIB total das empresas em 2013 mantiveram-se constantes em 0,52\% apesar dos esforços governamentais em propiciar um marco legal criado a partir da promulgação da Lei 11.196/05, que passou a ser conhecida como "Lei do Bem" (BRASIL, 2005), propiciando a concessão de incentivos fiscais às pessoas jurídicas que realizarem pesquisa, desenvolvimento tecnológico e inovação. Segundo o Relatório Anual de Incentivos Fiscais Ano-Base 2013 do MCTI, as empresas que utilizaram o benefício da Lei do Bem passou de 130 empresas participantes em 2006 para 1158 em 2013, e um aumento de $11 \%$ em relação ao ano-base de 2012. Cabe ressaltar que, do total de 1.158 empresas que participaram dos incentivos fiscais da Lei do Bem, no ano-base de 2013, os gastos totais com investimentos em atividades de $P, D \& 1$ registraram o valor de $\mathrm{R} \$ 9,04$ bilhões de reais (valor bruto), com usufruto de renúncia fiscal no valor de $\mathrm{R} \$ 2,14$ bilhões de reais (valor bruto) (BRASIL, 2013, p. 11). Assim, pode-se afirmar que o Brasil passou a contar com um there was an increase of $2.8 \%$ in government expenditure on $R \& D$, yet still below the annual inflation $(6.20 \%$ according to the INPC - National Consumer Price Index - data from the IBGE's website, in August 2016). The growth percentage of R\&D spending in relation to the total GDP increased from $1.15 \%$ to $1.24 \%$, but this behavior is still lower than that found in most economies of higher GDP. They have more significant results in terms of technological production - according to OECD data, more than $2 \%$ (OECD, 2013). Spending on $R \& D$ in relation to the total GDP of the companies in 2013 remained steady at $0.52 \%$, despite government efforts to provide a legal framework on the grounds of the enactment of Law 11,196 05, which became known as the "Good Act" (BRAZIL, 2005). This law provided for the granting of tax incentives to companies that undertake research, technological development and innovation. According to the Annual Tax Incentives Report - Base-Year 2013 - of the Ministry of Science, Technology and Innovation, the number of companies that benefited from the Good Act went from 130 in 2006 to 1,158 in 2013. Compared to the base-year of 2012, there was an $11 \%$ increase. It should be noted that of the total of 1,158 companies that participated in the tax incentive programs of the Good Act, in the base-year of 2013, the total spending on investments in R,D\&l recorded $\mathrm{R} \$ 9.04$ billion reais (gross value), with tax exemptions of $\mathrm{R} \$ 2.14$ billion reais (gross value) (BRASIL, 2013, p. 11). Therefore, it could be said that Brazil began to rely on a more integrated 
sistema mais integrado e coerente para a indução da inovação nas empresas nacionais a partir das sansões da Lei do Bem (Lei no 11.196/2005) e da Lei da Inovação (Lei n 10.973/2004).

No Gráfico 20.1, verifica-se que o Ministério da Saúde é o que menos investiu em P\&D em 2013, com 6,1 \%, o que pode em parte explicar o crescente déficit na balança comercial de insumos para o complexo da saúde. Por sua vez, os Ministérios da Educação e da Ciência, Tecnologia e Inovação investem em P\&D, respectivamente 51,4\% e 30,2 \% em 2013.

Ações de investimentos têm sido feitas pelo Ministério da Saúde, onde se saltou de R\$ 1 milhão em 2003 com 8 projetos para $\mathrm{R} \$ 88$ milhões em 2008 com um total de 250 projetos. $O$ incentivo se deu por meio do lançamento de editais, em âmbito nacional, bem como pela contratação direta de estudos. A elaboração dos editais segue os temas apontados na Agenda Nacional de Prioridades de Pesquisa em Saúde, considerando, especialmente, as necessidades de estudos para aperfeiçoamento das políticas, as lacunas de conhecimento existentes nas áreas e a disponibilidade de recursos financeiros. O Fomento Nacional tem o propósito de se aproximar das necessidades de saúde da população expressas na Política Nacional de Saúde, além de estabelecer a implantação da Política Nacional de Ciência, Tecnologia e Inovação em Saúde (PNCTIS) (BRASIL, 2016).

A região do Brasil que obteve mais Recursos dos governos estaduais aplicados em ciência e tecnologia no período entre 2008 e 2013 foi a região Sudeste, conforme and coherent system for the fostering of innovations in the national companies after the sanctions of the Good Act (Law No 11,196/2005) and the Innovation Act (Law No 10,973/2004).

In Graph 20.1, it can be seen that the Ministry of Health was the one with the lowest investment in R\&D in 2013, with $6.1 \%$ - which can partly explain the increased deficit in the balance of trade of health inputs. Conversely, the Ministries of Education and Science, Technology and Innovation invested in R\&D, respectively, $51.4 \%$ and $30.2 \%$ in 2013.

Some initiatives have been taken by the Ministry of Health, increasing investments from $\mathrm{R} \$ 1$ million in 2003 , with 8 projects, to $R \$ 88$ million in 2008, with a total of 250 projects. The incentive was granted through a call for bids, at national level and by means of contracting of studies. The bids considered the themes listed in the National Agenda of Priorities in Health Research, in particular, studies of policy improvements, of existing knowledge gaps in some fields and of the availability of financial resources. The National Incentive aims at meeting the health demands of the population as described in the National Health Policy, and at establishing the implementation of the National Policy on Science, Technology and Innovation in Health (PNCTIS) (BRAZIL, 2016).

The Southeast is the Major Region of

Brazil which obtained most resources from state governments applied to science and technology in the period 
Tabela 20.2. Entretanto, para o período mencionado o aumento dos recursos nas regiões foi desigual como segue: Norte $(138,9 \%)$, Nordeste $(109,3 \%)$, Sudeste $(102,7 \%)$, Sul (114,6 \%) e Centro-Oeste (303,2\%). São Paulo é sem dúvida o Estado que mais investe em $P \& D$, sendo que em 2013 foi de 8.786,68 milhões de reais, o que representou $41,5 \%$ em relação ao total dos recursos aplicados no Brasil, que totalizaram 15 bilhões de reais. Em seguida vem os Estados do Rio de Janeiro e Minas Gerais com investimentos na ordem de 959,99 e 701,08 milhões de reais respectivamente, o que representa $6,4 \%$ e $4,7 \%$ do total do Brasil. Assim, os três estados representam $52,6 \%$ de todos os recursos de todos os governos estaduais aplicados em ciência e tecnologia em 2013.

\section{Conclusão}

Os indicadores analisados sugerem que o aumento dos investimentos públicos em CT\&l, muito embora tenham surgidos significantes avanços no número de publicações cientificas produzidas em relação à produção mundial, bem como no crescente aumento da formação de mão de obra especializada, não foi suficiente para gerar uma quantidade razoável de inovações e novas tecnologias em setores industriais, de modo a gerar maior riqueza com os produtos exportados pelo Brasil. between 2008 and 2013, according to Table 20.2. However, for the period mentioned, the increase of resources among the regions was uneven: North (138.9\%), Northeast (109.3\%), Southeast (102.7\%), South (114.6\%) and Central-West (303.2\%). São Paulo is undoubtedly the state that invests more in R\&D. In 2013, the investment stood at $8,786.68$ billion reais, which represented $41.5 \%$ of the resources applied in Brazil, which totaled 15 billion reais. Next, come the states of Rio de Janeiro and Minas Gerais with investments of 959.99 and 701.08 million reais, respectively, representing $6.4 \%$ and $4.7 \%$ of Brazil's total. Thus, the three states account for $52.6 \%$ of the resources from all state governments allocated to science and technology in 2013.

\section{Final Remarks}

The indicators analyzed suggest that the increase in public investments in ST\&l, despite the significant advances in the number of scientific publications produced in relation to the world production, and the increased growth in the training of experts, was not enough to generate a reasonable amount of innovations and new technologies in industrial sectors in order to produce more wealth through the goods exported by Brazil.

\section{Referências/References}

BRASIL. Ministério da Ciência, Tecnologia e Inovação (MCTI). Secretaria de Desenvolvimento Tecnológico e Inovação - SETEC. Relatório Anual de Incentivos Fiscais, Lei do Bem, Ano-Base 2013. Brasília, DF, 2013. Disponível em/ Available from: < http://www.mct.gov.br/index.php/content/view/8563.html >. Acesso em/Cited: 20 ago. 2016. 
BRASIL, Lei n. 11.196, de 21 de novembro de 2005. Institui o Regime Especial de Tributação para a Plataforma de Exportação de Serviços de Tecnologia da Informação - REPES, o Regime Especial de Aquisição de Bens de Capital para Empresas Exportadoras - RECAP e o Programa de Inclusão Digital; dispõe sobre incentivos fiscais para a inovação tecnológica e dá outras providências. Diário Oficial [da] República Federativa do Brasil. Poder Executivo, Brasília, DF, 22 nov. 2005. Disponível em/ Available from: <http://www.planalto.gov.br/ccivil_03/_ato20042006/2005/lei/l11196.htm >. Acesso em/Cited: 20 ago. 2016.

BRASIL. Portal do Ministério da Saúde. Fomento Nacional. Disponível em/ Available from: < http://portalsaude. saude.gov.br/index.php/o-ministerio/principal/leia-mais-o-ministerio/245-sctie-raiz/decit-raiz/decitdepartamento-de-ciencia-e-tecnologia/l3-decit/8675-cursos-de-capacitacao-em-ats-no-brasil >. Acesso em/Cited: 20 de ago. 2016.

MACEDO, M. M.; VIOTTI, E. B. Indicadores de ciência, tecnologia e inovação no Brasil. Campinas: Editora Unicamp, 2003.

OCDE. Painel de Avaliação da OCDE para Ciência, Tecnologia e Indústria em 2013. Coordenadoria da OCDE para a Ciência, Tecnologia e Indústria. 2013. Disponível em/ Available from: < www.oecd.org/sti >. Acesso em/Cited: 20 ago. 2016.

\section{Gerson Rosenberg}

Pesquisador e professor conveniado na FACC/ UFRJ. Graduado em Eng. Química pela Escola de Química/UFRJ (1983). Tecnologista em Saúde Pública da Fundação Oswaldo Cruz - Fiocruz (1996). Mestre em Tecnologia de Processos Químicos e Bioquímicos pela EQ/ UFRJ - Universidade Federal do Rio de Janeiro (2002) e doutor em Tecnologia de Processos Químicos e Bioquímicos pela EQ-UFRJ Universidade Federal do Rio de Janeiro (2007). Pós-doutorado pelo Instituto de Economia Universidade Federal do Rio de Janeiro (2009).
Researcher and associate professor at FACCl UFRJ. Bachelor in Chemical Engineering from the School of Chemistry / UFRJ (1983). Technologist in Public Health from the Oswaldo Cruz Foundation - Fiocruz (1996). Master in Technology of Chemical and Biochemical Processes from EQ / UFRJ - Federal University of Rio de Janeiro (2002) and PhD in Technology of Chemical and Biochemical Processes from the EQ-UFRJ - Federal University of Rio de Janeiro (2007). Post-doctoral degree from the School of Economics - Federal University of Rio de Janeiro

(2009).

\section{Pierre Ohayon}

Professor Titular da Faculdade de Administração e Ciências Contábeis da Universidade Federal do Rio de Janeiro. Possui graduação em Administração de Empresas pela Universidade de São Paulo (1976), Mestrado com Distinção e Louvor em Administração pela Universidade de São Paulo (1984) e Doutorado com distinção em Administração pela Universidade de São Paulo
Full Professor at the School of Business and Accounting of the Federal University of Rio de Janeiro. Bachelor in Business Administration from the University of São Paulo (1976), Master's Summa Cum Laude in Business Administration from the University of São Paulo (1984) and

Doctoral degree with honors in Business Administration from the University of São Paulo

(1986).

(1986). 
Tabela 20.1 - Investimentos nacionais em pesquisa e desenvolvimento, por setores, em relação ao Produto Interno Bruto - PIB - 2012-2013

Table 20.1 - National investments in research and development, by sectors, vis-à-vis Gross Domestic Product - GDP - 2012-2013

\begin{tabular}{|c|c|c|c|c|c|c|}
\hline \multirow[t]{2}{*}{$\begin{array}{l}\text { Setores/ } \\
\text { Sectors }\end{array}$} & \multicolumn{2}{|c|}{$\begin{array}{l}\text { P\&D (em milhões } \\
\text { de reais correntes)/ } \\
R \& D \text { (in millions } \\
\text { of current Reais) }\end{array}$} & \multicolumn{2}{|c|}{$\begin{array}{l}\text { Percentual em } \\
\text { relação ao total } \\
\text { de P\&D/ } \\
\text { Percent vis-à-vis } \\
\text { total } R \& D\end{array}$} & \multicolumn{2}{|c|}{$\begin{array}{c}\text { Percentual P\&D } \\
\text { em relação } \\
\text { ao PIB/ } \\
\text { Percent of } R \& D \\
\text { vis-à-vis GDP }\end{array}$} \\
\hline & 2012 & $\begin{array}{c}2013 \\
(1)\end{array}$ & 2012 & $\begin{array}{c}2013 \\
(1)\end{array}$ & 2012 & $\begin{array}{c}2013 \\
(1)\end{array}$ \\
\hline Total/Total & 54254,6 & 63748,6 & 100,0 & 100,0 & 1,15 & 1,24 \\
\hline $\begin{array}{l}\text { Dispêndios públicos/ } \\
\text { Public expenditures }\end{array}$ & 29802,9 & 36783,7 & 54,93 & 57,70 & 0,63 & 0,71 \\
\hline $\begin{array}{l}\text { Dispêndios federais/ } \\
\text { Federal expenditures }\end{array}$ & 20020,7 & 25802,5 & 36,90 & 40,47 & 0,42 & 0,50 \\
\hline $\begin{array}{l}\text { Orçamento/ } \\
\text { Budget }\end{array}$ & 12013,9 & 16080,8 & 22,14 & 25,22 & 0,25 & 0,31 \\
\hline $\begin{array}{l}\text { Pós-graduação/ } \\
\text { Post-graduation }\end{array}$ & 8006,8 & 9721,7 & 14,76 & 15,25 & 0,17 & 0,19 \\
\hline $\begin{array}{l}\text { Dispêndios estaduais/ } \\
\text { State expenditures }\end{array}$ & 9782,2 & 10981,3 & 18,03 & 17,23 & 0,21 & 0,21 \\
\hline $\begin{array}{l}\text { Orçamento/ } \\
\text { Budget }\end{array}$ & 3165,4 & 3345,8 & 5,83 & 5,25 & 0,07 & 0,06 \\
\hline $\begin{array}{l}\text { Pós-graduação/ } \\
\text { Post-graduation }\end{array}$ & 6616,8 & 7635,5 & 12,20 & 11,98 & 0,14 & 0,15 \\
\hline $\begin{array}{l}\text { Dispêndios empresariais/ } \\
\text { Enterprise expenditures }\end{array}$ & 24451,7 & 26964,9 & 45,07 & 42,30 & 0,52 & 0,52 \\
\hline $\begin{array}{l}\text { Empresas privadas e esta- } \\
\text { tais/ } \\
\text { Private and government } \\
\text { enterprises }\end{array}$ & 23368,4 & 25722,4 & 43,07 & 40,35 & 0,50 & 0,50 \\
\hline $\begin{array}{l}\text { Pós-graduação (Instituições privadas)/ } \\
\text { Post-graduation (Private Institutions) }\end{array}$ & 1083,4 & 1242,5 & 2,00 & 1,95 & 0,02 & 0,02 \\
\hline
\end{tabular}

Fonte/Source: Dispêndio nacional em pesquisa e desenvolvimento ( $P \& D)$ em valores correntes, em relação ao total de P\&D e ao produto interno bruto (PIB), por setor institucional, 2000-2013. Brasília, DF: Ministério da Ciência, Tecnologia e Inovação, 2015. Disponível em/Available from: <http://www. mct.gov.br/index.php/content/view/29144.html>. Acesso em: abr. 2016/Cited: Apr. 2016.

(1) Dados preliminares obtidos por e-mail./ (1) Preliminary data received by e-mail. (2) Os valores para os dispêndios empresariais consideram a inclusão na amostra da PINTEC 2011 da seção D (Eletricidade e gás) e a divisão de serviço 71 (Serviços de arquitetura e engenharia; testes e análises técnicas) da CNAE./(2) The values for enterprise expenditures consider the inclusion of section $D$ (electricity and gas) and Service Division 71 (Architectural and engineering; tests and technical analysis) of CNAE in the sample for the PINTEC 2011 Survey. 


\section{Tabela 20.2 - Recursos dos governos estaduais aplicados em ciência e tecnologia - 2008-2013 \\ Table 20.2 - State government resources invested in science and technology - 2008-2013}

\begin{tabular}{|c|c|c|c|c|c|c|}
\hline \multirow{3}{*}{$\begin{array}{c}\text { Grandes Regiões e } \\
\text { Unidades da Federação/ } \\
\text { Major Regions and } \\
\text { Federation Units }\end{array}$} & \multirow{2}{*}{\multicolumn{6}{|c|}{$\begin{array}{l}\text { Valor ( } 1000000 \mathrm{R} \$) / \\
\text { Value }(1,000,000 \mathrm{R} \$)\end{array}$}} \\
\hline & & & & & & \\
\hline & 2008 & 2009 & 2010 & 2011 & 2012 & $\begin{array}{c}2013 \\
(1)\end{array}$ \\
\hline Brasil/Brazil & 7138,00 & 8424,82 & 10201,79 & 11871,64 & 13650,56 & 15006,60 \\
\hline Norte/North & 245,76 & 345,12 & 429,85 & 427,39 & 515,06 & 587,27 \\
\hline Rondônia & 2,93 & 37,90 & 52,13 & 63,33 & 77,71 & 76,18 \\
\hline Acre & 31,42 & 37,93 & 32,98 & 46,58 & 49,03 & 52,85 \\
\hline Amazonas & 91,16 & 104,59 & 128,85 & 118,71 & 129,40 & 176,04 \\
\hline Roraima & 6,12 & 8,38 & 4,94 & 4,77 & 10,08 & 16,77 \\
\hline Pará & 73,55 & 122,71 & 173,04 & 153,33 & 211,81 & 187,68 \\
\hline Amapá & 11,75 & 10,18 & 11,29 & 6,77 & 10,10 & 12,61 \\
\hline Tocantins & 28,82 & 23,43 & 26,61 & 33,91 & 26,93 & 65,14 \\
\hline Nordeste/Northeast & 732,46 & 938,82 & 1296,60 & 1245,05 & 1538,93 & 1532,96 \\
\hline Maranhão & 18,91 & 26,53 & 75,09 & 33,20 & 32,75 & 75,61 \\
\hline Piauí & 8,60 & 36,08 & 45,92 & 52,00 & 55,34 & 88,42 \\
\hline Ceará & 185,20 & 192,38 & 266,28 & 219,78 & 265,98 & 260,44 \\
\hline Rio Grande do Norte & 29,53 & 87,21 & 104,45 & 89,94 & 85,28 & 152,66 \\
\hline Paraíba & 24,32 & 98,30 & 122,01 & 135,74 & 174,02 & 165,94 \\
\hline Pernambuco & 142,58 & 147,83 & 146,21 & 236,56 & 192,01 & 160,06 \\
\hline Alagoas & 13,35 & 21,55 & 30,44 & 21,46 & 37,37 & 38,57 \\
\hline Sergipe & 17,17 & 23,58 & 19,06 & 22,89 & 82,00 & 53,12 \\
\hline Bahia & 292,80 & 305,37 & 487,15 & 433,48 & 614,19 & 538,13 \\
\hline Sudeste/Southeast & 5225,42 & 5871,09 & 6936,81 & 8487,91 & 9514,37 & 10590,91 \\
\hline Minas Gerais & 403,80 & 443,82 & 556,97 & 662,89 & 733,00 & 701,08 \\
\hline Espírito Santo & 27,39 & 69,06 & 85,20 & 116,20 & 144,79 & 143,15 \\
\hline Rio de Janeiro & 491,81 & 522,82 & 685,21 & 800,87 & 844,28 & 959,99 \\
\hline São Paulo & 4302,42 & 4835,38 & 5609,43 & 6907,95 & 7792,29 & 8786,68 \\
\hline Sul/South & 780,58 & 1000,52 & 1182,31 & 1305,83 & 1545,62 & 1675,35 \\
\hline Paraná & 425,31 & 572,25 & 600,07 & 617,81 & 759,53 & 771,52 \\
\hline Santa Catarina & 278,00 & 302,39 & 328,01 & 396,30 & 424,20 & 482,72 \\
\hline Rio Grande do Sul & 77,27 & 125,88 & 254,22 & 291,72 & 361,90 & 421,10 \\
\hline Centro-Oeste/Central-West & 153,80 & 269,28 & 356,23 & 405,47 & 536,58 & 620,13 \\
\hline Mato Grosso do Sul & 17,57 & 16,13 & 39,48 & 40,23 & 107,86 & 120,31 \\
\hline Mato Grosso & 59,16 & 80,51 & 105,97 & 131,56 & 127,21 & 154,65 \\
\hline Goiás & 31,15 & 39,59 & 44,46 & 101,34 & 149,47 & 174,52 \\
\hline Distrito Federal/Federal District & 45,91 & 133,05 & 166,32 & 132,35 & 152,05 & 170,64 \\
\hline
\end{tabular}

Fonte/Source: Brasil: dispêndios dos governos estaduais em ciência e tecnologia (C\&T) por região e unidade da federação, 2000-2013. Brasília, DF: Ministério da Ciência, Tecnologia e Inovação, 2015. Tab. 2.3.3. Disponível em/Available from: <http://www.mct.gov.br/index.php/content/view/8842. html>. Acesso em: abr. 2016/Cited: Apr. 2016.

(1) Dados preliminares obtidos por e-mail./ (1) Preliminary data received by e-mail. 
Tabela 20.3 - Indicadores selecionados dos cursos

de pós-graduação - 2001-2014

Table 20.3 - Selected indicators in master's and doctoral programs - 2001-2014

\begin{tabular}{|c|c|c|c|c|c|c|}
\hline \multirow{3}{*}{$\begin{array}{l}\text { Ano/ } \\
\text { Year }\end{array}$} & \multicolumn{3}{|c|}{$\begin{array}{l}\text { Alunos novos/ } \\
\text { New students }\end{array}$} & \multicolumn{3}{|c|}{$\begin{array}{l}\text { Alunos matriculados ao final do ano/ } \\
\text { Students enrolled at the end of the year }\end{array}$} \\
\hline & \multicolumn{2}{|c|}{$\begin{array}{c}\text { Mestrado/ } \\
\text { Master's programs }\end{array}$} & \multirow{2}{*}{$\begin{array}{l}\text { Doutorado/ } \\
\text { Doctoral } \\
\text { programs }\end{array}$} & \multicolumn{2}{|c|}{$\begin{array}{c}\text { Mestrado/ } \\
\text { Master's programs }\end{array}$} & \multirow{2}{*}{$\begin{array}{c}\text { Doutorado/ } \\
\text { Doctoral } \\
\text { programs }\end{array}$} \\
\hline & $\begin{array}{l}\text { Acadêmico/ } \\
\text { Academic }\end{array}$ & $\begin{array}{l}\text { Profissional/ } \\
\text { Professional }\end{array}$ & & $\begin{array}{l}\text { Acadêmico/ } \\
\text { Academic }\end{array}$ & $\begin{array}{l}\text { Profissional/ } \\
\text { Professional }\end{array}$ & \\
\hline 2001 & 26394 & 1680 & 9101 & 62353 & 2956 & 35134 \\
\hline 2002 & 29410 & 2156 & 9935 & 63990 & 4350 & 37728 \\
\hline 2003 & 32878 & 2452 & 11343 & 66951 & 5065 & 40213 \\
\hline 2004 & 34272 & 2795 & 9462 & 69190 & 5809 & 41261 \\
\hline 2005 & 36044 & 2914 & 9784 & 73805 & 6301 & 43942 \\
\hline 2006 & 38948 & 3272 & 10559 & 79050 & 6798 & 46572 \\
\hline 2007 & 41403 & 3684 & 11214 & 84356 & 7638 & 49667 \\
\hline 2008 & 42788 & 4654 & 12858 & 88295 & 9073 & 52750 \\
\hline 2009 & 46004 & 4847 & 14155 & 93016 & 10135 & 57917 \\
\hline 2010 & - & - & - & 98611 & 10213 & 64588 \\
\hline 2011 & - & - & - & 105240 & 12505 & 71890 \\
\hline $\begin{array}{l}2012 \\
2013\end{array}$ & $\begin{array}{l}- \\
-\end{array}$ & - & - & $\begin{array}{l}109515 \\
111156\end{array}$ & $\begin{array}{l}14724 \\
18417\end{array}$ & $\begin{array}{l}79478 \\
88575\end{array}$ \\
\hline 2014 & - & - & - & 115558 & 21973 & 94850 \\
\hline
\end{tabular}

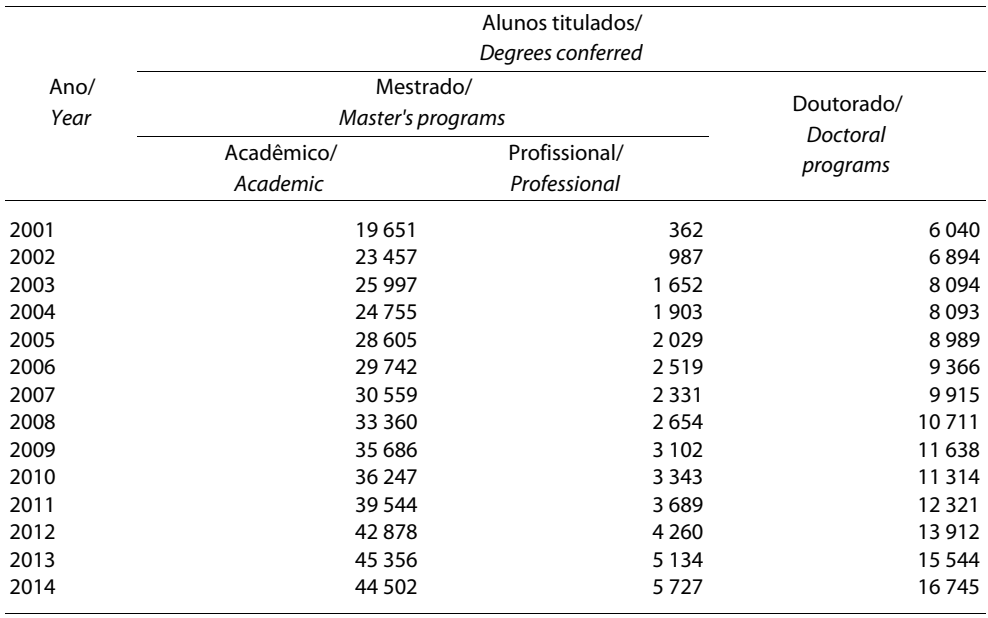

Fonte/Source: Brasil: alunos matriculados e titulados nos cursos de mestrado e doutorado, ao final do ano, 1998-2014. Brasília, DF: Ministério da Ciência, Tecnologia e Inovação, 2016. Disponível em/ Available from: <http://www.mct.gov.br/index.php/content/view/2072/Indicadores_sobre_o_ensino_ de_pos_graduacao.html>. Acesso em: abr. 2016/Cited:Apr. 2016. 
Tabela 20.4 - Instituições, grupos de pesquisa, pesquisadores e doutores em ciência e tecnologia - 2004/2014

Table 20.4 - Institutions, research groups, researchers and doctors in science and technology - 2004/2014

\begin{tabular}{|c|c|c|c|c|c|}
\hline Especificação/Item & 2004 & 2006 & 2008 & 2010 & 2014 \\
\hline Instituições/Institutions & 335 & 403 & 422 & 452 & 492 \\
\hline Grupos de pesquisa/Research groups & 19470 & 21024 & 22797 & 27523 & 35424 \\
\hline Pesquisadores/Researchers & 77649 & 90320 & 104018 & 128892 & 180262 \\
\hline Doutores/Doctors & 47973 & 57586 & 66785 & 81726 & 116427 \\
\hline
\end{tabular}

Fonte/Source: Brasil: instituições, grupos, pesquisadores e pesquisadores doutores, cadastrados no diretório dos grupos de pesquisa do CNPq, 1993/2014. Brasília, DF: Ministério da Ciência, Tecnologia e Inovação, 2016. Tab. 3.6.1. Disponível em/Available from: <http://www.mct.gov.br/index.php/ content/view/2073/Indicadores_dos_grupos_de_pesquisa.html>. Acesso em: abr. 2016/Cited:Apr. 2016.

\section{Tabela 20.5 - Artigos brasileiros e do mundo publicados em periódicos científicos internacionais indexados pela Scopus e percentual do Brasil em relação ao mundo em número de artigos e de citações recebidas - 2000-2013}

Table 20.5 - Brazilian and World papers published in international scientific journals indexed by Scopus and Brazilian relative contribution to World publication and citation - 2000-2013

\begin{tabular}{lcccc}
\hline & \multicolumn{2}{c}{$\begin{array}{c}\text { Número de artigos/ } \\
\text { Papers }\end{array}$} & \multicolumn{2}{c}{$\begin{array}{c}\text { Brasil em relação ao mundo (\%)/ } \\
\text { Brazil in relation to the World (\%) }\end{array}$} \\
\cline { 2 - 5 } Year & $\begin{array}{c}\text { Bundo/ } \\
\text { Brasil/ }\end{array}$ & $\begin{array}{c}\text { Número de artigos/ } \\
\text { Number of papers }\end{array}$ & $\begin{array}{c}\text { Citações recebidas/ } \\
\text { Citations }\end{array}$ \\
\hline 2000 & & & & \\
2001 & 13739 & 1170591 & 1,2 & 0,9 \\
2002 & 14425 & 1221254 & 1,2 & 0,9 \\
2003 & 16619 & 1270808 & 1,3 & 1,1 \\
2004 & 18744 & 1341271 & 1,4 & 1,1 \\
2005 & 21935 & 1484839 & 1,5 & 1,3 \\
2006 & 24852 & 1669046 & 1,5 & 1,4 \\
2007 & 32151 & 1758652 & 1,8 & 1,5 \\
2008 & 34765 & 1853442 & 1,9 & 1,8 \\
2009 & 40118 & 1935132 & 2,1 & 1,8 \\
2010 & 43959 & 2035770 & 2,2 & 1,8 \\
2011 & 47256 & 2144982 & 2,2 & 1,8 \\
2012 & 51060 & 2278411 & 2,2 & 1,9 \\
2013 & 56195 & 2345088 & 2,4 & 1,9 \\
\hline
\end{tabular}

Fonte/Source: SCImago Journal \& Country Rank. New York: Elsevier, [2016]. Disponível em: <http:// www.scimagojr.com/>. Acesso em: abr. 2016/Available from : abr. 2016/Cited: Apr. 2016. 
Tabela 20.6 - Pedidos depositados e decisões dos processos

sobre patentes - 2010-2015

Table 20.6 - Patent applications filed and decisions of the files

about patents - 2010-2015

\begin{tabular}{|c|c|c|c|c|c|c|}
\hline Especificação/Item & 2010 & 2011 & 2012 & $\begin{array}{c}2013 \\
(1)\end{array}$ & $\begin{array}{c}2014 \\
(1)\end{array}$ & $\begin{array}{c}2015 \\
(1)\end{array}$ \\
\hline Pedidos depositados/Applications filed & 28099 & 31881 & 33569 & 34050 & 33086 & 33043 \\
\hline Privilégio de invenção/Invention & 24986 & 28658 & 30435 & 30884 & 30270 & 30219 \\
\hline Modelo de utilidade/Utility model & 3005 & 3134 & 3010 & 3032 & 2710 & 2718 \\
\hline Certificado de adição/Certificate & 108 & 89 & 124 & 134 & 106 & 106 \\
\hline Decisões/Decisions (2) & 19417 & 30471 & 32378 & 15865 & 22283 & 15737 \\
\hline Patentes arquivadas/Archived patents & 12401 & 24368 & 27766 & 10372 & 16574 & 8978 \\
\hline Patentes concedidas/Granted patents & 3622 & 3813 & 3138 & 3325 & 3123 & 3895 \\
\hline Patentes indeferidas/Denied patents & 3394 & 2290 & 1474 & 2168 & 2586 & 2864 \\
\hline
\end{tabular}

Fonte/Source: Instituto Nacional da Propriedade Industrial - INPI, Assessoria de Assuntos Econômicos AECON.

(1) Dados preliminares./(1)Preliminary data. (2) Não abrangem todas as categorias de decisões./Not include all categories of decisions.

\section{Gráfico 20.1 - Dispêndios do governo federal em pesquisa e desenvolvimento, por instituições - 2013}

Graph 20.1 - Federal government expenditures on research and development, by institution - 2013

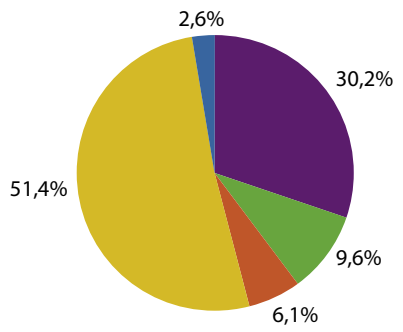

Ministério da Ciência e Tecnologia/ Ministry of Science and Technology
Ministério da Agricultura, Pecuária e Abastecimento/ Ministry of Agriculture, Livestock and Supply
Ministério da Saúde/ Ministry of Health
Ministério da Educação/ Ministry of Education
Outras/

Others

Fonte/Source: Ministério da Ciência, Tecnologia e Inovação.

(1) Dados preliminares obtidos por e-mail./ (1) Preliminary data received by e-mail. 


\section{Gráfico 20.2 - Artigos brasileiros publicados em periódicos científicos internacionais indexados pela Scopus e respectivo percentual em relação ao mundo - 2000-2013}

Graph 20.2 - Brazilian papers published in international scientific journals indexed by

Scopus and respective percent contribution in relation to the world - 2000-2013

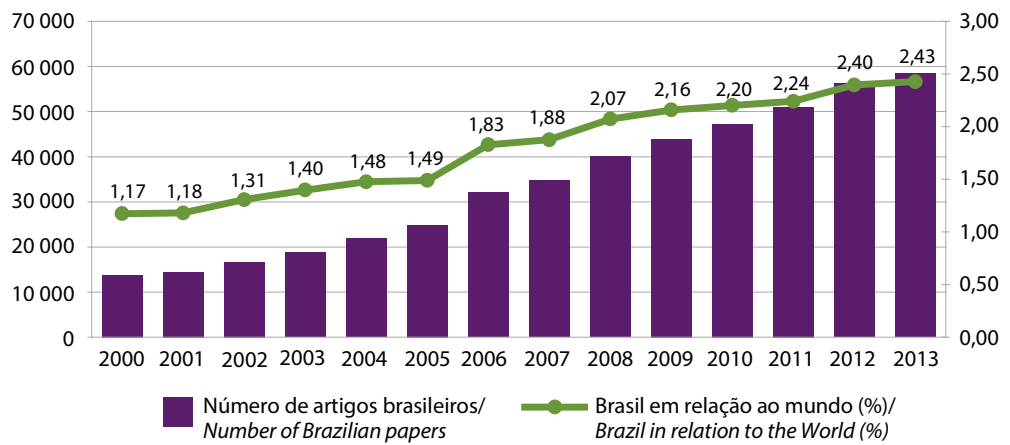

Fonte/Source: SCImago Journal \& Country Rank. New York: Elsevier, [2016]. Disponível em/Available from: <http://www.scimagojr.com/>. Acesso em: abr. 2016/Cited:Apr. 2016.

Nota: Dados preliminares obtidos por e-mail./Note: Preliminary data received by e-mail. 


\section{Poder Judiciário Judicial Power}

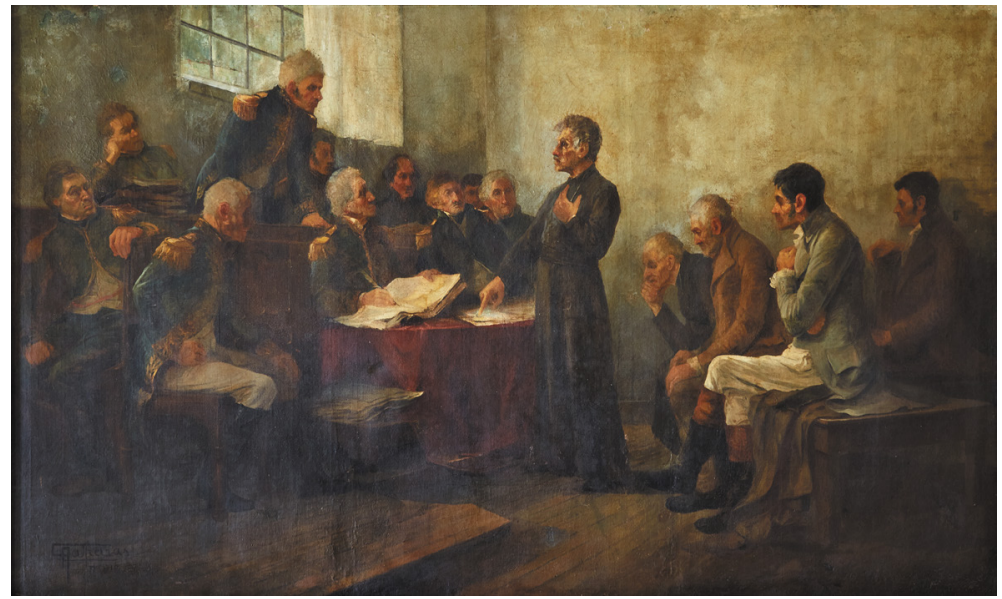

Julgamento de Padre Miguelinho/ Father Miguelinho's trial, 1918 Antônio Diogo da Silva Parreiras 



\section{Poder Judiciário}

Existem modos diversos de promover a justiça nas relações entre as pessoas. É possível solucionar conflitos por meios consensuais, intermediados ou não por conciliadores ou mediadores profissionais, no âmbito ou não das instituições do Estado, assim como há maneiras formais de demandar soluções para desacordos ou divergências. $O$ Poder Judiciário é a principal via estatal responsável pela entrega da justiça. Além dele, compõem o sistema de Justiça uma série de outras instituições, como o Ministério Público, a Defensoria Pública, a Advocacia pública ou privada, além daqueles que formulam e encaminham ao Judiciário seus pedidos formais por soluções de conflitos, sejam eles pessoas ou instituições, privadas ou partes do próprio Estado.

Este intricado arranjo de normas, instituições e rituais faz da tarefa de compreender a justiça brasileira um trabalho dos mais desafiadores. Para lidar com tal complexidade, uma primeira decisão deste capítulo se dá com a escolha por enfocar especificamente o Poder Judiciário. Ainda que seja apenas uma parte do sistema dedicado à entrega da justiça, é possível afirmar,

\section{Judiciary Power}

There are several ways to promote justice in the relations among people. You can solve conflicts by consensual means, mediated by conciliators or professional mediators or not, under the state institutions or not, just as there are formal ways to ask for solutions to disagreements or differences. The Judicial Power is the main government sphere responsible for the delivery of justice. Additionally, other institutions such as the Public Ministry and the Public Defender's Office also compose the justice system. Public or private law offices are instruments used by those who formulate and forward to the Justice System formal requests for conflict resolution, whether as individuals or institutions, private entities or as parts of the State.

The complex arrangement of rules, institutions and rituals makes the task of understanding the Brazilian Justice work a great challenge. In order to deal with such complexity, we have chosen to focus specifically on the Judicial Power as the starting point of this chapter. Although it is only one part of the system dedicated to the delivery of justice, one can say without much 
sem grandes chances de equívoco, que o Poder Judiciário é a principal expressão institucional da justiça brasileira.

\section{As Estatísticas Judiciais Oficiais no Brasil}

A principal fonte de estatísticas oficiais de amplo espectro sobre o Poder Judiciário Brasileiro é o Sistema de Estatísticas do Poder Judiciário (SIESPJ). Seu produto mais relevante é o Relatório Justiça em Números 1, já em sua décima edição2. Todo o Sistema está sob a responsabilidade do Conselho Nacional de Justiça, e consiste na principal fonte primária para uma série de decisões em termos de política judiciária no Brasil, já estando incorporado nas rotinas dos tribunais e das unidades judiciárias.

\section{Além de atualizar os principais indicadores da Justiça, nesta segunda oportunidade de contribuir para o Brasil em Números, optou-se por abordagens mais específicas dos dados existentes, possibilitando maiores aprofundamentos, sobretudo das informações sobre a litigiosidade no Brasil. Duas dimensões principais da litigiosidade serão enfocadas, a saber, a diferenciação entre processos que tramitam em primeira e em segunda instância, e a distinção entre as fases de um mesmo processo dedicadas, primeiro, ao conhecimento e, em seguida, à execução. Tais escolhas são}

\footnotetext{
' Para mais informações, veja: http://www.cnj.jus.br/ pesquisas-judiciarias

${ }^{2}$ Embora sejam compiladas estatísticas judiciais desde 1989, em 2009 passou a viger a Resolução n. 76, do Conselho Nacional de Justiça, que alterou os conceitos até então empregados para a coleta das estatísticas oficiais do Judiciário. Por tal motivo, consideram-se comparáveis apenas os dados coletados a partir desta dada, perfazendo um total de seis anos.
}

chance of misunderstanding, that the Judicial Power is the main institutional expression of the Brazilian justice.

\section{The Official Judicial Statistics in Brazil}

The main source of broad-spectrum official statistics on the Brazilian Judicial Power is the National System of Statistics of the Judicial Branch (SIESPJ). Its most important product is Justice: Facts and Figures Report1, now in its tenth edition2. The entire system is under the responsibility of the National Council of Justice and it is the main primary source for several decisions in terms of judicial policy in Brazil, having been incorporated into the routines of the courts and judicial units.

In this second opportunity to contribute to the Brazil in Figures report, in addition to the mandatory update in the Brazilian main justice indicators, we have decided to present more specific approaches regarding existing data, enabling greater insights, especially considering information on litigation. Two main litigation dimensions will be focused on. The first one is the distinction between ongoing lawsuits at first and second instance. The second will be the distinction between phases of the same lawsuits, firstly within the acknowledgment phase and secondly, within the enforcement phase. Such

\footnotetext{
${ }^{1}$ For more information, see: http://www.cnj.jus.br/ pesquisas-judiciarias

${ }^{2}$ Although judicial statistics have been compiled since 1989, only in 2009 came into effect Resolution no. 76, of the National Coucil of Justice, which changed the concepts then adopted for the collection of official judicial statistics. For this reason, data are considered comparable only when collected from that date on, for six years now.
} 
oportunas pois é a partir delas que são definidas importantes políticas institucionais em prol da melhoria da entrega da justiça no Brasil.

\section{A estrutura Judiciária em 2014}

As grandes linhas dos números mais recentes do Poder Judiciário, referentes a 2014, pouco mudaram quando comparadas aos dados disponíveis no período imediatamente anterior (2013). Em termos estruturais, em um ano, as despesas do Judiciário cresceram na ordem de 4,3\% (tabela 21.1). Tal montante equivale a $1,2 \%$ do Produto Interno Bruto (PIB) nacional e a 2,3\% dos gastos totais da União, dos Estados e dos Municípios, equivalendo a dizer que o Poder Judiciário custa R\$ 337,00 por habitante.

Também não há mudanças significativas na composição destas despesas, pois, desde o início da série histórica, cerca de $90 \%$ do que a Justiça brasileira despende é utilizado para pagamento de pessoal. $O$ ano de 2014 também não revela mudanças no nível de concentração das despesas por ramos de Justiça, sendo a Justiça Estadual responsável por $55 \%$ do total gasto pelo Judiciário, seguida pela trabalhista $(20,8 \%)$ e pela Justiça Federal $(12,7 \%)$, os três maiores ramos tanto em termos de recursos humanos e orçamentários, quanto em termos do número de processos judiciais recebidos e julgados (tabela 21.2).

Uma novidade pouco alentadora refere-se ao significativo decréscimo das arrecadações advindas da atividade jurisdicional. Ainda que o Poder Judiciário tenha retornado aos cofres públicos $39,4 \%$ do total dos recursos que despendeu neste choices are appropriate because they are sources of important institutional policies aimed at improving the delivery of justice in Brazil.

\section{Judicial Structure}

The broad figures of the Judiciary, referring to 2014, changed very little when compared to the data available from the previous year (2013). Structurally, in a year, Judicial expenses increased in the order of $4.3 \%$ (Table 21.1). This amount is equivalent to $1.2 \%$ of the Gross Domestic Product (GDP) and to $2.3 \%$ of total expenditures of Brazil, its states and municipalities. That means to say the Judicial Power has a per capita cost of $\mathrm{R} \$ 337.00$.

There are no significant changes in the composition of expenditure. Since the beginning of the historical series, about $90 \%$ of the whole Brazilian Justice expenses are used for staff payment. The year 2014 also shows no changes in the level of expenditure concentration by Justice Branches. The State Courts are responsible for $55 \%$ of the total spending by the judiciary, followed by Labour Justice (20.8\%) and Federal Justice $(12,7 \%)$. These are the three major branches both in terms of human and budgetary resources and in terms of the number of received and judged lawsuits (table 21.2).

Rather discouraging news refers to the significant decrease in collections arising from judicial activity. Although the judiciary has returned to public coffers $39.4 \%$ of total resources spent in the same year, this 
mesmo ano, tal percentual foi o menor desde 2009, com queda de dez pontos percentuais em relação a 2013 (tabela 21.1).

Havia 17.558 magistrados ativos ao final de $2014,85,8 \%$ deles atuando nas cerca de 15 mil unidades judiciárias de primeira instância, a porta de entrada da grande maioria das ações judiciais3. Embora o número de magistrados seja crescente em quase toda a série histórica, ainda há 4,9 mil cargos de magistrados vagos, o que representa $22 \%$ do total de 22,5 mil cargos criados por lei. Em relação à população brasileira, são 8 magistrados para cada 100 mil habitantes, número inferior a países como Colômbia $(11,7)$, Federação Russa (24,2), Portugal (18), Espanha $(10,7)$ Itália $(10,2)$ e Estados Unidos da América, com nove 9 magistrados (CNJ, 2011).

Além dos juízes, ao final de 2014, cerca de 278,7 mil servidores trabalhavam direta ou indiretamente em prol da solução dos conflitos. Somados apenas os que lidam diretamente com processos judiciais, são 218,2 mil, ou $78 \%$ do total da força de trabalho. Destes, 182,6 mil (84\%) trabalham nas unidades judiciárias de primeira instância. Além dos magistrados e dos servidores, devem ser somados à força de trabalho judiciária 139,3 mil trabalhadores auxiliares, entre funcionários terceirizados (51\%), estagiários (43\%), conciliadores (5\%) e juízes leigos (1\%).

Cada magistrado possui, em média, 13 servidores em auxílio à atividade de julgar processos. A primeira instância dos tribunais, onde tramitaram $92 \%$

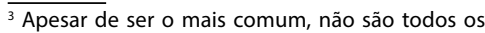
processos que ingressam na $1^{\mathrm{a}}$ instância. São possíveis processos iniciados na segunda instância, e também nos tribunais superiores.
}

percentage was the lowest since 2009, down ten points compared to 2013 (Table 21.1).

There were 17,558 active judges at the end of $2014,85.8 \%$ of them working in about 15,000 judicial units of first instance, the vast majority of the entrance door for pending lawsuits ${ }^{3}$. Although the number of judges is increasing in almost the entire series, there are still 4,900 vacant positions of judges, representing $22 \%$ of the total of 22,500 positions created by law. Regarding the Brazilian population, there are eight judges for every 100 thousand inhabitants, fewer than countries like Colombia (11.7), Russian Federation (24.2), Portugal (18), Spain (10.7), Italy $(10,2)$ and the United States of America, with nine 9 magistrates (CNJ, 2011).

In addition to the number of judges at the end of 2014 , about 278,700 civil servants worked directly or indirectly in favour of conflict resolution. There are 218,200 of them who deal directly with lawsuits, meaning $78 \%$ of the total workforce. Out of these, 182,600 (84\%) work in the judicial units of First Instance. In addition to the judges and workers, another 139,300 auxiliary workers form the legal labour force, for example: outsourced employees (51\%), trainees $(43 \%)$, conciliators $(5 \%)$ and lay judges (1\%).

Each Brazilian judge has the aid of an average of 13 civil servants to the activity of judging lawsuits. The first instance of the courts, in which $92 \%$ of

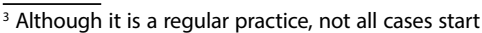
in the 1st instance. Some start in 2 nd instances and even in higher courts.
} 
dos processos, concentra $86 \%$ dos magistrados e $84 \%$ dos servidores especificamente dedicados ao trabalho de tramitar e julgar processos. Ainda que haja claras desproporções entre recursos humanos e carga de processos entre as instâncias, este é um dos pontos em que são necessários aprimoramentos nas estatísticas oficiais, além de estudos mais detalhados.

\section{A litigiosidade brasileira em 2014 Brazilian Litigation in 2014}

A estrutura disponível aos 90 tribunais que atualmente fazem parte do Sistema de Estatísticas do Poder Judiciário (SIESPJ) é dedicada principalmente a receber, distribuir, julgar (fase de conhecimento) e fazer cumprir (fase de execução) o que foi decidido pelos magistrados. $\mathrm{O}$ ano de 2014 iniciou já com 70,8 milhões de processos aguardando desfechos. Ao longo do período, ainda ingressaram mais 28,9 milhões de novas ações e foram julgados 28,5 milhões. Estima-se, portanto, que, ao final do ano de 2014, o total de processos pendentes de finalização tenha aumentado em meio ponto percentual, ultrapassando, assim, 71,2 milhões de processos pendentes em dezembro daquele ano.

Apesar de o cenário ser desfavorável, é possível afirmar que a produtividade dos magistrados e dos servidores é elevada. Em média, cada magistrado soluciona 7 processos por dia, o que equivale a um ritmo diário de $113 \mathrm{mil}$ casos solucionados. Mesmo que isso indique elevada capacidade produtiva das cortes brasileiras, são necessárias ações sistemáticas e coordenadas para que, em algum momento, o estoque de processos pendentes de julgamento possa começar a diminuir de modo the cases are processed, concentrates $86 \%$ of judges and $84 \%$ of civil servants dedicated to legal cases as they move through and are judged. Although there is a clear imbalance between the available human resources and the caseload between instances, this is one of the points which require improvements in the official statistics, besides detailed studies.

The infrastructure available in the 90 courts that are currently part of the SIESPJ is dedicated primarily to receive, distribute, judge (acknowledgment stage) and enforce (execution stage) judges' rulings and verdicts. Year 2014 has started with a backlog of 70.8 million pending lawsuits. Over the yearly period, additional 28.9 million new lawsuits were added. Unfortunately, only 28.5 million of the grand total was cleared. Therefore, it is estimated that at the end of 2014, the total lawsuit backlog had increased by half a percentage point, exceeding, thus, 71.2 million pending lawsuits in December of that year.

Although the scenario is unfavourable, it is clear that the judges and civil servants productivity is high. On average, each judge solves seven cases each day, which is equivalent to a daily rate of 113,000 judgments and rulings for the whole Brazilian Justice. Even if this indicates the high performance of Brazilian courts, systematic and coordinated actions are needed so that, at some point, the stock of pending lawsuits can begin to decline significantly. As a result of the 
significativo. Como consequência do aumento contínuo de processos, a Taxa de Congestionamento4 do Poder Judiciário foi de $71,4 \%, 0,8$ pontos percentuais maior em relação a 2013. Isso significa que a cada 100 processos que tramitaram em 2014, apenas 28,6 foram finalizados no período de um ano.

A taxa de congestionamento é, atualmente, o indicador que mais se aproxima da descrição da realidade de lentidão dos processos judiciais. $\mathrm{Na}$ ausência de um indicador de tempo que, em nível nacional, permita aferir o número de dias do início ao final de um processo5, é com base no conceito de congestionamento que se infere que $o$ Judiciário é lento em demasia.

Ao decompor o congestionamento dos tribunais brasileiros, é possível concluir, por exemplo, que a Justiça Estadual é mais congestionada que as demais $(74,2 \%)$, e a trabalhista a menos dentre os três maiores ramos (49,8\% - tabela 21.2). É também facilmente perceptível que a fase de execução, aquela que se dá posteriormente à decisão final do juiz, dedicada a tornar concretos direitos assegurados na fase de conhecimento, apresenta os piores congestionamentos, pois, de cada 100 processos de execução iniciados, apenas 14,4 são finalizados no período de 12 meses (taxa de congestionamento de $85,6 \%$ ). Enfocadas apenas as execuções fiscais, processos destinados à recuperação

\footnotetext{
${ }^{4} \mathrm{~A}$ Taxa de Congestionamento mede a efetividade do Judiciário em um período, levando-se em conta o total de casos novos que ingressaram, os casos baixados e o estoque. É calculado pela divisão do total de casos baixados pela soma de casos novos e pendentes em um mesmo período.

${ }^{5}$ A próxima edição do relatório Justiça em Números conterá, pela primeira vez, informações oficiais e abrangentes sobre o tempo do processo no Brasil.
}

continuing increase in new lawsuits, the Congestion Rate 4 of the Judiciary was $71.4 \% ; 0.8$ percentage points higher than in 2013. This means that out of every 100 lawsuits that went through the judiciary workflow in 2014 , only 28.6 were completed within one year.

The congestion rate is the indicator that most closely matches the description of the slow reality of court proceedings nowadays. Since we still do not have a national time indicator that can measure the number of days between the start and the end of a lawsuits5, the concept of congestion rate demonstrates that, in general terms, the Brazilian judiciary is way too slow.

When breaking down the Brazilian courts congestion rate, it is possible to notice, for instance, that congestion is highest in the State justice $(74.2 \%)$ and lowest in Labour among the three major justice branches $(49.8 \%$ - Table 21.2 ). It is also apparent that the enforcement phase, the one that comes immediately after the final ruling by the judge, has the worst congestion rate. For each 100 enforcement proceedings started, only 14.4 are finalized in 12 months (85.6\% congestion charge). Regarding tax foreclosures, which are lawsuits aimed to recover public outstanding

${ }^{4}$ The Congestion Rate measures the effectiveness
of the Judiciary in a given period, taking into
consideration the total number of new cases, of cases
cleared and the backlog of old cases. It is calculated
by the division of the total cases cleared by the sum of
new and pending cases in the same period.
${ }^{5}$ The next edition of the report Justice in Figures will
present, for the first time, official and comprehensive
information about the length of processes in Brazil. 
de créditos públicos inscritos em dívida ativa, como impostos não pagos6, o congestionamento alcança o maior patamar observado, pois apenas nove de cada 100 processos são finalizados ${ }^{7}$.

Outra constatação importante refere-se às diferenças na tramitação das ações judiciais na primeira e na segunda instâncias. Enquanto no primeiro grau a taxa de congestionamento é de $73 \%$, no segundo é de $50 \%$. Tal disparidade é maior na Justiça Estadual e bem menos acentuada nas Justiças Federal (diferença de apenas três pontos percentuais) e do Trabalho, ramo em que a diferença entre as instâncias foi de 15 pontos percentuais.

As comparações entre indicadores separados entre as instâncias de julgamento são importantes meios para ponderar sobre os critérios de priorização dos recursos nas gestões internas dos tribunais. Como visto na seção anterior, há desproporções estruturais entre as instâncias, que são injustificáveis pelo volume de trabalho. A carga de trabalho dos juízes de primeiro grau (6.521 processos por ano) é o dobro daquela a que estão sujeitos os desembargadores (3.305). Não é à toa que o congestionamento entre as instâncias é tão desproporcional. Ainda que os juízes e os servidores das instâncias iniciais julguem em média mais processos que os do 20 grau, as diferenças em produtividade ainda são bem menores que a observada na carga de trabalho. Enquanto desembargadores solucionaram 1.414 processos, em média, em 2014, os demais juízes baixaram debts such as unpaid taxes6, congestion rate reaches the highest observed level, since only nine out of 100 cases are finalized? ${ }^{7}$.

Another important finding relates to differences in the lawsuits processing within first and second instances. While in first instance, the congestion charge is $73 \%$, in the second it is $50 \%$. This disparity is greater in State Justice and less pronounced in the Federal Justice, with a difference of only three percentage points. In Labour branch, the difference between instances was 15 percent.

Comparisons between indicators from judgment instances are important ways to ponder about the resource prioritization criteria in the courts internal efforts. As seen in the previous section, there are structural disproportions between first and second instances that cannot be justified by the workload. The first instance judges' workload $(6,521$ lawsuits per year) is twice as that of second instance magistrates $(3,305)$. It is no surprise that congestion between instances is so disproportionate. Although the judges and workers of the first instances judge more processes than those of the second do, differences in productivity are still much lower than that observed in the workload. While second instance magistrates solved 1,414 cases on

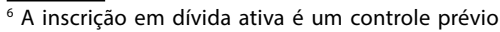
de legalidade da dívida, que torna o débito apto à cobrança.

${ }^{7}$ Para maiores aprofundamentos, veja o relatório "Custo unitário do processo de execução fiscal" (IPEA/ CNJ, 2011)
}

${ }^{6}$ The oustanding debt is a way of previous control of debt legality, which makes it entitled to charge.

${ }^{7}$ For further information, see the report "Custo unitário do processo de execução fiscal" (IPEA/CNJ, 2011) 
pouco mais de 1.700. Embora maior, para se desvencilharem da totalidade de processos existentes em 2014, a produtividade dos juízes de primeira instância deveria ser quase quatro vezes maior, de 6.521 processos por ano. Já na segunda instância, os juízes teriam carga de trabalho de apenas 3.305 processos se fossem efetivamente entregar todos os casos que aguardam solução.

Além de uma agenda de priorização baseada nas diferenças hierárquicas e de poder entre as unidades, também é preciso reponderar a atenção das autoridades do Poder Judiciário para as duas principais fases de um processo, a saber, aquela em que são conhecidos e reconhecidos os direitos pleiteados, daquela em que tais direitos são efetivados por meio da execução. Embora pareça uma mera calibragem da atenção dos gestores, é preciso compreender que a fase de execução depende de fatores que vão mais além do trabalho do magistrado, sendo o momento em que são precisos mecanismos mais eficientes de busca de pessoas e de bens, envolvendo articulações entre instituições, com grande espaço para soluções informacionais e tecnológicas.

Para pontuar com ainda mais ênfase essas diferenças, é oportuno revelar que, em média, magistrados de primeiro grau julgaram 1.234 processos de conhecimento e apenas 431 processos de execução por ano, justificando os já mencionados congestionamentos tão díspares. E, neste caso, não se pode afirmar que tal desproporção se justifica por desigualdades na destinação dos recursos, já que estão sendo comparados apenas processos da primeira instância. average in 2014, the first instance judges concluded just over 1,700 . Although greater, in order to clear all existing processes in 2014, the productivity of first instance judges should be almost four times higher: 6,521 cases per year. In the second instance, the judges would have a 3,305 cases workload if they effectively delivered all cases awaiting solution.

In addition to a prioritization schedule based on hierarchical and power differences between the units, it is also necessary to reconsider the attention of the judicial authorities to the two main stages of a lawsuit, namely, when the claimed rights are acknowledged and when such rights are effected by means of enforcement and foreclosure. Although it seems to be a simple matter of adjustment of management attention, it is necessary to understand that the enforcement phase depends on factors that go beyond the magistrate's work. The development of more efficient mechanisms for the search of persons and goods involving joint efforts between institutions, with great space for informational and technological solutions.

To emphasize these differences, it is appropriate to state that, on average, first-degree judges ruled on 1,234 acknowledgment lawsuits and only 431 enforcement lawsuits a year, justifying the aforementioned disparate congestion. In this case, it cannot be said that this disproportion is justified by inequalities in the resources allocation, since we are comparing only first instance lawsuits. 
Tais disparidades entre as fases de um processo conformam um importante exemplo da necessidade de se diversificar os focos da atuação institucional. Além da adoção de critérios alocativos mais condizentes com as diferenças entre as instâncias, é preciso desenvolver meios mais efetivos de fazer valer os direitos já garantidos por uma decisão judicial. Isso é crucial, sobretudo porque de nada adiantará garantir um direito ao cidadão, mas fazê-lo aguardar ainda mais tempo do que já esperou para que esse direito tenha efeitos concretos.

\section{Do diagnóstico às políticas judiciárias}

Do diagnóstico apresentado, é possível derivar que há excesso de processos no Brasil, que há pontos de estrangulamento de fácil identificação, sobretudo na justiça estadual, na primeira instância e na fase de execução. Quando há dados disponíveis, eles sugerem que as desproporções entre graus e fases de julgamentos também podem derivar de desigualdades na distribuição de recursos, muito embora ainda não haja informações suficientes para conclusões robustas a esse respeito, sobretudo pela ausência de algumas aberturas dos dados de estrutura por instância e fases do processo.

Tais diagnósticos, já conhecidos dos gestores judiciais, têm motivado soluções as mais diversas. Há soluções que endereçam a melhoria estrutural dos trâmites processuais, sendo uma das mais relevantes a implantação de processos judiciais eletrônicos. Ao uniformizar e automatizar seus trâmites, as comunicações dos seus atos e a própria transmissão das peças que
Such disparities between the phases of a lawsuit confirm the need to diversify the focus of institutional performance. In addition to the adoption of more consistent allocation criteria in relation to the differences between the instances, it is necessary to develop more effective means of enforcing rights already guaranteed by a judicial ruling. This is crucial, especially because it will help secure the rights of all citizens without them having to wait even longer than they already have in order to see that right have concrete effects.

\section{From Diagnosis to Judicial Policies}

From the presented diagnosis, it is possible to see that there is an excessive amount of lawsuits in Brazil. There are some easily identified bottlenecks, particularly in state courts in the first instance and in the enforcement phase. When supporting data is available, it is suggested that the imbalance between instances $\left(1^{\text {st }}\right.$ and $\left.2^{\text {nd }}\right)$ and lawsuit phases (acknowledgment and enforcement) can also derive from inequalities in the resources distribution, even though at this moment there is not enough information to draw firm conclusions in this respect, particularly due to the absence of some structural data by instance and stages.

Such diagnoses, already known by judicial managers, have motivated the most diverse solutions. There are those who address the structural improvement of the lawsuit workflow. One of the most significant is the deployment of electronic lawsuits. By standardizing and automating its procedures, communications of acts and the actual transmission of the 
compõem as ações judiciais, esta via de atuação institucional empenha-se, sobretudo, em reduzir o tempo total até a solução dos conflitos. Do total de processos que chegaram às varas e tribunais em 2014, quase a metade (45\%) já ingressaram e tramitaram eletronicamente, um crescimento de 14 pontos percentuais em relação a 2013.

Além de investimentos em medidas estruturais, com destaque para o processo eletrônico e seus óbvios ganhos de produtividade, o Poder Judiciário tem apostado também na redução do número de conflitos que se transformam em ações judiciais, especialmente pelo incentivo aos meios consensuais de solução de conflitos. Esse tema está estampado em metas nacionais do Judiciário ${ }^{8}$, campanhas, é objeto de mutirões anuais desde 2006, constou de prêmios institucionais, de numerosas capacitações e também de cooperações formais junto a órgãos de outros poderes e também com instituições privadas ligadas aos grandes litígios.

O ano de 2016 é promissor nesse quesito, pois também passará a viger o Novo Código de Processo Civil (CPC), legislação que elevou a importância da conciliação e da mediação, tornando obrigatória a fase conciliatória, além de antecipá-la para logo após o início do processo. No âmbito das estatísticas judiciárias, já em 2016 será possível saber quantos processos foram finalizados por meio da conciliação, algo até então desconhecido.

\section{Além de otimizar a máquina estatal ao solucionar conflitos sem,}

\footnotetext{
${ }^{8}$ A exemplo da Meta Nacional n³ de 2015, estabelecendo a necessidade de aumento dos casos solucionados por conciliação.
}

documents that make up lawsuits, this institutional action strives, above all, to reduce the total conflict resolution time. From the total amount of lawsuits that reached the local courts and tribunals in 2014, almost half (45\%) have already started and been electronically processed, an increase of 14 percentage points compared to 2013.

In addition to investments in structural measures, especially the electronic process and its obvious productivity gains, the Judiciary has also bet on reducing the number of conflicts that result in lawsuits, especially by encouraging consensual means of conflict resolution. This theme is included in the National Judiciary Goals ${ }^{8}$. Since 2006, it has been the subject of annual joint efforts consisting of institutional awards, staff training and also the development of formal cooperation with other governmental powers and private institutions linked to the great disputes.

The year 2016 is a promising one in this regard, since the new Civil Procedure Code (CPC) also will come into effect. This regulation increased the importance of conciliation and mediation, making the conciliatory stage mandatory, anticipating it to right after the lawsuit inception. Within the scope of judicial statistics, in 2016 it will be possible to know how many lawsuits were concluded through conciliation efforts, something unknown hitherto.

In addition to the state machinery optimization aimed at solving conflicts

\footnotetext{
${ }^{8}$ The National Goal \# 3, 2015, establishing the need for increase in cases resolved by conciliation.
} 
necessariamente, ter de passar por todas as fases de uma ação judicial, o novo CPC também ampliou as formas de resolver muitos processos de natureza parecida. Os já existentes processos com repercussão geral e aqueles reconhecidos como recursos repetitivos, são, na verdade, filtros prévios definidos nos tribunais superiores que, ao identificarem repetições de demandas, suspendem o trâmite de todos os processos parecidos, em todos os tribunais, até que uma ação representativa deste tipo de controvérsia seja julgada por eles. Uma vez julgado nos tribunais superiores, a todos os demais processos suspensos poderá ser dado o mesmo desfecho, com grande economia de recursos. $O$ que até então funcionava apenas com processos que alcançavam o Supremo Tribunal Federal (STF) e o Superior Tribunal de Justiça (STJ), a partir do novo CPC passará a ser aplicado nos demais tribunais superiores e nas segundas instâncias de todas as justiças.

O contexto mais recente do Poder Judiciário apresenta-se envolto a uma série de inovações, com efeitos ainda pouco sentidos, mas com muitas possibilidades promissoras de recaracterização da forma de se prestar o serviço do Judiciário. Além das mudanças no Judiciário, estão sendo melhoradas, como se comentou ao longo do capítulo, também as estatísticas judiciais brasileiras, com efeitos a serem sentidos já em 2016. Haverá informações ainda mais detalhadas sobre o conjunto do Poder Judiciário, pois será possível conhecer o tempo do processo, até a sentença e até a baixa definitiva do processo. Também saber-se-á de modo padronizado e contínuo quantos processos foram solucionados por meio da conciliação e quantos estão sobrestados ou suspensos without necessarily having to go through all the stages of a lawsuit, the new CPC has also expanded the ways to solve many similar cases. Existing general impact lawsuits and those defined as repetitive appeals are previous filters set in the higher courts, to identify demand repetitions, suspend the processing of all similar cases in all courts until they judge a representative lawsuit of this dispute type. Once ruled in higher courts, all other suspended lawsuits can have the same outcome, with great economy of resources. What has so far worked only with lawsuits that reached the Supreme Federal Court (STF) and the Superior Court of Justice (STJ), from the new CPC inception will be applied in other higher courts and in the second justice instance.

The most recent context of the Judicial Power shows a series of innovations, with still few noticeable effects, however, open to many promising possibilities for redefining the way to provide judicial services. In addition to structural and procedural improvements in the Brazilian judiciary, the judicial statistics are getting even better, as commented throughout the chapter, with practical results in 2016 already. There will be more detailed information available about the entire judiciary; it will be possible to know the lawsuit time until the judgment and ruling and until the final lawsuit clearance. Additionally, it will be possible to know how many cases have been 
por motivo de repercussão geral e recurso repetitivo. Ao identificá-los desta forma, será possível qualificar a informação do congestionamento, pois será possível mensurar o impacto causado na taxa de congestionamento em virtude dos processos suspensos, sobrestados ou em arquivo provisório. resolved through conciliation and how many lawsuits have been suspended for general impact and/or repetitive use reasons. By identifying them in this way, we can assess the congestion information; it will also be possible to measure the impact on the congestion rate because of suspended or temporary filed lawsuits.

\section{Referências/References}

Conselho Nacional de Justiça (CNJ). “Justiça em Números 2015: Ano-Base 2014". Brasília: Departamento de Pesquisas Judiciárias, Conselho Nacional de Justiça, 2015.

. “Justiça em Números 2014: Ano-Base 2013". Brasília: Departamento de Pesquisas Judiciárias,

Conselho Nacional de Justiça, 2014.

“Estudo Comparado sobre Recursos, Litigiosidade e Produtividade: a prestação jurisdicional no contexto internacional". Brasília: Departamento de Pesquisas Judiciárias, Conselho Nacional de Justiça, 2011.

Instituto de Pesquisa Econômica Aplicada (IPEA) e Conselho Nacional de Justiça (CNJ). "Custo unitário do processo de execução fiscal". Brasília, 2011.

Varella, Santiago. “Poder Judiciário”. In. Instituto Brasileiro de Geografia e Estatística (IBGE), Brasil em Números, 2015.

\section{Fernanda Paixão}

Diretora Executiva do Departamento de Pesquisas Judiciárias do Conselho Nacional de Justiça (CNJ) e especialista em administração

$$
\text { judiciária }
$$

Executive Director at the Judicial Research Department of the Brazilian National Council of Justice (CNJ)

Court administration specialist

\section{Santiago Varella}

Diretor de Projetos do Departamento de Pesquisas Judiciárias do Conselho Nacional de Justiça (CNJ), Mestre e Doutor em Sociologia pela Universidade de Brasília
Project Director at the Judicial Research Department of the Brazilian National Council of Justice (CNJ)

Master and PhD in Sociology from the University of Brasilia (UnB)

\section{Gabriela Soares}

Mestre em Estatística pela Universidade Federal do Rio de Janeiro e Pesquisadora do Departamento de Pesquisas Judiciárias do Conselho Nacional de Justiça (CNJ)
Researcher at the Judiciary Research Department of the Brazilian National Council of Justice (CNJ) Master in Statistics from the Federal University of Rio de Janeiro (UFRJ) 


\section{Igor Stemler}

Graduado em Estatística pela Universidade de Researcher at the Judiciary Research Department

Brasília e Pesquisador do Departamento de of the Brazilian National Council of Justice (CNJ)

Pesquisas Judiciárias do Conselho Nacional de Statistician from the University of Brasilia (UnB)

Translated by: Marcelo Conforto 


\section{Tabela 21 .1 - Informações de estrutura, recursos humanos e litigiosidade - 2010-2014}

Table 21.1 - Information on structure, human resources and litigiousness - 2010-2014

\begin{tabular}{|c|c|c|c|c|c|}
\hline Especificação/Item & 2010 & 2011 & 2012 & 2013 & 2014 \\
\hline \multicolumn{6}{|l|}{ Despesas/ } \\
\hline Expenditures & 53002586554 & 60209196928 & 64567339755 & 65588405261 & 68385447621 \\
\hline \multicolumn{6}{|l|}{$\begin{array}{l}\text { Despesa com recursos } \\
\text { humanos/Expenditure }\end{array}$} \\
\hline with human resources & 47422117787 & 53982982370 & 57309284379 & 58872655562 & 61185097511 \\
\hline \multicolumn{6}{|l|}{ Gasto com recursos } \\
\hline humanos/Expense with & & & & & \\
\hline human resources (\%) & 89,47 & 89,66 & 88,76 & 89,76 & 89,47 \\
\hline \multicolumn{6}{|l|}{ Receitas/ } \\
\hline Revenue & 25261572582 & 27788819166 & 26238859760 & 32751497765 & 26928475585 \\
\hline \multicolumn{6}{|l|}{$\begin{array}{l}\text { Receitas/Despesas/ } \\
\text { Revenue/Expenditures }\end{array}$} \\
\hline (\%) & 47,66 & 46,15 & 40,64 & 49,93 & 39,38 \\
\hline \multicolumn{6}{|l|}{ Magistrados/ } \\
\hline Judges & 16397 & 16413 & 16138 & 16477 & 16927 \\
\hline \multicolumn{6}{|l|}{ Servidores/ } \\
\hline Servants & 230781 & 263365 & 271593 & 276773 & 278707 \\
\hline \multicolumn{6}{|l|}{$\begin{array}{l}\text { Força de trabalho } \\
\text { auxiliar/Auxiliary }\end{array}$} \\
\hline workforce & 94233 & 102201 & 125528 & 135984 & 139298 \\
\hline \multicolumn{6}{|l|}{$\begin{array}{l}\text { Carga de Trabalho por } \\
\text { magistrado/Caseload }\end{array}$} \\
\hline perjudge & 5309 & 5544 & 5961 & 6041 & 6130 \\
\hline \multicolumn{6}{|l|}{ Casos novos/ } \\
\hline New cases & 24005741 & 26077097 & 28032551 & 28557871 & 28878663 \\
\hline \multicolumn{6}{|l|}{ Casos pendentes/ } \\
\hline Pending cases & 60737579 & 62013807 & 64451285 & 67131040 & 70828587 \\
\hline \multicolumn{6}{|l|}{ Processos baixados/ } \\
\hline Closed cases & 24132797 & 25794463 & 27697102 & 28098166 & 28498708 \\
\hline \multicolumn{6}{|l|}{$\begin{array}{l}\text { Processos baixados por } \\
\text { caso novo/Closed cases }\end{array}$} \\
\hline per new case (\%) & 100,53 & 98,92 & 98,80 & 98,39 & 98,68 \\
\hline \multicolumn{6}{|l|}{ Taxa de } \\
\hline \multicolumn{6}{|l|}{ Congestionamento/ } \\
\hline Backlog rate (\%) & 71,52 & 70,85 & 70,05 & 70,64 & 71,42 \\
\hline
\end{tabular}

Fonte/Source : Justiça em números 2011-2015. Brasília, DF: Conselho Nacional de Justiça - CNJ, 20112015. Disponível em/Available from: <http://www.cnj.jus.br/>. Disponível em: abr. 2016/Cited:Apr. 2016. 


\section{Tabela 21.2 - Informações de estrutura, recursos humanos e}

litigiosidade, por ramos de Justiça - 2014

Table 21.2 - Information on structure, human resources and

litigiousness, by courts of justice - 2014

(continua/to be continued)

$\begin{array}{lccc}\text { Justiça/ } & \text { Estadual/ } & \text { Federal/ } & \text { Trabalhista/ } \\ \text { Justice } & \text { State } & \text { Federal } & \text { Labor }\end{array}$

Despesas/

Expenditures

37598870632

8710192624

14203126022

Despesa com recursos humanos/

Expenditure with human resources

$33467967734 \quad 7825802337 \quad 13273548609$

Gasto com recursos humanos/

Expense with human resources (\%)

89,01

89,85

93,46

Receitas/

Revenue

$14295729309 \quad 9840393655 \quad 2754506625$

Receitas/Despesas/

Revenue/Expenditures (\%)

38,02

112,98

19,39

Magistrados/

Judges

11631

1751

3400

Servidores/

Servants

179711

28786

41217

Força de trabalho auxiliar/

Auxiliary workforce

92048

18279

14108

Carga de Trabalho por magistrado/

Caseload perjudge

6866

7510

2685

Casos novos/

New cases

Casos pendentes/

Pending cases

57206736

8484488

4396590

Processos baixados/

Closed cases

19945948

3699229

4210711

Processos baixados por caso novo/

Closed cases per new case (\%)

99,03

91,29

105,52

Taxa de Congestionamento/

Backlog rate (\%)

74,21

70,49

49,80 


\section{Tabela 21.2 - Informações de estrutura, recursos humanos e}

litigiosidade, por ramos de Justiça - 2014

Table 21.2 - Information on structure, human resources and

litigiousness, by courts of justice - 2014

(conclusão/concluded)

\section{Justiça/ \\ Justice}

Eleitoral/

Electoral
Militar Estadual/

State Military
Tribunais

Superiores/

Superior Courts

Despesas/

Expenditures

4782707761

116899056

2973651526

Despesa com recursos humanos/

Expenditure with human resources

4023122069

102623034

2492033728

Gasto com recursos humanos/

Expense with human resources (\%)

84,12

87,79

83,80

Receitas/

Revenue

Receitas/Despesas/

Revenue/Expenditures (\%)

3,19

Magistrados/

Judges

3180

40

Servidores/

Servants

Força de trabalho auxiliar/

Auxiliary workforce

9263

112

5194

Carga de Trabalho por magistrado/

Caseload perjudge

Casos novos/

New cases

Casos pendentes/

Pending cases

Processos baixados/

Closed cases

Processos baixados por caso novo/

Closed cases per new case (\%)

Taxa de Congestionamento/

Backlog rate (\%)

36,42

33,43

58,79

Fonte/Source :Justiça em números 2015. DF: Conselho Nacional de Justiça - CNJ, 2015. Disponível em/ Available from: <http://www.cnj.jus.br/>.Acesso em: abr.2016/Cited:Apr. 2016. 


\section{Gráfico 21.1 - Percentual de processos tramitados, por ramos de Justiça - 2014}

Graph 21.1 - Percentage of cases proceeded, by courts of Justice - 2014

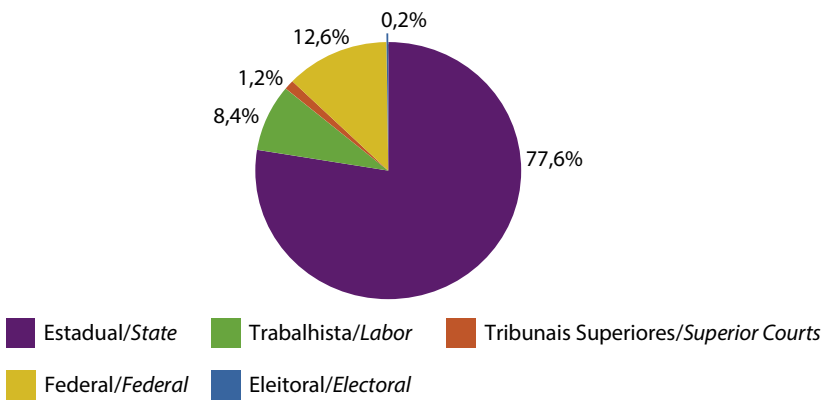

Fonte/Source:Justiça em números 2015. Brasília, DF: Conselho Nacional de Justiça - CNJ, 2015. Disponível em/Available from: <http://www.cnj.jus.br/>. Disponível em: abr. 2016/Cited:Apr. 2016.

Gráfico 21.2 - Percentual da despesa, por ramos de Justiça - 2014 Graph 21.2 - Percentage of expenditures, by courts of justice - 2014

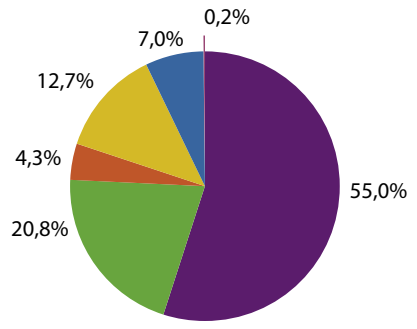
Estadual/State
Trabalhista/Labor
Tribunais Superiores/Superior Courts
Federal/Federal
Eleitoral/Electoral
Militar Estadual/State Military

Fonte/Source: Justiça em números, 2015. DF: Conselho Nacional de Justiça - CNJ, 2015. Disponível em/Available from: <http://www.cnj.jus.br/>.Acesso em: abr. 2016/Cited:Apr. 2016. 



\section{Meio Ambiente}

\section{Environment}

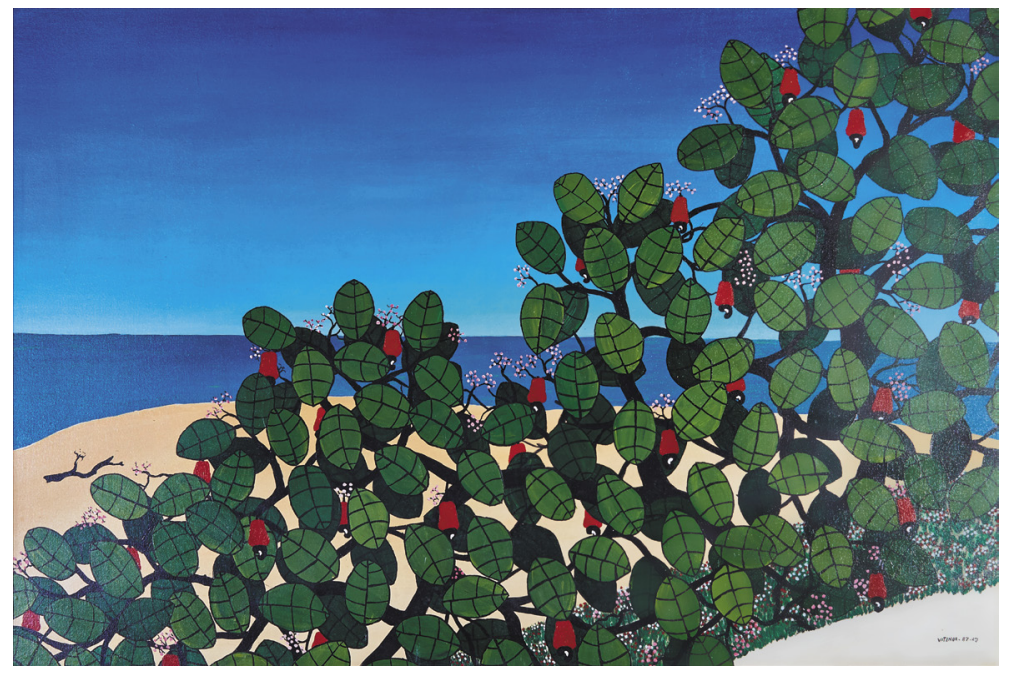

Cajueiro nas Dunas/Cashew tree on the dunes, 1987 Vatenor de Oliveira Silva 



\section{Meio Ambiente}

\section{Environment}

\section{Desmatamento na Amazônia e Unidades de Conservação no Brasil}

\begin{abstract}
A Amazônia brasileira é a maior floresta tropical da Terra. Abriga uma proporção substancial da biodiversidade terrestre global (Dirzo e Raven, 2003), armazena grandes estoques de carbono (Gibbs et al., 2007) e desempenha uma função fundamental na regulação do clima global e regional (Foley et al., 2007), entre uma infinidade de serviços ecossistêmicos (MEA, 2005). $O$ desmatamento em combinação com mudanças climáticas representa uma grande ameaça para a biodiversidade da Amazônia, além de colocar em risco a capacidade deste bioma em ofertar serviços ecossistêmicos em longo prazo (Malhi et al, 2008; Nepstad et al, 2008; Marengo e Espinoza, 2016).
\end{abstract}

O bioma brasileiro Amazônia ocupa quase a metade do território nacional, abrangendo $75,2 \%$ da área nacional de florestas naturais (SFB, 2016). Apesar das altas taxas de desmatamento no passado, o bioma ainda mantém $81,7 \%$ da sua cobertura florestal original (SFB, 2016).

\section{Deforestation in the Amazon and Conservation Units in Brazil}

The Brazilian Amazon is the largest tropical forest on Earth. It harbors a substantial proportion of global terrestrial biodiversity (Dirzo and Raven, 2003), it stores large stocks of carbon (Gibbs et al., 2007), and it plays a critical function in regulating the global and regional climate (Foley et al., 2007), among a myriad of other ecosystem services (MEA, 2005). Deforestation in combination with climate change represents a major threat to the Brazilian Amazon's biodiversity, in addition to putting at risk the capacity of this biome to deliver ecosystem services in the long run (Malhi et al., 2008; Nepstad et al., 2008; Marengo and Espinoza, 2016).

Brazil's Amazon biome occupies nearly half of the national territory, encompassing $75.2 \%$ of the national natural forest area (SFB, 2016). Despite high rates of deforestation in the past, this biome still maintains $81.7 \%$ of its original forest cover (SFB, 2016). 
Desde 1988, o desmatamento em corte raso (isto é, remoção total da cobertura florestal) tem sido monitorado pelo Instituto Nacional de Pesquisas Espaciais (INPE) por meio do projeto PRODES (INPE, 2016). Por sinal, o PRODES recebeu grande reconhecimento internacional pelo seu desempenho no rastreamento do desmatamento e transparência na divulgação dos resultados (Kintisch, 2007). O PRODES emprega imagens de sensoriamento remoto e técnicas de processamento de imagem digital para monitoramento de manchas de desmatamento em corte raso maiores que 6,25 ha (INPE, 2016). Numa base anual, o PRODES relata sua avaliação do desmatamento ocorrido em toda a Amazônia Legal (a wall-to-wall assessment), uma região sócio-geográfica, de aproximadamente 521,7 milhões de hectares, na qual o bioma Amazônia está inserido (INPE, 2016).

O desmatamento na Amazônia Legal é espacialmente concentrado em sua fronteira agrícola, ao longo das bordas leste e sul da floresta. Ao longo deste "arco do desmatamento", a maior parte do desmatamento ocorreu nos eixos das principais estradas, como a rodovia Transamazônica (BR-230) (Fearnside, 2005; Fearnside e Lima de Alencastro Graça, 2006; Banerjee; Macpherson; Alavalapati, 2009). Os estados de Mato Grosso, Rondônia e Pará possuem 81\% de toda a área desmatada acumulada na Amazônia Legal entre 1988 e 2015 (INPE, 2015). Para o estado do Pará, Brito e Barreto (2015) relataram que $39 \%$ do território se encontrava em situação fundiária irregular, onde se concentrava $71 \%$ da área desmatada acumulada no estado.
Since 1988, clear-cut deforestation (that is, full forest cover removal) has been monitored by the National Institute for Space Research (INPE) through the PRODES project (INPE, 2016). In fact, PRODES has received much international recognition for its deforestation tracking performance and transparency reporting (Kintisch, 2007). PRODES employs remote-sensing images and digital image-processing techniques for monitoring clearcut deforestation patches larger than 6.25 ha (INPE, 2016). On an annual basis, PRODES reports a wall-to-wall deforestation assessment for the Legal Amazon, a socio-geographic region, of approximately 521.7 million ha, wherein lies the Brazilian Amazon (INPE, 2016).

Deforestation in the Legal Amazon is spatially concentrated in its agricultural frontier, along the eastern and southern edges of the forest. Along this "arc of deforestation", the vast majority of clearing has occurred along the axes of major roads, such as the Transamazon highway (BR-230) (Fearnside, 2005; Fearnside e Lima De Alencastro Graca, 2006; Banerjee; Macpherson; Alavalapati, 2009). The states of Mato Grosso, Rondônia and Pará accounted for $81 \%$ of all the accumulated deforested area in the Legal Amazon from 1988 to 2015 (INPE, 2015). For the state of Pará, Brito and Barreto (2015) reported that 39\% of the territory was under irregular land tenure status, and concentrated $71 \%$ of the accumulated deforested area in the state. 
Com efeito, a regularização fundiária é uma necessidade urgente e vital para a proteção da Amazônia (Duchelle et al, 2014; Reydon et al, 2015). A ausência de direitos de propriedade bem definidos e protegidos na Amazônia é muito propícia para a invasão e especulação de terras, o que é um dos principais indutores ao desmatamento e frequentemente conduz a violentos conflitos por terra (Reydon et al., 2015). O Programa Terra Legal, parte da lei mais abrangente de reforma agrária $\mathrm{n}^{\circ} 11.952$ / 2009, configura uma das principais iniciativas nacionais para tratar desta questão. O programa tem como meta a regularização fundiária de até 300.000 ocupações informais em terras públicas na Amazônia Legal. É coordenado pelo Ministério do Desenvolvimento Agrário e conta com o apoio do Instituto Nacional de Colonização e Reforma Agrária (Incra). Um de seus grandes desafios é o combate à "grilagem", o fenômeno generalizado de invasão de terras e subsequente obtenção de títulos falsos de posse (Brito e Barreto, 2011).

A criação de gado e a produção de soja são as formas dominantes de uso da terra para fins agropecuários no Brasil e na sua Amazônia Legal (IBGE, 2016). As pastagens são responsáveis por $65 \%$ da área desmatada na Amazônia Legal (Inpe; Embrapa, 2014). Nesta região, o tamanho do rebanho bovino aumentou $70 \%$ entre 2000 e 2013 , enquanto acresceu apenas $7 \%$ no resto do país durante o mesmo período (Barreto, 2015). Atualmente, o desmatamento e a pecuária fazem do Brasil um dos líderes mundiais em termos de emissões de gases de efeito estufa (GEE). Em 2013, a mudança no uso da terra e a pecuária foram responsáveis por $55 \%$ e $24,5 \%$,
In fact, land tenure regularization is a vital and much urgent need in view of protecting the Brazilian Amazon (Duchelle et al., 2014; Reydon et al., 2015). The lack of clear and protected property rights in the Brazilian Amazon is very conducive to land grabbing and speculation, which is a main driver of deforestation and often leads to violent land conflicts (Reydon et al., 2015). The Legal Land (Terra Legal) program, as part of the broader land reform Law 11,952/2009, comprises one of the core national initiatives to address this issue. The program is aimed at expediting land titles up to 300,000 informal occupations in public land in the Legal Amazon. It is coordinated by the Ministry of Agrarian Development and assisted by the Land Reform and Settlement Institute (Incra). A main challenge faced by the program is fighting against "grilagem", the widespread phenomenon of land grabbing and subsequent registration of false land titles (Brito and Barreto, 2011).

Soybean fields and cattle ranching are the dominant agricultural land use types in Brazil as well as in its Legal Amazon (IBGE, 2016). Pastures account for $65 \%$ of the deforested area in the Legal Amazon (Inpe; Emprapa, 2014). In this region, the cattle herd size increased by $70 \%$ between 2000 and 2013, while it increased by only $7 \%$ in the rest of the country over the same period (Barreto, 2015). Currently, deforestation and cattle ranching render Brazil as one of the leading countries in terms of greenhouse gas emissions (GGE). In 2013, land use change and cattle ranching accounted for $55 \%$ and 
respectivamente, das emissões de gases de efeito estufa do Brasil (Piatto et al., 2015).

No bioma Cerrado, parte das pastagens foi substituída em grande escala pelo cultivo de soja, o que pode ter motivado o avanço das pastagens sobre o bioma Amazônia, acentuando-se assim o desmatamento. Mais recentemente, outra onda de substituição de pastagens no bioma Cerrado vem ocorrendo, mas desta vez associada especialmente à expansão dos canaviais (Jepson, 2005; Brannstrom et al., 2008; Lapola et al., 2014). Isso levanta a questão de se a produção de um combustível "ecologicamente correto" (isto é, o álcool de cana-de-açúcar) poderia paradoxalmente contribuir para o desmatamento na Amazônia (Martinelli et al, 2010; de Souza Ferreira Filho e Horridge, 2014).

Quanto à dinâmica temporal do uso da terra, houve um aumento substancial nas taxas de desmatamento anual na Amazônia Legal entre 2001 e 2003 (Gráfico 22.1). À luz da pressão pública aguda, o governo brasileiro teve que entrar em ação (Banerjee; Macpherson; Alavalapati, 2009). Em março de 2014, o governo lançou o Plano de Ação para Prevenção e Controle do Desmatamento na Amazônia (PPCDAm). Pela primeira vez, o desmatamento foi combatido por vários ministérios, sob a coordenação do Chefe de Gabinete da Presidência da República, ao invés de ser uma questão exclusiva do Ministério do Meio Ambiente. No mesmo ano, a capacidade de combate ao desmatamento foi intensificada por meio da criação do programa de Detecção de Desmatamento em Tempo Real (DETER),
$24.5 \%$, respectively, of Brazil's greenhouse gas emissions (GGE) (Piatto et al., 2015).

In the Cerrado biome, part of the pastures has been replaced by large-scale cropping of soybean, which may have enlarged their domain in the Amazon biome, thereby furthering deforestation (Reydon et al., 2015). More recently, there has been another replacement trend for pastures in the Cerrado biome, only this time by especially sugarcane crop fields. That raises the question whether the production of an "eco-friendly" fuel (that is, sugar cane alcohol) could paradoxically lead to further deforestation in the Amazon (Martinelli et al., 2010; De Souza Ferreira Filho e Horridge, 2014).

As regards the temporal dynamics of land use change, there was a substantial increase in annual deforestation rates in the Legal Amazon between 2001 and 2003 (graph 22.1). In light of acute public pressure, the government was forced into action. In March 2014, the government launched the Action Plan for Prevention and Control of Deforestation in the Amazon (PPCDAm). For the first time, several ministries got involved in the fight against deforestation, under the coordination of the President's chief of staff, and the issue was no longer solely addressed by the Ministry of Environment. At the same year, enforcement capacity was further increased through the creation 
um sistema de alerta de desmatamento baseado no sensor do satélite MODIS. No Plano Nacional sobre Mudança do Clima, anunciado na Conferência de Mudanças Climáticas 2009 da ONU em Copenhague, o governo se comprometeu a reduzir o desmatamento em $80 \%$ até 2020 (com base na taxa média de desmatamento do período entre 1995 e 2005).

Com efeito, houve um decréscimo considerável nas taxas anuais de desmatamento entre 2004 e 2012 (Gráfico 22.1). Porém, em paralelo às melhorarias no monitoramento $\mathrm{e}$ aplicação das leis, vários outros fatores podem ter contribuído para esse quadro. Mais especificamente, a expansão das áreas públicas de proteção; flutuações na lucratividade da soja e pecuária; os boicotes nas cadeias de soja e carne contra produtores que promovessem o desmatamento (a "Moratória da Soja" e o "Acordo de Gado"); e a criação do programa Municípios Verdes, que impôs restrições de crédito agrícola para os municípios com as maiores taxas de desmatamento (Macedo et al, 2012;. Nepstad et al, 2014;. Gibbs et al, 2015).

Também existem indícios de que estas reduções no desmatamento foram superestimadas. Por exemplo, a frequência anual de manchas pequenas de desmatamento em corte raso $(6,25-50,00$ hectare) permaneceu relativamente inalterada durante 0 período de avaliação de 2002 a 2009 (Rosa et al., 2012). Considerando-se que o PRODES só detecta manchas de corte raso maiores que 6,25 ha e que o PPCDAm prioriza manchas maiores (> $100 \mathrm{ha}$ ), então faz sentido se esperar que os "grileiros" tenham alterado seu of the "Detection of Deforestation in Real Time" (DETER) program, an alert monitoring system based on the MODIS satellite sensor. The Brazilian National Plan on Climate Change, announced at the 2009 UN Climate Change Conference in Copenhagen, pledged to achieve an $80 \%$ deforestation reduction by 2020 (based on the average deforestation rate over the period 1995-2005).

Indeed, there was a sharp decrease in annual deforestation rates between 2004 and 2012 (graph 22.1). Nonetheless, in addition to improving monitoring and enforcement, several other factors may have contributed to this scenario. More specifically, the expansion of protected public areas; fluctuations in the profitability of soy and cattle production; the boycott of soy and beef supply chains against deforesters (the "Soy Moratorium" and the "Cattle Agreement"); and the creation of the Critical County program, which imposed agricultural credit restrictions on the counties with the highest deforestation rates (Macedo et al., 2012; Nepstad et al., 2014; Gibbs et al., 2015).

There are also indications that these declines in deforestation were overestimated. For instance, the annual frequency of small clear-cut patches (6.25-50.00 ha) remained more or less unchanged during an assessment period from 2002 through 2009 (Rosa et al., 2012). Considering that PRODES only detects patches larger than 6.25 ha and that PPCDAm prioritizes larger patches (>100 ha), then it is reasonable to expect that land grabbers may have changed their 
comportamento de modo a concentrar o desmatamento em manchas menores (Rosa et al., 2012).

A partir de 2012, as taxas de desmatamento voltaram a subir, embora se mantendo bem inferiores àquela de 2004. Estes aumentos podem estar relacionados com a desvalorização da moeda do Brasil, o real, o que torna commodities de exportação mais lucrativas (Fearnside, 2015), e o surgimento de novos mercados de carne, como a Rússia e China (de Souza e de Marco Junior, 2015).

Fearnside (2015) advertiu que o desmatamento pode se agravar nos próximos anos, considerando que, entre outras razões, o orçamento do governo no combate ao desmatamento foi reduzido em $72 \%$ e planos para estradas e outros projetos de infraestrutura para a Amazônia estavam em andamento, enquanto a criação de novas áreas protegidas estava essencialmente paralisada.

As principais áreas protegidas públicas no Brasil são as Terras Indígenas e Unidades de Conservação. O SNUC (Sistema Nacional de Unidades de Conservação), instituído pela Lei no 9.985 / 2000, estabelece critérios e diretrizes para a criação e gestão de Unidades de Conservação (UCs). Uma característica central do SNUC é a diferenciação entre áreas de uso sustentável e áreas de proteção integral (tabela 22.3). A Reserva Particular do Patrimônio Natural (RPPN) representa a única categoria de proteção no âmbito do SNUC para propriedades privadas. Enquanto a criação de unidades de RPPN funciona numa base voluntária, os proprietários privados behavior toward concentrating deforestation into smaller patches (Rosa et al., 2012).

From 2012 onwards, deforestation rates began to rise again, although remaining much lower than that of 2004. These increases might be related to the depreciation of Brazil's currency, the real, which makes export commodity more profitable (Fearnside, 2015), and to the rise of new meat markets, such as Russia and China (De Souza e De Marco Junior, 2015).

Fearnside (2015) warns that deforestation might aggravate in the years to come considering that, among other reasons, government expenditure on enforcing environmental laws were cut by $72 \%$ and plans for Amazonian roads and other infra-structure projects were underway, while the creation of new official protected areas was essentially paralyzed.

The main public protected areas in Brazil are the Indigenous Lands and the Conservation Units. The SNUC (National System of Conservation Units), instituted by Law No. 9,985/2000, provides criteria and guidelines for the creation and management of Conservation Units. One main feature of the SNUC is the differentiation between sustainable use and strictly protected areas (table 22.3). The Private Natural Heritage Reserve (RPPN) represents the only conservation unit category under the SNUC for privately owned lands. While the creation of RPPN units works on a voluntarily basis, private 
devem obedecer ao Novo Código Florestal, instituído pela Lei no 12,651 / 2012, que dispõe sobre a proteção de áreas de vegetação nativa em terras de propriedade privada.

No Brasil, a área de florestas naturais ocupa 456,1 milhões de hectares $(53,7 \%$ do território nacional), dos quais $16,7 \%$ representa UCs federais (tabela 22.3). Este número sobe para $34 \%$ quando se considera o total de UCs federais, estaduais e municipais (SFB, 2016). $O$ bioma Amazônia, sozinho, concentra $73,25 \%$ da área total ocupada por UCs. As Terras Indígenas e UCs representam $33,1 \%$ e $32,6 \%$, respectivamente, da cobertura florestal natural nesse bioma (SFB, 2016).

Os conflitos fundiários (ocupações irregulares de terras públicas) e a falta de recursos humanos e materiais são os principais obstáculos para a consolidação e efetiva proteção das UCs (Fearnside, 2003; Rylands e Brandon, 2005; Soares-Filho et al, 2010; Nolte et al, 2013). No entanto, as UCs e Terras Indígenas têm contribuído um pouco na redução do desmatamento (Soares-Filho et al., 2010), talvez por desencorajar a especulação de terras, dado que é muito improvável que, no futuro, torne-se possível a reivindicação de posse sobre terras dentro destas áreas protegidas (Nepstad et al., 2014).

Enquanto a Amazônia brasileira abriga uma grande parcela da biodiversidade terrestre do planeta e se destaca na oferta de serviços ecossistêmicos, ela está entre as regiões menos desenvolvidas do país (IBGE, 2016). Aparentemente, o desmatamento não trouxe qualquer melhoria socioeconômica substancial landowners must abide by the Brazil's New Forest Code, established by Law No. 12,651/2012, which provides for the protection of native vegetation areas on privately owned lands.

Brazil's natural forest area occupies 456.1 million ha $53.7 \%$ of the national territory), out of which $16.7 \%$ represents federal Conservation Units (table 22.3). This figure adds up to $34 \%$ as federal, state and municipal Conservation Units are taken altogether (SFB, 2016). The Amazon biome, alone, contains $73.25 \%$ of the total area occupied by Conservation Units. Indigenous Lands and Conservation Units represent 33.1\% and $32.6 \%$, respectively, of the natural forest cover in this biome (SFB, 2016).

Unresolved land tenure conflicts (irregular occupations of public areas) and lack of human and material resources are major obstacles to consolidating and effectively protecting Conservation Units (Fearnside, 2003; Rylands e Brandon, 2005; SoaresFilho et al., 2010; Nolte et al., 2013). Nonetheless, Conservation Units and Indigenous Lands have been somewhat active in reducing deforestation (Soares-Filho et al., 2010), perhaps by discouraging land speculation, given that it is very unlikely that, in the future, it will ever be possible to claim ownership over lands within those protected areas (Nepstad et al., 2014).

While the Brazilian Amazon hosts a great share of the world's terrestrial biodiversity and excels at delivering ecosystem services, it is among the least developed regions in the country (IBGE, 2016). Deforestation appears not to have brought any substantial long term socioeconomic improvement for 
em longo prazo para os municípios localizados no arco do desmatamento (Rodrigues et al., 2009).

\section{Na Amazônia, a degradação das} pastagens ocorre normalmente alguns anos após a instalação (Dias-Filho e Andrade, 2006). É menos dispendioso para os produtores cortar e queimar novas áreas de floresta do que rejuvenescer os solos e replantar campos de pousio (Tollefson, 2015). Assim, o desmatamento avança ao passo que pastagens são abandonadas. (Dias-Filho e Andrade, 2006). Em 2010, o governo lançou o programa Agricultura de Baixo Carbono $(A B C)$, que financia empréstimos a juros baixos para atividades agropecuárias e florestais, como sistemas agroflorestais, que promovam benefícios socioecológicos tais como o sequestro de carbono, melhorias na capacidade do solo para a fixação de nitrogênio e a reabilitação de pastagens degradadas. No entanto, este programa tem tido muito pouca adesão, o que pode estar atrelado a vários fatores. Por exemplo, a fraca publicidade e a existência de exigências ambientais mais rigorosas do que outros empréstimos agrícolas (Angelo, 2012); a falta de conhecimento técnico necessário para o acesso aos empréstimos (Nepstad et al., 2014); limitações de logística e infraestrutura, falta de assistência técnica e dificuldades para treinar técnicos e produtores (Embrapa, 2016).

Para atividades florestais em propriedades privadas, o Código Florestal exige que os proprietários conservem vegetação nativa em suas propriedades por meio da proteção de uma Reserva Legal ( $R L)$, a qual ocupa $80 \%$ da área da propriedade no bioma Amazônia. O Código Florestal the municipalities located within the arc of deforestation (Rodrigues et al., 2009).

In the Amazon, pasture degradation typically occurs a few years after installation (Dias-Filho and Andrade, 2006). It is less costly for farmers to slash and burn forest than to rejuvenate soils and replant fallowed fields (Tollefson, 2015). So deforestation advances as former pasturelands are abandoned (DiasFilho and Andrade, 2006). In 2010, the government launched the Low Carbon Agriculture program $(A B C)$, which funds low-interest loans for activities, such as agroforestry, that promote socioecological benefits as, for instance, carbon sequestration, improving soil nitrogen uptake and rehabilitating degraded pastureland.

Nonetheless, this program has had little uptake so far, which might be associated to several factors. For instance, poor publicity and stricter environmental requirements than other agricultural loans (Angelo, 2012), lack of technical expertise required to access the loans (Nepstad et al., 2014), infrastructure and logistics limitations, lack of technical assistance, and difficulties to train technicians and producers (Embrapa, 2016).

Regarding forest activities on privately owned lands, Brazil's Forest Code requires landowners to conserve native vegetation on their rural properties by setting aside a Legal Reserve (LR), which occupies $80 \%$ of the property area in the Amazon. The Forest 
prevê também práticas de exploração madeireira na Reserva Legal. Mais especificamente, a exploração de florestas e formações secundárias, em regime de manejo florestal sustentável, seja em áreas de domínio público ou privado, exige a aprovação de um Plano de Manejo Florestal Sustentável pela autoridade florestal competente. Um dos principais obstáculos para o manejo florestal legal na Amazônia é a competição contra a exploração ilegal de madeira. Estima-se que $80 \%$ de toda exploração madeireira na Amazônia é ilegal (Sistema Ambiental Paulista, 2016).

Finalmente, mecanismos de incentivos positivos para evitar o desmatamento são urgentemente necessários (Nepstad et al., 2014). Uma iniciativa promissora é a Redução de Emissões por Desmatamento e Degradação em Países em Desenvolvimento (REDD), do programa da Convenção Quadro das Nações Unidas sobre o Clima. Outros programas na temática do REED são novos ou estão em desenvolvimento, incluindo o Fundo Amazônia e o programa de Agricultura de Baixo Carbono. A eficiência, limitações e falhas dessas iniciativas ainda terão de ser avaliadas.
Code also provides for selective logging practices in Legal Reserve. More specifically, exploring forests and secondary formations under a sustainable forest management regime, either within public or private domain, requires approval of a Sustainable Forest Management Plan by the competent forestry authority. A major obstacle for legal forest management in the Amazon is how to be competitive against illegal logging. About 80 percent of all logging in the Amazon is estimated to be illegal (Sistema Ambiental Paulista, 2016).

Finally, mechanisms to provide positive incentives for avoiding deforestation are urgently needed (Nepstad et al., 2014). A promising initiative is the Reduced Emissions from Deforestation and Degradation in Developing Countries (REDD) program of the United Nations Framework Convention on Climate. Other REED related programs are new or under development, including the Amazon Fund and the Low Carbon Agriculture programs. The efficiency, limitations and shortcomings of these initiatives are yet to be seen.

\section{Referências/References}

ANGELO, C. 2012. Nature News. Brazil's fund for low-carbon agriculture lies fallow. Disponível em/Available from: http://www.nature.com/news/brazil-s-fund-for-low-carbon-agriculture-lies-fallow-1.11111.

BANERJEE, O.; MACPHERSON, A.J.; ALAVALAPATI, J. Toward a Policy of Sustainable Forest Management in Brazil A Historical Analysis. The Journal of Environment \& Development, v. 18, n. 2, p. 130-153, Jun 2009.

BARRETO, P. (2015). Como reduzir a contribuição da pecuária brasileira para as mudanças climáticas? Belém, Brasil: Instituto do Homem e Meio Ambiente da Amazônia. 
BRANNSTROM, C. et al. Land change in the Brazilian Savanna (Cerrado), 1986-2002: Comparative analysis and implications for land-use policy. Land Use Policy, v. 25, n. 4, p. 579-595, Oct 2008. ISSN 0264-8377. Disponível em/ Available from: <<Go to ISI $>$ ://WOS:000256289700011 >.

BRITO, B., \& BARRETO, P. (2011). A regularização fundiária avançou na Amazônia? Os dois anos do programa Terra Legal. Belém, Brasil: Instituto do Homem e Meio Ambiente da Amazônia.

BRITO, B., \& BARRETO, P. (2015). Regularização fundiária no Pará: Afinal qual é o problema? Belém, Brazil: Instituto do Homem e Meio Ambiente da Amazônia.

DE SOUZA FERREIRA FILHO, J. B.; HORRIDGE, M. Ethanol expansion and indirect land use change in Brazil. Land Use Policy, v. 36, p. 595-604, Jan 2014. ISSN 0264-8377. Disponível em/Available from: < <Go to ISI>:// WOS:000329881400058>.

DE SOUZA, R. A.; DE MARCO JUNIOR, P. The Red Queen race in Brazilian Amazon deforestation: the necessity of a sustainable economy to zero deforestation. Natureza \& Conservacao, v. 13, n. 2, p. 190-192, Jul-Dec 2015. ISSN 1679-0073. Disponível em/Available from: <<Go to ISI $>$ ://WOS:000368260400015 >.

DIAS-FILHO, M.B.; ANDRADE, C.M. Pastagens no Trópico Húmido. Belém: EMBRAPA Amazônia Oriental, 2006.

DIRZO, R.; RAVEN, P. H. Global state of biodiversity and loss. Annual Review of Environment and Resources, v. 28, p. 137-167, 2003 2003. ISSN 1543-5938. Disponível em/Available from: <<Go to ISI>://WOS:000220102700005 >.

DUCHELLE, A. E. et al. Linking Forest Tenure Reform, Environmental Compliance, and Incentives: Lessons from REDD plus Initiatives in the Brazilian Amazon. World Development, v. 55, p. 53-67, Mar 2014. ISSN 0305-750X.

EMBRAPA. (2006). Technical note: ABC Sector Plan - Sector Plan on Climate Change Mitigation and Adaptation to Consolidate a Low-Carbon Economy in Agriculture. Disponível em/Available from: https://www.embrapa.br/en/ tema-agricultura-de-baixo-carbono/nota-tecnica.

FEARNSIDE, P. M . Deforestation in Brazilian Amazonia: History, rates, and consequences. Conservation Biology, v. 19, n. 3, p. 680-688, Jun 2005. ISSN 0888-8892. Disponível em/Available from: < <Go to ISI>:// WOS:000229448100016>.

FEARNSIDE, P. M . Deforestation soars in the Amazon. Nature, v. 521, n. 7553, p. 423-423, May 28 2015. ISSN 0028 0836. Disponível em/Available from: <<Go to ISI >://WOS:000355286600020 >.

FEARNSIDE, P. M. Conservation policy in Brazilian Amazonia: Understanding the dilemmas. World Development, v. 31, n. 5, p. 757-779, May 2003. ISSN 0305-750X. Disponível em/Available from: <<Go to ISI>:// WOS:000182806300001 >.

FEARNSIDE, P. M.; LIMA DE ALENCASTRO GRACA, P. M. BR-319: Brazil's manaus-porto velho highway and the potential impact of linking the arc of deforestation to central Amazonia. Environmental Management, v. 38, n. 5, p. 705-716, Nov 2006. ISSN 0364-152X. Disponível em/Available from: < <Go to ISI>://WOS:000241104500001 >. 
FOLEY, J. A. et al. Amazonia revealed: forest degradation and loss of ecosystem goods and services in the Amazon Basin. Frontiers in Ecology and the Environment, v. 5, n. 1, p. 25-32, Feb 2007. ISSN 1540-9295. Disponível em/ Available from: $<<$ Go to $|S|>: / / W O S: 000244042300021>$.

GIBBS, H. K. et al. Brazil's Soy Moratorium. Science, v. 347, n. 6220, p. 377-378, Jan 23 2015. ISSN 0036-8075. Disponível em/Available from: $<<$ Go to $|S|>: / / W O S: 000348225800019>$.

GIBBS, H. K. et al. Monitoring and estimating tropical forest carbon stocks: making REDD a reality. Environmental Research Letters, v. 2, n. 4, Oct-Dec 2007. ISSN 1748-9326. Disponível em/Available from: < <Go to ISI>:// WOS:000253653000028 >.

IBGE. Instituto Brasileiro de Geografia e Estatística. 2016. Disponível em/Available from: http://www.ibge.gov.br/ home/.

INPE. Instituto Nacional de Pesquisas Espaciais. 2016. Disponível em/Available from: http://www.inpe.br/.

INPE/EMBRAPA. Instituto de Pesquisa Espacial do Brasil/Empresa Brasileira de Pesquisa Agropecuária. 2014. TerraClass (2012). Mapeamento do uso e cobertura da terra na Amazônia Legal brasileira. Brasília: MAPA, MMA e MCTI.

JEPSON, W. A disappearing biome? Reconsidering land-cover change in the Brazilian savanna. Geographical Journal, v. 171, p. 99-111, Jun 2005. ISSN 0016-7398. Disponível em/Available from: <<Go to ISI>:// WOS:000230553200001 >.

KINTISCH, E. Carbon emissions - Improved monitoring of rainforests helps pierce haze of deforestation. Science, v. 316, n. 5824, p. 536-537, Apr 27 2007. ISSN 0036-8075. Disponível em/Available from:<<Go to ISI>:// WOS:000245983100011 >.

LAPOLA, D. M. et al. Pervasive transition of the Brazilian land-use system. Nature Climate Change, v. 4, n. 1, p. 2735, Jan 2014. ISSN 1758-678X. Disponível em/Available from: <<Go to ISI $: / /$ WOS:000333666600012 >.

MACEDO, M. N. et al. Decoupling of deforestation and soy production in the southern Amazon during the late 2000s. Proceedings of the National Academy of Sciences of the United States of America, v. 109, n. 4, p. 13411346, Jan 24 2012. ISSN 0027-8424. Disponível em/Available from: < <Go to ISI >://WOS:000299412600068>.

MALHI, Y. et al. Climate change, deforestation, and the fate of the Amazon. Science, v. 319, n. 5860, p. 169-172, Jan 11 2008. ISSN 0036-8075. Disponível em/Available from: <<Go to ISI >://WOS:000252246400030 >.

MARENGO, J. A.; ESPINOZA, J. C. Extreme seasonal droughts and floods in Amazonia: causes, trends and impacts. International Journal of Climatology, v. 36, n. 3, p. 1033-1050, Mar 15 2016. ISSN 0899-8418. Disponível em/ Available from: <<Go to |S|>://WOS:000371541400001 >.

MARTINELLI, L. A. et al. The false dichotomy between preservation of the natural vegetation and food production in Brazil. Biota Neotropica, v. 10, n. 4, p. 323-330, Oct-Dec 2010. ISSN 1676-0603. Disponível em/Available from: $<<$ Go to ISI $>$ ://WOS:000208655400037 >. 
MEA. Millennium Ecosystem Assessment. 2005. Ecosystem and human wellbeing: synthesis. Washington, DC: Island Press, 2005.

NEPSTAD, D. et al. Slowing Amazon deforestation through public policy and interventions in beef and soy supply chains. Science, v. 344, n. 6188, p. 1118-1123, Jun 6 2014. ISSN 0036-8075. Disponível em/Available from: < <Go to ISI>://WOS:000336791200038 >.

NEPSTAD, D. C. et al. Interactions among Amazon land use, forests and climate: prospects for a near-term forest tipping point. Philosophical Transactions of the Royal Society B-Biological Sciences, v. 363, n. 1498, p. 17371746, May 27 2008. ISSN 0962-8436. Disponível em/Available from:<<Go to ISI >://WOS:000254577500003 >.

NOLTE, C.; AGRAWAL, A.; BARRETO, P. Setting priorities to avoid deforestation in Amazon protected areas: are we choosing the right indicators? Environmental Research Letters, v. 8, n. 1, Jan-Mar 2013. ISSN 1748-9326. Disponível em/Available from: <<Go to ISI $>$ ://WOS:000316998300102 >.

PIATTO, M.; COSTA, C. JR.; PINTO, L. F. G. 2015. Análise das emissões de GEE do setor agropecuário e o impacto das políticas públicas nas mudanças climáticas, São Paulo.

REYDON, B. P.; FERNANDES, V. B.; TELLES, T. S. Land tenure in Brazil: The question of regulation and governance. Land Use Policy, v. 42, p. 509-516, Jan 2015. ISSN 0264-8377. Disponível em/Available from: <<Go to ISI>:// WOS:000347018700047 >.

RODRIGUES, A. S. L. et al. Boom-and-Bust Development Patterns Across the Amazon Deforestation Frontier. Science, v. 324, n. 5933, p. 1435-1437, Jun 12 2009. ISSN 0036-8075. Disponível em/Available from: < <Go to ISI>://WOS:000266878700043 >.

ROSA, I. M. D.; SOUZA, C., JR.; EWERS, R. M. Changes in Size of Deforested Patches in the Brazilian Amazon. Conservation Biology, v. 26, n. 5, p. 932-937, Oct 2012. ISSN 0888-8892. Disponível em/Available from: <<Go to ISI>://WOS:000308484500019 >.

RYLANDS, A. B.; BRANDON, K. Brazilian protected area. Conservation Biology, v. 19, n. 3, p. 612-618, Jun 2005. ISSN 0888-8892. Disponível em/Available from: <<Go to ISI $>$ :/WOS:000229448100006 >.

SFB. Serviço Florestal Brasileiro. 2016. Disponível em/Available from: http://www.florestal.gov.br/

SISTEMA AMBIENTAL PAULISTA. 2016. Secretaria do Meio Ambiente do Governo do Estado de São Paulo. Madeira legal Vs. Madeira ilegal. Disponível em/Available from: http://www.ambiente.sp.gov.br/madeiralegal/madeiralegal-vs-madeira-ilegal/.

SOARES-FILHO, B. et al. Role of Brazilian Amazon protected areas in climate change mitigation. Proceedings of the National Academy of Sciences of the United States of America, v. 107, n. 24, p. 10821-10826, Jun 152010. ISSN 0027-8424. Disponível em/Available from: $<<$ Go to ISI >://WOS:000278807400011 >.

TOLLEFSON, J. Battle for the Amazon. Nature, v. 520, n. 7545, p. 20-23, Apr 2 2015. ISSN 0028-0836. Disponível em/Available from: $<<$ Go to ISI $>$ ://WOS:000352027700017 $>$. 


\section{Rafael Kuster Oliveira}

Engenheiro Ambiental pela PUCPR.

Especialista em Sistema de Gestão Ambiental pela PUCPR. Mestre em Manejo e Ecologia de Florestas pela Universidade de Freiburg, Alemanha. Doutor em Engenharia Florestal pela UFPR. Professor do curso de Engenharia Ambiental da PUCPR desde 2012.
Bachelor in Environmental Engineering from the Pontifical University of Paraná - PUCPR. Specialist in System of Environmental Management from PUCPR. Master in Forest Ecology and Management from the University of Freiburg, Germany. PhD in Forest Engineering from the Federal University of Paraná - UFPR. Professor of the Environmental Engineering course at PUCPR since 2012.

\section{Fabiana De Nadai Andreoli}

Possui graduação em Engenharia Civil pela Universidade Federal do Espírito Santo (1993), mestrado em Engenharia Ambiental pela Universidade Federal do Espírito Santo (1996) e doutorado em Educação pela Pontifícia Universidade Católica do Paraná (2011). Atua como professora na Pontifícia Universidade Católica do Paraná. Pesquisadora dos grupos de pesquisas em Engenharia Ambiental e Educação em Engenharia. Coordenadora do curso de engenharia Ambiental da PUCPR desde 2008. Auditora e Consultora Ambiental. Sócia da empresa de consultoria ambiental Andreoli Engenheiros Associados.
Bachelor in Civil Engineering from the Federal University of Espírito Santo - UFES (1993), Master in Environmental Engineering from UFES (1996) and PhD in Education from PUCPR (2011). She is currently a professor at PUCPR. Researcher of the research groups on Environmental Engineering and Education in Engineering. Coordinator of the Environmental Engineering course at PUCPR since 2008. Environmental auditor and adviser. Partner in Andreoli Engenheiros Associados, an environmental advisory company.

\section{Antonio Rioyei Higa}

Engenheiro Florestal pela Universidade de São Paulo (1975), Mestre em Ciências Florestais pela Universidade de São Paulo (1980), PhD on Forestry pela The Australian National University (1990) e Pós-Doutor pela Universidade de Freiburg, Alemanha (2008). Chefe geral da Embrapa Florestal (atual Centro Nacional de Pesquisa de Florestas). Desde 1998 é professor da Universidade Federal do Paraná. É bolsista de Produtividade do CNPq (2014-2017).
Bachelor in Forest Engineering from the University of São Paulo - USP (1975), Master in Forest Sciences from USP (1980), PhD in Forestry from the Australian National University (1990) and Post-PhD from the University of Freiburg, Germany (2008). General manager of Embrapa Florestal (currently National Center of Forest Research). He has been a professor at UFPR since 1998. He is currently Fellow Researcher on Productivity of the National Council of Technological and Scientific Development - CNPq (2014-2017).

\section{Cleverson Vitório Andreoli}

Engenheiro Agrônomo (Universidade Federal do Paraná 1980), Mestre em Ciências do Solo

(Universidade Federal do Paraná 1989) e Doutor em Meio Ambiente e Desenvolvimento (Universidade Federal do Paraná 1999). Atualmente é professor e pesquisador no mestrado profissional em Governança e Sustentabilidade do ISAE/FGV e diretor da empresa de consultoria ambiental Andreoli Engenheiros Associados. Trabalhou 35 anos na
Bachelor in Agricultural Engineering from the UFPR (1980), Master in Soil Sciences from

UFPR (1989) and PhD in Environment and Development from UFPR (1999). He is currently a professor and researcher of the Master Program in Governance and Sustainability at the Higher Institute of Administration and Economy of Mercosur - ISAE/FGV and director of Andreoli Engenheiros Associados. He worked for 35 years at the Sanitation Company of Paraná-SANEPAR. 
Tabela 22.1 - Número de violações do padrão primário nacional de qualidade do ar de PM10, nas Regiões Metropolitanas de Salvador (Camaçari), Belo Horizonte, Vitória, Rio de Janeiro, São Paulo, Curitiba e Porto Alegre 1995-2013

Table 22.1 - Number of violations of the national primary standard of air quality of PM10 in the metropolitan areas of Salvador (Camaçari), Belo Vitoria, Rio de Janeiro, São Paulo, Curitiba and Porto Alegre 1995-2013

\begin{tabular}{|c|c|c|c|c|c|c|c|}
\hline $\begin{array}{l}\text { Anos/ } \\
\text { Years }\end{array}$ & $\begin{array}{c}\text { Salvador } \\
\text { (Camaçari) } \\
\text { (1) }\end{array}$ & $\begin{array}{c}\text { Belo } \\
\text { Horizonte }\end{array}$ & Vitória & $\begin{array}{l}\text { Rio de } \\
\text { Janeiro }\end{array}$ & São Paulo & Curitiba & $\begin{array}{l}\text { Porto } \\
\text { Alegre }\end{array}$ \\
\hline 1995 & ... & 7 & .. & $\ldots$ & ... & .. & $\ldots$ \\
\hline 1996 & ... & 10 & .. & $\ldots$ & $\ldots$ & .. & $\ldots$ \\
\hline 1997 & $\ldots$ & 11 & .. & $\ldots$ & 162 & .. & ... \\
\hline 1998 & ... & 7 & .. & $\ldots$ & 26 & .. & ... \\
\hline 1999 & $\ldots$ & 10 & .. & 59 & 61 & .. & ... \\
\hline 2000 & ... & 4 & .. & 64 & 38 & .. & $\ldots$ \\
\hline 2001 & ... & - & - & 33 & 42 & .. & $\ldots$ \\
\hline 2002 & $\ldots$ & - & - & 43 & 23 & & 1 \\
\hline 2003 & ... & 1 & 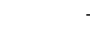 & 19 & 28 & 6 & 2 \\
\hline 2004 & 1 & ... & - & 11 & 7 & 11 & 3 \\
\hline 2005 & - & 1 & 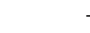 & 1 & 1 & 4 & 4 \\
\hline 2006 & - & 2 & 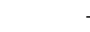 & 15 & 2 & $s$ & 5 \\
\hline 2007 & - & - & 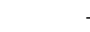 & 11 & 4 & 19 & 1 \\
\hline 2008 & - & 4 & - & 2 & 2 & 24 & 2 \\
\hline 2009 & 1 & - & 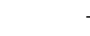 & 9 & 1 & 3 & - \\
\hline 2010 & - & 2 & - & 10 & 6 & 4 & - \\
\hline 2011 & - & 7 & - & 7 & 1 & 6 & 3 \\
\hline 2012 & - & $\ldots$ & 16 & 25 & - & 7 & - \\
\hline 2013 & ... & $\ldots$ & 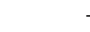 & 23 & - & .. & $\ldots$ \\
\hline
\end{tabular}

Fontes/Sources: 1. Fundação Estadual do Meio Ambiente - FEAM (MG). 2. Instituto Ambiental do Paraná - IAP. 3. Fundação Estadual de Proteção Ambiental Henrique Luiz Roessler. 4. Instituto Estadual do Ambiente - INEA (RJ). 5. Instituto do Meio Ambiente e Recursos Hídricos - INEMA. 6. Companhia Ambiental do Estado de São Paulo-CETESB. 7. Secretaria Municipal de Meio Ambiente (Vitória, ES).

(1) Na Região Metropolitana de Salvador, houve monitoramento somente nos municípios vizinhos ao Polo Industrial de Camaçari (10 pontos distribuídos nos municípios de: Câmara, Gravatá, Cobre, Sitio, Lamarão, Concórdia, Escola, Machadinho, Leandrinho e Areias)./(1) In the Metropolitan Area of Salvador, only the neighboring municipalities of the Camaçari Industrial Complex were monitored (10 points distributed in the municipalities of: Câmara, Gravatá, Cobre, Sitio, Lamarão, Concórdia, Escola, Machadinho, Leandrinho e Areias). 
Tabela 22.2 - Média anual da Demanda Bioquímica de Oxigênio - DBO, em corpos de água selecionados, nas Unidades da Federação de Pernambuco, Bahia, Espírito Santo, Minas Gerais, Rio de Janeiro, São Paulo, Paraná e Rio Grande do Sul - 2003-2013

Tabela 22.2 - Annual Average of Biochemical Oxygen Demand - BOD, in selected water bodies of Pernambuco, Bahia, Espírito Santo, Minas Gerais, Rio de Janeiro,

São Paulo, Paraná and Rio Grande do Sul States - 2003-2013

(continua/to be continued)

\begin{tabular}{|c|c|}
\hline $\begin{array}{l}\text { Unidades da Federação e } \\
\text { e corpos de água selecionados/ } \\
\text { Federation Units and selected water }\end{array}$ & $\begin{array}{c}\text { Média anual da Demanda Bioquímica } \\
\text { de Oxigênio - DBO }(\mathrm{mg} / \mathrm{l}) / \\
\text { Annual Average of Biochemical Oxygen Demand - BOD }(\mathrm{mg} / \mathrm{l})\end{array}$ \\
\hline & 20032004200520062007200820092010201120122013 \\
\hline
\end{tabular}

\section{Pernambuco}

Bacia do Rio Capibaribe/

Capibaribe River Basin

Bacia do Rio Igarassu/

Igarassu River Basin

Bacia do Rio Ipojuca/

Ipojuca River Basin

Bahia (1)

Bacia do Rio Paraguaçu/

Paraguaçu River Basin

Bacia do Rio São Francisco/

São Francisco River Basin

Rio Jequitinhonha/

Jequitinhonha River

\section{Espírito Santo}

Bacia do Rio Doce/

Doce River Basin

Rio Jucu/

Jucu River

Rio Santa Maria da Vitória/

Santa Maria da Vitória River

\section{Minas Gerais}

Bacia do Rio das Velhas/

Das Velhas River Basin

Bacia do Rio Doce/

Doce River Basin

Rio Jequitinhonha/

Jequitinhonha River

Rio de Janeiro

Rio Paraíba do Sul/

Paraíba do Sul River

\section{São Paulo}

Represa Billings/Alto Tietê/

Billings Dam/Upper Tietê

\begin{tabular}{rrrrrrrrrrr|}
5,1 & 3,1 & 4,3 & 4,2 & 4,8 & 4,2 & 5,1 & 4,3 & 5,5 & 8,4 & 10,7 \\
\hline 26,6 & 8,3 & 5,7 & 3,7 & 2,4 & 2,8 & 1,6 & 5,6 & 6,9 & 5,3 & 7,1 \\
\hline 15,8 & 3,9 & 3,7 & 4,1 & 5,1 & 4,4 & 7,7 & 4,5 & 13,2 & 10,8 & 16,1
\end{tabular}

\begin{tabular}{llllll|llllllll} 
& 8,2 & 12,9 & 3,7 & $\ldots$ & $\ldots$ & 8,6 & 10,0 & 3,4 & 5,8 & 6,4 & 5,0 \\
& & & & & & & & & & \\
$\ldots$ & $\ldots$ & $\ldots$ & $\ldots$ & $\ldots$ & 3,5 & 1,9 & 1,0 & 2,1 & 1,9 & 2,1 \\
& & & & & & & & & & \\
$\ldots$ & $\ldots$ & $\ldots$ & $\ldots$ & $\ldots$ & 2,8 & 1,5 & 1,2 & 2 & 2 & 2,2
\end{tabular}

\begin{tabular}{llll|lllllll}
$\ldots$ & $\ldots$ & $\ldots$ & $\ldots$ & 1,2 & 2,5 & 2,8 & 4,8 & 2,0 & 3,1 & $\ldots$ \\
$\ldots$ & $\ldots$ & $\ldots$ & $\ldots$ & 3,2 & 1,7 & 1,9 & 3,9 & 1,6 & 2,0 & $\ldots$ \\
& & & & & & & & & & \\
$\ldots$ & $\ldots$ & $\ldots$ & $\ldots$ & 1,2 & 2,4 & 1,8 & 6,7 & 1,9 & 2,9 & $\ldots$
\end{tabular}

$\begin{array}{lllllllllll}7,5 & 5,5 & 8,0 & 5,0 & 6,7 & 5,7 & 3,9 & 2,8 & 4,2 & 4,9 & \ldots\end{array}$

$\begin{array}{lllllllllll}1,6 & 1,6 & 1,5 & 1,6 & 1,3 & 1,5 & 1,3 & 1,1 & 2,1 & 2,0 & \ldots\end{array}$

$\begin{array}{lllllllllll}2,1 & 2,6 & 2,1 & 2,0 & 2,1 & 2,0 & 2,5 & 1,4 & 2,7 & 2,5 & \ldots\end{array}$

$\begin{array}{lllllllll}2,3 & 2,2 & 2,0 & 2,1 & 2,0 & 2,3 & 2,1 & 2,2 & 2,4\end{array}$

$\begin{array}{lllllllllll}7,0 & 5,5 & 5,1 & 5,0 & 5,4 & 5,6 & 5,8 & 7,4 & 6,9 & 7,4 & 10,9\end{array}$ 
Tabela 22.2 - Média anual da Demanda Bioquímica de Oxigênio - DBO, em corpos de água selecionados, nas Unidades da Federação de Pernambuco, Bahia, Espírito Santo, Minas Gerais, Rio de Janeiro, São Paulo, Paraná e Rio Grande do Sul - 2003-2013

Tabela 22.2 - Annual Average of Biochemical Oxygen Demand - BOD, in selected water bodies of Pernambuco, Bahia, Espírito Santo, Minas Gerais, Rio de Janeiro, São Paulo, Paraná and Rio Grande do Sul States - 2003-2013

(conclusão/concluded)

\begin{tabular}{|c|c|c|c|c|c|c|c|c|c|c|c|}
\hline \multirow{2}{*}{$\begin{array}{c}\text { Unidades da Federação e } \\
\text { e corpos de água selecionados/ } \\
\text { Federation Units and selected water } \\
\text { bodies }\end{array}$} & \multicolumn{11}{|c|}{$\begin{array}{c}\text { Média anual da Demanda Bioquímica } \\
\text { de Oxigênio - DBO }(\mathrm{mg} / \mathrm{l}) / \\
\text { Annual Average of Biochemical Oxygen Demand - BOD }(\mathrm{mg} / \mathrm{l})\end{array}$} \\
\hline & 2003 & 2004 & 2005 & 2006 & 2007 & 2008 & 2009 & 2010 & 2011 & 2012 & 22013 \\
\hline \multicolumn{12}{|l|}{ São Paulo } \\
\hline $\begin{array}{l}\text { Represa Guarapiranga/Alto Tietê/ } \\
\text { Guarapiranga Dam/Alto Tietê }\end{array}$ & 4,2 & 4,2 & 3,7 & 3,0 & 3,4 & 3,8 & 4,1 & 4,5 & 5,4 & 4,2 & 6,8 \\
\hline $\begin{array}{l}\text { Zona Metropolitan/Alto Tietê/ } \\
\text { Metropolitan Area/Upper Tietê }\end{array}$ & 40,8 & 36,1 & 33,2 & 37,0 & 35,9 & 40,9 & 35,7 & 35,8 & 27,7 & 34,3 & 39,2 \\
\hline \multicolumn{12}{|l|}{ Paraná } \\
\hline $\begin{array}{l}\text { Rio Iguaçu/Zona Metropolitana (2) } \\
\text { Iguaçu River/Metropolitan Area (2) }\end{array}$ & 13,8 & 8,6 & 9,5 & 9,0 & 20,4 & 13,0 & 11,3 & 10,2 & 6,3 & 15,4 & ... \\
\hline $\begin{array}{l}\text { Bacia do Rio Tibagi/ } \\
\text { Tibagi River Basin }\end{array}$ & 3,2 & 4,9 & 3,7 & 2,2 & 2,1 & 2,4 & 2,4 & 2,0 & 2,2 & 2,1 & ... \\
\hline \multicolumn{12}{|l|}{ Rio Grande do Sul } \\
\hline Rio Caí/ & 1,1 & 1,3 & 1,3 & 1,4 & 1,5 & 1,4 & 1,2 & 1,2 & 2,0 & 2,1 & ... \\
\hline \multicolumn{12}{|l|}{ Caí River } \\
\hline Rio Gravataí/ & 2,2 & 3,2 & 3,8 & 4,0 & 2,9 & 2,9 & 3,6 & 3,0 & 2,4 & 2,6 & ... \\
\hline \multicolumn{12}{|l|}{ Gravataí River } \\
\hline Rio dos Sinos/ & 4,1 & 2,8 & 3,2 & 9,0 & 2,6 & 2,7 & 3,8 & 3,0 & 2,8 & 4,0 & ... \\
\hline Dos Sinos River & & & & & & & & & & & \\
\hline
\end{tabular}

Fontes/Sources: 1. Instituto do Meio Ambiente e Recursos Hídricos - INEMA (BA). 2. Instituto Estadual de Meio Ambiente e Recursos Hídricos - IEMA (ES). 3. Instituto Mineiro de Gestão das Águas - IGAM (MG). 4. Instituto das Águas do Paraná. 5. Agência Estadual de Meio Ambiente e Recursos Hídricos CPRH (PE). 6. Instituto Estadual do Ambiente - INEA (RJ). 7. Fundação Estadual de Proteção Ambiental Henrique Luiz Roessler - FEPAM (RS). 8. Companhia Ambiental do Estado de São Paulo - CETESB.

Nota: Limite CONAMA da DBO para águas destinadas ao abastecimento público, após tratamento convencional: $5 \mathrm{mg} / \mathrm{l} . /$ Note: CONAMA limit of BOD for public watter supply after conventional treatment: $5 \mathrm{mg} / \mathrm{l}$.

(1) O INEMA iniciou o monitoramento a partir do mês de julho de 2011./(1) INEMA started monitoring from July 2011. (2) Refere-se ao subsistema 2 da Região Metropolitana, segundo o Instituto das Águas do Paraná./ (2) Refers to subsystem 2 of the Metropolitan Area, according to the Water Institute of Paraná.

DBO menor ou igual a $5 \mathrm{mg} / \mathrm{l} /$ $B O D$ less than or equal to $5 \mathrm{mg} / \mathrm{l}$
DBO maior que $5 \mathrm{mg} / \mathrm{l} /$

$B O D$ greater than or equal to $5 \mathrm{mg} / \mathrm{l}$ 
Tabela 22.3 - Número e Área das Unidades de Conservação Federais - 2014 Table 22.3 - Number and areas of Federal Conservation Units - 2014

\begin{tabular}{|c|c|c|}
\hline $\begin{array}{l}\text { Tipo de Uso e Categorias de Manejo/ } \\
\text { Type of Use and Management Categories }\end{array}$ & $\begin{array}{l}\text { Número/ } \\
\text { Number }\end{array}$ & $\begin{array}{l}\text { Área }\left(\mathrm{Km}^{2}\right) / \\
\text { Area }\left(\mathrm{Km}^{2}\right)\end{array}$ \\
\hline \multicolumn{3}{|l|}{ TOTAL / } \\
\hline Total & 954 & (1) 763844 \\
\hline \multicolumn{3}{|l|}{ Proteção Integral/ } \\
\hline Integral Protection & 143 & 369164 \\
\hline \multicolumn{3}{|l|}{ Estação Ecológica/ } \\
\hline Ecological Station & 32 & 74691 \\
\hline \multicolumn{3}{|l|}{ Monumento Natural/ } \\
\hline Natural monument & 3 & 443 \\
\hline \multicolumn{3}{|l|}{ Parque Nacional/ } \\
\hline National Park & 71 & 252978 \\
\hline \multicolumn{3}{|l|}{ Refúgio de Vida Silvestre/ } \\
\hline Wildlife Refuge & 7 & 2017 \\
\hline \multicolumn{3}{|l|}{ Reserva Biológica/ } \\
\hline Biological Reserve & 30 & 39034 \\
\hline \multicolumn{3}{|l|}{ Uso Sustentável/ } \\
\hline Sustainable Use & 811 & 394681 \\
\hline \multicolumn{3}{|l|}{ Floresta Nacional/ } \\
\hline National Forest & 65 & 163913 \\
\hline \multicolumn{3}{|l|}{ Reserva Extrativista/ } \\
\hline Extractive Reserve & 62 & 124362 \\
\hline \multicolumn{3}{|l|}{ Reserva de Desenvolvimento Sustentável/ } \\
\hline Sustainable Development Reserve & 2 & 1026 \\
\hline \multicolumn{3}{|l|}{ Reserva de Fauna/ } \\
\hline Fauna Reserve & - & - \\
\hline \multicolumn{3}{|l|}{ Área de Proteção Ambiental/ } \\
\hline Environmental Protection Area & 32 & 100101 \\
\hline \multicolumn{3}{|l|}{ Área de Relevante Interesse Ecológico/ } \\
\hline Area ofrelevant ecological interest & 16 & 447 \\
\hline \multicolumn{3}{|l|}{ Reservas Particulares do Patrimônio Natural - RPPN/ } \\
\hline Private Reserves of Natural Heritage - PRNH & 634 & 4831 \\
\hline
\end{tabular}

Fonte/Source: Ministério do Meio Ambiente. Cadastro Nacional de Unidades de Conservação - CNUC. Nota: Dados atualizados até outubro de 2014./Note: Updated data until October 2014.

(1) Considerando sobreposição mapeada a área passa a ser de $758.734 \mathrm{Km}^{2} /(1)$ Considering overlayed mapping, the area is $758734 \mathrm{~km}^{2}$. 


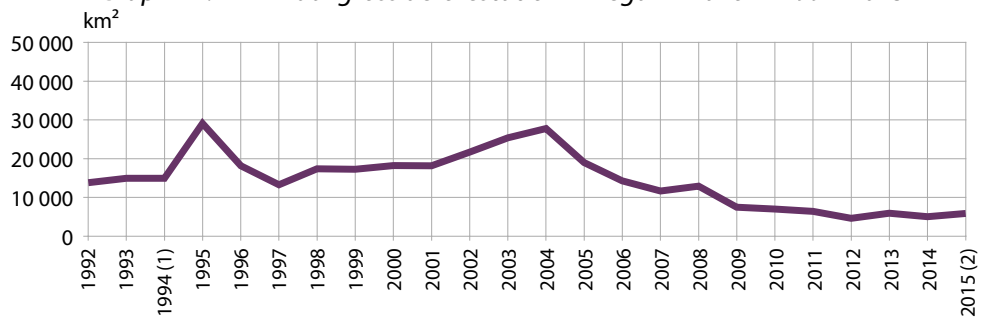

Fonte/Source: Projeto Prodes: monitoramento da floresta amazônica brasileira por satélite. São José dos Campos: Instituto Nacional de Pesquisas Espaciais - INPE, [2015]. Disponível em/Available from: <http:// www.inpe.br/\#>. Acesso em: jan. 2016/Cited: Jan. 2016.

Nota: Dados de 1ㅇ de agosto.(1) Dados referentes ao período entre agosto de 1992 e agosto de 1994 (taxa para 2 anos). (2) Dados estimados para o ano de 2015./Note: Data from August 10.(1) Data for the period between August 1992 and August 1994 (rate for 2 years). (2) Estimated data for 2015.

\section{Gráfico 22.2 - Focos de calor no Brasil, na Amazônia Legal e em Unidades de Conservação e Terras Indígenas - 2008-2015}

Graph 22.2 - Hotspots in Brazil, Legal Amazon and in Conservation Units, Indigenous Lands - 2008-2015

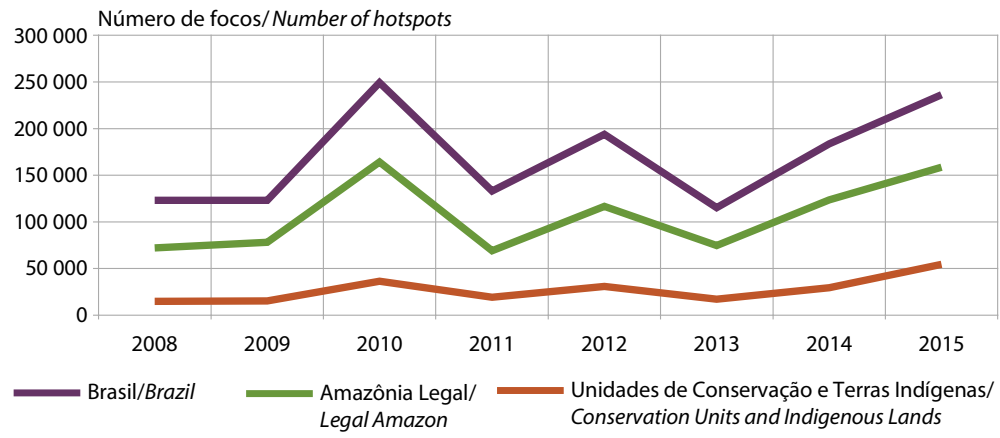

Fontes/Sources: 1. Monitoramento de queimadas e incêndios por satélite em tempo quase-real. São José dos Campos: Instituto Nacional de Pesquisas Espaciais - INPE, 2016. Disponível em/Available from: <http:// www.inpe.br/queimadas/estatisticas.php>. Acesso em: jan. 2016/Cited: Jan. 2016. 2. Queimadas: monitoramento de focos. Cachoeira Paulista: Instituto Nacional de Pesquisas Espaciais - INPE, Centro de Previsão de Tempo e Estudos Climáticos - CPTEC, 2016. Disponivel em/Available from: <http://www.inpe.br/queimadas/ estatisticas.php>. Acesso em: jan. 2016/Cited: Jan. 2016.

Notas: 1 . Os dados de focos de calor foram obtidos pelo satétite AQUA M-T.

2. Para o Brasil, o total corresponde à soma dos focos apresentados por cada bioma. cador.

3. Os focos apresentados como sem informação ou indeterminado não foram computados no indi-

4. Foram consideradas as Unidades de Conservação federais e estaduais./

Notes: 1. Data about hotspots were obtained by AQUA M-T.

2. For Brazil, the total is the sum of hotspots for each biome.

3. Hotspots presented as not informed or indeterminate were not included in the indicator.

4. Considering federal and state Conservation Units. 


\section{Gráfico 22.3 - Comercialização de agrotóxicos e afins, por área plantada - Brasil - 2001/2013 \\ Graph 22.3 - Commercialization of pesticides and the like, by planted area - Brazil - 2001/2013}

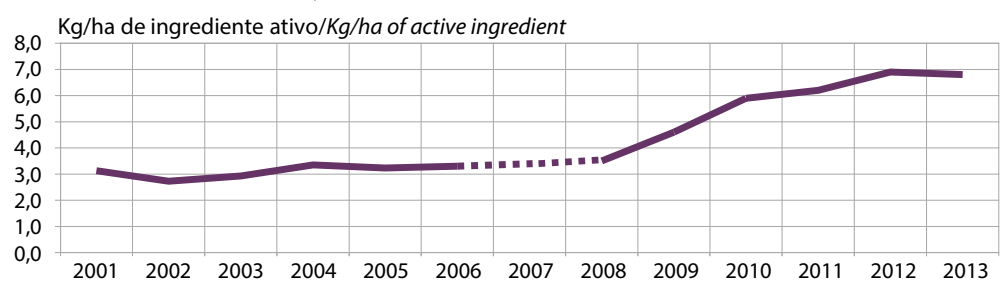

Fontes/Sources: 1. Relatórios de comercialização de agrotóxicos: boletim anual de produção, importação, exportação e vendas de agrotóxicos no Brasil 2000-2013. Brasília, DF: Instituto Brasileiro do Meio Ambiente e dos Recursos Naturais Renováveis - IBAMA, [2016]. Disponível em/Available from: <http://www.ibama.

gov.br/areas-tematicas-qa/relatorios-de-comercializacao-de-agrotoxicos/pagina-3>. Acesso em: abr. 2016/ Cited: Apr. 2016. 2. Levantamento sistemático da produção agrícola: pesquisa mensal de previsão e acompanhamento das safras agrícolas no ano civil 2000-2005. Rio de Janeiro: IBGE, v. 12-17, 2000-2005. Disponível em/Available from:<http://biblioteca.ibge.gov.br/index.php/biblioteca-catalogo?view=detalhes\&id=76>. Acesso em: abr. 2016/Cited: Apr. 2016. 3. Produção agrícola municipal 2009-2013. In: IBGE. Sidra: sistema IBGE de recuperação automática. Rio de Janeiro, 2014. Disponível em/Available from: <http://www.sidra. ibge.gov.br/bda/pesquisas/pam/default.asp>. Acesso em: fev. 2015/Cited:Feb.2015. 4. Relatórios de comercialização de agrotóxicos: boletim anual de produção, importação, exportação e vendas de agrotóxicos no Brasil. 2009 - 2012. Brasília, DF: Instituto Brasileiro do Meio Ambiente e dos Recursos Naturais Renováveis IBAMA, [2016]. Disponível em/Available from: <http://www.ibama.gov.br/areas-tematicas-qa/relatoriosde-comercializacao-de-agrotoxicos/pagina-3>. Acesso em: abr. 2016/Cited:Feb. 2016.

Nota: Sem informação para os anos de 2006, 2007 e 2008./Note: Data not avaiable for years 2006, 2007 e 2008. 


\section{Gráfico 22.4 - Proporção de material reciclado em atividades industriais \\ selecionadas - 1994/2014}

Graph 22.4 - Proportion of recycled material in selected industrial activities

$1994 / 2014$

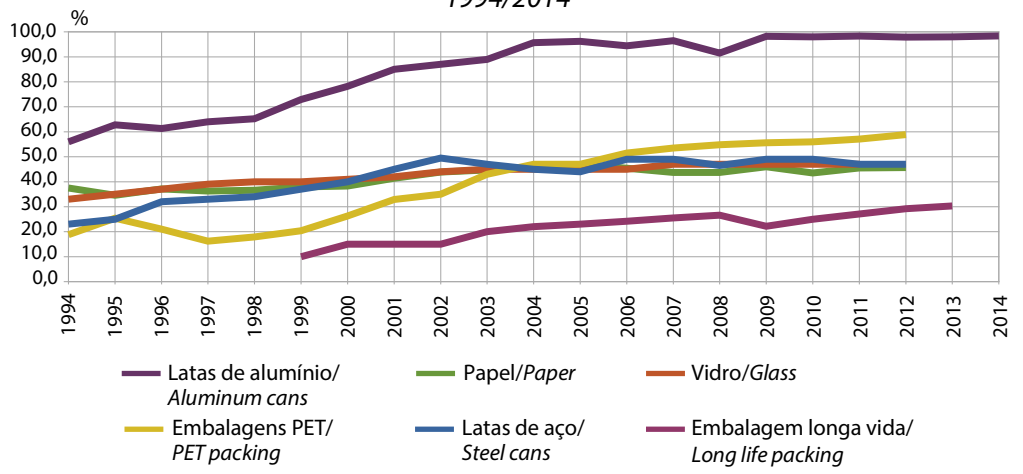

Fontes/Sources: 1. Associação Brasileira do Alumínio - ABAL. 2. Associação Brasileira de Papel e Celulose Bracelpa. 3. Associação Técnica Brasileira das Indústrias Automáticas de Vidro - ABividro. 4. Associação Brasileira da Indústria do PET - ABıPEt. 5. Associação Brasileira de Embalagem de Aço - ABEAço. 6. Associação Brasileira da Indústria de Leite Longa Vida - ABLV. 7. Compromisso Empresarial para Reciclagem - CEMPRE. Nota: Não há informação sobre reciclagem de latas de alumínio no ano de 2013. O último ano da informação para reciclagem de vidro é 2011; para reciclagem de papel, embalagens PET e latas de aço é 2012; para reciclagem de embalagem longa vida é 2013 e para reciclagem de latas de alumínio é 2014. Note: No information available on the recycling of aluminum cans in year 2013. The latest figures on glass recycling date back to 2011; on paper, PET and steel can recycling, to 2012; on long-life packaging recycling, to 2013 and on aluminum can recycling, to 2014. 


\section{Referências References}

ANUÁRIO DA INDÚSTRIA AUTOMOBILÍSTICA BRASILEIRA 2016. São Paulo: Associação Nacional dos Fabricantes de Veículos Automotores - Anfavea, 2016. Disponível em: <http://http://www.anfavea.com.br>. Acesso em: jan. 2016.

ANUÁRIO ESTATÍSTICO AQUAVIÁRIO 2013. Brasília, DF: Agência Nacional de Transportes Aquaviários - Antaq, [2016]. Disponível em: <http://www.antaq.gov.br/portal/anuarios/ anuario2012/index.htm>. Acesso em: abr. 2016.

ANUÁRIO ESTATÍSTICO AQUAVIÁRIO 2014. Brasília, DF: Agência Nacional de Transportes Aquaviários - Antaq, [2016]. Disponível em: <http://www.antaq.gov.br/Portal/ Estatisticas_Anuarios.asp>. Acesso em: abr. 2016.

ANUÁRIO ESTATÍSTICO DE TURISMO 2015. Ano base 2014. Brasília, DF: Ministério do Turismo, v. 42, 2015. Disponível em: <http://www.dadosefatos.turismo.gov.br/ dadosefatos/home.html>. Acesso em: abr. 2016.

ANUÁRIO ESTATÍSTICO DO BRASIL 2015. Rio de Janeiro: IBGE, v. 75, 2016. Disponível em: $<$ http://biblioteca.ibge.gov.br/index.php/biblioteca-catalogo?view=detalhes\&id=720>. Acesso em: abr. 2016.

BALANÇO ENERGÉTICO NACIONAL 2015: ano base 2014. Brasília, DF: Ministério de Minas e Energia, Empresa de Pesquisa Energética - EPE, 2015. Disponível em: <https://ben. epe.gov.br/default.aspx>. Acesso em: abr. 2016.

BOLETIM ESTATÍSTICO DA CONFEDERAÇÃO NACIONAL DOS TRANSPORTES. Brasília, DF, nov. 2015. Disponível em: <http://www.cnt.org.br/boletim/boletim-estatistico-cnt>. Acesso em: abr. 2016.

BOLETIM ESTATÍSTICO DE PESSOAL E INFORMAÇÕES ORGANIZACIONAIS. Brasília, DF: Ministério do Planejamento, Orçamento e Gestão, v. 21, n. 37, jan. 2016. Disponível em: <http://www.servidor.gov.br/arquivos-publicacoes>. Acesso em: jan. 2016.

BRASIL. Secretaria de Comércio Exterior. AliceWeb: sistema de análise das informações de comércio exterior. Brasília, DF: Secex, 2015. Disponível em: <http://aliceweb.mdic. gov.br/>. Acesso em: jan. 2016. 
BRASIL: alunos matriculados e titulados nos cursos de mestrado e doutorado, ao final do ano, 1998-2014. Brasília, DF: Ministério da Ciência, Tecnologia e Inovação, 2016. Disponível em: <http://www.mct.gov.br/index.php/content/view/2072/Indicadores_ sobre_o_ensino_de_pos_graduacao.html . Acesso em: jan. 2016.

BRASIL: dispêndios dos governos estaduais em ciência e tecnologia (C\&T) por região e unidade da federação, 2000-2013. Brasília, DF: Ministério da Ciência, Tecnologia e Inovação, 2015. Disponível em: <http://www.mct.gov.br/index.php/content/view/8842. html>. Acesso em: abr. 2016.

BRASIL: instituições, grupos, pesquisadores e pesquisadores doutores, cadastrados no diretório dos grupos de pesquisa do CNPq, 1993/2014. Brasília, DF: Ministério da Ciência, Tecnologia e Inovação, 2016. Disponível em: <http://www.mct.gov.br/index.php/ content/view/2073/Indicadores_dos_grupos_de_pesquisa.html >. Acesso em:abr. 2016.

DISPÊNDIO nacional em pesquisa e desenvolvimento (P\&D) em valores correntes, em relação ao total de P\&D e ao produto interno bruto (PIB), por setor institucional, 20002013. Brasília, DF: Ministério da Ciência, Tecnologia e Inovação, 2015. Disponível em: <http://www.mct.gov.br/index.php/content/view/29144.html>. Acesso em:abr. 2016.

ESTATÍSTICAS eleitorais 2014. Estatísticas de eleitorado. Comparecimento e votação. In: ELEIÇÕES. Brasília, DF: Tribunal Superior Eleitoral - TSE, 2014. Disponível em: <http:// www.tse.jus.br/hotSites/estatistica_2014/resultados/comparecimento.html>. Acesso em: abr. 2016.

ESTATÍSTICAS eleitorais 2014. Estatísticas de eleitorado. Quantitativo. In: ELEIÇÕES. Brasília, DF: Tribunal Superior Eleitoral - TSE, 2014. Disponível em: <http://www.tse.jus. br/eleicoes/estatisticas/estatisticas-eleitorais-2014-eleitorado>. Acesso em: abr. 2016.

ESTUDO da demanda turística internacional 2010-2014. Brasília, DF: Ministério do Turismo, 2015. Disponível em: <http://www.dadosefatos.turismo.gov.br/dadosefatos/ demanda_turistica/internacional/>. Acesso em: abr. 2016.

EVOLUÇÃO do transporte ferroviário de cargas. Brasília, DF: Agência Nacional de Transportes Terrestres - ANTT, 2015. Disponível em: <http://www.antt.gov.br/index.php/ content/view/15884/evolucao_do_transporte_ferroviario.html>. Acesso em: abr. 2016.

INDICADORES IBGE: contas nacionais trimestrais: indicadores de volume e valores correntes out./dez. 2014. Rio de Janeiro: IBGE, [2016]. Disponível em: <ftp://ftp.ibge. gov.br/Contas_Nacionais/>. Acesso em: abr. 2016.

ÍNDICE nacional de preços ao consumidor amplo - IPCA 2002-2015. In: IBGE. Sidra: sistema IBGE de recuperação automática. Rio de Janeiro, [2015]. Disponível: <http:// www.sidra.ibge.gov.br/bda/>. Acesso em: abr. 2016.

ÍNDICE nacional de preços ao consumidor amplo - IPCA 2015. In: IBGE. Sidra: sistema IBGE de recuperação automática. Rio de Janeiro, [2015]. Disponível em: $<$ http://www. sidra.ibge.gov.br/bda/>. Acesso em: abr. 2016. 
INFORMAÇÕES de saúde. Assistência à saúde. Imunizações. In: BRASIL. Ministério da Saúde. Datasus. Brasília, DF, [2015]. Disponível em: <http://www2.datasus.gov.br/ Datasus/index.php>. Acesso em: fev. 2016.

. Epidemiológicas e morbidade. In: BRASIL. Ministério da Saúde. Datasus. Brasília, DF, [2015]. Disponível em: <http://www2.datasusgov.br/datasus/index.php $>$. Acesso em: fev. 2016.

. Internações hospitalares do [Sistema Único de Saúde]-SUS. In: BRASIL. Ministério da Saúde. Ministério da Saúde. Datasus. Brasília, DF, [2015]. Disponível em: <http:// www2.datasus.gov.br/datasus/index.php>. Acesso em: fev. 2016.

. Mortalidade: dados preliminares. In: BRASIL. Ministério da Saúde. Datasus. Brasília, DF, [2015]. Disponível em: <http://www2.datasus.gov.br/datasus/index.php>. Acesso em: fev. 2016.

JUSTIÇA EM NÚMEROS 2011-2015. Brasília, DF: Conselho Nacional de Justiça - CNJ, 20112015. Disponível em: <http://www.cnj.jus.br/>. Disponível em: abr. 2016.

LEVANTAMENTO sistemático da produção agrícola: pesquisa mensal de previsão e acompanhamento das safras agrícolas no ano civil 2000-2005. Rio de Janeiro: IBGE, v. 12-17, 2000-2005. Disponível em:<http://biblioteca.ibge.gov.br/index.php/bibliotecacatalogo?view=detalhes\&id=76>. Acesso em: abr. 2016.

MONITORAMENTO de queimadas e incêndios por satélite em tempo quase-real. São José dos Campos: Instituto Nacional de Pesquisas Espaciais - INPE, 2016. Disponível em: <http://www.inpe.br/queimadas/estatisticas.php>. Acesso em: jan. 2016.

PESQUISA ANUAL DO COMÉRCIO 2013. Rio de Janeiro: IBGE, v. 25, 2013. Disponível em: $<$ http://biblioteca.ibge.gov.br/index.php/biblioteca-catalogo?view=detalhes\&id=755>. Acesso em: jan. 2016.

PESQUISA da pecuária municipal 2013-2014. In: IBGE. Sidra: sistema IBGE de recuperação automática. tab. 74, 3939. Rio de Janeiro, [2015]. Disponível em: <http://www.sidra.ibge. gov.br/>. Acesso em: abr. 2016.

PESQUISA da pecuária municipal 2014. In: IBGE. Sidra: sistema IBGE de recuperação automática. tab. 3940. Rio de Janeiro, [2015]. Disponível em: <http://www.sidra.ibge. gov.br/>. Acesso em: abr. 2016.

PESQUISA DE ESTOQUES 2005-2015. Rio de Janeiro: IBGE, n. 1, pt. 1, jan./jun. 2005-2015. Disponível em: <ftp://ftp.ibge.gov.br/Estoque>. Acesso em: jan. 2016.

PESQUISA INDUSTRIAL ANUAL. Empresa 2013. Rio de janeiro: IBGE, v. 32, n. 1, 2013. Disponível em: <http://www.ibge.gov.br/home/estatistica/economia/industria/pia/ empresas/2013/defaultempresa.shtm >. Acesso em: abr. 2016.

PESQUISA mensal de comércio 2007-2014. In: IBGE. Sidra: sistema IBGE de recuperação automática. Rio de Janeiro, [2016]. Disponível em: <http://www.sidra.ibge.gov.br/bda/>. Acesso em: abr. 2016. 
PESQUISA trimestral do abate de animais 2009-2014. In: IBGE. Sidra: sistema IBGE de recuperação automática. tab. 1092, 1093, 1094. Rio de Janeiro, [2009-2015]. Disponível em: <http://www.sidra.ibge.gov.br/>. Acesso em: jan. 2016.

POTENCIAL hidrelétrico brasileiro por bacia hidrográfica. Rio de Janeiro: Centrais Elétricas Brasileiras - Eletrobras, [2015]. Disponível em: <https://www.eletrobras.com/elb/data/ Pages/LUMIS21D128D3PTBRIE.htm>. Acesso em: abr. 2016.

PRODUÇÃO agrícola municipal 2004-2014. In: IBGE. Sidra: sistema IBGE de recuperação automática. Rio de Janeiro, [2015]. tab. 1002, 1612. Disponível em:<http://www.sidra. ibge.gov.br/bda/>. Acesso em: jan. 2016.

PRODUÇÃO agrícola municipal 2009-2013. In: IBGE. Sidra: sistema IBGE de recuperação automática. Rio de Janeiro, 2014. Disponível em: <http://www.sidra.ibge.gov.br/bda/ pesquisas/pam/default.asp >. Acesso em: fev. 2015.

PRODUÇÃO agrícola municipal 2014. In: IBGE. Sidra: sistema IBGE de recuperação automática. tab. 99, 106, 839, 1000, 1001, 1002, 1612, 1613. Rio de Janeiro, [2015]. Disponível em:<http://www.sidra.ibge.gov.br/bda/>. Acesso em: jan. 2016.

PRODUÇÃO da extração vegetal e da silvicultura 2013-2014. In: IBGE. Sidra: sistema IBGE de recuperação automática. tab. 291, 292. Rio de Janeiro, [2015]. Disponível em: <http://www.sidra.ibge.gov.br/>. Acesso em: abr. 2016.

PRODUÇÃO da extração vegetal e da silvicultura 2014. In: IBGE. Sidra: sistema IBGE de recuperação automática. Rio de Janeiro, [2015]. tab. 5930. Disponível em: <http://www. sidra.ibge.gov.br/bda/>. Acesso em: jan. 2016.

PROJETO Prodes: monitoramento da floresta amazônica brasileira por satélite. São José dos Campos: Instituto Nacional de Pesquisas Espaciais - INPE, [2015]. Disponível em: <http://www.inpe.br/\#>. Acesso em: jan. 2016.

QUEIMADAS: monitoramento de focos. Cachoeira Paulista: Instituto Nacional de Pesquisas Espaciais - INPE, Centro de Previsão de Tempo e Estudos Climáticos - CPTEC, 2016. Disponivel em: <http://www.inpe.br/queimadas/estatisticas.php>. Acesso em: jan. 2016.

RECEITA e despesa cambial turística. Brasília, DF: Ministério do Turismo, [2015]. Disponível em: <http://www.dadosefatos.turismo.gov.br/dadosefatos/estatisticas_ indicadores/receita_cambial/>. Acesso em: abr. 2016.

RELATÓRIO resumido de execução orçamentária 2014 e 2015. Brasília, DF: Tesouro Nacional, [2015]. Disponível em: <https://www.tesouro.fazenda.gov.br/-/relatorioresumido-de-execucao-orcamentaria>. Acesso em: abr. 2016.

REPOSITÓRIO de dados eleitorais. In: ELEIÇÕES. Brasília, DF: Tribunal Superior Eleitoral - TSE, 2014. Disponível em: <http://agencia.tse.jus.br/estatistica/sead/odsele/consulta_ cand/consulta_cand_2014.zip >. Acesso em: abr. 2016. 
RESENHA energética brasileira: exercício de 2014. Brasília, DF: Ministério de Minas e Energia, Empresa de Pesquisa Energética - EPE, 2015. Disponível em: $<$ https://ben.epe. gov.br/default.aspx>. Acesso em: abr. 2016.

SCIMAGO JOURNAL \& COUNTRY RANK. New York: Elsevier, [2016]. Disponível em: $<$ http://www.scimagojr.com/>. Acesso em: abr. 2016.

SÉRIES históricas. Despesa da União por grupo de natureza 2009-2014. Brasília, DF: Ministério da Fazenda, Sistema Integrado de Administração Financeira do Governo Federal - SIAFI, [2015]. Disponível em: <http://www.tesouro.fazenda.gov.br/-/serieshistoricas>. Acesso em: abr. 2016.

SÉRIES temporais. In: BANCO CENTRAL DO BRASIL. SGS: sistema gerenciador de séries temporais. Brasília, DF, [2015]. Disponível em: <http://www4.bcb.gov.br/pec/series/ port/aviso.asp>. Acesso em: jan. 2015.

- Tabelas especiais. Dívida líquida e necessidades de financiamento do setor público. In: BANCO CENTRAL DO BRASIL. SGS: sistema gerenciador de séries temporais. Brasília, DF, [2015]. Disponível em: <http://www4.bcb.gov.br/pec/series/port/aviso. asp>. Acesso em: jan. 2016.

. Setor externo. In: BANCO CENTRAL DO BRASIL. SGS: sistema gerenciador de séries temporais. Brasília, DF, [2015]. Disponível em: <http://www4.bcb.gov.br/pec/ series/port/aviso.asp>. Acesso em: jan. 2016.

STATISTICS by country. Paris: International Energy Agency - IEA, [2015]. Disponível em: <http://www.iea.org/>. Acesso em: abr. 2016. 



\section{Equipe \\ Staff}

\section{Editor/Editor}

Paulo Rabello de Castro

Centro de Documentação e Disseminação de Informações - CDDI David Wu Tai

\section{Coordenação Executiva/Executive Coordination}

Isabela Mateus de Araujo Torres

Desenvolvimento do Projeto/Project Development

Flávio Axel Lima Freire

Ruy Lemme Cartier

Versão para o inglês/English Version

Aline Milani Romeiro Pereira

Gisele Flores Caldas Manhães

La-Fayette Côrtes Neto

Coordenação de Produção/Production Coordination

Marise Maria Ferreira

Gerência de Editoração/Publishing Management

Katia Vaz Cavalcanti

Diagramação/Desktop Publishing

Elizabeth Santos da Fontoura

Helena Maria Mattos Pontes

Gerência de Documentação/Documentation Management

Normalização Bibliográfica/Bibliographic standards

Solange de Oliveira Santos

Vera Lucia Punzi Barcelos Capone

Preparação das Informações do IBGE/Preparation of IBGE's Information

Coordenações da Diretoria de Geociências e da Diretoria de Pesquisas

Impressão e Acabamento/Printing and finishes

Gerência de Gráfica, em 2016 
Esta publicação foi impressa pela Gráfica do Instituto Brasileiro de Geografia e Estatística - IBGE sobre papel couché matte $90 \mathrm{~g} / \mathrm{m}^{2}$ em 2016 . 
Se o assunto é Brasil, procure o IBGE. 


\section{Brasil em números 2016 - A arte de mostrar o Brasil}

0 Brasil em Números, em versão bilíngue, reúne informaçōes que permitem traçar uma síntese da realidade brasileira em seus múltiplos aspectos. É destinado a todos aqueles que desejam conhecer melhor 0 País de forma prática e ilustrada.

Sob a forma de tabelas e gráficos, os capítulos apresentam dados sobre o território nacional, características demográficas e socioeconômicas da população, preços, contas nacionais, atividades agropecuárias, industriais, comerciais, de serviços, finanças públicas, comércio exterior, ciência e tecnologia e estatísticas básicas do Governo, incluindo dados comparativos entre os estados brasileiros.

Como fonte de informaçóes, destacam-se os dados advindos de uma grande gama de pesquisas demográficas, sociais, econômicas, estruturais e conjunturais realizadas pelo IBGE. E para complementar a compreensão da realidade brasileira, são também utilizados dados produzidos pelo Banco Central do Brasil, Agências Reguladoras e Ministérios Federais e algumas de suas instituições vinculadas.

0 presente volume é ilustrado com obras do Museu Pinacoteca do Estado do Rio Grande do Norte, localizado no município de Natal, no estado do Rio Grande do Norte. A intenção é agregar aspectos culturais e regionais, por meio de imagens e gravuras que abrem cada capítulo.

Além disso, a obra é enriquecida com artigos de renomados professores, técnicos e pesquisadores, oferecendo ao leitor uma reflexão acerca de cada tema abordado. Com seu formato compacto, a publicação busca promover uma leitura prazerosa e instrutiva.

\section{Brazil in figures 2016 - The art of portraying Brazil}

Brazil in Figures, a bilingual publication, brings together information that provides an overview of the Brazilian reality in its multiple aspects. It is designed for all those who wish to better understand the Country in a practical and illustrated way.

Grouped in chapters with tables and graphs, the book presents data about the national territory, demographic and socioeconomic characteristics of the population, prices, national accounts, agriculture, industry, trade, services, public finances, foreign trade, science and technology and basic statistics of the Government, including comparative data among the Brazilian states.

The publication highlights, as its source, data coming from a wide range of demographic, social, economic, long-term and short-term surveys conducted by the IBGE. Besides, in order to provide further information on the Brazilian reality, data produced by the Central Bank of Brazil, Regulatory Agencies, Federal Ministries and some of their associated institutions are also included.

This volume of Brazil in Figures is illustrated with art pieces from the Pinacotheca of the State of Rio Grande do Norte, located in the municipality of Natal. The intention is to add cultural and regional aspects through images that open each chapter.

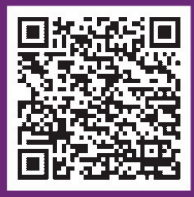

Enriched with articles written by renowned professors, technicians and researchers, the publication is intended to foster a reflection on each of the addressed subjects. With its compact format, this work aims at providing the reader with an instructive and pleasant reading.

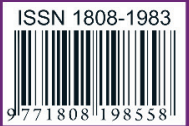

